\title{
An Electroreductive Approach to Radical Silylation via the Activation of Strong $\mathrm{Si}-\mathrm{Cl}$ Bond
}

\author{
Lingxiang Lu, Juno C. Siu, Yihuan Lai, Song Lin* \\ Department of Chemistry and Chemical Biology, Cornell University, Ithaca, NY 14853, USA. \\ Correspondence to: songlin@cornell.edu \\ Supporting Information
}




\section{Table of contents}

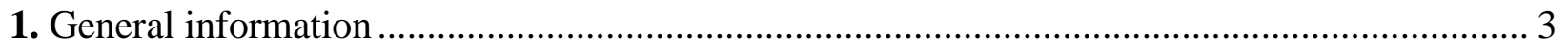

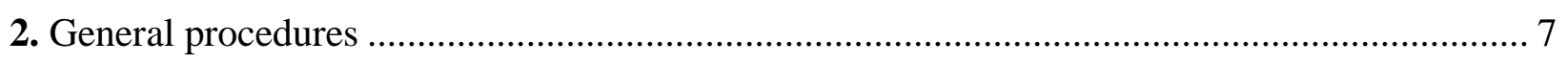

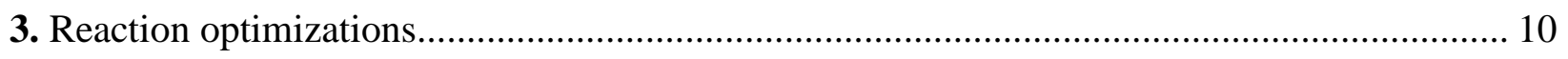

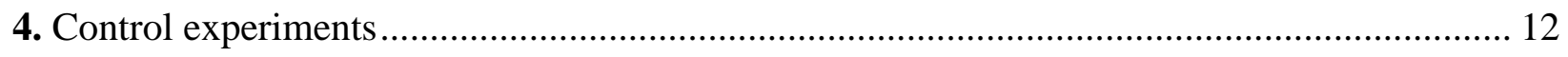

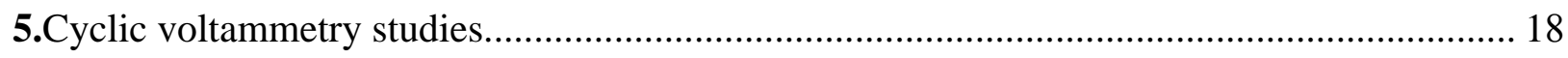

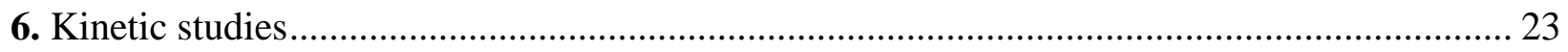

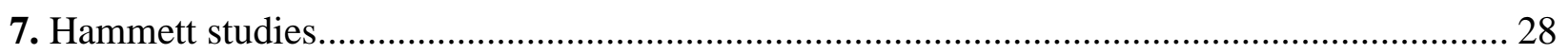

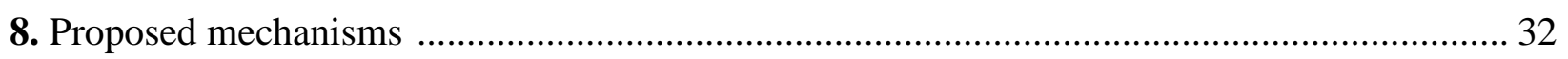

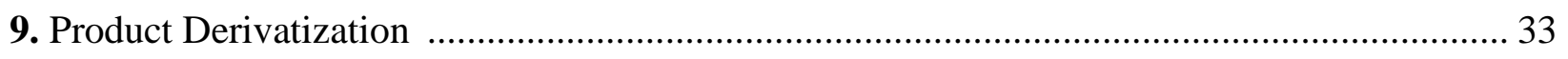

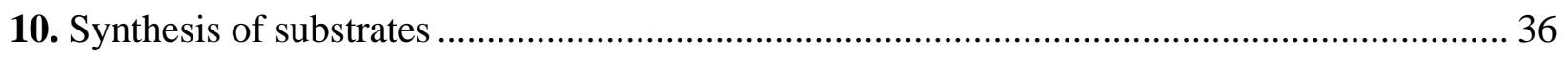

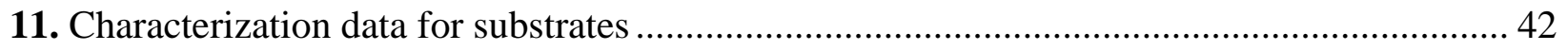

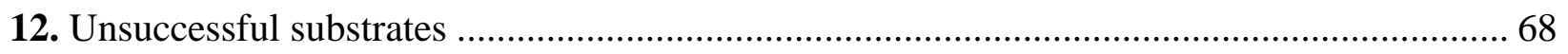

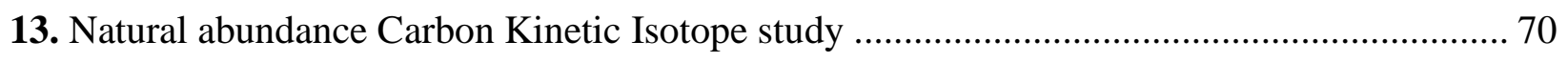

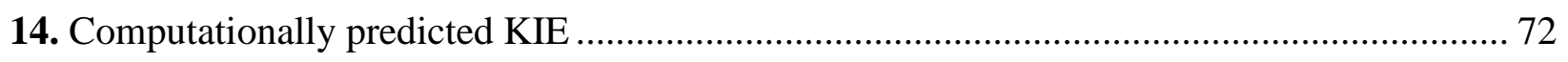

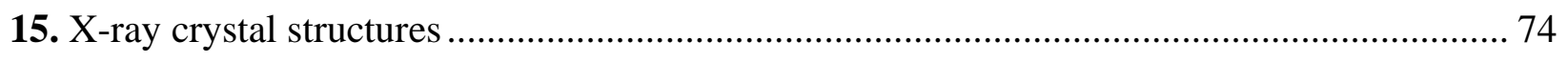

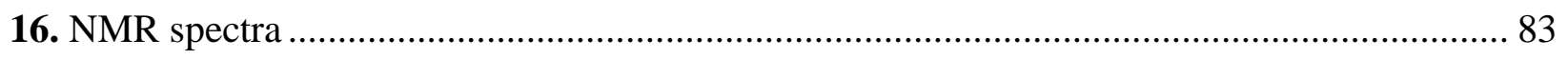

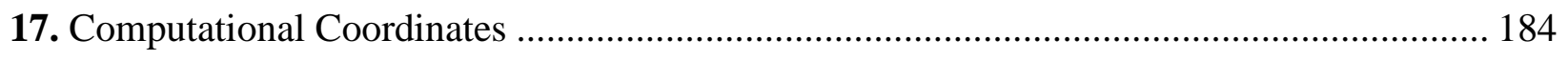

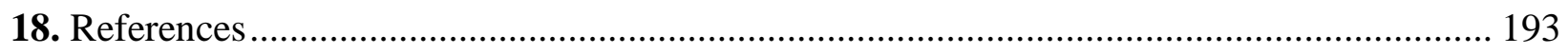




\section{General information}

All electrochemical reactions were performed in an IKA Electrasyn vial unless otherwise noted. The threads on the vial was wrapped with PTFE tape and reinforced with parafilm. All reactions were conducted under a nitrogen atmosphere unless otherwise noted. Flash chromatography was performed using silica gel P60 from SiliCycle. Commercial reagents were purchased from Sigma Aldrich, Alfa Aesar, Acros, TCI, AK Scientific, Combi-Blocks and Oakwood and used as received with the following exceptions: toluene, dichloromethane, diethyl ether, and acetonitrile were dried with molecular sieves. With the exception of THF in which all was dried with the sodium metal and benzophenone method.

\section{Nuclear magnetic resonance spectroscopy (NMR)}

All proton NMR spectra were recorded on either a Varian-mercury $300(300 \mathrm{MHz})$, VarianMercury $400(400 \mathrm{MHz})$, Inova $500(500 \mathrm{MHz})$ or Inova $600(600 \mathrm{MHz})$ spectrometers at $20^{\circ} \mathrm{C}$. Chemical shifts for proton are reported in parts per million downfield from tetramethylsilane and are reference to residual protium in the NMR solvent according to values reported in literature: $\delta\left(\mathrm{CDCl}_{3}\right)=7.26 \mathrm{ppm}, \delta\left(\mathrm{CD}_{3} \mathrm{CN}\right)=2.33 \mathrm{ppm}$. Carbon $\left({ }^{13} \mathrm{C}\left\{{ }^{1} \mathrm{H}\right\} \mathrm{NMR}\right)$ was referenced to the carbon resonances of the solvent according to values reported in literature: $\delta\left(\mathrm{CDCl}_{3}\right)=77.16$ brook.

\section{Mass spectrometry}

All mass spectra were obtained on a ThermoFisher Scientific Exactive series DART Mass Spectrometer. Input temperature were set between $100^{\circ} \mathrm{C}$ to $350^{\circ} \mathrm{C}$ depending on the molecule. Most of the ionization were done with helium as an ionization gas.

\section{Infrared (IR) spectra}

All IR spectra were obtained using a Bruker Tensor II Infrared FTIR spectrometer.

\section{Gas chromatography (GC)}

All GC analyses were performed on a Shimadzu Nexus GC-2030 gas chromatograph with an FID detector or an Agilent 6890A gas chromatograph with a MS detector.

\section{Electrolysis methods}

Electrolysis experiments were performed in a $5 \mathrm{~mL}$ or $10 \mathrm{~mL}$ vial and powered by IKA Electrasyn 2.0. Graphite electrode was supplied by IKA and Mg electrode was cut from a Mg plate $(99.95 \%$ $\mathrm{Mg}$ ). All potential is with reference to $\mathrm{Mg}^{2+/ 0}(-2.36 \mathrm{~V}$ vs SHE, that is $-2.60 \mathrm{~V}$ vs SCE). To convert the potential vs $\mathrm{Mg}^{2+/ 0}$ to $\mathrm{SCE}, 2.60 \mathrm{~V}$ is subtracted (eg. $-0.70 \mathrm{~V}$ vs $\mathrm{Mg}^{2+/ 0}=-3.30 \mathrm{~V}$ vs SCE).

\section{Abbreviations}

$\mathrm{Ac}=$ acetyl group, $\mathrm{Acac}=$ acetylacetonate, $\mathrm{Ar}=$ an aryl group, $\mathrm{Bn}=$ benzyl group,${ }^{t} \mathrm{Bu}=$ tert butyl group, $\mathrm{DMF}=$ dimethylformamide, $\mathrm{EA}=$ ethyl acetate, $\mathrm{F}=$ faraday, $\mathrm{Hex}=$ hexanes, HMPA 
= hexamethylphosphoramide, $\mathrm{LOO}=$ leave-one-out, $\mathrm{MeCN}=$ acetonitrile, $\mathrm{MeLi}=$ methyl lithium, $\mathrm{MeOH}=$ methanol, OTIPS = Triisopropylsilyl ether, SAR = structure-activity relationship, TBA $=$ tetrabutylammonium, $\mathrm{TEA}=$ triethylamine, $\mathrm{TES}=$ triethylsilyl, TFSI = trifluoromethanesulfonimide, THF = tetrahydrofuran, TMS = trimethylsilyl, $\mathrm{Ph}=$ phenyl group, ${ }^{i} \mathrm{Pr}=$ isopropyl group.

\section{Safety when handling chemicals and reaction setup}

$\mathrm{TBAClO}_{4}$ : $\mathrm{TBAClO}_{4}$ is classified as an oxidizer that should be handled with care and avoid heat or ignition. MSDS is available on https://www.sigmaaldrich.com/catalog/product/aldrich/86885. In our reaction, TBATFSI and some other electrolytes are also competent.

Reaction setup and workup: In our procedure, the reaction mixtures are prepared in a $\mathrm{N}_{2}$ glovebox before being transferred out to a bench top for electrolysis. We have tried to carry out the entire procedure outside of the glovebox by sparging the solution with $\mathrm{N}_{2}$ before electrolysis, and the reaction still proceeds with high yields (unoptimized; about 10-20\% lower than optimal yields). THF should be dried with 3 4 $\AA$ molecular sieves or over sodium/benzophenone before use. A convenient way to evaluate the dryness of the solvent is to observe whether there are any bubbles on the surface of the $\mathrm{Mg}$ electrode. If continuous bubbling is observed and $\mathrm{Mg}$ surface turns to grey/black quickly, it indicates that hydrochloric acid was formed from the hydrolysis of chlorosilanes and will result in messier reactions as well as safety hazards. In disilylation reactions, $\mathrm{Mg}^{0}$ will precipitate on the graphite cathode after $2 \mathrm{~F}$ of charge or at the stage when alkenes are almost consumed. In general, $\mathrm{Mg}$ will coat the carbon electrode uniformly as a shiny layer and is normal sign of a successful reaction and the work-up of the reaction can be handled safely using routine procedures. However, if the solvent is not dry or if $\mathrm{HCl}$ is formed during the reaction (eg. alcohol substrates which react with chlorosilane first to form $\mathrm{Si}-\mathrm{O}$ and release $\mathrm{HCl}$ ), $\mathrm{Mg}^{0}$ will plate out as black micro sized solids (see picture below), which is combustible when the electrode is exposed to air and needs to be handled with care. In this case, a good way to quench $\mathrm{Mg}^{0}$ is adding around $1 \mathrm{~mL}$ of water before working up the reaction.
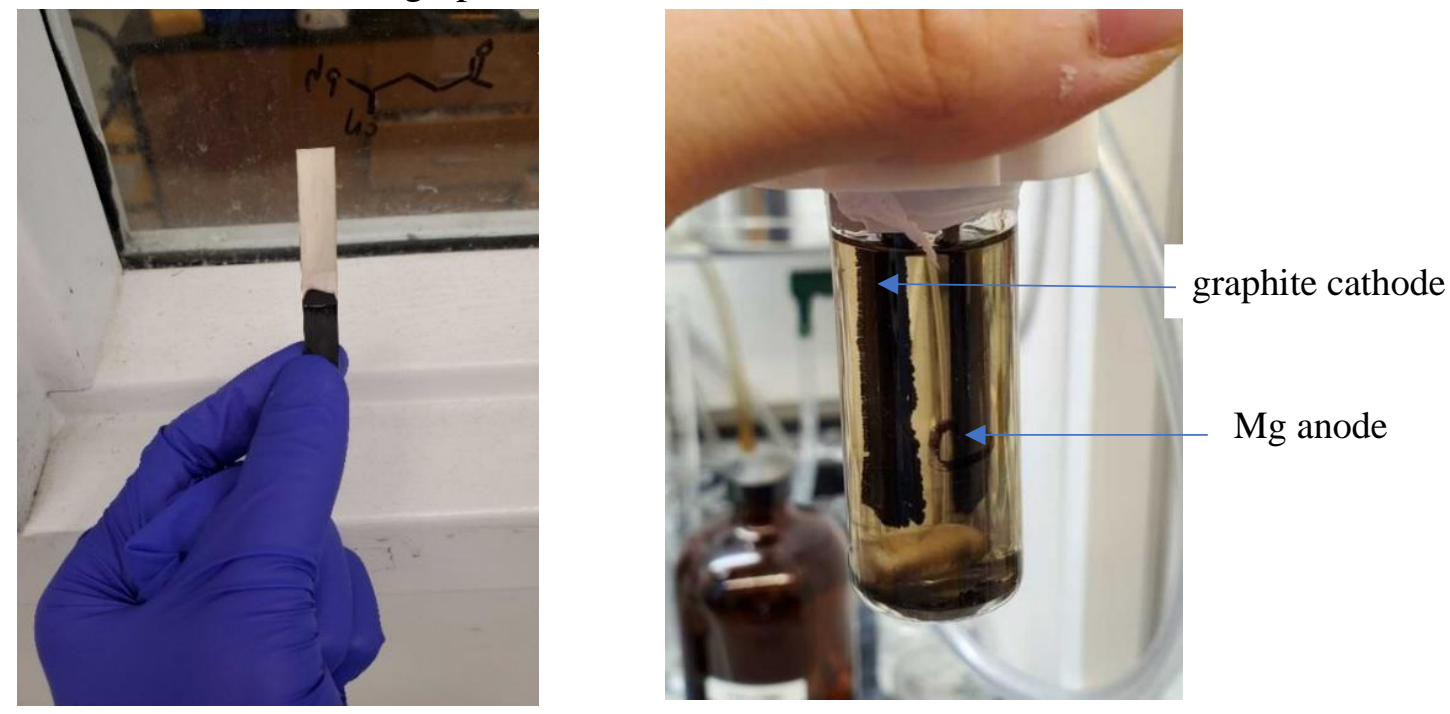

Figure S1: Graphite electrode after reaction. Left: in dry THF. Right: in wet THF or $\mathrm{HCl}$ formed during the reaction (electrode on the left is graphite). 


\section{Additional literature background}

There are currently no or limited literature examples for the types of transformations that we described in this work. The known examples use fundamentally different strategies and show significant limitations in reaction scope and/or selectivity due primarily to the harsh conditions or limited availability of silicon reagents employed.

Disilylation: The disilylation of alkenes (styrenes) with chlorosilanes have some limited precedents in the literature. These examples all employ stoichiometric strongly reducing agents (e.g., $\mathrm{Li}$ powder or wire, $\mathrm{Cp}_{2} \mathrm{TiCl}_{2}+$ Grignard reagent) and are proposed to undergo direct activation of the styrene to a radical anion or dianion before silylation. Only 1-3 unfunctionalized substrates were investigated in these studies, and we expect these reactions to display limited synthetic utility due to the strongly reducing agents needed and the intrinsic mechanism. See refs 14-15, 19a in the main text. In addition to Oestreich's work (ref. 20 in main text), inter- or intramolecular disilylation of alkenes has been reported using designer substrates with $\mathrm{Si}-\mathrm{Si}$ motifs via Pt or Pd catalysis. See refs. 19 b-d in the main text. In addition, disilylation with $\mathrm{HSiCl}_{3}$ and $\mathrm{SiCl}_{4}$ (4 equiv of each) as reagents was realized via phosphine catalyzed $\mathrm{Si}-\mathrm{H}$ cleavage under high temperature $\left(180^{\circ} \mathrm{C}\right)$, see ref. 1 . The reaction shows moderate to good selectivity over hydrosilylation; functional group compatibility was not explored. The harsh reaction conditions of these methods have limited the scope of the substrates and synthetic applications.

Hydrosilylation: There are two examples of hydrosilylation using chlorosilanes (ref. 2, 3). In ref. 2, only 2- and 4-vinylpyridine was used as the substrates by means of $\mathrm{Mg}$ reduction of the alkene to the radical anion. In ref. 3, polychlorosilanes (e.g., $\mathrm{SiCl}_{4}$ and $\mathrm{Me}_{2} \mathrm{SiCl}_{2}$ ) were reported to react with styrene with moderate yields (37-74\%) via a proposed anionic pathway. One example of cyclohexene hydrosilylation was reported (48\% yield with respect to TMSCl) with unclear mechanism. In general, there are limited examples of transition-metal-free hydrosilylation of alkenes in the literature, which predominantly use either hydrosilanes together with a radical initiator (e.g., refs. 4, 35 in the main text) or transfer hydrosilylation agents derived from cyclohexadiene/dihydropyridine (refs. 36 in the main text).

Allylic silylation: There are two examples of silylation of allylic ethers using chlorosilanes, both using a Grignard reagent together with a transition metal catalyst to generate the allyl Grignard reagent followed by nucleophilic substitution with a chlorosilane. See refs. 4,5 . There is only one example of silylation of allylic alcohol via palladium catalyzed pathway, in which $\mathrm{Si}$-Si bond was activated by $\mathrm{OH}$ group and generate silyl anion intermediate, see refs 6 .

Electroreduction of chlorosilanes to form $\mathrm{Si}-\mathrm{C}$ bond: $\mathrm{TMSCl}$ has been shown to react with phenylacetylene under electroreductive conditions (refs. 17e, $18 \mathrm{~b}$ in the main text). It was proposed $\mathrm{TMS}^{-}$generated from reduction of TMSCl deprotonates phenylacetylene, which then underwent substitution with TMSCl. However, based on reduction potentials, it is also likely that 
phenylacetylene is directed reduced to the acetylide without participation of TMSCl. Various other silylation products were also found. (Equation 14, ref. 18b in the main text). In addition, Ishikawa has reported the electroreductive of aryldichlorosilanes and their reactions with dienes via a silyl anion pathway, (ref. 18a in the main text). This reaction shows low yields (up to 28\%) and low selectivity (substantial polymers and disilanes observed).

Electrogeneration of silyl radicals: Jouikov has shown to use an EPR cell to generate TES radical via electroreductive generation of $\mathrm{TES}^{-}$and tandem electrooxidation to TES radical (ref. 30 in the main text). This study focuses on mechanistic understanding instead of the synthetic utility.

Full substrate table of disilylation

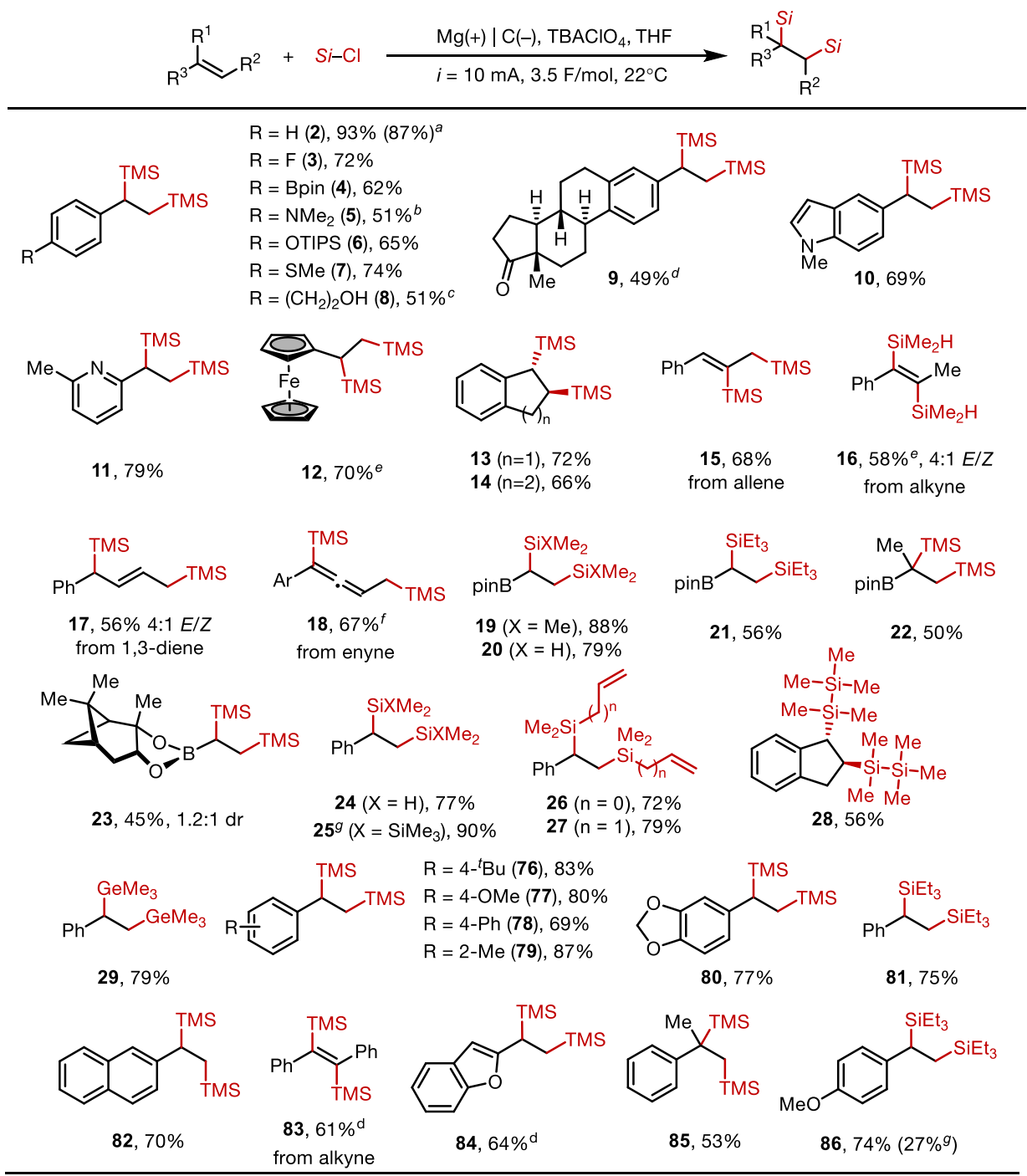

Reaction conditions: $1.0 \mathrm{mmol}$ alkene, 3.0 equiv chlorosilane, $0.2 \mathrm{M} \mathrm{TBACIO}_{4}$ in $9 \mathrm{~mL} \mathrm{THF}$, electrolysis at $22^{\circ} \mathrm{C}$ under a constant current of $10 \mathrm{~mA}$ (current density $=1.2 \mathrm{~mA} / \mathrm{cm}^{2}$ ) until $3.5 \mathrm{~F}$ total charge is passed. ${ }^{2} \mathrm{TMSBr}$ instead of TMSCl. ${ }^{b} 5.0 \mathrm{~F}$ total charge. ${ }^{c} \mathrm{TMSCl}=4.0$ equiv, $4.0 \mathrm{~F}$ total charge. ${ }^{d} \mathrm{TMSCl}=4.0$ equiv, $2.2 \mathrm{~F}$ total charge. ${ }^{e} 2.2 \mathrm{~F}$ total charge. ${ }^{f}$ Yield determined by ${ }^{1} \mathrm{H}$ NMR using 1,3,5-trimethoxybenzene as internal standard. ${ }_{9}$ Isolated yield of $\mathrm{SiEt}_{3}$ dimer (87, hexaethyldisilane), the yield is based on $1 \mathrm{mmol}$ of excess $\mathrm{SiEt}_{3} \mathrm{Cl}(0.135 \mathrm{mmol}$ dimer divide by $0.5 \mathrm{mmol}$ theoretical yield). Abbreviations: TBA, tetrabutylammonium; TMS, trimethylsilyl.

Scheme S1: Full substrate table of electrochemical disilylation 


\section{General procedures}

\section{General procedure 1 (GP 1) Disilylation}

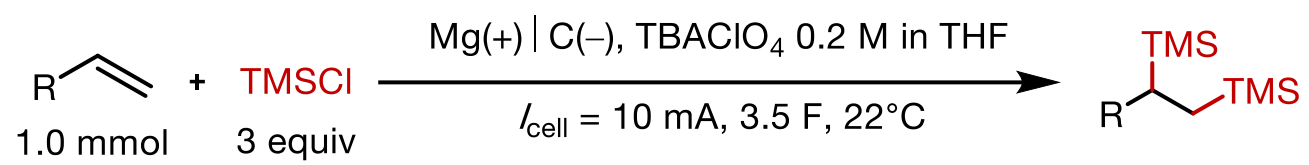

Magnesium and graphite electrodes were polished with 500 grit silicon carbide sandpaper until a shiny finish was obtained. The cap and electrodes were then transferred into the glovebox. In an oven dried $10 \mathrm{~mL}$ Electrasyn vial, tetrabutylammonium perchlorate $\left(\mathrm{TBAClO}_{4}, 720 \mathrm{mg}, 0.2 \mathrm{M}\right)$, alkene substrate ( $1 \mathrm{mmol}, 1$ equiv), trimethylsilyl chloride ( $324 \mathrm{mg}, 3 \mathrm{mmol}, 3$ equiv) and THF (9 $\mathrm{mL}$ ) was added in the glovebox. The cap was then screwed tight and sealed before transferring out and mounting on to the Electrasyn device. Electrolysis was initiated following the parameters specified below. Reaction usual starts as a clear colorless solution then eventually develops an opaque solution with grey microparticle. After electrolysis, the reaction mixture was added to hexanes $(10 \mathrm{~mL})$ to precipitate $\mathrm{TBAClO}_{4}$. The resultant mixture was then filtrated through a short silica plug (8 cm thick, ca. $10 \mathrm{~g}$ ) and flushed with $5 \%$ diethyl ether/Hex (100 mL). The crude was concentrated in vacuum and purified with column chromatography. This reaction is also completely scalable to the smaller $5 \mathrm{~mL}$ electrasyn vials with no issues.

\section{Electrolysis parameters}

\begin{tabular}{|l|l|l|l|l|}
\hline Current & Anode & Cathode & Stir rate & Duration \\
\hline $10 \mathrm{~mA}$ & $\mathrm{Mg}$ & Graphite & $1200 \mathrm{rpm}$ & $3.5 \mathrm{~F} \mathrm{~mol}^{-1}$ \\
\hline
\end{tabular}

\section{General procedure 2 (GP 2) Hydrosilylation}

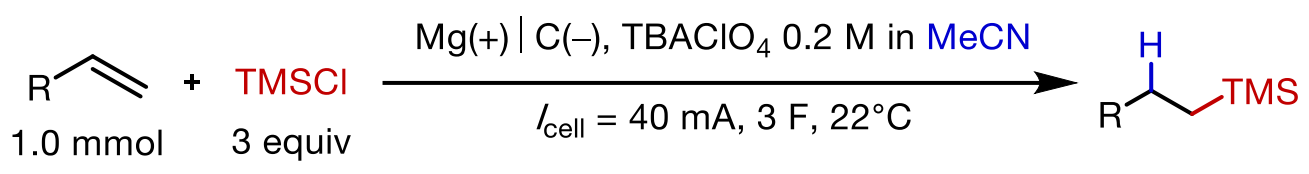

Magnesium and graphite electrodes were polished with 500 grit silicon carbide sandpaper until a shiny finish was obtained. The cap and electrodes were then transferred into the glovebox. In an oven dried $5 \mathrm{~mL}$ Electrasyn vial, $\mathrm{TBAClO}_{4}(370 \mathrm{mg}, 0.2 \mathrm{M})$, alkene substrate (1 mmol, 1 equiv), trimethylsilyl chloride (324 mg, $3 \mathrm{mmol}, 3$ equiv) and $\mathrm{MeCN}$ ( $4 \mathrm{~mL}$ ) was added in the glovebox. The cap was then screwed tight and sealed before transferring out and mounting on to the Electrasyn device. Electrolysis was initiated following the parameters specified below. Reaction usual starts as a clear colorless solution then eventually becomes an opaque solution with grey and white solids. After electrolysis, the reaction mixture was added hexanes $(10 \mathrm{~mL})$ to precipitate $\mathrm{TBAClO}_{4}$. The resultant mixture was then filtrated through a short silica plug (4 cm thick, ca. $5 \mathrm{~g}$ ) and flushed with $5 \%$ diethyl ether/Hex $(100 \mathrm{~mL})$. The crude was concentrated in vacuum and purified with column chromatography.

Electrolysis parameters 


\begin{tabular}{|l|l|l|l|l|}
\hline Current & Anode & Cathode & Stir rate & Duration \\
\hline $40 \mathrm{~mA}$ & $\mathrm{Mg}$ & Graphite & $1200 \mathrm{rpm}$ & $3 \mathrm{~F} \mathrm{~mol}^{-1}$ \\
\hline
\end{tabular}

\section{General procedure 3 (GP 3) Silacycles}

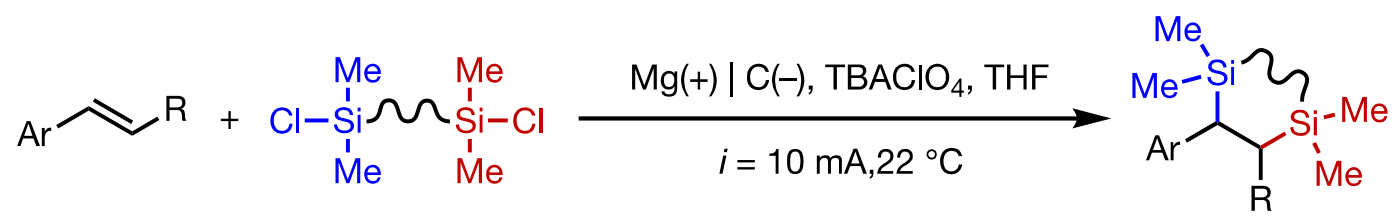

Magnesium and graphite electrodes were polished with 500 grit silicon carbide sandpaper until a shiny finish was obtained. The cap and electrodes were then transferred into the glovebox. In an oven dried $5 \mathrm{~mL}$ Electrasyn vial, tetrabutylammonium perchlorate $\left(\mathrm{TBAClO}_{4}, 370 \mathrm{mg}, 0.2 \mathrm{M}\right.$ ), alkene substrate $(0.5 \mathrm{mmol}, 1$ equiv), dichlorosilane $(0.75 \mathrm{mmol}, 1.5$ equiv) and THF $(4 \mathrm{~mL})$ was added in the glovebox. The cap was then screwed tight and sealed before transferring out and mounting on to the Electrasyn device. Electrolysis was initiated following the parameters specified below. Reaction usual starts as a clear colorless solution then eventually develops an opaque solution with grey microparticle. After electrolysis, the reaction mixture was added hexanes (10 $\mathrm{mL}$ ) to precipitate $\mathrm{TBAClO}_{4}$. The resultant mixture was then filtrated through a short silica plug $(4 \mathrm{~cm}$ thick) and flushed with $5 \%$ diethyl ether/Hex $(100 \mathrm{~mL})$. The crude was concentrated in vacuum and purified with column chromatography.

\section{Electrolysis parameters}

\begin{tabular}{|l|l|l|l|l|}
\hline Current & Anode & Cathode & Stir rate & Duration \\
\hline $10 \mathrm{~mA}$ & $\mathrm{Mg}$ & Graphite & $1200 \mathrm{rpm}$ & $3 \mathrm{~F} \mathrm{~mol}^{-1}$ \\
\hline
\end{tabular}

\section{Picture}
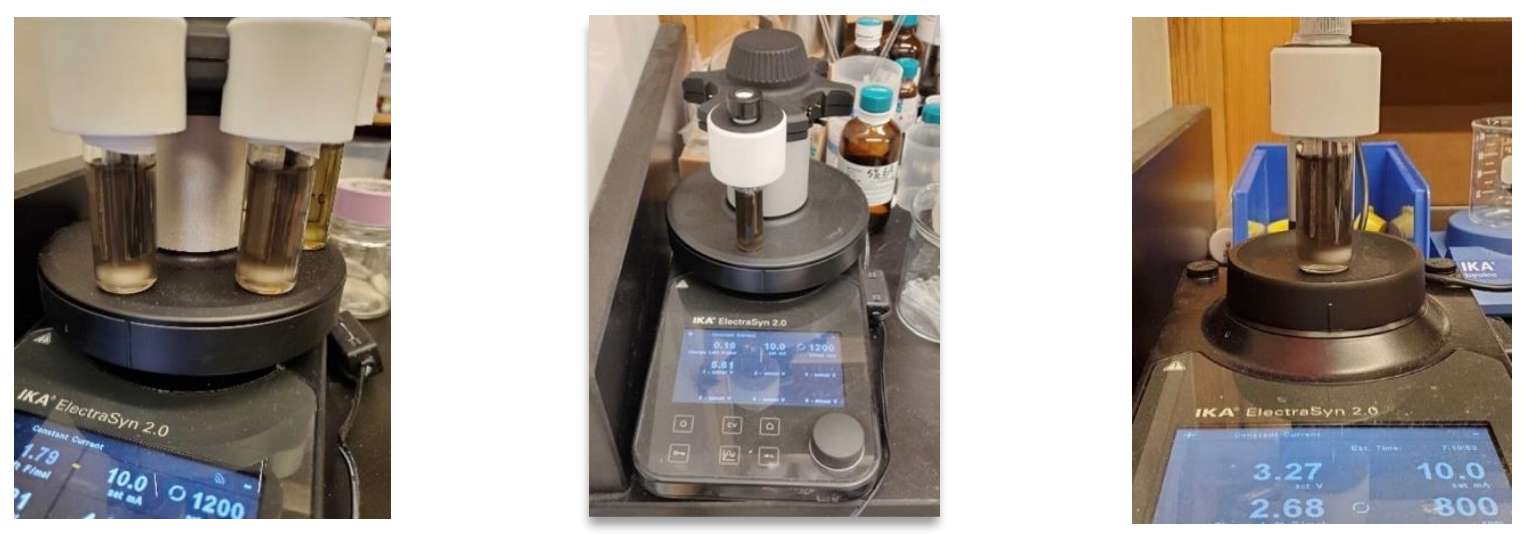

Figure S2: Reaction setup

\section{General procedure 4 (GP 4) Alternating polarity electrolysis}



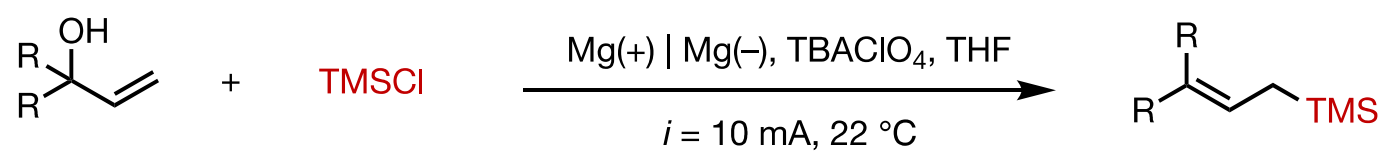

Alternating Potential Frequency $=5 \mathrm{~s}^{-1}$

Magnesium electrodes were polished with 500 grit silicon carbide sandpaper until a shiny finish was obtained. The cap and electrodes were then transferred into the glovebox. In an oven dried 5 mL Electrasyn vial, tetrabutylammonium perchlorate $\left(\mathrm{TBAClO}_{4}, 360 \mathrm{mg}, 0.2 \mathrm{M}\right)$, alkene substrate ( $0.5 \mathrm{mmol}, 1$ equiv), chlorosilane $(1.5 \mathrm{mmol}, 3$ equiv) and THF ( $4 \mathrm{~mL})$ was added in the glovebox. The cap was then screwed tight and sealed before transferring out and mounting on to the Electrasyn device. Electrolysis was initiated following the parameters specified below. Reaction usual starts as a clear colorless solution then eventually develops an opaque solution with grey microparticle. After electrolysis, the reaction mixture was added hexanes $(10 \mathrm{~mL})$ to precipitate $\mathrm{TBAClO}_{4}$. The resultant mixture was then filtrated through a short silica plug (4 cm thick) and flushed with $5 \%$ diethyl ether/Hex $(100 \mathrm{~mL})$. The crude was concentrated in vacuum and purified with column chromatography.

Electrolysis parameters

\begin{tabular}{|l|l|l|l|l|l|}
\hline Current & Anode & Cathode & $\begin{array}{l}\text { Potential Switch } \\
\text { frequency }\end{array}$ & Stir rate & Duration \\
\hline $10 \mathrm{~mA}$ & $\mathrm{Mg}$ & $\mathrm{Mg}$ & $5 \mathrm{~s}^{-1}$ & $1200 \mathrm{rpm}$ & $4 \mathrm{~F} \mathrm{~mol}^{-1}$ \\
\hline
\end{tabular}




\section{Reaction optimizations}

\begin{tabular}{|c|c|c|}
\hline $\begin{array}{l}\mathrm{Ph} \\
1.0 \mathrm{mmol}\end{array}$ & $\underset{3 \text { equiv }}{\mathrm{TMSCl}} \frac{\mathrm{Mg}(+) \mid \mathrm{C}(-), \mathrm{TBAClO}_{4} 0.2 \mathrm{M} \text { in THF}}{I_{\text {cell }}=10 \mathrm{~mA}, 3.0 \mathrm{~F}, 22^{\circ} \mathrm{C}}$ & $\underbrace{T M S}$ \\
\hline Entry & Variation from optimal conditions & $\%$ Yield $^{a}$ \\
\hline 1 & none & 94 \\
\hline 2 & 2 equiv $\mathrm{TMSCl}$ & 79 \\
\hline 3 & $\mathrm{TMSBr}$ instead of TMSCl & 87 \\
\hline 4 & TMSOAC instead of TMSCI & 31 \\
\hline 5 & TBATFSI instead of $\mathrm{TBACIO}_{4}{ }^{b}$ & 77 \\
\hline 6 & LiTFSI instead of $\mathrm{TBACIO}_{4}$ & 46 \\
\hline 7 & $\mathrm{LiClO}_{4}$ instead of $\mathrm{TBAClO}_{4}$ & 50 \\
\hline 8 & $\mathrm{TBABF}_{4}$ instead of $\mathrm{TBAClO}_{4}$ & 22 \\
\hline 9 & TBAPF $_{6}$ instead of $\mathrm{TBAClO}_{4}$ & $<5$ \\
\hline 10 & LiOTf instead of $\mathrm{TBACIO}_{4}$ & $<5$ \\
\hline 11 & Pt instead of C & 99 \\
\hline 12 & $\mathrm{Mg}$ instead of $\mathrm{C}$ & 82 \\
\hline 13 & $\mathrm{Ni}$ instead of $\mathrm{C}$ & 93 \\
\hline 14 & $\mathrm{Zn}$ instead of $\mathrm{Mg}$ & $<5$ \\
\hline 15 & Al instead of Mg & $<5^{c}$ \\
\hline 16 & $I_{\text {cell }}=20 \mathrm{~mA}$ & 93 \\
\hline 17 & $I_{\text {cell }}=30 \mathrm{~mA}$ & 91 \\
\hline 18 & no electricity & $<1$ \\
\hline 19 & $0.5 \mathrm{mmol}$ scale in $5 \mathrm{~mL}$ vial & 92 \\
\hline 20 & 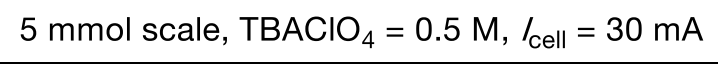 & 89 \\
\hline
\end{tabular}

Table S1: Optimisation for the disilylation reaction. 


\begin{tabular}{|c|c|c|c|}
\hline $1.0 \mathrm{mmol}$ & 3 equiv & & B \\
\hline Entry & & Variation from optimal conditions & $\%$ Yield A:B (SM) ${ }^{a}$ \\
\hline 1 & & none & $81: 3(0)$ \\
\hline 2 & & With 0.2 equiv $\mathrm{Et}_{3} \mathrm{NHCl}$ & $80: 3(0)$ \\
\hline 3 & & 2 equiv $\mathrm{TMSCl}, 0.2$ equiv $\mathrm{Et}_{3} \mathrm{NHCl}$ & $43: 15(0)$ \\
\hline 4 & & 4 equiv TMSCl, 0.2 equiv $\mathrm{Et}_{3} \mathrm{NHCl}$ & $83: 6(0)$ \\
\hline 5 & & $8 \mathrm{ml} \mathrm{MeCN}$, LiOTf, $10 \mathrm{~mA}$ & $7: 5(56)$ \\
\hline 6 & & $8 \mathrm{ml} \mathrm{MeCN}, 20 \mathrm{~mA}, 0.2$ equiv $\mathrm{Et}_{3} \mathrm{NHCl}, 4 \mathrm{~F}$ & $41: 15(0)$ \\
\hline 7 & & $10 \mathrm{~mA}, 0.2$ equiv $\mathrm{Et}_{3} \mathrm{NHCl}$ & $15: 14(29)$ \\
\hline 8 & & $20 \mathrm{~mA}, 0.2$ equiv $\mathrm{Et}_{3} \mathrm{NHCl}$ & $68: 16(0)$ \\
\hline 9 & & $8 \mathrm{ml} \mathrm{EtOAc,} 10 \mathrm{~mA}$ & $16: 0(7)$ \\
\hline 10 & & $8 \mathrm{ml} \mathrm{MeCN}, 10 \mathrm{~mA}$ & $18: 14(29)$ \\
\hline 11 & & $8 \mathrm{ml} \mathrm{MeCN}, 20 \mathrm{~mA}$ & $40: 21(21)$ \\
\hline 12 & & $20 \mathrm{~mA}, 0.5$ equiv $\mathrm{Et}_{3} \mathrm{NHCl}$ & $54: 6(12)$ \\
\hline 13 & & $8 \mathrm{ml} \mathrm{MeCN}, 0.1 \mathrm{M} \mathrm{TBAClO}{ }_{4}, 10 \mathrm{~mA}$ & $5: 0(37)$ \\
\hline 14 & & $4 \mathrm{~F}$ & $79: 3(0)$ \\
\hline 15 & & Constant $E_{\text {cell }}=0.65 \mathrm{~V}$ for 5 hours & $25: 0(0)$ \\
\hline
\end{tabular}

aYield obtained by ${ }^{1} \mathrm{H}$-NMR using 1,3,5 trimethoxybenzene as internal standard.

Table S2: Optimisation for the hydrosilylation reaction. 


\section{Control experiments}

\section{I) Controlled potential electrolysis}

An identical procedure was followed as described in GP 1 with the exception that a Mg wire was inserted through the septum into the reaction solution as a reference electrode and excess amount of anhydrous $\mathrm{MgCl}_{2}$ (ca. $50 \mathrm{mg}$ ) was added to saturate the reaction solution with $\mathrm{Mg}^{2+}$. Cathodic potential was controlled by a BASi Epsilon potentiostat. After $2.5 \mathrm{~F}$ charge passed, the reaction was terminated and the yield of the product was determined by ${ }^{1} \mathrm{H}$ NMR using 1,3,5trimethoxybenzene as internal standard.

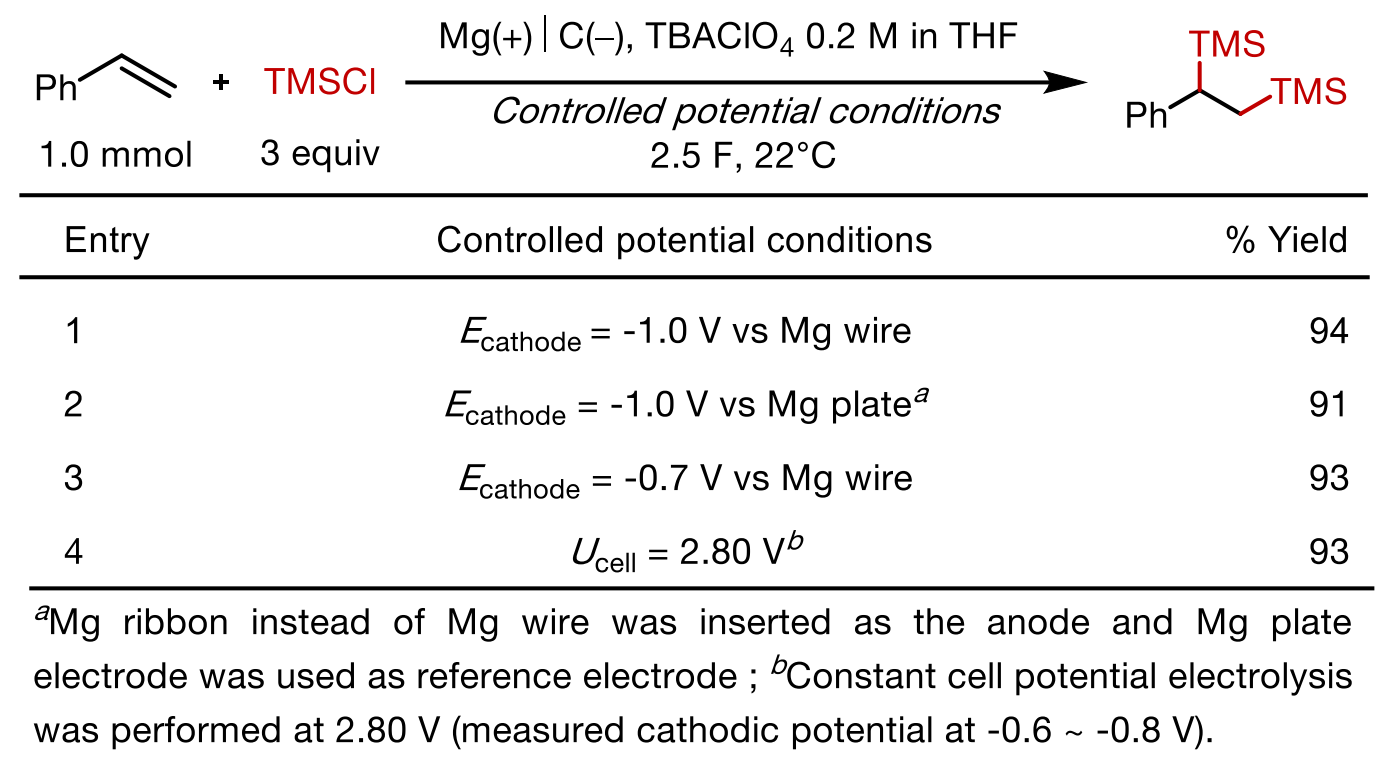

Table S3: Controlled potential electrolysis

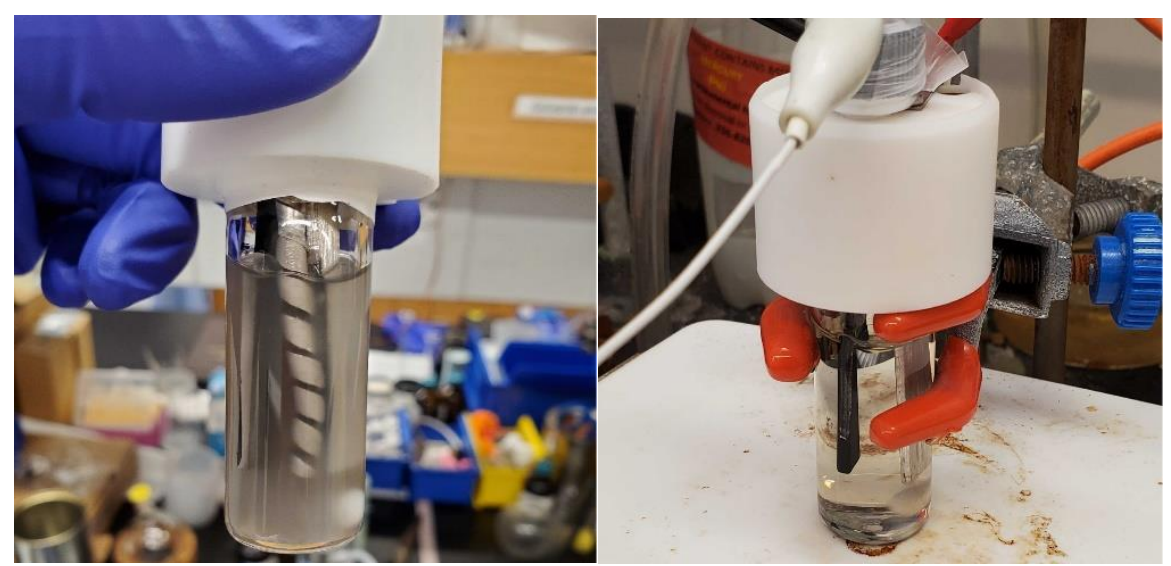

Figure S3: Reaction setup for controlled potential electrolysis 


\section{II) Divided cell electrolysis}

A flame dried, $10 \mathrm{~mL} \mathrm{H}$-type divided cell with glass frit was equipped with a stir bar respectively. A carbon felt cathode $\left(1.0 \times 0.5 \mathrm{~cm}^{2}\right)$ was connected to the electrical feedthrough via a $9 \mathrm{~cm}$ by 2 $\mathrm{mm}$ diameter graphite rod and fitted a threaded Teflon cap. $\mathrm{Zn} / \mathrm{Mg}$ plate anode was attached to a septum using a three-inch long needle and the conductor to the power supply. The cell and electrodes were then transferred into the glovebox. Styrene (105 mg, $1 \mathrm{mmol}, 1$ equiv), TMSCl (324 mg, $3 \mathrm{mmol}, 3$ equiv), and $\mathrm{TBAClO}_{4}(2.5 \mathrm{mmol}, 855 \mathrm{mg})$ were added into cathode chamber while the anodic chamber was added $\mathrm{TBAClO}_{4}(2.5 \mathrm{mmol}, 855 \mathrm{mg}) .5 .0 \mathrm{~mL}$ of THF was added to the anodic chamber and cathodic chamber respectively ( $10 \mathrm{~mL}$ in total). Subsequently, the cap was screwed tight and sealed before transferring out for electrolysis. The electrodes were connected to a DC power supply with a constant cell potential of $31.0 \mathrm{~V}$. The reaction was turned off when current dropped to $0 \mathrm{~mA}$ (ca. $12 \mathrm{~h}$ ). The reaction mixture of each chamber was collected and washed with $5 \%$ diethyl ether/Hex $(10.0 \mathrm{~mL} \times 3)$. The combined mixture was then filtrated on a silica plug $(4 \mathrm{~cm}$ thick, ca. $5 \mathrm{~g})$ and flushed with $5 \%$ diethyl ether/Hex $(100 \mathrm{~mL})$. The crude was concentrated on vacuum and the yield of the product was determined by ${ }^{1} \mathrm{H}$ NMR using 1,3,5trimethoxybenzene as internal standard.

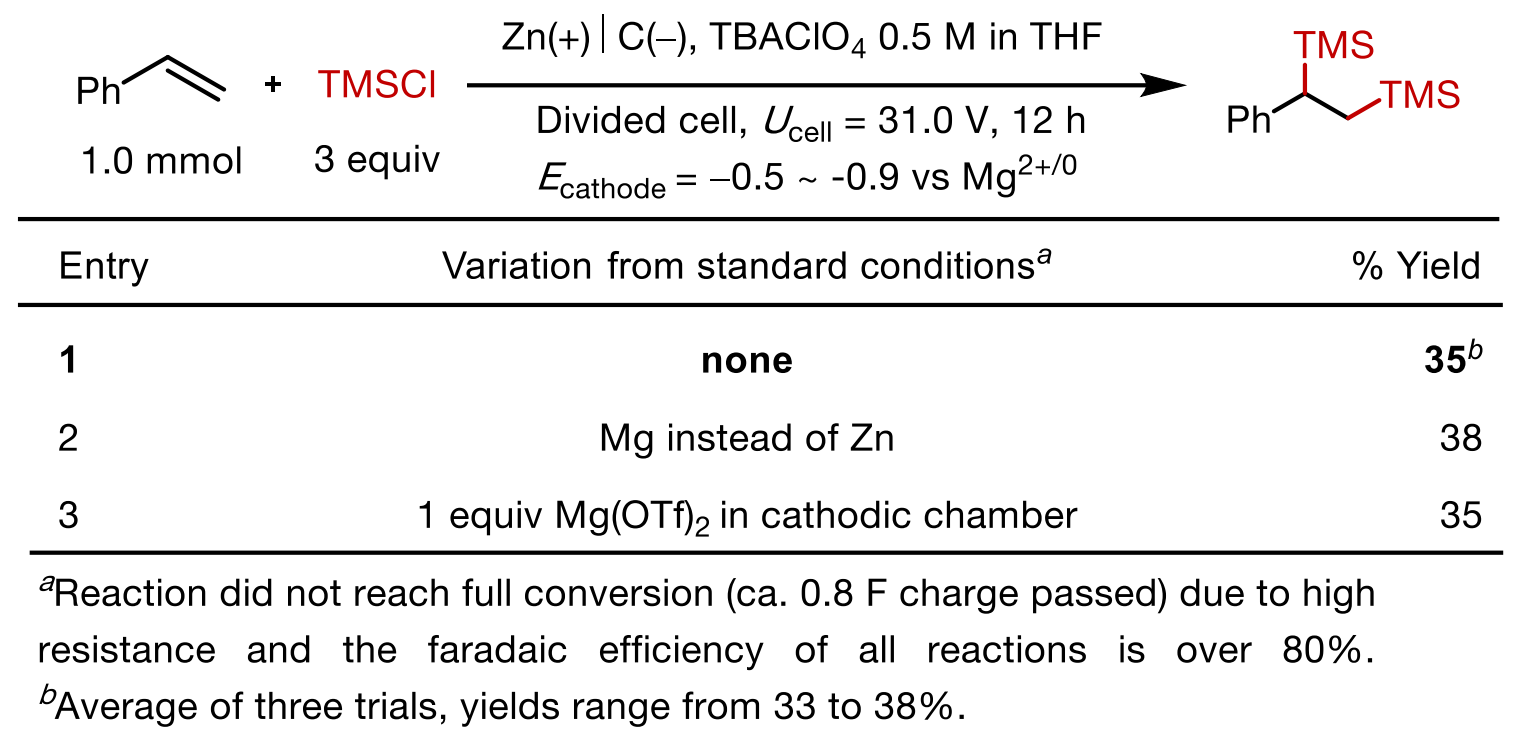

Table S4: Divided cell experiments 

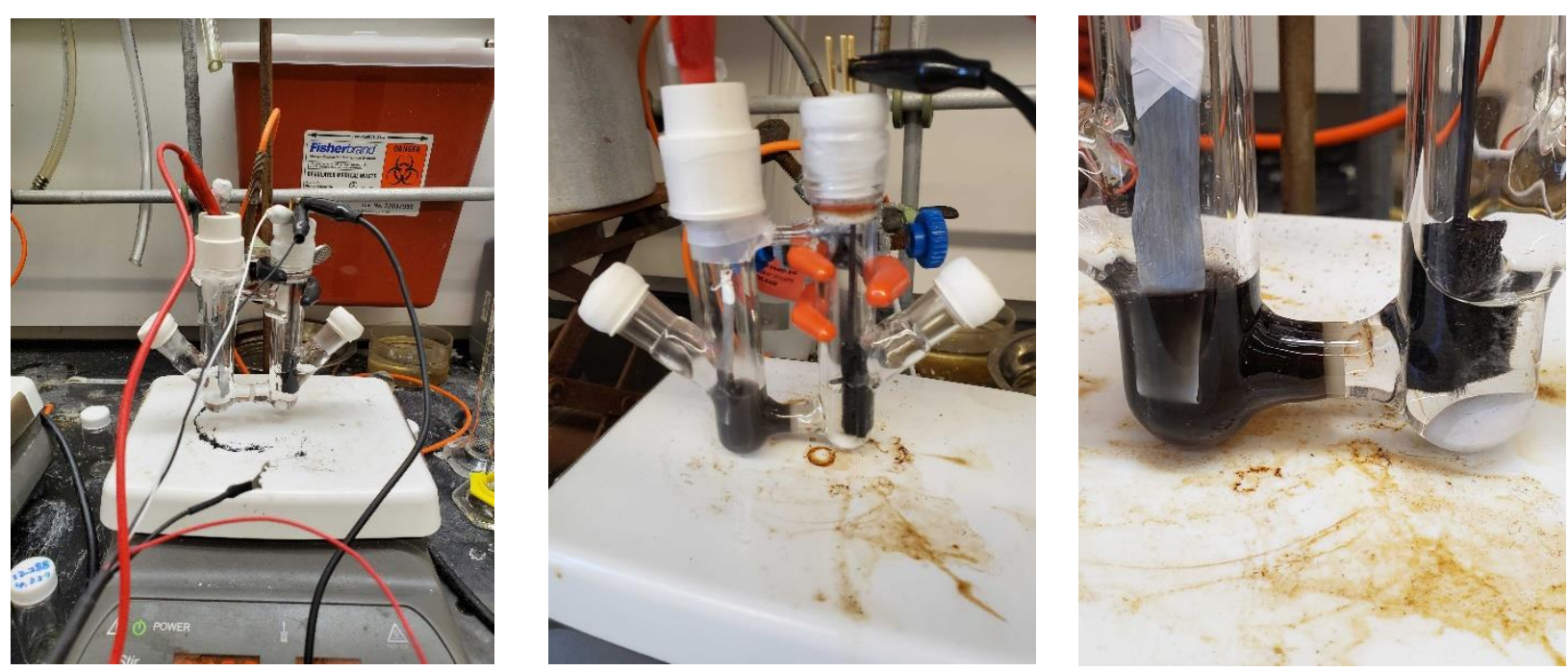

Figure S4: Reaction setup for divided cell experiments

\section{III) Radical Probe experiments}

Radical probe experiments were performed as described in GP 1 with the exception that the experiment using substrate $\mathbf{3 0}$ was stopped at $2.5 \mathrm{~F}$ to avoid further silylation of the product $\mathbf{3 2}$ and 4.0 equivalent of $\mathrm{TMSCl}$ is added to achieve higher conversion of the substrate 31. Anionic ring opening of cyclopropane ( $c f .30)$ is unlikely to happen, given this process is kinetically unfavorable. ${ }^{7}$<smiles>C=C(c1ccccc1)C1CC1c1ccccc1</smiles>

30

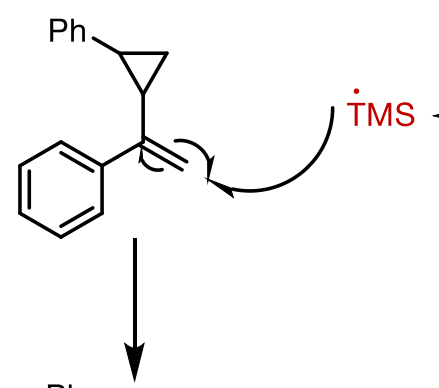

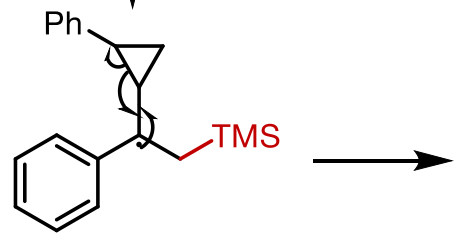

TMSCI 3.0 equiv

$\mathrm{Mg}(+) \mid \mathrm{C}(-) \mathrm{TBAClO}_{4}$ in THF $0.2 \mathrm{M}$

$10 \mathrm{~mA}, 2.5 \mathrm{~F}, 22^{\circ} \mathrm{C}$

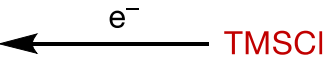<smiles>[As]CC(=CCC([As])c1ccccc1)c1ccccc1</smiles>

$32,73 \%$<smiles>CC(C)(C)CC(=CCCc1ccccc1)c1ccccc1</smiles>

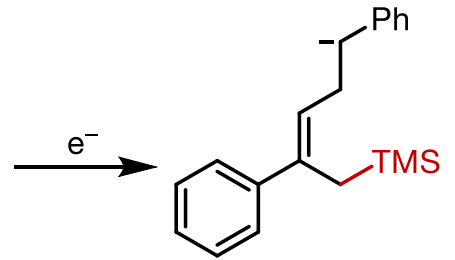


Scheme S2: Radical probe experiments (substrate 30)

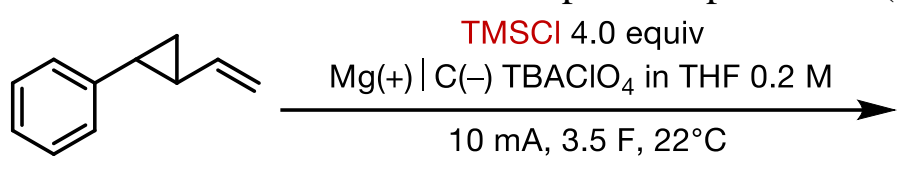

31<smiles>CC(CC=CCS(C)(=O)=O)c1ccccc1</smiles>

$33,55 \%$

Trims

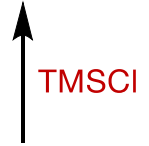<smiles>CS(C)(=O)=O</smiles><smiles>CC(C)[C-]Cc1ccccc1</smiles>

Scheme S3: Radical probe experiments (substrate 31)

IV) Anionic probe experiments

The anionic probe experiments were performed as described in GP 1 with the exception that the reaction was stopped after $2.5 \mathrm{~F}$ of charge passed.

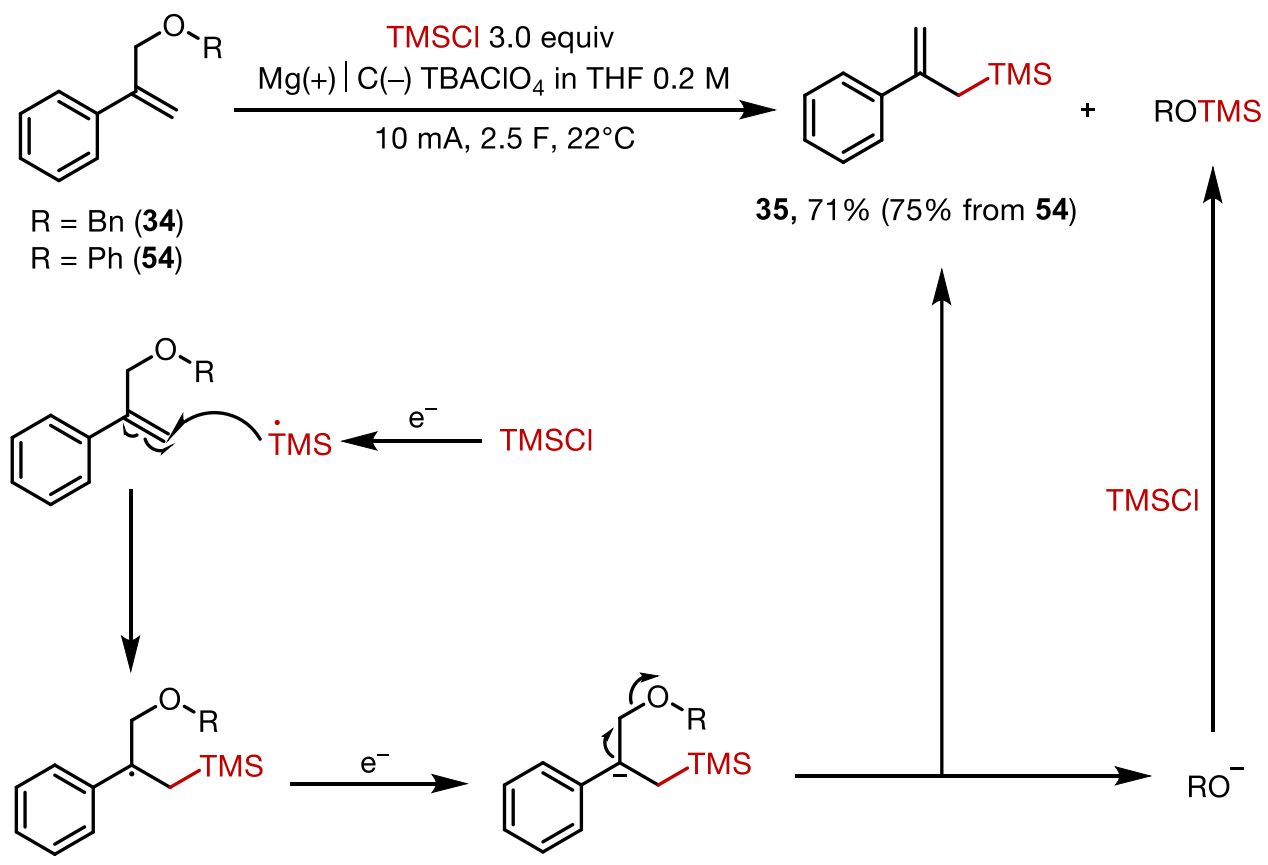

Scheme S4: Anionic probe experiments 


\section{V) Anionic TMS control experiments:}

Anionic TMS compound was synthesized following a literature procedure ${ }^{8}$ as a TMSLi solution in HMPA or HMPA/THF. To a stirring solution of hexamethyldisilane $(1.25 \mathrm{mmol}, 183 \mathrm{mg}, 1.25$ equiv) in HMPA $(2 \mathrm{~mL})$ or HMPA/THF $\left(1: 1\right.$ ratio, $1 \mathrm{~mL}$ for each) at $0^{\circ} \mathrm{C}$ was dropwise added MeLi ( $1 \mathrm{mmol}, 0.63 \mathrm{~mL} 1.6 \mathrm{M}$ solution in diethyl ether). The mixture was stirred for $15 \mathrm{~min}$ at $0^{\circ} \mathrm{C}$ to produce a deep red solution of TMSLi. To the TMSLi solution was portion-wise added a mixture of styrene ( $1 \mathrm{mmol}, 104 \mathrm{mg}, 1$ equiv) and TMSCl (2 mmol, $217 \mathrm{mg}, 2$ equiv) in HMPA $(0.2 \mathrm{~mL})$ or HMPA/THF (1:1 ratio, $0.1 \mathrm{~mL}$ for each) solution. The reaction solution was stirred for an hour, quenched in air and was then filtrated on a silica plug ( $4 \mathrm{~cm}$ thick, ca. $5 \mathrm{~g}$ ) and flushed with $5 \%$ diethyl ether/Hex $(10 \mathrm{~mL})$. The crude was concentrated on vacuum and the yield of the product was determined by ${ }^{1} \mathrm{H}$ NMR using 1,3,5-trimethoxybenzene as internal standard.

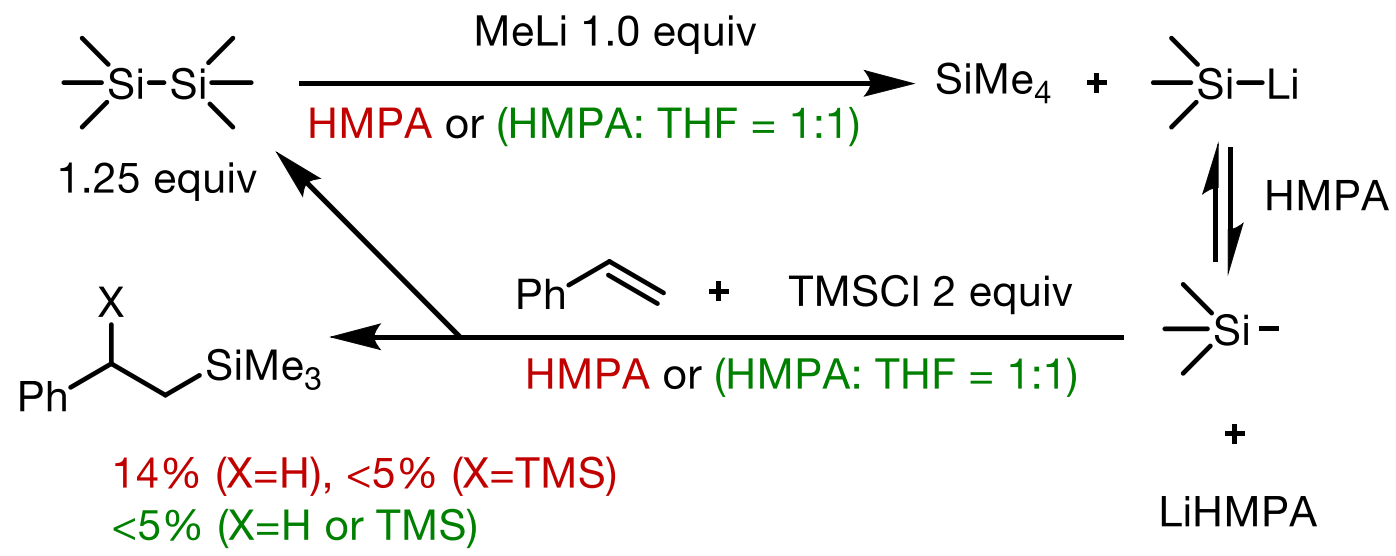

Scheme S5: Anionic TMS control experiments

In both reaction conditions, disilylated product was not detected, which indicates anionic TMS pathway is unlikely to occur for the disilylation reaction. HMPA is necessary for the formation and stabilization of TMSLi and no deep red colour was observed when adding MeLi to hexamethyldisilane solution in THF. Further diluting the styrene and TMSCl solution (ie. in $3 \mathrm{~mL}$ instead of $0.2 \mathrm{~mL}$ HMPA solution) or adding it dropwise resulted in diminished yields for silylated species ( $<10 \%$ yield for hydrosilylation), suggesting hydrosilylation reactions only occur in highy concentrated conditions. The detection of hydrosilyated product when only HMPA is used was consistent with the reported literature that HMPA can assist hydrosilylation of alkenes or alkynes. ${ }^{9}$

\section{VI) Reactive magnesium complex control}

An identical procedure was followed as described in GP 1 with the exception of entry 1, where ten equivalents of $\mathrm{Mg}$ powder from a fresh opened bottle was added to the ElectraSyn vial and stirred overnight. In entry 2 4, styrene and TMSCl was added accordingly after the electrolysis was over via a syringe and stirred overnight. The yield was determined by ${ }^{1} \mathrm{H}-\mathrm{NMR}$ using $1,3,5-$ trimethoxybenzene as internal standard. 


\begin{tabular}{|c|c|c|}
\hline 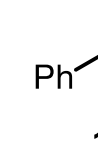 & conditions & $\underbrace{T M S}_{2}$ \\
\hline Entry & Conditions & NMR yield (\%) \\
\hline 1 & $\begin{array}{l}\text { No electrical input. 1, TMSCl, Mg powder } \\
\text { TBACIO }_{4}, \mathrm{THF}, 12 \mathrm{~h}, 22^{\circ} \mathrm{C}\end{array}$ & $\begin{array}{r}<1 \% \\
(1 \text { recovered })\end{array}$ \\
\hline 2 & $\begin{array}{c}\mathrm{Mg}(+) \mid \mathrm{C}(-), \mathrm{TBAClO}_{4}, \mathrm{THF}, i=10 \mathrm{~mA}, 2 \mathrm{~F} \\
\text { then, add } 1 \text { and } \mathrm{TMSCl}, 12 \mathrm{~h}, 22^{\circ} \mathrm{C}\end{array}$ & $\begin{array}{r}<1 \% \\
(1 \text { recovered })\end{array}$ \\
\hline 3 & $\begin{array}{c}\text { 1, } \mathrm{Mg}(+) \mid \mathrm{C}(-), \mathrm{TBAClO}_{4}, \mathrm{THF}, i=10 \mathrm{~mA}, 2 \mathrm{~F} \\
\text { then, add } \mathrm{TMSCl}, 12 \mathrm{~h}, 22^{\circ} \mathrm{C}\end{array}$ & $\begin{array}{r}<1 \% \\
(1 \text { consumed })\end{array}$ \\
\hline 4 & $\begin{array}{c}\mathrm{TMSCl}, \mathrm{Mg}(+) \mid \mathrm{C}(-), \mathrm{TBAClO}_{4}, \mathrm{THF}, i=10 \mathrm{~mA}, 2 \mathrm{~F} \\
\text { then, add } 1,12 \mathrm{~h}, 22^{\circ} \mathrm{C}\end{array}$ & $\begin{array}{r}<1 \% \\
(1 \text { recovered })\end{array}$ \\
\hline
\end{tabular}

Table S5: Reactive magnesium complex control experiments 


\section{Cyclic voltammetry studies}

All cyclic voltammetry studies were conducted inside the glovebox with the Pine WaveNow® Potentiostat/Galvanostat System. Measurements were performed in $0.2 \mathrm{M} \mathrm{TBAClO}_{4}$ in THF using a divided three-compartment cell. $\mathrm{Mg}(\mathrm{OTf})_{2}$, which bears a redox innocent anion, was used as the $\mathrm{Mg}^{2+}$ source instead of $\mathrm{MgCl}_{2}$ due to its higher solubility in THF. Control experiments revealed no difference of $\mathrm{Mg}(\mathrm{OTf})_{2}$ and $\mathrm{MgCl}_{2}$ in the scan range of $-2.0 \sim 1.0 \mathrm{~V}$ vs $\mathrm{Mg}^{2+/ 0}$. Scan rate is 50 $\mathrm{mV} / \mathrm{s}$ unless specified.

Supporting Electrolyte: $\mathrm{TBAClO}_{4}$ was recrystallized in ethyl acetate for three times and dried under vacuum at $60^{\circ} \mathrm{C}$ overnight.

Solvents: THF was first dried overnight with potassium hydroxide. Then reflux in sodium metal and benzophenone under nitrogen for 5 hours. The quality of the solvent was verified by the deep purple colour when acetophenone was added.

Working electrode: The working electrode is a $3 \mathrm{~mm}$ diameter glassy carbon working electrode. Polished with $0.3 \mu \mathrm{m}$ aluminum oxide and then sonicated in distilled water before drying and transferring into the glovebox.

Reference electrode: The reference electrode consisted of a magnesium wire submerged in a saturated solution of magnesium chloride and THF. The $\mathrm{Mg}$ wire was polished with sand paper and washed with diethyl ether before transferring into the glovebox.

Counter electrode: The counter electrode is a platinum wire that was previous burnt for 30 seconds with a butane torch.

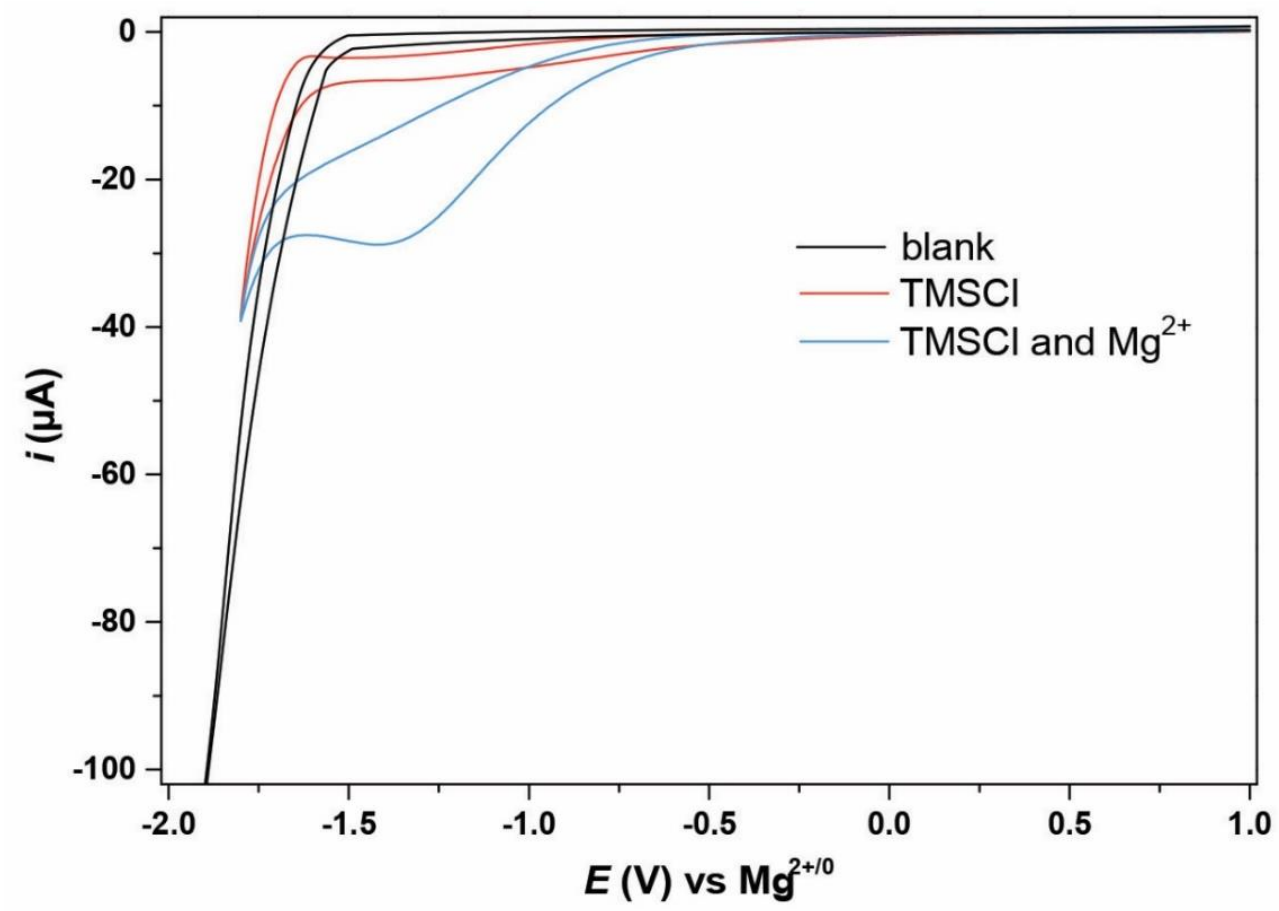

Figure S5: Cyclic voltammetry of TMSCl, and TMSCl with $\mathrm{Mg}^{2+}$. TMSCl showed onset of a reductive event at $c a$. $-0.5 \mathrm{~V}$ and a current enhancement was observed for the reduction of TMSCl upon addition of $\mathrm{Mg}^{2+}$, indicating that $\mathrm{Mg}^{2+}$ may facilitate the reduction of TMSCl . 

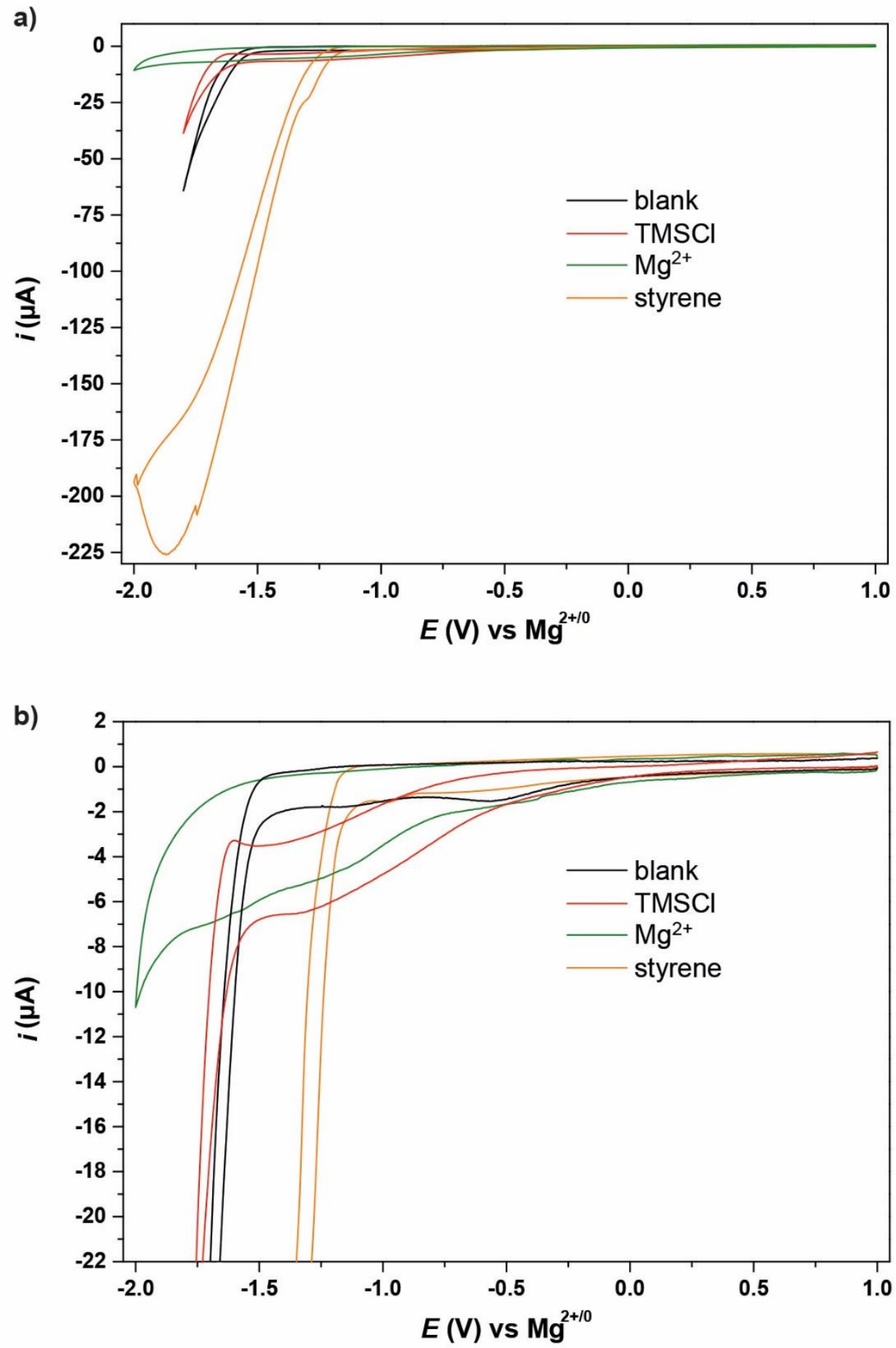

Figure S6: Cyclic voltammetry of TMSCl, $\mathrm{Mg}^{2+}$ and styrene. a) full scale; b) zoom. The cathodic potential of this reaction was measured to vary between $-0.7 \mathrm{~V}$ and $-1.0 \mathrm{~V}$. In this range, only TMSCl and $\mathrm{Mg}^{2+}$ can be reduced. 
a)

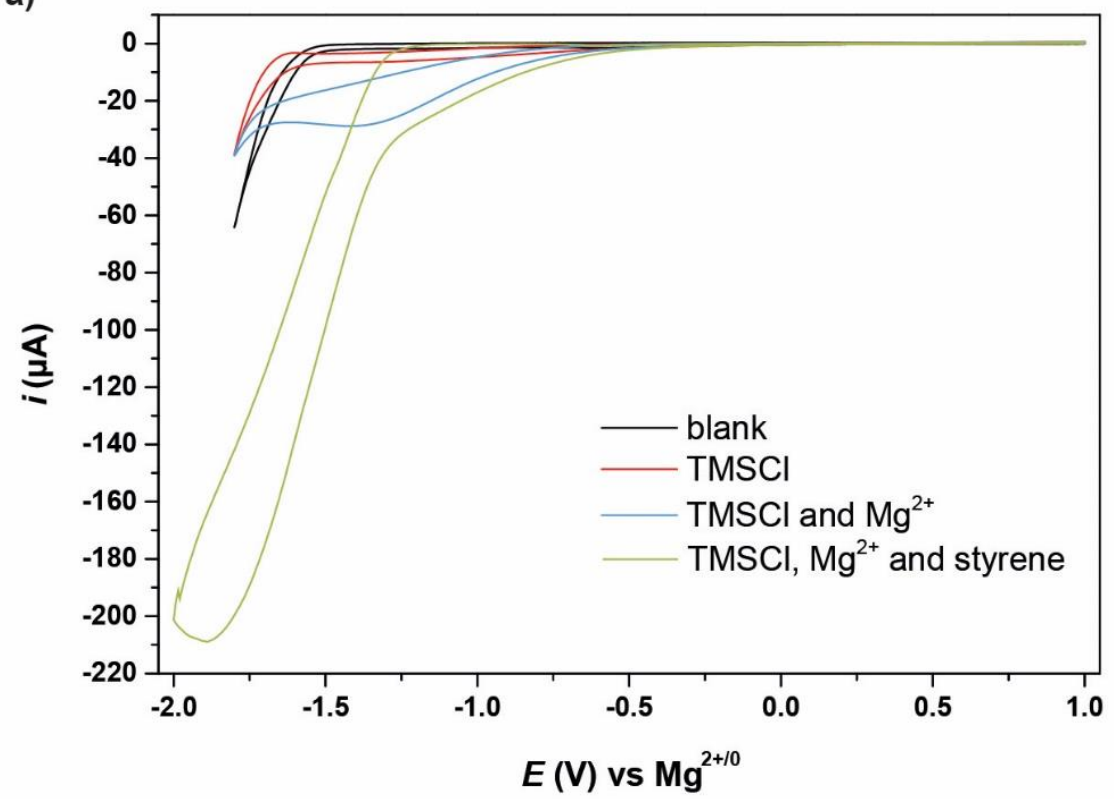

b)

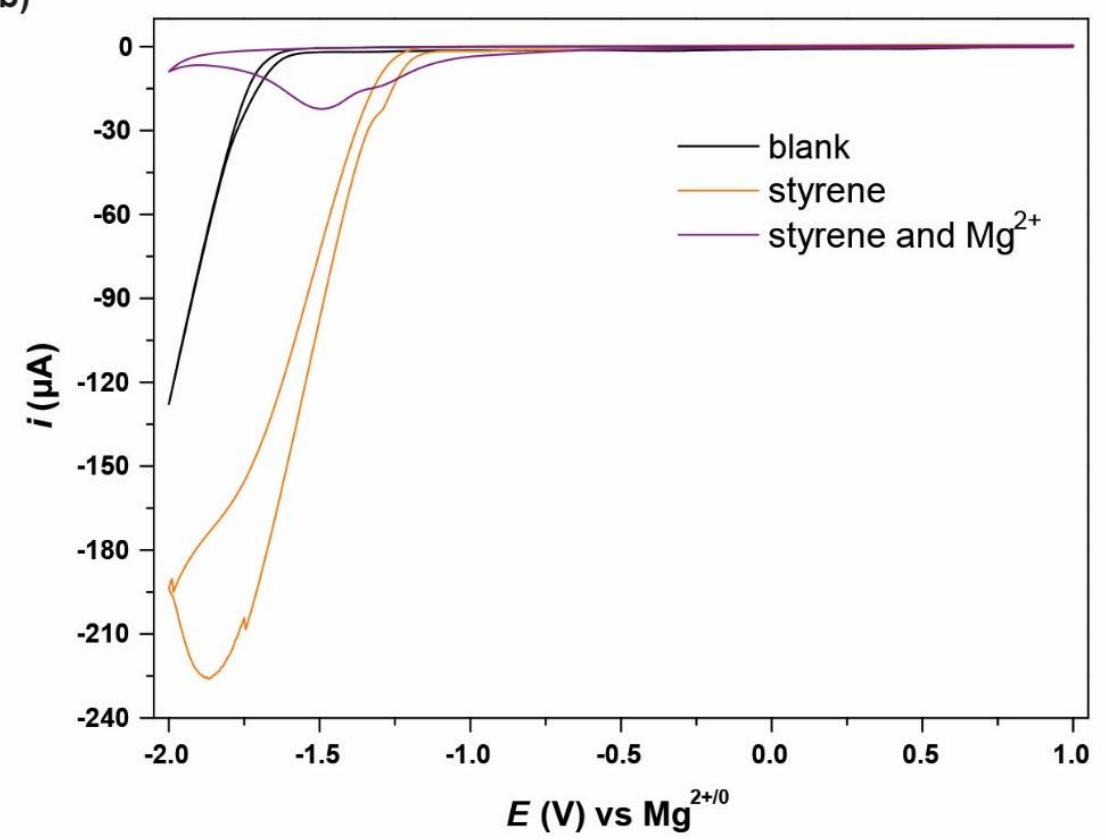

Figure S7: a) Cyclic voltammetry of TMSCl; $\mathrm{TMSCl}$ and $\mathrm{Mg}^{2+}$; $\mathrm{TMSCl}, \mathrm{Mg}^{2+}$ and styrene. b) cyclic voltammetry of styrene; styrene and $\mathrm{Mg}^{2+}$. The addition of $\mathrm{Mg}^{2+}$ to $\mathrm{TMSCl}$ significantly increased the reducing current (also see Figure S5), while the addition of $\mathrm{Mg}^{2+}$ to styrene resulted in electrode passivation (also see Figure S8). This result indicates $\mathrm{Mg}^{2+}$ may assist the reduction of TMSCl. These two figures support an ECEC type mechanism because the current at the potential of reactivity ( $c a .-0.7 \mathrm{~V}$ ) when all components are mixed is greater than the sum of the currents from the individual components. The absence of a second peak at a more negative potential shows that the second reduction is more facile than the first. 


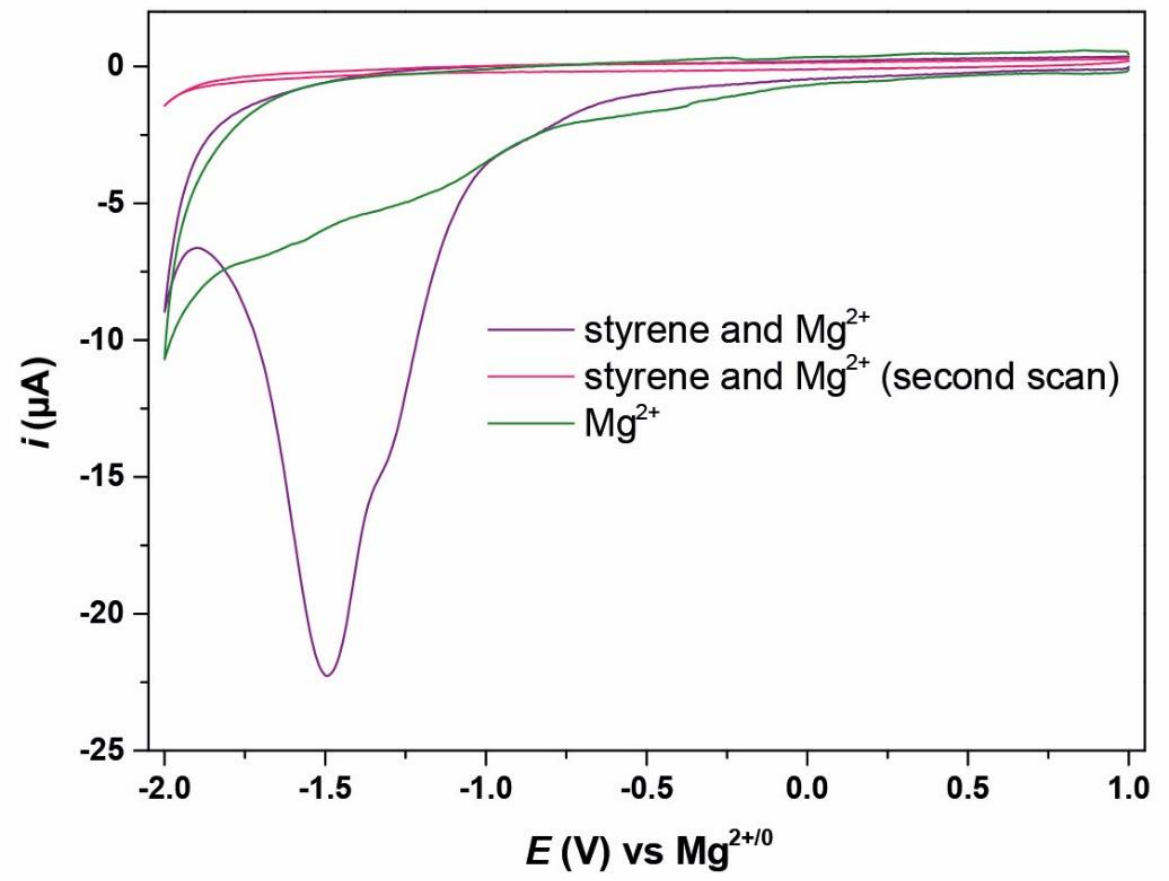

Figure S8: Cyclic voltammetry of $\mathrm{Mg}^{2+}$ and styrene; $\mathrm{Mg}^{2+} \cdot \mathrm{Mg}^{2+}$ can assist the reduction of styrene as the peak potential $\mathrm{E}_{\mathrm{p}}$ shifts from $-1.8 \mathrm{~V}$ to $-1.5 \mathrm{~V}$ but the onset potential $\mathrm{E}$ is more negative than $-1.0 \mathrm{~V}$, thus further suggests styrene is not likely to be reduced in this reaction.

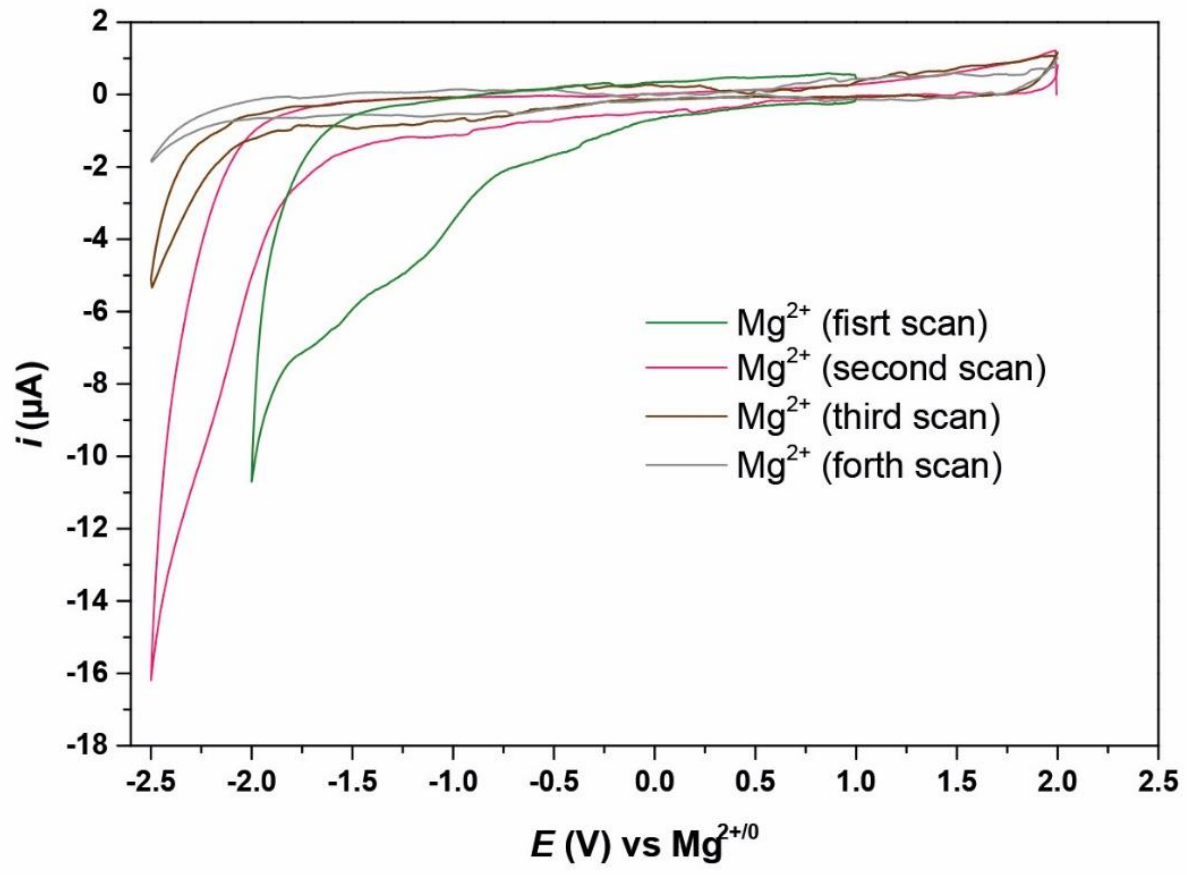

Figure S9: Multiple CV scans of $\mathrm{Mg}^{2+}$. Electrode passivation during CV scans is caused by $\mathrm{Mg}^{2+}$ and the $\mathrm{Mg}^{2+}$ passivation is not observed in the presence of $\mathrm{TMSCl}$. 

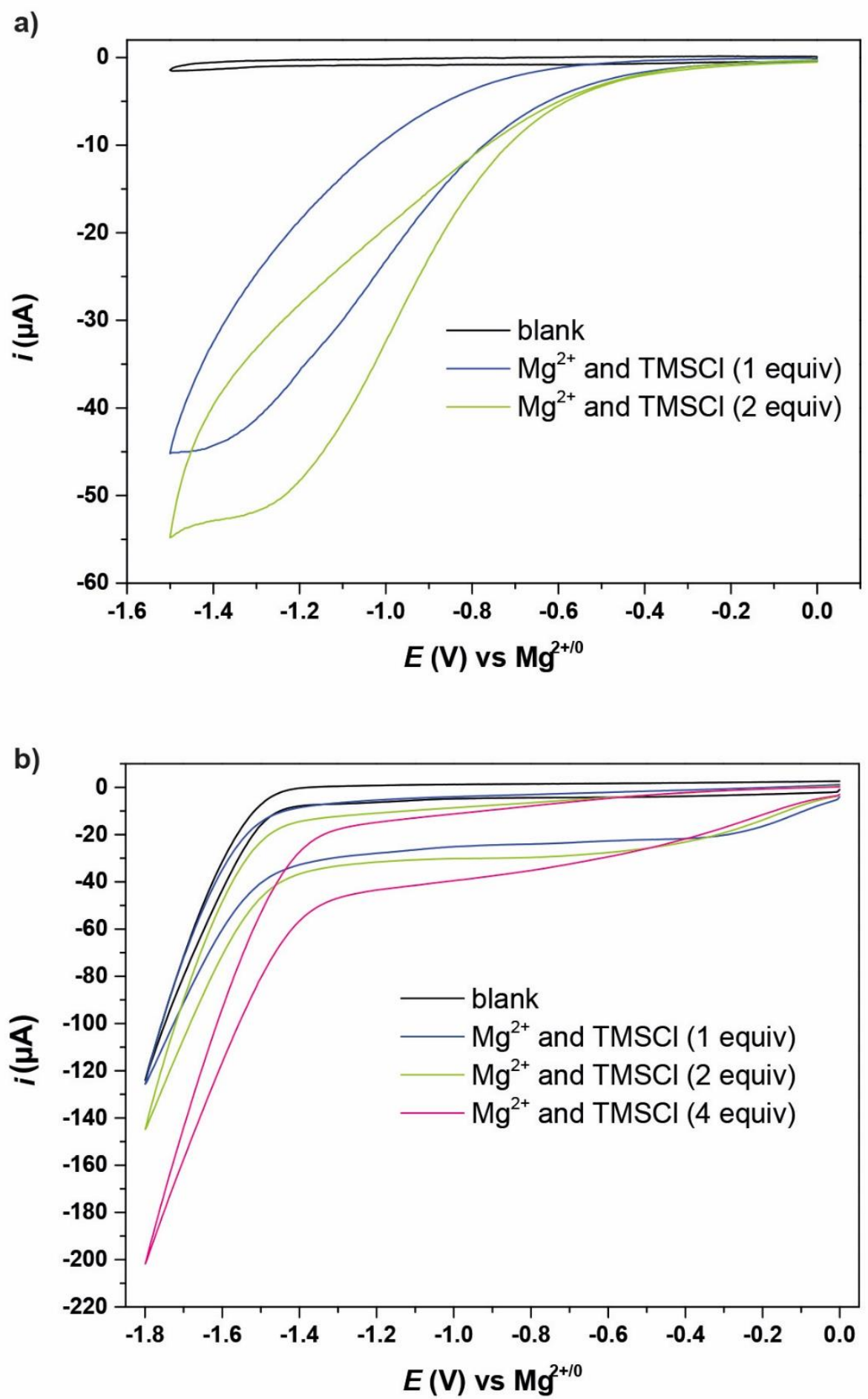

Figure S10: Cyclic voltammetry of $\mathrm{Mg}^{2+}$ and various equivalents of TMSCl. a) scan rate $=50$ $\mathrm{mV} / \mathrm{s}$, a third scan was not performed due to TMS radical may react with the surface of the working electrode after multiple scans. b) scan rate $=500 \mathrm{mV} / \mathrm{s}$. 


\section{Kinetic studies}

The reactions were setup as described in GP 1 using styrene as the model substrate under constant current conditions. Hexadecane $(113 \mathrm{mg}, 0.5 \mathrm{mmol})$ was added as an internal standard. A nitrogenfilled balloon was used to sustain a nitrogen atmosphere. $10 \mu \mathrm{l}$ aliquots were drawn from the reaction at various charges passed $(0.1,0.2,0.3,0.4,0.5,0.75,1.0,1.5,2.1,2.3,2.5,3$ and $4 \mathrm{~F})$ and diluted with $2 \mathrm{~mL}$ HPLC grade hexanes. The resultant solution was then flushed through a syringe filter and analyzed on a GC-FID instrument. Signals were identified by comparison with the retention time of authentic samples. Areas of the product was calibrated about the hexadecane internal standard. Strong linearity $\left(\mathrm{R}^{2}>0.999\right)$ was found for the desired product (2), indicating a zero-order rate in product formation. Varying reaction currents $(10 \sim 30 \mathrm{~mA})$, TMSCl concentration

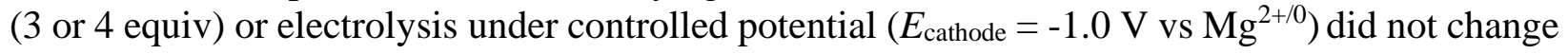
overall yield or reaction order. The reaction rates were found to be proportional to applied current, suggesting that electron transferred process be presumably the rate-determining step of the overall reaction. The Faradaic efficiency of the styrene disilylation reaction was found to be $92 \%$.

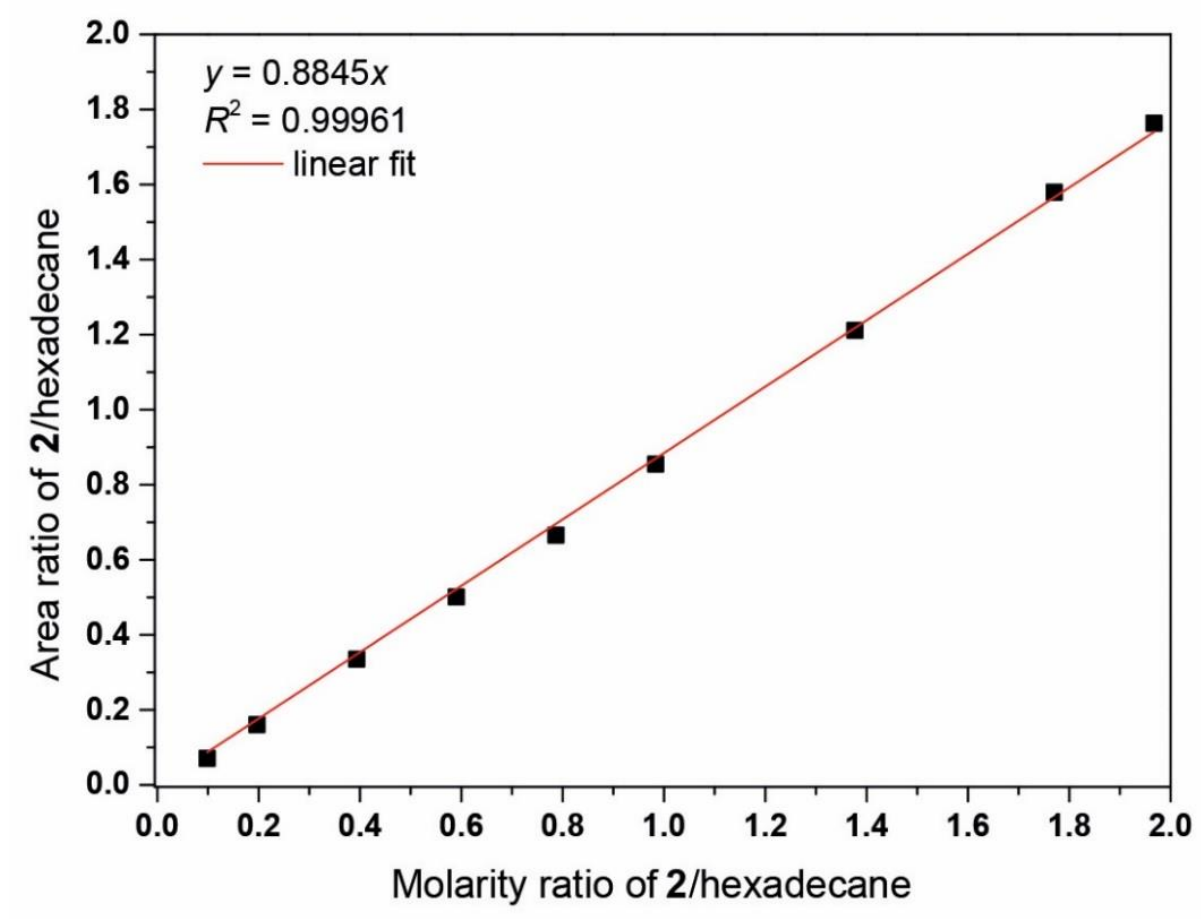

Figure S11: Calibration curve of the desired product (2) over hexadecane

\begin{tabular}{|l|l|l|l|}
\hline Time $(\mathrm{h})$ & Yield $(20 \mathrm{~mA})$ & Yield $(10 \mathrm{~mA})$ & Yield $(30 \mathrm{~mA})$ \\
\hline 0.0893 & & & 0.040559799 \\
\hline 0.134 & 0.040907032 & 0.016176911 & \\
\hline 0.1787 & & & 0.088751955 \\
\hline 0.268 & 0.086888806 & 0.035518088 & 0.139083875 \\
\hline 0.402 & 0.134602693 & 0.055328716 & \\
\hline
\end{tabular}




\begin{tabular}{|l|l|l|l|}
\hline 0.447 & & & 0.234641303 \\
\hline 0.536 & 0.174449935 & 0.075672273 & \\
\hline 0.67 & 0.226532199 & 0.098624776 & \\
\hline 0.893 & & & 0.477077308 \\
\hline 1.005 & 0.345352938 & 0.1580475 & \\
\hline 1.34 & 0.466846199 & 0.219806247 & 0.711611106 \\
\hline 1.787 & & & 0.920259485 \\
\hline 2.01 & 0.702396391 & 0.342919456 & \\
\hline 2.68 & 0.921167131 & 0.472625881 & 0.932974829 \\
\hline 2.814 & 0.940624446 & 0.4955838 & \\
\hline 3.082 & 0.954713563 & 0.5414994 & \\
\hline 3.35 & 0.954926466 & 0.587414553 & \\
\hline 3.573 & & & 0.943743258 \\
\hline 4.02 & 0.952343685 & 0.704150901 & \\
\hline 5.36 & 0.945836758 & 0.913257946 & \\
\hline 5.628 & & 0.935435304 & \\
\hline 6.164 & & 0.94047145 & \\
\hline 6.7 & & 0.944415263 & \\
\hline 8.04 & & 0.93791021 & \\
\hline 10.72 & & 0.945763031 & \\
\hline
\end{tabular}

Table S6: Yield vs reaction time at constant current conditions

\begin{tabular}{|l|l|l|l|l|l|}
\hline $10 \mathrm{~mA}$ & & $20 \mathrm{~mA}$ & & $30 \mathrm{~mA}$ & \\
\hline $\begin{array}{l}\text { Equiv } \\
\text { Charge }\end{array}$ & Yield & $\begin{array}{l}\text { Equiv } \\
\text { Charge }\end{array}$ & yield & $\begin{array}{l}\text { Equiv } \\
\text { Charge }\end{array}$ & Yield \\
\hline 0.05 & 0.016177 & 0.1 & 0.040907 & 0.1 & 0.04056 \\
\hline 0.1 & 0.035518 & 0.2 & 0.086889 & 0.2 & 0.088752 \\
\hline 0.15 & 0.055329 & 0.3 & 0.134603 & 0.3 & 0.139084 \\
\hline 0.2 & 0.075672 & 0.4 & 0.17445 & 0.5 & 0.234641 \\
\hline 0.25 & 0.098625 & 0.5 & 0.226532 & 0.75 & 0.354501 \\
\hline 0.3 & 0.12099 & 0.75 & 0.345353 & 1 & 0.477077 \\
\hline 0.4 & 0.170408 & 1 & 0.466846 & 1.5 & 0.711611 \\
\hline 0.5 & 0.219806 & 1.5 & 0.702396 & 2 & 0.920259 \\
\hline 0.75 & 0.342919 & 2 & 0.921167 & 3 & 0.932975 \\
\hline 1 & 0.472626 & 2.1 & 0.940624 & 4 & 0.943743 \\
\hline 1.25 & 0.587415 & 2.3 & 0.954714 & & \\
\hline 1.5 & 0.704151 & 2.5 & 0.954926 & & \\
\hline 1.75 & 0.818396 & 3 & 0.952344 & & \\
\hline 2 & 0.913258 & 4 & 0.945837 & & \\
\hline 2.1 & 0.935435 & & & & \\
\hline
\end{tabular}




\begin{tabular}{|l|l|l|l|l|l|}
\hline 2.2 & 0.944712 & & & & \\
\hline 2.3 & 0.940471 & & & & \\
\hline 2.5 & 0.944415 & & & & \\
\hline 3 & 0.93791 & & & & \\
\hline 3.5 & 0.935684 & & & & \\
\hline 4 & 0.945763 & & & & \\
\hline
\end{tabular}

Table S7: Yield vs charge at constant current conditions

\begin{tabular}{|l|l|l|l|}
\hline 4 equiv TMSCl & $20 \mathrm{~mA}$ & $\mathrm{Ec}=-1.0 \mathrm{~V}$ vs Mg & \\
\hline Equiv Charge & Yield & Equiv Charge & Yield \\
\hline 0.1 & 0.035769053 & 0.43 & 0.18771947 \\
\hline 0.2 & 0.081251115 & 0.51 & 0.224740323 \\
\hline 0.3 & 0.129729611 & 0.6 & 0.297500216 \\
\hline 0.4 & 0.180171506 & 0.81 & 0.366719387 \\
\hline 0.5 & 0.228345565 & 1 & 0.460719006 \\
\hline 0.75 & 0.358938821 & 1.25 & 0.584611591 \\
\hline 1 & 0.492534788 & 1.495 & 0.685584824 \\
\hline 1.5 & 0.74431985 & 1.98 & 0.92226203 \\
\hline 2 & 0.963728161 & 2.2 & 0.92962093 \\
\hline 2.1 & 0.977886046 & 2.5 & 0.927924167 \\
\hline 2.3 & 0.978691389 & & \\
\hline 2.5 & 0.973849478 & & \\
\hline 3 & 0.961691563 & & \\
\hline 4 & 0.960080342 & & \\
\hline
\end{tabular}

Table S8: Yield vs charge with 4 equivalents of TMSCl or controlled potential electrolysis. 


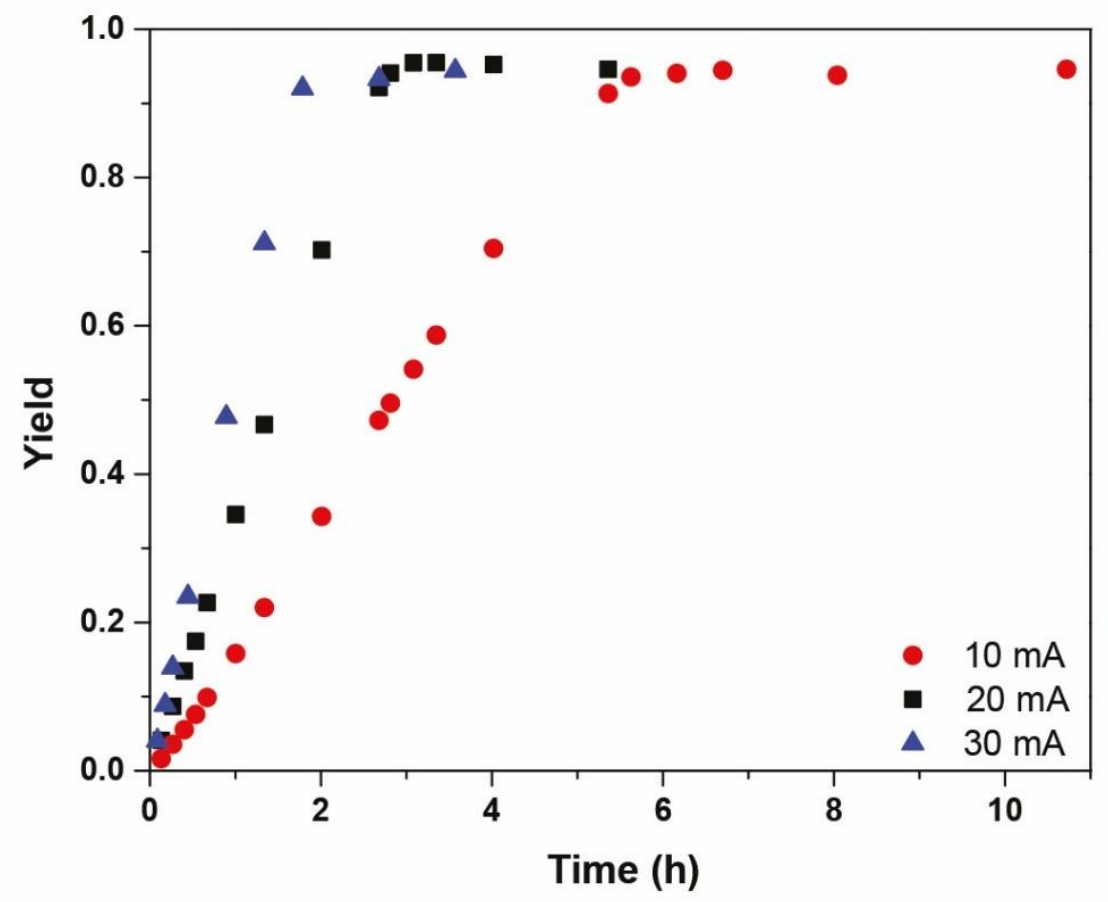

Figure S12: Yield vs reaction time at different currents

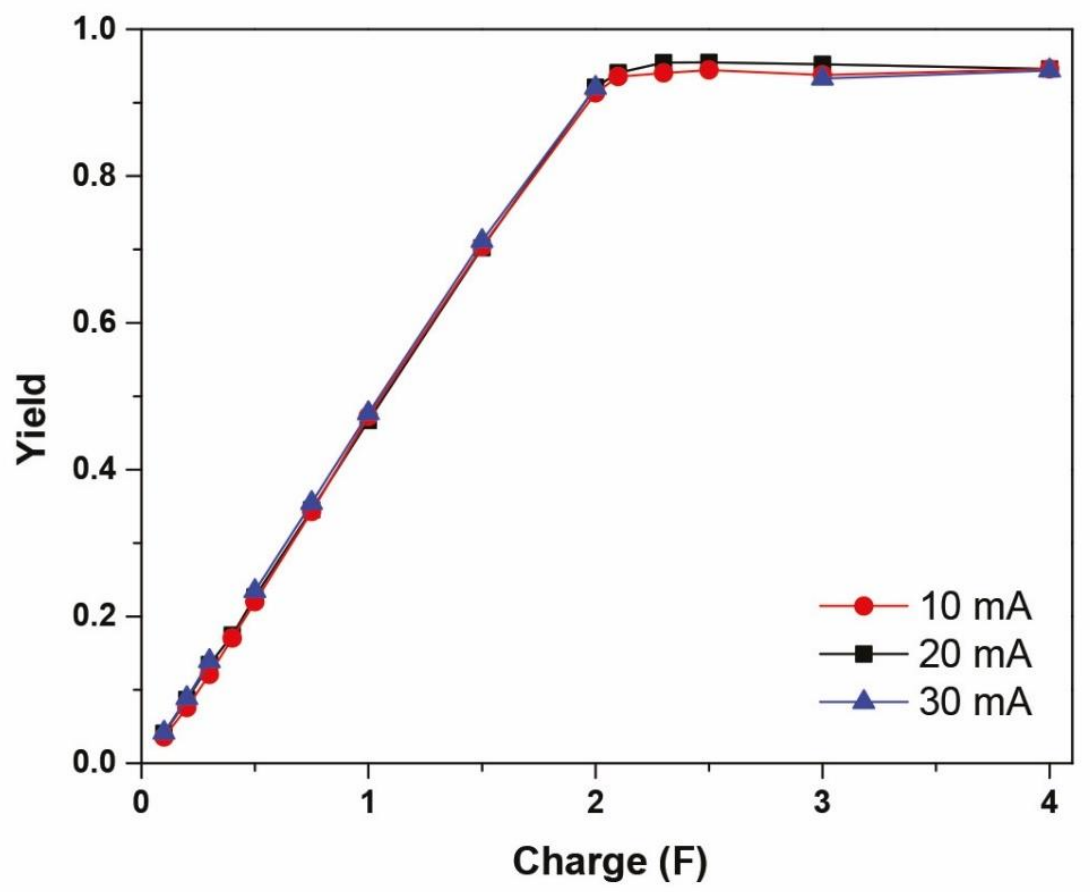

Figure S13: Yield vs charge at different currents 


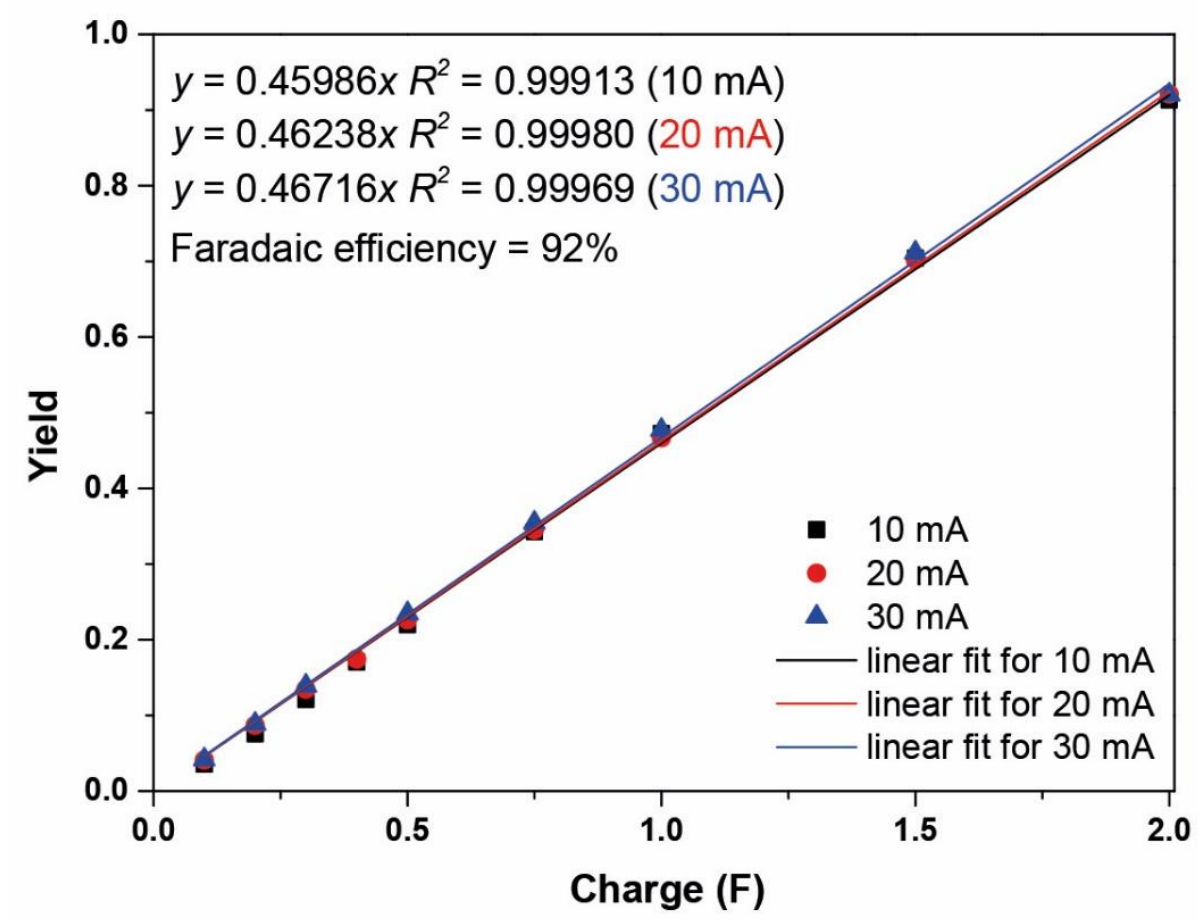

Figure S14: Calculation of Faradaic efficiency

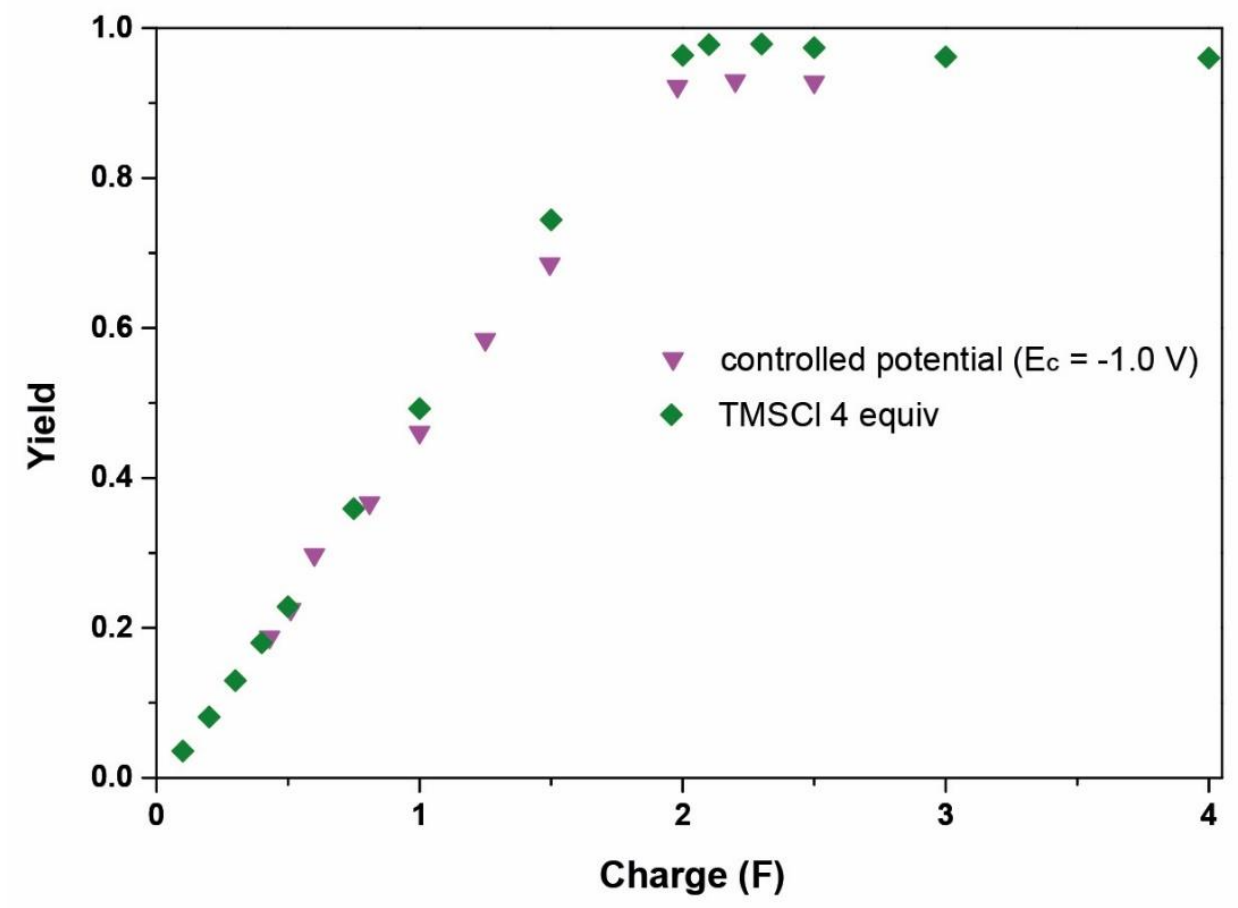

Figure S15: Yield vs charge with controlled potential or 4 equivalents of TMSCl 


\section{Hammett studies}

Hammett studies were conducted by running competing reactions of an alkene and a standard (2vinyltoluene) to around $30 \%$ overall conversion. The reactions were setup as described in GP 1 with the expectation that alkene substrate $(0.5 \mathrm{mmol})$ was added together with 2-vinyltoluene $(59.1$ $\mathrm{mg}, 0.5 \mathrm{mmol})$. The reaction was terminated when $0.6 \mathrm{~F}$ of charge passed and the yields of alkene substrate and standard were determined by ${ }^{1} \mathrm{H}-\mathrm{NMR}$ using 1,3,5-trimethoxybenzne as an internal standard. Each experiment was reproduced for at least twice and the average yield was used for Hammett plots. The rate ratio $\left(\mathrm{k}_{\mathrm{rel}}\right)$ was determined by dividing the yield of alkene by the yield of standard. The $\left(\mathrm{k}_{\mathrm{rel}}\right)$ of $\mathrm{N}, \mathrm{N}$-dimethyl-4-vinylaniline is unavailable due to the yield of desired product is less than $2 \%$.

\begin{tabular}{|l|l|l|l|l|l|l|}
\hline $\begin{array}{l}\text { Functional } \\
\text { groups }\end{array}$ & $\boldsymbol{\sigma}$ & $\boldsymbol{\sigma}^{*}$ & $\boldsymbol{\sigma}-$ & Ratio to std(2-Me styrene) & Error & $\lg \left(\mathrm{k}_{\text {rel }}\right)$ \\
\hline $\mathrm{H}$ & 0 & 0 & 0 & 1.008 & $\begin{array}{c}+/- \\
0.002\end{array}$ & 0.003460532 \\
\hline$p$-Me & -0.17 & 0.11 & -0.17 & 0.141 & $\begin{array}{c}+/- \\
0.005\end{array}$ & -0.850780887 \\
\hline$p$-OMe & -0.27 & 0.24 & -0.26 & 0.032 & $\begin{array}{l}+/- \\
0.003\end{array}$ & -1.494850022 \\
\hline$p$-F & 0.06 & -0.08 & -0.03 & 0.55 & $+/-0.03$ & -0.259637311 \\
\hline$p-\mathrm{SMe}$ & 0 & 0.43 & 0.06 & 14.5 & $+/-0.03$ & 1.161368002 \\
\hline$p$-Ph & -0.01 & 0.46 & 0.02 & 33 & $+/-8$ & 1.51851394 \\
\hline$p$-tBu & -0.2 & 0.13 & -0.13 & 0.139 & $\begin{array}{l}+/- \\
0.002\end{array}$ & -0.8569852 \\
\hline$m-\mathrm{OMe}$ & 0.12 & -0.02 & & 1.42 & $+/-0.02$ & 0.152288344 \\
\hline$m-\mathrm{F}$ & 0.34 & -0.05 & & 50 & $+/-10$ & 1.698970004 \\
\hline$p-\mathrm{NMe} 2$ & -0.83 & & & $<0.02$ & $\mathrm{NA}$ & $\mathrm{NA}$ \\
\hline
\end{tabular}

Table S9: Results of Hammett studies ${ }^{10,11}$ 

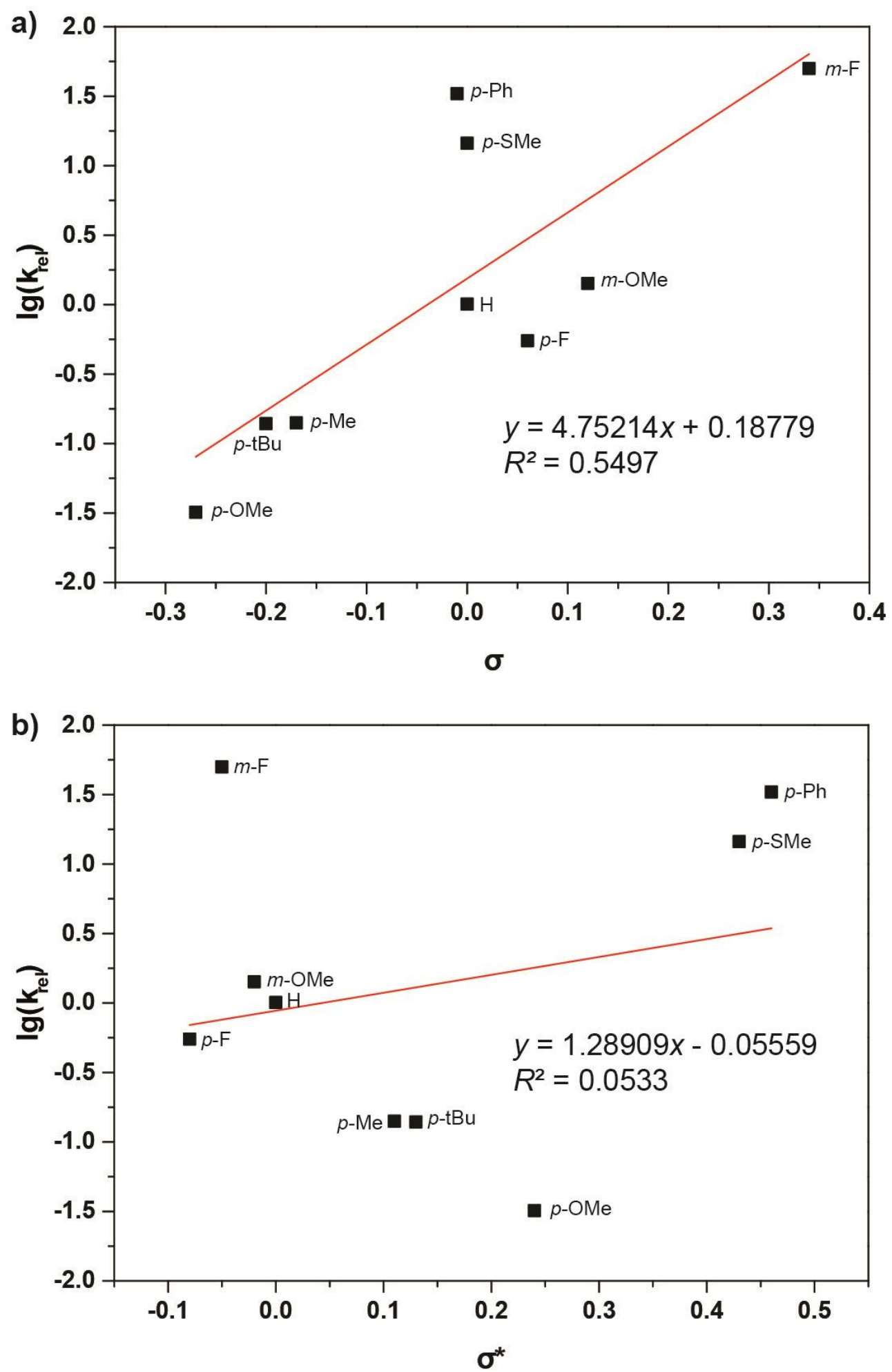

Figure S16: Hammett plot with (a) $\sigma$ and (b) $\sigma^{*}$ values. The two outliner data points (p-Ph, p$\mathrm{SMe}$ ) in the top figure are radical stabilizing substituents with large $\sigma^{*}$ values. 


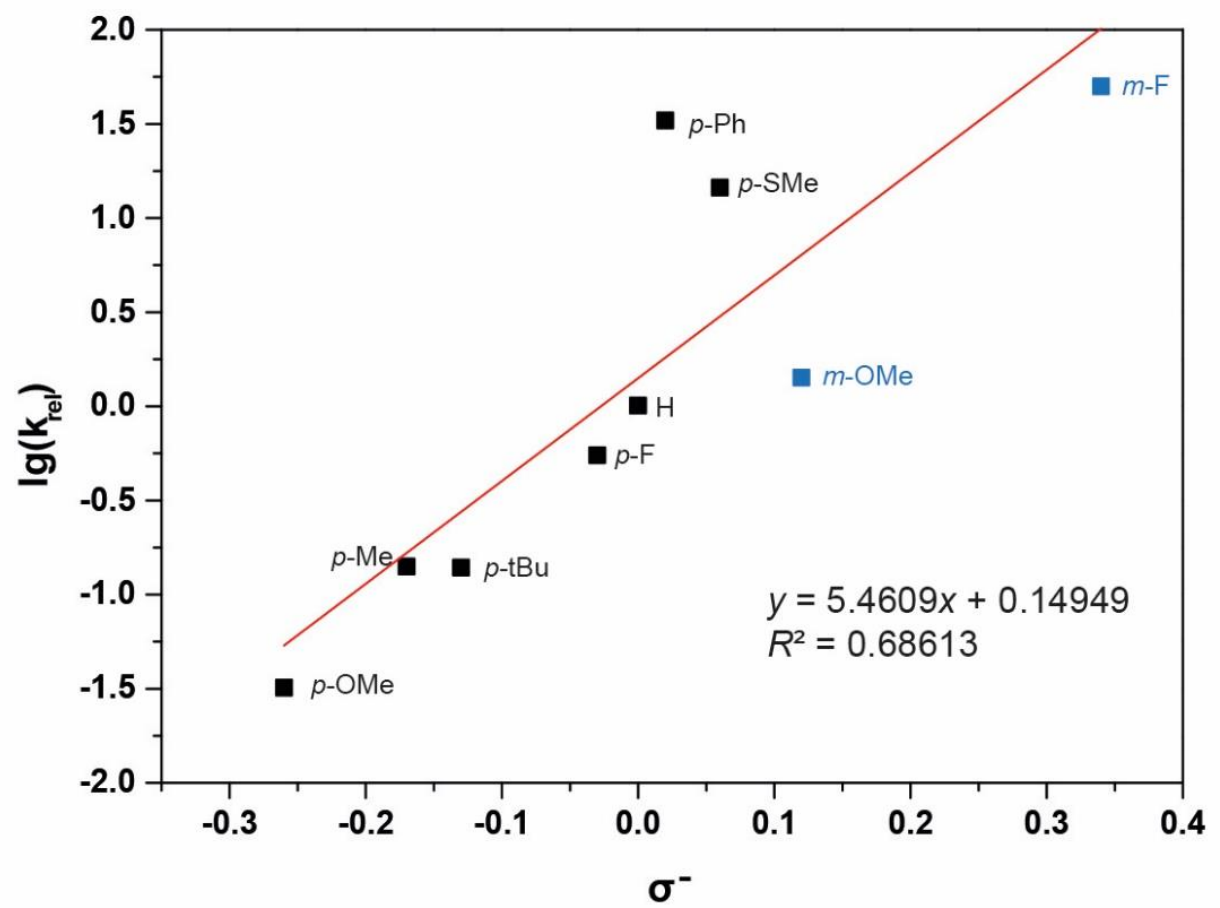

Figure S17: Hammett plot with $\sigma^{-}$values. For meta-substituents (blue dots), $\sigma^{-}$value is unavailable, thus $\sigma$ values of $\mathrm{m}-\mathrm{OMe}$ and $\mathrm{m}-\mathrm{F}$ (assuming meta-position does not have resonance effect) are used for the Hammett plot. If the $\sigma^{-}$values of $\mathrm{m}-\mathrm{OMe}$ and $\mathrm{m}-\mathrm{F}$ are calculated based on the pKa of phenols, the correlation $\left(\mathrm{R}^{2}\right)$ is worse.

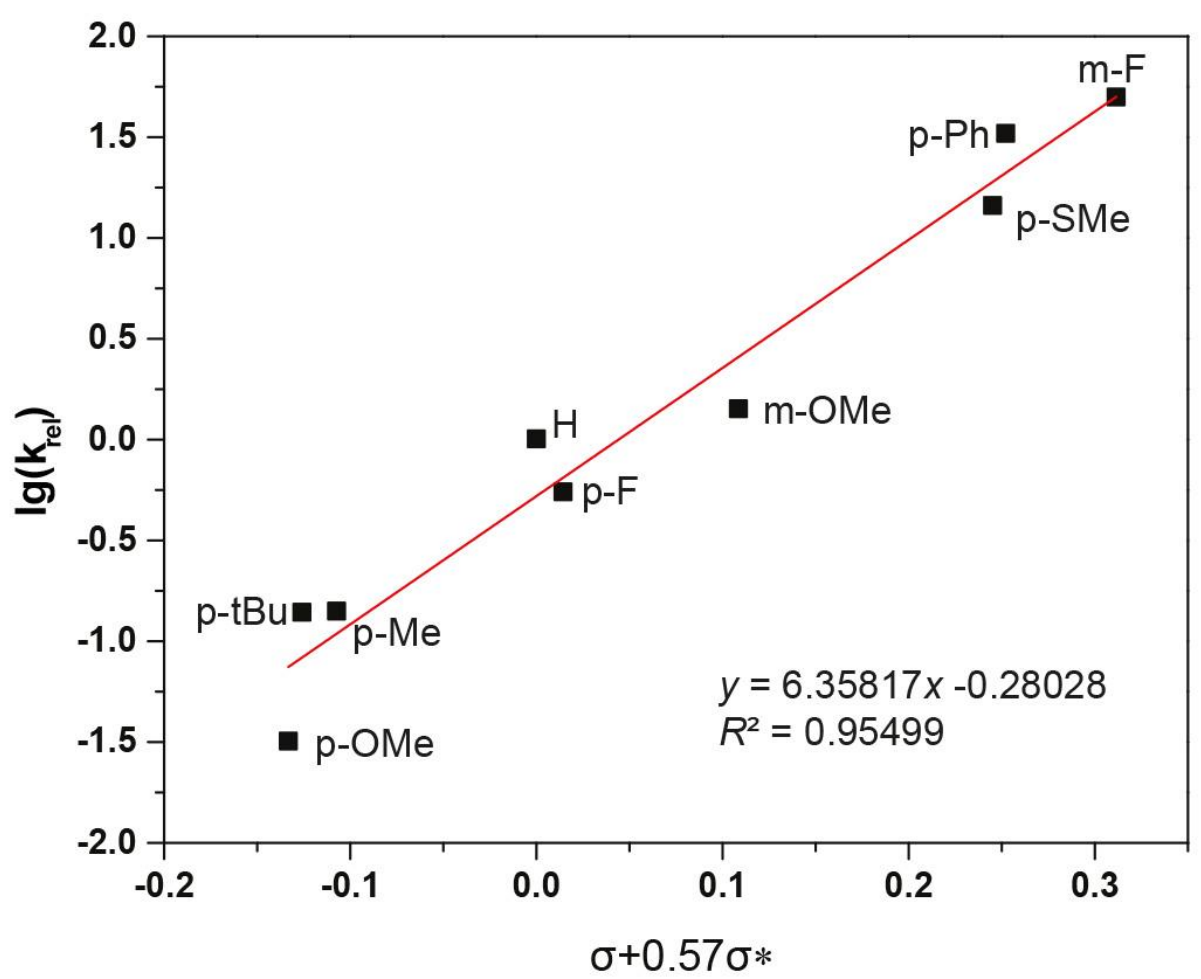

Figure S18: Hammett plot with the combination of $\sigma$ and $\sigma^{*}$ 


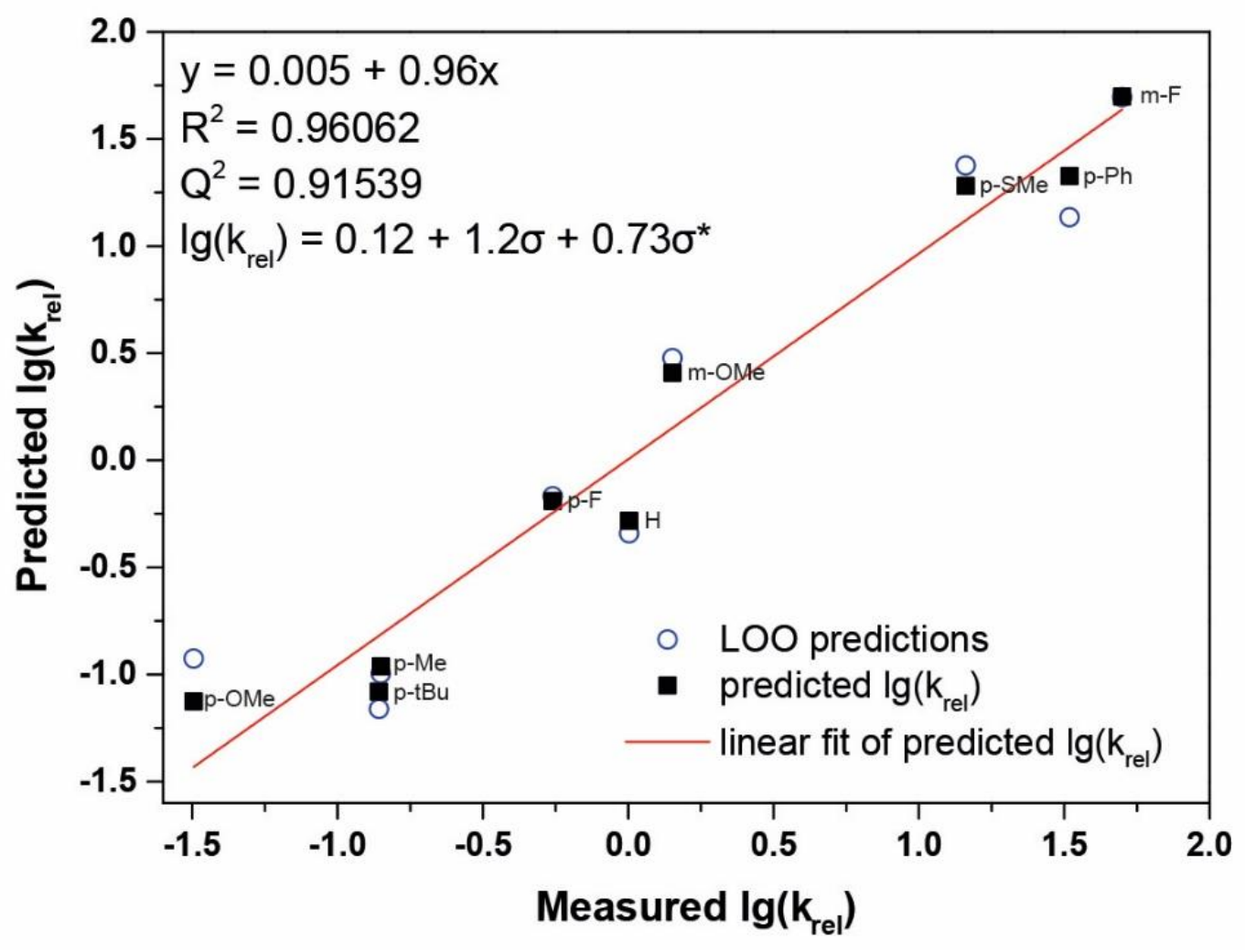

Figure S19: Hammett plot using multiple linear regression model.

Models were validated by LOO cross-validation techniques, which is an iterative process in which a single data point is excluded, and the remaining data points are used to predict the excluded point. This is done for every data point and the plotted predicted values are fit with a linear regression to give the $\mathrm{R}^{2}$ value, which is referred to as $\mathrm{Q}^{2}$. 


\section{Proposed Mechanisms}

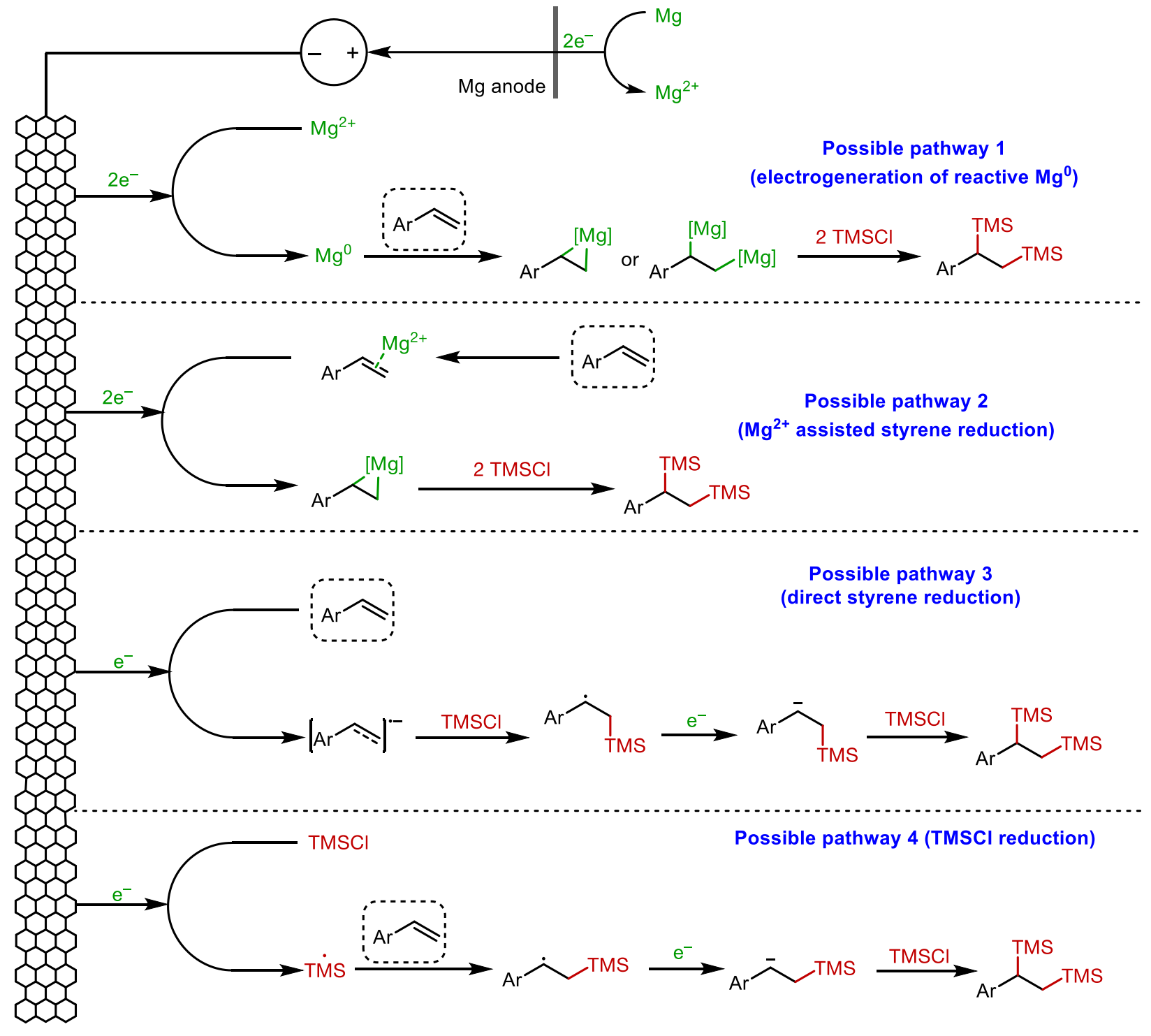

C cathode

Scheme S6: Proposed possible mechanisms 


\section{Product Derivatization}

\section{I) Electrophilic fluorination of allyl silanes (derivatization procedure DP1)}

Following a modified procedure of a reported literature. ${ }^{12}$ To a $4 \mathrm{~mL}$ vial equipped with a stir bar was added allyl silane $\left(0.2 \mathrm{mmol}, 1.0\right.$ equiv) and Selectfluor ${ }^{\circledR}$ fluorination reagent $(0.22 \mathrm{mmol}$, $80.0 \mathrm{mg}, 1.1$ equiv). The vial was purged with $\mathrm{N}_{2}$ for $5 \mathrm{~min}$ and $2 \mathrm{~mL}$ anhydrous $\mathrm{MeCN}$ was added and stirred at room temperature for 2 hours (reaction completion monitored by TLC). The reaction crude was added to $10 \mathrm{~mL}$ hexanes, passed through a plug of silica $(5 \mathrm{~cm}$ deep in a pipet) and flushed with $20 \mathrm{~mL} \mathrm{10 \%} \mathrm{diethyl} \mathrm{ether/hexanes} \mathrm{solution.} \mathrm{The} \mathrm{filtrate} \mathrm{was} \mathrm{concentrated} \mathrm{in} \mathrm{vacuum}$ and purified via column chromatography. For NMR spectrum of allyl fluorides, see section 10 and 11 .

$$
\begin{aligned}
& \mathrm{RiR}_{3}^{\prime} \frac{\text { Selectfluoro }^{\circledR} 1.1 \text { equiv, MeCN }}{22^{\circ} \mathrm{C}, 2 \mathrm{~h}} \longrightarrow \\
& \text { 35, } R=P h, R^{\prime}=M e \\
& \text { 71, 67\% ( } \mathrm{R}=\mathrm{Ph}) \\
& \text { 62, } \mathrm{R}=\mathrm{CH}_{2} \mathrm{OSiEt}_{3}, \mathrm{R}^{\prime}=\mathrm{Et} \\
& \text { 72, } 79 \%\left(\mathrm{R}=\mathrm{CH}_{2} \mathrm{OSiEt}_{3}\right)
\end{aligned}
$$

Scheme S7: Fluorination of allyl silanes

\section{II) Hydrolysis of silyl hydrides (DP2)}

Following a reported procedure ${ }^{13}$. To a $4 \mathrm{~mL}$ vial equipped with a stir bar was added water $(8$ mmol, 10 equiv, $90 \mu \mathrm{L}),\left[\mathrm{Rh}(\mathrm{CO})_{2} \mathrm{Cl}\right]_{2}(0.0016 \mathrm{mmol}, 0.62 \mathrm{mg}, 2 \mathrm{~mol} \%)$, THF $(2 \mathrm{~mL})$. The vial was purged under $\mathrm{N}_{2}$ for $5 \mathrm{~min}$ before the addition of silanes via syringe $(0.4 \mathrm{mmol}$ substrate, 0.8 $\mathrm{mmol} \mathrm{Si}-\mathrm{H}$ bond, 1 equiv). Upon the addition of silanes, hydrogen evolution was observed and they reaction was quench with $2 \mathrm{~mL}$ DCM after $1 \mathrm{~h}$. The reaction mixture was then passed through a pipet with $\mathrm{MgSO}_{4}$ and celite to remove water and precipitate. The pipet was rinsed with $20 \mathrm{~mL}$ of diethyl ether, and the filtrate was concentrated under vacuum and purified via column chromatography. For the NNR spectrum of silyl hydroxide compounds, see section 10 and 11.

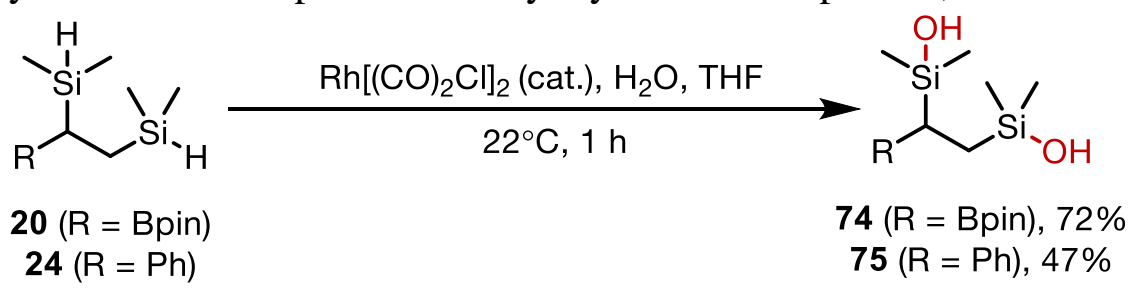

Scheme S8: Hydrolysis of Si-H bond

\section{III) Oxidation of bis(pinacol) ester (DP3)}

Following a literature procedure. ${ }^{14}$ In a $20 \mathrm{~mL}$ scintillation vial equipped with a stir bar and (1(4,4,5,5-tetramethyl-1,3,2-dioxaborolan-2-yl)ethane-1,2-diyl)bis(triethylsilane) $(0.2 \mathrm{mmol}, 73.6$ $\mathrm{mg}$ ) was added THF $(6 \mathrm{~mL})$. The reaction vial was cooled to $0^{\circ} \mathrm{C}$, charged with $3 \mathrm{M}$ sodium hydroxide $(1.0 \mathrm{~mL})$ and $30 \%$ hydrogen peroxide $1.0 \mathrm{~mL}$ ) and allowed to stir at room temperature 
for 3 hours. The vial was then cooled to $0^{\circ} \mathrm{C}$ and quenched with sodium thiosulfate $(0.50 \mathrm{~mL})$. The reaction solution was diluted with diethyl ether, and both organic and aqueous phase was passed through a silica gel and $\mathrm{MgSO}_{4}$ large volume pipet to remove the aqueous layer. The organic extract was concentrated under reduced pressure, and the crude reaction mixture purified via column chromatography. For the NNR spectrum of the final product, see section 10 and 11.

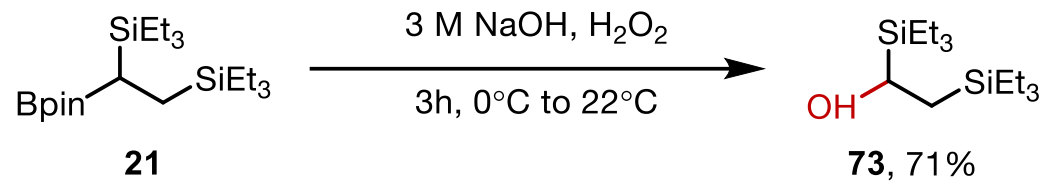

Scheme S9: Oxidation of bis(pinacol) ester.

\section{IV) Tamao-Fleming oxidation of C-Si bond (DP4)}

Following a modified reported procedure ${ }^{15}$. To a $25 \mathrm{~mL}$ round bottom flask equipped with a stir bar was added potassium fluoride (KF, $8 \mathrm{mmol}, 450 \mathrm{mg}, 8$ equiv), potassium bicarbonate $\left(\mathrm{KHCO}_{3}\right.$, $9 \mathrm{mmol}, 900 \mathrm{mg}, 9$ equiv) and $12 \mathrm{~mL}$ of $\mathrm{THF} / \mathrm{MeOH}(1 / 1)$. The suspension was cooled to $0^{\circ} \mathrm{C}$, following the addition of (1-phenylethane-1,2-diyl)bis(dimethylsilane) (1 mmol, $222 \mathrm{mg}, 1$ equiv) and $5 \mathrm{~mL}$ of $\mathrm{H}_{2} \mathrm{O}_{2}$. The reaction was allowed to warm to room temperature and stirred at $50^{\circ} \mathrm{C}$ overnight before quenching with $7 \mathrm{~mL}$ of saturated $\mathrm{Na}_{2} \mathrm{~S}_{2} \mathrm{O}_{3}$ solution. The mixture was transferred to a $125 \mathrm{~mL}$ separation funnel and was extracted with diethyl ether $(25 \mathrm{~mL} \times 3)$, washed with saturated $\mathrm{NaCl}$ solution, dried over $\mathrm{MgSO}_{4}$. The solution was filtrated, concentrated under vacuum and purified via column chromatography. Product is a white solid and the ${ }^{1} \mathrm{H} /{ }^{13} \mathrm{C} \mathrm{NMR}$ spectrum is consistent with reported literatures (see section 10 and 11 for details).

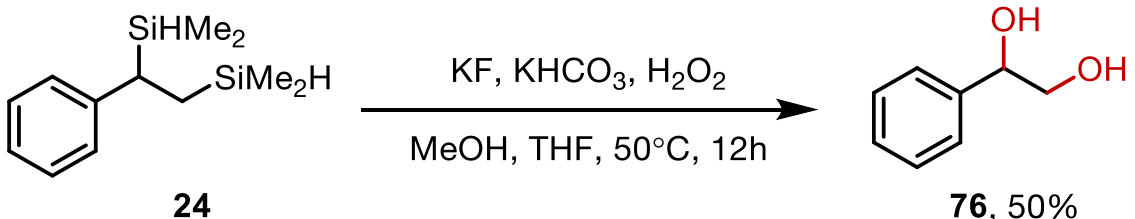

Scheme S10: Tamao-Fleming oxidation of C-Si bond.

\section{V) Electrooxidation of disilanes}

Following a literature procedure. ${ }^{16}$ To a $5 \mathrm{~mL}$ of Electrasyn vial equipped with a stir bar was added tetraethylammonium p-toluenesulfonate (TBAOTs, $1.20 \mathrm{~g}$ ), silane $(1.0 \mathrm{mmol}, 250 \mathrm{mg})$ and $4 \mathrm{~mL}$ $\mathrm{MeOH}$. Electrolysis was performed with graphite as both anode and cathode with a constant current of $5 \mathrm{~mA}$ and the Electrasyn vial was purged with $\mathrm{N}_{2}$ for $5 \mathrm{~min}$ before electrolysis. After electrolysis completion, the solution was poured to $10 \mathrm{~mL}$ mixture of n-pentane/diethyl ether (1/1) to precipitate the electrolyte out. The mixture was then filtrated through a silica plug $(4 \mathrm{~cm}$ length, $5 \mathrm{~g}$ ) and flushed with $50 \mathrm{~mL} 10 \%$ diethyl ether/pentane. The filtrate was concentrated under reduced pressure (200 mbar) and added with internal standard. ${ }^{1} \mathrm{H}$ NMR revealed $51 \%$ yield of styrene with $13 \%$ methoxy substituted product (internal standard, dibromomethane, $\delta=4.90 \mathrm{ppm}$ ). ${ }^{1} \mathbf{H}$ NMR $\left(500 \mathrm{MHz}, \mathrm{CDCl}_{3}\right)$ : styrene (1), $\delta 6.71(\mathrm{dd}, \mathrm{J}=17.6,10.9 \mathrm{~Hz}, 1 \mathrm{H}), 5.74(\mathrm{dd}, \mathrm{J}=17.7$, $1.0 \mathrm{~Hz}, 1 \mathrm{H}), 5.23(\mathrm{dd}, \mathrm{J}=10.8,1.0 \mathrm{~Hz}, 1 \mathrm{H})$; internal standard, $4.90(\mathrm{~s}, 2 \mathrm{H})$; substitution product: $4.19(\mathrm{t}, \mathrm{J}=7.6 \mathrm{~Hz}, 1 \mathrm{H}), 3.12(\mathrm{~s}, 3 \mathrm{H}), 1.36-1.24(\mathrm{~m}, 2 \mathrm{H}),-0.09(\mathrm{~s}, 9 \mathrm{H})$. 


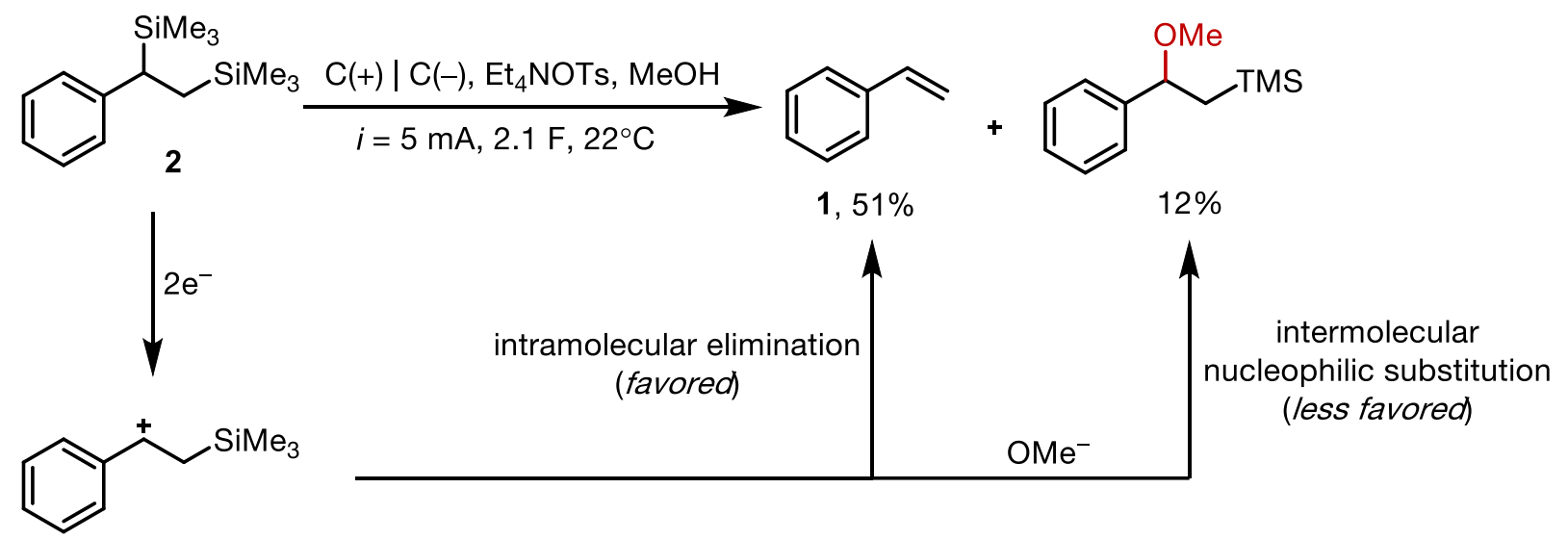

$\beta$-Silicon effect

stablized carbon cation

Scheme S11: Electrochemical oxidation of benzylic C-Si

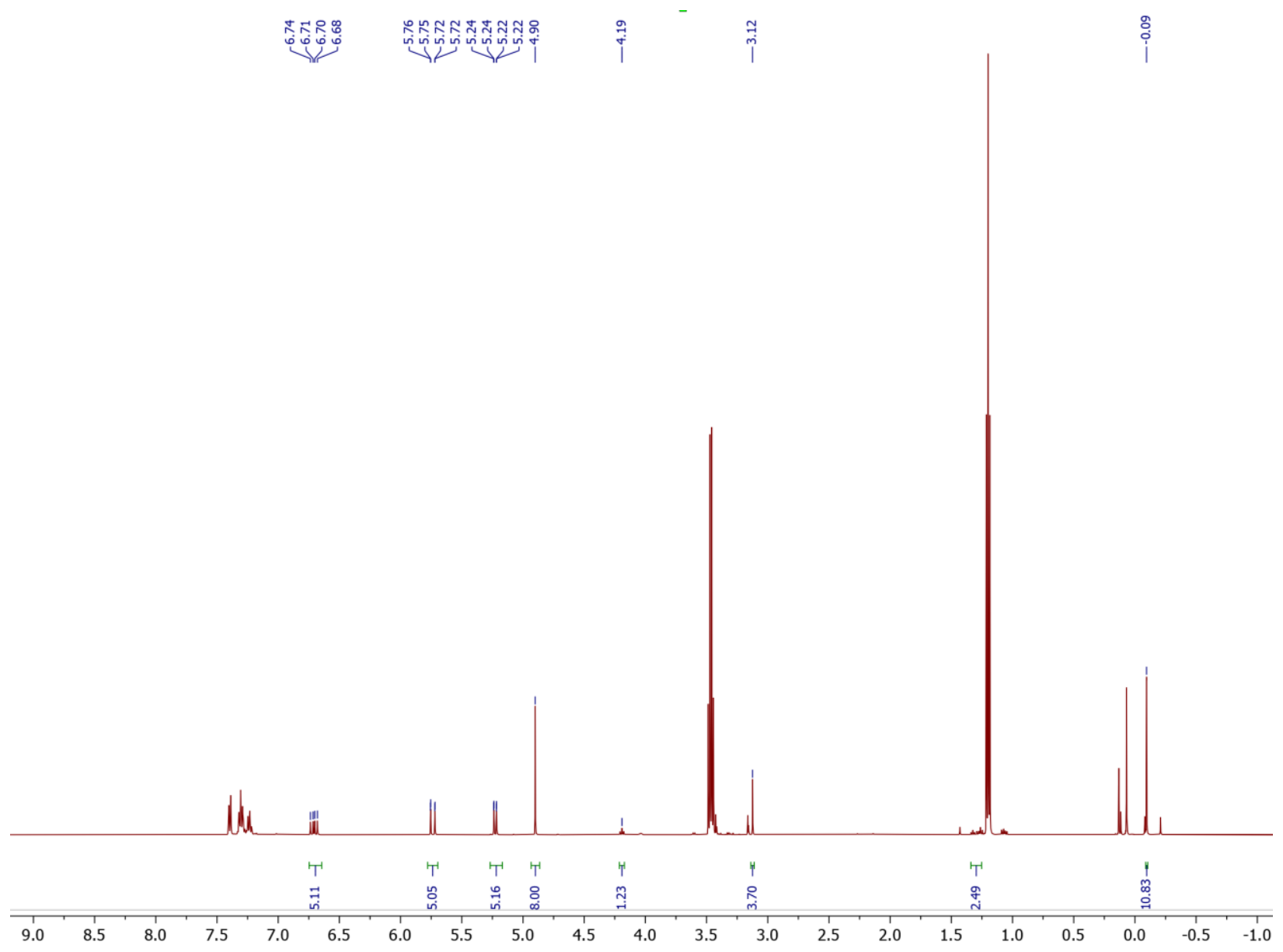

Figure S20: Crude ${ }^{1} \mathrm{H}$ NMR of C-Si oxidation 


\section{Synthesis of substrates}

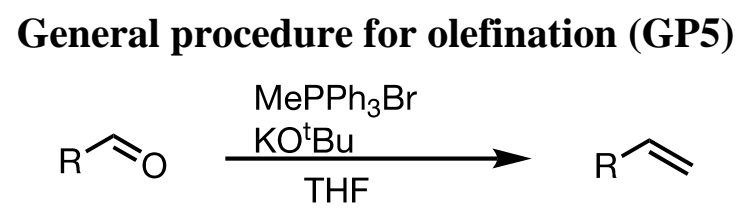

To an oven dried round bottom flask equipped with a stir bar under a $\mathrm{N}_{2}(\mathrm{~g})$ atmosphere was added $\mathrm{MePPh} 3 \mathrm{Br}$ (1.60 eq.). The flask was evacuated and back filled with $\mathrm{N}_{2}(\mathrm{~g})$ and dry THF $(0.1 \mathrm{M})$ was added. Then potassium tert-butoxide (1.6 eq.) was added. After stirring for $30 \mathrm{mins}$, the ketone substrate was added (1.0 eq.). The reaction was allowed to stir at $\mathrm{rt}$ for $18 \mathrm{~h}$ before being diluted with hexane $(30 \mathrm{~mL})$ and filtered through a plug of silica $(5 \mathrm{~cm}$ deep). The crude can sometimes be used directly without further purification or it can be purified via column chromatography.<smiles>C=Cc1ccc(-c2ccccc2)cc1</smiles>

Following GP5 with [1,1'-biphenyl]-4-carbaldehyde. The crude was purified with hexanes andobtained as a white solid $(1.65 \mathrm{~g}, 92 \%)$. The NMR spectrum is consistent with literature reports.

methyl(4-vinylphenyl)sulfane<smiles>C=Cc1ccc(SC)cc1</smiles>

Following GP5 with 4-(methylthio)benzaldehyde. The crude was purified with 5\% EA/Hex and obtained as a light-yellow oil $(1.40 \mathrm{~g}, 95 \%)$. The NMR spectrum is consistent with literature reports.

\section{5-vinylbenzo $[d][1,3]$ dioxole<smiles>C=Cc1ccc2c(c1)OCO2</smiles>

Following GP5 with benzo[ $d][1,3]$ dioxole-5-carbaldehyde. The crude was purified with $5 \%$ EA/Hex and obtained as a colorless oil $(1.04 \mathrm{~g}, 70 \%)$. The NMR spectrum is consistent with literature reports.

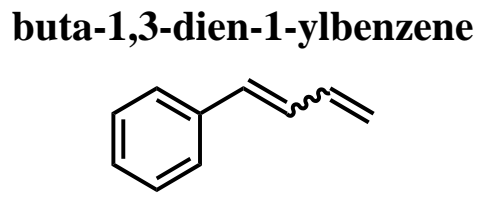

Following GP5 with 3-phenylacrylaldehyde. The crude was purified with hexanes and obtained as a colorless oil $(820 \mathrm{mg}, 63 \%)$. The NMR spectrum is consistent with literature reports. 


\section{$\mathrm{N}, \mathrm{N}$-dimethyl-4-vinylaniline}<smiles>C=Cc1ccc(N(C)C)cc1</smiles>

Following GP5 with 4-(dimethylamino)benzaldehyde. The crude was purified with 3\% EA, 1\% TEA/Hex and obtained as a colorless oil (1.14 g, 78\%). The NMR spectrum is consistent with literature reports.

\section{2-methyl-6-vinylpyridine<smiles>C=Cc1cccc(C)n1</smiles>

Following GP5 with 6-methylpicolinaldehyde. The crude was purified with 5\% EA, 1\% TEA/Hex and obtained as a light-yellow oil ( $775 \mathrm{mg}, 65 \%)$. The NMR spectrum is consistent with literature reports.

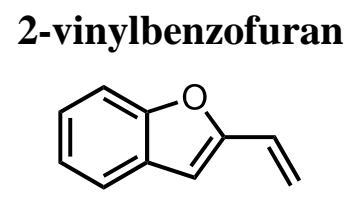

Following GP5 with benzofuran-2-carbaldehyde. The crude was purified with 5\% EA/Hex and obtained as a colorless oil ( $995 \mathrm{mg}, 69 \%)$. The NMR spectrum is consistent with literature reports.

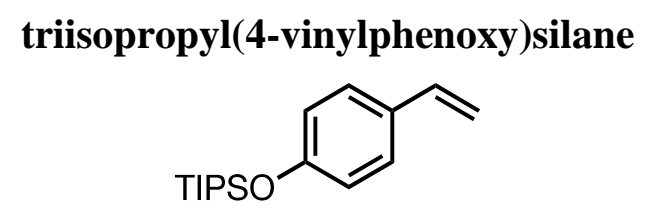

Following GP5 with 4-((triisopropylsilyl)oxy)benzaldehyde. The crude was purified with 5\% EA/Hex and obtained as a colorless oil $(1.94 \mathrm{~g}, 70 \%)$. The NMR spectrum is consistent with literature reports.

\section{((2-methylenepropane-1,3-diyl)bis(oxy))dibenzene<smiles>C=C(COc1ccccc1)COc1ccccc1</smiles>

Following a modified reported procedure with 4 equivalents of phenol and sodium hydroxide added. ${ }^{17}$ The crude was purified with 3\% 5\% EA/Hex and obtained as a colorless oil (1.02 g, $43 \%$ ). The NMR spectrum is consistent with literature reports.

propa-1,2-dien-1-ylbenzene 


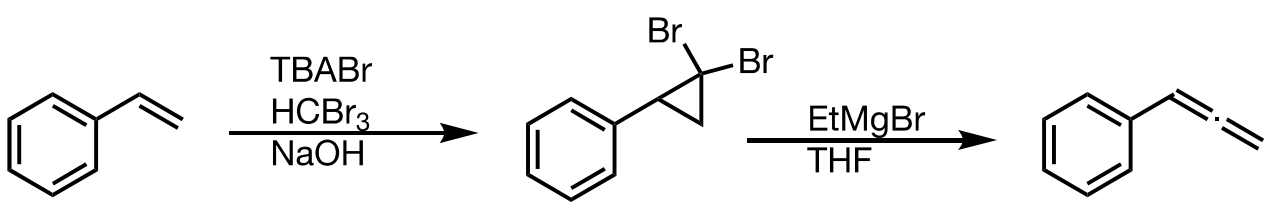

Following a standard procedure starting from styrene ${ }^{18}$, the allene was obtained as a clear colorless oil (540 mg, 75\%) over two steps. ${ }^{1} \mathbf{H}$ NMR $\left(300 \mathrm{MHz}, \mathrm{CDCl}_{3}\right) \delta 7.30(\mathrm{~s}, 4 \mathrm{H}), 7.20(\mathrm{dt}, J=8.7$, $4.1 \mathrm{~Hz}, 1 \mathrm{H}), 6.17(\mathrm{t}, J=6.8 \mathrm{~Hz}, 1 \mathrm{H}), 5.15(\mathrm{~d}, J=6.8 \mathrm{~Hz}, 2 \mathrm{H})$. The NMR spectrum is consistent with literature reports.

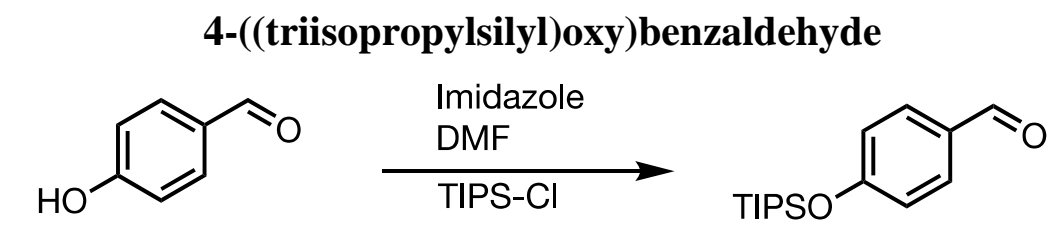

Following a reported procedure. ${ }^{19}$ The titled compound was obtained as a colorless oil (4.12 g, 99\%). The NMR spectrum is consistent with literature reports.

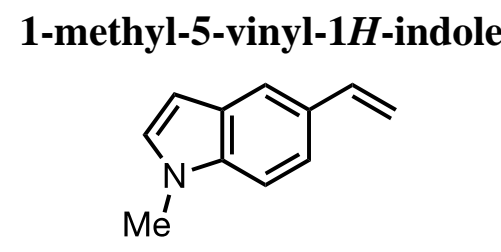

Following a reported procedure. ${ }^{20} \mathrm{~A}$ white solid was obtained (629 mg, 40\%). The NMR spectrum is consistent with literature reports.

\section{2-(4-vinylphenyl)ethan-1-ol}<smiles>C=Cc1ccc(CCO)cc1</smiles>

Following a reported procedure. ${ }^{21}$ A clear light-yellow liquid was obtained $(1.08 \mathrm{~g}, 74 \%)$. The NMR spectrum is consistent with literature reports.

\section{Scheme S12}

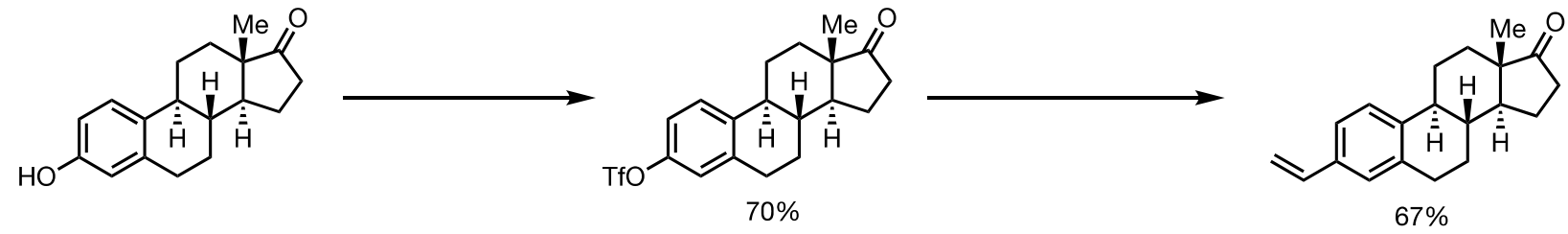

$(8 R, 9 S, 13 S, 14 S)-13-m e t h y l-17-0 x 0-7,8,9,11,12,13,14,15,16,17-d e c a h y d r o-6 H$ cyclopenta $[a]$ phenanthren-3-yl trifluoromethanesulfonate 
Step 1: Following a reported procedure. ${ }^{22}$ A white solid was obtained (2.82 g, 70\%). The NMR spectrum is consistent with literature reports.

$(8 R, 9 S, 13 S, 14 S)$-13-methyl-3-vinyl-6,7,8,9,11,12,13,14,15,16-decahydro-17Hcyclopenta[a]phenanthren-17-one

Step 2: Following a reported procedure. ${ }^{23}$ A white solid was obtained (1.32 g, 67\%). The NMR spectrum is consistent with literature reports.

\section{Synthesis of 3-methyleneoxetane}

\section{Scheme S13}
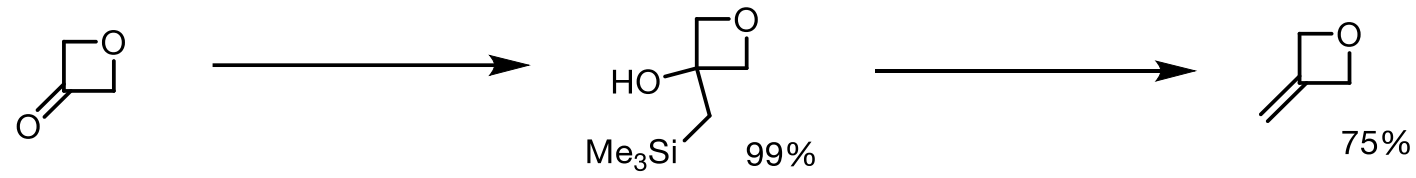

Step 1: Following a reported procedure. ${ }^{24}$ A colorless liquid was obtained (2.38 g, 99\%). The NMR spectrum is consistent with literature reports.

Step 2: Following a reported procedure. ${ }^{20}$ A clear colorless solution was obtained $(75 \%)$ and the concentration was determined to be $0.74 \mathrm{M}$ using dichloromethane as internal standard. The NMR spectrum is consistent with literature reports.

\section{Scheme S14}

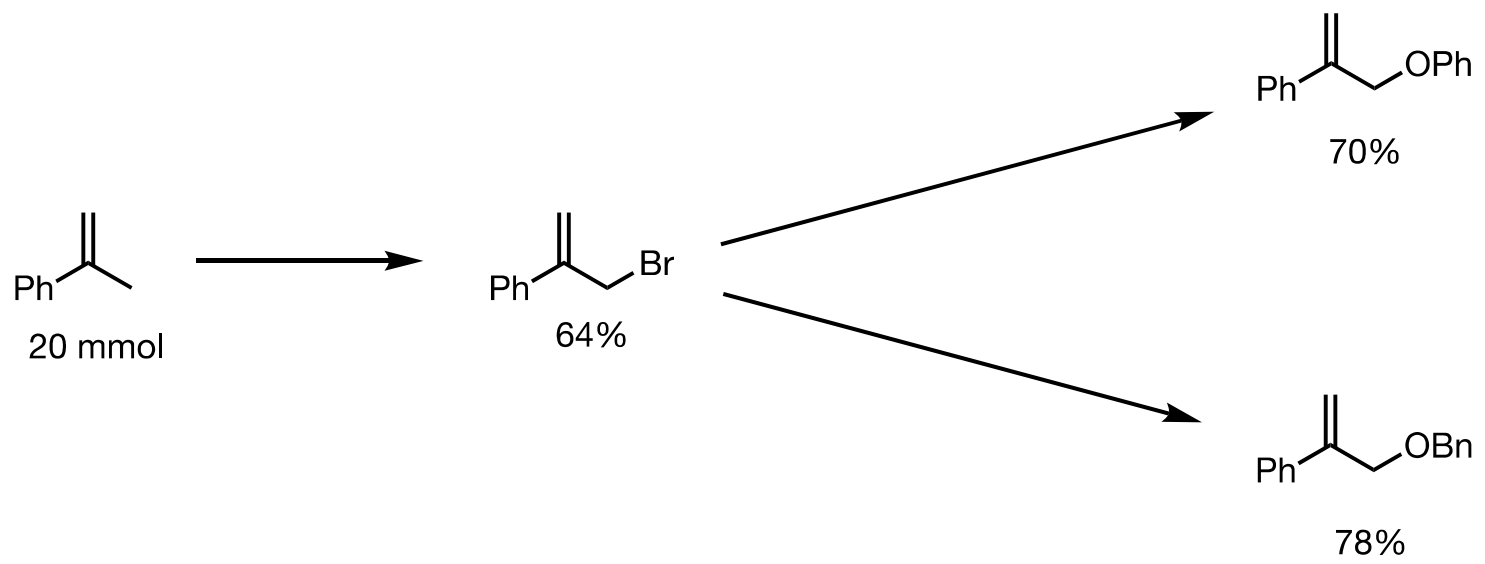

(3-bromoprop-1-en-2-yl)benzene

Step 1: Following a reported procedure. ${ }^{25}$ A clear liquid was obtained $(2.52 \mathrm{~g}, 64 \%)$. The NMR spectrum is consistent with literature reports.

\section{(3-phenoxyprop-1-en-2-yl)benzene}

Step 2: Following a reported procedure. ${ }^{26}$ A clear liquid was obtained (941 mg, 70\%). The NMR spectrum is consistent with literature reports. 


\section{(3-(benzyloxy)prop-1-en-2-yl)benzene}

Step 3: Following a reported procedure. ${ }^{27}$ A clear liquid was obtained (1.13 g, 78\%). The NMR spectrum is consistent with literature reports.

Scheme S15

\section{Synthesis of (2-vinylcyclopropyl)benzene}<smiles>C=CCC1CC1c1ccccc1</smiles>

Step 1,2,3: Following a reported procedure. ${ }^{28}$ A clear liquid was obtained (497 mg, 34\%). The NMR spectrum is consistent with literature reports.

\section{Synthesis of 1,4-dichloro-1,1,2,2,3,3,4,4-octamethyltetrasilane and 1,3-dichloro-1,1,2,2,3,3- hexamethyltrisilane}

\section{Scheme S16}

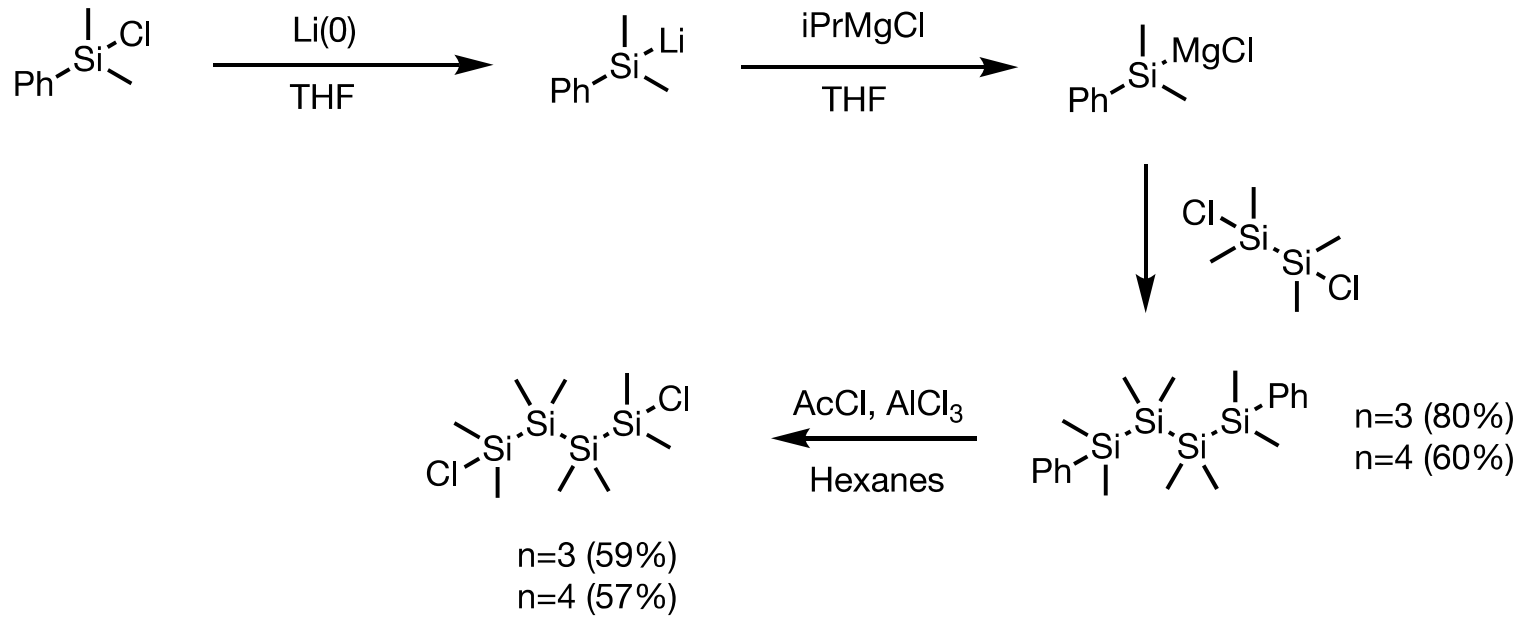

Step 1

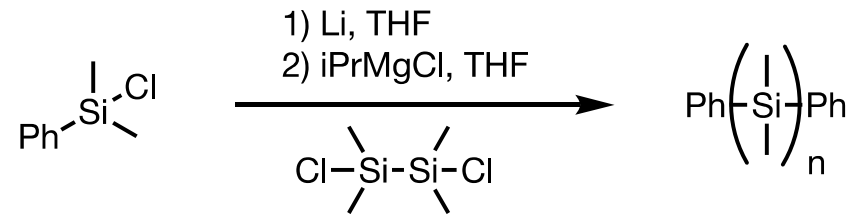

Following reported procedure ${ }^{29}$, the crude products were used directly in the next step without purification.

\section{Step 2}

$$
\mathrm{Ph}\left(\begin{array}{c}
\mathrm{I} \\
\mathrm{Si} \\
\mathrm{I}
\end{array}\right)_{\mathrm{n}} \mathrm{Ph} \underset{\text { Hexanes }}{\stackrel{\mathrm{AcCl}, \mathrm{AlCl}_{3}}{\longrightarrow}} \mathrm{Cl}\left(\begin{array}{c}
\mathrm{I} \\
\mathrm{Si} \\
\mathrm{l}
\end{array}\right)_{\mathrm{n}}^{\mathrm{Cl}}
$$


Following reported procedure ${ }^{30}, 1,4$-dichloro-1,1,2,2,3,3,4,4-octamethyltetrasilane $(57 \%)$ and 1,3dichloro-1,1,2,2,3,3-hexamethyltrisilane (59\%) were obtained as colorless oils. The products were purified by a very gentle Kugelrohr vacuum distillation. When $n=3{ }^{1} \mathbf{H}$ NMR $\left(400 \mathrm{MHz}, \mathrm{C}_{6} \mathrm{D}_{6}\right) \delta$ 0.39 (s, 12H), 0.15 (s, 6H). When n =4 ${ }^{\mathbf{1}} \mathbf{H}$ NMR (400 MHz, $\left.\mathrm{C}_{6} \mathrm{D}_{6}\right) \delta 0.40(\mathrm{~s}, 12 \mathrm{H}), 0.20(\mathrm{~s}, 12 \mathrm{H})$.

\section{((2-((benzyloxy)methyl)allyl)oxy)benzene}<smiles>C=C(COc1ccccc1)COc1ccccc1</smiles>

Following a literature procedure. ${ }^{31,32}$ A clear colorless oil was obtained $(810 \mathrm{mg}, 32 \%)$. The NMR spectrum is consistent with literature reports.

\section{4-vinyltetrahydro-2H-pyran-4-ol}<smiles>C=CC1(O)CCOCC1</smiles>

Following a literature procedure. ${ }^{33}$ A clear colorless oil was obtained (917 mg, 71\%). The NMR spectrum is consistent with literature reports.

\section{4-vinyltetrahydro-2H-thiopyran-4-ol}<smiles>C=CC1(O)CCSCC1</smiles>

Synthesized from the same procedure as 4-vinyltetrahydro-2H-pyran-4-ol. A clear colorless oil was obtained (730 mg, 51\%). ${ }^{\mathbf{1}} \mathbf{H}$ NMR $\left(400 \mathrm{MHz}, \mathrm{CDCl}_{3}\right) \delta$ 5.97-5.90 (dd, $\left.1 \mathrm{H}\right)$, 5.27-5.22 (dd, $1 \mathrm{H})$, 5.09-5.06 (dd, 1H), 3.04-2.98 (m, 2H), 2.46-2.42 (m, 2H), 1.86-1.84 (dd, 4H), 1.37 (s, 1H).

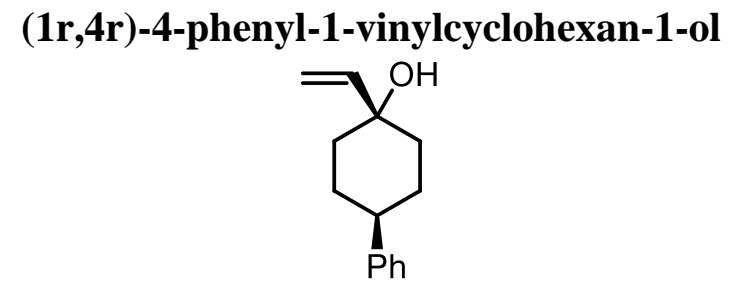

Following a literature procedure. ${ }^{34}$ A clear colorless oil was obtained $(820 \mathrm{mg}, 41 \%)$. The NMR spectrum is consistent with literature reports. 


\section{Characterisation data for substrates}

\section{(1-phenylethane-1,2-diyl)bis(trimethylsilane) (2):}<smiles>CCC(C)c1ccccc1</smiles>

Following general procedure 1 with styrene $(104 \mathrm{mg})$. The crude was purified with pure hexanes and a clear colorless oil was obtained $(232 \mathrm{mg}, 93 \%) .{ }^{\mathbf{1}} \mathbf{H} \mathbf{~ N M R}\left(500 \mathrm{MHz}, \mathrm{CDCl}_{3}\right) \delta 7.24-7.15$ $(\mathrm{m}, 2 \mathrm{H}), 7.08-6.98(\mathrm{~m}, 3 \mathrm{H}), 2.07(\mathrm{dd}, J=13.1,2.4 \mathrm{~Hz}, 1 \mathrm{H}), 1.06(\mathrm{dd}, J=15.1,13.0 \mathrm{~Hz}, 1 \mathrm{H})$, $0.84(\mathrm{dd}, J=15.0,2.3 \mathrm{~Hz}, 1 \mathrm{H}),-0.08(\mathrm{~s}, 9 \mathrm{H}),-0.21(\mathrm{~s}, 9 \mathrm{H}) .{ }^{13} \mathbf{C} \mathbf{N M R}\left(126 \mathrm{MHz}, \mathrm{CDCl}_{3}\right) \delta 145.3$, 127.97, 127.93, 124.3, 31.7, 16.1, -0.9, -3.2. IR (FTIR): 3086, 3057, 3032, 3018, 2953, 2898, 2872, 1598, 1494, 1415, 1374, 1248, 1215, $836 \mathrm{~cm}^{-1}$. HRMS (DART) exact mass calculated for $\mathrm{C}_{13} \mathrm{H}_{23} \mathrm{Si}_{2}\left[\mathrm{M}-\mathrm{CH}_{3}\right]^{+} \mathrm{m} / \mathrm{z}=235.1333$, found $\mathrm{m} / \mathrm{z}=235.1336 . \mathrm{R}_{\mathrm{f}}=0.8(\mathrm{Hex})$. The compound is consistent with previous reports.

\section{(1-(4-fluorophenyl)ethane-1,2-diyl)bis(trimethylsilane) (3):}<smiles>CCC(C)c1ccc(F)cc1</smiles>

Following general procedure 1 with 4-fluorostyrene $(122 \mathrm{mg})$. The crude was purified with pure n-pentane and a clear colorless oil was obtained (192 mg, 72\%). ${ }^{1} \mathbf{H}$ NMR $\left(500 \mathrm{MHz}, \mathrm{CDCl}_{3}\right) \delta$ $7.09-6.80(\mathrm{~m}, 4 \mathrm{H}), 2.05$ (dd, $J=13.1,2.4 \mathrm{~Hz}, 1 \mathrm{H}), 0.99$ (dd, $J=15.1,13.1 \mathrm{~Hz}, 1 \mathrm{H}), 0.83$ (dd, $J$ $=15.1,2.4 \mathrm{~Hz}, 1 \mathrm{H}),-0.09(\mathrm{~s}, 9 \mathrm{H}),-0.21(\mathrm{~s}, 9 \mathrm{H}) .{ }^{13} \mathbf{C ~ N M R}\left(126 \mathrm{MHz}, \mathrm{CDCl}_{3}\right) \delta 161.4,159.5$, $140.9,140.9,128.88,128.82,114.8,114.6,30.9,16.3,-0.9,-3.3 .{ }^{19} \mathbf{F ~ N M R}\left(470 \mathrm{MHz}, \mathrm{CDCl}_{3}\right) \delta$ -119.98. ${ }^{29} \mathrm{Si} \mathrm{NMR}\left(99 \mathrm{MHz}, \mathrm{CDCl}_{3}\right) \delta 4.27,4.25,2.94$. IR (FTIR): 2954, 2928, 2898, 2871, 1506, $1248,1222,1158,1101,838,757,690 \mathrm{~cm}^{-1}$. HRMS (DART) exact mass calculated for $\mathrm{C}_{13} \mathrm{H}_{22} \mathrm{FSi}_{2}$ $\left[\mathrm{M}-\mathrm{CH}_{3}\right]^{+} \mathrm{m} / \mathrm{z}=253.1239$, found $\mathrm{m} / \mathrm{z}=253.1244 . \mathrm{R}_{\mathrm{f}}=0.7$ (Hex). The compound is consistent with previous reports.

\section{(1-(4-(4,4,5,5-tetramethyl-1,3,2-dioxaborolan-2-yl)phenyl)ethane-1,2- diyl)bis(trimethylsilane) (4):}<smiles>CC(C)c1ccc(B2OC(C)(C)C(C)(C)O2)cc1</smiles>

Following general procedure 1 with 4,4,5,5-tetramethyl-2-(4-vinylphenyl)-1,3,2-dioxaborolane (233 mg). The crude was purified with pure hexanes through a $25 \mathrm{~g}$ Florisil column and a white solid was obtained $(233 \mathrm{mg}, 62 \%) .{ }^{1} \mathbf{H}$ NMR $\left(500 \mathrm{MHz}, \mathrm{CDCl}_{3}\right) \delta 7.66-7.60(\mathrm{~m}, 2 \mathrm{H}), 7.02(\mathrm{~d}, J$ $=7.9 \mathrm{~Hz}, 2 \mathrm{H}), 2.10(\mathrm{dd}, J=13.0,2.3 \mathrm{~Hz}, 1 \mathrm{H}), 1.34(\mathrm{~s}, 12 \mathrm{H}), 1.08(\mathrm{dd}, J=15.1,12.9 \mathrm{~Hz}, 1 \mathrm{H}), 0.83$ $(\mathrm{dd}, J=15.1,2.3 \mathrm{~Hz}, 1 \mathrm{H}),-0.10(\mathrm{~s}, 9 \mathrm{H}),-0.21(\mathrm{~s}, 9 \mathrm{H}) .{ }^{13} \mathbf{C} \mathbf{N M R}\left(126 \mathrm{MHz}, \mathrm{CDCl}_{3}\right) \delta 149.1$, 
134.5, 127.4, 83.6, 32.4, 25.11, 25.04, 15.8, -0.9, -3.2. IR (FTIR): 3079, 3042, 2980, 2951, 2897, 2874, 1608, 1400, 1369, 1328, 1291, 1265, 1247, 1164, 1149, 1102, 1018, 962, 852, $834 \mathrm{~cm}^{-1}$. HRMS (DART) exact mass calculated for $\mathrm{C}_{20} \mathrm{H}_{38} \mathrm{O}_{2} \mathrm{BSi}_{2}[\mathrm{M}+\mathrm{H}]^{+} \mathrm{m} / \mathrm{z}=377.2498$, found $\mathrm{m} / \mathrm{z}=$ 377.2502. $\mathrm{R}_{\mathrm{f}}=0.8(5 \% \mathrm{EA} / \mathrm{Hex})$.

\section{4-(1,2-bis(trimethylsilyl)ethyl)- $N, N$-dimethylaniline (5):}<smiles>CCC(CC(C)(C)C)c1ccc(N(C)C)cc1</smiles>

Following general procedure 1 with $N, N$-dimethyl-4-vinylaniline $(147 \mathrm{mg})$ and stopped at $5 \mathrm{~F}$ of charge. The crude was purified with $3 \%$ ethyl acetate, $1 \%$ triethylamine/hexanes and then a short path distillation was performed to remove any residual starting material. A clear colorless oil was obtained (149 mg, 51\%). ${ }^{1} \mathbf{H}$ NMR $\left(500 \mathrm{MHz}, \mathrm{CDCl}_{3}\right) \delta 6.88(\mathrm{~d}, J=8.7 \mathrm{~Hz}, 2 \mathrm{H}), 6.64(\mathrm{~d}, J=8.7$ $\mathrm{Hz}, 2 \mathrm{H}), 2.89$ (s, 6H), 1.95 (dd, $J=13.1,2.5 \mathrm{~Hz}, 1 \mathrm{H}), 0.98$ (dd, $J=15.0,13.0 \mathrm{~Hz}, 1 \mathrm{H}), 0.80$ (dd, $J=15.0,2.5 \mathrm{~Hz}, 1 \mathrm{H}),-0.09$ (s, 9H), -0.20 (s, 9H). ${ }^{13} \mathbf{C ~ N M R}\left(126 \mathrm{MHz}, \mathrm{CDCl}_{3}\right) \delta 148.1,133.5$, $128.5,113.0,41.2,30.1,16.2,-0.9,-3.1 .{ }^{29} \mathrm{Si} \mathrm{NMR}\left(99 \mathrm{MHz}, \mathrm{CDCl}_{3}\right) \delta 3.71,2.68$. IR (FTIR): 3087, 3004, 2951, 2894, 2867, 2797, 1739, 1726, 1695, 1612, 1516, 1478, 1444, 1408, 1346, 1226, 1163, 947, $832 \mathrm{~cm}^{-1}$. HRMS (DART) exact mass calculated for $\mathrm{C}_{16} \mathrm{H}_{32} \mathrm{NSi}_{2}[\mathrm{M}+\mathrm{H}]^{+} \mathrm{m} / \mathrm{z}=$ 294.2073, found $\mathrm{m} / \mathrm{z}=294.2067 . \mathrm{R}_{\mathrm{f}}=0.8(5 \% \mathrm{EA} / \mathrm{Hex})$.

\section{(1-(4-((triisopropylsilyl)oxy)phenyl)ethane-1,2-diyl)bis(trimethylsilane) (6):}

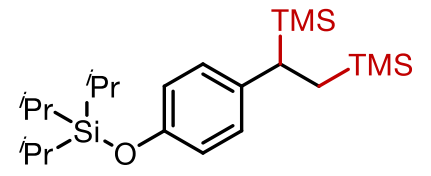

Following general procedure 1 with triisopropyl(4-vinylphenoxy)silane ( $277 \mathrm{mg})$. The crude was purified with $1 \%$ ethyl acetate/hexanes and a clear colorless oil was obtained $(274 \mathrm{mg}, 65 \%) .{ }^{1} \mathbf{H}$ NMR $\left(500 \mathrm{MHz}, \mathrm{CDCl}_{3}\right) \delta 6.85(\mathrm{~d}, J=8.6 \mathrm{~Hz}, 2 \mathrm{H}), 6.74(\mathrm{~d}, J=8.5 \mathrm{~Hz}, 2 \mathrm{H}), 1.99(\mathrm{dd}, J=13.3$, $2.3 \mathrm{~Hz}, 1 \mathrm{H}), 1.23(\mathrm{dt}, J=14.9,7.4 \mathrm{~Hz}, 3 \mathrm{H}), 1.08(\mathrm{~d}, J=7.4 \mathrm{~Hz}, 18 \mathrm{H}), 0.98(\mathrm{dd}, J=15.0,13.3 \mathrm{~Hz}$, $1 \mathrm{H}), 0.80(\mathrm{dd}, J=15.0,2.3 \mathrm{~Hz}, 1 \mathrm{H}),-0.11(\mathrm{~s}, 9 \mathrm{H}),-0.21(\mathrm{~s}, 9 \mathrm{H}) .{ }^{13} \mathbf{C} \mathbf{N M R}\left(126 \mathrm{MHz}, \mathrm{CDCl}_{3}\right) \delta$ 153.0, 137.7, 128.6, 119.7, 30.6, 18.0, 16.3, 12.8, -0.9, -3.2. ${ }^{29} \mathrm{Si} \mathbf{N M R}\left(99 \mathrm{MHz}, \mathrm{CDCl}_{3}\right) \delta 14.68$, 3.83, 2.86. IR (FTIR): 2947, 2894, 2867, 1605, 1504, 1463, 1258, 1246, 1171, 1102, 1013, 997 , 912, 881, 831, 772, 748, $682 \mathrm{~cm}^{-1}$. HRMS (DART) exact mass calculated for $\mathrm{C}_{23} \mathrm{H}_{47} \mathrm{OS} \mathrm{OS}_{3}[\mathrm{M}+\mathrm{H}]^{+}$ $\mathrm{m} / \mathrm{z}=423.2929$, found $\mathrm{m} / \mathrm{z}=423.2930 . \mathrm{R}_{\mathrm{f}}=0.6(2 \% \mathrm{EA} / \mathrm{Hex})$.

\section{(1-(4-(methylthio)phenyl)ethane-1,2-diyl)bis(trimethylsilane) (7):}<smiles>CCCCC(c1ccc(SC)cc1)C(C)(C)C</smiles>

Following general procedure 1 with 4-methylthiostyrene $(150 \mathrm{mg})$. The crude was purified with $2.5 \%$ ethyl acetate/hexanes and a clear colorless oil was obtained (220 mg, $74 \%)$. ${ }^{1} \mathbf{H}$ NMR (500 
$\left.\mathrm{MHz}, \mathrm{CDCl}_{3}\right) \delta 7.15-7.09(\mathrm{~m}, 2 \mathrm{H}), 6.97-6.91(\mathrm{~m}, 2 \mathrm{H}), 2.46(\mathrm{~s}, 3 \mathrm{H}), 2.03(\mathrm{dd}, J=13.0,2.4 \mathrm{~Hz}$, $1 \mathrm{H}), 1.01$ (dd, $J=15.1,13.0 \mathrm{~Hz}, 1 \mathrm{H}), 0.82(\mathrm{dd}, J=15.1,2.4 \mathrm{~Hz}, 1 \mathrm{H}),-0.09$ (s, 9H), -0.20 (s, 9H). ${ }^{13} \mathbf{C}$ NMR $\left(126 \mathrm{MHz}, \mathrm{CDCl}_{3}\right) \delta 142.7,133.1,128.4,127.1,31.2,16.7,16.0,-0.9,-3.2$. IR (FTIR): 3072, 3017, 2953, 2920, 2896, 2872, 1492, 1438, 1406, 1247, 1102, $837 \mathrm{~cm}^{-1}$. HRMS (DART) exact mass calculated for $\mathrm{C}_{15} \mathrm{H}_{28} \mathrm{SSi}_{2}[\mathrm{M}+\mathrm{H}]^{+} \mathrm{m} / \mathrm{z}=297.1523$, found $\mathrm{m} / \mathrm{z}=297.1525$. $\mathrm{R}_{\mathrm{f}}=0.8$ (5\% EA/Hex).

\section{2-(4-(1,2-bis(trimethylsilyl)ethyl)phenyl)ethan-1-ol (8):}<smiles>CCC(C)c1ccc(CCO)cc1</smiles>

Following general procedure 1 with 2-(4-vinylphenyl)ethan-1-ol (148 mg) and 4 equivalents of TMSCl. The reaction was stopped at $4.0 \mathrm{~F}$ of charge and the crude was stirred in $0.5 \mathrm{M} \mathrm{HCl}$ in ether solution for one hour to remove the TMS protecting group on alcohol and purified with $30 \%$ ethyl acetate/hexanes and a white solid was obtained (150 mg, 51\%). ${ }^{1} \mathbf{H} \mathbf{~ N M R}\left(500 \mathrm{MHz}, \mathrm{CDCl}_{3}\right)$ $\delta 7.05(\mathrm{~d}, J=8.1 \mathrm{~Hz}, 2 \mathrm{H}), 6.99-6.93(\mathrm{~m}, 2 \mathrm{H}), 3.82(\mathrm{q}, J=6.4 \mathrm{~Hz}, 2 \mathrm{H}), 2.81(\mathrm{t}, J=6.6 \mathrm{~Hz}, 2 \mathrm{H})$, $2.04(\mathrm{dd}, J=13.0,2.4 \mathrm{~Hz}, 1 \mathrm{H}), 1.35(\mathrm{t}, J=6.0 \mathrm{~Hz}, 1 \mathrm{H}), 1.02(\mathrm{dd}, J=15.1,13.0 \mathrm{~Hz}, 1 \mathrm{H}), 0.83(\mathrm{dd}$, $J=15.0,2.4 \mathrm{~Hz}, 1 \mathrm{H}),-0.09(\mathrm{~s}, 9 \mathrm{H}),-0.23(\mathrm{~s}, 9 \mathrm{H}) .{ }^{13} \mathbf{C} \mathbf{N M R}\left(126 \mathrm{MHz}, \mathrm{CDCl}_{3}\right) \delta 143.5,133.9$, 128.6, 128.2, 64.0, 38.9, 31.2, 16.2, -1.0, -3.2. IR (FTIR): 3335, 3020, 3002, 2952, 2896, 2871, 1510, 1413, 1330, 1285, 1247, 1176, 1143, 1109, 1099, 1046, 1019, $837 \mathrm{~cm}^{-1}$. HRMS (DART) exact mass calculated for $\mathrm{C}_{15} \mathrm{H}_{27} \mathrm{OSi}_{2}\left[\mathrm{M}-\mathrm{CH}_{3}\right]^{+} \mathrm{m} / \mathrm{z}=279.1595$, found $\mathrm{m} / \mathrm{z}=279.1600 . \mathrm{R}_{\mathrm{f}}=0.3$ (20\% EA/Hex).

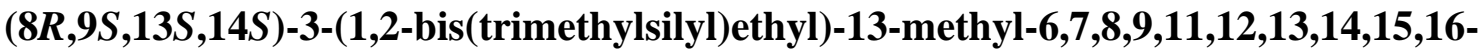
decahydro-17H-cyclopenta[ $[a]$ phenanthren-17-one (9):

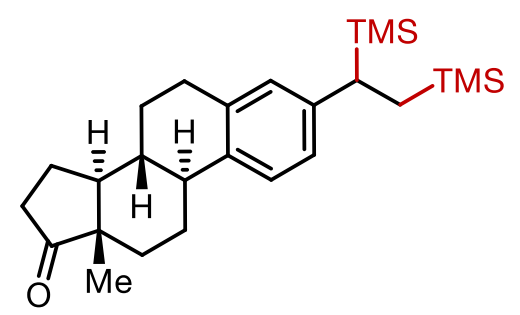

Following general procedure 1 with estrone $(280 \mathrm{mg})$ and 4.0 equivalents of TMSCl. The reaction was stopped at $2.2 \mathrm{~F}$ of charge and the crude was purified with $10 \%$ ethyl acetate in hexanes and a clear colorless oil was obtained $(209 \mathrm{mg}, 49 \%) .{ }^{1} \mathbf{H} \mathbf{~ N M R}\left(500 \mathrm{MHz}, \mathrm{CDCl}_{3}\right) \delta 7.09(\mathrm{~d}, J=8.0$ $\mathrm{Hz}, 1 \mathrm{H}), 6.79(\mathrm{~d}, J=8.0 \mathrm{~Hz}, 1 \mathrm{H}), 6.72(\mathrm{~d}, J=2.0 \mathrm{~Hz}, 1 \mathrm{H}), 2.87(\mathrm{dt}, J=8.9,5.5 \mathrm{~Hz}, 2 \mathrm{H}), 2.52(\mathrm{dd}$, $J=18.9,8.7 \mathrm{~Hz}, 1 \mathrm{H}), 2.45-2.37(\mathrm{~m}, 1 \mathrm{H}), 2.28(\mathrm{td}, J=11.0,4.1 \mathrm{~Hz}, 1 \mathrm{H}), 2.22-1.89(\mathrm{~m}, 5 \mathrm{H})$, $1.74-1.37(\mathrm{~m}, 6 \mathrm{H}), 1.04(\mathrm{ddd}, J=15.1,12.8,2.4 \mathrm{~Hz}, 1 \mathrm{H}), 0.95(\mathrm{~s}, 3 \mathrm{H}), 0.84(\mathrm{dt}, J=15.1,2.2 \mathrm{~Hz}$, $1 \mathrm{H}),-0.06$ (s, 9H), $-0.18(\mathrm{~s}, 9 \mathrm{H}) .{ }^{13} \mathbf{C ~ N M R}\left(126 \mathrm{MHz}, \mathrm{CDCl}_{3}\right) \delta 221.2,142.3,135.5,135.4,128.3$, 125.6, 125.4, 124.6, 50.8, 48.2 , 44.4, 38.5, 36.0, 31.8, 30.9, 29.6, 26.9, 25.8, 21.8, 16.0, 14.1, -0.8, -3.1. ${ }^{29} \mathrm{Si}$ NMR $\left(99 \mathrm{MHz}, \mathrm{CDCl}_{3}\right) \delta 3.82,2.91$. IR (FTIR): 2950, 2933, 2895, 2870, 1742, 1608, 1563, 1497, 1454, 1436, 1408, 1372, 1340, 1246, 1217, 1099, 1084, 1052, 1007, $837 \mathrm{~cm}^{-1}$. HRMS (DART) exact mass calculated for $\mathrm{C}_{26} \mathrm{H}_{43} \mathrm{OSi}_{2}[\mathrm{M}+\mathrm{H}]^{+} \mathrm{m} / \mathrm{z}=427.2847$, found $\mathrm{m} / \mathrm{z}=427.2852$. $\mathrm{R}_{\mathrm{f}}$ $=0.4(10 \% \mathrm{EA} / \mathrm{Hex})$. 


\section{5-(1,2-bis(trimethylsilyl)ethyl)-1-methyl-1H-indole (10):}<smiles>Cn1ccc2cc(C(CC(C)(C)C)CS(C)(=O)=O)ccc21</smiles>

Following general procedure 1 with 1 -methyl-5-vinyl- $1 H$-indole $(157 \mathrm{mg})$. The crude was purified with $10 \%$ ethyl acetate/hexanes and a white solid was obtained (209 mg, 69\%). ${ }^{1} \mathbf{H}$ NMR (500 $\left.\mathrm{MHz}, \mathrm{CDCl}_{3}\right) \delta 7.24(\mathrm{~d}, J=1.7 \mathrm{~Hz}, 1 \mathrm{H}), 7.15(\mathrm{~d}, J=8.4 \mathrm{~Hz}, 1 \mathrm{H}), 6.97(\mathrm{~d}, J=3.0 \mathrm{~Hz}, 1 \mathrm{H}), 6.90$ $(\mathrm{dd}, J=8.5,1.7 \mathrm{~Hz}, 1 \mathrm{H}), 6.36(\mathrm{~d}, J=3.0 \mathrm{~Hz}, 1 \mathrm{H}), 3.75(\mathrm{~s}, 3 \mathrm{H}), 2.14(\mathrm{dd}, J=13.1,2.4 \mathrm{~Hz}, 1 \mathrm{H})$, $1.12(\mathrm{dd}, J=15.1,13.0 \mathrm{~Hz}, 1 \mathrm{H}), 0.92-0.84(\mathrm{~m}, 1 \mathrm{H}),-0.08(\mathrm{~s}, 9 \mathrm{H}),-0.23(\mathrm{~s}, 9 \mathrm{H}) .{ }^{13} \mathbf{C ~ N M R}(126$ $\left.\mathrm{MHz}, \mathrm{CDCl}_{3}\right) \delta 135.8,134.9,128.6,128.5,122.8,119.3,108.5,100.3,33.0,31.1,16.6,-0.8,-3.0$. IR (FTIR): 3015, 2951, 2895, 2871, 1514, 1488, 1444, 1422, 1336, 1308, 1245, 1158, 1130, 1099, 1080, $835 \mathrm{~cm}^{-1}$. HRMS (DART) exact mass calculated for $\mathrm{C}_{17} \mathrm{H}_{30} \mathrm{NSi}_{2}[\mathrm{M}+\mathrm{H}]^{+} \mathrm{m} / \mathrm{z}=304.1911$, found $\mathrm{m} / \mathrm{z}=304.1913 . \mathrm{R}_{\mathrm{f}}=0.5(10 \% \mathrm{EA} / \mathrm{Hex})$.

\section{2-(1,2-bis(trimethylsilyl)ethyl)-6-methylpyridine (11):}<smiles>CCCCCC(CS(C)(=O)=O)c1cccc(C)n1</smiles>

Following general procedure 1 with 2-methyl-6-vinylpyridine $(119 \mathrm{mg})$. The crude was purified with $1 \%$ ethyl acetate, $1 \%$ triethylamine/hexanes and a clear light-yellow oil was obtained (210 mg, 79\%). ${ }^{1} \mathbf{H}$ NMR $\left(500 \mathrm{MHz}, \mathrm{CDCl}_{3}\right) \delta 7.36(\mathrm{t}, J=7.7 \mathrm{~Hz}, 1 \mathrm{H}), 6.78(\mathrm{dd}, J=18.3,7.7 \mathrm{~Hz}, 2 \mathrm{H})$, $2.46(\mathrm{~s}, 3 \mathrm{H}), 2.37(\mathrm{dd}, J=12.9,2.2 \mathrm{~Hz}, 1 \mathrm{H}), 1.25(\mathrm{dd}, J=15.0,12.9 \mathrm{~Hz}, 1 \mathrm{H}), 0.80$ (dd, $J=15.0$, $2.2 \mathrm{~Hz}, 1 \mathrm{H}),-0.07$ (s, 9H), $-0.21(\mathrm{~s}, 9 \mathrm{H}) .{ }^{13} \mathbf{C ~ N M R}\left(126 \mathrm{MHz}, \mathrm{CDCl}_{3}\right) \delta$ 164.7, 157.2, 135.7, 118.7 , 118.6, 34.5, 24.7, 15.4, -1.1, -3.1. IR (FTIR): 3061, 2953, 2897, 2872, 1585, 1575, 1449, 1409, 1373, 1321, 1247, 1223, 1198, 1159, 1102, $837 \mathrm{~cm}^{-1}$. HRMS (DART) exact mass calculated for $\mathrm{C}_{14} \mathrm{H}_{28} \mathrm{NSi}_{2}[\mathrm{M}+\mathrm{H}]^{+} \mathrm{m} / \mathrm{z}=266.1755$, found $\mathrm{m} / \mathrm{z}=266.1757 . \mathrm{R}_{\mathrm{f}}=0.5(2 \% \mathrm{EA} / \mathrm{Hex})$.

\section{(1-Ferrocenoethane-1,2-diyl)bis(trimethylsilane) (12):}

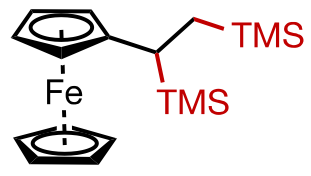

Following general procedure 1 with vinylferrocene $(212 \mathrm{mg})$. The reaction was stopped at $2.2 \mathrm{~F}$ of charge and the crude was purified with $3 \%$ ethyl acetate/hexanes and a clear orange oil was obtained (250 mg, 70\%). ${ }^{1} \mathbf{H}$ NMR $\left(500 \mathrm{MHz}, \mathrm{CDCl}_{3}\right) \delta 4.10(\mathrm{~s}, 5 \mathrm{H}), 4.02(\mathrm{~s}, 2 \mathrm{H}), 3.93(\mathrm{~s}, 1 \mathrm{H})$, $3.83(\mathrm{~s}, 1 \mathrm{H}), 1.70(\mathrm{~d}, J=10.4 \mathrm{~Hz}, 1 \mathrm{H}), 1.33(\mathrm{~d}, J=15.0 \mathrm{~Hz}, 1 \mathrm{H}), 1.00(\mathrm{dd}, J=15.2,10.5 \mathrm{~Hz}, 1 \mathrm{H})$, 0.14 (s, 9H), -0.15 (s, 9H). ${ }^{13} \mathbf{C}$ NMR $\left(126 \mathrm{MHz}, \mathrm{CDCl}_{3}\right) \delta$ 99.3, 68.3, 68.0, 66.5, 65.9, 65.6, 21.9, 18.2, 0.2, -1.9. IR (FTIR): 3093, 3047, 2952, 2894, 1455, 1411, 1247, 1150, 1106, 1074, 1043, $1025,1001,919,836 \mathrm{~cm}^{-1}$. HRMS (DART) exact mass calculated for $\mathrm{C}_{18} \mathrm{H}_{30} \mathrm{Si}_{2} \mathrm{Fe}[\mathrm{M}]^{+} \mathrm{m} / \mathrm{z}=$ 358.1230, found $\mathrm{m} / \mathrm{z}=358.1232 . \mathrm{R}_{\mathrm{f}}=0.7(5 \% \mathrm{EA} / \mathrm{Hex})$.

trans-2,3-dihydro-1H-indene-1,2-diyl)bis(trimethylsilane) (13): 


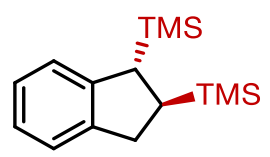

Following general procedure 1 with indene $(116 \mathrm{mg})$. The crude was purified with pure hexanes and a clear colorless oil was obtained $(188 \mathrm{mg}, 72 \%)$. Stereochemistry was determined by 2D NOESY NMR. ${ }^{1} \mathrm{H}$ NMR $\left(500 \mathrm{MHz}, \mathrm{CDCl}_{3}\right) \delta 7.19-6.87(\mathrm{~m}, 4 \mathrm{H}), 3.23-3.14(\mathrm{~m}, 1 \mathrm{H}), 2.90-$ $2.83(\mathrm{~m}, 1 \mathrm{H}), 2.41(\mathrm{~d}, J=2.2 \mathrm{~Hz}, 1 \mathrm{H}), 1.53-1.48(\mathrm{~m}, 1 \mathrm{H}),-0.01(\mathrm{~s}, 9 \mathrm{H}),-0.14(\mathrm{~s}, 9 \mathrm{H}) .{ }^{13} \mathbf{C}$ NMR $\left(126 \mathrm{MHz}, \mathrm{CDCl}_{3}\right) \delta 146.4,142.7,125.8,124.6,124.2,123.4,36.6,34.2,25.4,-2.8,-3.6$. IR (FTIR): 3068, 3019, 2954, 2896, 2866, 2846, 1602, 1482, 1136, 1112, 1087, 928, 875, $835 \mathrm{~cm}^{-1}$. HRMS (DART) exact mass calculated for $\mathrm{C}_{14} \mathrm{H}_{23} \mathrm{Si}_{2}\left[\mathrm{M}-\mathrm{CH}_{3}\right]^{+} \mathrm{m} / \mathrm{z}=247.1333$, found $\mathrm{m} / \mathrm{z}=$ 247.1339. $\mathrm{R}_{\mathrm{f}}=0.8$ (Hex).

\section{1,2,3,4-tetrahydronaphthalene-1,2-diyl)bis(trimethylsilane) (14):}<smiles>CS(=O)(=O)c1ccccc1[C@H]1CCc2ccccc21</smiles>

Following general procedure 1 with 1,2-dihydronapthalene $(130 \mathrm{mg})$. The crude was purified with pure hexanes and a clear colorless oil was obtained $(183 \mathrm{mg}, 66 \%)$. Stereochemistry was determined by coupling constants. ${ }^{1} \mathbf{H}$ NMR $\left(500 \mathrm{MHz}, \mathrm{CDCl}_{3}\right) \delta 7.16-6.72(\mathrm{~m}, 4 \mathrm{H}), 2.71$ (ddd, $J=15.6,6.7,4.8 \mathrm{~Hz}, 1 \mathrm{H}), 2.58(\mathrm{ddd}, J=15.5,8.3,5.0 \mathrm{~Hz}, 1 \mathrm{H}), 2.29(\mathrm{~d}, J=2.9 \mathrm{~Hz}, 1 \mathrm{H}), 2.03$ (dtd, $J=13.2,6.6,4.9 \mathrm{~Hz}, 1 \mathrm{H}), 1.54-1.45(\mathrm{~m}, 1 \mathrm{H}), 1.30(\mathrm{ddd}, J=9.1,6.6,2.9 \mathrm{~Hz}, 1 \mathrm{H}),-0.04(\mathrm{~s}, 9 \mathrm{H})$, $-0.06(\mathrm{~s}, 9 \mathrm{H}) .{ }^{13} \mathbf{C}$ NMR $\left(126 \mathrm{MHz}, \mathrm{CDCl}_{3}\right) \delta 139.8,137.7,128.3,128.3,125.4,123.7,30.5,30.3$, 24.0, 20.9, -1.6, -2.5. IR (FTIR): 3061, 3014, 2952, 2931, 2898, 2851, 1484, 1449, 1408, 1295, $1248,1218,1157,1100,938,918,835 \mathrm{~cm}^{-1}$. HRMS (DART) exact mass calculated for $\mathrm{C}_{16} \mathrm{H}_{29} \mathrm{Si}_{2}$ $[\mathrm{M}+\mathrm{H}]^{+} \mathrm{m} / \mathrm{z}=277.1802$, found $\mathrm{m} / \mathrm{z}=277.1806 . \mathrm{R}_{\mathrm{f}}=0.8(\mathrm{Hex})$.

\section{(Z)-(3-phenylprop-2-ene-1,2-diyl)bis(trimethylsilane) (15):}<smiles>CC(C)(C)CC(C)(C)C=Cc1ccccc1</smiles>

Following general procedure 1 with propa-1,2-dien-1-ylbenzene $(116 \mathrm{mg})$. The crude was purified with pure hexanes and a clear colorless oil was obtained (178 mg, 68\%). ${ }^{1} \mathbf{H}$ NMR $(500 \mathrm{MHz}$, $\left.\mathrm{CDCl}_{3}\right) \delta 7.35-7.27(\mathrm{~m}, 4 \mathrm{H}), 7.18(\mathrm{dq}, J=6.4,3.4,2.9 \mathrm{~Hz}, 1 \mathrm{H}), 6.63(\mathrm{~d}, J=1.9 \mathrm{~Hz}, 1 \mathrm{H}), 2.09-$ 2.05 (m, 2H), 0.16 (s, 9H), -0.09 (s, 9H). ${ }^{13} \mathbf{C}$ NMR (126 MHz, $\left.\mathrm{CDCl}_{3}\right) \delta 143.0,139.7,134.8$, 128.9, 128.2, 126.3, 20.6, 0.0, -0.8. ${ }^{29} \mathrm{Si}$ NMR $\left(99 \mathrm{MHz}, \mathrm{CDCl}_{3}\right) \delta 1.19,-2.42$. IR (FTIR): 3057, 3022, 2953, 2897, 1591, 1491, 1444, 1415, 1246, 1172, 1015, 926, 750, 729, $694 \mathrm{~cm}^{-1}$. HRMS (DART) exact mass calculated for $\mathrm{C}_{14} \mathrm{H}_{23} \mathrm{Si}_{2}\left[\mathrm{M}-\mathrm{CH}_{3}\right]^{+} \mathrm{m} / \mathrm{z}=247.1333$, found $\mathrm{m} / \mathrm{z}=247.1334$. $\mathrm{R}_{\mathrm{f}}=0.8$ (Hex).

(E)-(1-phenylprop-1-ene-1,2-diyl)bis(dimethylsilane) (16):<smiles>CCCCCC(C)=C(C)c1ccccc1</smiles> 
Following general procedure 1 with 1-phenyl-1-propyne $(116 \mathrm{mg})$ and dimethylchlorosilane (282 $\mathrm{mg}$ ). The reaction was stopped at $2.2 \mathrm{~F}$ of charge and the crude was purified with pure hexanes and a clear colorless oil was obtained $(136 \mathrm{mg}, 58 \%, \mathrm{E} / \mathrm{Z}=4: 1)$. Stereochemistry was determined by 2D NOESY NMR. Major isomer: ${ }^{1} \mathbf{H}$ NMR $\left(500 \mathrm{MHz}, \mathrm{CDCl}_{3}\right) \delta 7.24(\mathrm{td}, J=7.6,2.1 \mathrm{~Hz}, 2 \mathrm{H})$, $7.21-7.13(\mathrm{~m}, 1 \mathrm{H}), 6.91(\mathrm{dt}, J=6.0,1.5 \mathrm{~Hz}, 2 \mathrm{H}), 4.47(\mathrm{p}, J=3.7 \mathrm{~Hz}, 1 \mathrm{H}), 3.77-3.51(\mathrm{~m}, 1 \mathrm{H})$, $2.09(\mathrm{~s}, 3 \mathrm{H}), 0.06(\mathrm{~d}, J=3.8 \mathrm{~Hz}, 6 \mathrm{H}),-0.10(\mathrm{~d}, J=3.8 \mathrm{~Hz}, 6 \mathrm{H})$. Minor isomer : ${ }^{1} \mathbf{H}$ NMR $(500$ $\left.\mathrm{MHz}, \mathrm{CDCl}_{3}\right) \delta 7.34-7.27(\mathrm{~m}, 3 \mathrm{H}), 7.24(\mathrm{~m}, 2 \mathrm{H}), 6.88-6.83(\mathrm{~m}, 1 \mathrm{H}), 4.55-4.50(\mathrm{~m}, 2 \mathrm{H}), 1.60$ $(\mathrm{s}, 3 \mathrm{H}), 0.26(\mathrm{~d}, J=3.8 \mathrm{~Hz}, 6 \mathrm{H}), 0.03(\mathrm{~d}, J=3.6 \mathrm{~Hz}, 6 \mathrm{H}) .{ }^{13} \mathbf{C} \mathbf{N M R}\left(126 \mathrm{MHz}, \mathrm{CDCl}_{3}\right) \delta 155.1$, $155.0,151.3,149.3,145.7,144.7,128.2,127.8,127.5,125.7,125.4,21.9,20.3,-3.1,-3.4,-3.5$. ${ }^{29} \mathrm{Si}$ NMR (99 MHz, $\left.\mathrm{CDCl}_{3}\right) \delta$-22.30 (minor), -22.87, -24.84 (minor), -28.66. IR (FTIR): 3074, 3058, 3019, 2958, 2902, 2854, 1597, 1573, 1483, 1439, 1421, 1370, 1247, 1070, 1030, 836, $832 \mathrm{~cm}^{-1}$. HRMS (DART) exact mass calculated for $\mathrm{C}_{13} \mathrm{H}_{21} \mathrm{Si}_{2}[\mathrm{M}+\mathrm{H}]^{+} \mathrm{m} / \mathrm{z}=233.1176$, found $\mathrm{m} / \mathrm{z}$ $=233.1179 . \mathrm{R}_{\mathrm{f}}=0.6(\mathrm{Hex})$.

\section{(E)-(1-phenylbut-2-ene-1,4-diyl)bis(trimethylsilane) (17):}<smiles>CCCCC(C)(C)c1ccccc1</smiles>

Following general procedure 1 with $(E)$-buta-1,3-dien-1-ylbenzene $(130 \mathrm{mg})$. The crude was purified with pure hexanes and a clear colorless oil was obtained $(101 \mathrm{mg}, 36 \%$ for E isomer and $54.3 \mathrm{mg}, 20 \%$ for mixture of $\mathrm{E}$ and $\mathrm{Z}$ isomer). The $\mathrm{E} / \mathrm{Z}$ ratio is $4: 1$ which is obtained from the crude NMR. Stereochemistry was determined by 2D NOESY NMR. ${ }^{1} \mathbf{H}$ NMR $\left(500 \mathrm{MHz}, \mathrm{CDCl}_{3}\right)$ $\delta 7.24(\mathrm{dd}, J=13.8,6.1 \mathrm{~Hz}, 2 \mathrm{H}), 7.11-7.02(\mathrm{~m}, 3 \mathrm{H}), 5.63(\mathrm{ddt}, J=15.0,9.9,1.4 \mathrm{~Hz}, 1 \mathrm{H}), 5.39$ (dt, $J=15.4,8.0 \mathrm{~Hz}, 1 \mathrm{H}), 2.87(\mathrm{~d}, J=9.7 \mathrm{~Hz}, 1 \mathrm{H}), 1.50-1.44(\mathrm{~m}, 2 \mathrm{H}), 0.01(\mathrm{~s}, 9 \mathrm{H}),-0.04(\mathrm{~s}, 9 \mathrm{H})$. ${ }^{13}$ C NMR $\left(126 \mathrm{MHz} \mathrm{CDCl}_{3}\right) \delta 143.8,128.3,127.8,127.3,125.3,124.4,43.0,23.2,-1.7,-2.7 .{ }^{29} \mathbf{S i}$ NMR (99 MHz, $\mathrm{CDCl}_{3}$ ) $\delta 2.57,0.65$. IR (FTIR): 3077, 3058, 3017, 2954, 2897, 1695, 1598, 1490, 1408, 1302, 1247, 1190, 1090, 1030, 962, 927, 892, 831, 754, 699, $623 \mathrm{~cm}^{-1}$. HRMS (DART) exact mass calculated for $\mathrm{C}_{15} \mathrm{H}_{25} \mathrm{Si}_{2}\left[\mathrm{M}-\mathrm{CH}_{3}\right]^{+} \mathrm{m} / \mathrm{z}=261.1489$, found $\mathrm{m} / \mathrm{z}=261.1498$. $\mathrm{R}_{\mathrm{f}}=0.8$ (Hex).

\section{(1-(4-(tert-butyl)phenyl)buta-1,2-diene-1,4-diyl)bis(trimethylsilane) (18):}

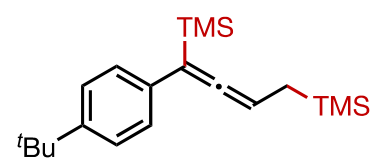

Following general procedure 1 with 1-(but-3-en-1-yn-1-yl)-4-(tert-butyl)benzene (184 mg). The crude was purified with pure hexanes and a light-yellow oil was obtained. There is 5\% 10\% impurities in the desired product that are difficult to remove due to low polarity and yield was determined by ${ }^{1} \mathrm{H}-\mathrm{NMR}$ using 1,3,5 trimethoxybenzene as internal standard (67\%). ${ }^{\mathbf{1}} \mathbf{H}$ NMR (500 $\left.\mathrm{MHz} \mathrm{CDCl}_{3}\right) \delta 7.50-6.91(\mathrm{~m}, 4 \mathrm{H}), 5.10(\mathrm{t}, J=8.3 \mathrm{~Hz}, 1 \mathrm{H}), 1.40(\mathrm{dd}, J=5.7,2.9 \mathrm{~Hz}, 2 \mathrm{H}), 1.31$ (s, 9H), 0.24 (s, 9H), 0.06 (s, 9H). $\left.{ }^{13} \mathbf{C ~ N M R ~ ( 1 2 6 ~ M H z , ~} \mathrm{CDCl}_{3}\right) \delta$ 209.4, 148.8, 135.3, 127.4, 125.4, 99.1, 83.5, 34.5, 31.5, 17.2, 0.1, -1.5. IR (FTIR): 3065, 3025, 3020, 2951, 2900, 1950, 1900, 1601, 1488, 1450, 1339, 1250, 1190, 1150, 1113, $830 \mathrm{~cm}^{-1}$. HRMS (DART) exact mass calculated for $\mathrm{C}_{20} \mathrm{H}_{34} \mathrm{Si}_{2}[\mathrm{M}+\mathrm{H}]^{+} \mathrm{m} / \mathrm{z}=315.1964$, found $\mathrm{m} / \mathrm{z}=315.1966 . \mathrm{R}_{\mathrm{f}}=0.7(\mathrm{Hex})$. 
(1-(4,4,5,5-tetramethyl-1,3,2-dioxaborolan-2-yl)ethane-1,2-diyl)bis(trimethylsilane) (19):<smiles>CC(C)C(C)SB1OC(C)(C)C(C)(C)O1</smiles>

Following general procedure 1 at 50\% scale and 4,4,5,5-tetramethyl-2-vinyl-1,3,2-dioxaborolane (77 mg). The crude was purified with $2 \%$ ethyl acetate/hexanes and a clear colorless oil was obtained (130 mg, 88\%). ${ }^{1} \mathbf{H}$ NMR $\left(500 \mathrm{MHz}, \mathrm{CDCl}_{3}\right) \delta 1.20(\mathrm{~d}, J=2.7 \mathrm{~Hz}, 12 \mathrm{H}), 0.74(\mathrm{dd}, J=$ $14.6,11.5 \mathrm{~Hz}, 1 \mathrm{H}), 0.42$ (dd, $J=14.7,1.3 \mathrm{~Hz}, 1 \mathrm{H}), 0.24(\mathrm{dd}, J=11.6,1.3 \mathrm{~Hz}, 1 \mathrm{H}), 0.00$ (s, 9H), $-0.07(\mathrm{~s}, 9 \mathrm{H}) .{ }^{13} \mathbf{C}$ NMR $\left(126 \mathrm{MHz}, \mathrm{CDCl}_{3}\right) \delta 82.8,25.5,25.1,10.6,-1.72,-1.76$. IR (FTIR): 2993, 2979, 2954, 2897, 2862, 1458, 1379, 1346, 1307, 1247, 1146, 1036, 990, 964, $830 \mathrm{~cm}^{-1}$. HRMS (DART) exact mass calculated for $\mathrm{C}_{14} \mathrm{H}_{33} \mathrm{BO}_{2} \mathrm{Si}_{2}\left[\mathrm{M}-\mathrm{CH}_{3}\right]^{+} \mathrm{m} / \mathrm{z}=285.1877$, found $\mathrm{m} / \mathrm{z}=285.1873$. $\mathrm{R}_{\mathrm{f}}=0.3(2 \% \mathrm{EA} / \mathrm{Hex})$.

(1-(4,4,5,5-tetramethyl-1,3,2-dioxaborolan-2-yl)ethane-1,2-diyl)bis(dimethylsilane) (20):

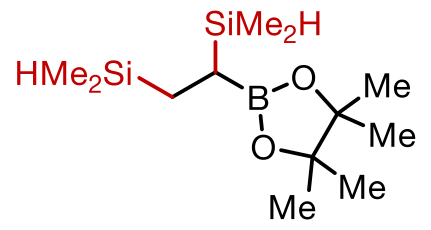

Following general procedure 1 with $154 \mathrm{mg}$ of vinylboronic acid pinacol ester. The product was purified with $3 \%$ ethyl acetate/hexanes and a colorless oil was obtained $(215 \mathrm{mg}, 79 \%)$. ${ }^{1} \mathbf{H}$ NMR $\left(500 \mathrm{MHz}, \mathrm{CDCl}_{3}\right) \delta 3.93-3.79(\mathrm{~m}, 2 \mathrm{H}), 1.23(\mathrm{~d}, J=1.8 \mathrm{~Hz}, 12 \mathrm{H}), 0.86$ (ddd, $J=14.5,11.7,2.7$ $\mathrm{Hz}, 1 \mathrm{H}), 0.55$ (ddd, $J=14.7,3.7,2.5 \mathrm{~Hz}, 1 \mathrm{H}), 0.42$ (dt, $J=11.8,2.5 \mathrm{~Hz}, 1 \mathrm{H}), 0.11$ (t, $J=3.9 \mathrm{~Hz}$, $6 \mathrm{H}), 0.06(\mathrm{t}, J=3.5 \mathrm{~Hz}, 6 \mathrm{H}) .{ }^{13} \mathbf{C}$ NMR $\left(126 \mathrm{MHz} \mathrm{CDCl}_{3}\right) \delta 83.0,25.2,25.0,8.9,-4.1,-4.29$, 4.33, -4.7. IR (FTIR): 2979, 2959, 2904, 2867, 2105, 1379, 1371, 1345, 1309, 1247, 1144, 1046, 1004, 992, 966, 871, $834 \mathrm{~cm}^{-1}$. HRMS (DART) exact mass calculated for $\mathrm{C}_{14} \mathrm{H}_{33} \mathrm{BO}_{2} \mathrm{Si}_{2}[\mathrm{M}-\mathrm{H}]^{+}$ $\mathrm{m} / \mathrm{z}=271.1720$, found $\mathrm{m} / \mathrm{z}=271.1718 . \mathrm{R}_{\mathrm{f}}=0.3(3 \% \mathrm{EA} / \mathrm{Hex})$.

\section{(1-(4,4,5,5-tetramethyl-1,3,2-dioxaborolan-2-yl)ethane-1,2-diyl)bis(triethylsilane) (21)}

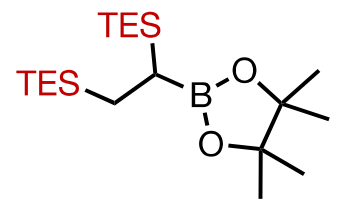

Following general procedure 1 with $154 \mathrm{mg}$ of 4,4,5,5-tetramethyl-2-vinyl-1,3,2-dioxaborolane. The crude was purified with $2 \%$ ethyl acetate/hexanes and a clear colorless oil was obtained (108 mg, 56\%). ${ }^{1} \mathbf{H}$ NMR (500 MHz, $\left.\mathrm{CDCl}_{3}\right) \delta 1.23(\mathrm{~d}, J=5.1 \mathrm{~Hz}, 12 \mathrm{H}), 1.02-0.84(\mathrm{~m}, 19 \mathrm{H}), 0.77$ $(\mathrm{dd}, J=14.9,11.9 \mathrm{~Hz}, 1 \mathrm{H}), 0.64-0.55(\mathrm{~m}, 6 \mathrm{H}), 0.49(\mathrm{~m}, 6 \mathrm{H}), 0.37(\mathrm{~d}, J=12.1 \mathrm{~Hz}, 1 \mathrm{H}) .{ }^{13} \mathbf{C ~ N M R}$ $\left(126 \mathrm{MHz} \mathrm{CDCl}_{3}\right) \delta 82.8,25.7,25.1,7.8,7.7,3.4,3.2 .{ }^{11} \mathbf{B} \mathbf{N M R}\left(160 \mathrm{MHz}, \mathrm{CDCl}_{3}\right) \delta 34.61$. IR (FTIR): 2977, 2950, 2909, 2874, 2807, 1459, 1416, 1376, 1341, 1305, 1270, 1240, 1211, 1144, 1119, 1044, 1005, 989, 967, 891, 850,835 $\mathrm{cm}^{-1}$. HRMS (DART) exact mass calculated for $\mathrm{C}_{20} \mathrm{H}_{45} \mathrm{BO}_{2} \mathrm{Si}_{2}\left[\mathrm{M}-\mathrm{C}_{2} \mathrm{H}_{5}\right]^{+} \mathrm{m} / \mathrm{z}=355.2654$, found $\mathrm{m} / \mathrm{z}=355.2662 . \mathrm{R}_{\mathrm{f}}=0.8(5 \% \mathrm{EA} / \mathrm{Hex})$. 


\section{(2-(4,4,5,5-tetramethyl-1,3,2-dioxaborolan-2-yl)propane-1,2-diyl)bis(trimethylsilane) (22):}

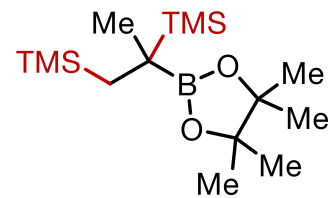

Following general procedure 1 at 50\% scale and 4,4,5,5-tetramethyl-2-(prop-1-en-2-yl)-1,3,2dioxaborolane $(84 \mathrm{mg})$. The crude was purified with $3 \%$ ethyl acetate/hexanes and a clear colorless oil was obtained (79 mg, 50\%). ${ }^{\mathbf{1}} \mathbf{H}$ NMR $\left(500 \mathrm{MHz}, \mathrm{CDCl}_{3}\right) \delta 1.29-1.19(\mathrm{~m}, 12 \mathrm{H}), 1.04(\mathrm{~s}, 3 \mathrm{H})$, $1.03-0.94(\mathrm{~m}, 1 \mathrm{H}), 0.47(\mathrm{~d}, J=14.4 \mathrm{~Hz}, 1 \mathrm{H}), 0.02(\mathrm{~s}, 9 \mathrm{H}),-0.00(\mathrm{~s}, 9 \mathrm{H}) .{ }^{13} \mathrm{C} \mathrm{NMR}(126 \mathrm{MHz}$, $\left.\mathrm{CDCl}_{3}\right) \delta 83.0,26.1,25.1,19.4,18.1,0.9,-3.5$. IR (FTIR): 2979, 2954, 2899, 2868, 2844, 1462, 1378, 1334, 1298, 1246, 1213, 1197, 1144, 1113, 1099, 1035, 970, $835 \mathrm{~cm}^{-1}$. HRMS (DART) exact mass calculated for $\mathrm{C}_{14} \mathrm{H}_{33} \mathrm{BO}_{2} \mathrm{Si}_{2}\left[\mathrm{M}-\mathrm{CH}_{3}\right]^{+} \mathrm{m} / \mathrm{z}=299.2034$, found $\mathrm{m} / \mathrm{z}=299.2033$. $\mathrm{R}_{\mathrm{f}}=$ $0.4(3 \% \mathrm{EA} / \mathrm{Hex})$.

(1-((3aR,4R,6R,7aS)-3a,5,5,7a-tetramethylhexahydro-4,6methanobenzo $[d][1,3,2]$ dioxaborol-2-yl)ethane-1,2-diyl)bis(trimethylsilane)(23):

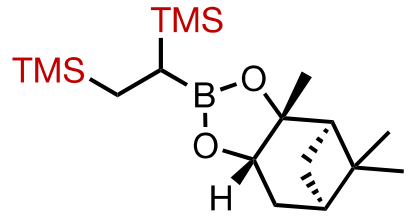

Following general procedure 1 at $50 \%$ scale with $103 \mathrm{mg}$ of (+)-vinylboronic acid pinanediol ester. The crude was purified with $1 \%$ ethyl acetate/hexanes. A clear colorless oil was obtained (79.8 mg, 45\%). ${ }^{1} \mathbf{H}$ NMR (500 MHz, $\left.\mathrm{CDCl}_{3}\right) \delta 4.23$ (ddd, $\left.J=10.6,8.8,2.2 \mathrm{~Hz}, 1 \mathrm{H}\right), 2.33$ (ddt, $J=14.2$, $8.9,2.5 \mathrm{~Hz}, 1 \mathrm{H}), 2.24-2.14(\mathrm{~m}, 1 \mathrm{H}), 2.05(\mathrm{td}, J=5.5,3.6 \mathrm{~Hz}, 1 \mathrm{H}), 1.90(\mathrm{dq}, J=5.8,2.9 \mathrm{~Hz}, 1 \mathrm{H})$, $1.82(\mathrm{tt}, J=14.1,2.7 \mathrm{~Hz}, 1 \mathrm{H}), 1.36(\mathrm{~s}, 3 \mathrm{H}), 1.27(\mathrm{~s}, 3 \mathrm{H}), 1.20(\mathrm{t}, J=10.7 \mathrm{~Hz}, 1 \mathrm{H}), 0.83(\mathrm{~s}, 3 \mathrm{H})$, 0.77 (ddd, $J=14.7,11.5,7.3 \mathrm{~Hz}, 1 \mathrm{H}), 0.48$ (ddd, $J=14.6,7.3,1.4 \mathrm{~Hz}, 1 \mathrm{H}), 0.29$ (ddd, $J=11.6$, 2.6, $1.5 \mathrm{~Hz}, 1 \mathrm{H}), 0.03$ (s, 9H), -0.04 (s, 9H). ${ }^{11} \mathbf{B}$ NMR (160 MHz, CDCl3) $\delta 34.17 .{ }^{13} \mathbf{C ~ N M R}(126$ $\left.\mathrm{MHz}, \mathrm{CDCl}_{3}\right) \delta 85.43,85.39,77.7,77.5,51.46,51.43,39.7,38.4,38.3,36.0,35.8,29.0,27.3,26.8$, 26.6, 24.2, 10.80, 10.73, -1.6, -1.7. IR (FTIR): 2984, 2951, 2916, 2870, 1475, 1450, 1366, 1336, 1314, 1278, 1245, 1208, 1122, 1078, 1028, 1009, 993, 939, 832, 750, $721 \mathrm{~cm}^{-1}$. HRMS (DART) exact mass calculated for $\mathrm{C}_{18} \mathrm{H}_{37} \mathrm{BO}_{2} \mathrm{Si}_{2}\left[\mathrm{M}-\mathrm{CH}_{3}\right]^{+} \mathrm{m} / \mathrm{z}=337.2190$, found $\mathrm{m} / \mathrm{z}=337.2192$. $\mathrm{R}_{\mathrm{f}}=$ $0.2(1 \% \mathrm{EA} / \mathrm{Hex})$.

(1-phenylethane-1,2-diyl)bis(dimethylsilane) (24):<smiles>CCCCC(C)c1ccccc1</smiles>

Following general procedure 1 with styrene $(104 \mathrm{mg})$ and dimethylchlorosilane $(282 \mathrm{mg})$. The crude was purified with pure hexanes and a clear colorless oil was obtained $(170 \mathrm{mg}, 77 \%) .{ }^{1} \mathbf{H}$ NMR $\left(500 \mathrm{MHz} \mathrm{CDCl}_{3}\right) \delta 7.36-6.90(\mathrm{~m}, 5 \mathrm{H}), 3.85(\mathrm{q}, J=3.6 \mathrm{~Hz}, 1 \mathrm{H}), 3.81-3.74(\mathrm{~m}, 1 \mathrm{H})$, $2.26-2.18(\mathrm{~m}, 1 \mathrm{H}), 1.15(\mathrm{ddd}, J=14.9,11.2,3.1 \mathrm{~Hz}, 1 \mathrm{H}), 1.04(\mathrm{dt}, J=15.0,4.0 \mathrm{~Hz}, 1 \mathrm{H}), 0.02$ $(\mathrm{dd}, J=3.7,1.3 \mathrm{~Hz}, 3 \mathrm{H}),-0.04(\mathrm{dd}, J=3.8,1.3 \mathrm{~Hz}, 3 \mathrm{H}),-0.07(\mathrm{dd}, J=3.7,1.8 \mathrm{~Hz}, 6 \mathrm{H}) .{ }^{13} \mathbf{C} \mathbf{N M R}$ 
$\left(126 \mathrm{MHz} \mathrm{CDCl}_{3}\right) \delta 144.8,128.2,127.8,124.7,29.9,15.2,-3.8,-4.0,-5.4,-6.1$. IR (FTIR): 3082, 3064, 3024, 2958, 2902, 2874, 1599, 1577, 1559, 1541, 1522, 1494, 1450, 1249, 1102, $876 \mathrm{~cm}^{-1}$. HRMS (DART) exact mass calculated for $\mathrm{C}_{12} \mathrm{H}_{21} \mathrm{Si}_{2}[\mathrm{M}-\mathrm{H}]^{+} \mathrm{m} / \mathrm{z}=221.1176$, found $\mathrm{m} / \mathrm{z}=$ 221.1179. $\mathrm{R}_{\mathrm{f}}=0.7($ Hex $)$.

\section{2,2'-(1-phenylethane-1,2-diyl)bis(1,1,1,2,2-pentamethyldisilane) (25):}

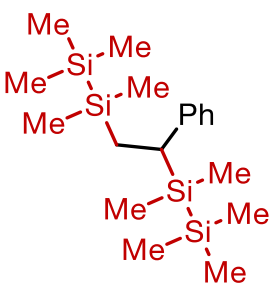

Following general procedure 1 with styrene (104 mg) and 1-chloro-1,1,2,2,2-pentamethyldisilane (500 mg). The crude was purified with pure hexanes and a clear colorless oil was obtained (329 mg, 90\%). ${ }^{1} \mathbf{H}$ NMR $\left(500 \mathrm{MHz}, \mathrm{CDCl}_{3}\right) \delta 7.19$ (t, $\left.J=7.8 \mathrm{~Hz}, 2 \mathrm{H}\right), 7.08-6.99$ (m, 3H), 2.27 (dd, $J=13.4,2.0 \mathrm{~Hz}, 1 \mathrm{H}), 1.21(\mathrm{dd}, J=15.1,13.4 \mathrm{~Hz}, 1 \mathrm{H}), 0.96(\mathrm{dd}, J=15.0,2.1 \mathrm{~Hz}, 1 \mathrm{H}), 0.03--$ $0.10(\mathrm{~m}, 24 \mathrm{H}),-0.14(\mathrm{~s}, 3 \mathrm{H}),-0.26(\mathrm{~s}, 3 \mathrm{H}) .{ }^{13} \mathbf{C ~ N M R}\left(126 \mathrm{MHz}, \mathrm{CDCl}_{3}\right) \delta 145.5,128.1,127.9$, $124.5,30.8,15.4,-1.6,-2.0,-3.4,-3.8,-5.2,-5.6 .{ }^{29} \mathrm{Si}$ NMR $\left(99 \mathrm{MHz}, \mathrm{CDCl}_{3}\right) \delta-11.38,-15.76$, 19.40, -19.71. IR (FTIR): 3081, 3062, 3024, 2948, 2893, 1599, 1494, 1450, 1402, 1243, 1098, 828, $791 \mathrm{~cm}^{-1}$. HRMS (DART) exact mass calculated for $\mathrm{C}_{17} \mathrm{H}_{35} \mathrm{Si}_{4}\left[\mathrm{M}-\mathrm{CH}_{3}\right]^{+} \mathrm{m} / \mathrm{z}=351.1810$, found $\mathrm{m} / \mathrm{z}=351.1820 . \mathrm{R}_{\mathrm{f}}=0.8(\mathrm{Hex})$.

\section{(1-phenylethane-1,2-diyl)bis(dimethyl(vinyl)silane) (26):}

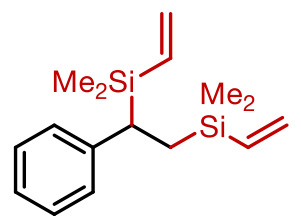

Following general procedure 1 with styrene $(118 \mathrm{mg}$ ) and chlorodimethyl(vinyl)silane (362 $\mathrm{mg})$. The crude was purified with pure hexanes and a clear colorless oil was obtained (197 mg, $72 \%) .{ }^{1} \mathbf{H}$ NMR $\left(500 \mathrm{MHz}, \mathrm{CDCl}_{3}\right) \delta 7.19(\mathrm{t}, J=7.7 \mathrm{~Hz}, 2 \mathrm{H}), 7.06(\mathrm{~d}, J=7.4 \mathrm{~Hz}, 1 \mathrm{H}), 7.03-6.98(\mathrm{~m}, 2 \mathrm{H})$, $6.12-5.78(\mathrm{~m}, 4 \mathrm{H}), 5.63(\mathrm{dd}, J=20.0,4.1 \mathrm{~Hz}, 1 \mathrm{H}), 5.54(\mathrm{dd}, J=20.0,4.2 \mathrm{~Hz}, 1 \mathrm{H}), 2.15(\mathrm{dd}, J=$ 13.0, 2.4 Hz, 1H), $1.10(\mathrm{dd}, J=15.1,13.0 \mathrm{~Hz}, 1 \mathrm{H}), 0.95(\mathrm{dd}, J=15.1,2.4 \mathrm{~Hz}, 1 \mathrm{H}),-0.02(\mathrm{~d}, J=$ $8.5 \mathrm{~Hz}, 6 \mathrm{H}),-0.18(\mathrm{~d}, J=7.8 \mathrm{~Hz}, 6 \mathrm{H}) .{ }^{13} \mathbf{C ~ N M R}\left(126 \mathrm{MHz}, \mathrm{CDCl}_{3}\right) \delta 144.7,139.6,137.6,132.7$, 131.2, 128.2, 128.0, 124.5, 30.7, 15.2, -2.4, -3.1, -4.6, -5.4. IR (FTIR): 3081, 3048, 3024, 3009, 2955, 2900, 2873, 1599, 1493, 1450, 1404, 1338, 1247, 1222, 1206, 1153, 1100, 1070, 1008, 950, 906, $832 \mathrm{~cm}^{-1}$. HRMS (DART) exact mass calculated for $\mathrm{C}_{16} \mathrm{H}_{27} \mathrm{Si}_{2}[\mathrm{M}+\mathrm{H}]^{+} \mathrm{m} / \mathrm{z}=275.1646$, found $\mathrm{m} / \mathrm{z}=275.1643$. $\mathrm{R}_{\mathrm{f}}=0.8(\mathrm{Hex})$.

(1-phenylethane-1,2-diyl)bis(allyldimethylsilane) (27): 


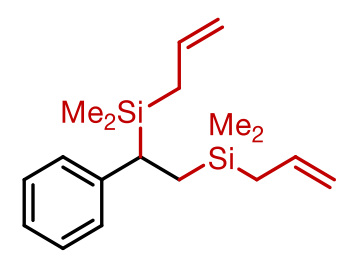

Following general procedure 1 with styrene $(104 \mathrm{mg})$ and allylchlorodimethylsilane (404mg). The crude was purified with pure hexanes and a clear colorless oil was obtained $(239 \mathrm{mg}, 79 \%)$. ${ }^{1} \mathbf{H}$ NMR $\left(500 \mathrm{MHz}, \mathrm{CDCl}_{3}\right) \delta 7.20(\mathrm{~m}, 2 \mathrm{H}), 7.09-7.00(\mathrm{~m}, 3 \mathrm{H}), 5.76-5.60(\mathrm{~m}, 2 \mathrm{H}), 4.85-4.72$ $(\mathrm{m}, 4 \mathrm{H}), 2.16(\mathrm{dd}, J=13.4,2.2 \mathrm{~Hz}, 1 \mathrm{H}), 1.46(\mathrm{~d}, J=8.1 \mathrm{~Hz}, 2 \mathrm{H}), 1.38-1.24(\mathrm{~m}, 2 \mathrm{H}), 1.08(\mathrm{dd}, J$ $=15.1,13.3 \mathrm{~Hz}, 1 \mathrm{H}), 0.89$ (dd, J = 15.1, $2.2 \mathrm{~Hz}, 1 \mathrm{H}),-0.04(\mathrm{~s}, 3 \mathrm{H}),-0.11(\mathrm{~s}, 3 \mathrm{H}),-0.22(\mathrm{~s}, 3 \mathrm{H})$, $0.25(\mathrm{~s}, 3 \mathrm{H}) .{ }^{13} \mathbf{C ~ N M R}\left(126 \mathrm{MHz}, \mathrm{CDCl}_{3}\right) \delta 144.6,135.3,135.1,128.2,128.0,124.6,113.2,112.8$, 30.2, 23.8, 21.8, 14.4, -2.9, -3.2, -5.35, -5.36. IR (FTIR): 3078, 3061, 3025, 2996, 2969, 2955, 2913, 2898, 2877, 1738, 1630, 1600, 1494, 1450, 1420, 1366, 1249, 1228, 1217, 1205, 1155, 1102, 1033, 991, 893, $834 \mathrm{~cm}^{-1}$. HRMS (DART) exact mass calculated for $\mathrm{C}_{15} \mathrm{H}_{25} \mathrm{Si}_{2}\left[\mathrm{M}-\mathrm{C}_{3} \mathrm{H}_{5}\right]^{+} \mathrm{m} / \mathrm{z}=$ 261.1489, found $\mathrm{m} / \mathrm{z}=261.1493$. $\mathrm{R}_{\mathrm{f}}=0.8(\mathrm{Hex})$.

\section{2,2'-((1S,2S)-2,3-dihydro-1H-indene-1,2-diyl)bis(1,1,1,2,2-pentamethyldisilane) (28):}

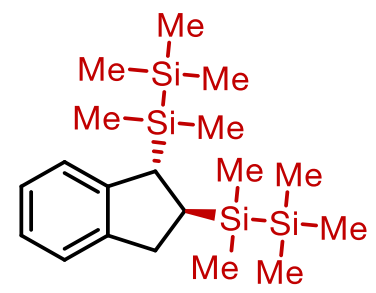

Following general procedure 1 with indene $(116 \mathrm{mg})$ and 1-chloro-1,1,2,2,2-pentamethyldisilane (500 mg). The crude was purified with pure hexanes and a clear colorless oil was obtained (211 mg, 56\%). ${ }^{1} \mathbf{H}$ NMR $\left(599 \mathrm{MHz}, \mathrm{CDCl}_{3}\right) \delta 7.12-7.07(\mathrm{~m}, 1 \mathrm{H}), 7.06-6.97(\mathrm{~m}, 3 \mathrm{H}), 3.27-3.19$ $(\mathrm{m}, 1 \mathrm{H}), 2.83(\mathrm{dd}, J=16.4,2.0 \mathrm{~Hz}, 1 \mathrm{H}), 2.58(\mathrm{~d}, J=2.0 \mathrm{~Hz}, 1 \mathrm{H}), 1.69(\mathrm{dt}, J=10.4,2.0 \mathrm{~Hz}, 1 \mathrm{H})$, $0.05(\mathrm{~d}, J=1.3 \mathrm{~Hz}, 3 \mathrm{H}), 0.04(\mathrm{~s}, 3 \mathrm{H}), 0.00(\mathrm{~s}, 3 \mathrm{H}),-0.02(\mathrm{~s}, 9 \mathrm{H}),-0.11(\mathrm{~s}, 3 \mathrm{H}),-0.12(\mathrm{~s}, 9 \mathrm{H}) .{ }^{13} \mathrm{C}$ NMR $\left(126 \mathrm{MHz}, \mathrm{CDCl}_{3}\right) \delta 146.7,142.4,126.0,124.9,124.6,123.7,36.5,35.3,26.2,-1.6,-1.7$, 4.36, -4.39, -4.8, -5.6. ${ }^{29} \mathrm{Si}$ NMR $\left(99 \mathrm{MHz} \mathrm{CDCl}_{3}\right) \delta-13.44,-13.81,-19.61,-19.97$. IR (FTIR): 3068, 3019, 2948, 2892, 2852, 1740, 1600, 1473, 1456, 1395, 1243, 1133, 1085, 1046, 927, 829 $\mathrm{cm}^{-1}$. HRMS (DART) exact mass calculated for $\mathrm{C}_{18} \mathrm{H}_{35} \mathrm{Si}_{4}\left[\mathrm{M}-\mathrm{CH}_{3}\right]^{+} \mathrm{m} / \mathrm{z}=363.1810$, found $\mathrm{m} / \mathrm{z}$ $=363.1817 . \mathrm{R}_{\mathrm{f}}=0.8($ Hex $)$.

\section{(1-phenylethane-1,2-diyl)bis(trimethylgermane) (29):}<smiles>CC(C)CC(CC(C)C)c1ccccc1</smiles>

Following general procedure 1 with styrene $(104 \mathrm{mg})$ and chlorotrimethylgermane (1020 mg). The crude was purified with pure hexanes and a clear colorless oil was obtained $(270 \mathrm{mg}, 79 \%)$. ${ }^{1} \mathbf{H}$ NMR $\left(500 \mathrm{MHz}, \mathrm{CDCl}_{3}\right) \delta 7.19(\mathrm{t}, J=7.7 \mathrm{~Hz}, 2 \mathrm{H}), 7.08-6.97(\mathrm{~m}, 3 \mathrm{H}), 2.37(\mathrm{dd}, J=13.9,2.9$ $\mathrm{Hz}, 1 \mathrm{H}), 1.38$ (t, $J=13.9 \mathrm{~Hz}, 1 \mathrm{H}), 1.08$ (dd, $J=14.1,2.9 \mathrm{~Hz}, 1 \mathrm{H}), 0.03(\mathrm{~s}, 9 \mathrm{H}),-0.09(\mathrm{~s}, 9 \mathrm{H}) .{ }^{13} \mathrm{C}$ NMR $\left(126 \mathrm{MHz}, \mathrm{CDCl}_{3}\right) \delta 145.6,128.0,127.2,124.3,33.6,17.4,-1.4,-3.8$. IR (FTIR): 3102, 
3081, 3061, 3023, 2970, 2907, 2801, 1600, 1493, 1450, 1416, 1340, 1234, 1214, 1098, 1070, 1022, 903, $819 \mathrm{~cm}^{-1}$. HRMS (DART) exact mass calculated for $\mathrm{C}_{13} \mathrm{H}_{23} \mathrm{Ge}_{2}\left[\mathrm{M}-\mathrm{CH}_{3}\right]^{+} \mathrm{m} / \mathrm{z}=327.0218$, found $\mathrm{m} / \mathrm{z}=327.0225$. $\mathrm{R}_{\mathrm{f}}=0.9(\mathrm{Hex})$.

(Z)-(2,5-diphenylpent-2-ene-1,5-diyl)bis(trimethylsilane) (32):<smiles>CS(=O)(=O)C/C(=C\CC(c1ccccc1)c1ccccc1)c1ccccc1</smiles>

Following general procedure 1 with (1-(2-phenylcyclopropyl)vinyl)benzene $(220 \mathrm{mg})$. The reaction was stopped at $2.5 \mathrm{~F}$ of charge and the crude was purified with pure hexanes and a clear colorless oil was obtained (268 mg, 73\%). ${ }^{1} \mathbf{H}$ NMR $\left(500 \mathrm{MHz}, \mathrm{CDCl}_{3}\right) \delta 7.25-7.00(\mathrm{~m}, 10 \mathrm{H})$, $5.43(\mathrm{t}, J=6.5 \mathrm{~Hz}, 1 \mathrm{H}), 2.66-2.52(\mathrm{~m}, 2 \mathrm{H}), 2.18(\mathrm{dd}, J=10.1,5.7 \mathrm{~Hz}, 1 \mathrm{H}), 2.00(\mathrm{~s}, 2 \mathrm{H}),-0.01$ (s, 9H), -0.12 (s, 9H). ${ }^{13} \mathrm{C}$ NMR (126 MHz, $\left.\mathrm{CDCl}_{3}\right) \delta 145.0,143.8,138.0,128.2,128.1,127.9$, 126.7, 126.5, 126.1, 124.5, 37.4, 29.4, 21.3, -0.7, -2.6. ${ }^{29} \mathrm{Si} \mathrm{NMR}\left(99 \mathrm{MHz}, \mathrm{CDCl}_{3}\right) \delta 3.42,1.90$. IR (FTIR): 3078, 3058, 3021, 2953, 2894, 1599, 1489, 1449, 1412, 1339, 1246, 1189, 1154, 1135 , 833, 774, 756, $696 \mathrm{~cm}^{-1}$. HRMS (DART) exact mass calculated for $\mathrm{C}_{23} \mathrm{H}_{35} \mathrm{Si}_{2}[\mathrm{M}+\mathrm{H}]^{+} \mathrm{m} / \mathrm{z}=$ 367.2277, found $\mathrm{m} / \mathrm{z}=367.2277 . \mathrm{R}_{\mathrm{f}}=0.4(\mathrm{Hex})$.

(5-phenylpent-2-ene-1,5-diyl)bis(trimethylsilane) (33):<smiles>CCCCCC([As])c1ccccc1</smiles>

Following general procedure 1 at 50\% scale with (2-vinylcyclopropyl)benzene (72 $\mathrm{mg}$ ) and 4 equivalents of TMSCl. The crude was purified with pure hexanes and a clear colorless oil was obtained $(79.8 \mathrm{mg}, 55 \%, \mathrm{E} / \mathrm{Z}$ or $\mathrm{Z} / \mathrm{E}=1.16: 1) .{ }^{1} \mathbf{H} \mathbf{~ N M R}\left(500 \mathrm{MHz}, \mathrm{CDCl}_{3}\right) \delta 7.25-7.19(\mathrm{~m}$, $2 \mathrm{H}), 7.11-7.05(\mathrm{~m}, 1 \mathrm{H}), 7.04-6.98(\mathrm{~m}, 2 \mathrm{H}), 5.31(\mathrm{dtt}, J=10.2,8.5,1.5 \mathrm{~Hz}, 1 \mathrm{H}), 5.22-5.14$ (m, 1H), $2.54-2.38(\mathrm{~m}, 2 \mathrm{H}), 2.06(\mathrm{dd}, J=10.8,5.1 \mathrm{~Hz}, 1 \mathrm{H}), 1.53-1.40(\mathrm{~m}, 2 \mathrm{H}), 0.01(\mathrm{~s}, 9 \mathrm{H})$, 0.04 (s, 9H). $\left.{ }^{13} \mathbf{C ~ N M R ~ ( 1 2 6 ~ M H z , ~} \mathrm{CDCl}_{3}\right) \delta 143.9,128.1,127.9,127.8,125.6,124.4,37.5,26.9$, 18.7, -1.6, -2.7. IR (FTIR): 3082, 3062, 3023, 2008, 2953, 2895, 2858, 2363, 2332, 1636, 1603, 1495, 1450, 1417, 1391, 1247, 1149, 1075, 1031, 986, 896, $834 \mathrm{~cm}^{-1}$. HRMS (DART) exact mass calculated for $\mathrm{C}_{17} \mathrm{H}_{30} \mathrm{Si}_{2}[\mathrm{M}+\mathrm{H}]^{+} \mathrm{m} / \mathrm{z}=291.1964$, found $\mathrm{m} / \mathrm{z}=291.1960 . \mathrm{R}_{\mathrm{f}}=0.8$ (Hex).

\section{trimethyl(2-phenylallyl)silane (35):}<smiles>C=C(CC(C)C)c1ccccc1</smiles>

Following general procedure 1 with (3-(benzyloxy)prop-1-en-2-yl)benzene (34, $224 \mathrm{mg}$ ) or (3phenoxyprop-1-en-2-yl)benzene $(\mathbf{5 4}, 210 \mathrm{mg})$. The reaction was stopped at $2.5 \mathrm{~F}$ crude was purified with pure hexanes and a clear colorless oil was obtained (135 mg, 71\%) or (142 mg,75\% from 65). ${ }^{1} \mathbf{H}$ NMR $\left(500 \mathrm{MHz}, \mathrm{CDCl}_{3}\right) \delta 7.53-7.18(\mathrm{~m}, 5 \mathrm{H}), 5.13(\mathrm{~d}, J=1.7 \mathrm{~Hz}, 1 \mathrm{H}), 4.89-4.85$ 
(m, 1H), $2.04-2.01(\mathrm{~m}, 2 \mathrm{H}),-0.10(\mathrm{~s}, 9 \mathrm{H})$. HRMS (DART) exact mass calculated for $\mathrm{C}_{12} \mathrm{H}_{19} \mathrm{Si}_{2}$ $[\mathrm{M}+\mathrm{H}]^{+} \mathrm{m} / \mathrm{z}=191.1256$, found $\mathrm{m} / \mathrm{z}=191.1251 . \mathrm{R}_{\mathrm{f}}=0.8(0 \%)$. Product is consistent with previous literature reports.

\section{4-(4-methoxyphenyl)-1,1,2,2,3,3-hexamethyl-1,2,3-trisilolane (36):}<smiles>COc1ccc(C(C[Si](C)(C)[Si](C)(C)C)[Si](C)(C)C)cc1</smiles>

Following general procedure 3 with 4-methoxystyrene $(67 \mathrm{mg})$ and 1,3-dichloro-1,1,2,2,3,3hexamethyltrisilane $(184 \mathrm{mg})$. The crude was purified with $0 \%$ to $6 \%$ ethyl acetate/hexanes over $15 \mathrm{CV}$ on the biotage and a clear colorless oil was obtained $(142 \mathrm{mg}, 92 \%) .{ }^{\mathbf{1}} \mathbf{H}$ NMR $(500 \mathrm{MHz}$, $\mathrm{CDCl} 3) \delta 7.02(\mathrm{~d}, \mathrm{~J}=8.6 \mathrm{~Hz}, 2 \mathrm{H}), 6.90-6.84(\mathrm{~m}, 2 \mathrm{H}), 3.83(\mathrm{~s}, 3 \mathrm{H}), 2.17$ (dd, J = 15.4, 3.8 Hz, $1 \mathrm{H}), 1.20(\mathrm{dd}, \mathrm{J}=13.8,3.8 \mathrm{~Hz}, 1 \mathrm{H}), 1.15-1.05(\mathrm{~m}, 1 \mathrm{H}), 0.29(\mathrm{~s}, 3 \mathrm{H}), 0.27(\mathrm{~s}, 6 \mathrm{H}), 0.20(\mathrm{~s}, 3 \mathrm{H})$, 0.11 (s, 3H), -0.16 (s, 3H). ${ }^{13} \mathbf{C}$ NMR (126 MHz, CDCl3) $\delta 156.5,138.5,127.2,113.4,55.2,33.1$, 20.2, -3.3, -3.7, -4.3, -7.2, -7.3, -7.6. ${ }^{29} \mathrm{Si} \mathrm{NMR}(99 \mathrm{MHz}, \mathrm{CDCl} 3) \delta-7.54,-12.05,-50.95$. Product is unstable on the NMR time scale. IR (FTIR): 3094, 3059, 3030, 2997, 2951, 2893, 2833, 2797, 1609, 1579, 1506, 1463, 1441, 1404, 1300, 1273, 1243, 1177, 1037, 992, 799, 779, 730, $692 \mathrm{~cm}^{-}$ 1. HRMS (DART) exact mass calculated for $\mathrm{C}_{15} \mathrm{H}_{29} \mathrm{OSi}_{3}[\mathrm{M}+\mathrm{H}]^{+} \mathrm{m} / \mathrm{z}=309.15207$, found $\mathrm{m} / \mathrm{z}=$ 309.15195. $\mathrm{R}_{\mathrm{f}}=0.48(2.5 \% \mathrm{EA} / \mathrm{Hex})$.

\section{3-(1,1,2,2,3,3-hexamethyl-1,2,3-trisilolan-4-yl)-1-phenyl-1H-pyrazole (37):}<smiles>C[Si]1(C)CC(c2ccn(-c3ccccc3)n2)[Si](C)(C)[Si]1(C)C</smiles>

Following general procedure 3 with 4-methoxystyrene $(85 \mathrm{mg})$ and 1,3-dichloro-1,1,2,2,3,3hexamethyltrisilane $(184 \mathrm{mg})$. The crude was purified with $0 \%$ to $5 \%$ ethyl acetate/hexanes over $15 \mathrm{CV}$ on the biotage and a clear colorless oil was obtained $(119 \mathrm{mg}, 69 \%) .{ }^{\mathbf{1}} \mathbf{H}$ NMR $(500 \mathrm{MHz}$, $\left.\mathrm{CDCl}_{3}\right) \delta 7.74-7.67(\mathrm{~m}, 2 \mathrm{H}), 7.63(\mathrm{~s}, 1 \mathrm{H}), 7.50(\mathrm{~s}, 1 \mathrm{H}), 7.48-7.41(\mathrm{~m}, 2 \mathrm{H}), 7.26(\mathrm{q}, J=8.6,7.3$ $\mathrm{Hz}, 1 \mathrm{H}), 2.10(\mathrm{dd}, J=15.3,3.9 \mathrm{~Hz}, 1 \mathrm{H}), 1.33-1.21(\mathrm{~m}, 1 \mathrm{H}), 1.02-0.87(\mathrm{~m}, 1 \mathrm{H}), 0.39-0.10(\mathrm{~m}$, $15 \mathrm{H}),-0.11(\mathrm{~s}, 3 \mathrm{H}) .{ }^{13} \mathbf{C ~ N M R}\left(126 \mathrm{MHz}, \mathrm{CDCl}_{3}\right) \delta 140.5,139.8,129.5,129.4,128.8,125.8,122.4$, 118.5, 23.1, 20.8, -3.2, -3.5, -4.1, -7.0, -7.3, -7.6. ${ }^{29} \mathrm{Si}$ NMR $\left(99 \mathrm{MHz}, \mathrm{CDCl}_{3}\right) \delta$-7.43, -11.97, 51.41. IR (FTIR): 3050, 2949, 2889, 2793, 1601, 1503, 1463, 1403, 1387, 1361, 1244, 1191, 1140, 1047, 1014, 952, 908, 831, 814, 783, 753, 729, $688 \mathrm{~cm}^{-1}$. HRMS (DART) exact mass calculated for $\mathrm{C}_{17} \mathrm{H}_{29} \mathrm{~N} 2 \mathrm{Si}_{3}[\mathrm{M}+\mathrm{H}]^{+} \mathrm{m} / \mathrm{z}=345.16330$, found $\mathrm{m} / \mathrm{z}=345.16318 . \mathrm{R}_{\mathrm{f}}=0.1(2.5 \% \mathrm{EA} / \mathrm{Hex})$.

\section{1,1,2,2,3,3-hexamethyl-4,5-diphenyl-2,3-dihydro-1H-1,2,3-trisilole (38):}




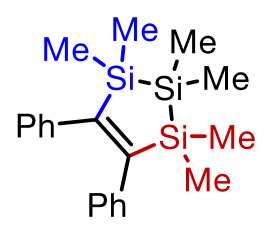

Following general procedure 3 with 4-methoxystyrene $(70 \mathrm{mg}$ ) and 1,3-dichloro-1,1,2,2,3,3hexamethyltrisilane $(184 \mathrm{mg})$. The crude was purified with $0 \%$ to $3 \%$ ethyl acetate/hexanes over $15 \mathrm{CV}$ on the biotage and a white crystalline solid was obtained $(74 \mathrm{mg}, 42 \%) .{ }^{1} \mathbf{H}$ NMR $(500 \mathrm{MHz}$, $\left.\mathrm{CDCl}_{3}\right) \delta 7.10(\mathrm{td}, J=7.7,1.8 \mathrm{~Hz}, 4 \mathrm{H}), 6.99(\mathrm{dd}, J=7.4,1.7 \mathrm{~Hz}, 2 \mathrm{H}), 6.81-6.75(\mathrm{~m}, 4 \mathrm{H}), 0.37$ $(\mathrm{d}, J=1.8 \mathrm{~Hz}, 6 \mathrm{H}), 0.21(\mathrm{~s}, 12 \mathrm{H}) .{ }^{13} \mathbf{C} \mathbf{N M R}\left(126 \mathrm{MHz}, \mathrm{CDCl}_{3}\right) \delta 162.4,144.1,127.8,127.4$, 124.7, -2.5, -8.2. ${ }^{29} \mathbf{S i} \mathbf{~ N M R}\left(99 \mathrm{MHz}, \mathrm{CDCl}_{3}\right.$ ) $\delta-11.55,-56.58$. IR (FTIR): 3068, 3011, 2956, 2894, 1595, 1568, 1480, 1436, 1403, 1243, 1068, 1028, 985, 856, 836, 824, 801, 783, 752, 730, $714,696,657 \mathrm{~cm}^{-1}$. HRMS (DART) exact mass calculated for $\mathrm{C}_{20} \mathrm{H}_{29} \mathrm{Si}_{3}[\mathrm{M}+\mathrm{H}]^{+} \mathrm{m} / \mathrm{z}=353.15716$, found $\mathrm{m} / \mathrm{z}=353.15703 . \mathrm{R}_{\mathrm{f}}=0.25(\mathrm{Hex})$.

\section{1,1,2,2,3,3,4-heptamethyl-5-phenyl-2,3-dihydro-1 $H$-1,2,3-trisilole (39):}

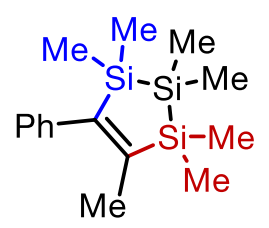

Following general procedure 3 with 1-phenyl-propyne (116 mg) and 1,3-dichloro-1,1,2,2,3,3hexamethyltrisilane $(184 \mathrm{mg})$. The crude was purified with pure hexanes over $5 \mathrm{CV}$ on the biotage and a clear colorless oil was obtained $(82 \mathrm{mg}, 56 \%) .{ }^{1} \mathbf{H}$ NMR $\left(500 \mathrm{MHz}, \mathrm{C}_{6} \mathrm{D}_{6}\right) \delta 7.25-7.16(\mathrm{~m}$, $2 \mathrm{H}), 7.10-7.03(\mathrm{~m}, 1 \mathrm{H}), 6.91-6.86(\mathrm{~m}, 2 \mathrm{H}), 1.75(\mathrm{~s}, 3 \mathrm{H}), 0.27(\mathrm{~s}, 6 \mathrm{H}), 0.25(\mathrm{~s}, 6 \mathrm{H}), 0.16(\mathrm{~s}, 6 \mathrm{H})$. ${ }^{13} \mathbf{C}$ NMR $\left(126 \mathrm{MHz}, \mathrm{C}_{6} \mathrm{D}_{6}\right) \delta 161.2,156.0,144.9,128.5,127.5,125.5,18.3,-2.5,-3.2,-8.3 .{ }^{29} \mathbf{S i}$ NMR $\left(99 \mathrm{MHz}, \mathrm{C}_{6} \mathrm{D}_{6}\right) \delta$-11.25, -12.59, -57.94. IR (FTIR): 3074, 3057, 3022, 2950, 2893, 2852, 2280, 1597, 1480, 1439, 1404, 1330, 1244, 1209, 1069, 1030, 1005, 980, 926, 894, 834, 809, 770, $754 \mathrm{~cm}^{-1}$. HRMS (DART) exact mass calculated for $\mathrm{C}_{15} \mathrm{H}_{27} \mathrm{Si}_{3}[\mathrm{M}+\mathrm{H}]^{+} \mathrm{m} / \mathrm{z}=291.14151$, found $\mathrm{m} / \mathrm{z}=291.14139$. $\mathrm{R}_{\mathrm{f}}=0.54(\mathrm{Hex})$.

\section{5-(4-methoxyphenyl)-1,1,2,2,3,3,4,4-octamethyl-1,2,3,4-tetrasilinane (40):}

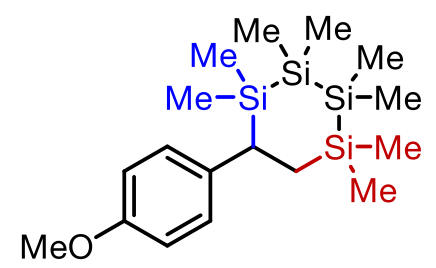

Following general procedure 3 with 4-methoxystyrene $(70 \mathrm{mg}$ ) and 1,4-dichloro-1,1,2,2,3,3,4,4octamethyltetrasilane $(227 \mathrm{mg}$ ). The crude was purified with $0 \%$ to $3 \%$ ethyl acetate/hexanes over $15 \mathrm{CV}$ on the biotage and a white crystalline solid was obtained (114 mg, 62\%). ${ }^{1} \mathbf{H}$ NMR (500 $\left.\mathrm{MHz}, \mathrm{CDCl}_{3}\right) \delta 7.05-6.97(\mathrm{~m}, 2 \mathrm{H}), 6.90-6.81(\mathrm{~m}, 2 \mathrm{H}), 3.82(\mathrm{~s}, 3 \mathrm{H}), 2.28-2.21(\mathrm{~m}, 1 \mathrm{H}), 1.38$ $(\mathrm{t}, J=14.0 \mathrm{~Hz}, 1 \mathrm{H}), 1.11-1.00(\mathrm{~m}, 1 \mathrm{H}), 0.48--0.30(\mathrm{~m}, 24 \mathrm{H}) .{ }^{13} \mathbf{C} \mathbf{~ N M R}\left(126 \mathrm{MHz}, \mathrm{CDCl}_{3}\right) \delta$ 156.7, 140.0, 128.0, 113.4, 55.4, 29.4, 20.2, -2.7, -3.7, -3.8, -6.1, -6.5, -6.6, -6.8, -7.2. ${ }^{29} \mathrm{Si}$ NMR $\left(99 \mathrm{MHz}, \mathrm{CDCl}_{3}\right) \delta$-12.79, -14.94, -48.01, -49.33. IR (FTIR): 3100, 3086, 2950, 2891, 2833, 1601, 
1579, 1506, 1463, 1440, 1403, 1326, 1300, 1273, 1245, 1200, 1177, 1149, 1103, 1037, 995, 834, $820,801,780,750,729,693 \mathrm{~cm}^{-1}$. HRMS (DART) exact mass calculated for $\mathrm{C}_{17} \mathrm{H}_{35} \mathrm{OSi}{ }_{4}[\mathrm{M}+\mathrm{H}]^{+}$ $\mathrm{m} / \mathrm{z}=367.17595$, found $\mathrm{m} / \mathrm{z}=367.17604$. $\mathrm{R}_{\mathrm{f}}=0.2(\mathrm{Hex})$. Compound is very unstable at room temperature and ambient conditions.

\section{2,2,5,5-tetramethyl-3-phenyl-1,2,5-oxadisilolane (41):}<smiles>C[Si]1(C)CC(c2ccccc2)[Si](C)(C)O1</smiles>

Following general procedure 1 with styrene $(104 \mathrm{mg})$. The crude was purified with 0 3\% ethyl acetate/ hexanes through a $25 \mathrm{~g}$ Forisil column to a semi pure product, which was further purified via short path distillation. A clear colorless oil was obtained $(87 \mathrm{mg}, 37 \%)$. ${ }^{\mathbf{1}} \mathbf{H}$ NMR $(500 \mathrm{MHz}$, $\left.\mathrm{CDCl}_{3}\right) \delta 7.29-7.23(\mathrm{~m}, 2 \mathrm{H}), 7.17-7.07(\mathrm{~m}, 3 \mathrm{H}), 2.51(\mathrm{dd}, J=13.2,7.4 \mathrm{~Hz}, 1 \mathrm{H}), 1.29-1.15$ $(\mathrm{m}, 2 \mathrm{H}), 0.32(\mathrm{~s}, 3 \mathrm{H}), 0.30(\mathrm{~s}, 3 \mathrm{H}), 0.23(\mathrm{~s}, 3 \mathrm{H}),-0.13(\mathrm{~s}, 3 \mathrm{H}),{ }^{13} \mathbf{C} \mathbf{N M R}\left(126 \mathrm{MHz}, \mathrm{CDCl}_{3}\right) \delta$ 144.5, 128.5, 126.4, 124.5, 33.1, 16.7, 1.0, 0.5, -0.4, -2.6. IR (FTIR): 3081, 3060, 3022, 2957, 2916, 2890, 2853, 1739, 1599, 1494, 1448, 1408, 1251, 1198, 1147, 1072, 1033, 914, 875, 853, $819 \mathrm{~cm}^{-1}$. HRMS (DART) exact mass calculated for $\mathrm{C}_{12} \mathrm{H}_{21} \mathrm{OSi}_{2}[\mathrm{M}+\mathrm{H}]^{+} \mathrm{m} / \mathrm{z}=237.1131$, found $\mathrm{m} / \mathrm{z}=237.1127 . \mathrm{R}_{\mathrm{f}}=0.6(3 \% \mathrm{EA} / \mathrm{Hex})$.

\section{1,1,4,4-tetramethyl-2-phenyl-1,4-disilinane (42):}

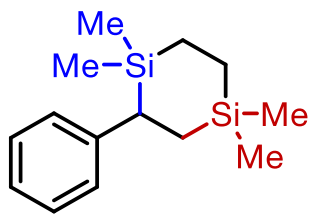

Following general procedure 1 with styrene (104 mg) and 1,2-bis(chlorodimethylsilyl)ethane (645 $\mathrm{mg})$. The crude was purified with pure hexanes and a clear colorless oil was obtained (214 $\mathrm{mg}$, 86\%). ${ }^{1} \mathbf{H}$ NMR $\left(500 \mathrm{MHz}, \mathrm{CDCl}_{3}\right) \delta 7.28-7.21(\mathrm{~m}, 2 \mathrm{H}), 7.11-7.04(\mathrm{~m}, 3 \mathrm{H}), 2.28$ (dd, J = 14.8, $2.9 \mathrm{~Hz}, 1 \mathrm{H}), 1.21(\mathrm{t}, J=14.4 \mathrm{~Hz}, 1 \mathrm{H}), 1.03-0.84(\mathrm{~m}, 3 \mathrm{H}), 0.76(\mathrm{td}, J=13.4,12.4,2.5 \mathrm{~Hz}, 1 \mathrm{H})$, $0.68(\mathrm{td}, J=14.4,13.3,2.9 \mathrm{~Hz}, 1 \mathrm{H}), 0.11(\mathrm{~s}, 3 \mathrm{H}), 0.05(\mathrm{~s}, 3 \mathrm{H}),-0.13(\mathrm{~d}, J=6.9 \mathrm{~Hz}, 6 \mathrm{H}) .{ }^{13} \mathbf{C ~ N M R}$ $\left(126 \mathrm{MHz}, \mathrm{CDCl}_{3}\right) \delta 147.1,128.0,127.0,124.2,31.0,17.0,9.5,9.0,-1.8,-3.0,-4.5,-8.0 .{ }^{29} \mathbf{S i}$ NMR $\left(99 \mathrm{MHz}, \mathrm{CDCl}_{3}\right) \delta 2.11,1.26$. IR (FTIR): 3079, 3056, 3021, 2958, 2898, 2861, 2794, 1598, 1492, 1448, 1404, 1244, 1199, 1190, 1157, 1056, 1037, 1029, 994, 824, 759, $732 \mathrm{~cm}^{-1}$. HRMS (DART) exact mass calculated for $\mathrm{C}_{14} \mathrm{H}_{25} \mathrm{Si}_{2}[\mathrm{M}+\mathrm{H}]^{+} \mathrm{m} / \mathrm{z}=249.1489$, found $\mathrm{m} / \mathrm{z}=249.1493$. $\mathrm{R}_{\mathrm{f}}$ $=0.8($ Hex $)$.

\section{(4-methoxyphenethyl)trimethylsilane (43):}<smiles>CCCc1ccc(OC)cc1</smiles>

Following general procedure 2 with 4-methoxystyrene $(134 \mathrm{mg})$. The crude was purified with $2.5 \%$ ethyl acetate/hexanes and a clear colorless oil was obtained (167 mg, 80\%). ${ }^{1} \mathbf{H}$ NMR (500 
$\left.\mathrm{MHz}, \mathrm{CDCl}_{3}\right) \delta 7.25-7.11(\mathrm{~m}, 2 \mathrm{H}), 7.00-6.77(\mathrm{~m}, 2 \mathrm{H}), 3.86(\mathrm{~s}, 3 \mathrm{H}), 2.84-2.51(\mathrm{~m}, 2 \mathrm{H}), 1.19$ $-0.71(\mathrm{~m}, 2 \mathrm{H}), 0.12(\mathrm{~s}, 9 \mathrm{H}) .{ }^{13} \mathbf{C}$ NMR $\left(126 \mathrm{MHz}, \mathrm{CDCl}_{3}\right) \delta 157.7,137.5,128.7,113.8,55.3,29.2$, 19.0, -1.6. ${ }^{29} \mathrm{Si}$ NMR $\left(99 \mathrm{MHz}, \mathrm{CDCl}_{3}\right) \delta$ 1.37. IR (FTIR): 3062, 3031, 2998, 2952, 2909, 2834 , $1612,1584,1511,1465,1441,1300,1245,1176,1127,1040,907,862,831,771,756,734,714$, $691 \mathrm{~cm}^{-1}$. HRMS (DART) exact mass calculated for $\mathrm{C}_{11} \mathrm{H}_{17} \mathrm{OSi}\left[\mathrm{M}-\mathrm{CH}_{3}\right]^{+} \mathrm{m} / \mathrm{z}=193.10432$, found $\mathrm{m} / \mathrm{z}=193.10437$. $R_{\mathrm{f}}=0.2(2.5 \% \mathrm{EA} / \mathrm{Hex})$. The compound is consistent with previous reports.

\section{1-phenyl-4-(2-(trimethylsilyl)ethyl)-1H-pyrazole (44):}

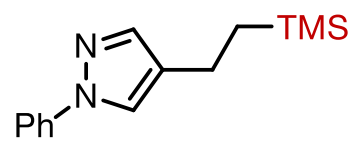

Following general procedure 2 with 1-phenyl-4-vinyl-1H-pyrazole $(170 \mathrm{mg})$. The crude was purified with 5\% ethyl acetate/hexanes and a pale yellow oil was obtained (122 $\mathrm{mg}, 50 \%)$. ${ }^{1} \mathbf{H}$ $\operatorname{NMR}\left(500 \mathrm{MHz}, \mathrm{CDCl}_{3}\right) \delta 7.75-7.66(\mathrm{~m}, 3 \mathrm{H}), 7.59(\mathrm{~s}, 1 \mathrm{H}), 7.45(\mathrm{ddd}, J=8.6,5.6,1.9 \mathrm{~Hz}, 2 \mathrm{H})$, $7.30-7.23(\mathrm{~m}, 1 \mathrm{H}), 2.65-2.53(\mathrm{~m}, 2 \mathrm{H}), 0.96-0.89(\mathrm{~m}, 2 \mathrm{H}), 0.07(\mathrm{~s}, 9 \mathrm{H}) .{ }^{13} \mathbf{C}$ NMR $(126 \mathrm{MHz}$, $\left.\mathrm{CDCl}_{3}\right) \delta 140.7,140.5,129.4,127.0,126.0,124.3,118.8,18.6,18.0,-1.6 .{ }^{29} \mathrm{Si}$ NMR $(99 \mathrm{MHz}$, $\mathrm{CDCl}_{3}$ ) $\delta$ 1.41. IR (FTIR): 3088, 3051, 2951, 2911, 2858, 1600, 1569, 1503, 1463, 1447, 1394, 1316, 1246, 1211, 1174, 1108, 1073, 1043, 1018, 994, 952, 831, 752, 688, 665, 652 $\mathrm{cm}^{-1}$. HRMS (DART) exact mass calculated for $\mathrm{C}_{14} \mathrm{H}_{21} \mathrm{~N}_{2} \mathrm{Si}[\mathrm{M}+\mathrm{H}]^{+} \mathrm{m} / \mathrm{z}=245.14685$, found $\mathrm{m} / \mathrm{z}=245.14675$. $\mathrm{R}_{\mathrm{f}}=0.3(5 \% \mathrm{EA} / \mathrm{hex})$.

\section{1-phenyl-4-(2-(trimethylsilyl)ethyl-1-d)-1H-pyrazole (45):}

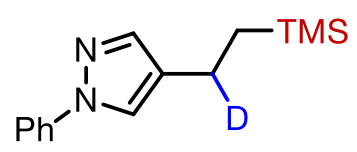

Following general procedure 2 with 1-phenyl-4-vinyl-1H-pyrazole $(170 \mathrm{mg})$ and $\mathrm{MeCN}-d_{3}(4 \mathrm{~mL})$. The crude was purified with 5\% ethyl acetate/hexanes and a pale yellow oil was obtained $(78 \mathrm{mg}$, $32 \%) .{ }^{1} \mathrm{H}$ NMR $\left(500 \mathrm{MHz}, \mathrm{CDCl}_{3}\right) \delta 7.75-7.66(\mathrm{~m}, 3 \mathrm{H}), 7.60(\mathrm{~s}, 1 \mathrm{H}), 7.49-7.41(\mathrm{~m}, 2 \mathrm{H}), 7.30$ $-7.23(\mathrm{~m}, 1 \mathrm{H}), 2.57(\mathrm{tt}, J=8.5,2.2 \mathrm{~Hz}, 1 \mathrm{H}), 0.92(\mathrm{~d}, J=8.7 \mathrm{~Hz}, 2 \mathrm{H}), 0.07$ (s, 9H). ${ }^{13} \mathbf{C}$ NMR $\left(126 \mathrm{MHz}, \mathrm{CDCl}_{3}\right) \delta 140.7,140.5,129.4,126.9,126.0,124.3,118.8,18.5,18.3,18.2,17.9,-1.6$. IR (FTIR): 3087, 3051, 2951, 2896, 1600, 1569, 1503, 1463, 1393, 1297, 1247, 1247, 1215, 1180 , $1145,1073,1046,1031,993,953,855,837,753,688 \mathrm{~cm}^{-1}$. HRMS (DART) exact mass calculated for $\mathrm{C}_{14} \mathrm{H}_{20} \mathrm{DN} 2 \mathrm{Si}[\mathrm{M}+\mathrm{H}]^{+} \mathrm{m} / \mathrm{z}=246.15313$, found $\mathrm{m} / \mathrm{z}=246.15295 . \mathrm{R}_{\mathrm{f}}=0.3(5 \% \mathrm{EA} / \mathrm{Hex})$.

\section{(1,2-diphenylvinyl)trimethylsilane (46):}<smiles></smiles>

Following general procedure 2 with diphenylacetylene (178 mg). The crude was purified with $0 \%$ ethyl acetate/hexanes and a clear colorless oil was obtained (130 mg, 51\%, E/Z = 1:1). ${ }^{1} \mathbf{H}$ NMR $\left(500 \mathrm{MHz}, \mathrm{CDCl}_{3}\right) \delta 7.69-6.92(\mathrm{~m}, 11 \mathrm{H}), 0.25(\mathrm{~s}, 9 \mathrm{H}) .{ }^{13} \mathbf{C} \mathbf{~ N M R}\left(126 \mathrm{MHz}, \mathrm{CDCl}_{3}\right) \delta 147.2$, $142.9,137.51,137.47,131.8,129.6,128.8,128.48,128.38,128.0,127.5,127.2,125.8,-1.5 .{ }^{29} \mathbf{S i}$ 
NMR (99 MHz, $\left.\mathrm{CDCl}_{3}\right) \delta$-2.84. IR (FTIR): 3074, 3056, 3022, 2955, 2897, 1600, 1572, 1492, $1445,1246,1072,1030,955,901,835,770,755,692 \mathrm{~cm}^{-1}$. HRMS (DART) exact mass calculated for $\mathrm{C}_{17} \mathrm{H}_{20} \mathrm{Si}[\mathrm{M}]^{+} \mathrm{m} / \mathrm{z}=252.13288$, found $\mathrm{m} / \mathrm{z}=252.13272$. $\mathrm{R}_{\mathrm{f}}=0.4$ (hexanes).

1-methyl-4-(2-(trimethylsilyl)ethyl)-1 $H$-indole (47):<smiles>Cn1ccc2c(CC[As])cccc21</smiles>

Following general procedure 2 with 1-phenyl-4-vinyl-1H-pyrazole $(157 \mathrm{mg})$. The crude was purified with 5\% ethyl acetate/hexanes and a clear slight yellow oil was obtained (116 mg, 50\%). ${ }^{1} \mathrm{H}$ NMR $\left(500 \mathrm{MHz}, \mathrm{CDCl}_{3}\right) \delta 7.34-7.25(\mathrm{~m}, 2 \mathrm{H}), 7.16-7.07(\mathrm{~m}, 2 \mathrm{H}), 6.65(\mathrm{~d}, J=3.1 \mathrm{~Hz}, 1 \mathrm{H})$, 3.87 (s, 3H), $3.09-3.02(\mathrm{~m}, 2 \mathrm{H}), 1.19-1.12(\mathrm{~m}, 2 \mathrm{H}), 0.21$ (s, 9H). ${ }^{13} \mathbf{C ~ N M R}\left(126 \mathrm{MHz}, \mathrm{CDCl}_{3}\right)$ $\delta$ 137.9, 136.8, 128.2, 127.4, 121.9, 117.8, 106.9, 99.2, 33.0, 27.6, 18.0, -1.6. ${ }^{29} \mathbf{S i}$ NMR (99 MHz, $\left.\mathrm{CDCl}_{3}\right) \delta$ 1.78. IR (FTIR): 3045, 2950, 2896, 2854, 2817, 1606, 1581, 1514, 1495, 1444, 1417, 1341, 1302, 1245, 1175, 1154, 1083, 906, 858, 830, 742, 710, $689 \mathrm{~cm}^{-1}$. HRMS (DART) exact mass calculated for $\mathrm{C}_{14} \mathrm{H}_{22} \mathrm{OSi}[\mathrm{M}+\mathrm{H}]^{+} \mathrm{m} / \mathrm{z}=232.15160$, found $\mathrm{m} / \mathrm{z}=232.15159 . \mathrm{R}_{\mathrm{f}}=0.25(5 \%$ $\mathrm{EA} / \mathrm{Hex})$.

\section{(4-(tert-butyl)phenethyl)trimethylsilane (48):}<smiles>CCCCc1ccc(C(C)(C)C)cc1</smiles>

Following general procedure 2 with diphenylacetylene $(160 \mathrm{mg})$. The crude was purified with $0 \%$ ethyl acetate/hexanes and a clear colorless oil was obtained (180 mg, 77\%). ${ }^{1} \mathbf{H}$ NMR (500 MHz, $\left.\mathrm{CDCl}_{3}\right) \delta 7.36-7.31(\mathrm{~m}, 2 \mathrm{H}), 7.17(\mathrm{~d}, J=7.9 \mathrm{~Hz}, 2 \mathrm{H}), 2.67-2.59(\mathrm{~m}, 2 \mathrm{H}), 1.35(\mathrm{~s}, 9 \mathrm{H}), 0.95-$ $0.87(\mathrm{~m}, 2 \mathrm{H}), 0.05(\mathrm{~d}, J=2.6 \mathrm{~Hz}, 9 \mathrm{H}) .{ }^{13} \mathrm{C} \mathrm{NMR}\left(126 \mathrm{MHz}, \mathrm{CDCl}_{3}\right) \delta 148.4,142.4,127.5,125.3$, 34.5, 31.6, 29.5, 18.7, -1.6. ${ }^{29} \mathrm{Si}$ NMR (99 MHz, $\left.\mathrm{CDCl}_{3}\right) \delta$ 1.46. IR (FTIR): 3092, 3054, 3022, 3000, 2953, 2906, 2870, 1515, 1462, 1412, 1363, 1247, 1202, 1193, 1173, 1132, 1109, 1018, 999, 908, 861, 829, 757, $689 \mathrm{~cm}^{-1}$. HRMS (DART) exact mass calculated for $\mathrm{C}_{14} \mathrm{H}_{23} \mathrm{Si}\left[\mathrm{M}-\mathrm{CH}_{3}\right]^{+} \mathrm{m} / \mathrm{z}$ $=219.15635$, found $\mathrm{m} / \mathrm{z}=219.15642 . \mathrm{R}_{\mathrm{f}}=0.5(\mathrm{Hex})$.

(1-(4,4,5,5-tetramethyl-1,3,2-dioxaborolan-2-yl)ethane-1,2-diyl)bis(trimethylsilane) (49):

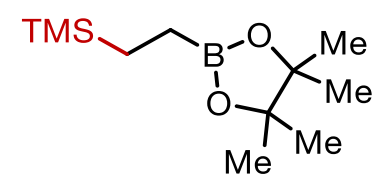

Following general procedure 2 at $50 \%$ scale, $77 \mathrm{mg}$ of vinylboronic acid pinacol ester was used. The crude first passed through florisil plug then concentrated. The crude was purified with $1 \% \mathrm{Et}_{3} \mathrm{~N} / \mathrm{Hexanes}$ to obtain a clear colorless oil (41mg, 35\%). ${ }^{1} \mathbf{H} \mathbf{~ N M R}\left(500 \mathrm{MHz}, \mathrm{CDCl}_{3}\right) \delta 1.25$ $(\mathrm{s}, 12 \mathrm{H}), 0.77-0.67(\mathrm{~m}, 2 \mathrm{H}), 0.58-0.51(\mathrm{~m}, 2 \mathrm{H}),-0.04(\mathrm{~s}, 9 \mathrm{H}) .{ }^{13} \mathbf{C ~ N M R}\left(126 \mathrm{MHz}, \mathrm{CDCl}_{3}\right) \delta$ 83.1, 25.0, 9.4, -2.0. IR (FTIR): 2979, 2954, 2929, 2900, 1467, 1415, 1370, 1360, 1321, 1246, $1147,1113,1007,955,862,830 \mathrm{~cm}^{-1}$. HRMS (DART) exact mass calculated for $\mathrm{C}_{11} \mathrm{H}_{25} \mathrm{BO}_{2} \mathrm{Si}$ $\left[\mathrm{M}-\mathrm{CH}_{3}\right]^{+} \mathrm{m} / \mathrm{z}=213.1428$, found $\mathrm{m} / \mathrm{z}=213.1431 . \mathrm{R}_{\mathrm{f}}=0.3(2 \% \mathrm{EA} / \mathrm{Hex})$. 


\section{(2-(benzo[b]thiophen-3-yl)ethyl)dimethyl(vinyl)silane (50):}

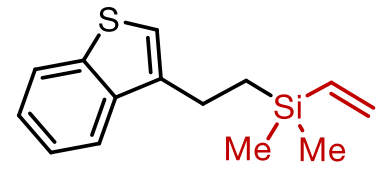

Following general procedure 2 with 3-vinylbenzothiophene $(160 \mathrm{mg})$ and dimethyl(vinyl)chlorosilane $(362 \mathrm{mg}$ ). The crude was purified with $0 \%$ ethyl acetate/hexanes and a clear colorless oil was obtained $(84 \mathrm{mg}, 34 \%)$. ${ }^{\mathbf{1}} \mathbf{H} \mathbf{N M R}\left(500 \mathrm{MHz}, \mathrm{CDCl}_{3}\right) \delta 7.95-7.90(\mathrm{~m}$, $1 \mathrm{H}), 7.84-7.79(\mathrm{~m}, 1 \mathrm{H}), 7.54-7.35(\mathrm{~m}, 2 \mathrm{H}), 7.16(\mathrm{t}, J=1.4 \mathrm{~Hz}, 1 \mathrm{H}), 6.29(\mathrm{dd}, J=20.3,14.7$ $\mathrm{Hz}, 1 \mathrm{H}), 6.10(\mathrm{dd}, J=14.7,3.7 \mathrm{~Hz}, 1 \mathrm{H}), 5.83(\mathrm{dd}, J=20.3,3.8 \mathrm{~Hz}, 1 \mathrm{H}), 2.97-2.90(\mathrm{~m}, 2 \mathrm{H}), 1.21$ $-1.13(\mathrm{~m}, 2 \mathrm{H}), 0.23(\mathrm{~s}, 6 \mathrm{H}) .{ }^{13} \mathrm{C}$ NMR $\left(126 \mathrm{MHz}, \mathrm{CDCl}_{3}\right) \delta 140.8,139.6,139.0,138.6,132.2$, 124.2, 123.8, 123.0, 121.8, 120.4, 22.9, 15.0, -3.4. ${ }^{29} \mathrm{Si}$ NMR (99 MHz, $\left.\mathrm{CDCl}_{3}\right) \delta$-5.32. IR (FTIR): 3047, 3008, 2954, 2899, 1592, 1459, 1427, 1404, 1248, 1008, 951, 912, 835, 763, 733, $705 \mathrm{~cm}^{-1}$. HRMS (DART) exact mass calculated for $\mathrm{C}_{13} \mathrm{H}_{15} \mathrm{SSi}\left[\mathrm{M}-\mathrm{CH}_{3}\right]^{+} \mathrm{m} / \mathrm{z}=231.06582$, found $\mathrm{m} / \mathrm{z}=$ 231.06584. $\mathrm{R}_{\mathrm{f}}=0.4(\mathrm{Hex})$

\section{allyl(2-(benzo[b]thiophen-5-yl)ethyl)dimethylsilane (51):}

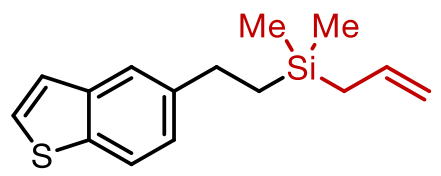

Following general procedure 2 with 5-vinylbenzothiophene $(160 \mathrm{mg})$ and allylchlorodimethylsilane (404 mg). The crude was purified with $2.5 \%$ ethyl acetate/hexanes and a clear colorless oil was obtained $(116 \mathrm{mg}, 45 \%) .{ }^{1} \mathbf{H} \mathbf{~ N M R}\left(500 \mathrm{MHz}, \mathrm{CDCl}_{3}\right) \delta 7.86(\mathrm{~d}, J=8.3$ $\mathrm{Hz}, 1 \mathrm{H}), 7.73(\mathrm{~d}, J=1.9 \mathrm{~Hz}, 1 \mathrm{H}), 7.47(\mathrm{~d}, J=5.4 \mathrm{~Hz}, 1 \mathrm{H}), 7.35(\mathrm{~d}, J=5.3 \mathrm{~Hz}, 1 \mathrm{H}), 7.29(\mathrm{dd}, J=$ 8.3, $1.8 \mathrm{~Hz}, 1 \mathrm{H}), 5.91$ (ddt, $J=16.5,10.1,8.1 \mathrm{~Hz}, 1 \mathrm{H}), 5.03-4.92(\mathrm{~m}, 2 \mathrm{H}), 2.94-2.77$ (m, 2H), $1.70-1.64(\mathrm{~m}, 2 \mathrm{H}), 1.10-1.03(\mathrm{~m}, 2 \mathrm{H}), 0.14(\mathrm{~s}, 6 \mathrm{H}) .{ }^{13} \mathbf{C} \mathbf{~ N M R}\left(126 \mathrm{MHz}, \mathrm{CDCl}_{3}\right) \delta 141.4$, $140.1,137.2,135.1,126.5,125.0,123.7,122.3,122.3,113.1,30.0,23.3,17.5,-3.6 .{ }^{29}$ Si NMR (99 $\mathrm{MHz}_{\mathrm{CDCl}}$ ) $\delta 1.50$. IR (FTIR): 3103, 3075, 3032, 2994, 2952, 2916, 2881, 2854, 1629, 1436, 1420, 1393, 1248, 1151, 1089, 1050, 1036, 992, 931, 889, 829, 809, 752, $689 \mathrm{~cm}^{-1}$. HRMS (DART) exact mass calculated for $\mathrm{C}_{12} \mathrm{H}_{15} \mathrm{SSi}\left[\mathrm{M}-\mathrm{C}_{3} \mathrm{H}_{5}\right]^{+} \mathrm{m} / \mathrm{z}=219.06582$, found $\mathrm{m} / \mathrm{z}=219.06582$. $\mathrm{R}_{\mathrm{f}}=$ $0.19(5 \%$ EA/Hex $)$.

\section{allyl(4-methoxyphenethyl)dimethylsilane (52):}<smiles>C=CC[Si](C)(C)CCc1ccc(OC)cc1</smiles>

Following general procedure 2 with 4-methoxystyrene $(134 \mathrm{mg})$ and allylchlorodimethylsilane (404 mg). The crude was purified with $0 \%$ ethyl acetate/hexanes and a clear colorless oil was obtained (220 mg, 93\%). ${ }^{1} \mathbf{H}$ NMR $\left(500 \mathrm{MHz} \mathrm{CDCl}_{3}\right) \delta 7.17-7.11(\mathrm{~m}, 2 \mathrm{H}), 6.90-6.82(\mathrm{~m}, 2 \mathrm{H})$, $5.82(\mathrm{ddt}, J=16.5,10.1,8.1 \mathrm{~Hz}, 1 \mathrm{H}), 4.92-4.83(\mathrm{~m}, 2 \mathrm{H}), 3.82(\mathrm{~s}, 3 \mathrm{H}), 2.65-2.58(\mathrm{~m}, 2 \mathrm{H}), 1.57$ $(\mathrm{dt}, J=8.1,1.2 \mathrm{~Hz}, 2 \mathrm{H}), 0.95-0.87(\mathrm{~m}, 2 \mathrm{H}), 0.04(\mathrm{~s}, 6 \mathrm{H}) .{ }^{13} \mathbf{C ~ N M R}\left(126 \mathrm{MHz}, \mathrm{CDCl}_{3}\right) \delta 157.8$, 137.4, 135.2, 128.8, 113.9, 113.0, 55.4, 29.1, 23.3, 17.2, -3.6. ${ }^{29} \mathrm{Si} \mathbf{N M R}\left(99 \mathrm{MHz}, \mathrm{CDCl}_{3}\right) \delta 1.36$. 
IR (FTIR): 3076, 3969, 3030, 2996, 2954, 2912, 2854, 2834, 1630, 1612, 1584, 1511, 1465, 1441, $1300,1245,1176,1155,1039,993,932,893,823 \mathrm{~cm}^{-1}$. HRMS (DART) exact mass calculated for $\mathrm{C}_{11} \mathrm{H}_{17} \mathrm{OSi}\left[\mathrm{M}+\mathrm{C}_{3} \mathrm{H}_{5}\right]^{+} \mathrm{m} / \mathrm{z}=193.10432$, found $\mathrm{m} / \mathrm{z}=193.10444$. $\mathrm{R}_{\mathrm{f}}=0.1(\mathrm{Hex})$.

\section{(1-phenylpropane-2,2-diyl)bis(dimethylsilane) (53):}

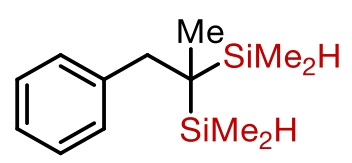

Following general procedure 2 with 3-phenyl-1-propyne $(116 \mathrm{mg}$ ) and chlorodimethylsilane (298 $\mathrm{mg}$ ). The crude was purified with $1 \%$ ethyl acetate/hexanes and a clear colorless oil was obtained $(106 \mathrm{mg}, 45 \%) .{ }^{1} \mathbf{H}$ NMR $\left(500 \mathrm{MHz}, \mathrm{CDCl}_{3}\right) \delta 7.39-7.16(\mathrm{~m}, 5 \mathrm{H}), 3.87-3.79(\mathrm{~m}, 2 \mathrm{H}), 2.80(\mathrm{~s}$, $2 \mathrm{H}), 1.12(\mathrm{~s}, 3 \mathrm{H}), 0.03(\mathrm{dd}, J=3.8,1.9 \mathrm{~Hz}, 12 \mathrm{H}) .{ }^{13} \mathbf{C} \mathbf{~ N M R}\left(126 \mathrm{MHz}, \mathrm{CDCl}_{3}\right) \delta 139.8,130.5$, $127.9,126.2,40.6,17.4,11.0,-5.34,-5.37$. Product is unstable on the NMR time scale. IR (FTIR): 3084, 3063, 3029, 2954, 2902, 2855, 2839, 2108, 1602, 1494, 1452, 1421, 1374, 1250, 1077, 1032, $888,834,760,700 \mathrm{~cm}^{-1}$. HRMS (DART) exact mass calculated for $\mathrm{C}_{13} \mathrm{H}_{23} \mathrm{Si}_{2}[\mathrm{M}-\mathrm{H}]^{+} \mathrm{m} / \mathrm{z}=$ 235.13328 , found $\mathrm{m} / \mathrm{z}=235.13322$. $\mathrm{R}_{\mathrm{f}}=0.25(\mathrm{Hex})$.

\section{trimethyl(2-(phenoxymethyl)allyl)silane (57):<smiles>C=C(COc1ccccc1)C[Hg]C</smiles>

Following general procedure 1 with ((2-methylenepropane-1,3-diyl)bis(oxy))dibenzene (240 mg). The reaction was stopped at $2.2 \mathrm{~F}$ of charge and the crude was purified with $2 \%$ ethyl acetate/hexanes and a clear colorless oil was obtained (108 $\mathrm{mg}$, 49\%). If more than $2 \mathrm{~F}$ of charge was passed with sufficient amount of TMSCl, the remaining phenol group can also eliminated to form a an allyl silane on the other side. ${ }^{1} \mathbf{H}$ NMR $\left(500 \mathrm{MHz}, \mathrm{CDCl}_{3}\right) \delta 7.32-7.24(\mathrm{~m}, 2 \mathrm{H}), 6.98$ $-6.90(\mathrm{~m}, 3 \mathrm{H}), 5.02(\mathrm{~d}, J=1.5 \mathrm{~Hz}, 1 \mathrm{H}), 4.82-4.77(\mathrm{~m}, 1 \mathrm{H}), 4.38(\mathrm{~s}, 2 \mathrm{H}), 1.66-1.63(\mathrm{~m}, 2 \mathrm{H})$, 0.07 (s, 9H). ${ }^{13} \mathbf{C}$ NMR $\left(126 \mathrm{MHz}, \mathrm{CDCl}_{3}\right) \delta 159.0,142.9,129.5,120.9,114.9,109.9,72.0,23.6$, -1.1. ${ }^{29} \mathrm{Si}$ NMR (99 MHz, $\mathrm{CDCl}_{3}$ ) $\delta$ 0.76. IR (FTIR): 3074, 3040, 2953, 2914, 2896, 1739, 1640 , $1599,1587,1494,1455,1418,1366,1302,1291,1240,1217,1170,1078,1046,1033,1014,994$, 882, $837 \mathrm{~cm}^{-1}$. HRMS (DART) exact mass calculated for $\mathrm{C}_{13} \mathrm{H}_{21} \mathrm{OSi}[\mathrm{M}+\mathrm{H}]^{+} \mathrm{m} / \mathrm{z}=221.1362$, found $\mathrm{m} / \mathrm{z}=221.1358$. $\mathrm{R}_{\mathrm{f}}=0.6(3 \% \mathrm{EA} / \mathrm{Hex})$.

\section{(2-((benzyloxy)methyl)allyl)trimethylsilane (58)}<smiles>C=C(COCc1ccccc1)C[Hg]C</smiles>

Following general procedure 1 with $254 \mathrm{mg}$ of ((2-((benzyloxy)methyl)allyl)oxy)benzene. The reaction was stopped at $2.2 \mathrm{~F}$ of charge and the crude was added dibromoethane as an internal standard. Crude NMR shows product with $23 \%$ yield and it is consistent with reported literature. ${ }^{35}$ ${ }^{1}$ H NMR (crude) $\left(400 \mathrm{MHz}, \mathrm{CDCl}_{3}\right) \delta 5.00(\mathrm{~d}, 1 \mathrm{H}), 4.79(\mathrm{~m}, 1 \mathrm{H}), 4.54(\mathrm{~s}, 2 \mathrm{H}), 3.93(\mathrm{~s}, 2 \mathrm{H}), 1.63$ $(\mathrm{m}, 2 \mathrm{H}), 0.07$ (s, 9H). internal standard: $4.90(\mathrm{~s}, 2 \mathrm{H})$.

triethyl((2-((triethylsilyl)methyl)allyl)oxy)silane (60): 
<smiles>C=C(COCC)COCC</smiles>

Following general procedure 1 with 3 -methyleneoxetane $(1.35 \mathrm{~mL}$ in $0.74 \mathrm{M}$ THF solution) and triethylchlorsilane $(450 \mathrm{mg})$. The crude was purified with $2 \%$ ethyl acetate/hexanes and a clear colorless oil was obtained. The semi pure product was then subjected to a Hickmann distillation to remove volatile impurities. (123 mg, 41\%). ${ }^{1} \mathbf{H} \mathbf{~ N M R}\left(599 \mathrm{MHz}, \mathrm{CDCl}_{3}\right) \delta 4.95(\mathrm{~d}, J=1.9 \mathrm{~Hz}$, $1 \mathrm{H}), 4.71-4.61(\mathrm{~m}, 1 \mathrm{H}), 3.98$ (t, $J=1.6 \mathrm{~Hz}, 2 \mathrm{H}), 1.49$ (d, $J=1.1 \mathrm{~Hz}, 2 \mathrm{H}), 0.96$ (dt, $J=17.3,7.9$ $\mathrm{Hz}, 18 \mathrm{H}), 0.62(\mathrm{q}, J=8.0 \mathrm{~Hz}, 6 \mathrm{H}), 0.54(\mathrm{q}, J=7.9 \mathrm{~Hz}, 6 \mathrm{H}) .{ }^{13} \mathbf{C ~ N M R}\left(126 \mathrm{MHz}, \mathrm{CDCl}_{3}\right) \delta 146.4$, 106.5, 66.8, 17.5, 7.5, 7.0, 4.6, 3.6. ${ }^{29} \mathrm{Si}$ NMR (99 $\left.\mathrm{MHz}^{\mathrm{CDCl}} \mathrm{CDC}_{3}\right) \delta 19.45,5.95$. IR (FTIR): 3094, 2953, 2910, 2875, 2732, 1645, 1458, 1415, 1379, 1158, 1123, 1085, 1005, 972, 879, $826 \mathrm{~cm}^{-1}$. HRMS (DART) exact mass calculated for $\mathrm{C}_{16} \mathrm{H}_{37} \mathrm{OSi}_{2}[\mathrm{M}+\mathrm{H}]^{+} \mathrm{m} / \mathrm{z}=301.2383$, found $\mathrm{m} / \mathrm{z}=$ 301.2383. $\mathrm{R}_{\mathrm{f}}=0.7(5 \% \mathrm{EA} / \mathrm{Hex})$.

trimethyl(2-(tetrahydro-4H-pyran-4-ylidene)ethyl)silane (64)<smiles>CS(=O)(=O)CC=C1CCOCC1</smiles>

Following general procedure 4 at $50 \%$ scale with $64 \mathrm{mg} 4$-vinyltetrahydro- $2 H$-pyran-4-ol. The crude was purified with $3 \%$ ethyl acetate/hexanes and a clear colorless oil was obtained (37.0 mg, $40 \%$ ). There is a small amount of TMS protected alcohol that have similar polarity to the product, which may come out with the product as a mixture. To remove this, 1 equiv (per O-TMS bond) of TBAF in THF was added and the solution was stirred for $15 \mathrm{~min}$ and purified through a short pipet with silica. ${ }^{1} \mathbf{H}$ NMR $\left(500 \mathrm{MHz}, \mathrm{CDCl}_{3}\right) \delta 5.25-5.17(\mathrm{~m}, 1 \mathrm{H}), 3.64(\mathrm{dt}, J=7.5,5.4 \mathrm{~Hz}, 4 \mathrm{H}), 2.24$ $-2.18(\mathrm{~m}, 4 \mathrm{H}), 1.41(\mathrm{~d}, J=8.6 \mathrm{~Hz}, 2 \mathrm{H}),-0.00(\mathrm{~s}, 9 \mathrm{H}) .{ }^{13} \mathbf{C} \mathbf{N M R}\left(126 \mathrm{MHz}, \mathrm{CDCl}_{3}\right) \delta 131.6$, 119.1, 70.0, 68.9, 37.2, 29.6, 17.9, -1.6. IR (FTIR): 2955, 2932, 2892, 2843, 1420, 1287, 1247 , 1231, 1206, 1151, 1100, 1044, 1022, 1004, 916, 855, $839 \mathrm{~cm}^{-1}$. HRMS (DART) exact mass calculated for $\mathrm{C}_{10} \mathrm{H}_{20} \mathrm{OSi}[\mathrm{M}+\mathrm{H}]^{+} \mathrm{m} / \mathrm{z}=185.1356$, found $\mathrm{m} / \mathrm{z}=185.1359 . \mathrm{R}_{\mathrm{f}}=0.4(5 \% \mathrm{EA} / \mathrm{Hex})$.

\section{trimethyl(2-(tetrahydro-4H-thiopyran-4-ylidene)ethyl)silane (65)}<smiles>CCCCC1CCSCC1</smiles>

Following general procedure 4 at $50 \%$ scale with $72 \mathrm{mg}$ of 4-vinyltetrahydro- $2 \mathrm{H}$-thiopyran-4-ol. The crude was purified with $3 \%$ ethyl acetate/hexanes and a clear colorless oil was obtained (37.6 mg, 37\%). ${ }^{1} \mathbf{H}$ NMR $\left(500 \mathrm{MHz}, \mathrm{CDCl}_{3}\right) \delta 5.21(\mathrm{tt}, J=8.7,1.1 \mathrm{~Hz}, 1 \mathrm{H}), 2.61$ (ddd, $J=11.2,7.1$, $4.3 \mathrm{~Hz}, 4 \mathrm{H}), 2.48-2.40(\mathrm{~m}, 4 \mathrm{H}), 1.41(\mathrm{~d}, J=8.6 \mathrm{~Hz}, 2 \mathrm{H}),-0.01(\mathrm{~s}, 9 \mathrm{H}) .{ }^{13} \mathrm{C} \mathrm{NMR}(126 \mathrm{MHz}$, $\left.\mathrm{CDCl}_{3}\right) \delta 133.6,120.6,39.2,31.4,30.3,30.2,17.9,-1.6$. IR (FTIR): 2951, 2917, 2901, 2827, 1426, 
1410, 1269, 1247, 1173, 1152, 1048, 968, 939, $850 \mathrm{~cm}^{-1}$. HRMS (DART) exact mass calculated for $\mathrm{C}_{10} \mathrm{H}_{20} \mathrm{SSi}[\mathrm{M}+\mathrm{H}]^{+} \mathrm{m} / \mathrm{z}=201.1128$, found $\mathrm{m} / \mathrm{z}=201.1129 . \mathrm{R}_{\mathrm{f}}=0.6(5 \% \mathrm{EA} / \mathrm{Hex})$.

\section{trimethyl(2-(4-phenylcyclohexylidene)ethyl)silane (66)}<smiles>CCCC=C1CCC(c2ccccc2)CC1</smiles>

Following general procedure 4 at $50 \%$ scale with $101 \mathrm{mg}$ of 4-phenyl-1-vinylcyclohexan-1-ol. The crude was purified with hexanes and a clear colorless oil was obtained (45.4 mg, 35\%). ${ }^{\mathbf{1}} \mathbf{H}$ NMR $\left(500 \mathrm{MHz} \mathrm{CDCl}_{3}\right) \delta 7.33-7.25(\mathrm{~m}, 2 \mathrm{H}), 7.23-7.14(\mathrm{~m}, 3 \mathrm{H}), 5.18(\mathrm{tt}, J=8.5,1.9 \mathrm{~Hz}, 1 \mathrm{H}), 2.67$ $(\mathrm{td}, J=12.1,10.4,3.6 \mathrm{~Hz}, 2 \mathrm{H}), 2.34-2.26(\mathrm{~m}, 1 \mathrm{H}), 2.25-2.15(\mathrm{~m}, 1 \mathrm{H}), 2.00-1.92(\mathrm{~m}, 2 \mathrm{H})$, $1.83(\mathrm{td}, J=13.4,4.1 \mathrm{~Hz}, 1 \mathrm{H}), 1.56-1.37(\mathrm{~m}, 4 \mathrm{H}), 0.01(\mathrm{~s}, 9 \mathrm{H}) .{ }^{\mathbf{1 3}} \mathbf{C} \mathbf{~ N M R}\left(126 \mathrm{MHz}, \mathrm{CDCl}_{3}\right) \delta$ 147.4, 135.7, 128.4, 127.0, 126.0, 117.8, 45.0, 37.2, 36.2, 35.1, 28.3, 18.1, -1.6. IR (FTIR): 3082, 3062, 3027, 2953, 2923, 2854, 2833, 1493, 1443, 1410, 1247, 1154, 1091, 1066, 1031, 1010, 972, $852 \mathrm{~cm}^{-1}$. HRMS (DART) exact mass calculated for $\mathrm{C}_{17} \mathrm{H}_{26} \mathrm{Si}[\mathrm{M}+\mathrm{H}]^{+} \mathrm{m} / \mathrm{z}=259.1877$, found $\mathrm{m} / \mathrm{z}=259.1880 . \mathrm{R}_{\mathrm{f}}=0.5(\mathrm{Hex})$.

\section{trimethyl((1-phenylcyclopentyl)methyl)silane (68):}<smiles>CCC1(c2ccccc2)CCCC1</smiles>

Following general procedure 1 at $50 \%$ scale with $97 \mathrm{mg}$ of (6-chlorohex-1-en-2-yl)benzene. The crude was purified with pure hexanes. There is around 5\% of impurities in the desired product that are difficult to remove due to low polarity and yield was determined by ${ }^{1} \mathrm{H}-\mathrm{NMR}$ using $1,3,5$ trimethoxybenzene as internal standard (43\%). ${ }^{1} \mathbf{H} \mathbf{~ N M R}\left(500 \mathrm{MHz}, \mathrm{CDCl}_{3}\right) \delta 7.35-7.24(\mathrm{~m}, 4 \mathrm{H})$, $7.23-7.11(\mathrm{~m}, 1 \mathrm{H}), 2.07-1.97(\mathrm{~m}, 2 \mathrm{H}), 1.82-1.67(\mathrm{~m}, 4 \mathrm{H}), 1.66-1.54(\mathrm{~m}, 2 \mathrm{H}), 1.12(\mathrm{~s}, 2 \mathrm{H})$, -0.32 (s, 9H). ${ }^{13} \mathbf{C ~ N M R ~}\left(126 \mathrm{MHz}, \mathrm{CDCl}_{3}\right) \delta 149.6,128.04,128.02,126.7,125.5,49.8,41.1,32.2$, 23.2, 0.0. IR (FTIR): 3085, 3060, 3024, 2951, 2874, 1600, 1495, 1446, 1414, 1246, 1192, 1058, 1032, 964, 944, 910, 856, $833 \mathrm{~cm}-1$. HRMS (DART) exact mass calculated for $\mathrm{C}_{15} \mathrm{H}_{24} \mathrm{Si}[\mathrm{M}+\mathrm{H}]^{+}$ $\mathrm{m} / \mathrm{z}=233.1726$, found $\mathrm{m} / \mathrm{z}=233.1723 . \mathrm{R}_{\mathrm{f}}=0.8($ Hex $)$.

\section{(3-fluoroprop-1-en-2-yl)benzene (69)}<smiles>C=C(CF)c1ccccc1</smiles>

Following derivatization procedure 1 with $95 \mathrm{mg}$ of trimethyl(2-phenylallyl)silane $(0.5 \mathrm{mmol}$ scale). The crude was purified with hexanes and a clear colorless oil was obtained $(45.9 \mathrm{mg}, 67 \%)$. ${ }^{1} \mathbf{H}$ NMR $\left(500 \mathrm{MHz}, \mathrm{CDCl}_{3}\right) \delta 7.47-7.45(\mathrm{~m}, 2 \mathrm{H}), 7.39-7.33(\mathrm{~m}, 3 \mathrm{H}), 5.62(\mathrm{~s}, 1 \mathrm{H}), 5.43(\mathrm{dt}, J$ 
$=2.3,1.2 \mathrm{~Hz}, 1 \mathrm{H}), 5.30(\mathrm{~d}, J=1.3 \mathrm{~Hz}, 1 \mathrm{H}), 5.20(\mathrm{~d}, J=1.3 \mathrm{~Hz}, 1 \mathrm{H})$. The compound was consistent with previous literature reports.

\section{triethyl((2-(fluoromethyl)allyl)oxy)silane (70)}

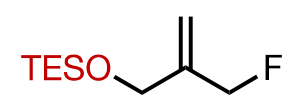

Following derivatization procedure 1 with $60 \mathrm{mg}$ of triethyl((2-((triethylsilyl)methyl)allyl)oxy)silane. The crude was purified with $2 \%$ ethyl acetate/hexanes and a clear colorless oil was obtained (32.4 mg, 79\%). ${ }^{1} \mathbf{H}$ NMR $\left(500 \mathrm{MHz}, \mathrm{CDCl}_{3}\right) \delta 5.28(\mathrm{q}, J=1.7 \mathrm{~Hz}$, $1 \mathrm{H}), 5.19(\mathrm{dq}, J=3.8,1.3 \mathrm{~Hz}, 1 \mathrm{H}), 4.93(\mathrm{~d}, J=1.2 \mathrm{~Hz}, 1 \mathrm{H}), 4.84(\mathrm{~d}, J=1.2 \mathrm{~Hz}, 1 \mathrm{H}), 4.22(\mathrm{~d}, J=$ $1.7 \mathrm{~Hz}, 2 \mathrm{H}), 0.97(\mathrm{t}, J=8.0 \mathrm{~Hz}, 9 \mathrm{H}), 0.63(\mathrm{q}, J=7.9 \mathrm{~Hz}, 6 \mathrm{H}) .{ }^{13} \mathbf{C} \mathbf{N M R}\left(126 \mathrm{MHz}, \mathrm{CDCl}_{3}\right) \delta$ 144.2, 144.0, 113.5, 113.4, 84.2, 82.9, 63.0, 6.9, 4.5. ${ }^{19} \mathbf{F ~ N M R}\left(470 \mathrm{MHz}, \mathrm{CDCl}_{3}\right) \delta-217.95$, 218.05, -218.15. IR (FTIR): 2955, 2912, 2877, 1459, 1415, 1362, 1240, 1117, 1084, 1047, 1001, 959, 920, $814 \mathrm{~cm}^{-1}$. HRMS (DART) exact mass calculated for $\mathrm{C}_{10} \mathrm{H}_{21} \mathrm{FOSi}[\mathrm{M}+\mathrm{H}]^{+} \mathrm{m} / \mathrm{z}=$ 205.1419, found $\mathrm{m} / \mathrm{z}=205.1421 . \mathrm{R}_{\mathrm{f}}=0.7$ (5\% EA/Hex).

\section{1,2-bis(triethylsilyl)ethan-1-ol (71)}<smiles>OC(C[As]=[Te])[I-][Te]</smiles>

Following derivatization procedure 3 with $76.8 \mathrm{mg}$ of (1-(4,4,5,5-tetramethyl-1,3,2-dioxaborolan2-yl)ethane-1,2-diyl)bis(triethylsilane). The crude was purified with 5\% ethyl acetate/hexanes and a clear colorless oil was obtained $(39.0 \mathrm{mg}, 71 \%)$. Brook rearrangement was proved to be unlikely to happen as the ${ }^{29} \mathrm{Si}$ NMR reveals $\mathrm{Si}$ is connected to carbon $(\delta \sim 5-8 \mathrm{ppm})$ instead of oxygen $(\delta \sim$ $20 \mathrm{ppm})$. This is also supported by the integration of ${ }^{1} \mathrm{H}$ NMRs as only one proton is connected to the carbon next to $\mathrm{OH}$ group and the phasing of 2D HSQC. ${ }^{1} \mathbf{H} \mathbf{~ N M R}\left(500 \mathrm{MHz}, \mathrm{CDCl}_{3}\right) \delta 3.67$ $(\mathrm{dd}, J=13.2,2.3 \mathrm{~Hz}, 1 \mathrm{H}), 0.97(\mathrm{dt}, J=18.0,8.0 \mathrm{~Hz}, 18 \mathrm{H}), 0.89(\mathrm{dd}, J=15.1,13.2 \mathrm{~Hz}, 1 \mathrm{H}), 0.75$ $(\mathrm{dd}, J=15.1,2.4 \mathrm{~Hz}, 1 \mathrm{H}), 0.68-0.54(\mathrm{~m}, 12 \mathrm{H}) .{ }^{13} \mathrm{C}$ NMR $\left(126 \mathrm{MHz}, \mathrm{CDCl}_{3}\right) \delta 61.8,16.2,7.8$, 7.7, 4.2, 1.6. ${ }^{29} \mathrm{Si}$ NMR (99 MHz, $\left.\mathrm{CDCl}_{3}\right) \delta$ 7.28, 4.90. IR: 3510, 2952, 2909, 2875, 1461, 1417 , 1379, 1267, 1239, 1167, 1115, 1016, 969, 943, $928 \mathrm{~cm}^{-1}$. HRMS (DART) exact mass calculated for $\mathrm{C}_{14} \mathrm{H}_{34} \mathrm{OSi}_{2}\left[\mathrm{M}-\mathrm{C}_{2} \mathrm{H}_{5}\right]^{+} \mathrm{m} / \mathrm{z}=245.1752$, found $\mathrm{m} / \mathrm{z}=245.1755 . \mathrm{R}_{\mathrm{f}}=0.4(5 \% \mathrm{EA} / \mathrm{Hex})$.

(1-(4,4,5,5-tetramethyl-1,3,2-dioxaborolan-2-yl)ethane-1,2-diyl)bis(dimethylsilanol) (72):

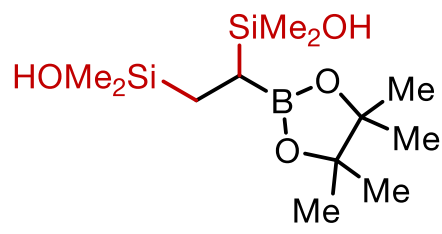

Following derivatization procedure 2 with $217 \mathrm{mg}$ of 37. The crude was purified with 3 5\% ethyl acetate/hexanes through a Florisil column and a clear colorless oil was obtained (174 $\mathrm{mg}, 72 \%)$. ${ }^{1} \mathbf{H}$ NMR $\left(500 \mathrm{MHz}, \mathrm{CDCl}_{3}\right) \delta 1.23(\mathrm{~s}, 12 \mathrm{H}), 0.89(\mathrm{qd}, \mathrm{J}=15.2,9.4 \mathrm{~Hz}, 2 \mathrm{H}), 0.59$ (dd, J = 10.6, $8.1 \mathrm{~Hz}, 1 \mathrm{H}), 0.24(\mathrm{~s}, 3 \mathrm{H}), 0.21(\mathrm{~s}, 3 \mathrm{H}), 0.12(\mathrm{~s}, 3 \mathrm{H}), 0.10$ (s, 3H). ${ }^{13} \mathrm{C}$ NMR $(126 \mathrm{MHz}, \mathrm{CDCl} 3) \delta$ 82.9, 25.1, 25.0, 11.3, 1.2, 0.5, 0.3, 0.0. IR: 2978, 2958, 2928, 2895, 1469, 1446, 1379, 1371, 1343, 1305, 1250, 1228, 1145, 1115, 1081, 1003, 976, 916, 875, $847 \mathrm{~cm}^{-1}$. HRMS (DART) exact mass 
calculated for $\mathrm{C}_{12} \mathrm{H}_{29} \mathrm{BO}_{4} \mathrm{Si}_{2}[\mathrm{M}-\mathrm{OH}]^{+} \mathrm{m} / \mathrm{z}=287.1670$, found $\mathrm{m} / \mathrm{z}=287.1665 . \mathrm{R}_{\mathrm{f}}=0.5(5 \%$ EA/Hex).

\section{(1-phenylethane-1,2-diyl)bis(dimethylsilanol) (73):}

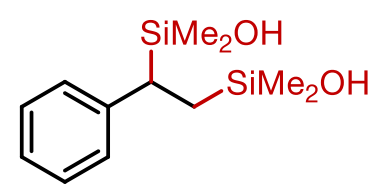

Following derivatization procedure 2, $178 \mathrm{mg}$ of $\mathbf{2 9}$ was added. The crude was purified with 3 5\% ethyl acetate/hexanes through a Florisil column and a clear colorless oil was obtained (95.3 mg, 47\%). ${ }^{1} \mathbf{H}$ NMR $\left(500 \mathrm{MHz}, \mathrm{CDCl}_{3}\right) \delta 7.30-7.22(\mathrm{~m}, 2 \mathrm{H}), 7.17-7.06(\mathrm{~m}, 3 \mathrm{H}), 2.51$ (dd, J = 13.2, $7.4 \mathrm{~Hz}, 1 \mathrm{H}), 1.30-1.14(\mathrm{~m}, 2 \mathrm{H}), 0.32(\mathrm{~s}, 3 \mathrm{H}), 0.30(\mathrm{~s}, 3 \mathrm{H}), 0.23(\mathrm{~s}, 3 \mathrm{H}),-0.13(\mathrm{~s}, 3 \mathrm{H}) .{ }^{13} \mathbf{C}$ NMR $\left(126 \mathrm{MHz}, \mathrm{CDCl}_{3}\right) \delta 144.5,128.5,126.4,124.5,33.1,16.7,1.0,0.5,-0.4,-2.6$. IR: 3082, 3060, 3022, 2957, 2915, 2890, 2851, 1599, 1494, 1448, 1408, 1251, 1197, 1147, 1072, 1033, 972, 914, $875,853,820 \mathrm{~cm}^{-1}$. HRMS (DART) exact mass calculated for $\mathrm{C}_{12} \mathrm{H}_{22} \mathrm{O}_{2} \mathrm{Si}_{2}[\mathrm{M}-\mathrm{OH}]^{+} \mathrm{m} / \mathrm{z}=$ 237.1131, found $\mathrm{m} / \mathrm{z}=237.1127$. $\mathrm{R}_{\mathrm{f}}=0.6(5 \% \mathrm{EA} / \mathrm{Hex})$.

\section{1-phenylethane-1,2-diol (74)}<smiles>OCC(O)c1ccccc1</smiles>

Following derivatization procedure 4 (DP4) with $222 \mathrm{mg}$ of (1-phenylethane-1,2diyl)bis(dimethylsilane). The crude was purified with $40 \%$ ethyl acetate/hexanes and a white solid was obtained (69.4 mg, 50\%). ${ }^{1} \mathbf{H}$ NMR $\left(500 \mathrm{MHz}^{\mathrm{CDCl}} \mathrm{CDC}_{3}\right) \delta .37$ (d, J=4.4 Hz, 4H), $7.35-7.27$ $(\mathrm{m}, 1 \mathrm{H}), 4.82(\mathrm{dd}, J=8.2,3.5 \mathrm{~Hz}, 1 \mathrm{H}), 3.76(\mathrm{dd}, J=11.4,3.5 \mathrm{~Hz}, 1 \mathrm{H}), 3.66(\mathrm{dd}, J=11.3,8.2 \mathrm{~Hz}$, 1H). ${ }^{13} \mathbf{C ~ N M R}\left(126 \mathrm{MHz}, \mathrm{CDCl}_{3}\right) \delta 140.6,128.7,128.2,126.2,74.8,68.2$. The compound was consistent with previous reports.

\section{(1-(4-(tert-butyl)phenyl)ethane-1,2-diyl)bis(trimethylsilane) (76):}<smiles>CCCCC(C)c1ccc(Br)cc1</smiles>

Following general procedure 1 with 4-tertbutylstyrene $(160 \mathrm{mg})$. The crude was purified with pure hexanes and a clear colorless oil was obtained $(254 \mathrm{mg}, 83 \%)$. ${ }^{\mathbf{1}} \mathbf{H} \mathbf{~ N M R}\left(500 \mathrm{MHz}, \mathrm{CDCl}_{3}\right) \delta 7.18$ $(\mathrm{d}, J=8.3 \mathrm{~Hz}, 2 \mathrm{H}), 6.91(\mathrm{~d}, J=8.3 \mathrm{~Hz}, 2 \mathrm{H}), 2.02(\mathrm{dd}, J=13.0,2.4 \mathrm{~Hz}, 1 \mathrm{H}), 1.28(\mathrm{~s}, 9 \mathrm{H}), 1.02$ $(\mathrm{dd}, J=15.0,12.9 \mathrm{~Hz}, 1 \mathrm{H}), 0.82(\mathrm{dd}, J=15.0,2.4 \mathrm{~Hz}, 1 \mathrm{H}),-0.08(\mathrm{~s}, 9 \mathrm{H}),-0.23(\mathrm{~s}, 9 \mathrm{H}) .{ }^{13} \mathbf{C}$ NMR $\left(126 \mathrm{MHz} \mathrm{CDCl}_{3}\right) \delta 147.0,141.8,127.5,124.7,34.3,31.6,30.9,16.3,-1.0,-3.1 .{ }^{29} \mathrm{Si}$ NMR (99 $\left.\mathrm{MHz} \mathrm{CDCl}_{3}\right) \delta 3.92,2.84$. IR (FTIR): 3032, 2954, 2899, 2869, 1739, 1718, 1514, 1364, 1247 , 1111, 1098, $837 \mathrm{~cm}^{-1}$. HRMS (DART) exact mass calculated for $\mathrm{C}_{18} \mathrm{H}_{35} \mathrm{Si}_{2}[\mathrm{M}+\mathrm{H}]^{+} \mathrm{m} / \mathrm{z}=$ 307.2272, found $\mathrm{m} / \mathrm{z}=307.2275 . \mathrm{R}_{\mathrm{f}}=0.8(\mathrm{Hex})$.

\section{(1-(4-methoxyphenyl)ethane-1,2-diyl)bis(trimethylsilane) (77):}


<smiles>CCC(C)c1ccc(OC)cc1</smiles>

Following general procedure 1 with 4-methoxystyrene $(134 \mathrm{mg})$. The crude was purified with $2.5 \%$ ethyl acetate/hexanes and a clear colorless oil was obtained (224 mg, 80\%). ${ }^{1} \mathbf{H}$ NMR (500 $\left.\mathrm{MHz} \mathrm{CDCl}_{3}\right) \delta 6.92(\mathrm{~d}, J=8.6 \mathrm{~Hz}, 2 \mathrm{H}), 6.75(\mathrm{~d}, J=8.6 \mathrm{~Hz}, 2 \mathrm{H}), 3.77(\mathrm{~s}, 3 \mathrm{H}), 2.00(\mathrm{dd}, J=13.2$, $2.4 \mathrm{~Hz}, 1 \mathrm{H}), 0.99(\mathrm{dd}, J=15.0,13.1 \mathrm{~Hz}, 1 \mathrm{H}), 0.81(\mathrm{dd}, J=15.0,2.4 \mathrm{~Hz}, 1 \mathrm{H}),-0.10$ (s, 9H), -0.21 $(\mathrm{s}, 9 \mathrm{H}) .{ }^{13} \mathbf{C} \mathbf{N M R}\left(126 \mathrm{MHz}, \mathrm{CDCl}_{3}\right) \delta 156.8,137.2,128.6,113.4,55.3,30.5,16.3,-0.9,-3.2$. IR (FTIR): 3063, 3014, 2953, 2907, 2873, 2835, 1606, 1511, 1486, 1462, 1414, 1247, 1224, 1205, $1176,1152,1125,1103,1039 \mathrm{~cm}^{-1}$. HRMS (DART) exact mass calculated for $\mathrm{C}_{14} \mathrm{H}_{25} \mathrm{OSi}_{2}$ [M$\left.\mathrm{CH}_{3}\right]^{+} \mathrm{m} / \mathrm{z}=265.1438$, found $\mathrm{m} / \mathrm{z}=265.1443 . \mathrm{R}_{\mathrm{f}}=0.8(5 \% \mathrm{EA} / \mathrm{Hex})$.

\section{(1-([1,1'-biphenyl]-4-yl)ethane-1,2-diyl)bis(trimethylsilane) (78):}<smiles>CCC(C)c1ccc(-c2ccccc2)cc1</smiles>

Following general procedure 1 with 4-vinyl-1,1'-biphenyl (180 mg). The crude was purified with pure hexanes and a white solid was obtained $(224 \mathrm{mg}, 69 \%) .{ }^{1} \mathbf{H} \mathbf{~ N M R}\left(500 \mathrm{MHz}, \mathrm{CDCl}_{3}\right) \delta 7.63$ $-7.58(\mathrm{~m}, 2 \mathrm{H}), 7.52-7.38(\mathrm{~m}, 4 \mathrm{H}), 7.30(\mathrm{~s}, 1 \mathrm{H}), 7.11-7.06(\mathrm{~m}, 2 \mathrm{H}), 2.12(\mathrm{dd}, J=13.0,2.3 \mathrm{~Hz}$, 1H), 1.09 (dd, $J=15.1,13.0 \mathrm{~Hz}, 1 \mathrm{H}), 0.87$ (dd, $J=15.1,2.3 \mathrm{~Hz}, 1 \mathrm{H}),-0.05$ (s, 9H), -0.18 (s, 9H). ${ }^{13} \mathrm{C}$ NMR $\left(126 \mathrm{MHz}, \mathrm{CDCl}_{3}\right) \delta 144.6,141.3,136.9,128.8,128.3,126.8,126.5,31.5,16.1$, -0.9, 3.1. IR (FTIR): 3080, 3059, 3028, 2953, 2896, 2873, 1602, 1519, 1486, 1449, 1411, 1247, 1222 , 1182, 1146, 1108, 1098, 1007, $835 \mathrm{~cm}^{-1}$. HRMS (DART) exact mass calculated for $\mathrm{C}_{20} \mathrm{H}_{31} \mathrm{Si}_{2}$ $[\mathrm{M}+\mathrm{H}]^{+} \mathrm{m} / \mathrm{z}=327.1959$, found $\mathrm{m} / \mathrm{z}=327.1962 . \mathrm{R}_{\mathrm{f}}=0.6(\mathrm{Hex})$.

(1-(o-tolyl)ethane-1,2-diyl)bis(trimethylsilane) (79):<smiles>Cc1ccccc1C(CCC(C)(C)C)C(C)(C)C</smiles>

Following general procedure 1 with 2-methylstyrene $(118 \mathrm{mg})$. The crude was purified with pure hexanes and a clear colorless oil was obtained $(290 \mathrm{mg}, 87 \%) .{ }^{1} \mathbf{H} \mathbf{~ N M R}\left(500 \mathrm{MHz}, \mathrm{CDCl}_{3}\right) \delta 7.08$ $(\mathrm{d}, J=7.8 \mathrm{~Hz}, 2 \mathrm{H}), 7.03(\mathrm{~d}, J=7.6 \mathrm{~Hz}, 1 \mathrm{H}), 6.99-6.92(\mathrm{~m}, 1 \mathrm{H}), 2.37(\mathrm{dd}, J=13.0,2.4 \mathrm{~Hz}, 1 \mathrm{H})$, $2.27(\mathrm{~s}, 3 \mathrm{H}), 1.10(\mathrm{dd}, J=15.0,13.0 \mathrm{~Hz}, 1 \mathrm{H}), 0.88(\mathrm{dd}, J=15.1,2.4 \mathrm{~Hz}, 1 \mathrm{H}),-0.06(\mathrm{~s}, 9 \mathrm{H}),-0.22$ $(\mathrm{s}, 9 \mathrm{H}) .{ }^{13} \mathbf{C} \mathbf{N M R}\left(126 \mathrm{MHz}, \mathrm{CDCl}_{3}\right) \delta 143.8,134.6,130.1,126.9,125.7,123.9,25.3,20.8,17.0$, -0.9, -3.0. ${ }^{29} \mathrm{Si}$ NMR $\left(99 \mathrm{MHz}, \mathrm{CDCl}_{3}\right) \delta$ 4.87, 2.67. IR (FTIR): 3063, 3016, 2953, 2904, 2870, 1486, 1461, 1410, 1378, 1366, 1427, 1218, 1100, $836 \mathrm{~cm}^{-1}$. HRMS (DART) exact mass calculated for $\mathrm{C}_{15} \mathrm{H}_{29} \mathrm{Si}_{2}[\mathrm{M}+\mathrm{H}]^{+} \mathrm{m} / \mathrm{z}=265.1802$, found $\mathrm{m} / \mathrm{z}=265.1806 . \mathrm{R}_{\mathrm{f}}=0.9(\mathrm{Hex})$.

(1-(benzo[d][1,3]dioxol-5-yl)ethane-1,2-diyl)bis(trimethylsilane) $(80)$ : 


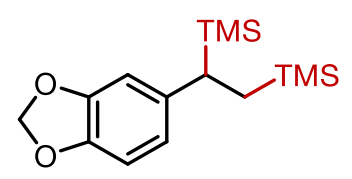

Following general procedure 1 with 5-vinylbenzo[ $d][1,3]$ dioxole $(148 \mathrm{mg})$. The crude was purified with pure hexanes and a clear colorless oil was obtained $(227 \mathrm{mg}, 77 \%) .{ }^{1} \mathbf{H}$ NMR $(500 \mathrm{MHz}$, $\left.\mathrm{CDCl}_{3}\right) \delta 6.66(\mathrm{~d}, J=8.0 \mathrm{~Hz}, 1 \mathrm{H}), 6.53(\mathrm{~d}, J=1.8 \mathrm{~Hz}, 1 \mathrm{H}), 6.46(\mathrm{dd}, J=7.9,1.8 \mathrm{~Hz}, 1 \mathrm{H}), 5.89(\mathrm{~d}$, $J=1.6 \mathrm{~Hz}, 2 \mathrm{H}), 1.99(\mathrm{dd}, J=13.1,2.4 \mathrm{~Hz}, 1 \mathrm{H}), 0.94(\mathrm{dd}, J=15.1,13.0 \mathrm{~Hz}, 1 \mathrm{H}), 0.80(\mathrm{dd}, J=$ $15.1,2.4 \mathrm{~Hz}, 1 \mathrm{H}),-0.08$ (s, 9H), -0.19 (s, 9H). ${ }^{13} \mathbf{C ~ N M R}\left(126 \mathrm{MHz}, \mathrm{CDCl}_{3}\right) \delta 147.3,144.5,139.3$, 120.5, 108.3, 107.9, 100.6, 31.4, 16.5, -0.9, -3.2. IR (FTIR): 3015, 2953, 2896, 2875, 2772, 1505, 1485, 1438, 1411, 1351, 1304, 1246, 1218, 1180, 1119, 1096, 1043, 942, 924, $836 \mathrm{~cm}^{-1}$. HRMS (DART) exact mass calculated for $\mathrm{C}_{15} \mathrm{H}_{27} \mathrm{O}_{2} \mathrm{Si}_{2}[\mathrm{M}+\mathrm{H}]^{+} \mathrm{m} / \mathrm{z}=295.1544$, found $\mathrm{m} / \mathrm{z}=295.1548$. $\mathrm{R}_{\mathrm{f}}=0.3(\mathrm{Hex})$.

\section{(1-phenylethane-1,2-diyl)bis(triethylsilane) (81):}

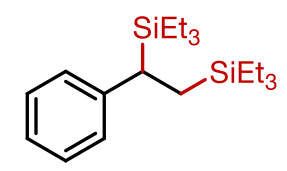

Following general procedure 1 with styrene $(104 \mathrm{mg})$ and triethylchlorosilane $(450 \mathrm{mg})$. The crude was purified with pure hexanes and a clear colorless oil was obtained $(251 \mathrm{mg}, 75 \%)$. ${ }^{\mathbf{1}} \mathbf{H}$ NMR $\left(500 \mathrm{MHz}, \mathrm{CDCl}_{3}\right) \delta 7.18(\mathrm{t}, J=7.6 \mathrm{~Hz}, 2 \mathrm{H}), 7.05(\mathrm{dd}, J=13.3,7.2 \mathrm{~Hz}, 3 \mathrm{H}), 2.21(\mathrm{dd}, J=13.5$, $2.0 \mathrm{~Hz}, 1 \mathrm{H}), 1.08(\mathrm{dd}, J=15.3,13.4 \mathrm{~Hz}, 1 \mathrm{H}), 0.88(\mathrm{t}, J=7.9 \mathrm{~Hz}, 10 \mathrm{H}), 0.79(\mathrm{t}, J=7.9 \mathrm{~Hz}, 9 \mathrm{H})$, $0.51(\mathrm{~m}, 6 \mathrm{H}), 0.29(\mathrm{~m}, 6 \mathrm{H}) .{ }^{13} \mathbf{C}$ NMR $\left(126 \mathrm{MHz} \mathrm{CDCl}_{3}\right) \delta 145.6,128.04,127.96,124.3,28.4$, 11.5, 7.7, 7.5, 3.7, 2.3. ${ }^{29} \mathrm{Si}$ NMR $\left(99 \mathrm{MHz} \mathrm{CDCl}_{3}\right) \delta 8.56,7.53$. IR (FTIR): 2950, 2908, 2874, 1599, 1493, 1452, 1415, 1377, 1238, 1102, 1007, 971, 904, 843, 760, 714, $698 \mathrm{~cm}^{-1}$. HRMS (DART) exact mass calculated for $\mathrm{C}_{18} \mathrm{H}_{33} \mathrm{Si}_{2}\left[\mathrm{M}-\mathrm{C}_{2} \mathrm{H}_{5}\right]^{+} \mathrm{m} / \mathrm{z}=305.2115$, found $\mathrm{m} / \mathrm{z}=305.2118$. $\mathrm{R}_{\mathrm{f}}=0.9(\mathrm{Hex})$.

\section{(1-(naphthalen-2-yl)ethane-1,2-diyl)bis(trimethylsilane) (82):}<smiles>CCC(C)c1ccc2ccccc2c1</smiles>

Following general procedure 1 with 2-vinylnapthalene $(154 \mathrm{mg})$. The crude was purified with pure hexanes and a white solid was obtained (211 mg, 70\%). ${ }^{\mathbf{1}} \mathbf{H} \mathbf{N M R}\left(500 \mathrm{MHz}, \mathrm{CDCl}_{3}\right) \delta 7.93-7.61$ $(\mathrm{m}, 3 \mathrm{H}), 7.51-7.34(\mathrm{~m}, 3 \mathrm{H}), 7.20(\mathrm{dd}, J=8.4,1.8 \mathrm{~Hz}, 1 \mathrm{H}), 2.26(\mathrm{dd}, J=13.0,2.3 \mathrm{~Hz}, 1 \mathrm{H}), 1.21$ $(\mathrm{dd}, J=15.1,13.0 \mathrm{~Hz}, 1 \mathrm{H}), 0.93(\mathrm{dd}, J=15.2,2.3 \mathrm{~Hz}, 1 \mathrm{H}),-0.05(\mathrm{~s}, 9 \mathrm{H}),-0.20(\mathrm{~s}, 9 \mathrm{H}) .{ }^{13} \mathbf{C}$ NMR $\left(126 \mathrm{MHz}, \mathrm{CDCl}_{3}\right) \delta 143.1,133.8,131.5,127.7,127.7,127.3,125.7,125.2,124.5,32.0,16.0$, 0.8, -3.1. ${ }^{29} \mathrm{Si}$ NMR $\left(99 \mathrm{MHz}, \mathrm{CDCl}_{3}\right) \delta$ 4.67, 3.13. IR (FTIR): 3055, 3016, 2950, 2896, 1738, 1246, 1230, 1217, 1093, 893, $839 \mathrm{~cm}^{-1}$. HRMS (DART) exact mass calculated for $\mathrm{C}_{18} \mathrm{H}_{29} \mathrm{Si}_{2}$ $[\mathrm{M}+\mathrm{H}]^{+} \mathrm{m} / \mathrm{z}=301.1802$, found $\mathrm{m} / \mathrm{z}=301.1806 . \mathrm{R}_{\mathrm{f}}=0.8(\mathrm{Hex})$.

\section{(E)-1,2-diphenyl-1,2-bis(trimethylsilyl)ethene (83):}


<smiles>CC(=C(c1ccccc1)S(C)(=O)=O)c1ccccc1</smiles>

Following general procedure 1 with diphenylacetylene $(178 \mathrm{mg})$ and 4 equivalents of TMSCl. The reaction was stopped at $2.2 \mathrm{~F}$ of charge and the crude was purified with pure hexanes and a white solid was obtained $(198 \mathrm{mg}, 61 \%) .{ }^{1} \mathbf{H}$ NMR $\left(500 \mathrm{MHz}, \mathrm{CDCl}_{3}\right) \delta 7.32-7.24(\mathrm{~m}, 4 \mathrm{H}), 7.23-7.16$ $(\mathrm{m}, 2 \mathrm{H}), 7.05(\mathrm{dt}, J=6.3,1.5 \mathrm{~Hz}, 4 \mathrm{H}),-0.36(\mathrm{~s}, 18 \mathrm{H}) .{ }^{13} \mathbf{C ~ N M R}\left(126 \mathrm{MHz}, \mathrm{CDCl}_{3}\right) \delta 159.3,146.0$, 128.2, 127.8, 125.7, 0.4. IR (FTIR): 3072, 3051, 3020, 2953, 2894, 1739, 1595, 1491, 1439, 1404, 1244, 1068, 1029, 915, 838, 796, 759, $701 \mathrm{~cm}^{-1}$. HRMS (DART) exact mass calculated for $\mathrm{C}_{20} \mathrm{H}_{28} \mathrm{Si}_{2}[\mathrm{M}]^{+} \mathrm{m} / \mathrm{z}=324.1724$, found $\mathrm{m} / \mathrm{z}=324.1728 . \mathrm{R}_{\mathrm{f}}=0.8(\mathrm{Hex})$.

\section{(1-(benzofuran-2-yl)ethane-1,2-diyl)bis(trimethylsilane) (84):}

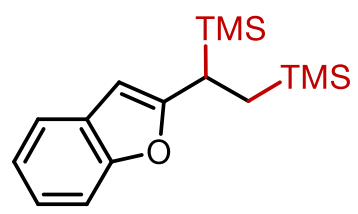

Following general procedure 1 with 2-vinylbenzofuran (144 mg) and 4 equivalents of TMSCl. The reaction was stopped at $2.2 \mathrm{~F}$ of charge and the crude was purified with pure hexanes and a clear colorless oil was obtained $(185 \mathrm{mg}, 64 \%) .{ }^{1} \mathbf{H} \mathbf{~ N M R}\left(500 \mathrm{MHz}, \mathrm{CDCl}_{3}\right) \delta 7.46-7.39(\mathrm{~m}, 1 \mathrm{H})$, $7.39-7.33(\mathrm{~m}, 1 \mathrm{H}), 7.18-7.10(\mathrm{~m}, 2 \mathrm{H}), 6.18(\mathrm{~s}, 1 \mathrm{H}), 2.29(\mathrm{dd}, J=13.0,2.3 \mathrm{~Hz}, 1 \mathrm{H}), 1.12(\mathrm{dd}, J$ $=15.0,13.0 \mathrm{~Hz}, 1 \mathrm{H}), 0.81(\mathrm{dd}, J=15.0,2.3 \mathrm{~Hz}, 1 \mathrm{H}), 0.02(\mathrm{~s}, 9 \mathrm{H}),-0.14(\mathrm{~s}, 9 \mathrm{H}) .{ }^{13} \mathrm{C}$ NMR $(126$ $\left.\mathrm{MHz} \mathrm{CDCl}_{3}\right) \delta 163.0,154.4,129.7,122.3,122.2,119.6,110.5,100.2,25.2,14.4,-1.4,-3.0$. IR (FTIR): 3063, 3035, 2954, 2897, 2880, 1576, 1455, 1417, 1250, 1179, 1094, 1010, 937, $839 \mathrm{~cm}^{-1}$. HRMS (DART) exact mass calculated for $\mathrm{C}_{16} \mathrm{H}_{27} \mathrm{OSi}_{2}[\mathrm{M}+\mathrm{H}]^{+} \mathrm{m} / \mathrm{z}=291.1595$, found $\mathrm{m} / \mathrm{z}=$ 291.1597. $\mathrm{R}_{\mathrm{f}}=0.6(2 \% \mathrm{EA} / \mathrm{Hex})$.

\section{(2-phenylpropane-1,2-diyl)bis(trimethylsilane) (85):}<smiles>CC(C)(C)c1ccccc1</smiles>

Following general procedure 1 with prop-1-en-2-ylbenzene $(118 \mathrm{mg})$. The crude was purified with pure hexanes and a clear colorless oil was obtained $(141 \mathrm{mg}, 53 \%) .{ }^{1} \mathbf{H} \mathbf{~ N M R}\left(500 \mathrm{MHz}, \mathrm{CDCl}_{3}\right)$ $\delta 7.28-7.19(\mathrm{~m}, 2 \mathrm{H}), 7.17-7.11(\mathrm{~m}, 2 \mathrm{H}), 7.10-7.03(\mathrm{~m}, 1 \mathrm{H}), 1.54-1.47(\mathrm{~m}, 1 \mathrm{H}), 1.40(\mathrm{~d}, J=$ $1.1 \mathrm{~Hz}, 3 \mathrm{H}), 0.86(\mathrm{~d}, J=14.9 \mathrm{~Hz}, 1 \mathrm{H}),-0.13(\mathrm{~s}, 9 \mathrm{H}),-0.21(\mathrm{~s}, 9 \mathrm{H}) .{ }^{13} \mathbf{C} \mathbf{N M R}\left(126 \mathrm{MHz}, \mathrm{CDCl}_{3}\right)$ $\delta$ 148.0, 127.6, 126.6, 123.9, 29.5, 24.0, 22.6, 1.4, -4.1. IR (FTIR): 3081, 3062, 3024, 2953, 2897 , 2873, 2855, 1600, 1494, 1450, 1411, 1247, 1223, 1181, 1143, 1101, 1070, 1032, 906, $836 \mathrm{~cm}^{-1}$. HRMS (DART) exact mass calculated for $\mathrm{C}_{15} \mathrm{H}_{29} \mathrm{Si}_{2}[\mathrm{M}+\mathrm{H}]^{+} \mathrm{m} / \mathrm{z}=265.1802$, found $\mathrm{m} / \mathrm{z}=$ 265.1805. $\mathrm{R}_{\mathrm{f}}=0.9(\mathrm{Hex})$.

\section{(1-(4-methoxyphenyl)ethane-1,2-diyl)bis(triethylsilane) (86):}


<smiles>CCCC(CCC)c1ccc(OC)cc1</smiles>

Following general procedure 1 with 4-methoxystyrene $(134 \mathrm{mg})$. The crude was purified with $2 \%$ ethyl acetate/hexanes and a clear colorless oil was obtained $(270 \mathrm{mg}, 74 \%)$. ${ }^{\mathbf{1}} \mathbf{H}$ NMR $(500 \mathrm{MHz}$, $\left.\mathrm{CDCl}_{3}\right) \delta 7.00-6.93(\mathrm{~m}, 2 \mathrm{H}), 6.78-6.71(\mathrm{~m}, 2 \mathrm{H}), 3.77(\mathrm{~s}, 3 \mathrm{H}), 2.14(\mathrm{dd}, \mathrm{J}=13.6,2.0 \mathrm{~Hz}, 1 \mathrm{H})$, $1.01(\mathrm{dd}, \mathrm{J}=15.2,13.5 \mathrm{~Hz}, 1 \mathrm{H}), 0.83(\mathrm{~m}, 19 \mathrm{H}), 0.55-0.44(\mathrm{~m}, 6 \mathrm{H}), 0.37-0.19(\mathrm{~m}, 6 \mathrm{H}) .{ }^{13} \mathrm{C}$ NMR $\left(126 \mathrm{MHz}, \mathrm{CDCl}_{3}\right) \delta 156.8,137.5,128.7,113.4,55.3,27.1,11.6,7.8,7.5,3.7,2.3$. IR (FTIR): 2950, 2908, 2874, 2833, 1609, 1579, 1508, 1462, 1441, 1416, 1377, 1326, 1300, 1281, 1246, 1177, 1143, 1107, 1041, 1013, 972, 854, 828, $808 \mathrm{~cm}^{-1}$. HRMS (DART) exact mass calculated for $\mathrm{C}_{21} \mathrm{H}_{41} \mathrm{OSi}_{2}[\mathrm{M}+\mathrm{H}]^{+} \mathrm{m} / \mathrm{z}=365.2691$, found $\mathrm{m} / \mathrm{z}=365.2690 . \mathrm{R}_{\mathrm{f}}=0.6(3 \% \mathrm{EA} / \mathrm{Hex})$.

\section{1,1,1,2,2,2-hexaethyldisilane (87):}

$$
\mathrm{Et}_{3} \mathrm{Si}-\mathrm{SiEt}_{3}
$$

Following general procedure 1 with 4-methoxystyrene $(134 \mathrm{mg})$. This side product was purified with pure hexanes and a clear colorless oil was obtained $(31.1 \mathrm{mg}, 27 \%) .{ }^{1} \mathbf{H}$ NMR $(500 \mathrm{MHz}$, $\left.\mathrm{CDCl}_{3}\right) \delta 0.93(\mathrm{t}, \mathrm{J}=8.0 \mathrm{~Hz}, 18 \mathrm{H}), 0.52(\mathrm{q}, \mathrm{J}=7.9 \mathrm{~Hz}, 12 \mathrm{H}) .{ }^{13} \mathbf{C} \mathbf{N M R}\left(126 \mathrm{MHz}, \mathrm{CDCl}_{3}\right) \delta 7.0$, 6.6. ${ }^{29} \mathrm{Si} \mathrm{NMR}\left(99 \mathrm{MHz}, \mathrm{CDCl}_{3}\right) \delta 8.85$. The compound was consistent with previous reports. 


\section{Unsuccessful substrates}

$$
\underset{1.0 \mathrm{mmol}}{\mathrm{R}}+\underset{3 \text { equiv }}{\mathrm{TMSCl}} \frac{\mathrm{Mg}(+) \mid \mathrm{C}(-), \mathrm{TBAClO}_{4} 0.2 \mathrm{M} \text { in THF }}{l_{\text {cell }}=10 \mathrm{~mA}, 3.5 \mathrm{~F}, 22^{\circ} \mathrm{C}}{ }_{\mathrm{R}}^{\mathrm{TMS}}
$$

\section{Unsuccessful alkenes}

inseparatable mixtures (yield $=5 \sim 30 \%$ )

Functional group not tolerated

P<smiles>C(=Cc1ccccc1)c1ccccc1</smiles><smiles>C=Cc1ccc(C(C)=O)cc1</smiles><smiles>C=Cc1ccc(C(=O)O)cc1</smiles><smiles>C=Cc1ccc(C#N)cc1</smiles><smiles>C=Cc1cccnc1</smiles><smiles>C=Cc1ccc2nc(C)ccc2c1</smiles><smiles>C=Cc1ccc(C(F)(F)F)cc1</smiles><smiles>C=Cc1ccc(NC(=O)OC(C)(C)C)cc1</smiles><smiles>CC(=O)OC=Cc1ccccc1</smiles>

Side reactions<smiles>C=Cc1ccccn1</smiles>

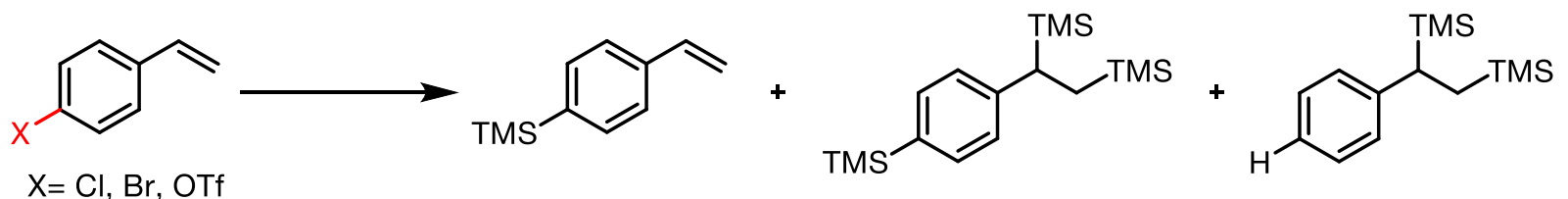

Low conversion or no reaction<smiles>C=CCCc1ccccc1</smiles><smiles>C=CCc1ccccc1</smiles><smiles>C1=CCCCCCC1</smiles><smiles>C=C(C)CCc1ccccc1</smiles>

Unsuccessful chlorosilanes (messy reactions)<smiles></smiles><smiles>C[Si]([Mg])(Cl)Cl</smiles><smiles></smiles><smiles>C[Si](C)(Cl)c1ccccc1</smiles>

Scheme S17: Unsuccessful substrates for disilylation 


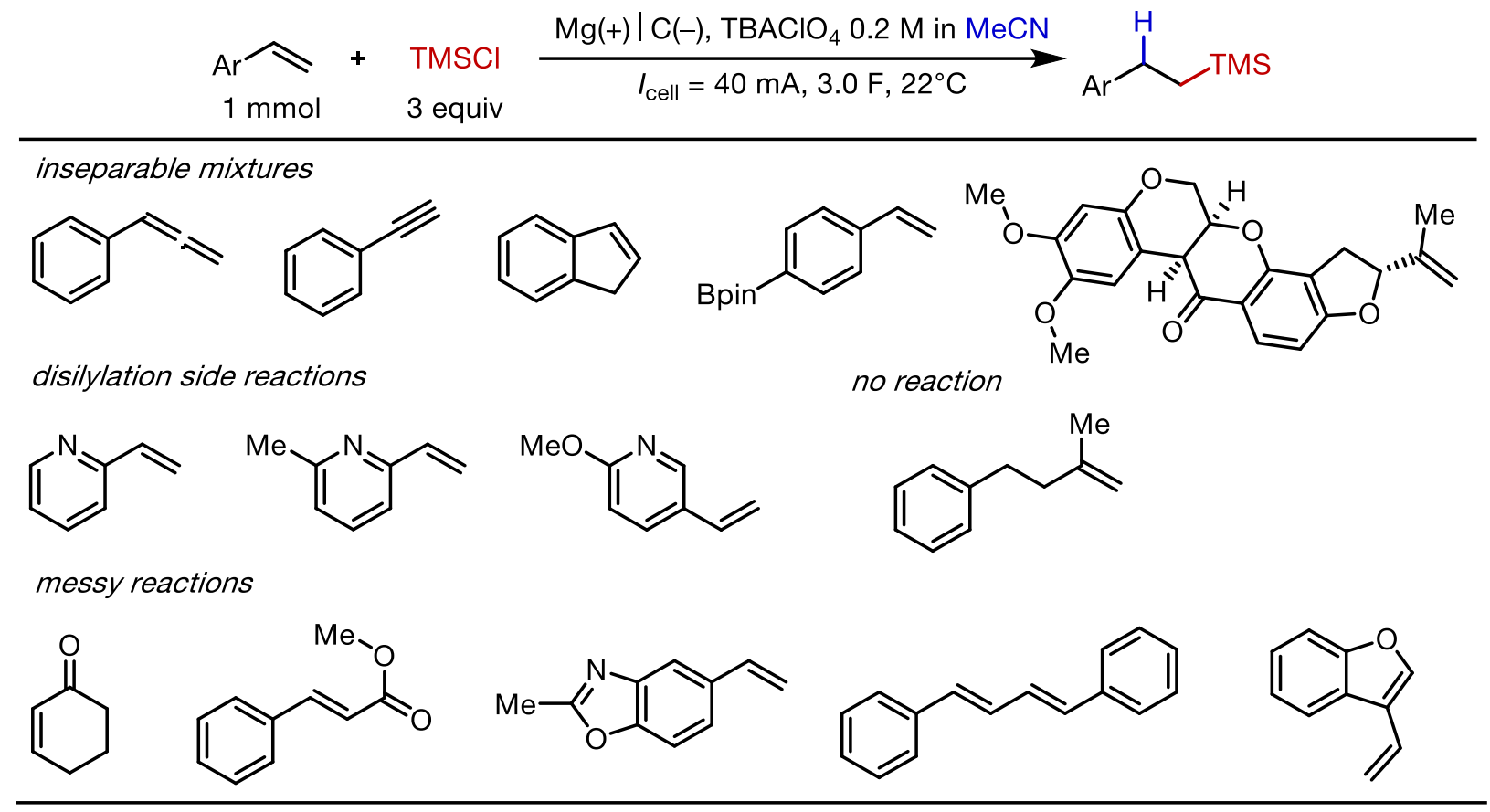

Scheme S18: Unsuccessful substrates for hydrosilylation 


\section{Natural abundance Carbon Kinetic Isotope study}

Based on the method outlined in GP 1, the reaction was scaled up 3 times. In an oven dried echem cell, 4-tert-butylstyrene (freshly distilled) $(268 \mathrm{mg}, 3 \mathrm{mmol}, 1 \mathrm{eq})$, trimethylsilylchloride (648 $\mathrm{mg}$, $9 \mathrm{mmol}, 3 \mathrm{eq}), \mathrm{TBAClO}_{4}(740 \mathrm{mg}, 0.2 \mathrm{M})$ and around $20 \mathrm{mg}$ of dodecane $(0.2 \mathrm{mmol}$, $0.2 \mathrm{eq})$ was added. Then THF (10 mL) was added and stirred until everything dissolved. The vessel was then sealed with the cap and transferred out of the glovebox for electrolysis. After a calibration test to determine the conversion efficiency. The amount of charge require to achieve around $80 \%$ conversion was used. After completion of electrolysis, a small sample was taken for GC analysis to determine conversion. The usual workup and purification the afforded the starting material. Then the starting material went through another bulb to bulb distillation under vacuum before analysis. $21 \mathrm{mg}$ of the sample was then taken and dissolved in $500 \mathrm{ul}\left(0.001 \mathrm{M} \mathrm{Cr}(\mathrm{acac})_{3}\right)$ of $\mathrm{CDCl}_{3}$ and analysed by the quantitative ${ }^{13} \mathrm{C}$ pulse sequence. A $21 \mathrm{mg}$ sample of the starting tertbutyl styrene that hasn't gone through fractionation was also analysed analogously.

\section{General method for ${ }^{13} \mathrm{C}$ measurements}

The spectrums were recorded on a $126 \mathrm{MHz}$ Bruker 500 using inverse gated decoupling and calibrated at $\pi / 2$ pulses. Following a general method outlined by the Singleton group. ${ }^{36} \mathrm{~A}$ relaxation delay of 70s was used with no noticeable difference in integration when a relaxation delay of 180s was used. Acquisition time was 10s. The spectrums were processed in Mestrenova 11 without the application of any window functions. A manual phase correction and an eighth order polynomial baseline correction was applied. Each peak was integrated between $\pm 10 \mathrm{~Hz}$.

Table S10. Raw data for ${ }^{13} \mathrm{C}$ KIE experiments

\begin{tabular}{|c|c|c|c|c|c|c|c|c|c|}
\hline carbon & 31 & 35 & 113 & 125.5 & 126 & 135 & 137 & 151 & conversion \\
\hline \multicolumn{10}{|c|}{ Experiment } \\
\hline \multirow{2}{*}{$\begin{array}{l}\text { Starting } \\
\text { material }\end{array}$} & 2947.99 & 955.92 & 989.23 & 2000.00 & 1997.28 & 1005.43 & 980.01 & 1008.80 & 0 \\
\hline & 2893.93 & 962.13 & 1011.34 & 2000 & 1990.16 & 1015.32 & 980.05 & 1008.49 & 0.84 \\
\hline \multirow[t]{2}{*}{ KIE } & 0.9900 & 1.0035 & 1.0122 & 1.0000 & 0.9981 & 1.0054 & 1.0000 & 0.9998 & \\
\hline & 2895.62 & 975.23 & 1028.3 & 2000 & 1980.36 & 992.67 & 989.41 & 1019.44 & 0.84 \\
\hline \multirow[t]{2}{*}{ KIE } & 0.9903 & 1.0110 & 1.0216 & 1.0000 & 0.9954 & 0.9931 & 1.0052 & 1.0058 & \\
\hline & 2854.72 & 942.94 & 1030.74 & 2000 & 1995.66 & 995.91 & 995.34 & 1005.47 & 0.84 \\
\hline \multirow[t]{2}{*}{ KIE } & 0.9828 & 0.9926 & 1.0229 & 1.0000 & 0.9996 & 0.9948 & 1.0085 & 0.9982 & \\
\hline & 2881.24 & 952.88 & 1020.69 & 2000 & 2010.83 & 1009.01 & 985.52 & 993.76 & 0.84 \\
\hline \multirow[t]{2}{*}{$\mathrm{KIE}$} & 0.9877 & 0.9983 & 1.0174 & 1.0000 & 1.0037 & 1.0019 & 1.0031 & 0.9919 & \\
\hline & 2862.5 & 937.26 & 1030.59 & 2000 & 1987.73 & 1005.04 & 999.67 & 1010.72 & 0.84 \\
\hline \multirow[t]{2}{*}{ KIE } & 0.9842 & 0.9894 & 1.0229 & 1.0000 & 0.9974 & 0.9998 & 1.0110 & 1.0010 & \\
\hline & 2878.5 & 967.51 & 1021.65 & 2000 & 1986.95 & 1001.12 & 998.45 & 1009.77 & 0.84 \\
\hline \multirow[t]{2}{*}{ KIE } & 0.9872 & 1.0066 & 1.0179 & 1.0000 & 0.9972 & 0.9977 & 1.0103 & 1.0005 & \\
\hline & 2889.7 & 948.86 & 1038.18 & 2000 & 2004.17 & 1010.17 & 988.2 & 1007.66 & 0.84 \\
\hline KIE & 0.9892 & 0.9960 & 1.0271 & 1.0000 & 1.0019 & 1.0026 & 1.0046 & 0.9994 & \\
\hline
\end{tabular}




\begin{tabular}{|l|l|l|l|l|l|l|l|l|l|}
\hline & 2874.3 & 938.19 & 1013.16 & 2000 & 1993.09 & 997.44 & 990.4 & 1006.98 & 0.84 \\
\hline KIE & 0.9864 & 0.9899 & 1.0132 & 1.0000 & 0.9989 & 0.9957 & 1.0058 & 0.9990 & \\
\hline & 2865.33 & 954.11 & 1018.24 & 2000 & 1992.02 & 994.03 & 994.86 & 999.81 & 0.84 \\
\hline KIE & 0.9847 & 0.9990 & 1.0160 & 1.0000 & 0.9986 & 0.9938 & 1.0083 & 0.9951 & \\
\hline
\end{tabular}

\begin{tabular}{|l|l|l|l|l|l|l|l|l|}
\hline & 31 & 35 & 113 & 125.5 & 126 & 135 & 137 & 151 \\
\hline 1 & 0.9900 & 1.0035 & 1.0122 & 1.0000 & 0.9981 & 1.0054 & 1.0000 & 0.9998 \\
\hline 2 & 0.9903 & 1.0110 & 1.0216 & 1.0000 & 0.9954 & 0.9931 & 1.0052 & 1.0058 \\
\hline 3 & 0.9828 & 0.9926 & 1.0229 & 1.0000 & 0.9996 & 0.9948 & 1.0085 & 0.9982 \\
\hline 4 & 0.9877 & 0.9983 & 1.0174 & 1.0000 & 1.0037 & 1.0019 & 1.0031 & 0.9919 \\
\hline 5 & 0.9842 & 0.9894 & 1.0229 & 1.0000 & 0.9974 & 0.9998 & 1.0110 & 1.0010 \\
\hline 6 & 0.9872 & 1.0066 & 1.0179 & 1.0000 & 0.9972 & 0.9977 & 1.0103 & 1.0005 \\
\hline 7 & 0.9892 & 0.9960 & 1.0271 & 1.0000 & 1.0019 & 1.0026 & 1.0046 & 0.9994 \\
\hline 8 & 0.9864 & 0.9899 & 1.0132 & 1.0000 & 0.9989 & 0.9957 & 1.0058 & 0.9990 \\
\hline 9 & 0.9847 & 0.9990 & 1.0160 & 1.0000 & 0.9986 & 0.9938 & 1.0083 & 0.9951 \\
\hline average & 0.9869 & 0.9985 & 1.0190 & 1.0000 & 0.9990 & 0.9983 & 1.0063 & 0.9990 \\
\hline stdev & 0.0027 & 0.007 & 0.005 & 0.0000 & 0.003 & 0.004 & 0.0035 & 0.0039 \\
\hline
\end{tabular}

Impurity peak around $34.588 \mathrm{ppm}$ and $31.308 \mathrm{ppm}$ prevented accurate measurement of KIE at those positions.

Experimentally measured

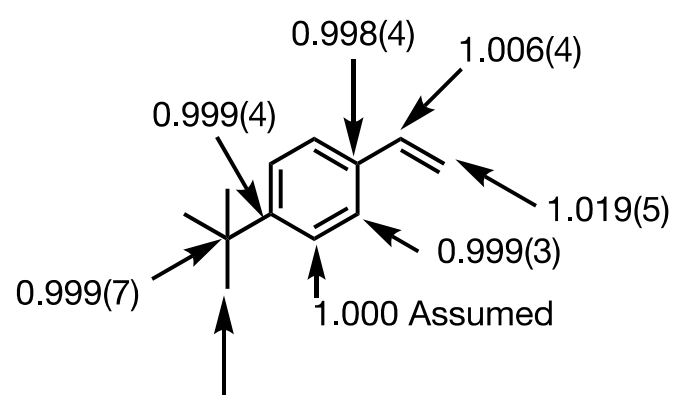

$0.987(3)$

Scheme S19: ${ }^{13} \mathrm{KIE}$ data using 4-tert-butylstyrene 


\section{Computationally predicted KIE}

Transition state search: All DFT calculations were run using Gaussian $09^{37}$ using the unrestricted M06- $1^{38}$ functional employing the 6-31G(d) basis set and solvation using the SMD solvation model. Transition states were found via bond scan of the bond forming atoms and then subjecting the highest energy structure for transition state search. The transition states were then verified by a single negative frequency associated with the bond forming coordinate. Additional saddle point calculations where radical addition to the benzylic positions were also conducted but none were found. The starting material (4-tBu-styrene) was optimized using the same functional, basis set, and solvent model. The lowest energy transition state structure was used in the prediction of ${ }^{13} \mathrm{C}$ KIEs with PyKIE $^{39}$ and taking the inverted parabola predictions.

Computationally predicted KIE, showing better consistency with the experimental data for the radical pathway:

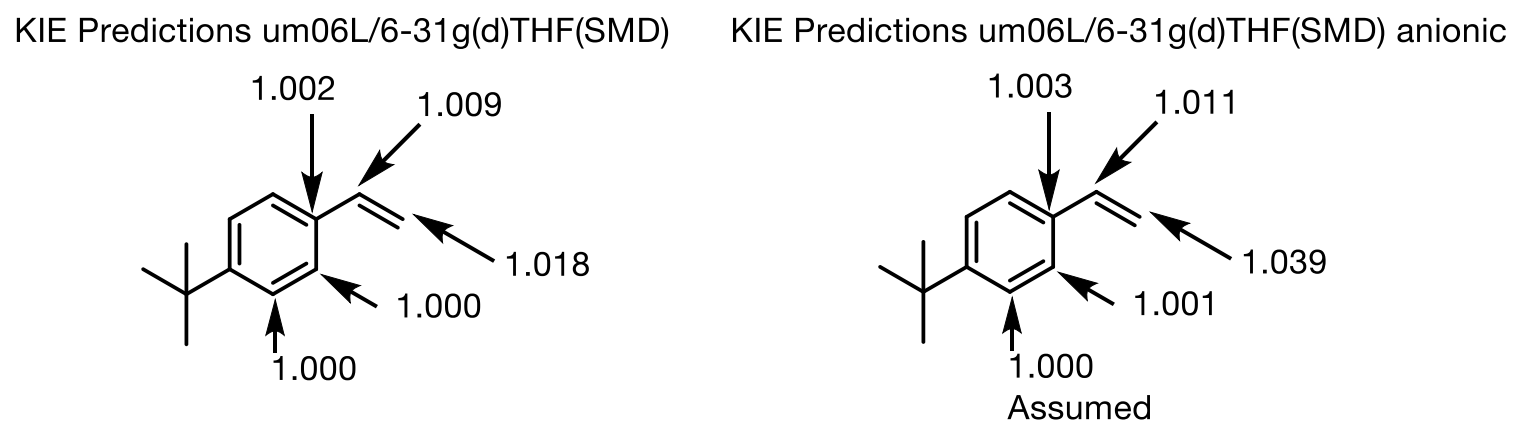

Scheme S20: predicted ${ }^{13} \mathrm{KIE}$ data

Computational predicted transition states of radical and anionic pathways, showing that silyl radical addition is consistently lower in energy than silyl anion addition.

\begin{tabular}{|l|l|l|}
\hline $\begin{array}{l}\text { All with SMD/THF } \\
\text { solvent modelling }\end{array}$ & $\begin{array}{l}\text { Transition } \\
\text { kcal/mol }\end{array}$ & states in \\
\hline Method & anionic & radical \\
\hline um062x/def2tzvp & 15.3 & 10.2 \\
\hline um061/def2tzvp & 12.5 & 8.7 \\
\hline uwb97xd/lanl2dz & 16.1 & 10.2 \\
\hline ub3lyp/6-311++g(d,p) & 18.2 & 12.4 \\
\hline ub3lyp/lanl2dz & 16.7 & 11.9 \\
\hline
\end{tabular}

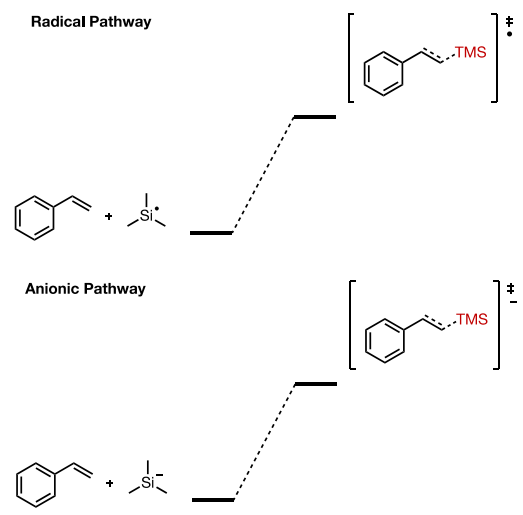




\section{Computational predictions on the energetics of the reaction}

All intermediates were optimized at the ub3lyp/6-311++g(d,p) level of theory with solvent modelling with the SMD (THF) method. It is found that with simple alkenes, the second reduction of the radical intermediate in energetically uphill and likely results in the elimination of TMS anion in the process.

Computational studies of intermediates at $u B 3 L Y P / 6-311++g(d, p) / T H F(S M D)$ level of theory

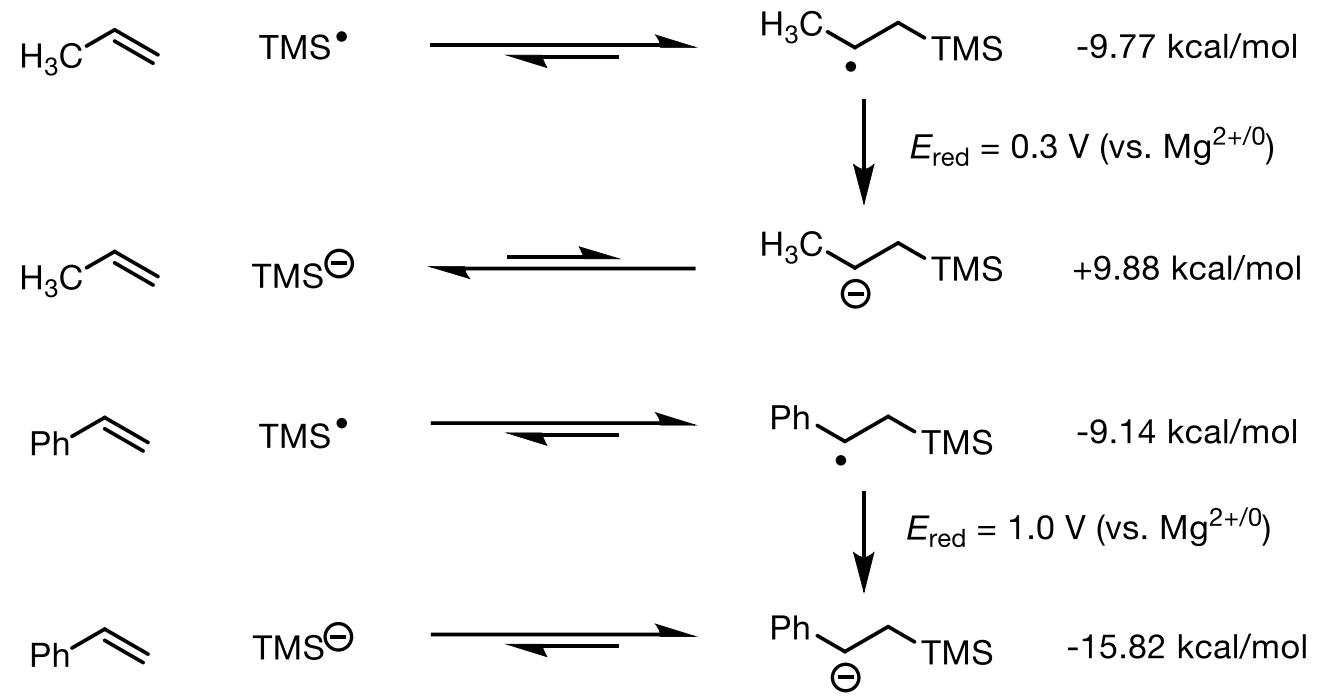

Scheme S21: Computational rationale for the lack of reactivity of simple alkenes. 


\section{X-ray crystal structures}

General information: Low-temperature X-ray diffraction data for (CCDC-1993270) were collected on a Rigaku XtaLAB Synergy diffractometer coupled to a RigakuHypix detector with $\mathrm{Cu} \mathrm{K} \alpha$ radiation $(\lambda=1.54184 \AA)$, from a PhotonJet micro- focus X-ray source at $100 \mathrm{~K}$. The diffraction images were processed and scaled using the CrysAlisPro software ${ }^{40}$. The structures were solved through intrinsic phasing using SHELXT ${ }^{41}$ and refined against F2 on all data by fullmatrix least squares with SHELXL $^{7}$ following established refinement strategies ${ }^{42}$ All nonhydrogen atoms were refined anisotropically. All hydrogen atoms bound to carbon were included in the model at geometrically calculated positions and refined using a riding model. The isotropic displacement parameters of all hydrogen atoms were fixed to 1.2 times the Ueq value of the atoms they are linked to (1.5 times for methyl groups). Details of the data quality and a summary of the residual values of the refinements are listed in Tables 11-12. The crystals were obtained by slow evaporation of the pure compound in hexanes at $-18^{\circ} \mathrm{C}$ over 3 days.

\section{Table S11. Crystal data and structure refinement for Rjes7.}

\begin{tabular}{ll} 
Identification code & rjcs7_abs \\
Empirical formula & $\mathrm{C} 20 \mathrm{H} 28 \mathrm{Si} 3$ \\
Formula weight & 352.69 \\
Temperature & $103(5) \mathrm{K}$ \\
Wavelength & $1.54184 \AA$ \\
Crystal system & Monoclinic \\
Space group & $\mathrm{P} 121 / \mathrm{c} 1$ \\
Unit cell dimensions & $\mathrm{a}=12.0621(2) \AA$ \\
& $\mathrm{b}=11.3639(2) \AA$ \\
& $\mathrm{c}=15.1141(2) \AA$ \\
Volume & $2071.73(6) \AA^{3}$ \\
Z & 4 \\
Density (calculated) & $1.131 \mathrm{Mg} / \mathrm{m}^{3}$ \\
Absorption coefficient & $2.071 \mathrm{~mm}{ }^{-1}$ \\
F(000) & 760 \\
Crystal size & $0.111 \times 0.105 \times 0.00 .006(2)^{\circ}$. \\
Theta range for data collection & 2.924 to $76.975^{\circ}$. \\
Index ranges & $-15<=\mathrm{h}<=14,-13<=\mathrm{k}<=14,-19<=1<=17$ \\
Reflections collected & 15249 \\
Independent reflections & $4148[\mathrm{R}(\mathrm{int})=0.0549]$ \\
Completeness to theta $=67.684^{\circ}$ & $99.9 \%$ \\
Absorption correction & $\mathrm{Gaussian}$ \\
\hline
\end{tabular}


Max. and min. transmission

Refinement method

Data / restraints / parameters

Goodness-of-fit on $\mathrm{F}^{2}$

Final R indices [I>2sigma(I)]

$\mathrm{R}$ indices (all data)

Extinction coefficient
0.982 and 0.858

Full-matrix least-squares on $\mathrm{F}^{2}$

4148 / 0 / 215

1.098

$\mathrm{R} 1=0.0483, \mathrm{wR} 2=0.1300$

$\mathrm{R} 1=0.0520, \mathrm{wR} 2=0.1328$

n/a

Largest diff. peak and hole 0.447 and -0.605 e. $\AA^{-3}$

Table S12. Atomic coordinates $\left(x 1^{4}\right)$ and equivalent isotropic displacement parameters $\left(\AA^{2} \times 1^{3}\right)$

for Rjcs7. U(eq) is defined as one third of the trace of the orthogonalized $\mathrm{U}^{\mathrm{ij}}$ tensor.

\begin{tabular}{|c|c|c|c|c|}
\hline & $\mathrm{x}$ & $\mathrm{y}$ & z & $\mathrm{U}(\mathrm{eq})$ \\
\hline $\operatorname{Si}(1)$ & $7417(1)$ & $3451(1)$ & $6189(1)$ & $23(1)$ \\
\hline $\operatorname{Si}(2)$ & $7388(1)$ & 3911(1) & $7699(1)$ & $23(1)$ \\
\hline $\operatorname{Si}(3)$ & $7562(1)$ & $5924(1)$ & $7429(1)$ & $22(1)$ \\
\hline $\mathrm{C}(1)$ & $8723(2)$ & $2671(2)$ & $5840(2)$ & $30(1)$ \\
\hline$C(2)$ & $6187(2)$ & $2590(3)$ & $5788(2)$ & $31(1)$ \\
\hline $\mathrm{C}(3)$ & $8603(2)$ & $3269(3)$ & $8315(2)$ & $33(1)$ \\
\hline $\mathrm{C}(4)$ & $6041(2)$ & $3539(2)$ & $8263(2)$ & $32(1)$ \\
\hline$C(5)$ & $6393(2)$ & $6849(3)$ & $7861(2)$ & $32(1)$ \\
\hline$C(6)$ & $8902(2)$ & $6562(2)$ & $7828(2)$ & $32(1)$ \\
\hline$C(7)$ & $7520(2)$ & $5961(2)$ & $6164(1)$ & $23(1)$ \\
\hline $\mathrm{C}(8)$ & $7552(2)$ & $7170(2)$ & $5779(1)$ & $23(1)$ \\
\hline $\mathrm{C}(9)$ & $6564(2)$ & $7748(2)$ & $5565(2)$ & $29(1)$ \\
\hline$C(10)$ & $6574(3)$ & $8909(3)$ & $5274(2)$ & $33(1)$ \\
\hline $\mathrm{C}(11)$ & $7565(3)$ & $9512(2)$ & $5190(2)$ & $32(1)$ \\
\hline$C(12)$ & $8546(3)$ & $8945(3)$ & $5400(2)$ & $34(1)$ \\
\hline $\mathrm{C}(13)$ & $8549(2)$ & $7789(2)$ & $5687(2)$ & $30(1)$ \\
\hline$C(14)$ & $7441(2)$ & $4974(2)$ & $5666(1)$ & $22(1)$ \\
\hline$C(15)$ & $7441(2)$ & $5020(2)$ & $4676(1)$ & $26(1)$ \\
\hline$C(16)$ & $6482(3)$ & $4812(3)$ & 4192(2) & $35(1)$ \\
\hline$C(17)$ & $6503(3)$ & $4829(3)$ & $3267(2)$ & $48(1)$ \\
\hline
\end{tabular}




$\begin{array}{lllll}\mathrm{C}(18) & 7491(4) & 5033(2) & 2824(2) & 49(1) \\ \mathrm{C}(19) & 8440(3) & 5227(3) & 3297(2) & 44(1) \\ \mathrm{C}(20) & 8425(3) & 5232(3) & 4217(2) & 34(1)\end{array}$

\section{Table S13. Bond lengths $[\AA \stackrel{\circ}{]}]$ and angles $\left[{ }^{\circ}\right]$ for Rjcs7.}

\begin{tabular}{ll}
\hline $\mathrm{Si}(1)-\mathrm{Si}(2)$ & $2.3416(7)$ \\
$\mathrm{Si}(1)-\mathrm{C}(1)$ & $1.883(3)$ \\
$\mathrm{Si}(1)-\mathrm{C}(2)$ & $1.878(3)$ \\
$\mathrm{Si}(1)-\mathrm{C}(14)$ & $1.903(2)$ \\
$\mathrm{Si}(2)-\mathrm{Si}(3)$ & $2.3335(8)$ \\
$\mathrm{Si}(2)-\mathrm{C}(3)$ & $1.884(3)$ \\
$\mathrm{Si}(2)-\mathrm{C}(4)$ & $1.883(3)$ \\
$\mathrm{Si}(3)-\mathrm{C}(5)$ & $1.876(3)$ \\
$\mathrm{Si}(3)-\mathrm{C}(6)$ & $1.871(3)$ \\
$\mathrm{Si}(3)-\mathrm{C}(7)$ & $1.912(2)$ \\
$\mathrm{C}(1)-\mathrm{H}(1 \mathrm{~A})$ & 0.9800 \\
$\mathrm{C}(1)-\mathrm{H}(1 \mathrm{~B})$ & 0.9800 \\
$\mathrm{C}(1)-\mathrm{H}(1 \mathrm{C})$ & 0.9800 \\
$\mathrm{C}(2)-\mathrm{H}(2 \mathrm{~A})$ & 0.9800 \\
$\mathrm{C}(2)-\mathrm{H}(2 \mathrm{~B})$ & 0.9800 \\
$\mathrm{C}(2)-\mathrm{H}(2 \mathrm{C})$ & 0.9800 \\
$\mathrm{C}(3)-\mathrm{H}(3 \mathrm{~A})$ & 0.9800 \\
$\mathrm{C}(3)-\mathrm{H}(3 \mathrm{~B})$ & 0.9800 \\
$\mathrm{C}(3)-\mathrm{H}(3 \mathrm{C})$ & 0.9800 \\
$\mathrm{C}(4)-\mathrm{H}(4 \mathrm{~A})$ & 0.9800 \\
$\mathrm{C}(4)-\mathrm{H}(4 \mathrm{~B})$ & 0.9800 \\
$\mathrm{C}(4)-\mathrm{H}(4 \mathrm{C})$ & 0.9800 \\
$\mathrm{C}(5)-\mathrm{H}(5 \mathrm{~A})$ & 0.9800 \\
$\mathrm{C}(5)-\mathrm{H}(5 \mathrm{~B})$ & 0.9800 \\
$\mathrm{C}(5)-\mathrm{H}(5 \mathrm{C})$ & 0.9800 \\
$\mathrm{C}(6)-\mathrm{H}(6 \mathrm{~A})$ & 0.9800 \\
$\mathrm{C}(6)-\mathrm{H}(6 \mathrm{~B})$ & 0.9800 \\
$\mathrm{C}(6)-\mathrm{H}(6 \mathrm{C})$ & \\
$\mathrm{C}(7)-\mathrm{C}(8)$ & \\
& \\
& \\
&
\end{tabular}




\begin{tabular}{|c|c|}
\hline$C(7)-C(14)$ & $1.355(3)$ \\
\hline $\mathrm{C}(8)-\mathrm{C}(9)$ & $1.399(4)$ \\
\hline$C(8)-C(13)$ & $1.400(4)$ \\
\hline $\mathrm{C}(9)-\mathrm{H}(9)$ & 0.9500 \\
\hline$C(9)-C(10)$ & $1.391(4)$ \\
\hline $\mathrm{C}(10)-\mathrm{H}(10)$ & 0.9500 \\
\hline$C(10)-C(11)$ & $1.384(4)$ \\
\hline $\mathrm{C}(11)-\mathrm{H}(11)$ & 0.9500 \\
\hline$C(11)-C(12)$ & $1.384(4)$ \\
\hline $\mathrm{C}(12)-\mathrm{H}(12)$ & 0.9500 \\
\hline$C(12)-C(13)$ & $1.383(4)$ \\
\hline $\mathrm{C}(13)-\mathrm{H}(13)$ & 0.9500 \\
\hline$C(14)-C(15)$ & $1.496(3)$ \\
\hline$C(15)-C(16)$ & $1.390(4)$ \\
\hline$C(15)-C(20)$ & $1.396(4)$ \\
\hline $\mathrm{C}(16)-\mathrm{H}(16)$ & 0.9500 \\
\hline$C(16)-C(17)$ & $1.399(4)$ \\
\hline $\mathrm{C}(17)-\mathrm{H}(17)$ & 0.9500 \\
\hline $\mathrm{C}(17)-\mathrm{C}(18)$ & $1.386(6)$ \\
\hline $\mathrm{C}(18)-\mathrm{H}(18)$ & 0.9500 \\
\hline $\mathrm{C}(18)-\mathrm{C}(19)$ & $1.368(6)$ \\
\hline $\mathrm{C}(19)-\mathrm{H}(19)$ & 0.9500 \\
\hline$C(19)-C(20)$ & $1.392(4)$ \\
\hline $\mathrm{C}(20)-\mathrm{H}(20)$ & 0.9500 \\
\hline $\mathrm{C}(1)-\mathrm{Si}(1)-\mathrm{Si}(2)$ & 112.98(9) \\
\hline$C(1)-S i(1)-C(14)$ & $107.38(12)$ \\
\hline $\mathrm{C}(2)-\mathrm{Si}(1)-\mathrm{Si}(2)$ & 114.79(9) \\
\hline $\mathrm{C}(2)-\mathrm{Si}(1)-\mathrm{C}(1)$ & $108.99(11)$ \\
\hline$C(2)-S i(1)-C(14)$ & $110.60(12)$ \\
\hline$C(14)-\operatorname{Si}(1)-\operatorname{Si}(2)$ & $101.67(7)$ \\
\hline $\operatorname{Si}(3)-\operatorname{Si}(2)-\operatorname{Si}(1)$ & $92.70(3)$ \\
\hline $\mathrm{C}(3)-\mathrm{Si}(2)-\mathrm{Si}(1)$ & $112.56(9)$ \\
\hline $\mathrm{C}(3)-\mathrm{Si}(2)-\mathrm{Si}(3)$ & $113.38(10)$ \\
\hline $\mathrm{C}(4)-\mathrm{Si}(2)-\mathrm{Si}(1)$ & $113.82(9)$ \\
\hline $\mathrm{C}(4)-\mathrm{Si}(2)-\mathrm{Si}(3)$ & 112.13(9) \\
\hline
\end{tabular}




\begin{tabular}{|c|c|}
\hline $\mathrm{C}(4)-\mathrm{Si}(2)-\mathrm{C}(3)$ & $111.14(13)$ \\
\hline $\mathrm{C}(5)-\mathrm{Si}(3)-\operatorname{Si}(2)$ & $114.85(9)$ \\
\hline$C(5)-S i(3)-C(7)$ & $108.44(11)$ \\
\hline $\mathrm{C}(6)-\mathrm{Si}(3)-\mathrm{Si}(2)$ & $113.63(9)$ \\
\hline$C(6)-\operatorname{Si}(3)-C(5)$ & $108.67(13)$ \\
\hline $\mathrm{C}(6)-\mathrm{Si}(3)-\mathrm{C}(7)$ & $109.66(12)$ \\
\hline $\mathrm{C}(7)-\mathrm{Si}(3)-\mathrm{Si}(2)$ & $101.20(7)$ \\
\hline $\mathrm{Si}(1)-\mathrm{C}(1)-\mathrm{H}(1 \mathrm{~A})$ & 109.5 \\
\hline $\mathrm{Si}(1)-\mathrm{C}(1)-\mathrm{H}(1 \mathrm{~B})$ & 109.5 \\
\hline $\mathrm{Si}(1)-\mathrm{C}(1)-\mathrm{H}(1 \mathrm{C})$ & 109.5 \\
\hline $\mathrm{H}(1 \mathrm{~A})-\mathrm{C}(1)-\mathrm{H}(1 \mathrm{~B})$ & 109.5 \\
\hline $\mathrm{H}(1 \mathrm{~A})-\mathrm{C}(1)-\mathrm{H}(1 \mathrm{C})$ & 109.5 \\
\hline $\mathrm{H}(1 \mathrm{~B})-\mathrm{C}(1)-\mathrm{H}(1 \mathrm{C})$ & 109.5 \\
\hline $\mathrm{Si}(1)-\mathrm{C}(2)-\mathrm{H}(2 \mathrm{~A})$ & 109.5 \\
\hline $\mathrm{Si}(1)-\mathrm{C}(2)-\mathrm{H}(2 \mathrm{~B})$ & 109.5 \\
\hline $\mathrm{Si}(1)-\mathrm{C}(2)-\mathrm{H}(2 \mathrm{C})$ & 109.5 \\
\hline $\mathrm{H}(2 \mathrm{~A})-\mathrm{C}(2)-\mathrm{H}(2 \mathrm{~B})$ & 109.5 \\
\hline $\mathrm{H}(2 \mathrm{~A})-\mathrm{C}(2)-\mathrm{H}(2 \mathrm{C})$ & 109.5 \\
\hline $\mathrm{H}(2 \mathrm{~B})-\mathrm{C}(2)-\mathrm{H}(2 \mathrm{C})$ & 109.5 \\
\hline $\mathrm{Si}(2)-\mathrm{C}(3)-\mathrm{H}(3 \mathrm{~A})$ & 109.5 \\
\hline $\mathrm{Si}(2)-\mathrm{C}(3)-\mathrm{H}(3 \mathrm{~B})$ & 109.5 \\
\hline $\mathrm{Si}(2)-\mathrm{C}(3)-\mathrm{H}(3 \mathrm{C})$ & 109.5 \\
\hline $\mathrm{H}(3 \mathrm{~A})-\mathrm{C}(3)-\mathrm{H}(3 \mathrm{~B})$ & 109.5 \\
\hline $\mathrm{H}(3 \mathrm{~A})-\mathrm{C}(3)-\mathrm{H}(3 \mathrm{C})$ & 109.5 \\
\hline $\mathrm{H}(3 \mathrm{~B})-\mathrm{C}(3)-\mathrm{H}(3 \mathrm{C})$ & 109.5 \\
\hline $\mathrm{Si}(2)-\mathrm{C}(4)-\mathrm{H}(4 \mathrm{~A})$ & 109.5 \\
\hline $\mathrm{Si}(2)-\mathrm{C}(4)-\mathrm{H}(4 \mathrm{~B})$ & 109.5 \\
\hline $\mathrm{Si}(2)-\mathrm{C}(4)-\mathrm{H}(4 \mathrm{C})$ & 109.5 \\
\hline $\mathrm{H}(4 \mathrm{~A})-\mathrm{C}(4)-\mathrm{H}(4 \mathrm{~B})$ & 109.5 \\
\hline $\mathrm{H}(4 \mathrm{~A})-\mathrm{C}(4)-\mathrm{H}(4 \mathrm{C})$ & 109.5 \\
\hline $\mathrm{H}(4 \mathrm{~B})-\mathrm{C}(4)-\mathrm{H}(4 \mathrm{C})$ & 109.5 \\
\hline $\mathrm{Si}(3)-\mathrm{C}(5)-\mathrm{H}(5 \mathrm{~A})$ & 109.5 \\
\hline $\mathrm{Si}(3)-\mathrm{C}(5)-\mathrm{H}(5 \mathrm{~B})$ & 109.5 \\
\hline $\mathrm{Si}(3)-\mathrm{C}(5)-\mathrm{H}(5 \mathrm{C})$ & 109.5 \\
\hline $\mathrm{H}(5 \mathrm{~A})-\mathrm{C}(5)-\mathrm{H}(5 \mathrm{~B})$ & 109.5 \\
\hline $\mathrm{H}(5 \mathrm{~A})-\mathrm{C}(5)-\mathrm{H}(5 \mathrm{C})$ & 109.5 \\
\hline
\end{tabular}




\begin{tabular}{|c|c|}
\hline $\mathrm{H}(5 \mathrm{~B})-\mathrm{C}(5)-\mathrm{H}(5 \mathrm{C})$ & 109.5 \\
\hline $\mathrm{Si}(3)-\mathrm{C}(6)-\mathrm{H}(6 \mathrm{~A})$ & 109.5 \\
\hline $\mathrm{Si}(3)-\mathrm{C}(6)-\mathrm{H}(6 \mathrm{~B})$ & 109.5 \\
\hline $\mathrm{Si}(3)-\mathrm{C}(6)-\mathrm{H}(6 \mathrm{C})$ & 109.5 \\
\hline $\mathrm{H}(6 \mathrm{~A})-\mathrm{C}(6)-\mathrm{H}(6 \mathrm{~B})$ & 109.5 \\
\hline $\mathrm{H}(6 \mathrm{~A})-\mathrm{C}(6)-\mathrm{H}(6 \mathrm{C})$ & 109.5 \\
\hline $\mathrm{H}(6 \mathrm{~B})-\mathrm{C}(6)-\mathrm{H}(6 \mathrm{C})$ & 109.5 \\
\hline $\mathrm{C}(8)-\mathrm{C}(7)-\mathrm{Si}(3)$ & $114.18(14)$ \\
\hline$C(14)-C(7)-\operatorname{Si}(3)$ & $122.65(16)$ \\
\hline$C(14)-C(7)-C(8)$ & $123.15(18)$ \\
\hline $\mathrm{C}(9)-\mathrm{C}(8)-\mathrm{C}(7)$ & $120.0(2)$ \\
\hline $\mathrm{C}(9)-\mathrm{C}(8)-\mathrm{C}(13)$ & $118.2(2)$ \\
\hline$C(13)-C(8)-C(7)$ & $121.6(2)$ \\
\hline $\mathrm{C}(8)-\mathrm{C}(9)-\mathrm{H}(9)$ & 119.6 \\
\hline$C(10)-C(9)-C(8)$ & $120.8(2)$ \\
\hline $\mathrm{C}(10)-\mathrm{C}(9)-\mathrm{H}(9)$ & 119.6 \\
\hline $\mathrm{C}(9)-\mathrm{C}(10)-\mathrm{H}(10)$ & 119.8 \\
\hline $\mathrm{C}(11)-\mathrm{C}(10)-\mathrm{C}(9)$ & $120.4(3)$ \\
\hline $\mathrm{C}(11)-\mathrm{C}(10)-\mathrm{H}(10)$ & 119.8 \\
\hline $\mathrm{C}(10)-\mathrm{C}(11)-\mathrm{H}(11)$ & 120.4 \\
\hline $\mathrm{C}(10)-\mathrm{C}(11)-\mathrm{C}(12)$ & 119.1(2) \\
\hline $\mathrm{C}(12)-\mathrm{C}(11)-\mathrm{H}(11)$ & 120.4 \\
\hline $\mathrm{C}(11)-\mathrm{C}(12)-\mathrm{H}(12)$ & 119.5 \\
\hline$C(13)-C(12)-C(11)$ & $121.1(3)$ \\
\hline $\mathrm{C}(13)-\mathrm{C}(12)-\mathrm{H}(12)$ & 119.5 \\
\hline $\mathrm{C}(8)-\mathrm{C}(13)-\mathrm{H}(13)$ & 119.8 \\
\hline$C(12)-C(13)-C(8)$ & $120.5(3)$ \\
\hline $\mathrm{C}(12)-\mathrm{C}(13)-\mathrm{H}(13)$ & 119.8 \\
\hline $\mathrm{C}(7)-\mathrm{C}(14)-\mathrm{Si}(1)$ & $121.53(15)$ \\
\hline$C(7)-C(14)-C(15)$ & $121.79(19)$ \\
\hline $\mathrm{C}(15)-\mathrm{C}(14)-\mathrm{Si}(1)$ & $116.57(15)$ \\
\hline$C(16)-C(15)-C(14)$ & $121.3(2)$ \\
\hline $\mathrm{C}(16)-\mathrm{C}(15)-\mathrm{C}(20)$ & $118.4(2)$ \\
\hline $\mathrm{C}(20)-\mathrm{C}(15)-\mathrm{C}(14)$ & $120.2(2)$ \\
\hline $\mathrm{C}(15)-\mathrm{C}(16)-\mathrm{H}(16)$ & 119.7 \\
\hline$C(15)-C(16)-C(17)$ & $120.6(3)$ \\
\hline
\end{tabular}




$\begin{array}{ll}\mathrm{C}(17)-\mathrm{C}(16)-\mathrm{H}(16) & 119.7 \\ \mathrm{C}(16)-\mathrm{C}(17)-\mathrm{H}(17) & 119.9 \\ \mathrm{C}(18)-\mathrm{C}(17)-\mathrm{C}(16) & 120.1(3) \\ \mathrm{C}(18)-\mathrm{C}(17)-\mathrm{H}(17) & 119.9 \\ \mathrm{C}(17)-\mathrm{C}(18)-\mathrm{H}(18) & 120.2 \\ \mathrm{C}(19)-\mathrm{C}(18)-\mathrm{C}(17) & 119.6(2) \\ \mathrm{C}(19)-\mathrm{C}(18)-\mathrm{H}(18) & 120.2 \\ \mathrm{C}(18)-\mathrm{C}(19)-\mathrm{H}(19) & 119.6 \\ \mathrm{C}(18)-\mathrm{C}(19)-\mathrm{C}(20) & 120.8(3) \\ \mathrm{C}(20)-\mathrm{C}(19)-\mathrm{H}(19) & 119.6 \\ \mathrm{C}(15)-\mathrm{C}(20)-\mathrm{H}(20) & 119.8 \\ \mathrm{C}(19)-\mathrm{C}(20)-\mathrm{C}(15) & 120.5(3) \\ \mathrm{C}(19)-\mathrm{C}(20)-\mathrm{H}(20) & 119.8\end{array}$

Symmetry transformations used to generate equivalent atoms:

Table S14.Anisotropic displacement parameters $\left(\AA^{2} \times 1^{3}\right)$ for Rjes7.

The anisotropic displacement factor exponent takes the form: $-2 p^{2}\left[h^{2} a^{* 2} U^{11}+\ldots+2 h k a^{*} b^{*} U^{12}\right]$

\begin{tabular}{lcccccc}
\hline & $\mathrm{U}^{11}$ & $\mathrm{U}^{22}$ & $\mathrm{U}^{33}$ & $\mathrm{U}^{23}$ & $\mathrm{U}^{13}$ & $\mathrm{U}^{12}$ \\
\hline $\mathrm{Si}(1)$ & $28(1)$ & $21(1)$ & $18(1)$ & $0(1)$ & $2(1)$ & $0(1)$ \\
$\mathrm{Si}(2)$ & $29(1)$ & $22(1)$ & $18(1)$ & $0(1)$ & $1(1)$ & $1(1)$ \\
$\mathrm{Si}(3)$ & $26(1)$ & $22(1)$ & $17(1)$ & $0(1)$ & $2(1)$ & $0(1)$ \\
$\mathrm{C}(1)$ & $39(1)$ & $28(1)$ & $24(1)$ & $-3(1)$ & $2(1)$ & $6(1)$ \\
$\mathrm{C}(2)$ & $35(1)$ & $30(1)$ & $30(1)$ & $1(1)$ & $1(1)$ & $-8(1)$ \\
$\mathrm{C}(3)$ & $40(2)$ & $34(1)$ & $26(1)$ & $-2(1)$ & $-1(1)$ & $8(1)$ \\
$\mathrm{C}(4)$ & $39(1)$ & $32(1)$ & $27(1)$ & $3(1)$ & $4(1)$ & $-5(1)$ \\
$\mathrm{C}(5)$ & $37(1)$ & $33(1)$ & $27(1)$ & $-1(1)$ & $6(1)$ & $5(1)$ \\
$\mathrm{C}(6)$ & $33(1)$ & $34(1)$ & $28(1)$ & $-1(1)$ & $-1(1)$ & $-6(1)$ \\
$\mathrm{C}(7)$ & $23(1)$ & $25(1)$ & $19(1)$ & $1(1)$ & $1(1)$ & $0(1)$ \\
$\mathrm{C}(8)$ & $30(1)$ & $24(1)$ & $15(1)$ & $-1(1)$ & $4(1)$ & $-1(1)$ \\
$\mathrm{C}(9)$ & $29(1)$ & $30(1)$ & $28(1)$ & $4(1)$ & $-1(1)$ & $0(1)$ \\
$\mathrm{C}(10)$ & $40(2)$ & $30(1)$ & $30(1)$ & $6(1)$ & $4(1)$ & $6(1)$ \\
$\mathrm{C}(11)$ & $50(2)$ & $23(1)$ & $23(1)$ & $3(1)$ & $5(1)$ & $-1(1)$ \\
& & & & $\mathrm{S} 80$ & & \\
& & & & & &
\end{tabular}




$\begin{array}{lllllll}\mathrm{C}(12) & 39(2) & 31(1) & 33(1) & 4(1) & 5(1) & -9(1) \\ \mathrm{C}(13) & 31(1) & 31(1) & 29(1) & 5(1) & 4(1) & -3(1) \\ \mathrm{C}(14) & 23(1) & 25(1) & 19(1) & -1(1) & 2(1) & 0(1) \\ \mathrm{C}(15) & 35(1) & 21(1) & 20(1) & 0(1) & 2(1) & 1(1) \\ \mathrm{C}(16) & 47(2) & 30(1) & 27(1) & 2(1) & -6(1) & -1(1) \\ \mathrm{C}(17) & 80(2) & 35(2) & 28(1) & 2(1) & -21(2) & 0(2) \\ \mathrm{C}(18) & 100(3) & 28(1) & 18(1) & 0(1) & 8(2) & 2(2) \\ \mathrm{C}(19) & 74(2) & 30(1) & 28(1) & 5(1) & 21(1) & 6(1) \\ \mathrm{C}(20) & 45(2) & 31(1) & 26(1) & 2(1) & 8(1) & -2(1)\end{array}$

Table S15. Hydrogen coordinates $\left(\times 1^{4}\right)$ and isotropic displacement parameters $\left(\AA^{2} \times 10^{3}\right)$ for Rjes7.

\begin{tabular}{|c|c|c|c|c|}
\hline & $\mathrm{x}$ & $\mathrm{y}$ & $\mathrm{z}$ & $\mathrm{U}(\mathrm{eq})$ \\
\hline $\mathrm{H}(1 \mathrm{~A})$ & 9369 & 3105 & 6059 & 45 \\
\hline $\mathrm{H}(1 \mathrm{~B})$ & 8753 & 2628 & 5193 & 45 \\
\hline $\mathrm{H}(1 \mathrm{C})$ & 8727 & 1873 & 6087 & 45 \\
\hline $\mathrm{H}(2 \mathrm{~A})$ & 6182 & 1814 & 6071 & 47 \\
\hline $\mathrm{H}(2 \mathrm{~B})$ & 6232 & 2495 & 5145 & 47 \\
\hline $\mathrm{H}(2 \mathrm{C})$ & 5504 & 3012 & 5940 & 47 \\
\hline $\mathrm{H}(3 \mathrm{~A})$ & 8666 & 3645 & 8896 & 50 \\
\hline $\mathrm{H}(3 \mathrm{~B})$ & 9285 & 3406 & 7977 & 50 \\
\hline $\mathrm{H}(3 \mathrm{C})$ & 8492 & 2421 & 8392 & 50 \\
\hline $\mathrm{H}(4 \mathrm{~A})$ & 5981 & 2684 & 8330 & 49 \\
\hline $\mathrm{H}(4 \mathrm{~B})$ & 5421 & 3829 & 7906 & 49 \\
\hline $\mathrm{H}(4 \mathrm{C})$ & 6021 & 3911 & 8848 & 49 \\
\hline $\mathrm{H}(5 \mathrm{~A})$ & 5684 & 6488 & 7696 & 48 \\
\hline $\mathrm{H}(5 \mathrm{~B})$ & 6439 & 7640 & 7606 & 48 \\
\hline $\mathrm{H}(5 \mathrm{C})$ & 6444 & 6900 & 8507 & 48 \\
\hline $\mathrm{H}(6 \mathrm{~A})$ & 8929 & 6523 & 8475 & 48 \\
\hline $\mathrm{H}(6 \mathrm{~B})$ & 8957 & 7385 & 7638 & 48 \\
\hline $\mathrm{H}(6 \mathrm{C})$ & 9521 & 6113 & 7579 & 48 \\
\hline $\mathrm{H}(9)$ & 5879 & 7343 & 5619 & 35 \\
\hline
\end{tabular}




$\begin{array}{lrrrr}\mathrm{H}(10) & 5896 & 9291 & 5132 & 40 \\ \mathrm{H}(11) & 7573 & 10304 & 4990 & 39 \\ \mathrm{H}(12) & 9229 & 9356 & 5347 & 41 \\ \mathrm{H}(13) & 9231 & 7413 & 5823 & 36 \\ \mathrm{H}(16) & 5805 & 4658 & 4492 & 42 \\ \mathrm{H}(17) & 5840 & 4700 & 2942 & 57 \\ \mathrm{H}(18) & 7508 & 5039 & 2195 & 58 \\ \mathrm{H}(19) & 9118 & 5359 & 2993 & 53 \\ \mathrm{H}(20) & 9090 & 5381 & 4536 & 41\end{array}$

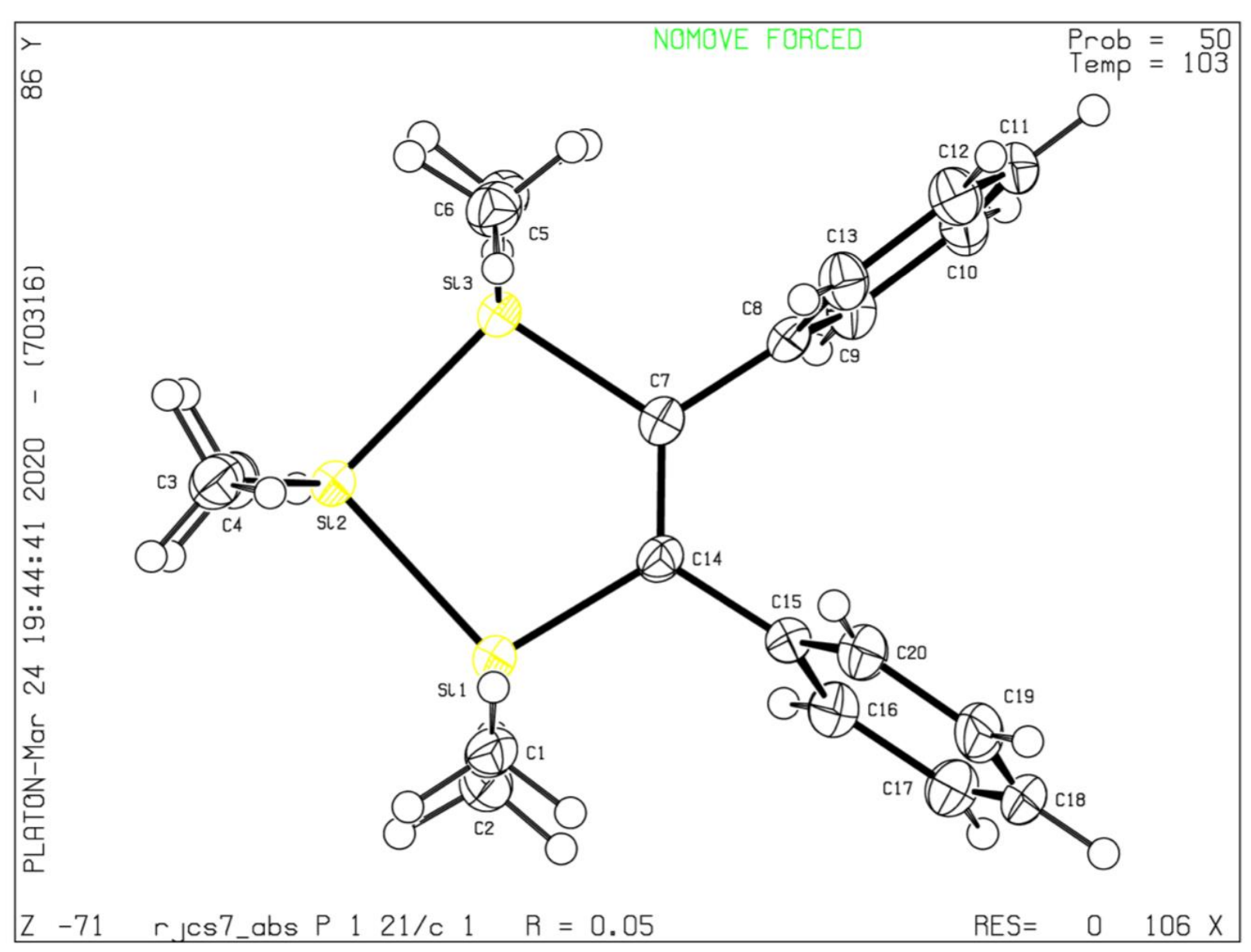




\section{NMR spectra}<smiles>CC(C)C(C)Cc1ccccc1</smiles>

(2)
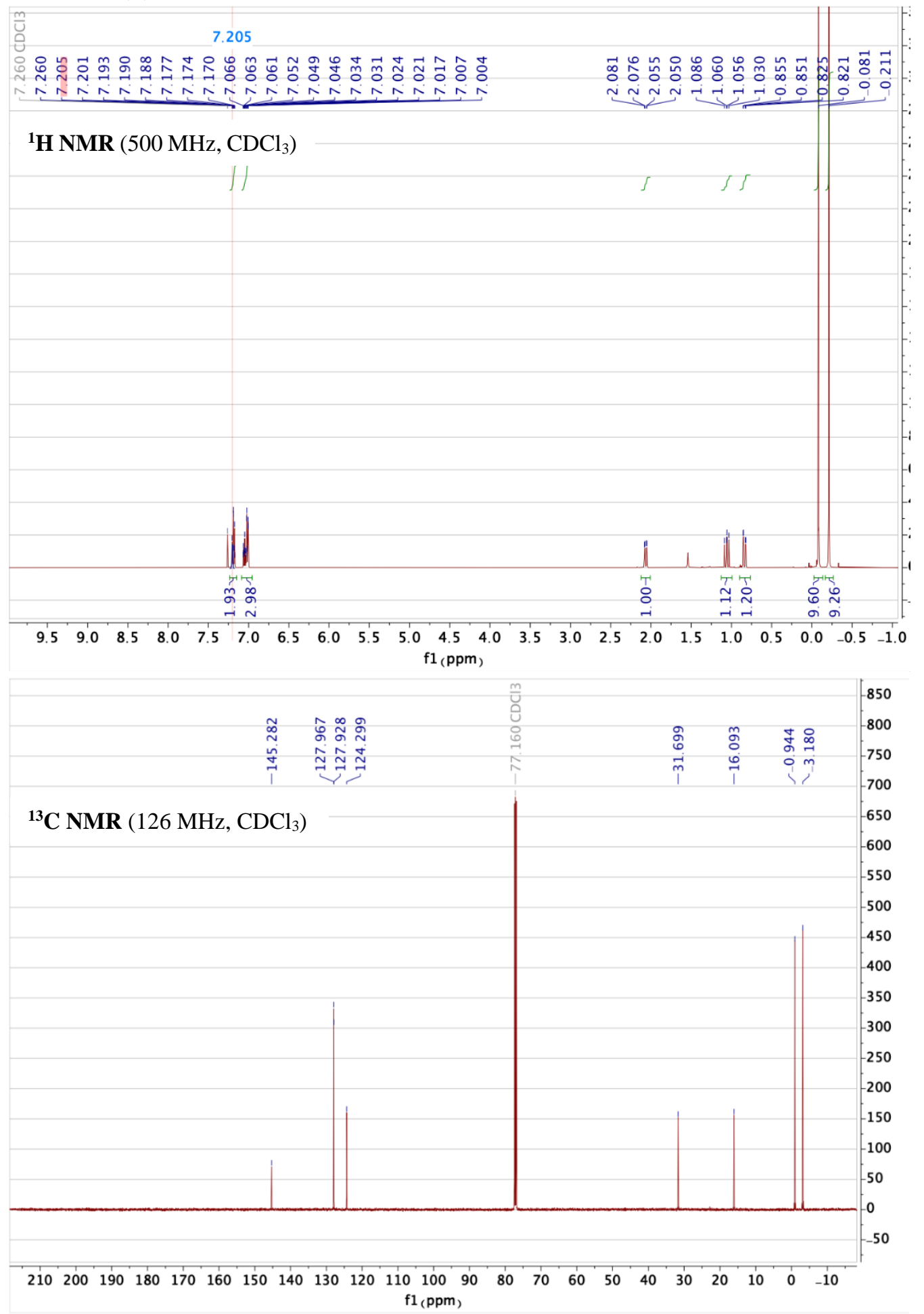
<smiles>CCC(C)c1ccc(F)cc1</smiles>
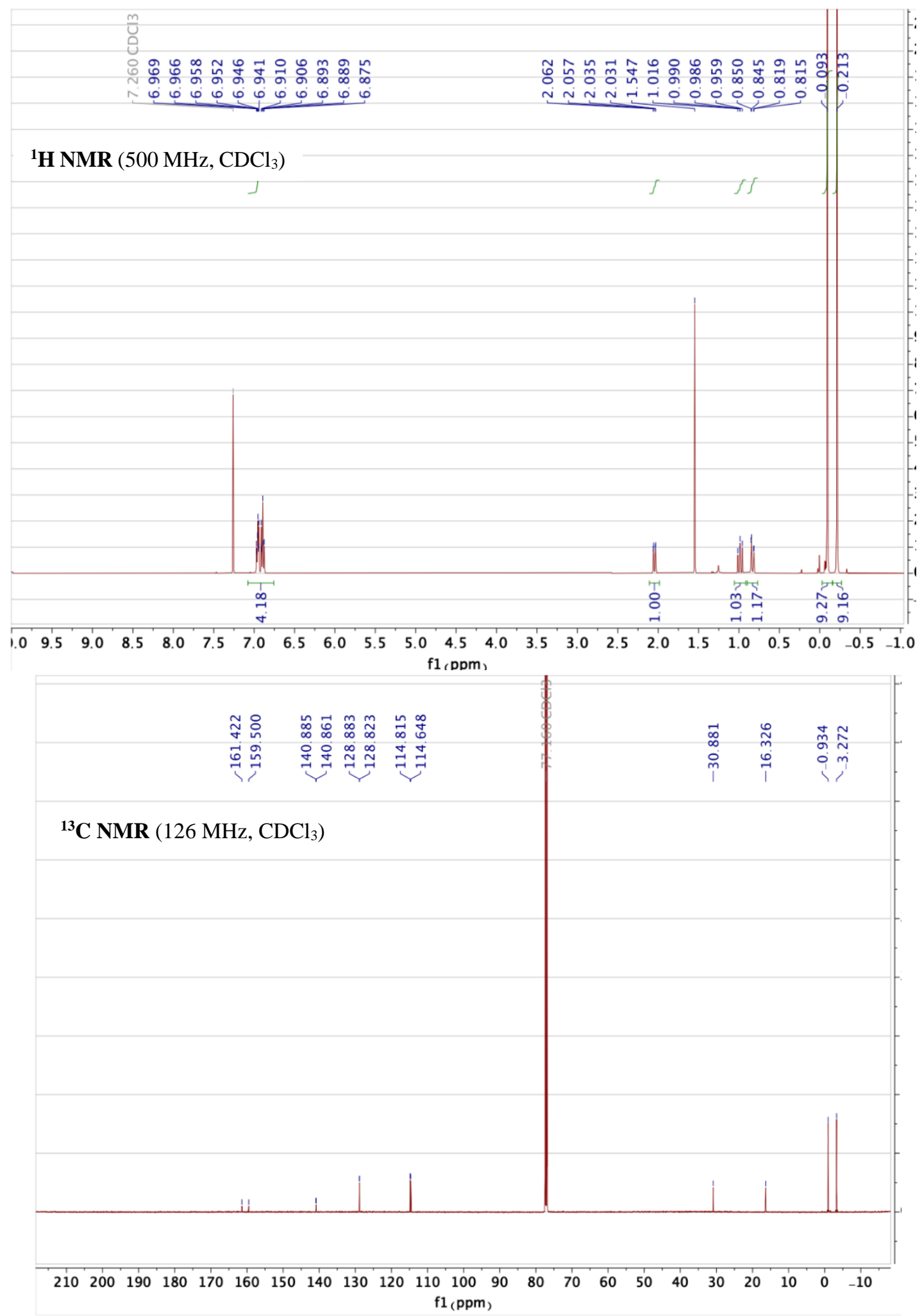


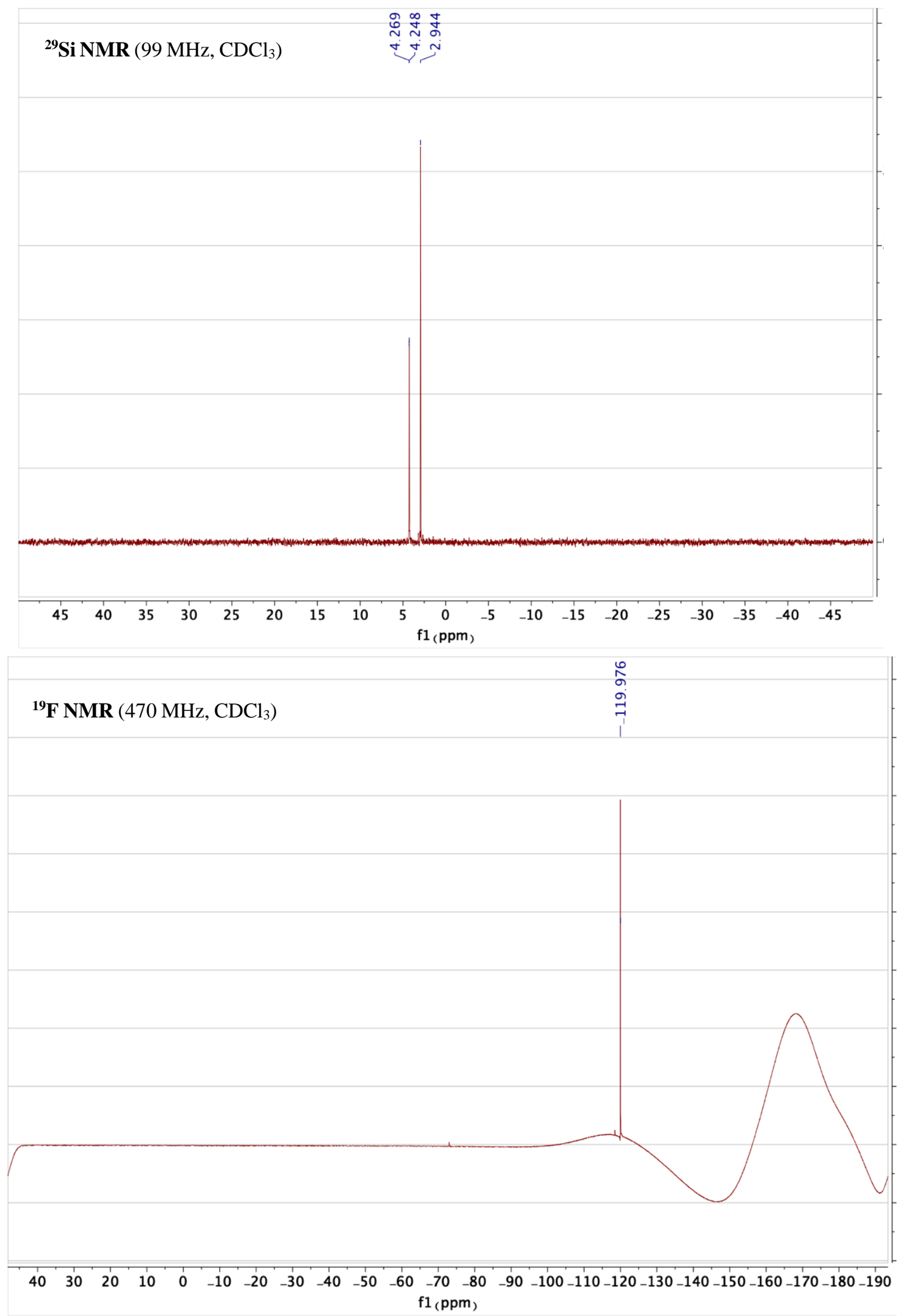


<smiles>CC(C)(C)CC(CCS(C)(=O)=O)c1ccc(Cc2ccccc2)cc1</smiles>
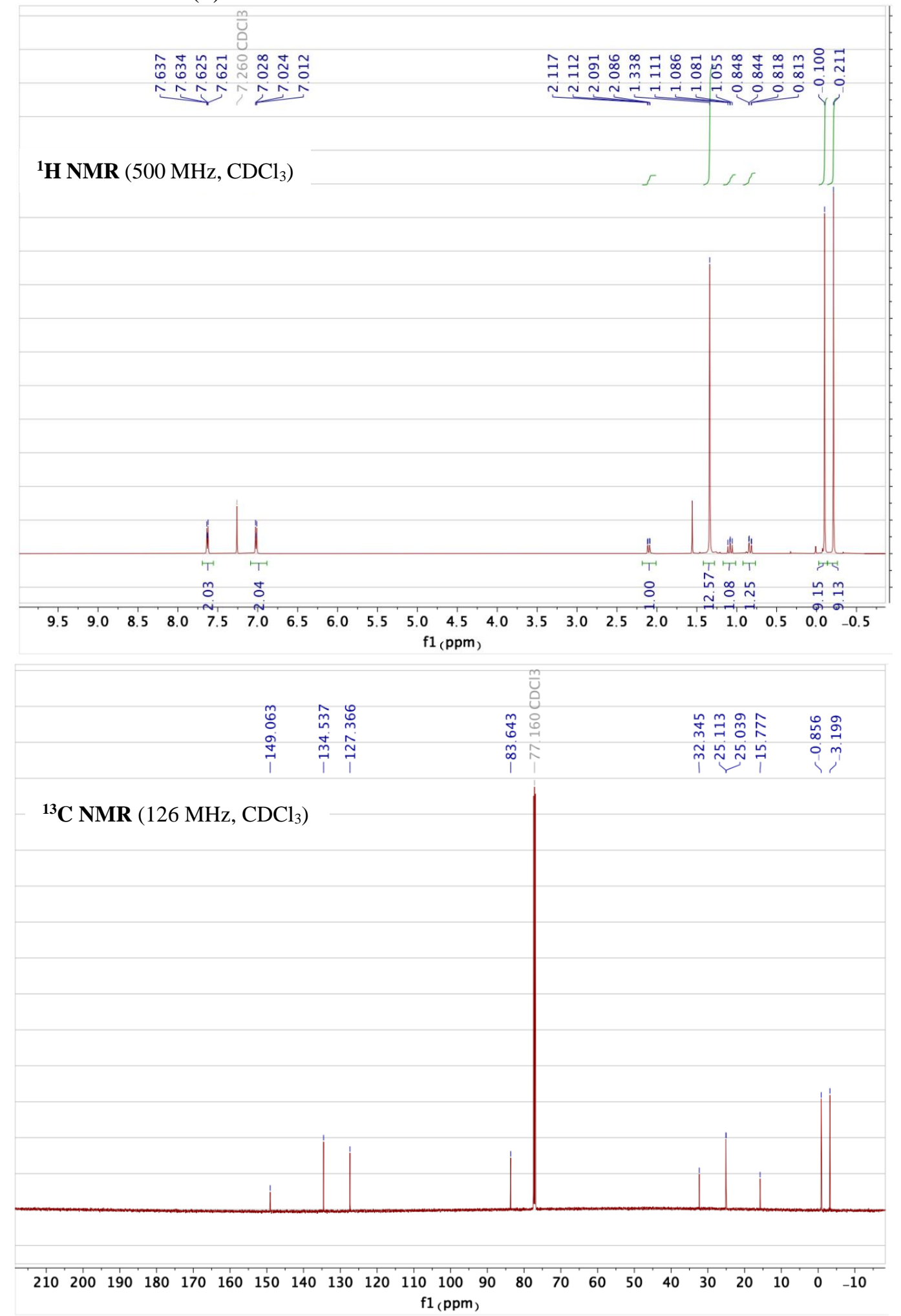
<smiles>CN(C)c1ccc(C(C[As])C(C)(C)C)cc1</smiles>
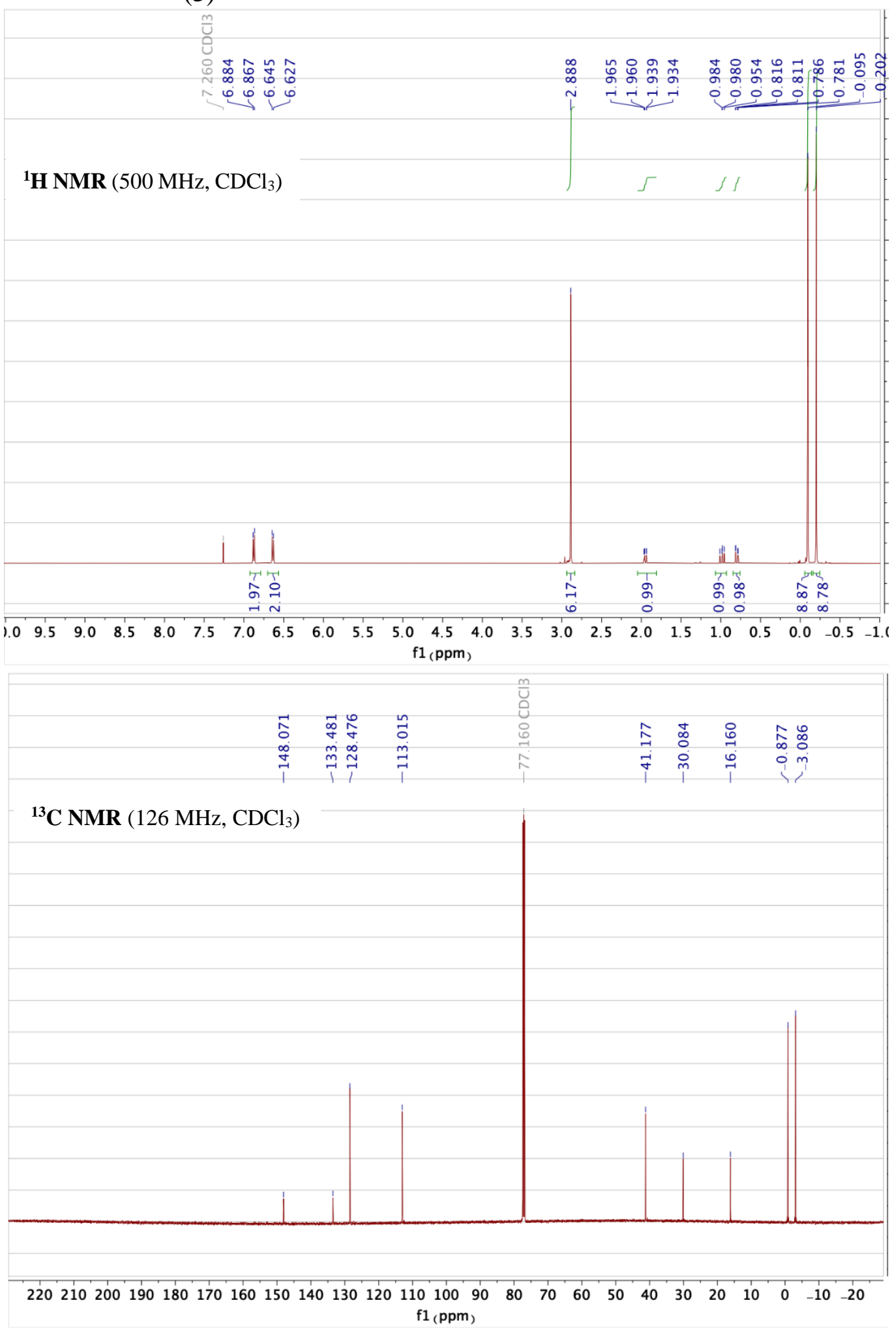

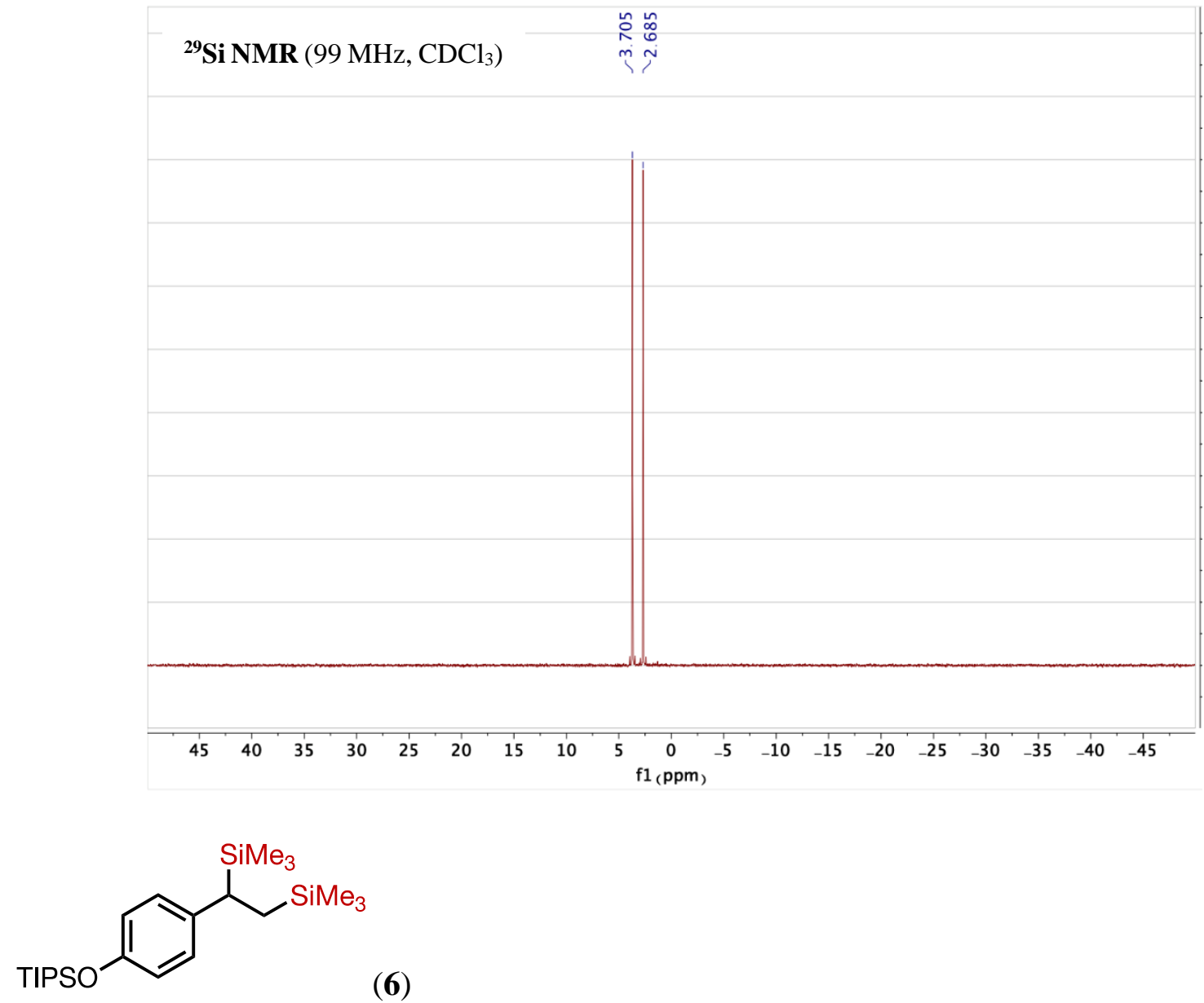

(6)

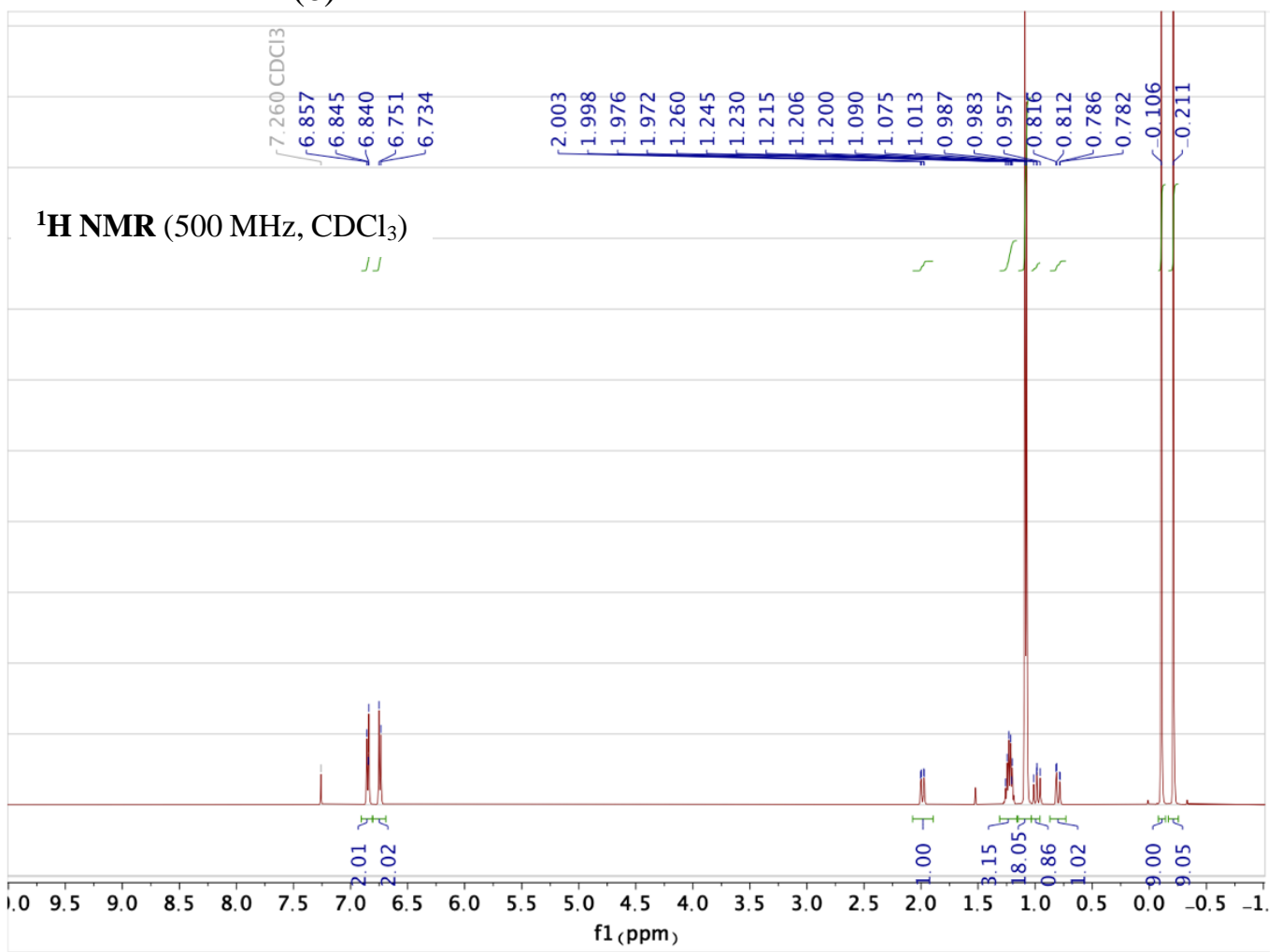




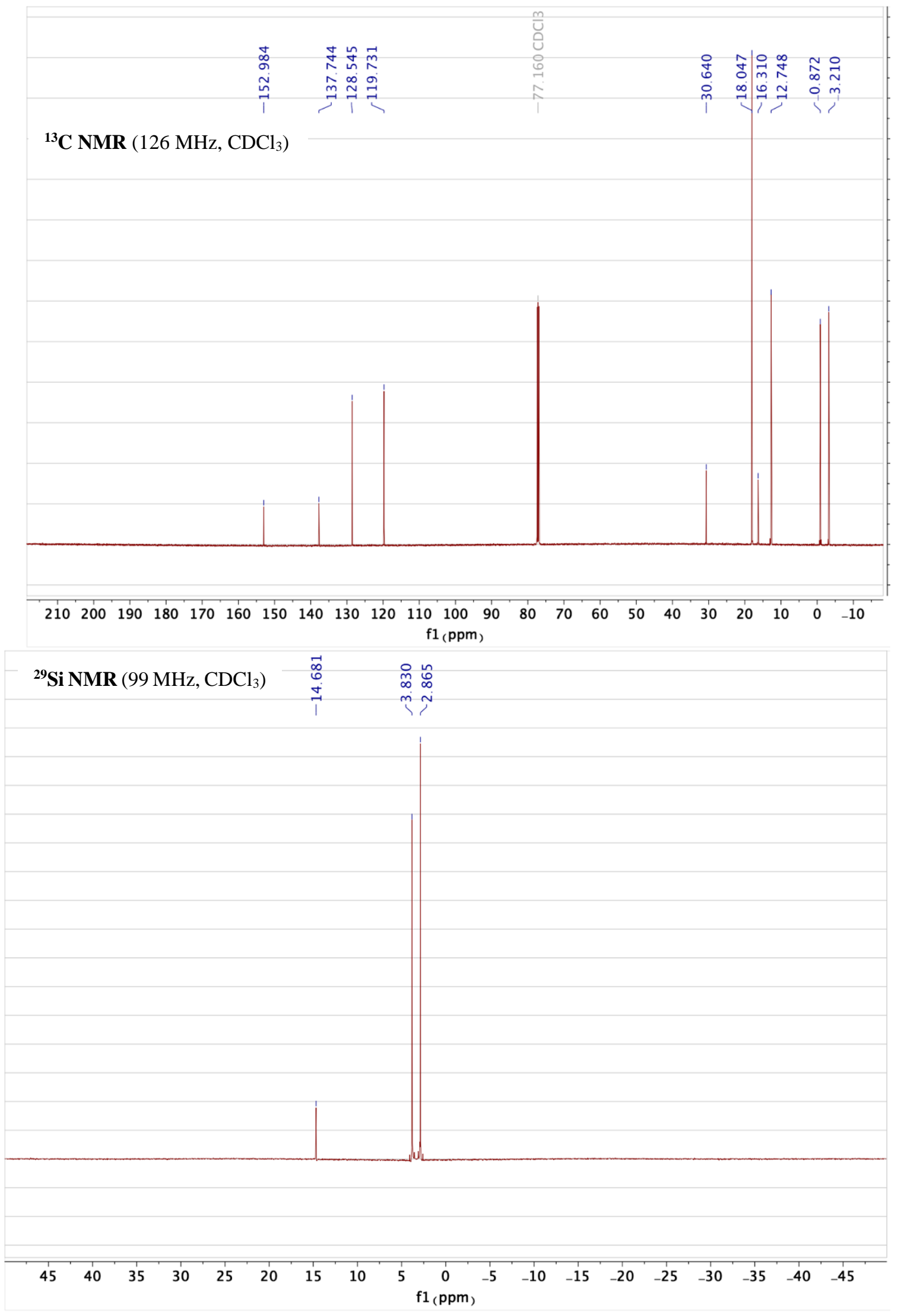


<smiles>CSc1ccc(C(C[AsH2])[SiH2]C(C)C)cc1</smiles>
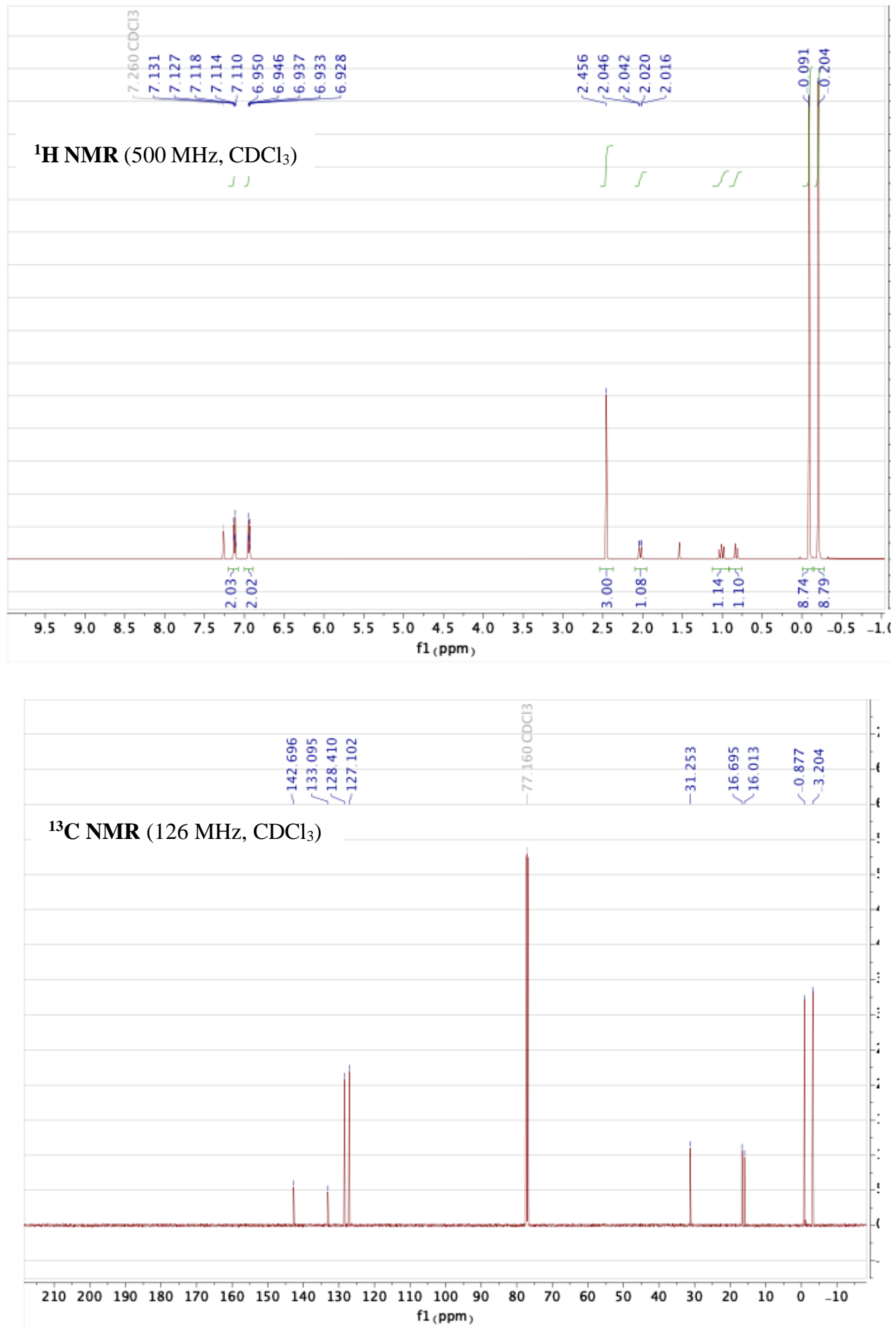
<smiles>CCC(CCS(C)(=O)=O)c1ccc(CCO)cc1</smiles>

(8)

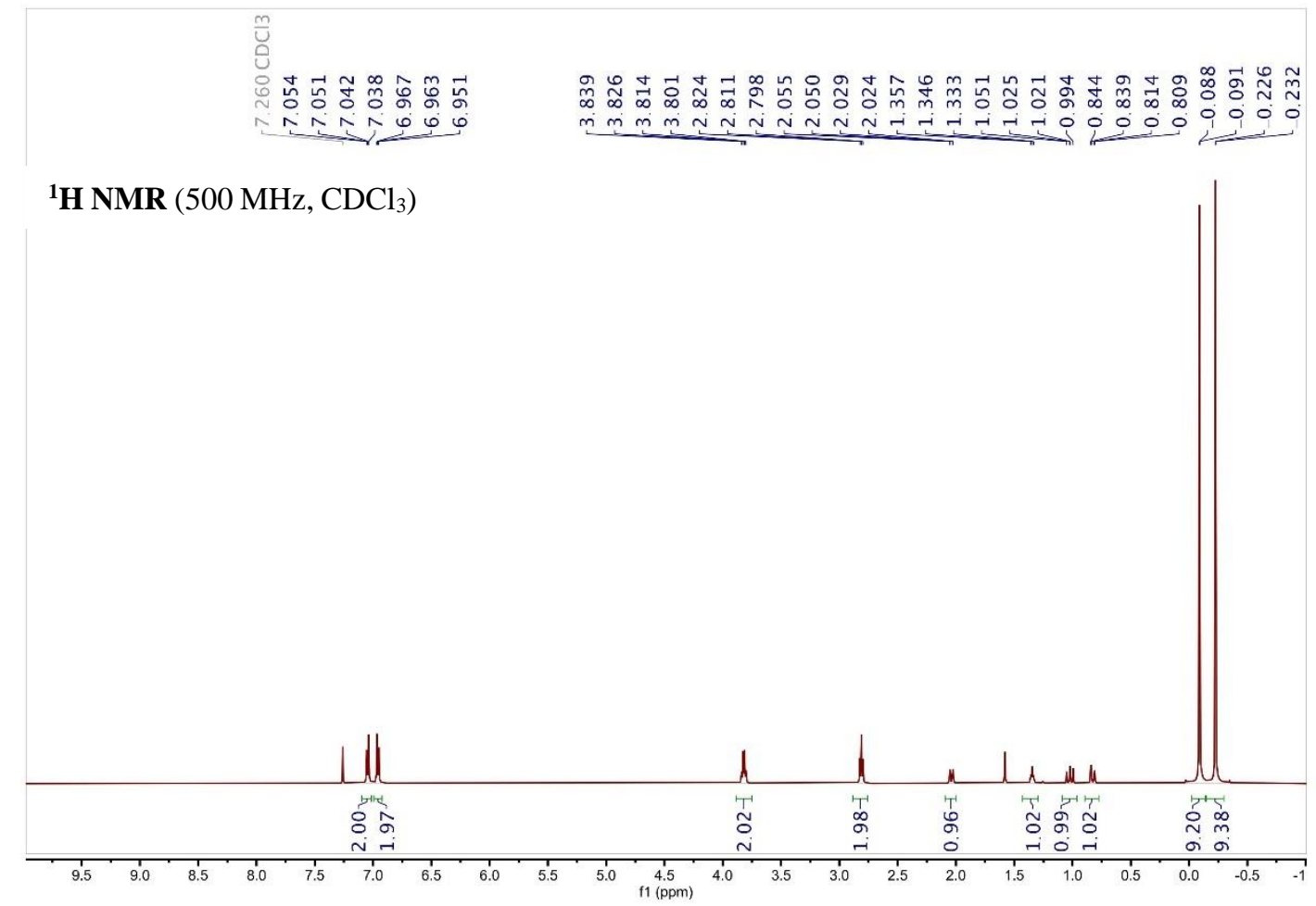

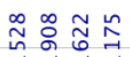

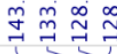

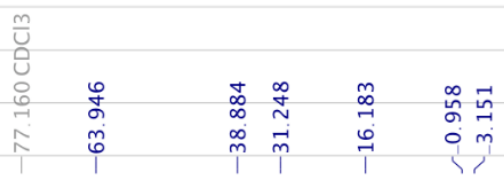

${ }^{13}$ C NMR (126 MHz, $\left.\mathrm{CDCl}_{3}\right)$

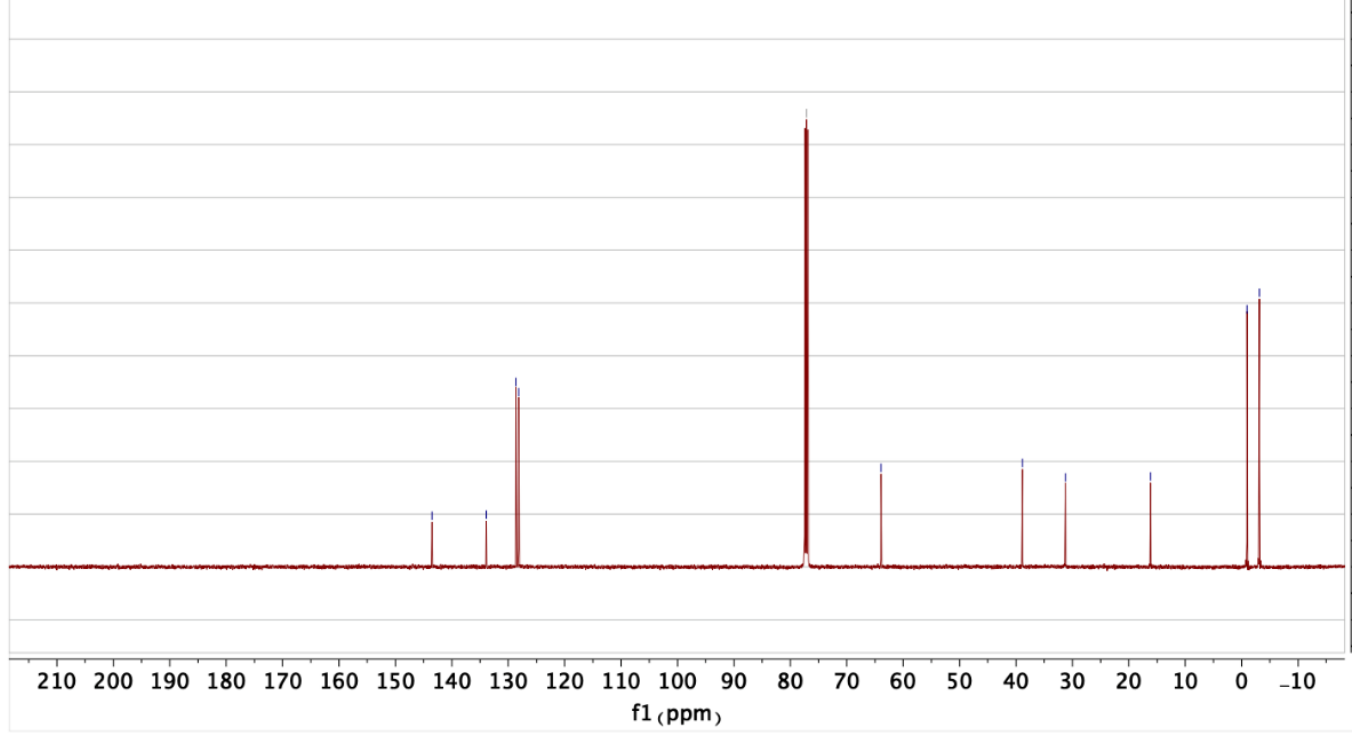




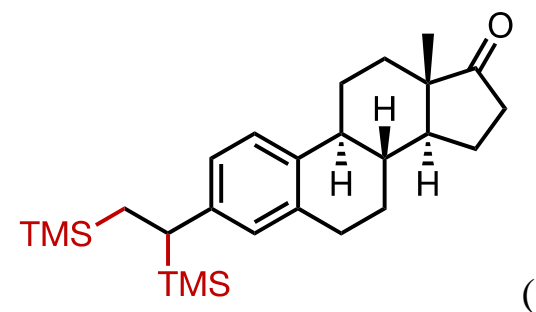

(9)

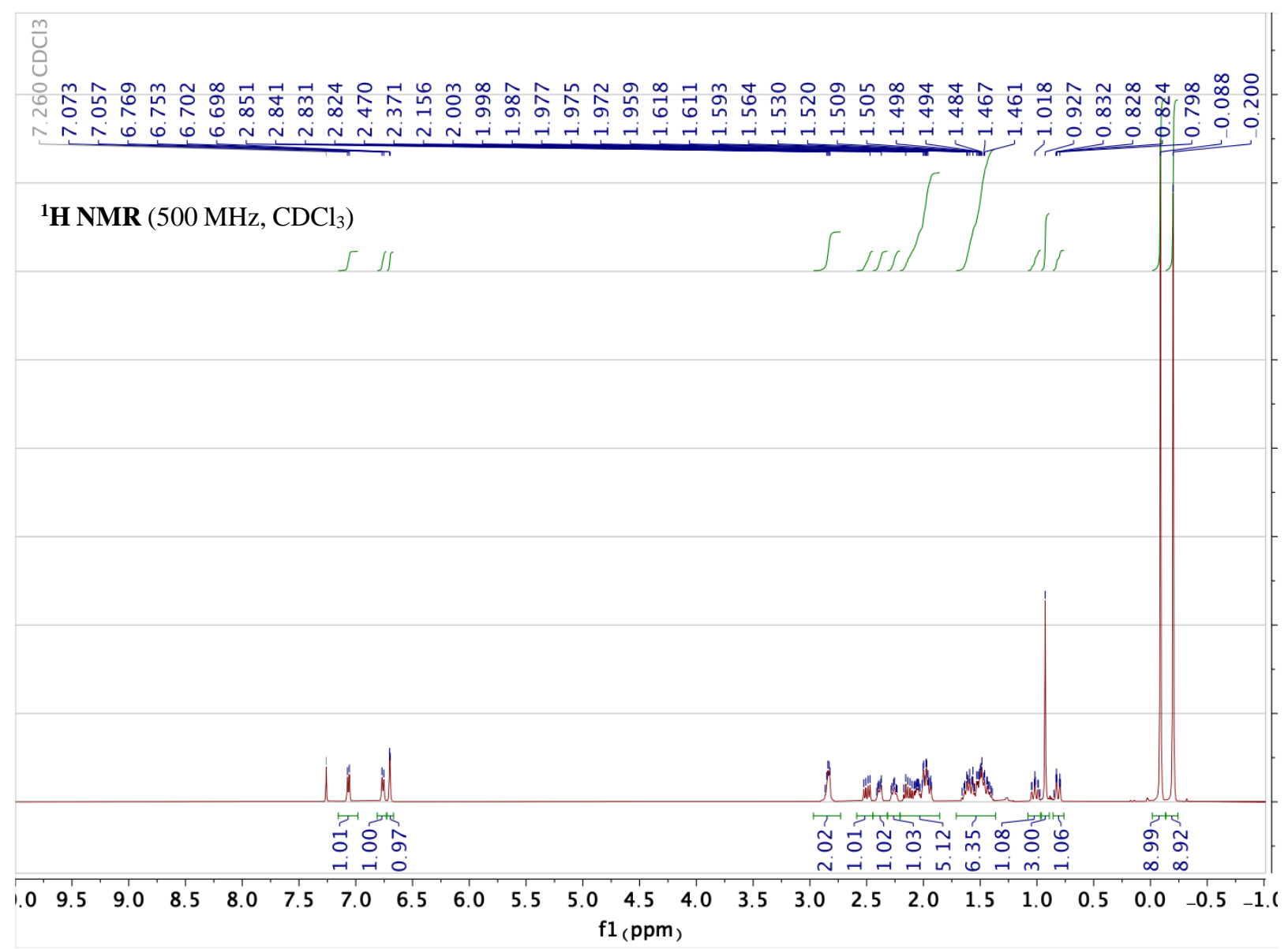


${ }^{13} \mathbf{C}$ NMR (126 MHz, $\left.\mathrm{CDCl}_{3}\right)$

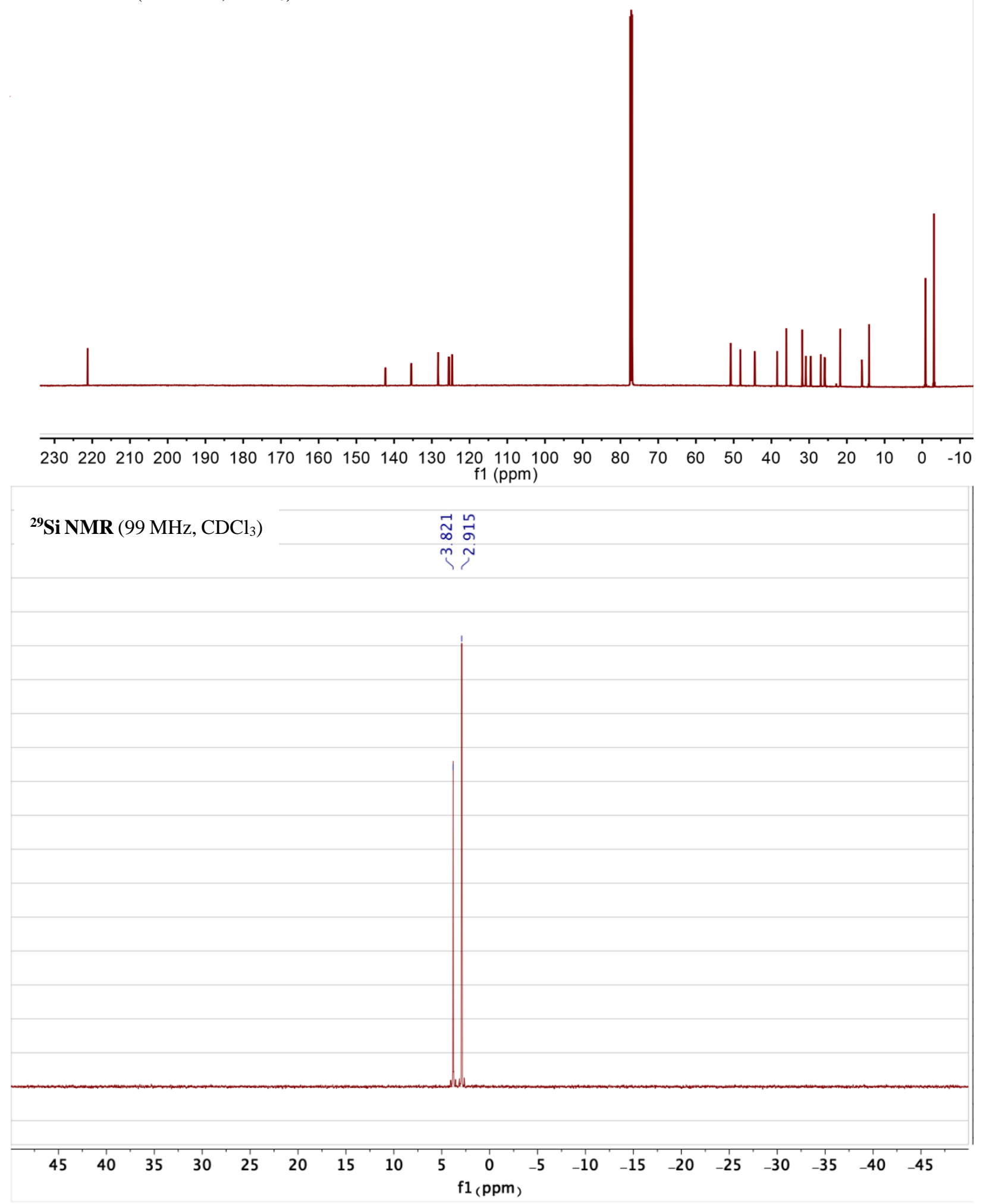


TMS

(10)
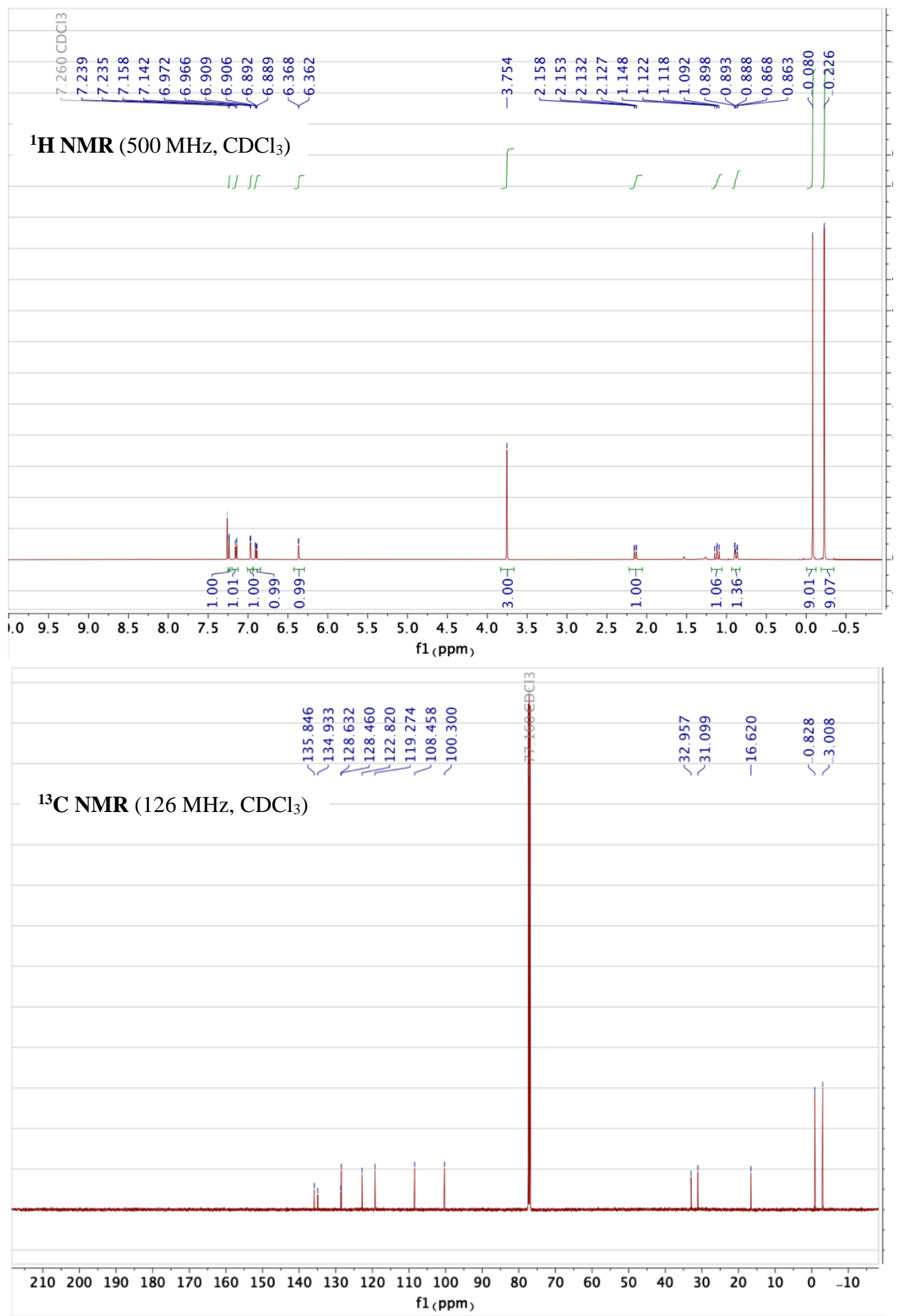
$\overbrace{T_{M S}}$

(11)
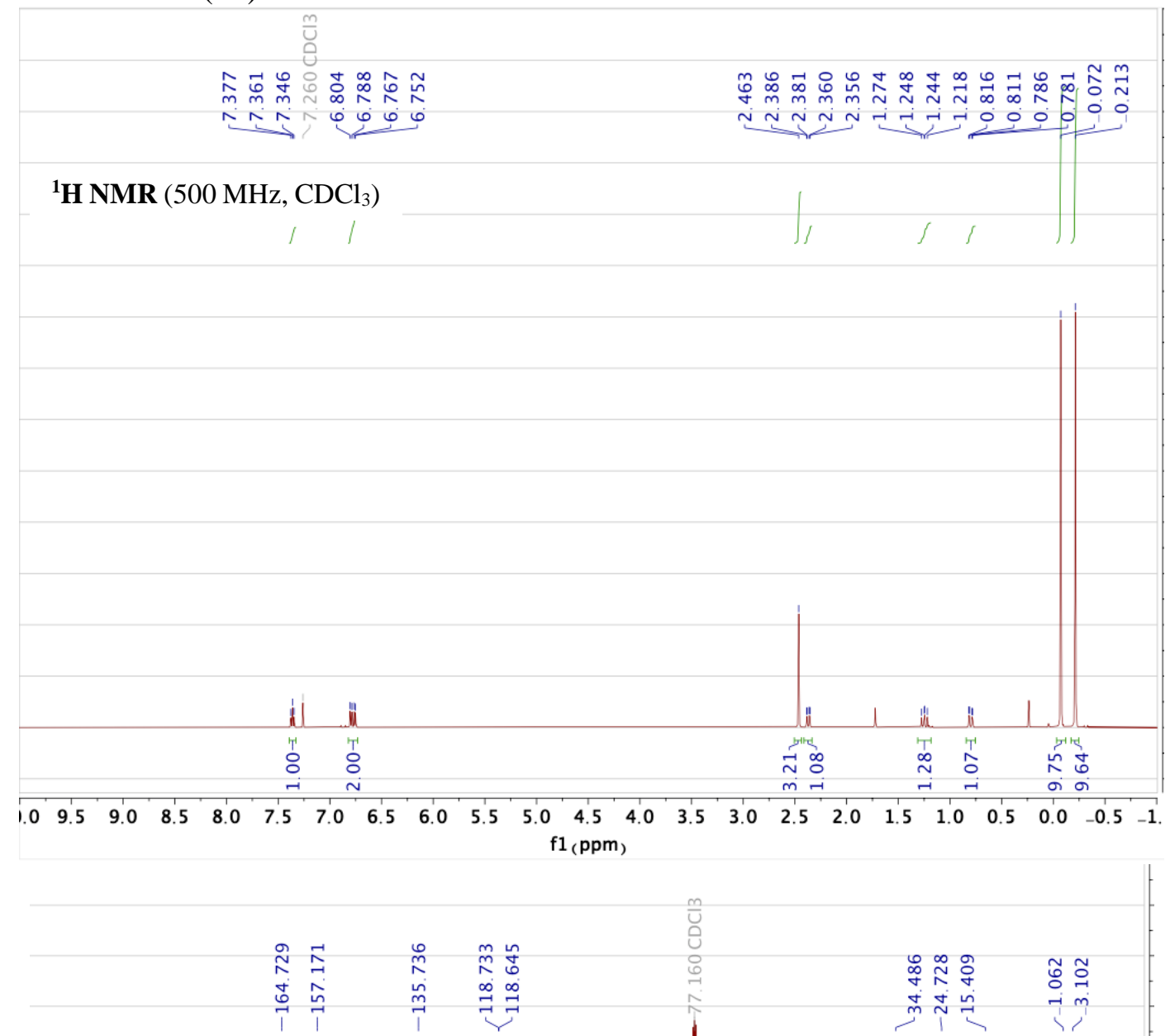

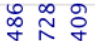

응

${ }^{13} \mathbf{C}$ NMR $\left(126 \mathrm{MHz}, \mathrm{CDCl}_{3}\right)$

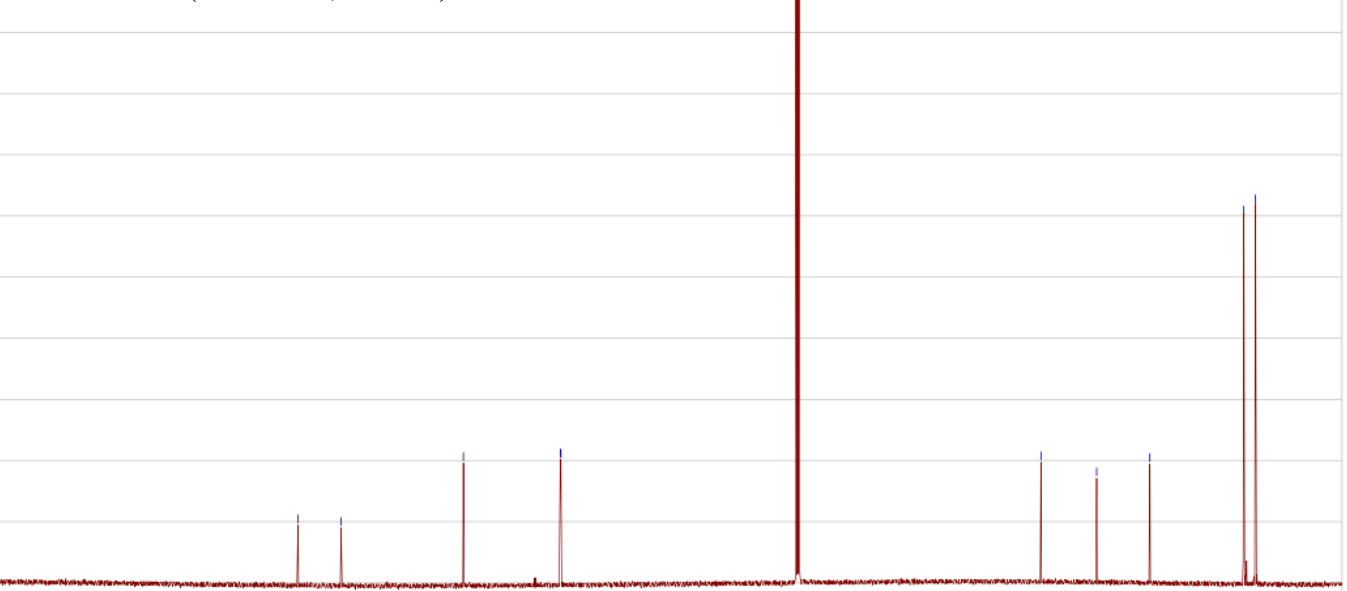

$\begin{array}{lllllllllllllllllllllll}210 & 200 & 190 & 180 & 170 & 160 & 150 & 140 & 130 & 120 & 110 & 100 & 90 & 80 & 70 & 60 & 50 & 40 & 30 & 20 & 10 & 0 & -10\end{array}$ 


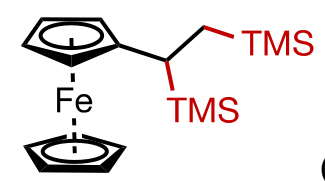

(12)
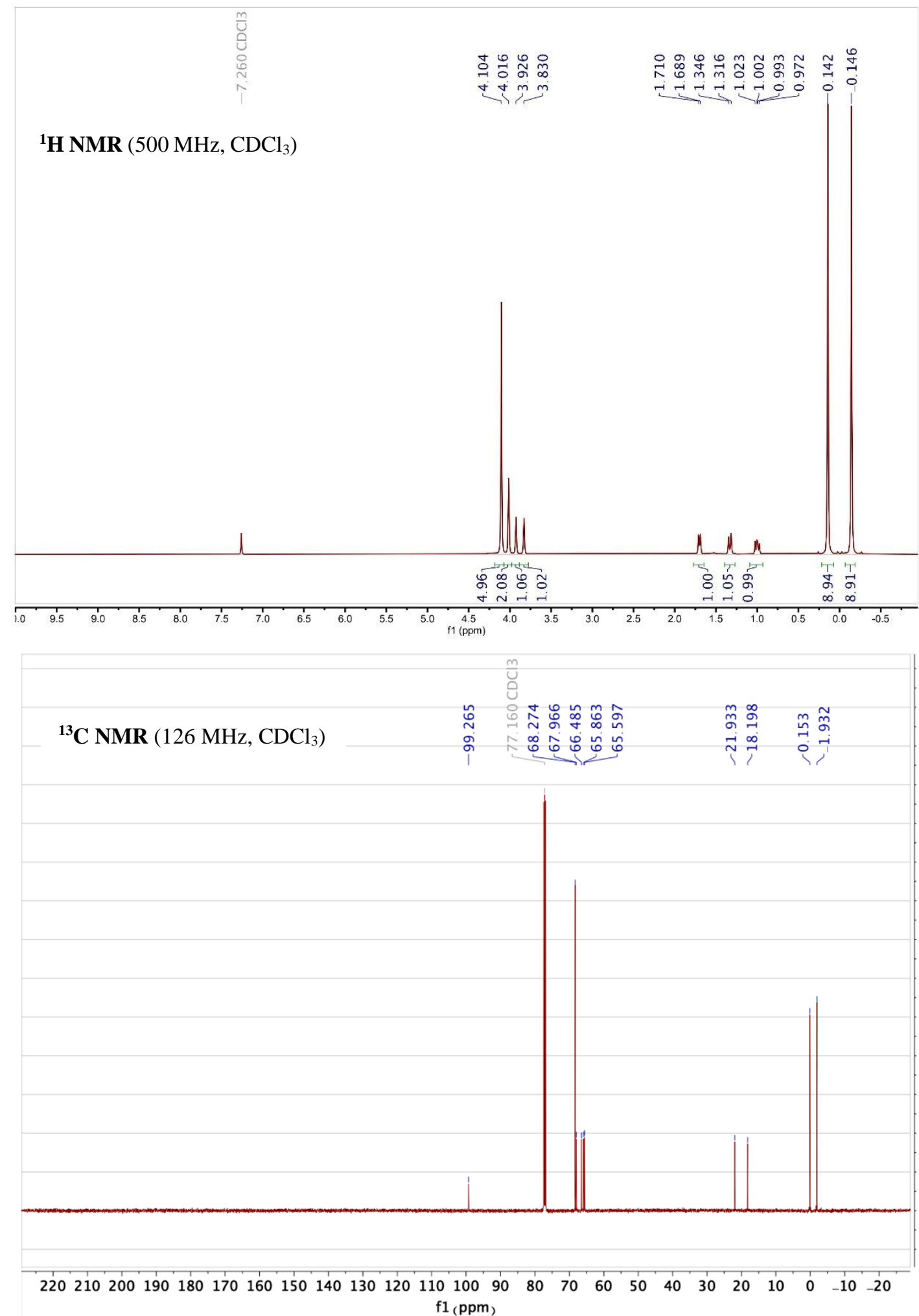
<smiles>CC(C)(C)[C@@H]1Cc2ccccc2[C@@H]1S(C)(=O)=O</smiles>

(13)
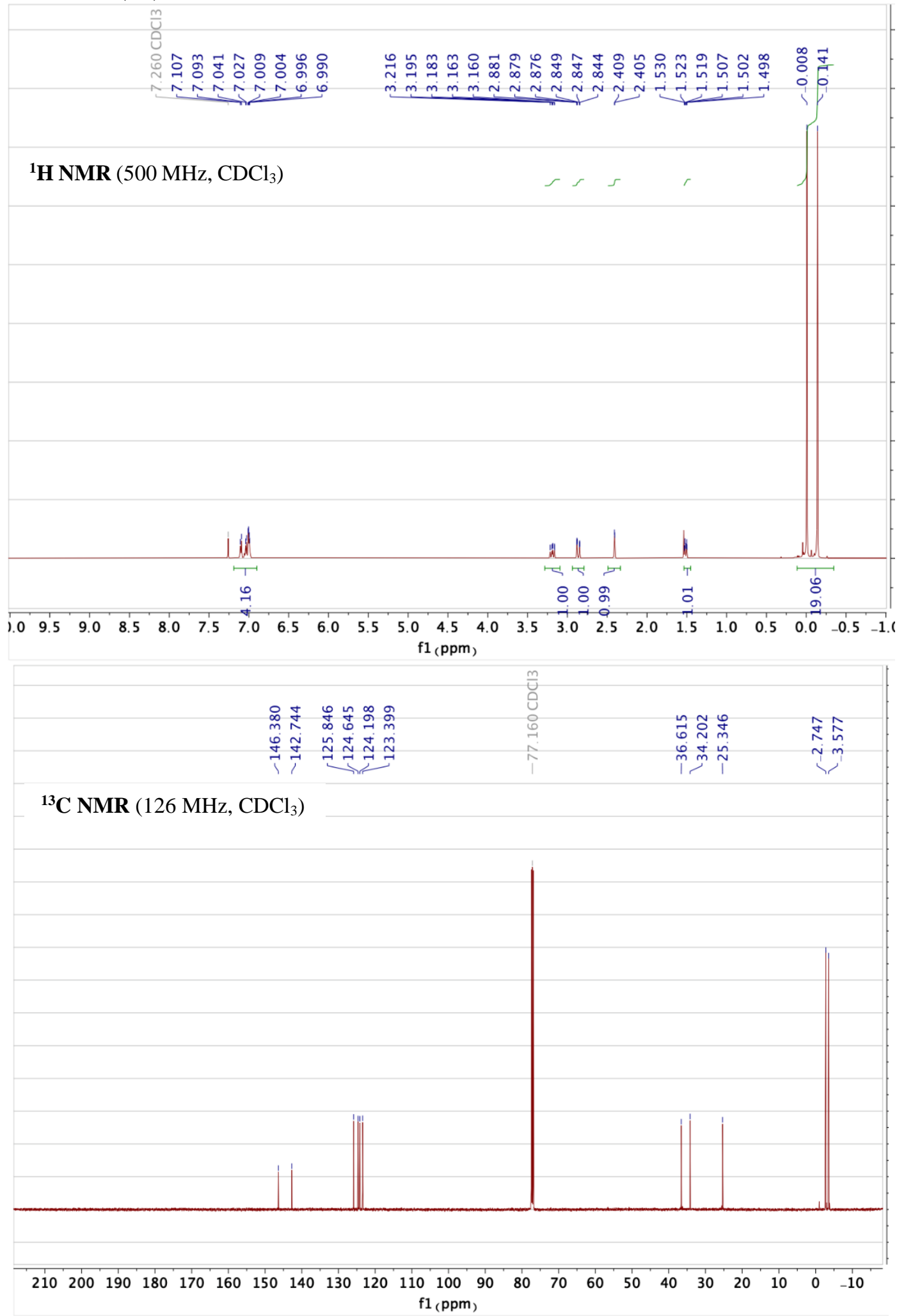
<smiles>CC(C)(C)[C@@H]1c2ccccc2CC[C@@H]1S(C)(=O)=O</smiles>

(14)

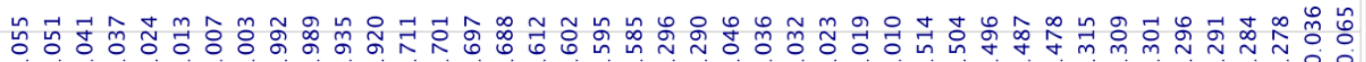
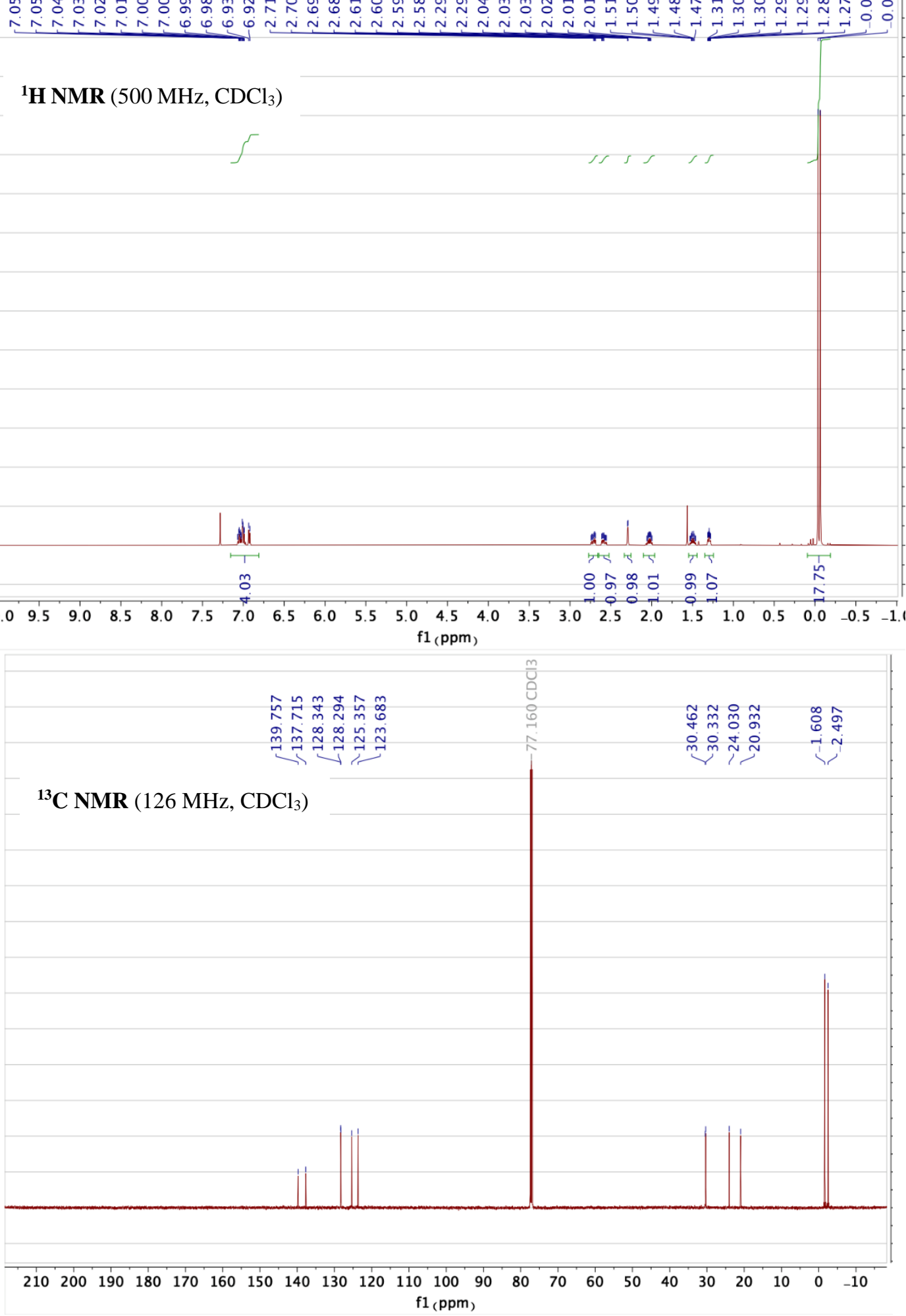

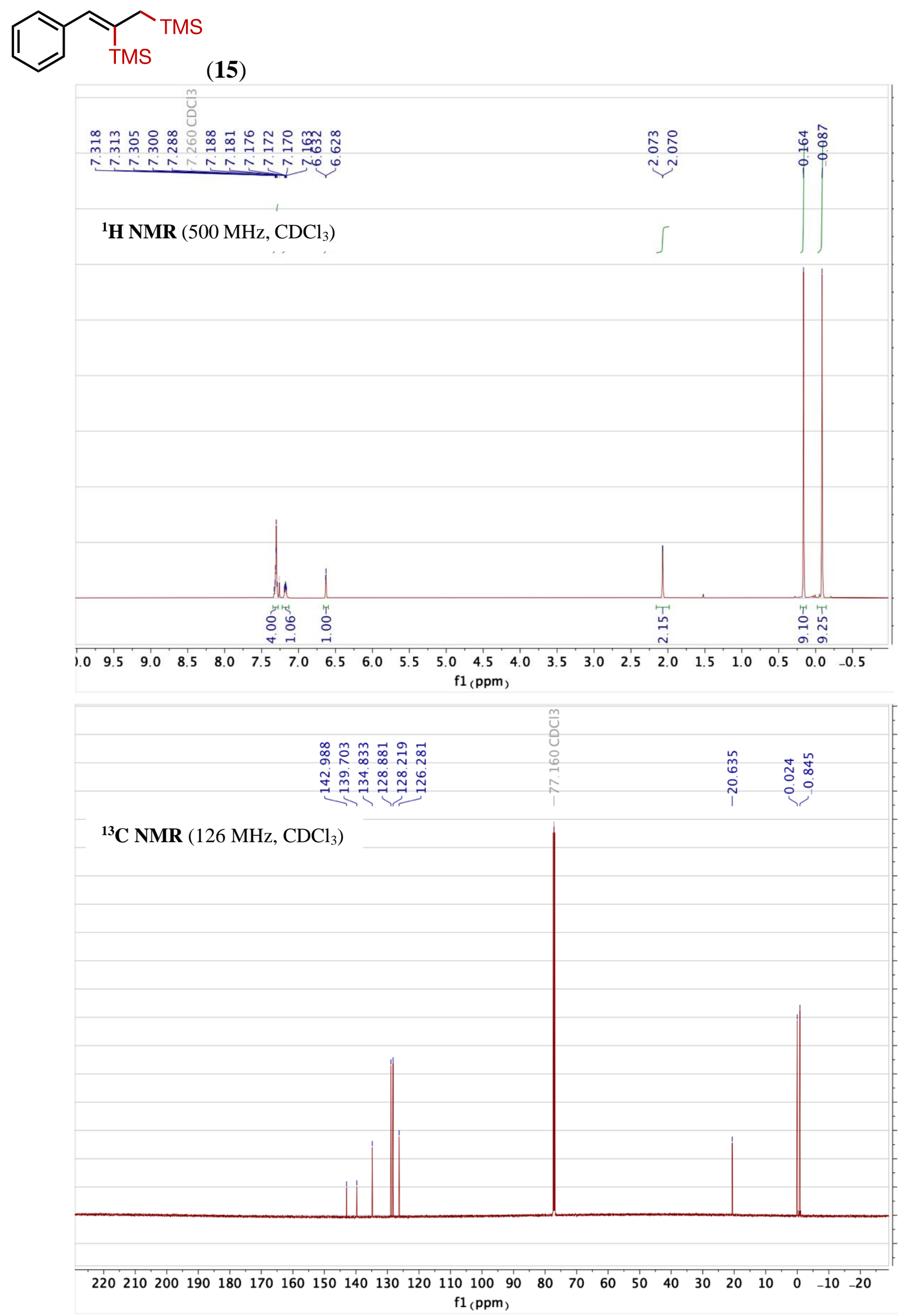


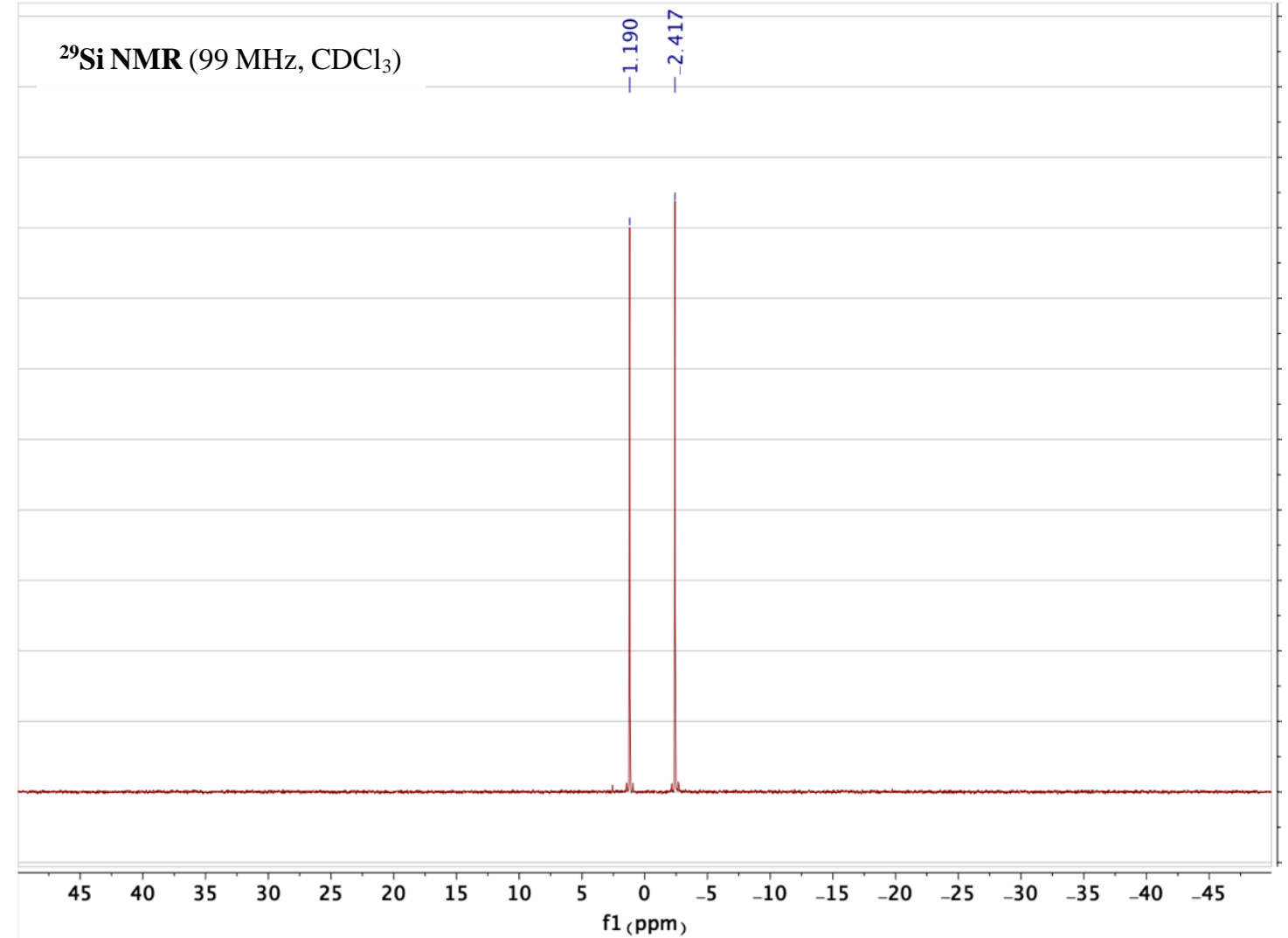

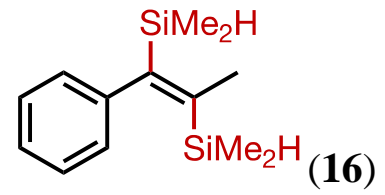



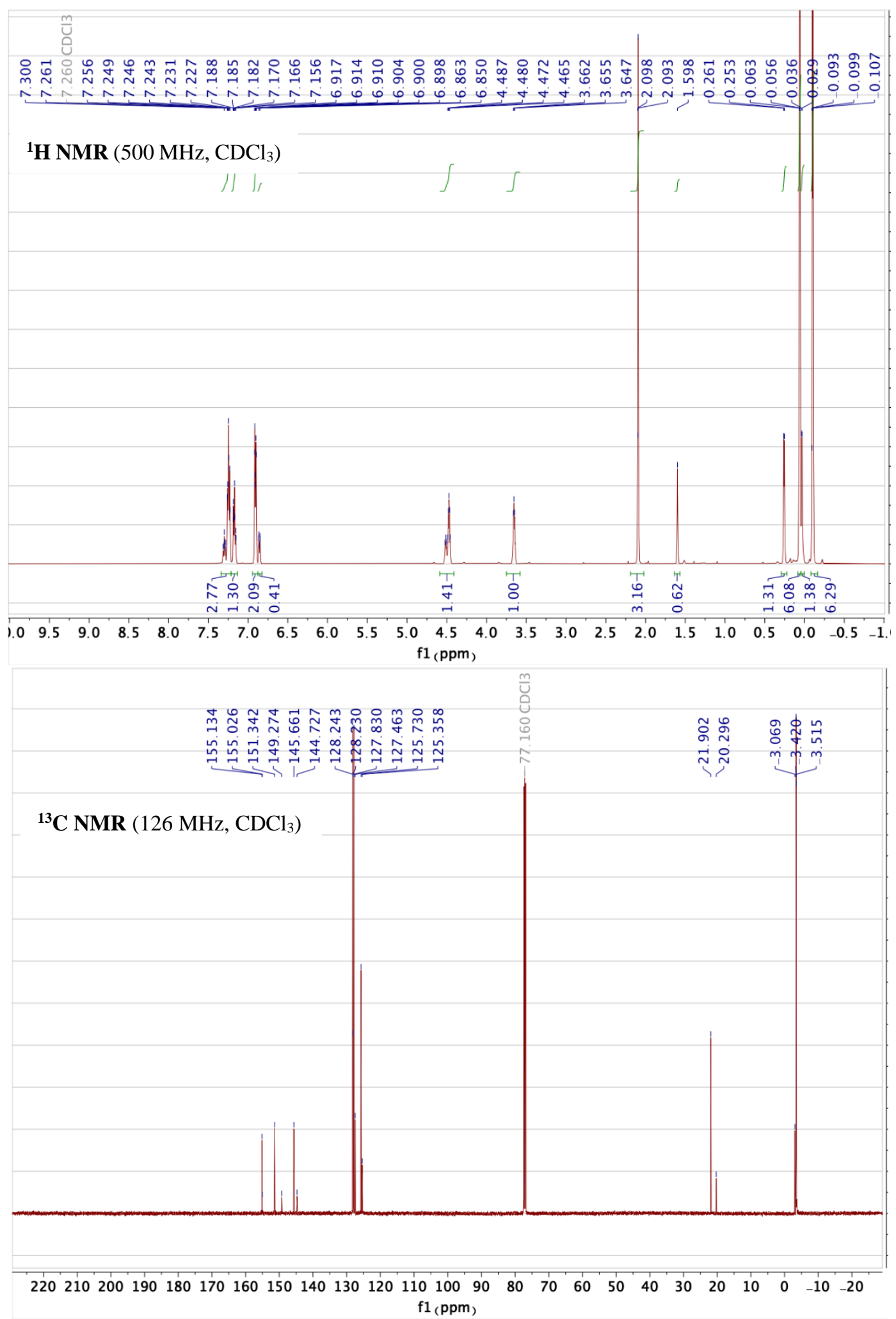


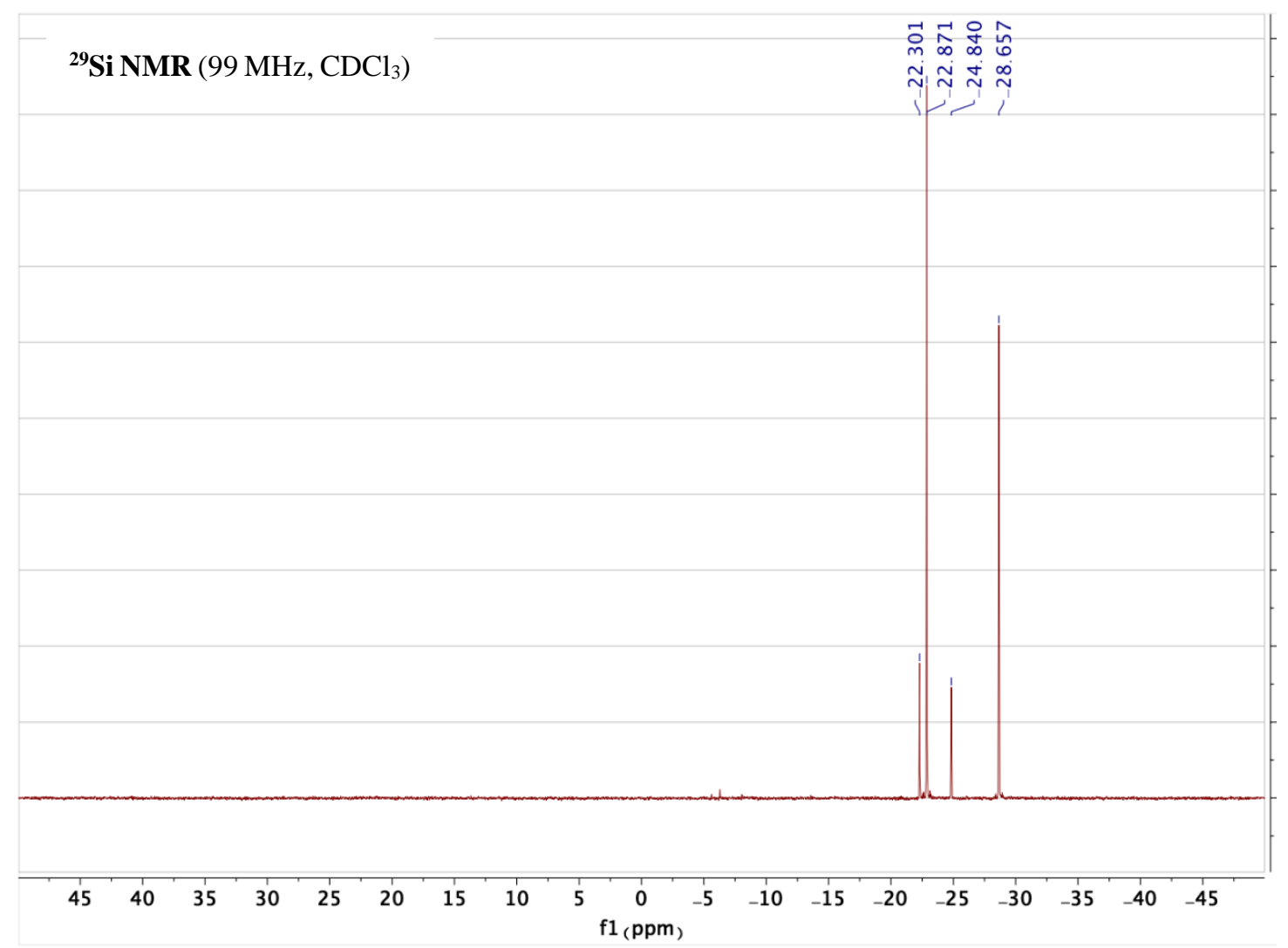

\section{NOESY}

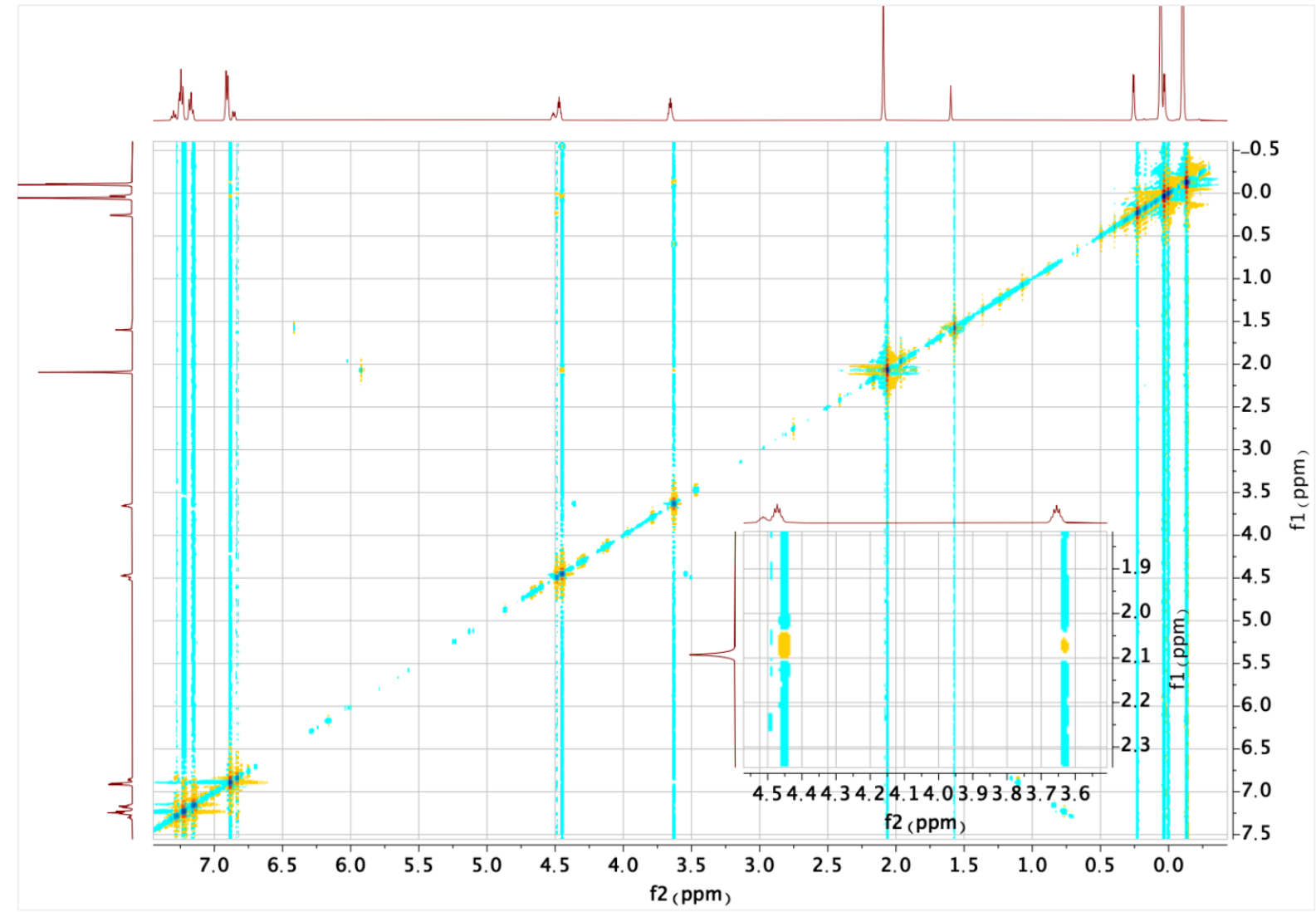




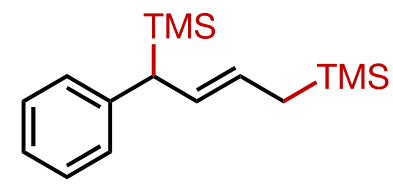

(17)

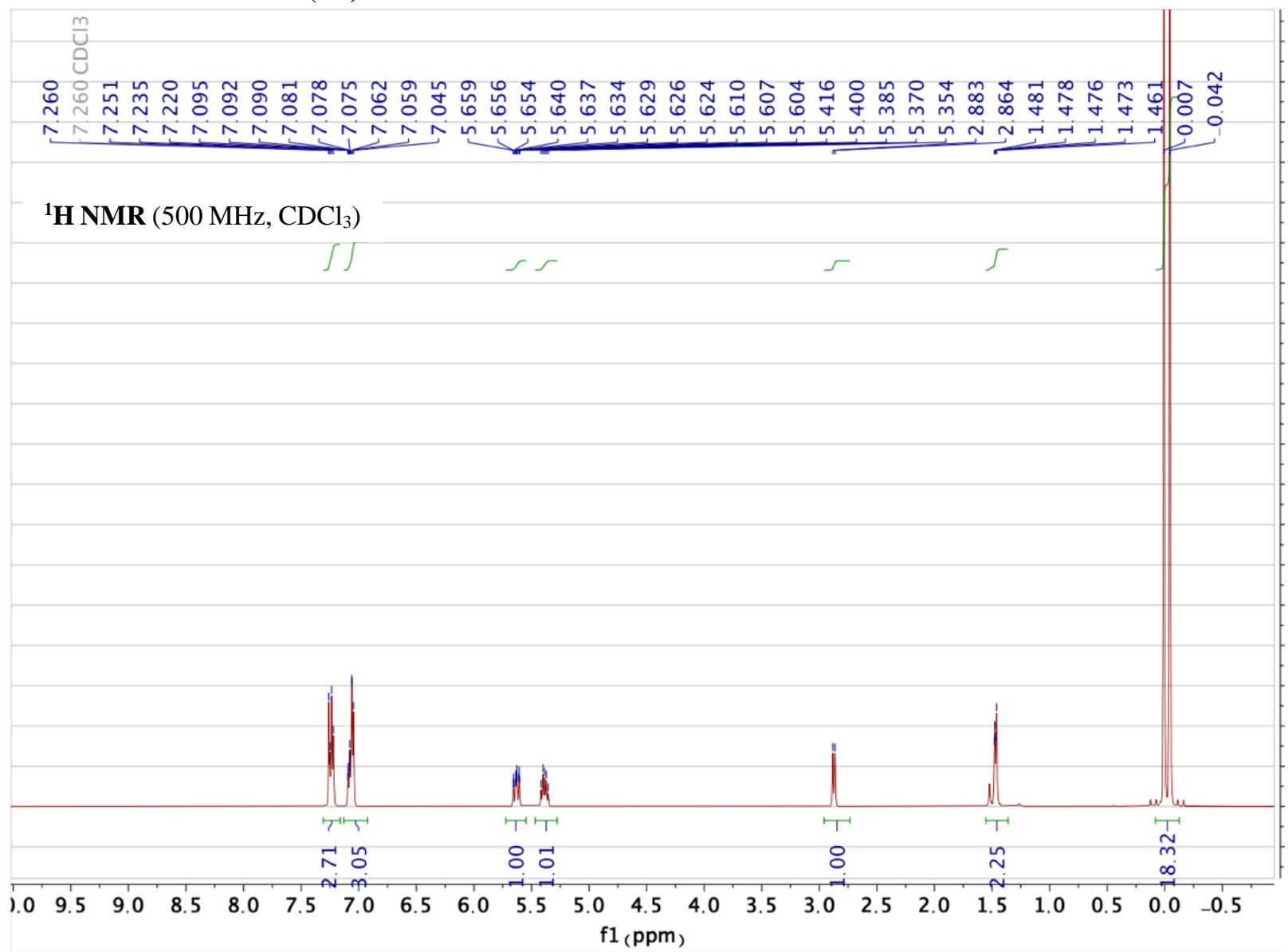




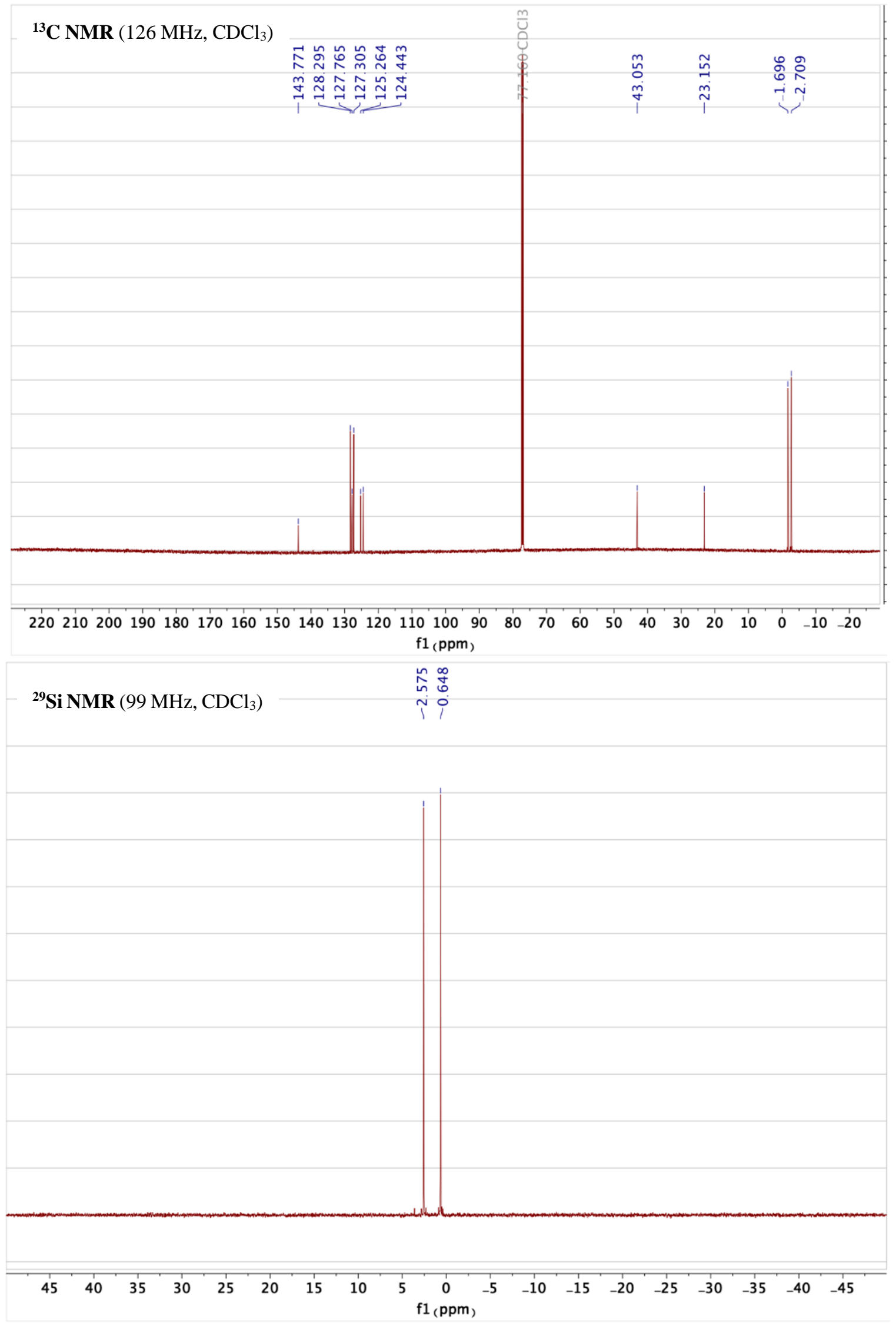




\section{NOESY}

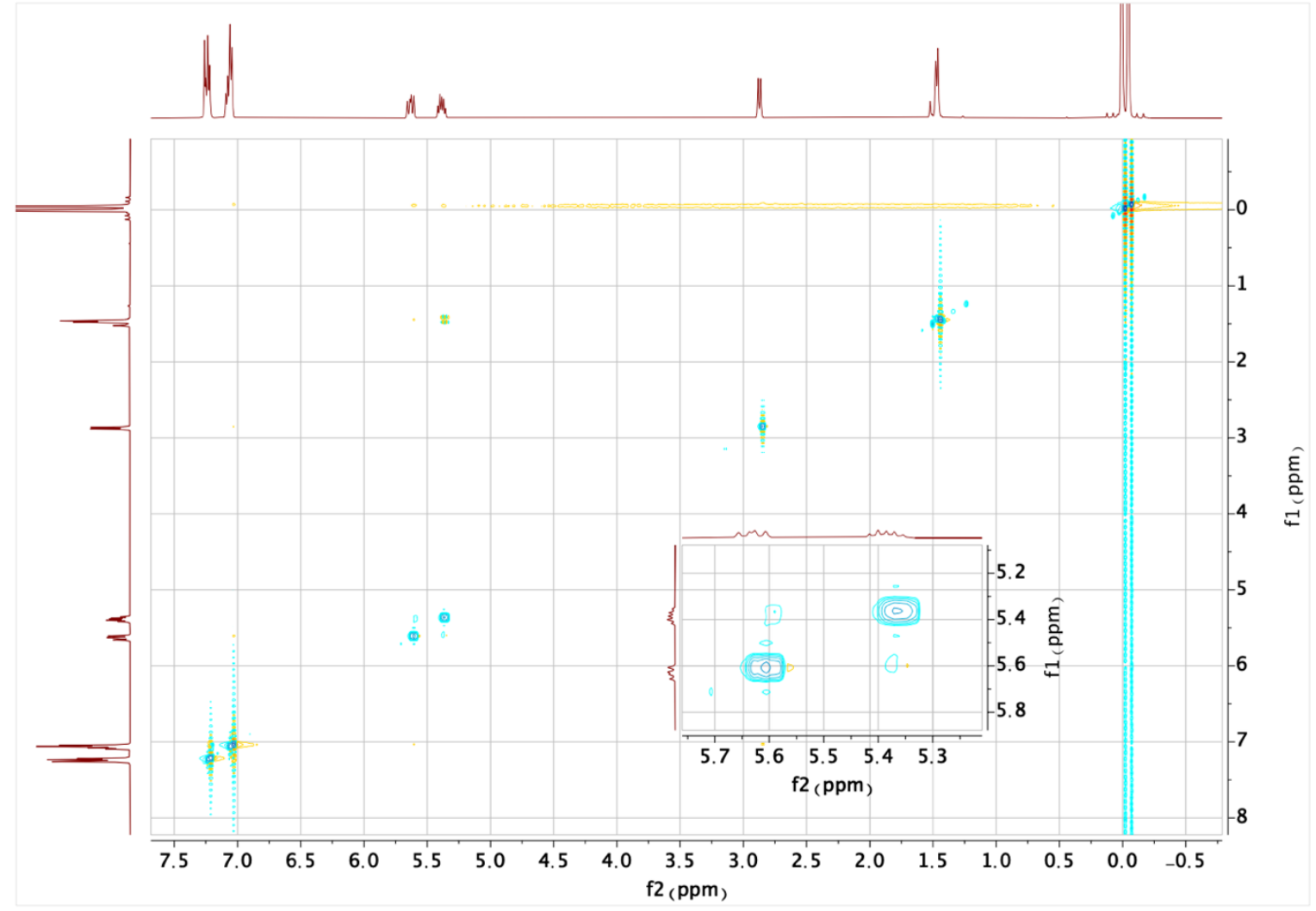

\section{Crude NMR}

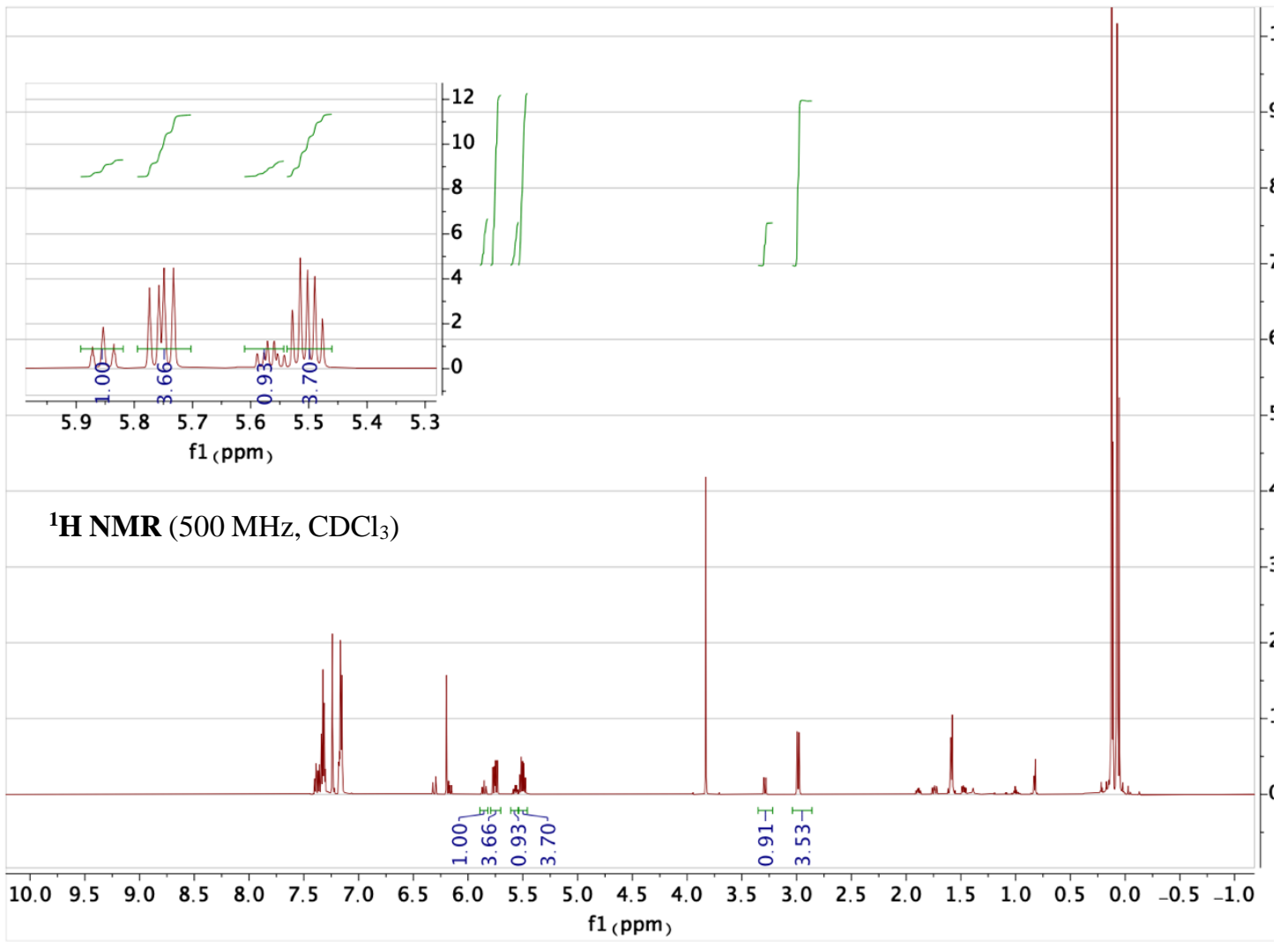


<smiles>CC(C)(C)c1ccc(C(=CC[As](C)(=O)=O)c2ccc(C(C)(C)C)cc2)cc1</smiles>

(18)

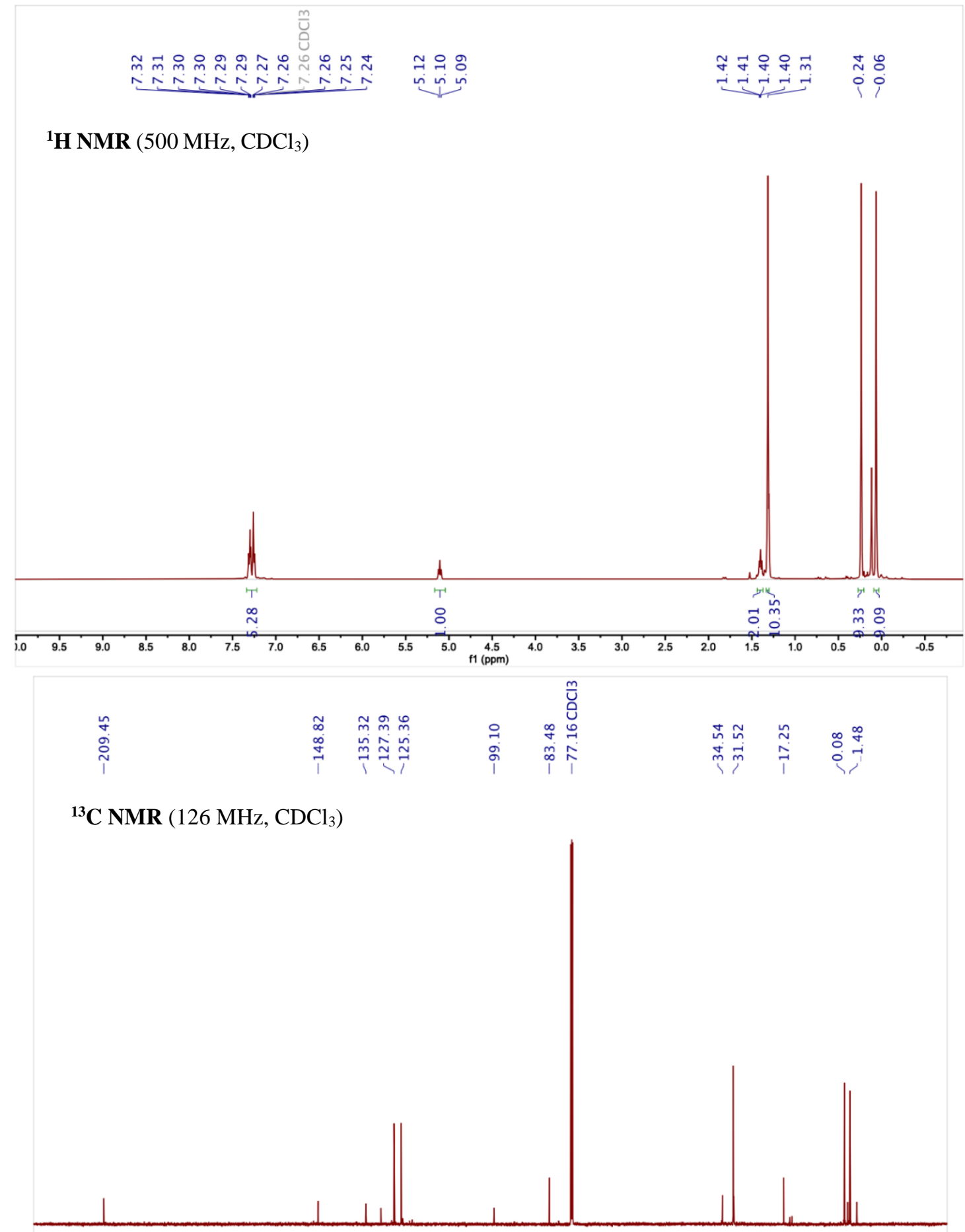

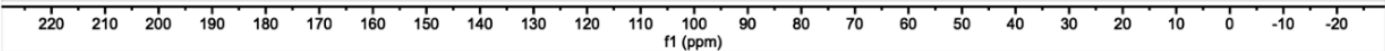



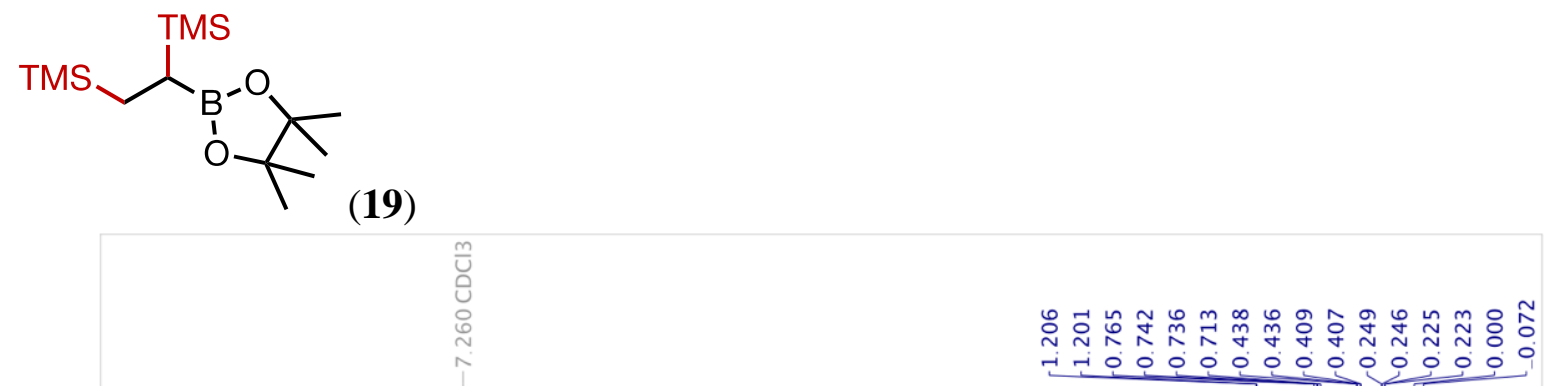

${ }^{1} \mathbf{H}$ NMR $\left(500 \mathrm{MHz}, \mathrm{CDCl}_{3}\right)$

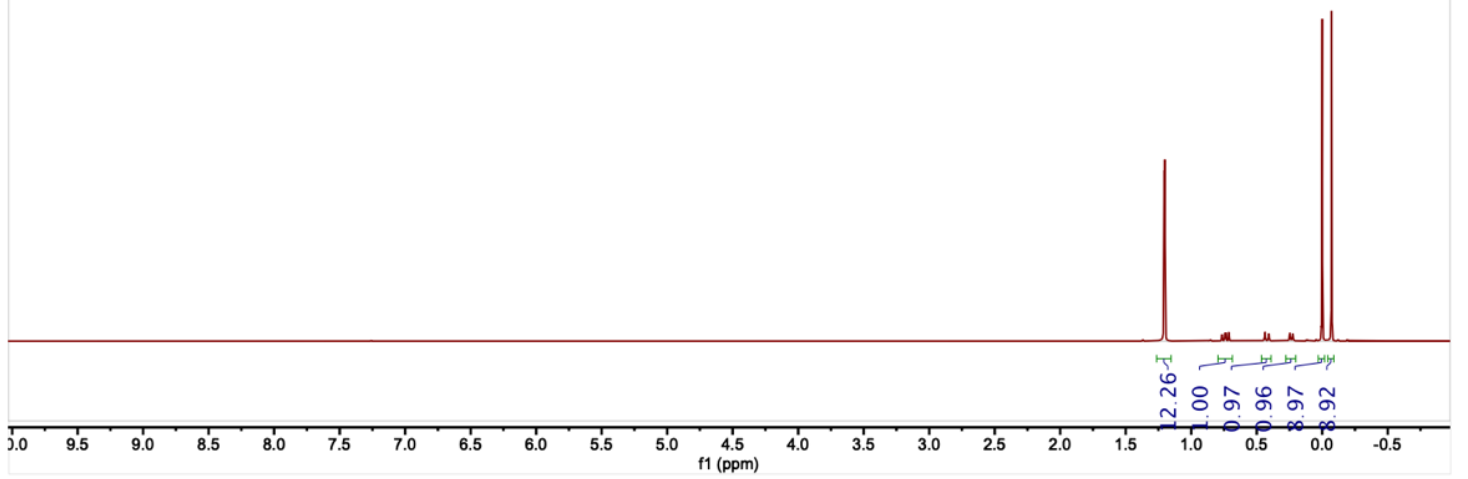

${ }^{13} \mathbf{C}$ NMR $\left(126 \mathrm{MHz}, \mathrm{CDCl}_{3}\right)$

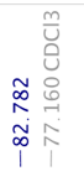

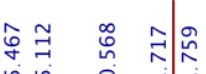

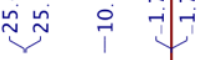

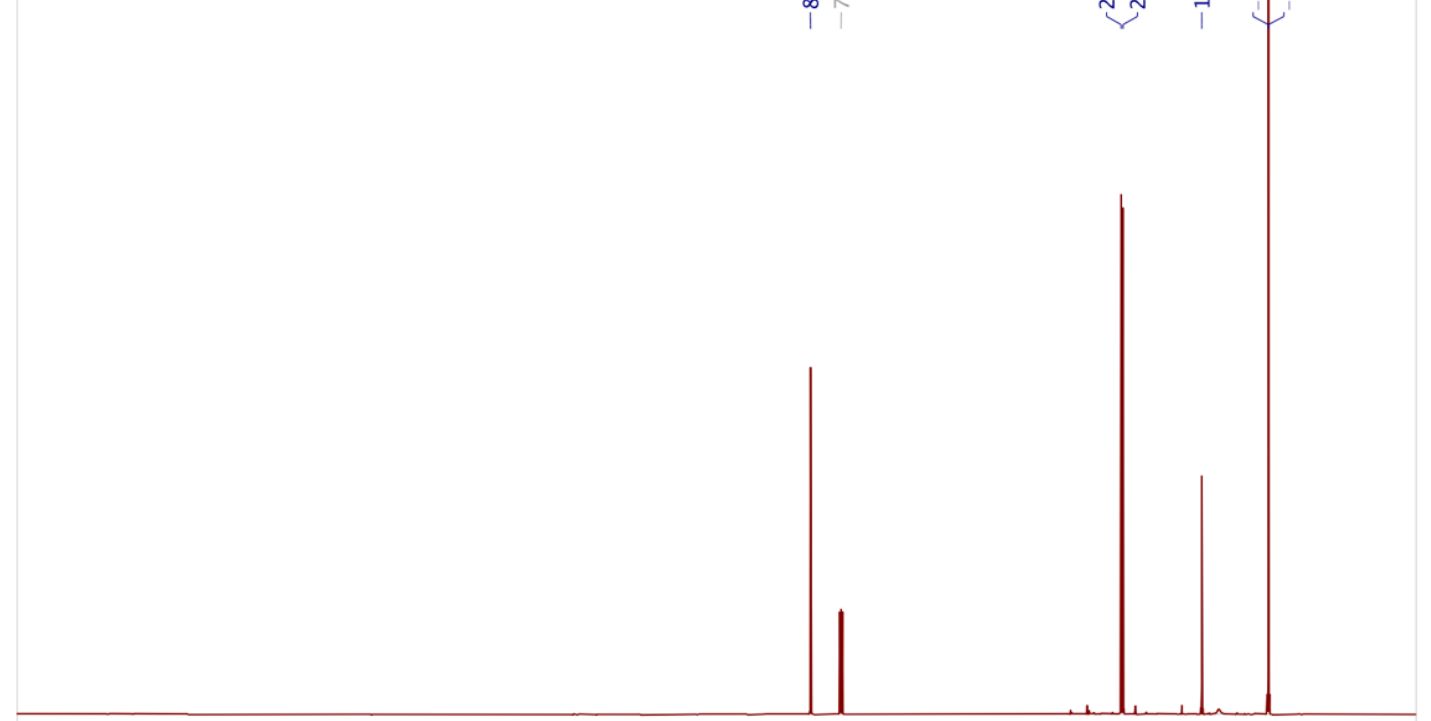

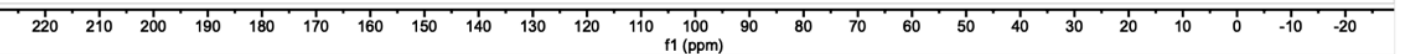



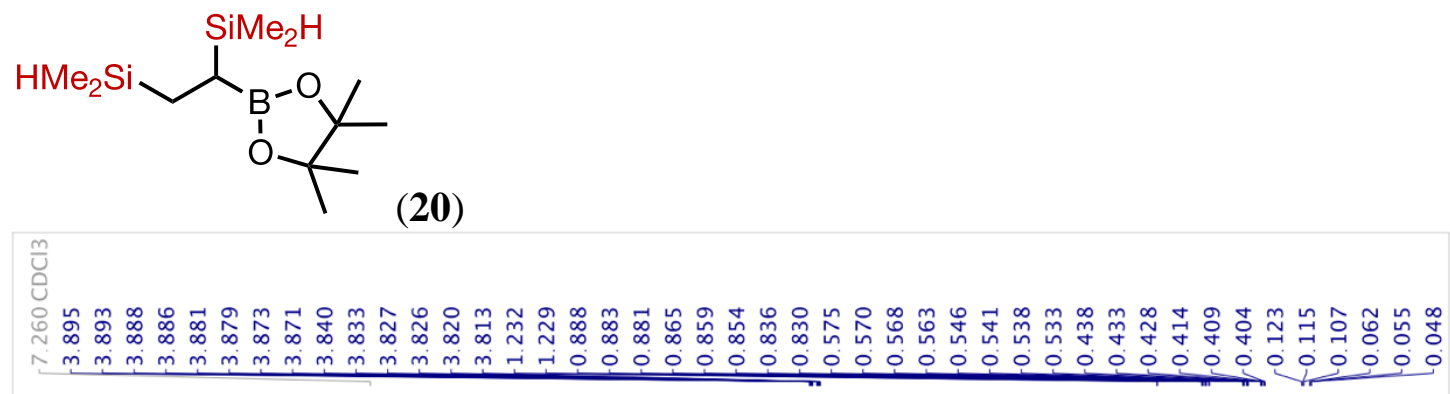

${ }^{1} \mathbf{H}$ NMR $\left(500 \mathrm{MHz}, \mathrm{CDCl}_{3}\right)$

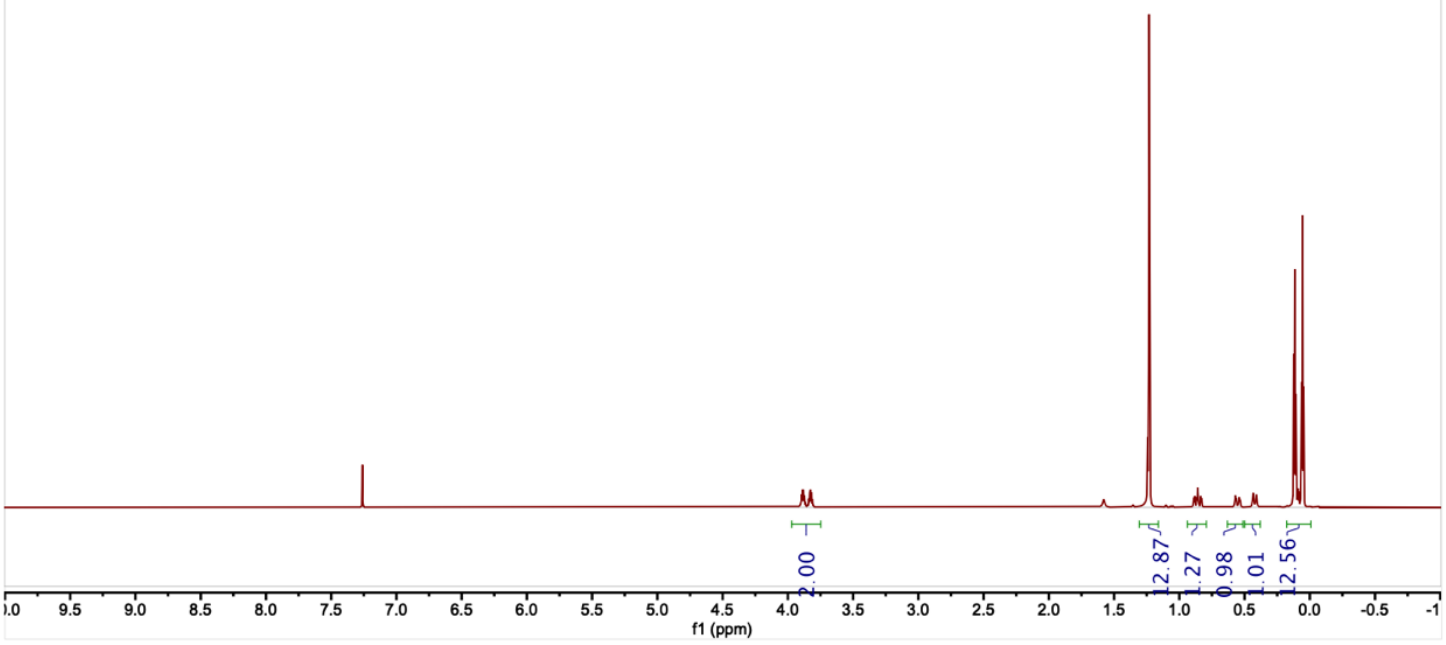

${ }^{13} \mathbf{C ~ N M R}\left(126 \mathrm{MHz}, \mathrm{CDCl}_{3}\right)$
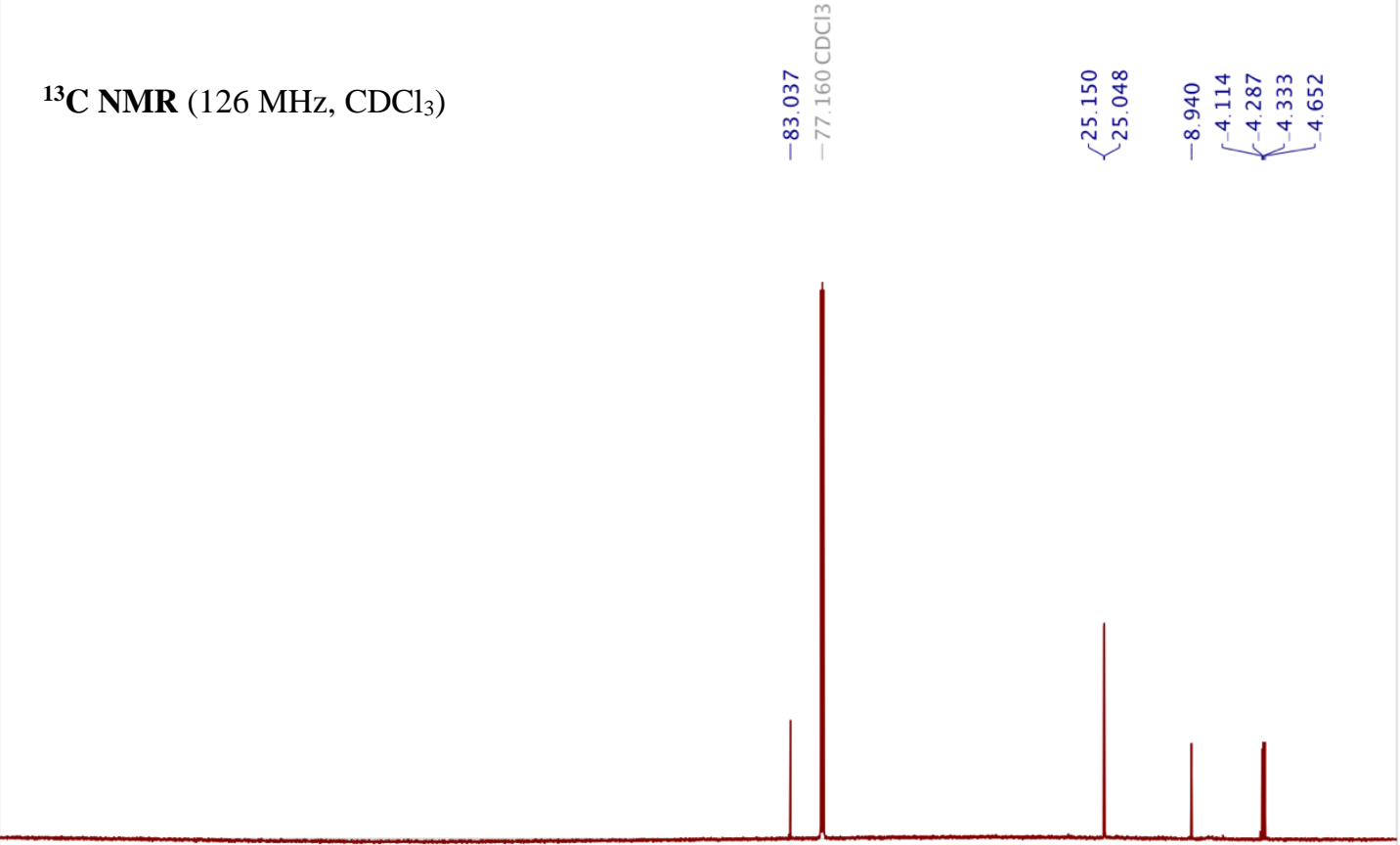

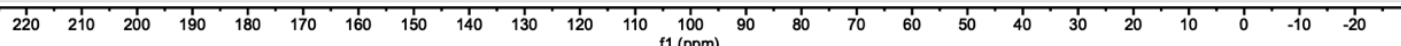



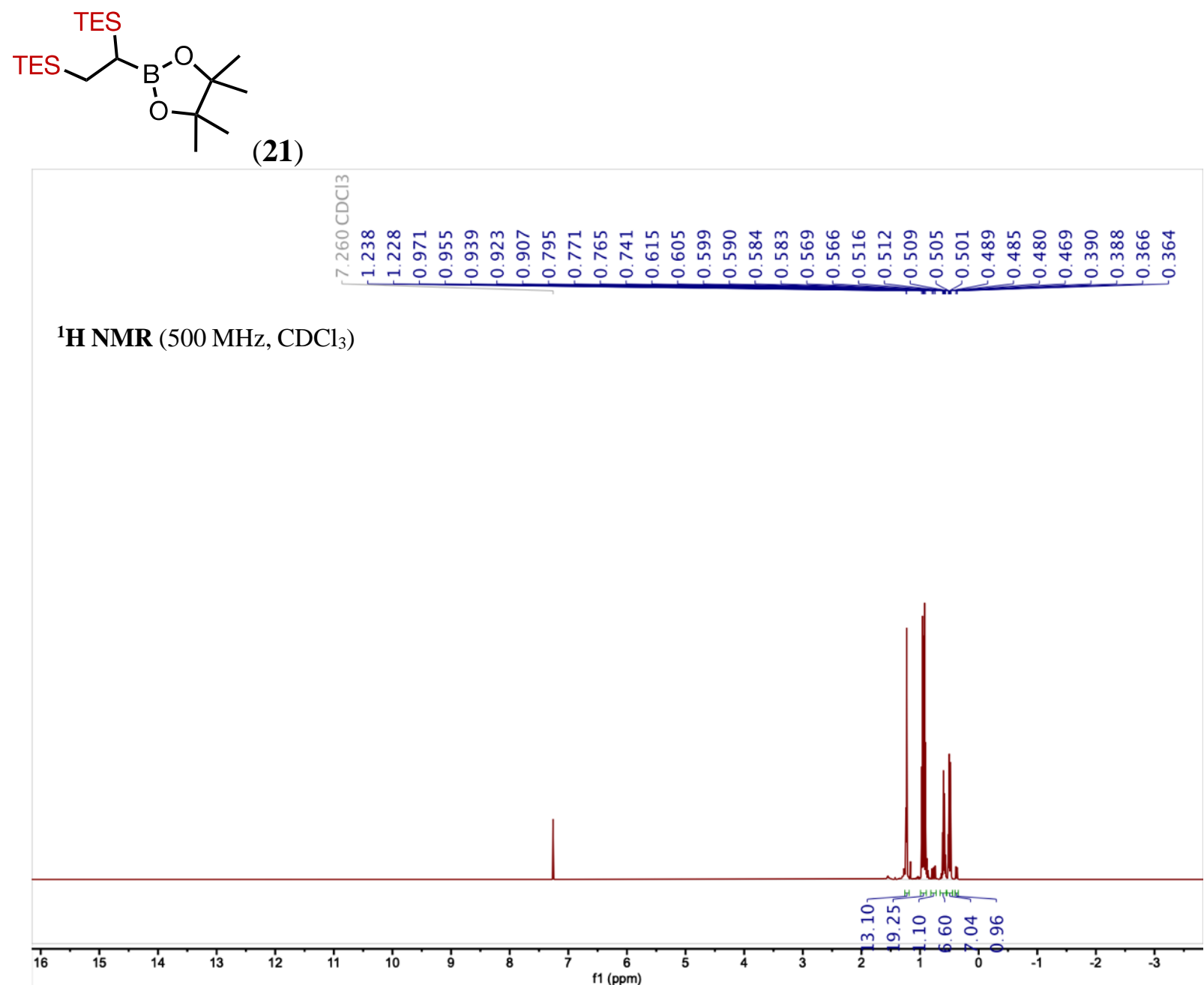
${ }^{13} \mathrm{C}$ NMR (126 MHz, $\left.\mathrm{CDCl}_{3}\right)$
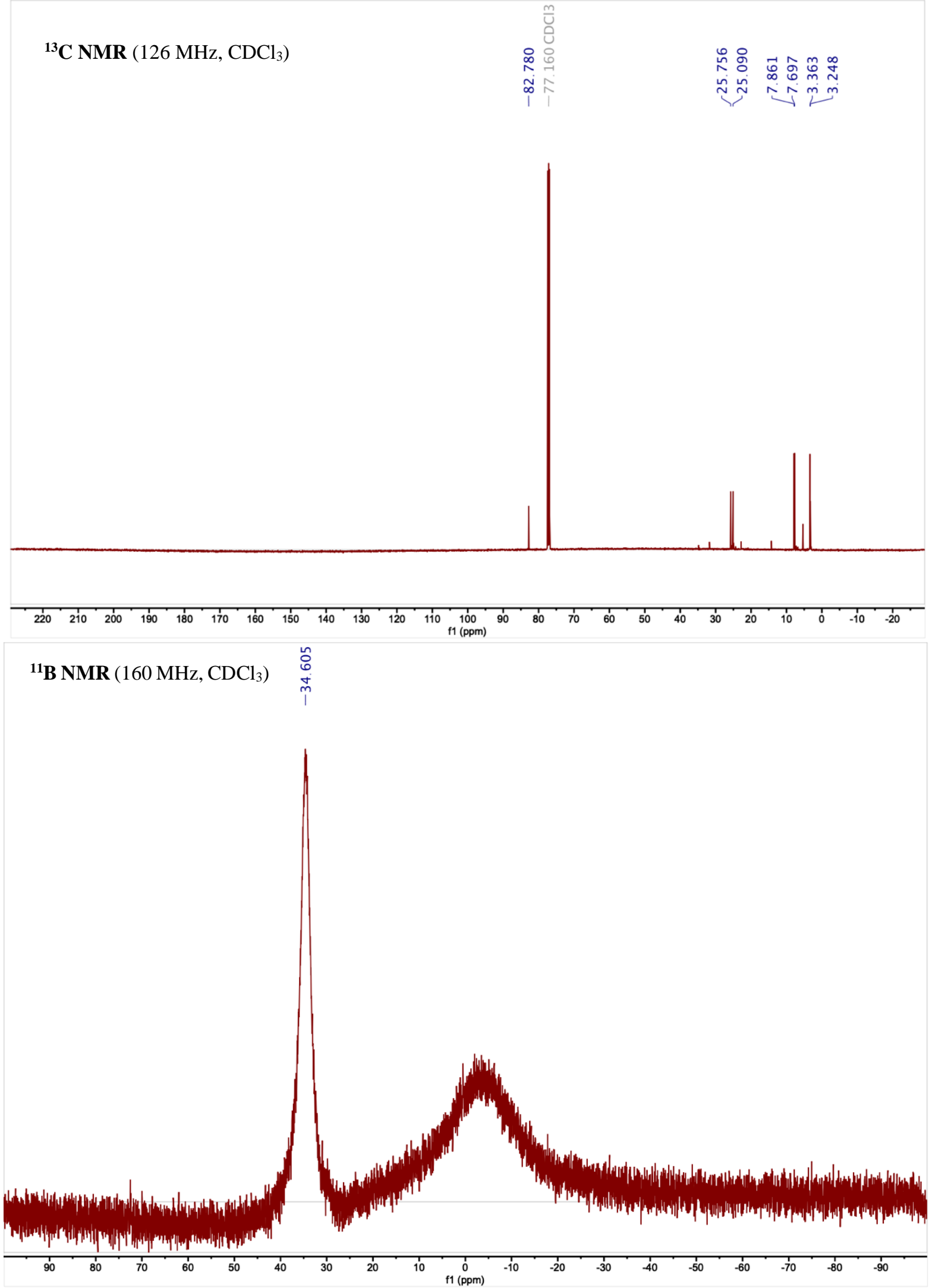

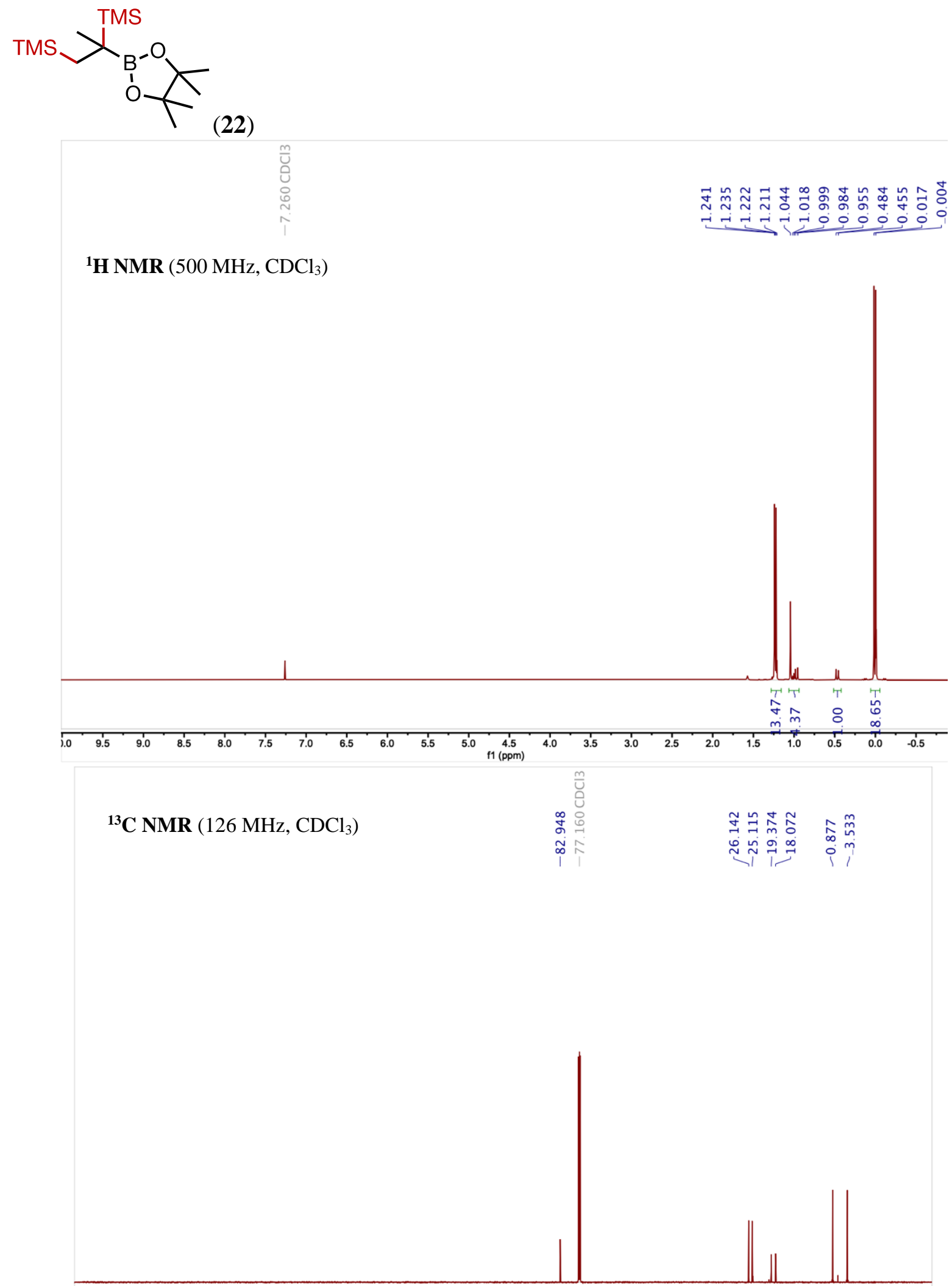

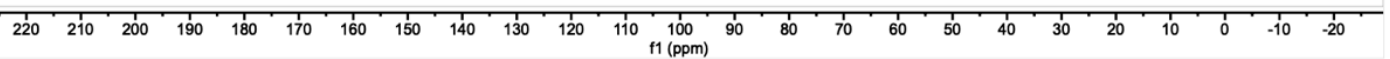



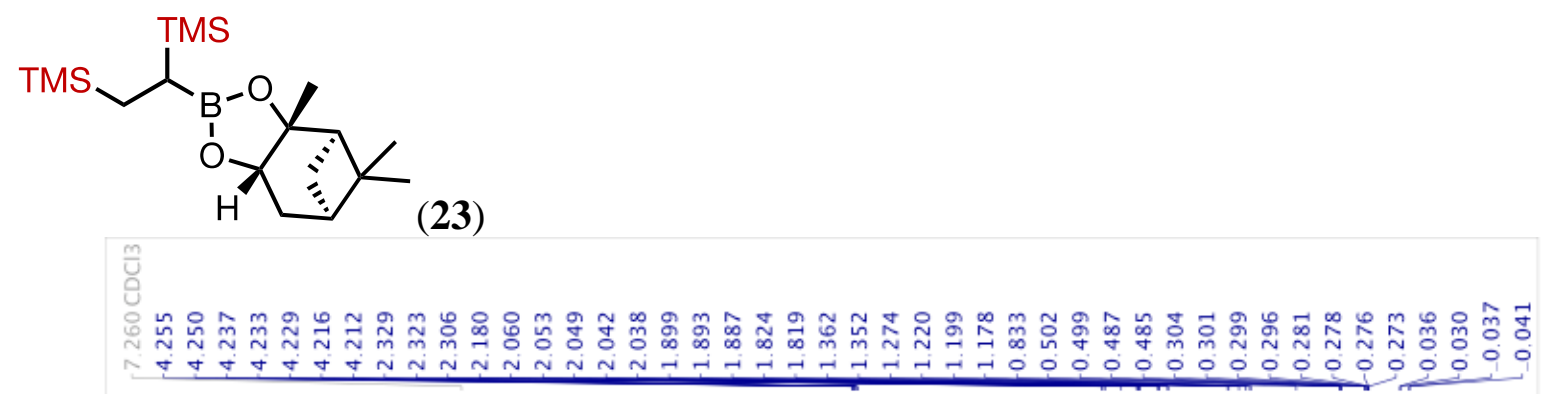

${ }^{1} \mathbf{H}$ NMR $\left(500 \mathrm{MHz}, \mathrm{CDCl}_{3}\right)$

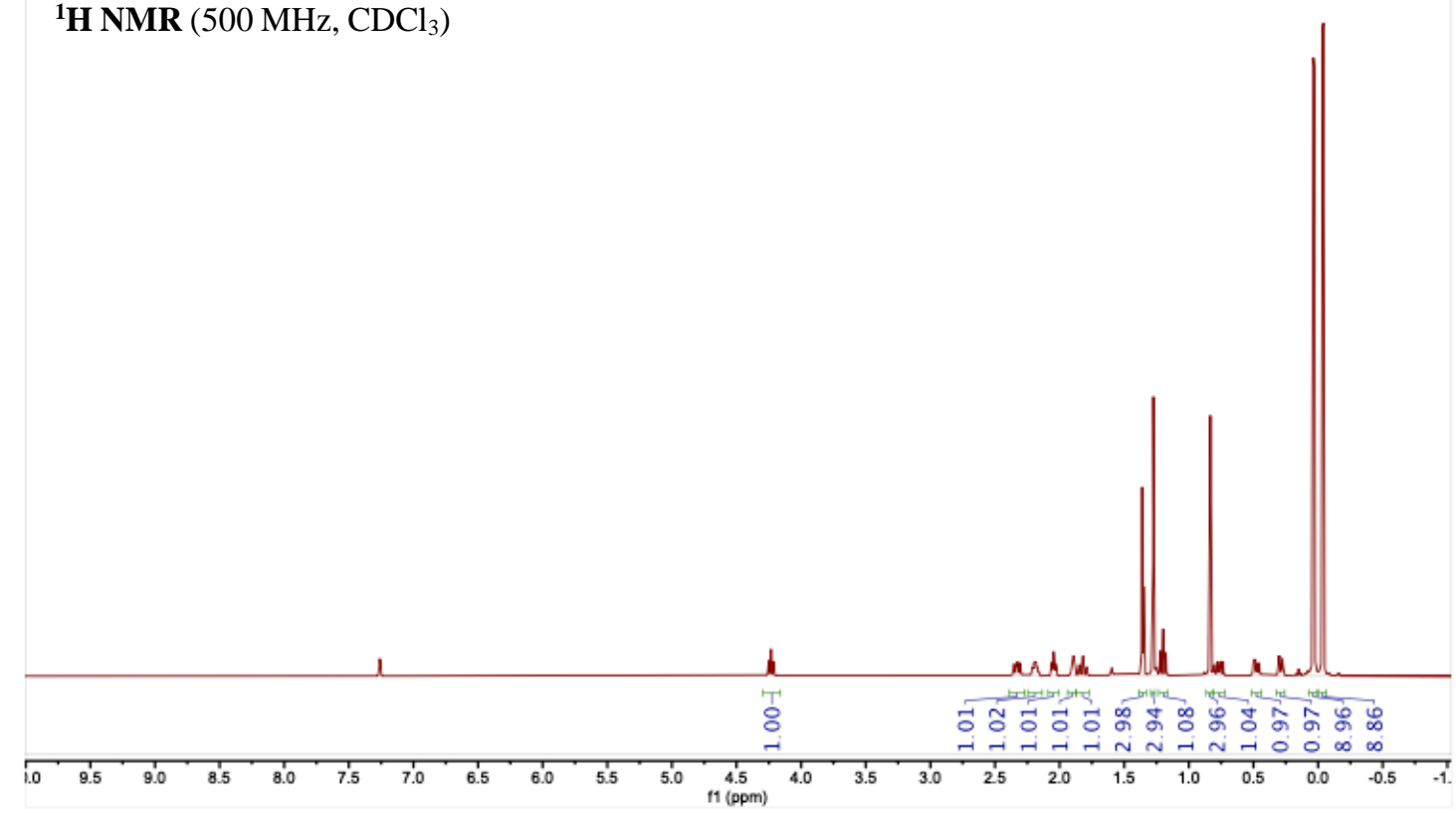

${ }^{13} \mathrm{C}$ NMR (126 MHz, $\left.\mathrm{CDCl}_{3}\right)$

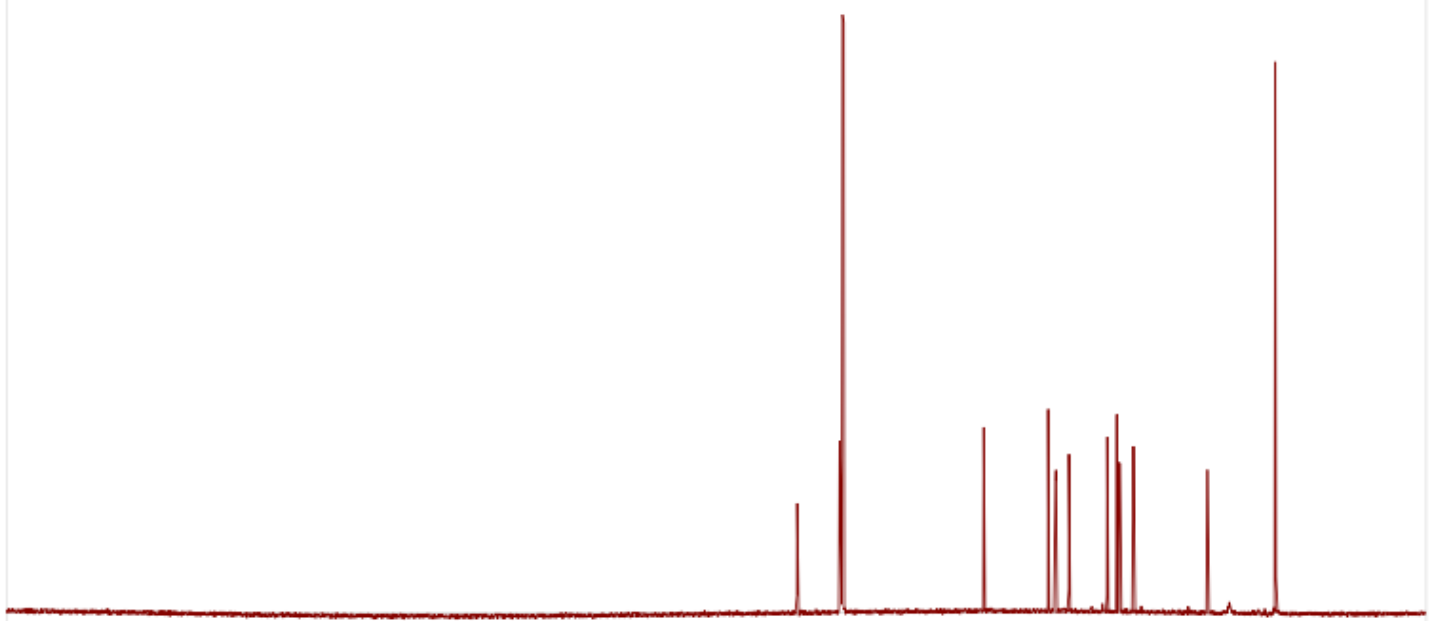

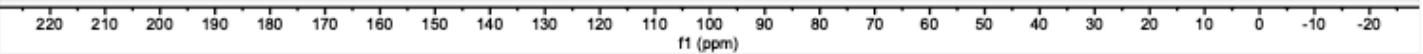




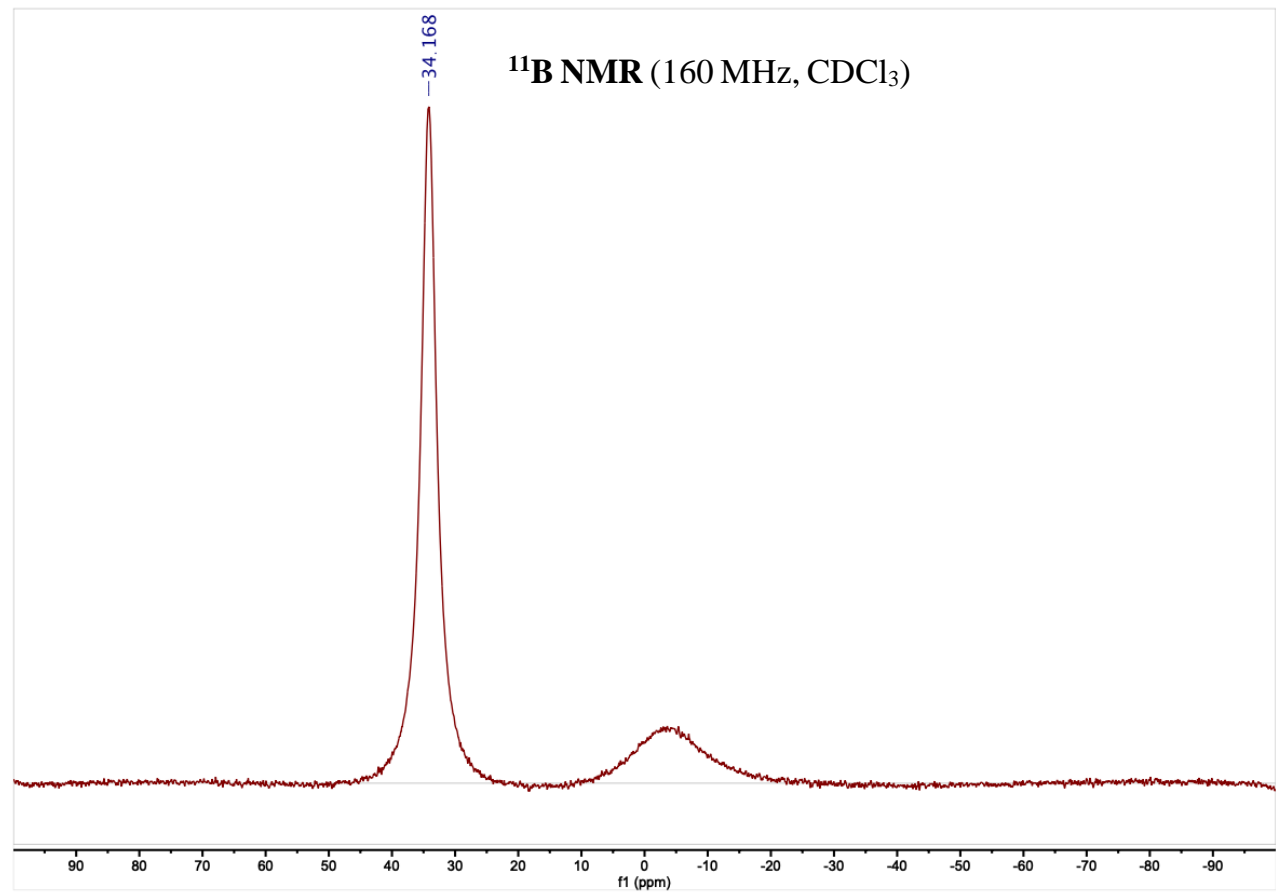<smiles>C[SiH2]CC([SiH2]C)c1ccccc1</smiles>

(24)

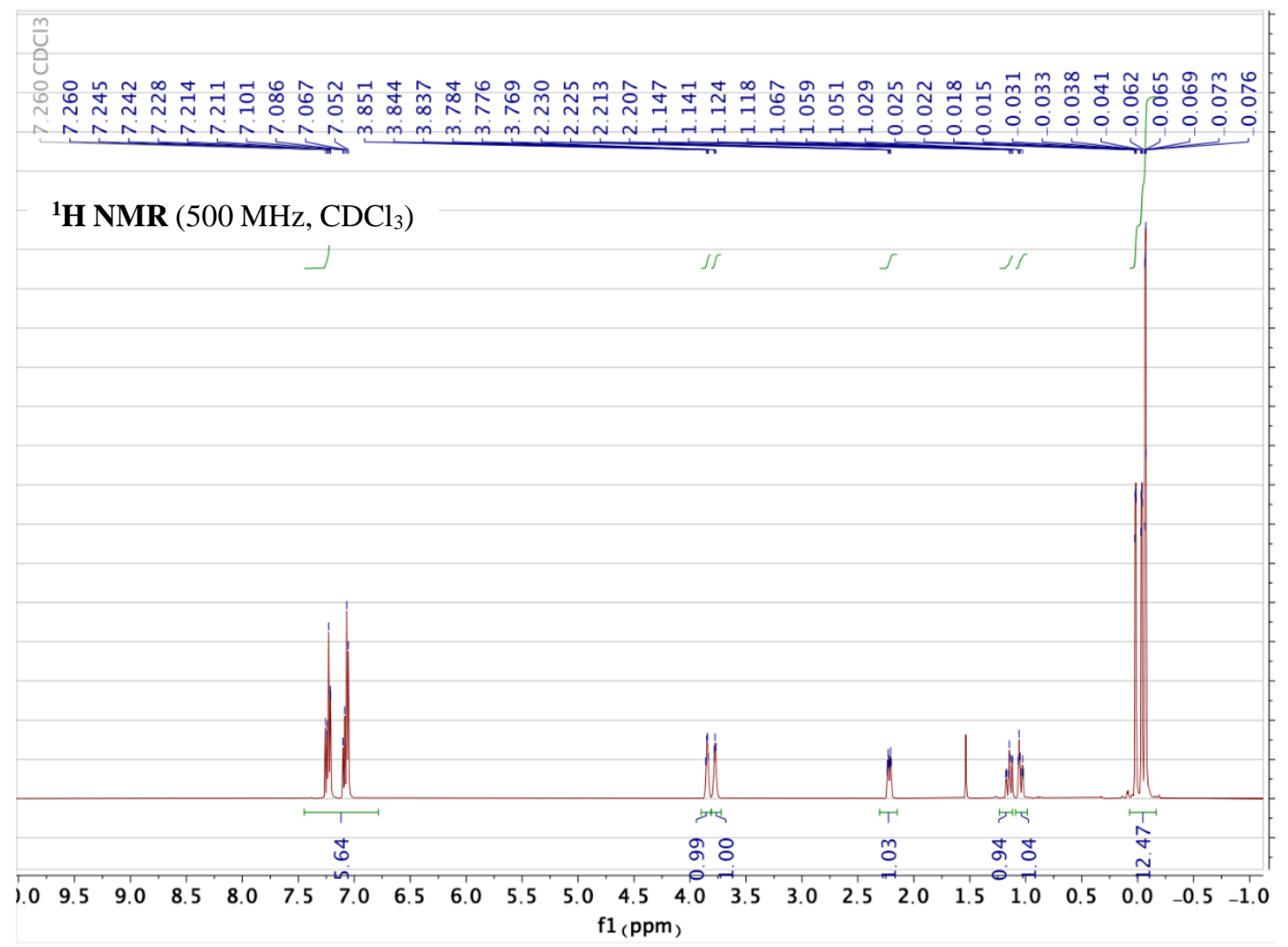




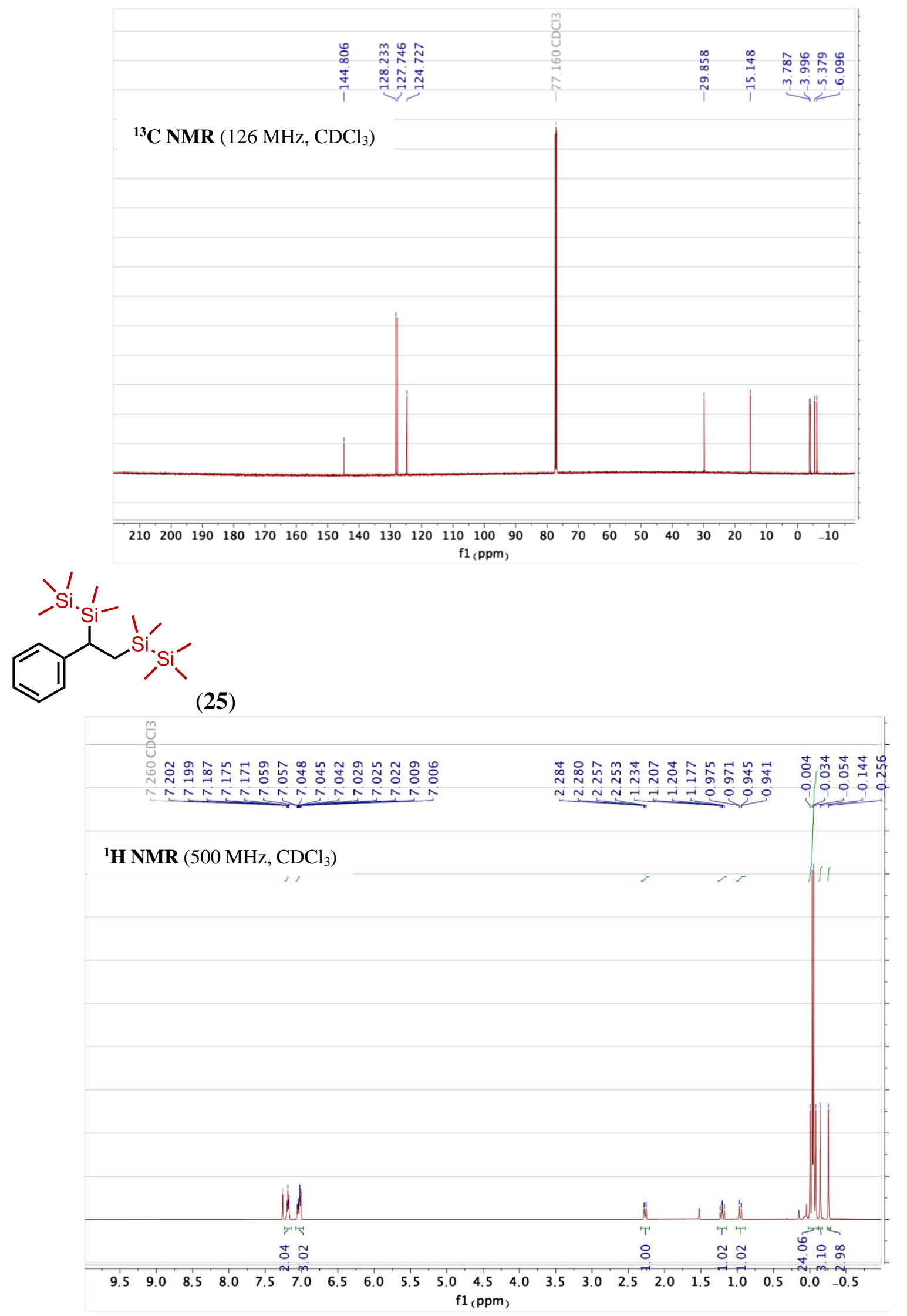

S114 


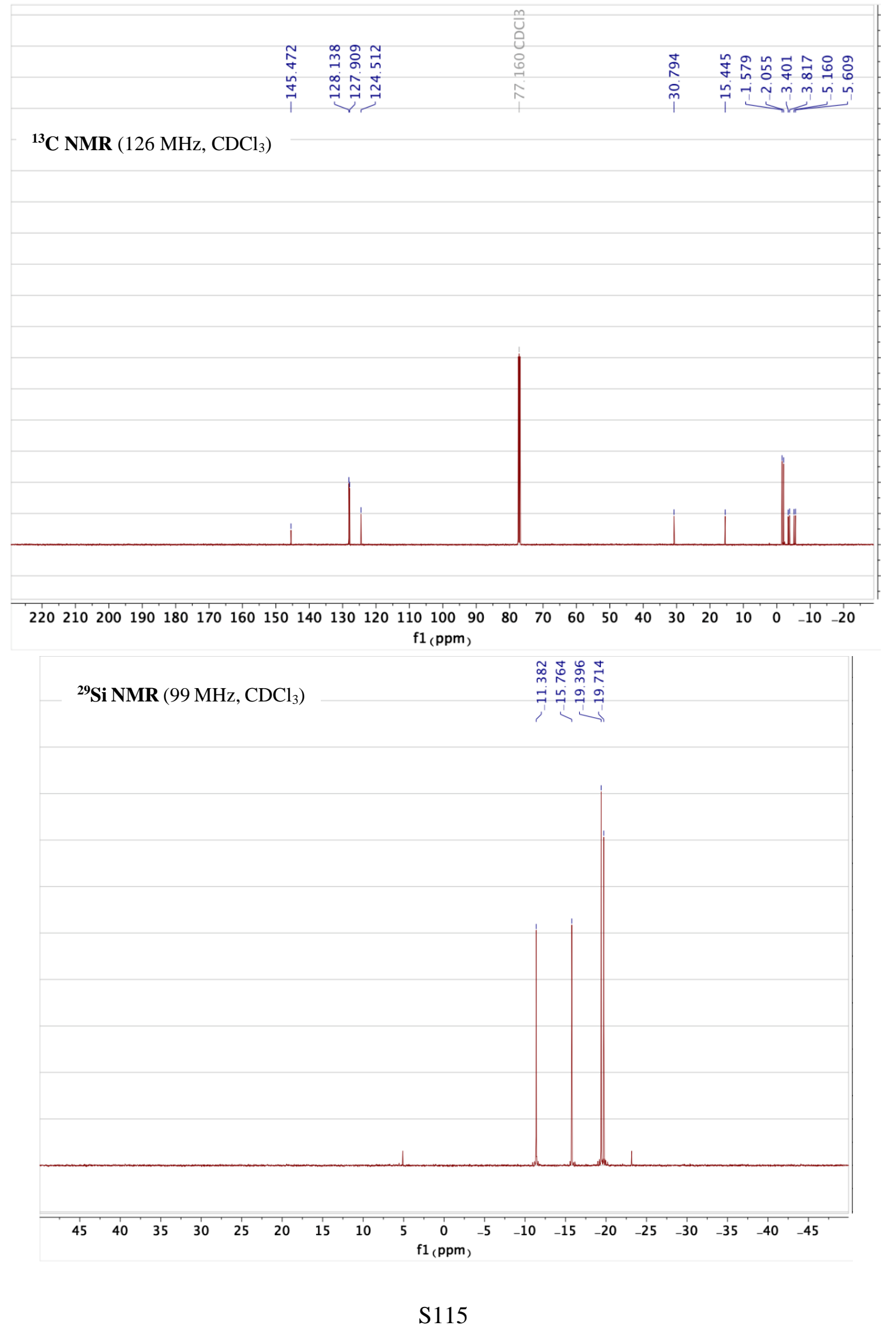



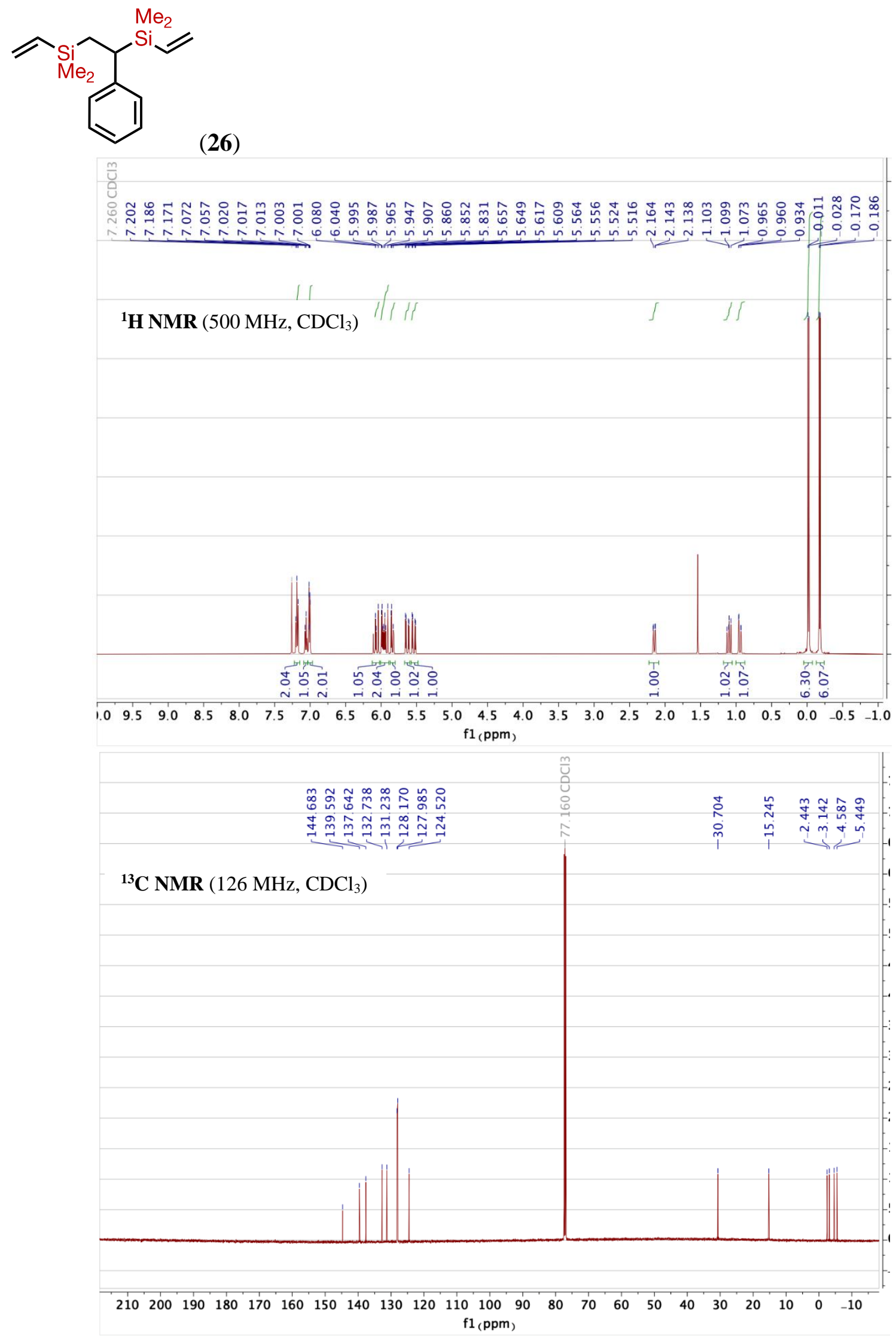

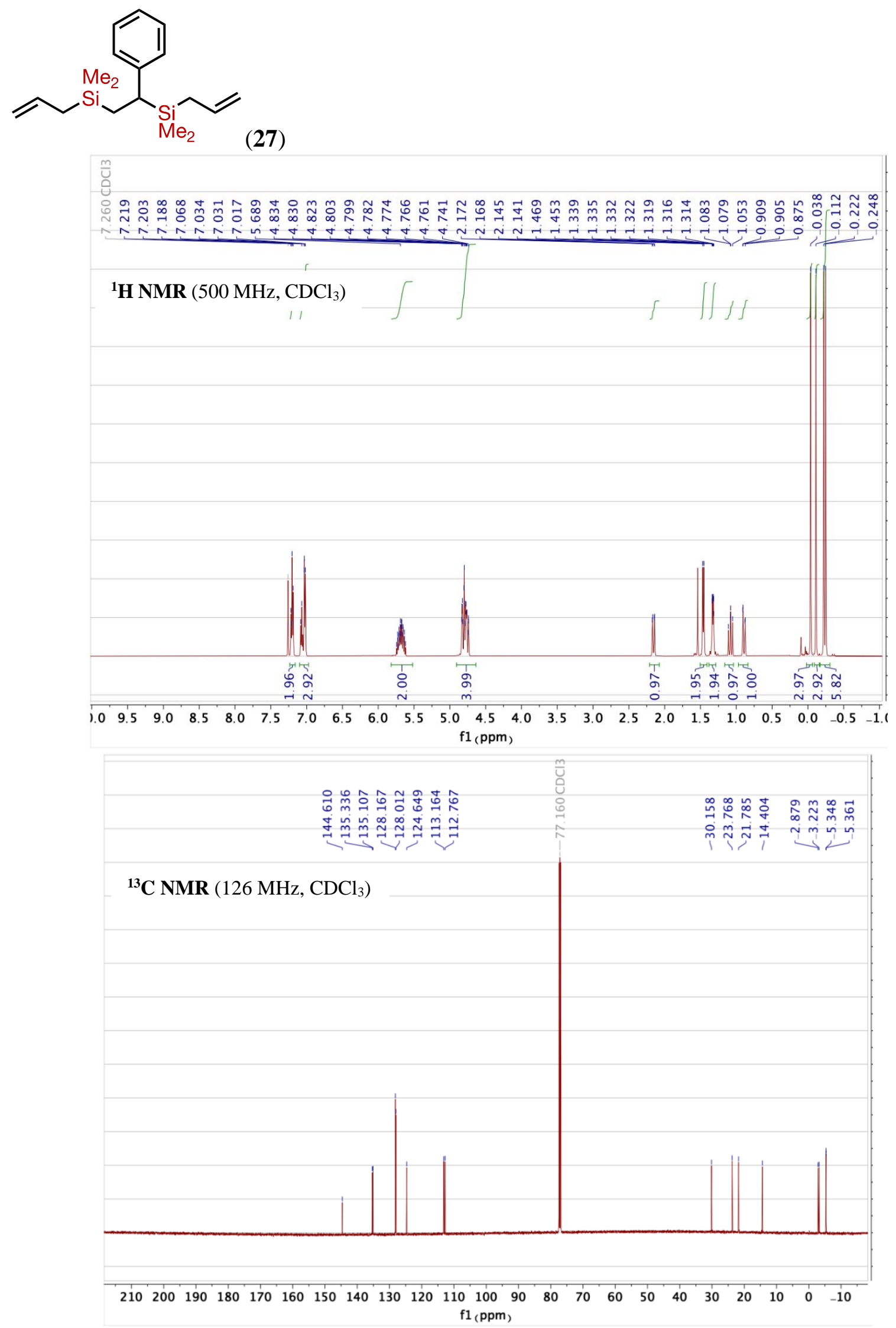


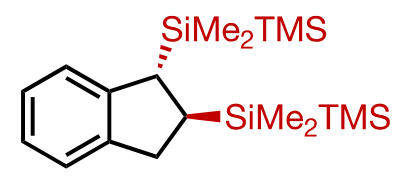

(28)
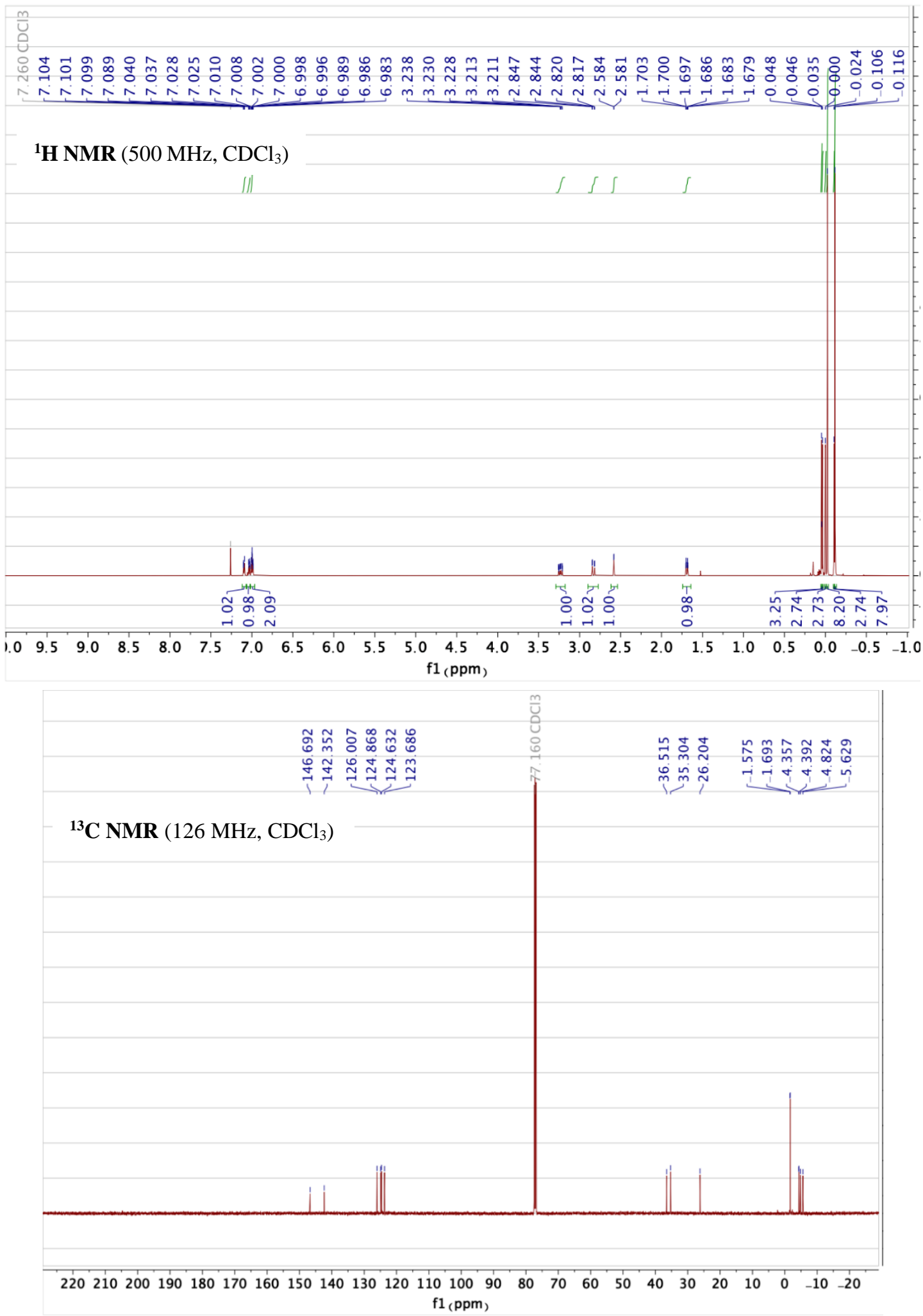
${ }^{29} \mathrm{Si} \mathrm{NMR}\left(99 \mathrm{MHz}, \mathrm{CDCl}_{3}\right)$

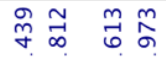

mi

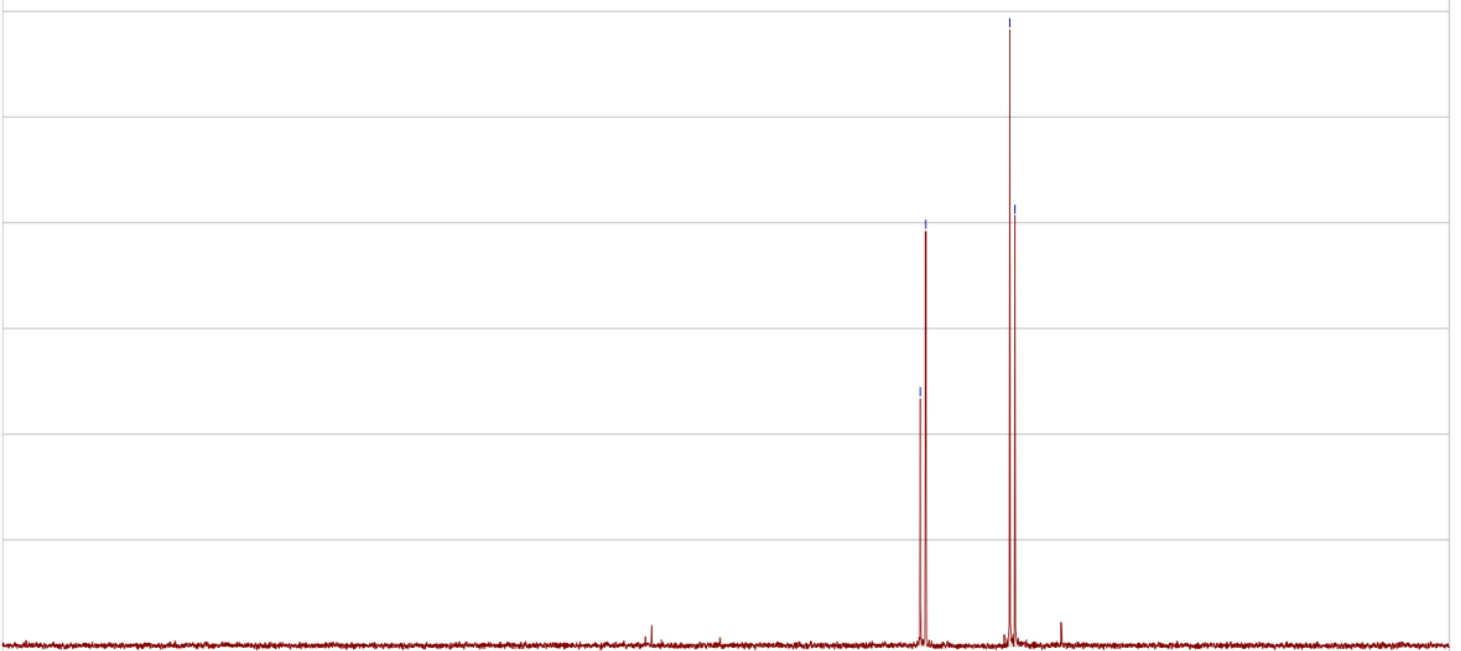

$45 \quad 40$

$40 \quad 35$

$30 \quad 25$

$20 \quad 15$

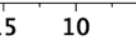

5
$\mathrm{f1}(\mathrm{ppm})$

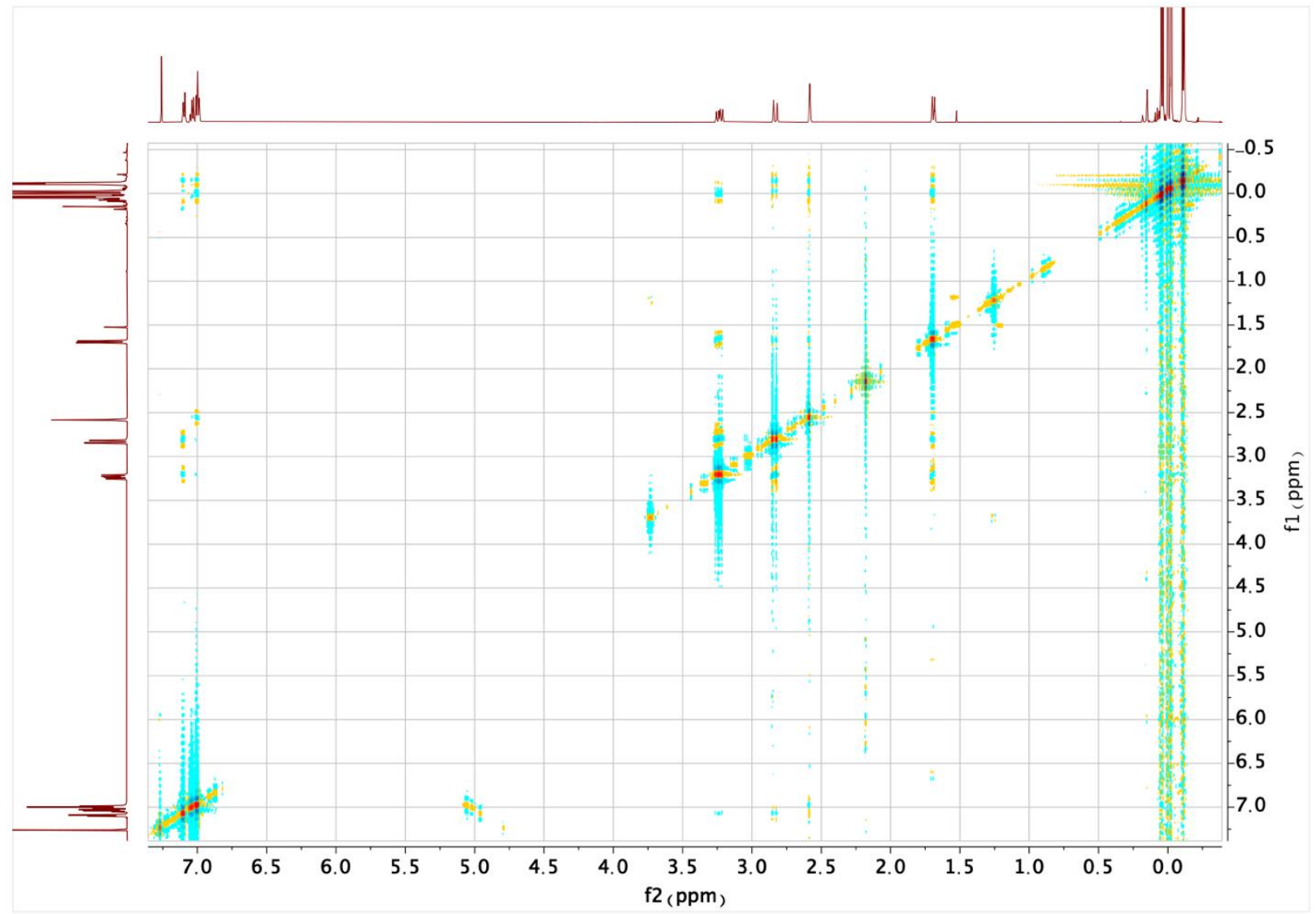


<smiles></smiles>

(29)
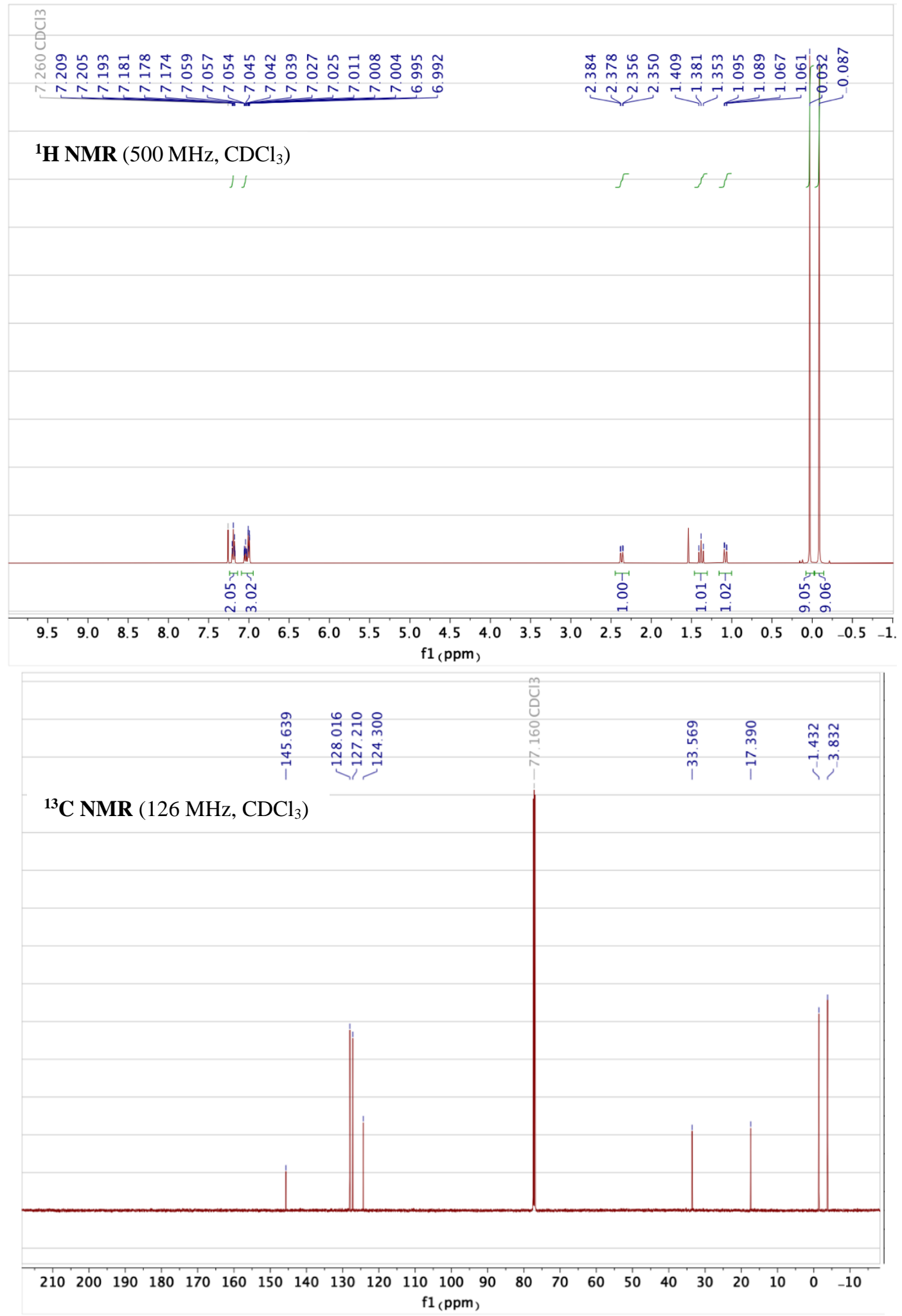


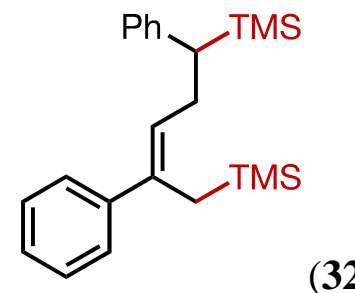

(32)

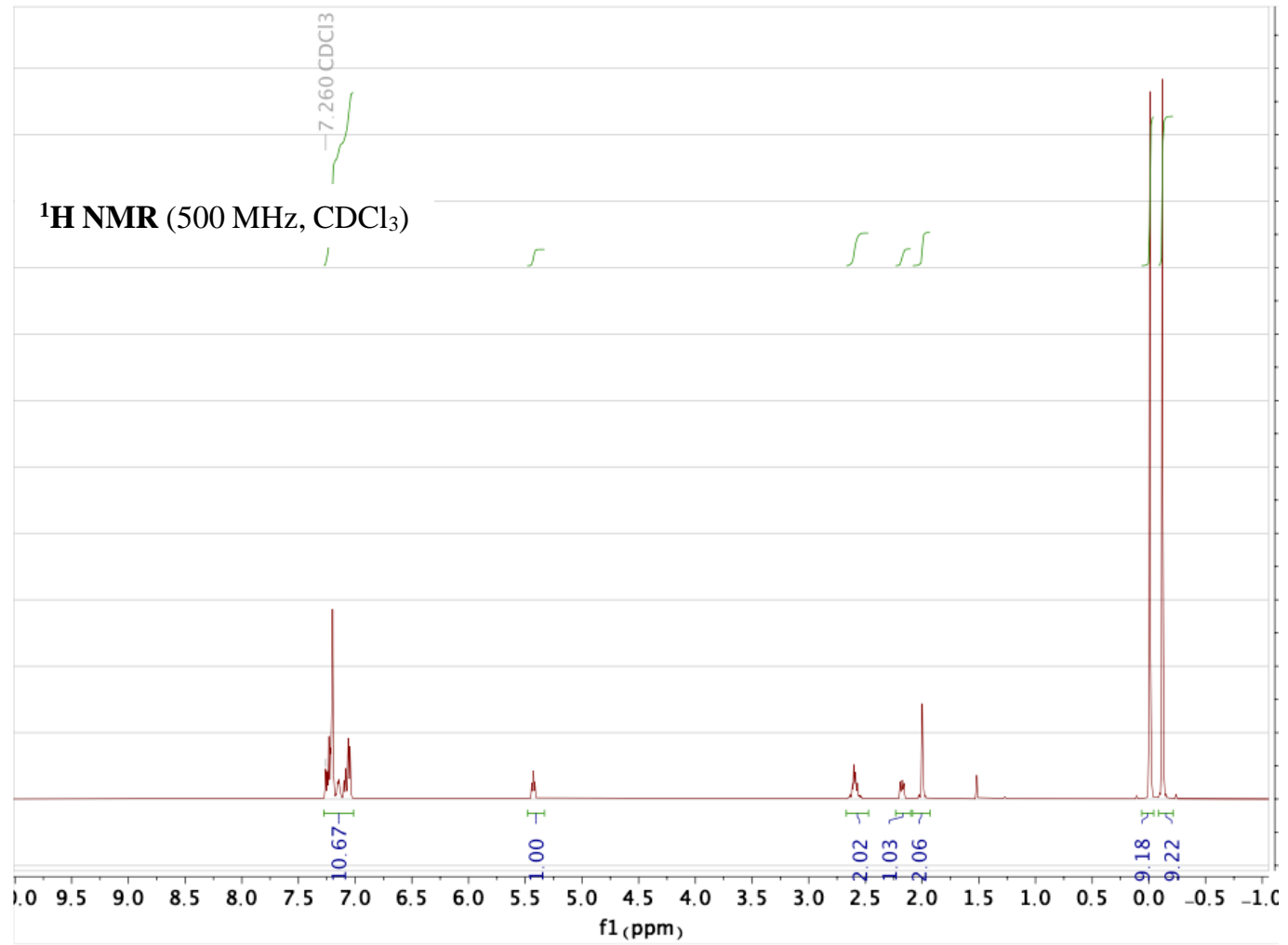




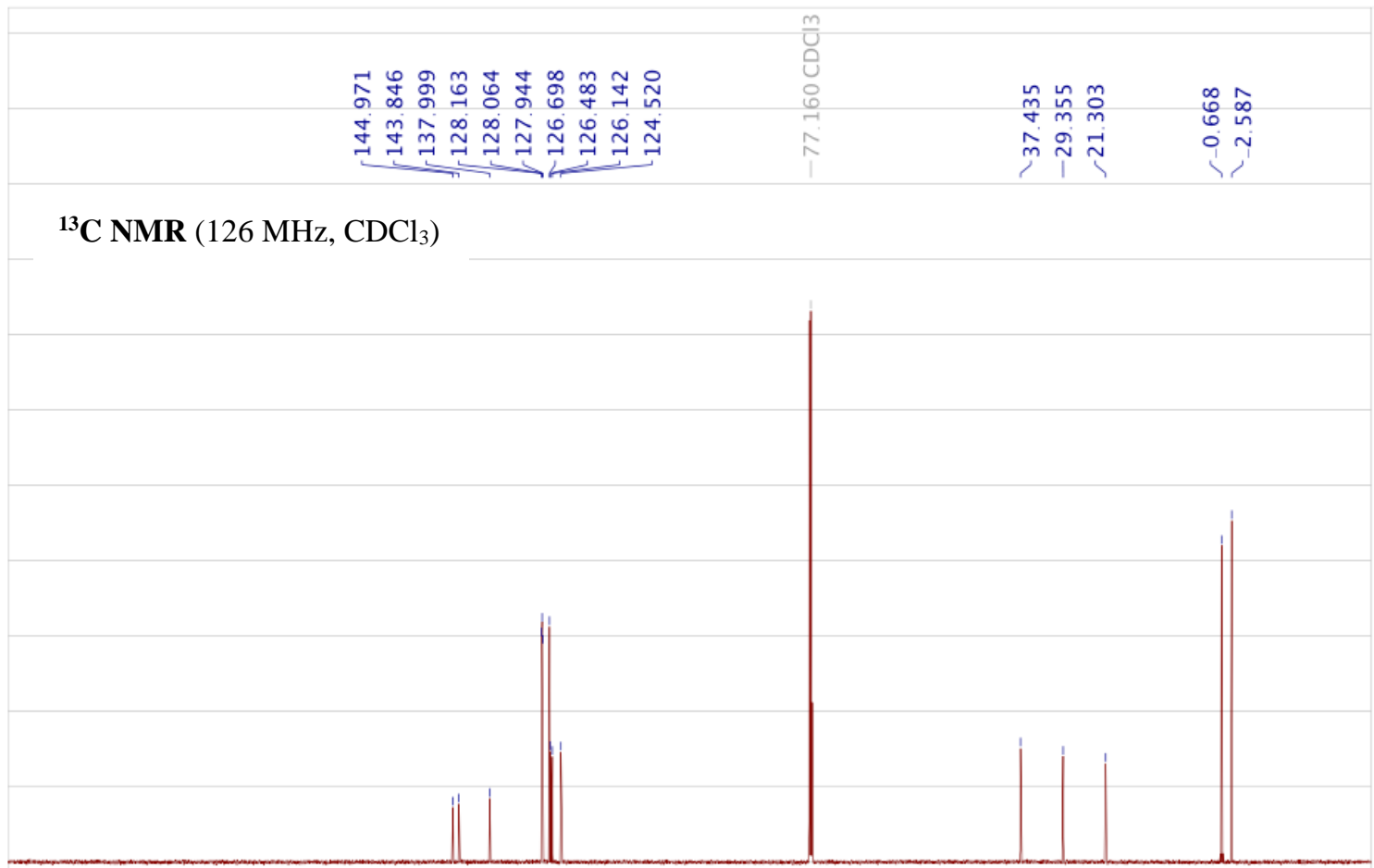

$\begin{array}{lllllllllllllllllllllllllll}220 & 210 & 200 & 190 & 180 & 170 & 160 & 150 & 140 & 130 & 120 & 110 & 100 & 90 & 80 & 70 & 60 & 50 & 40 & 30 & 20 & 10 & 0 & -10 & -20\end{array}$ $\mathrm{f} 1$ (ppm)

${ }^{29} \mathrm{Si} \mathrm{NMR}\left(99 \mathrm{MHz}, \mathrm{CDCl}_{3}\right)$

₹

mir

$\begin{array}{lllllllllllllllllll}45 & 40 & 35 & 30 & 25 & 20 & 15 & 10 & 5 & 0 & -5 & -10 & -15 & -20 & -25 & -30 & -35 & -40 & -45\end{array}$ 


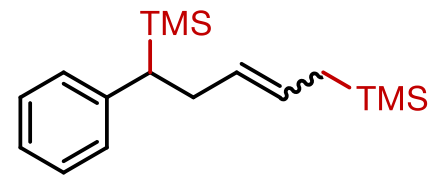

(33)

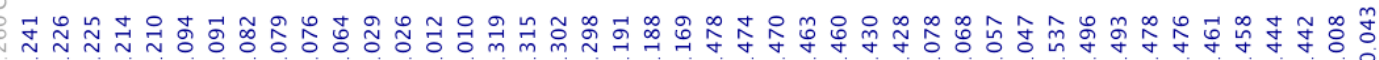

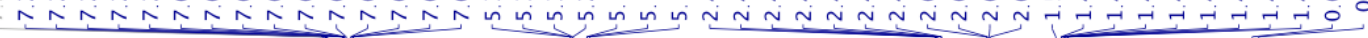

${ }^{1} \mathbf{H}$ NMR $\left(500 \mathrm{MHz}, \mathrm{CDCl}_{3}\right)$

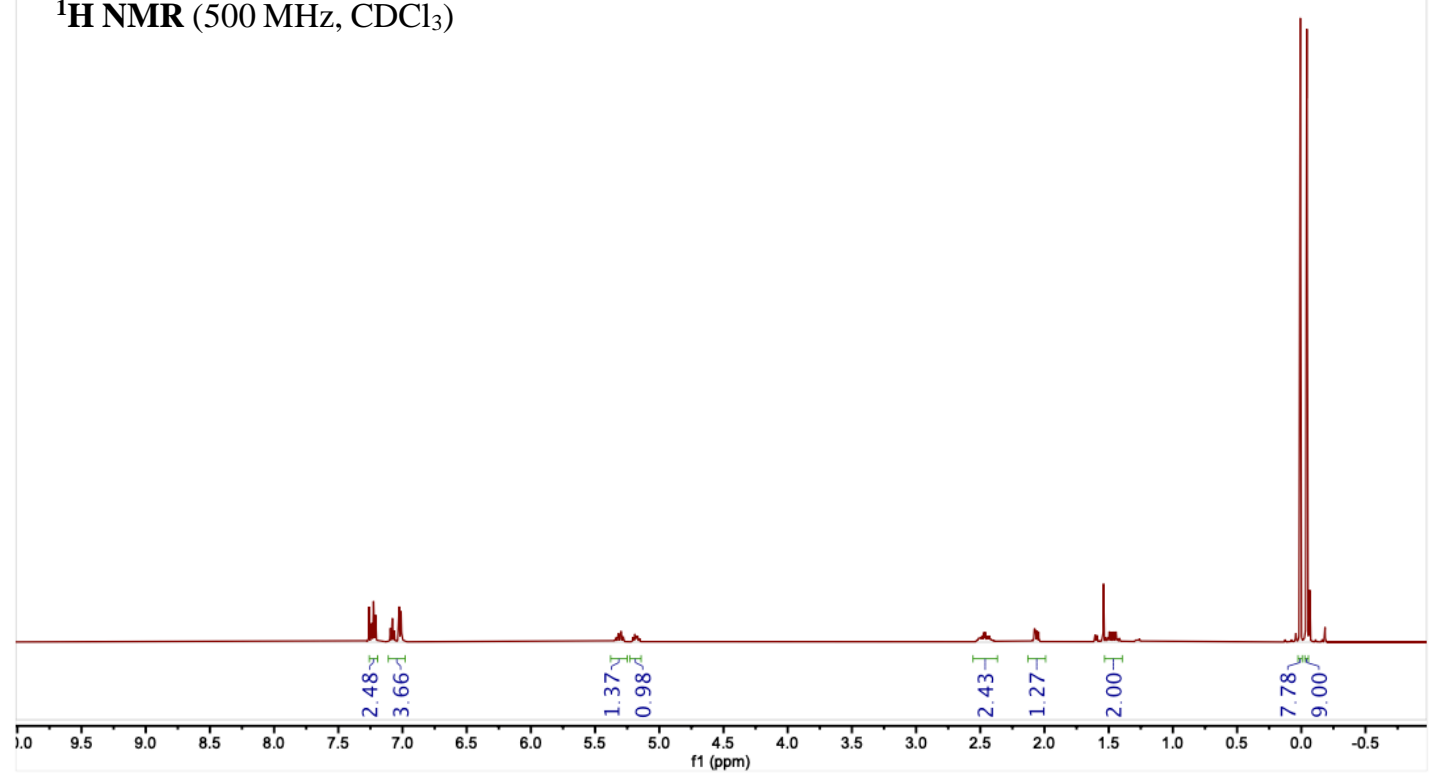

${ }^{13} \mathbf{C}$ NMR $\left(126 \mathrm{MHz}, \mathrm{CDCl}_{3}\right)$

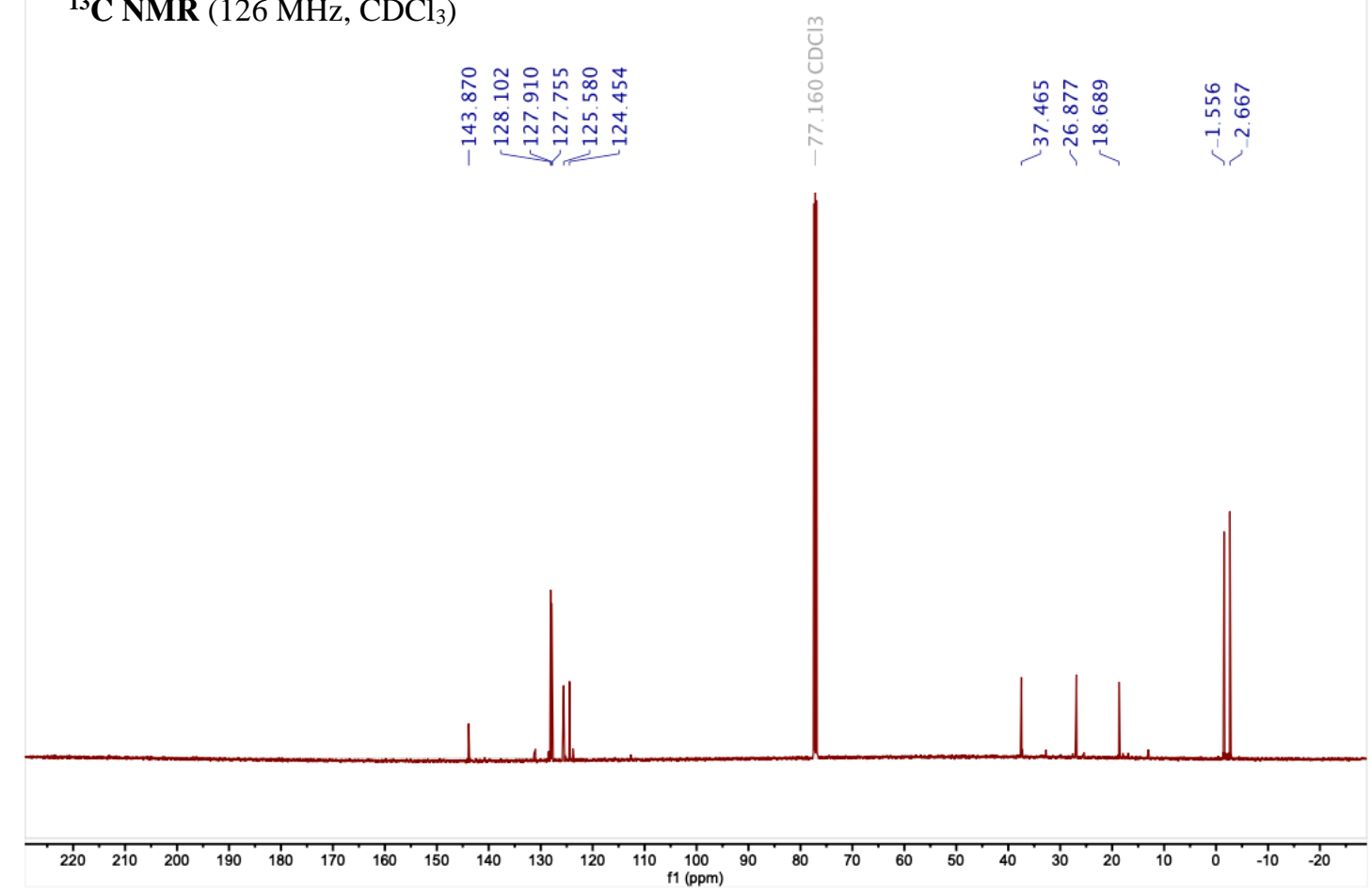

Trace amount of the other isomer cannot be separated and results in integration off. 


\section{Crude mixture}
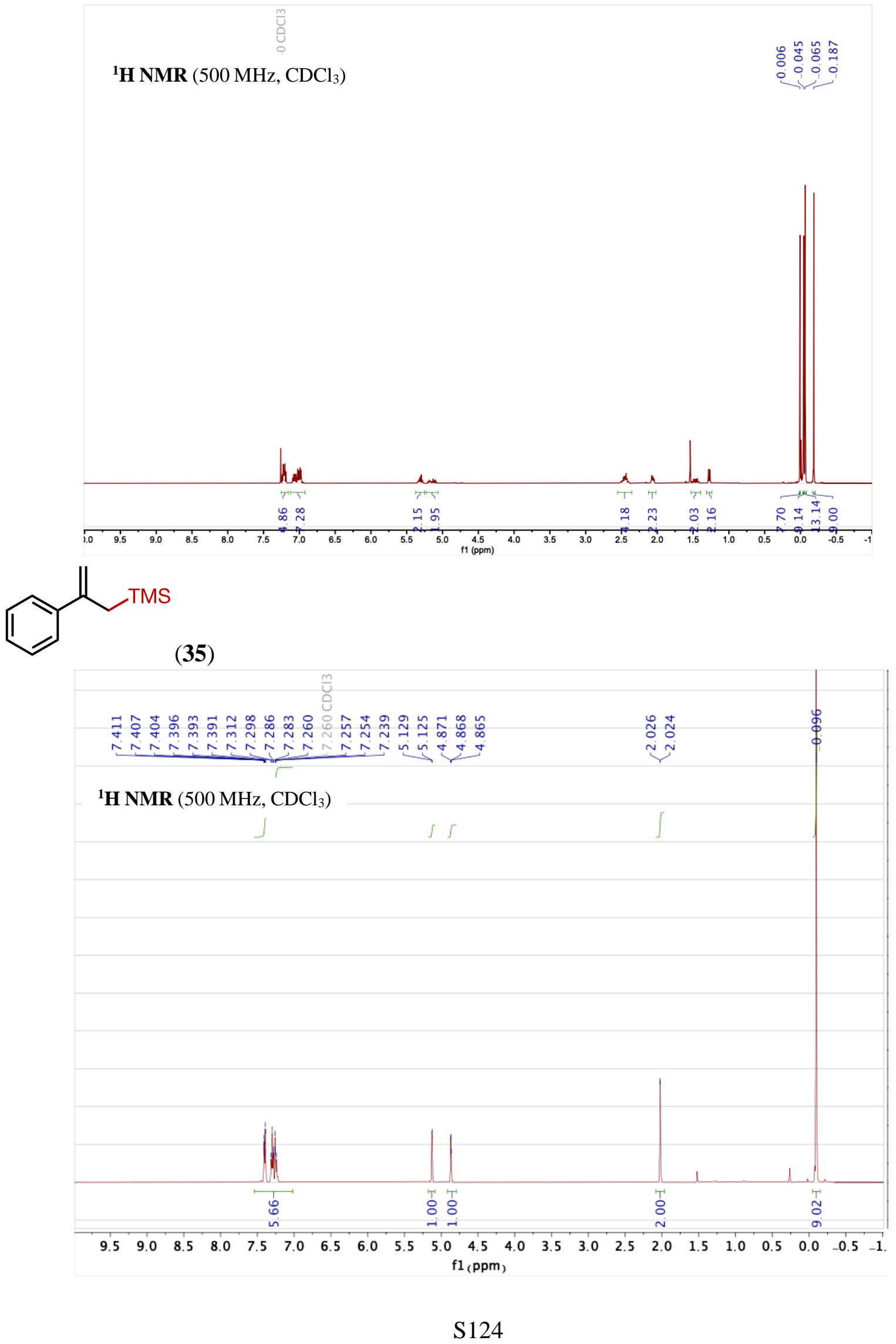


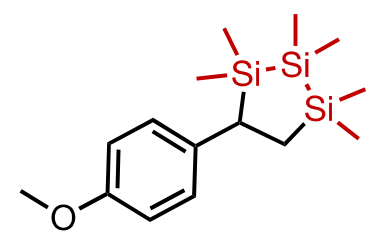

(36)

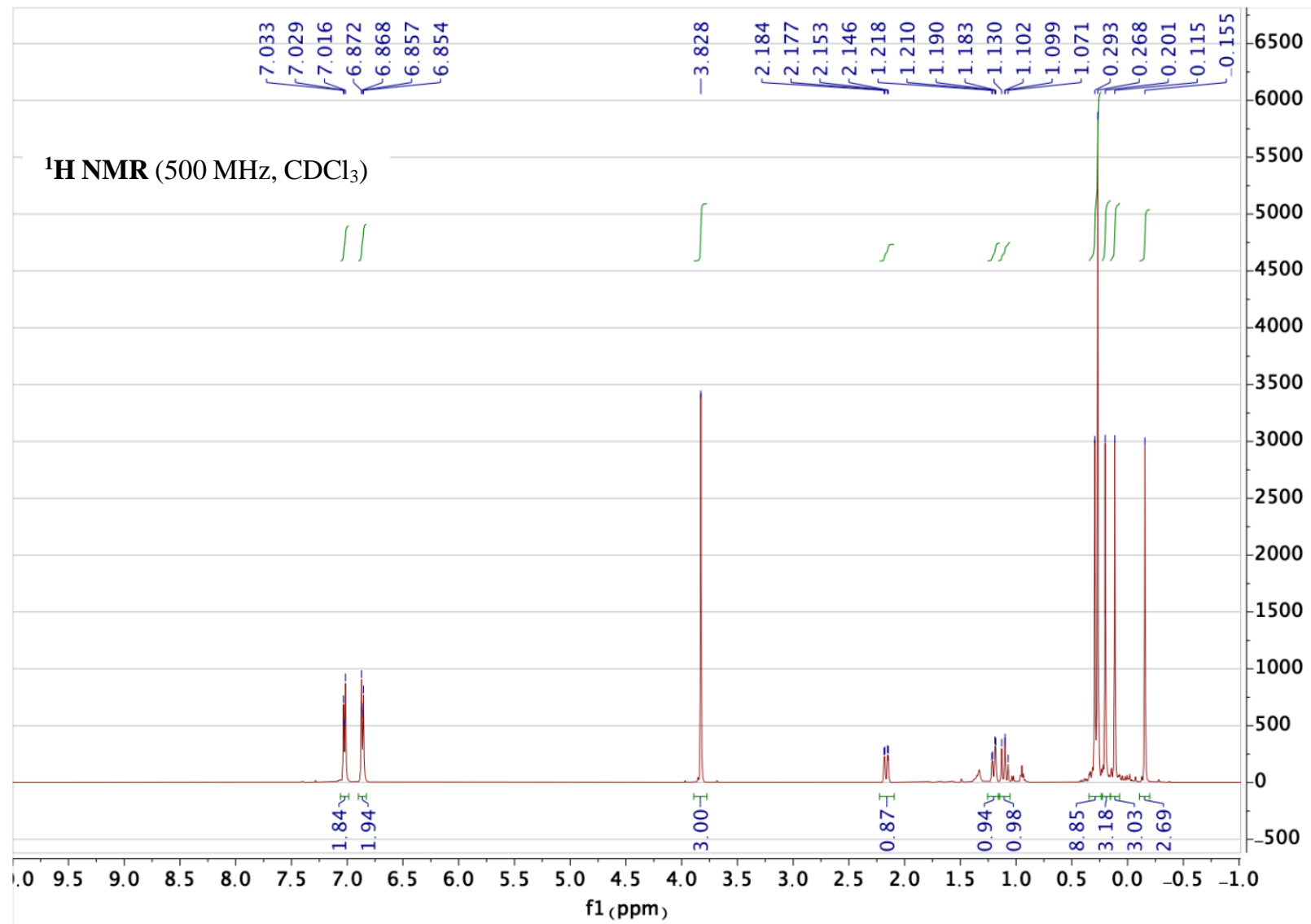



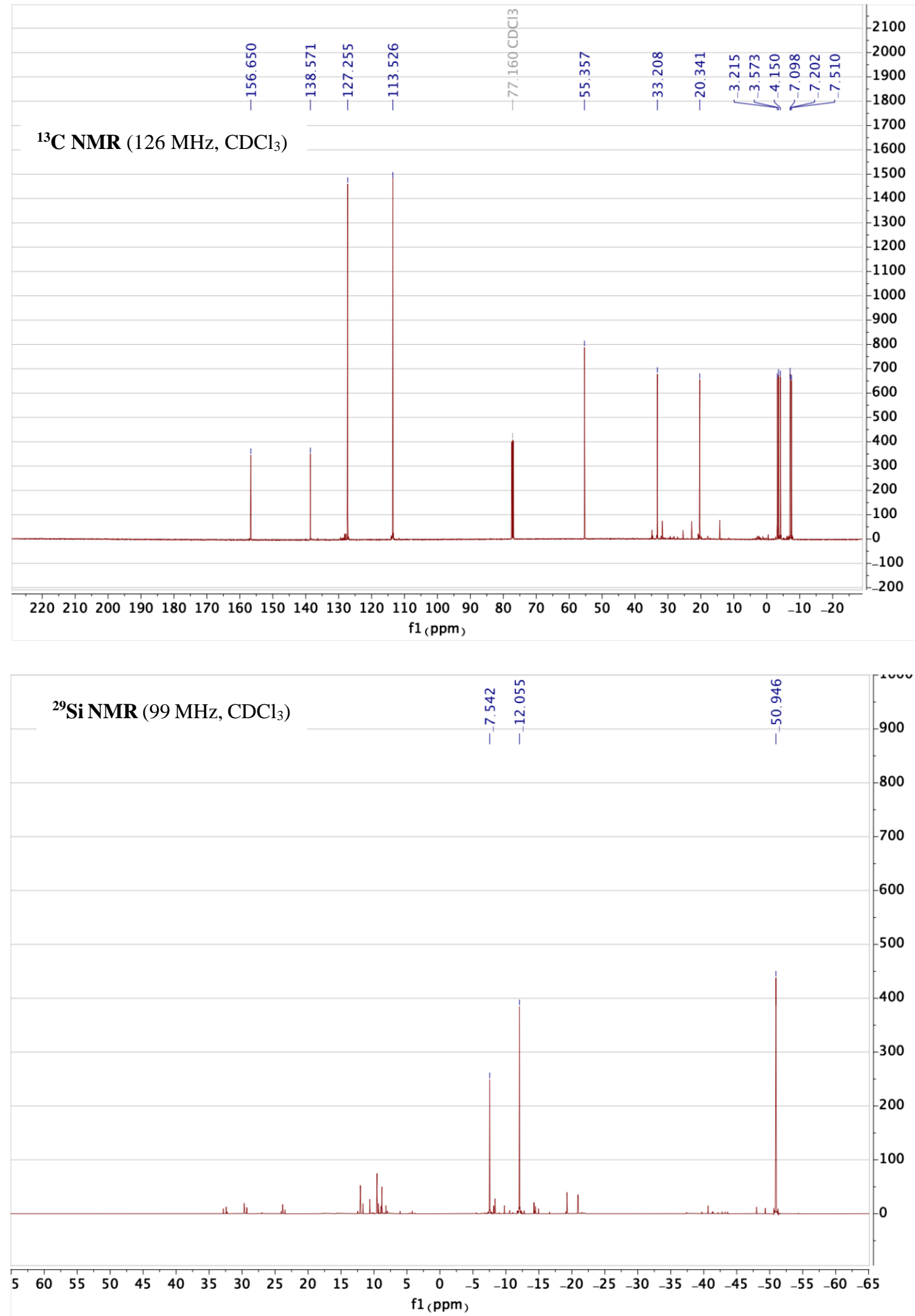

Product unstable on the timescale of NMR. 
<smiles>C[Si](C)(C)CC(c1ccn(-c2ccccc2)n1)[Si](C)(C)[Si](C)(C)[Si](C)(C)C</smiles>

(37)

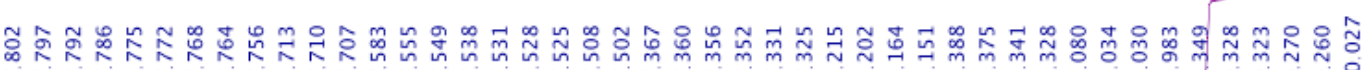

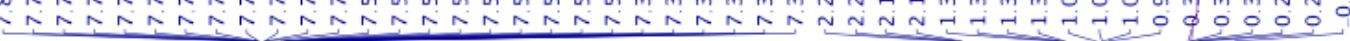

${ }^{\mathbf{1}} \mathbf{H} \mathbf{N M R}\left(500 \mathrm{MHz}, \mathrm{CDCl}_{3}\right)$

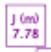

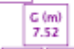

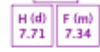

7.58
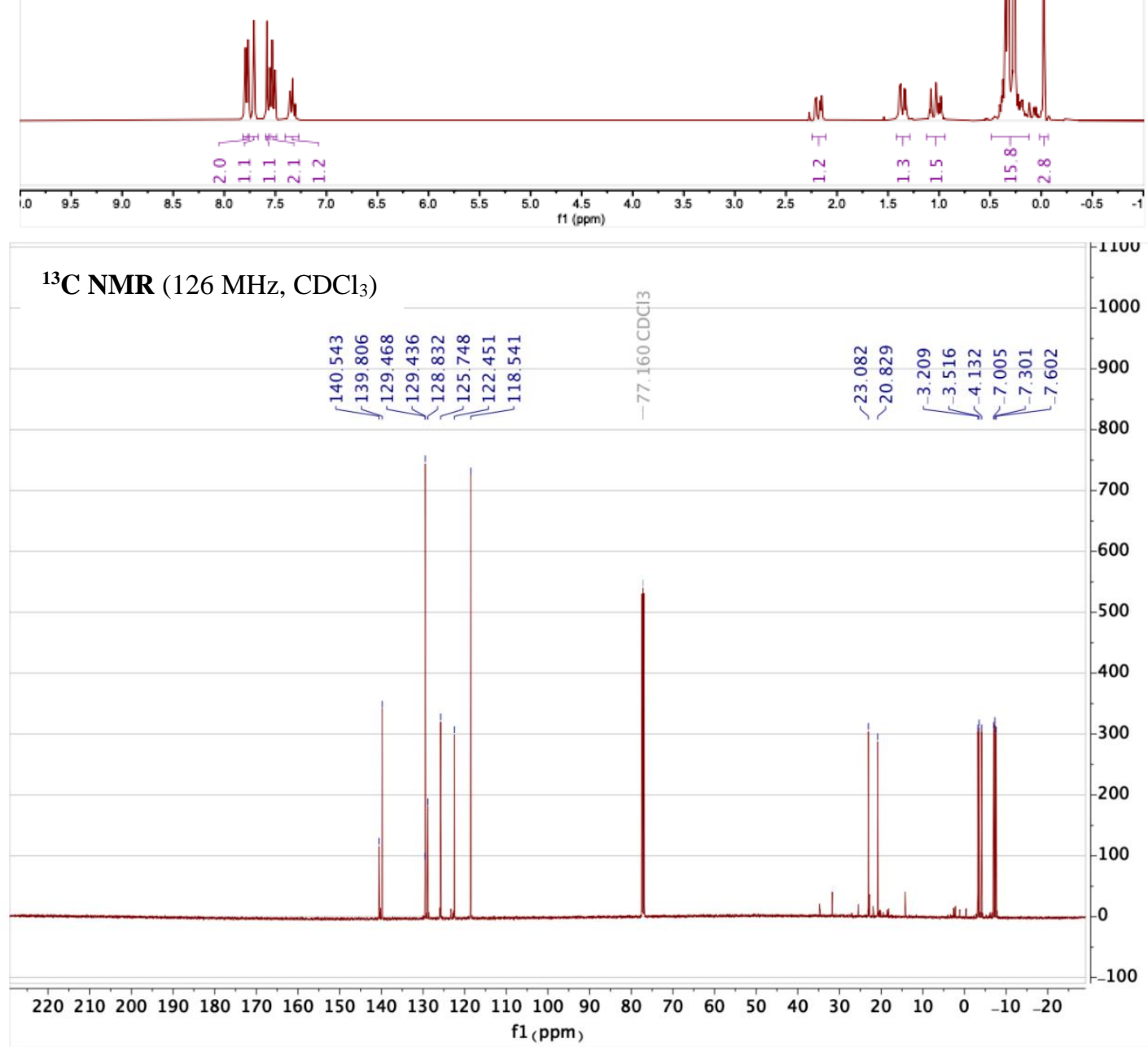

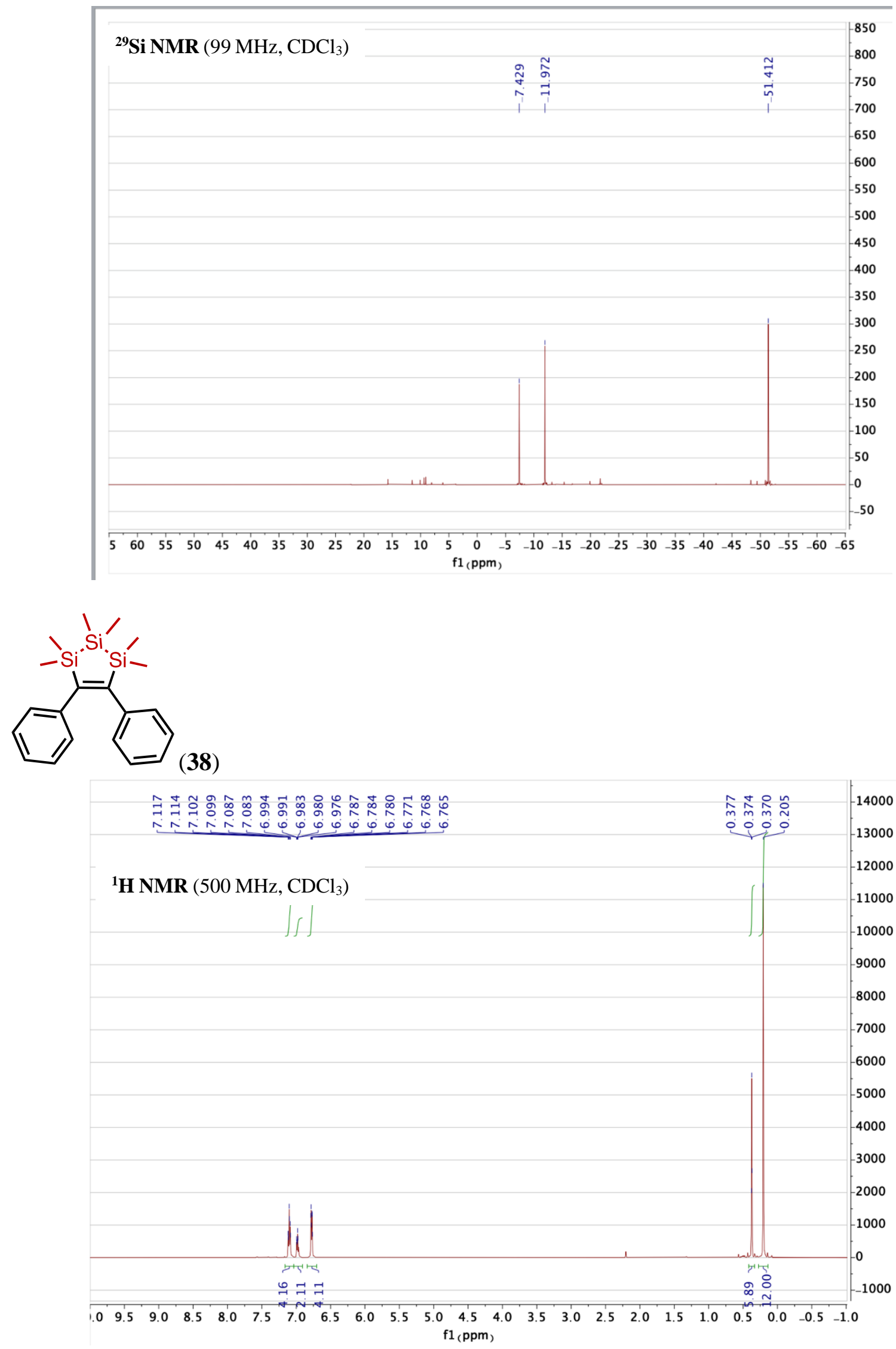


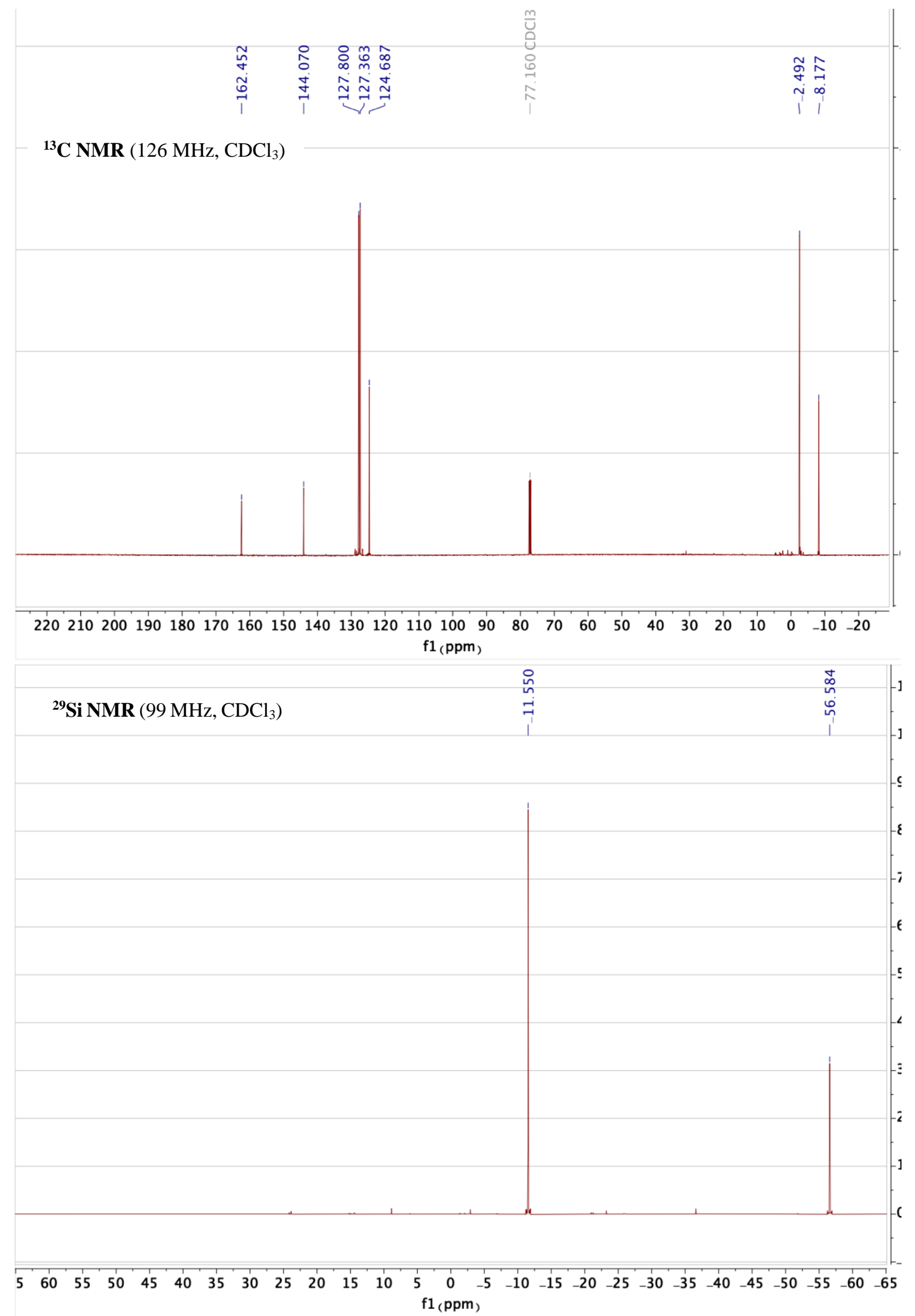




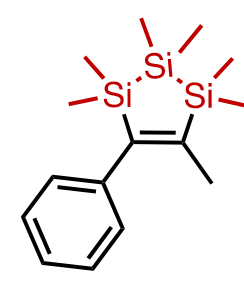

(39)
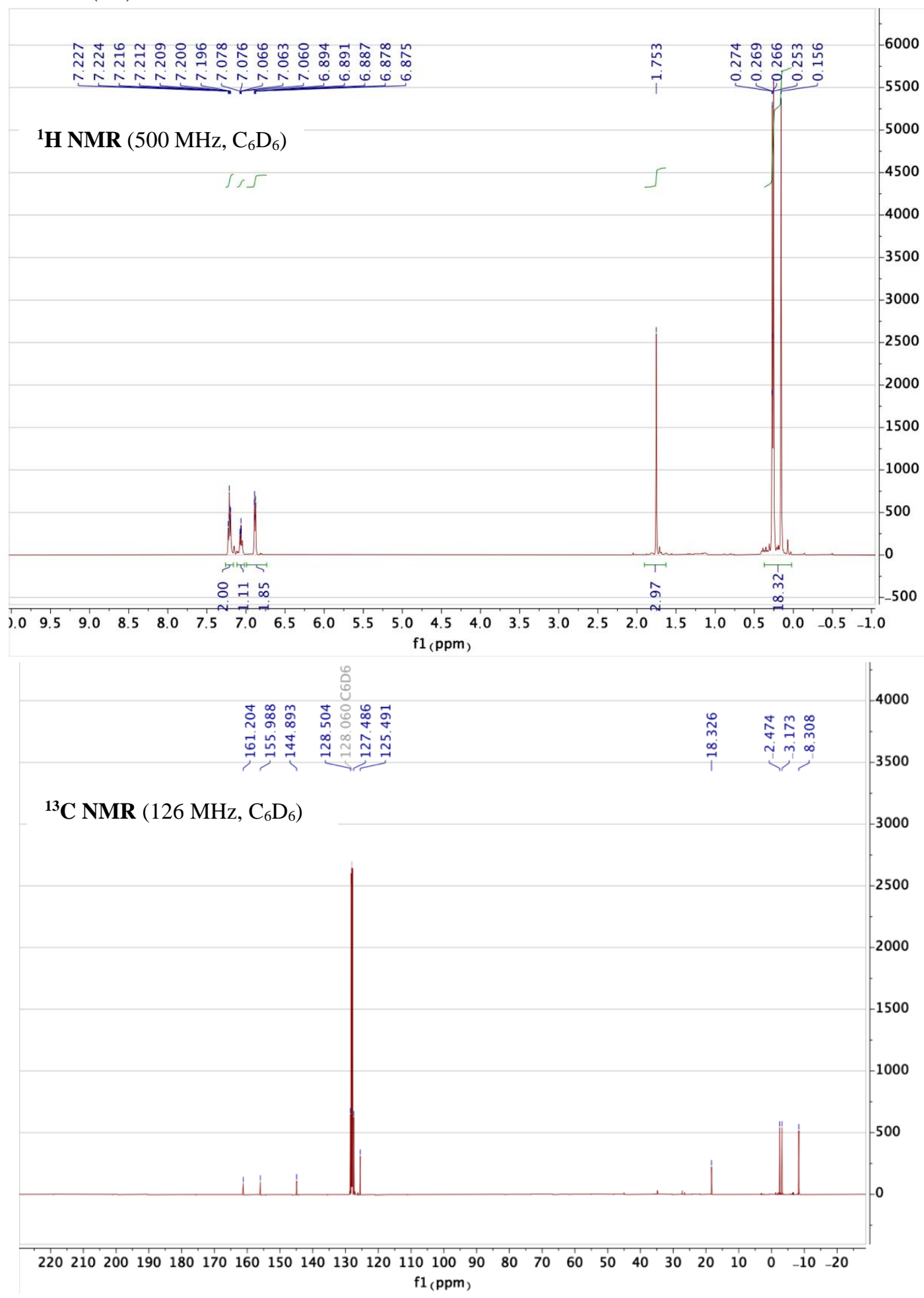

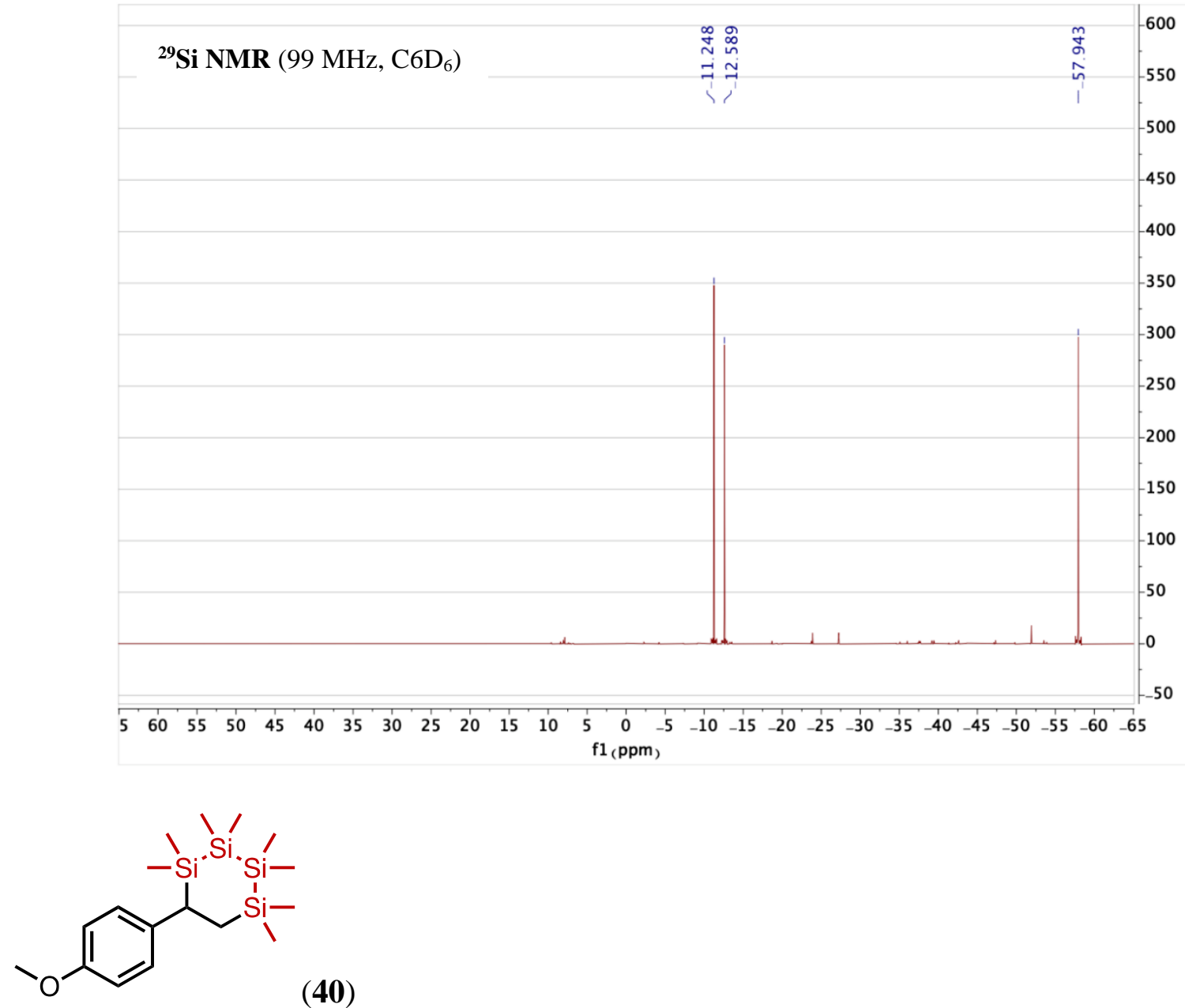

(40)

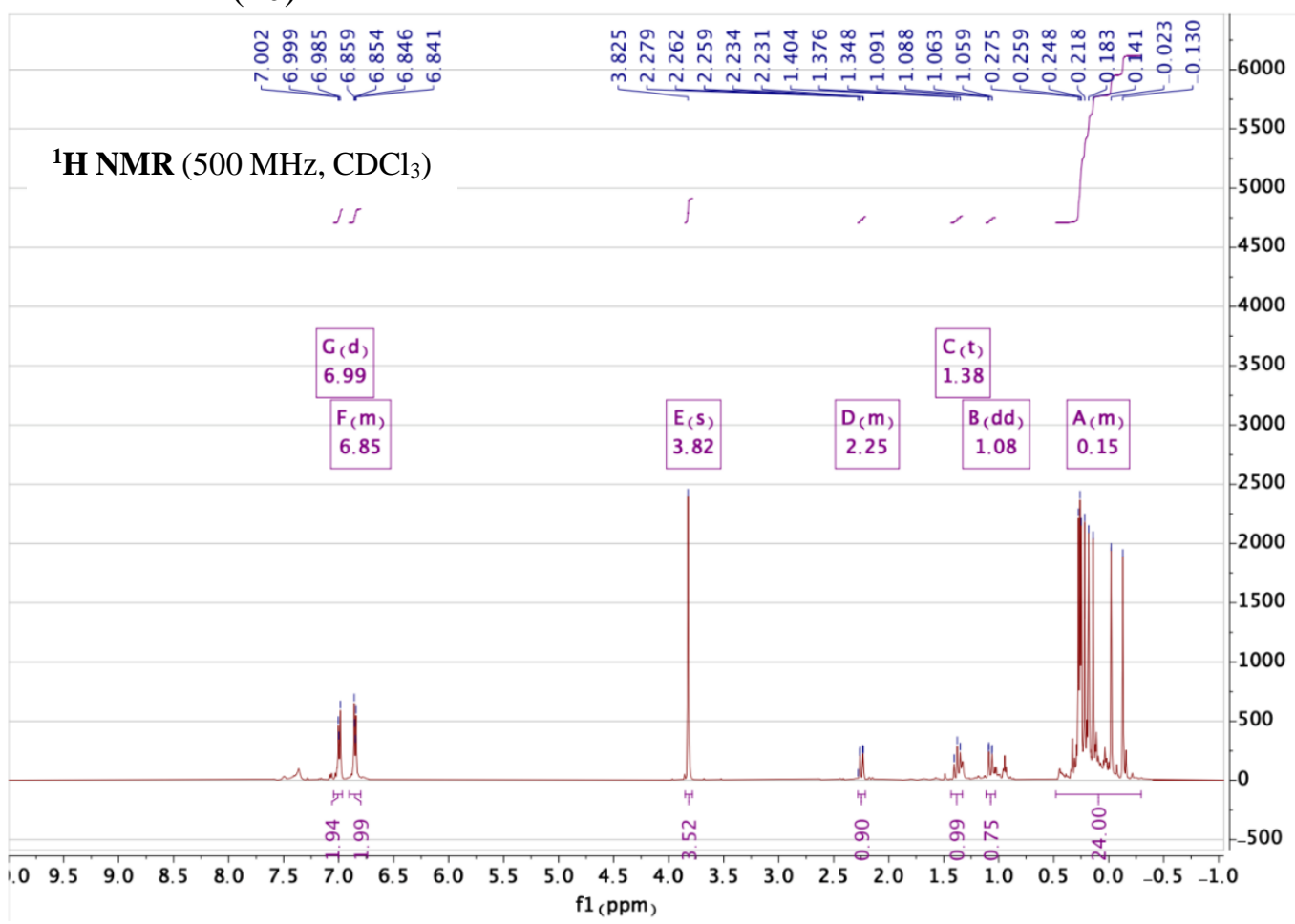




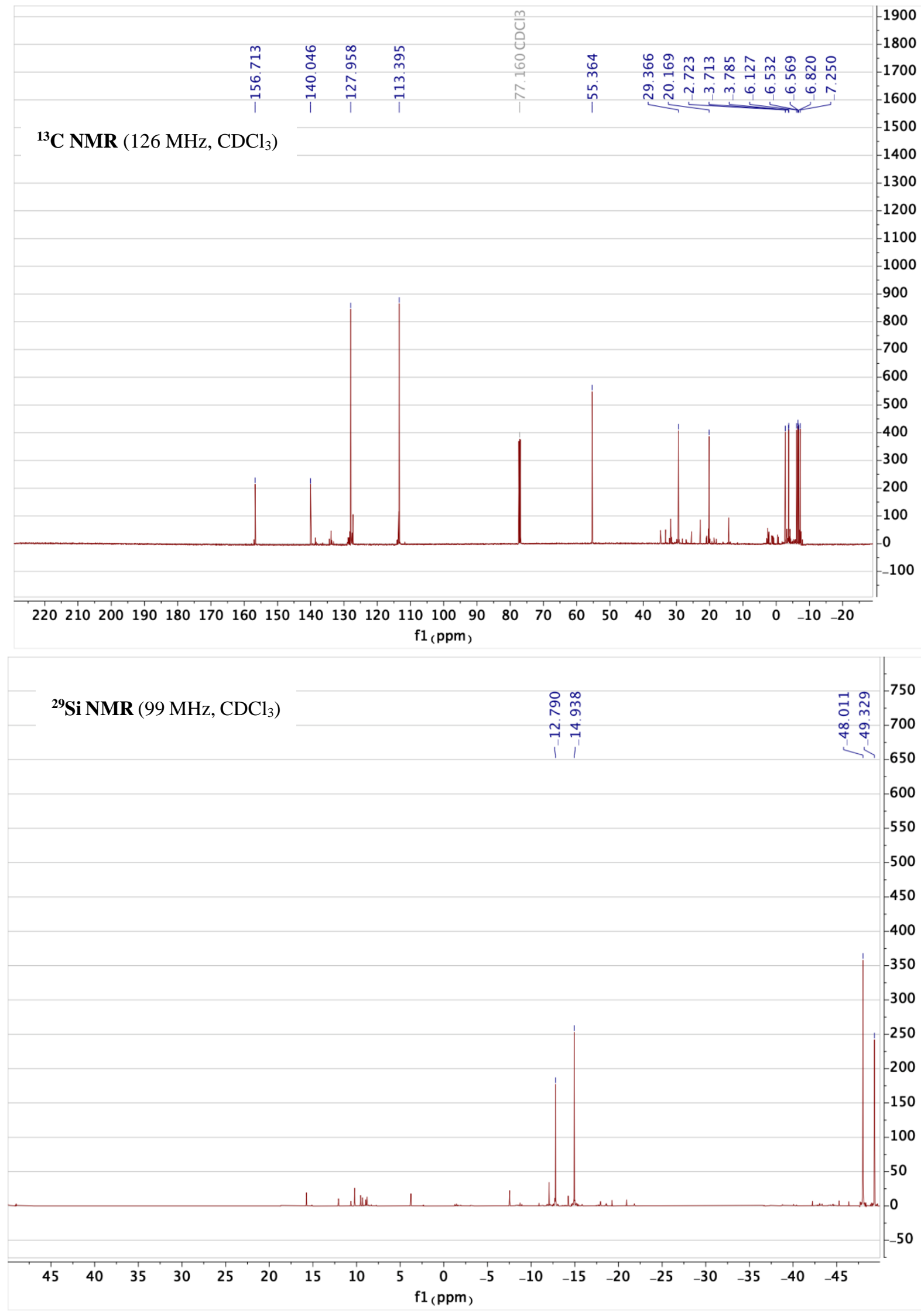


<smiles>C[Si]1(C)CC(c2ccccc2)[Si](C)(C)O1</smiles>

(41)
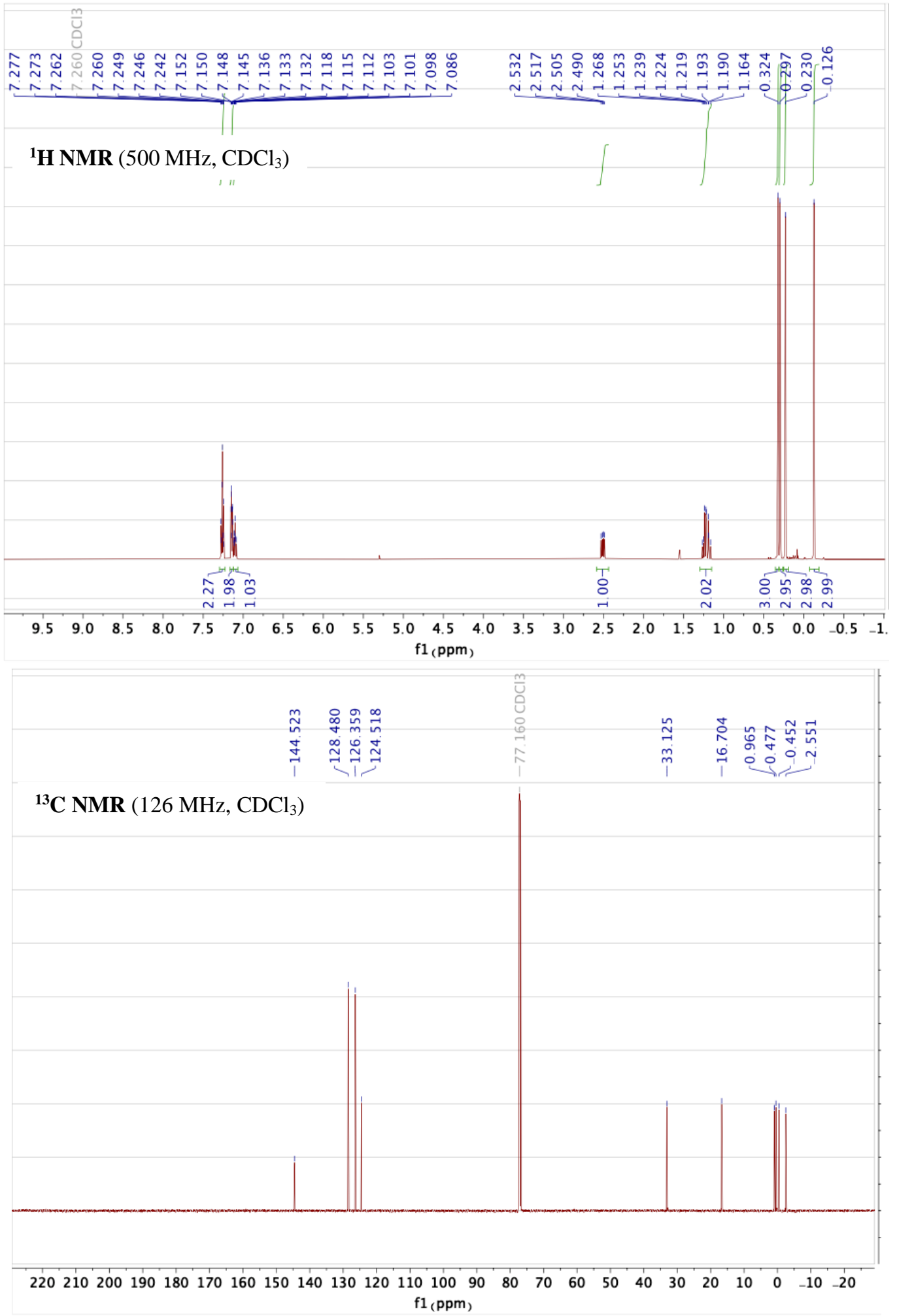


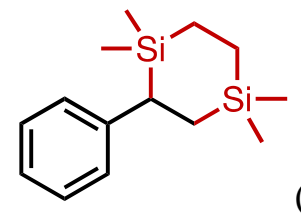

(42)

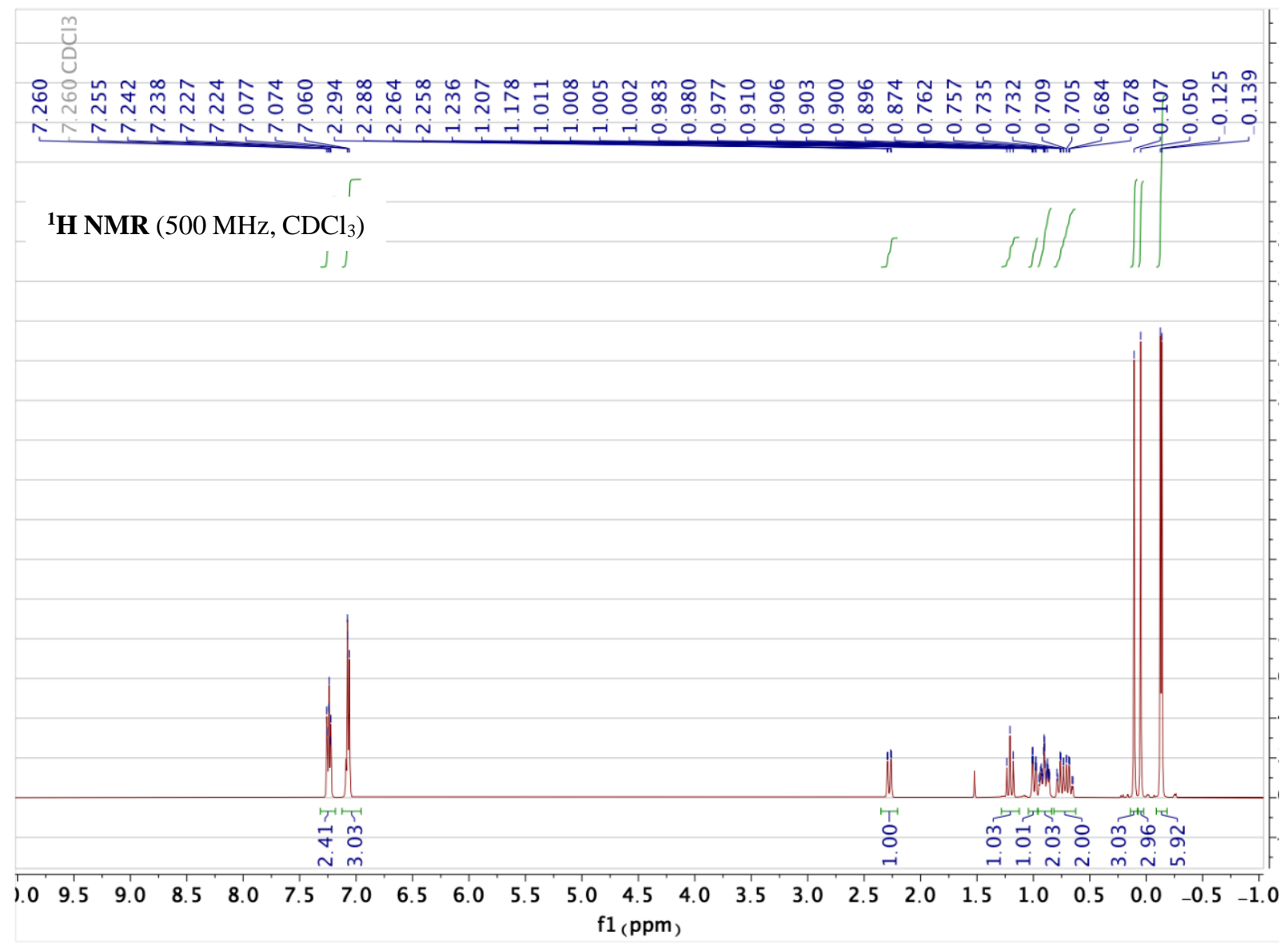




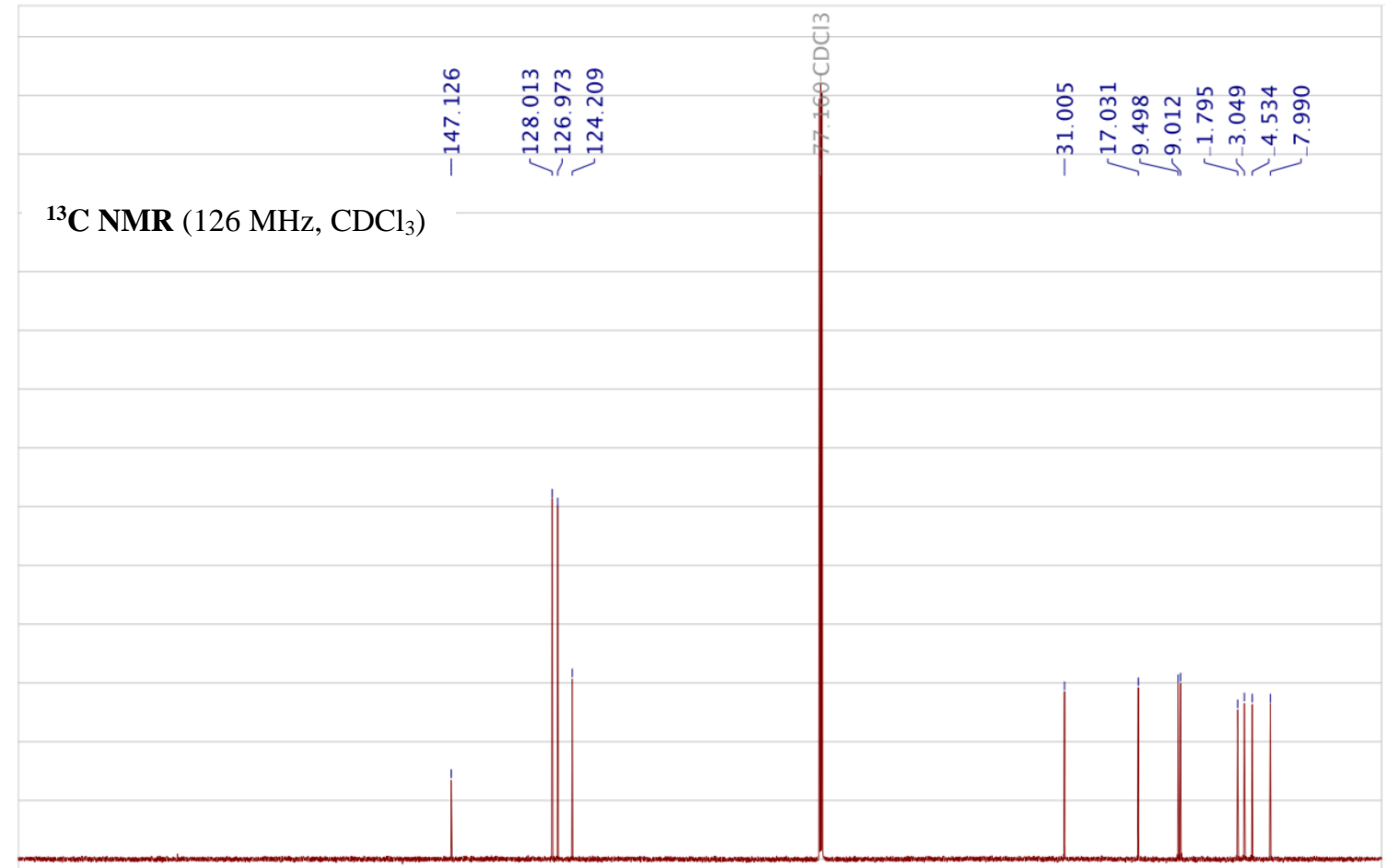

$\begin{array}{llllllllllllllllllllllllllll}220 & 210 & 200 & 190 & 180 & 170 & 160 & 150 & 140 & 130 & 120 & 110 & 100 & 90 & 80 & 70 & 60 & 50 & 40 & 30 & 20 & 10 & 0 & -10 & -20\end{array}$ f1 ( $p p m)$

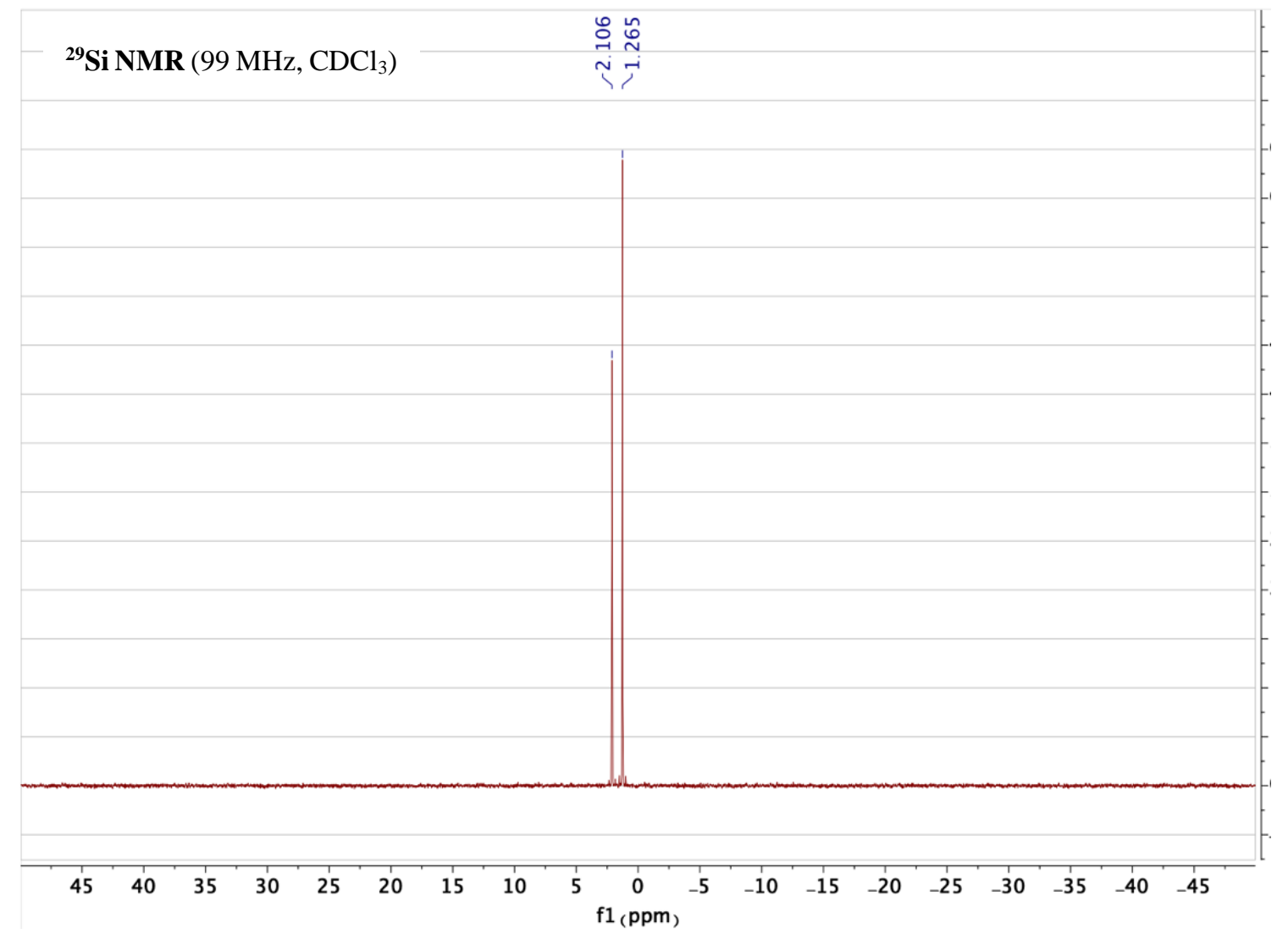




\section{Hydrosilylated Products}<smiles>COc1ccc(CC[AsH2])cc1</smiles>

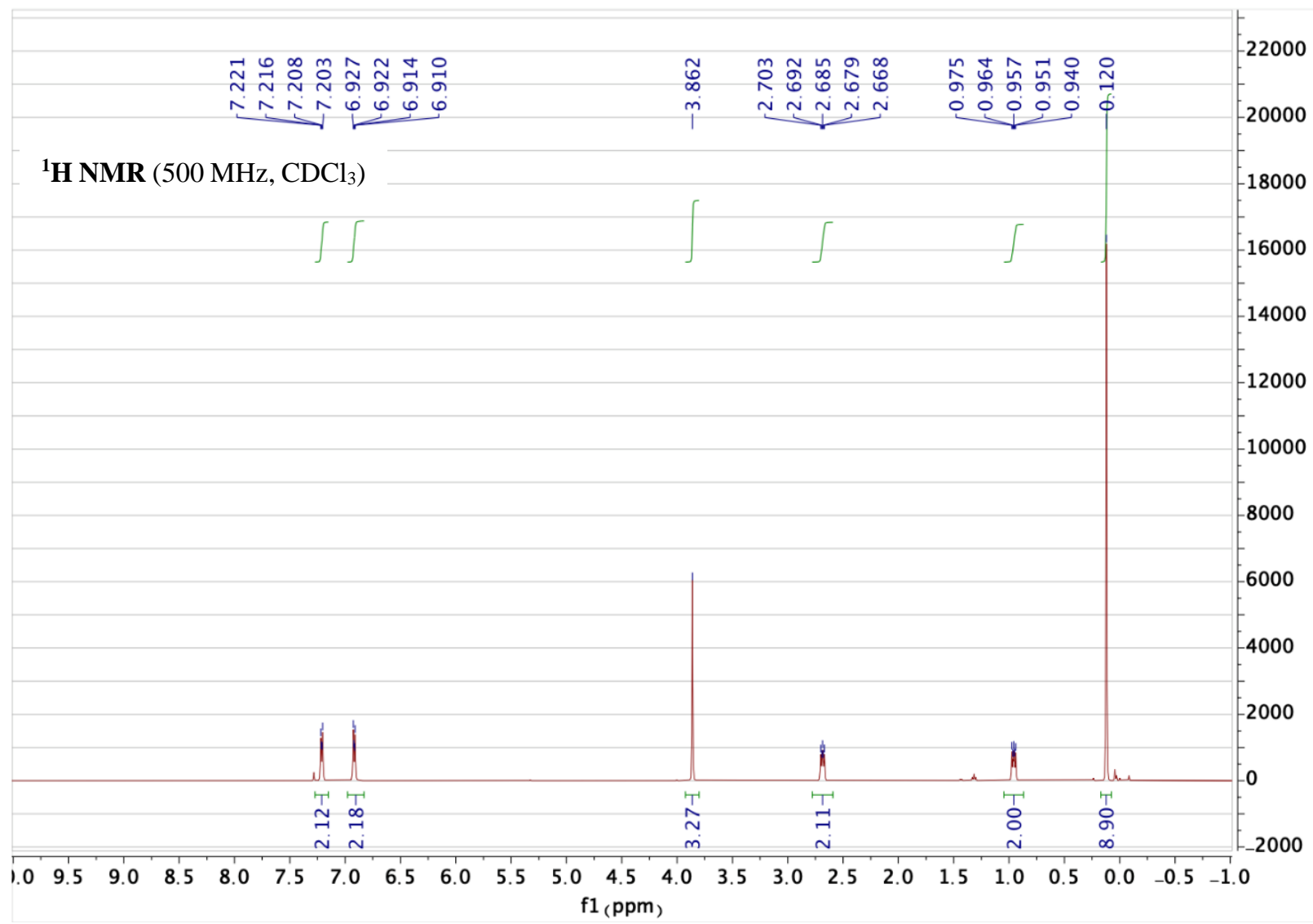



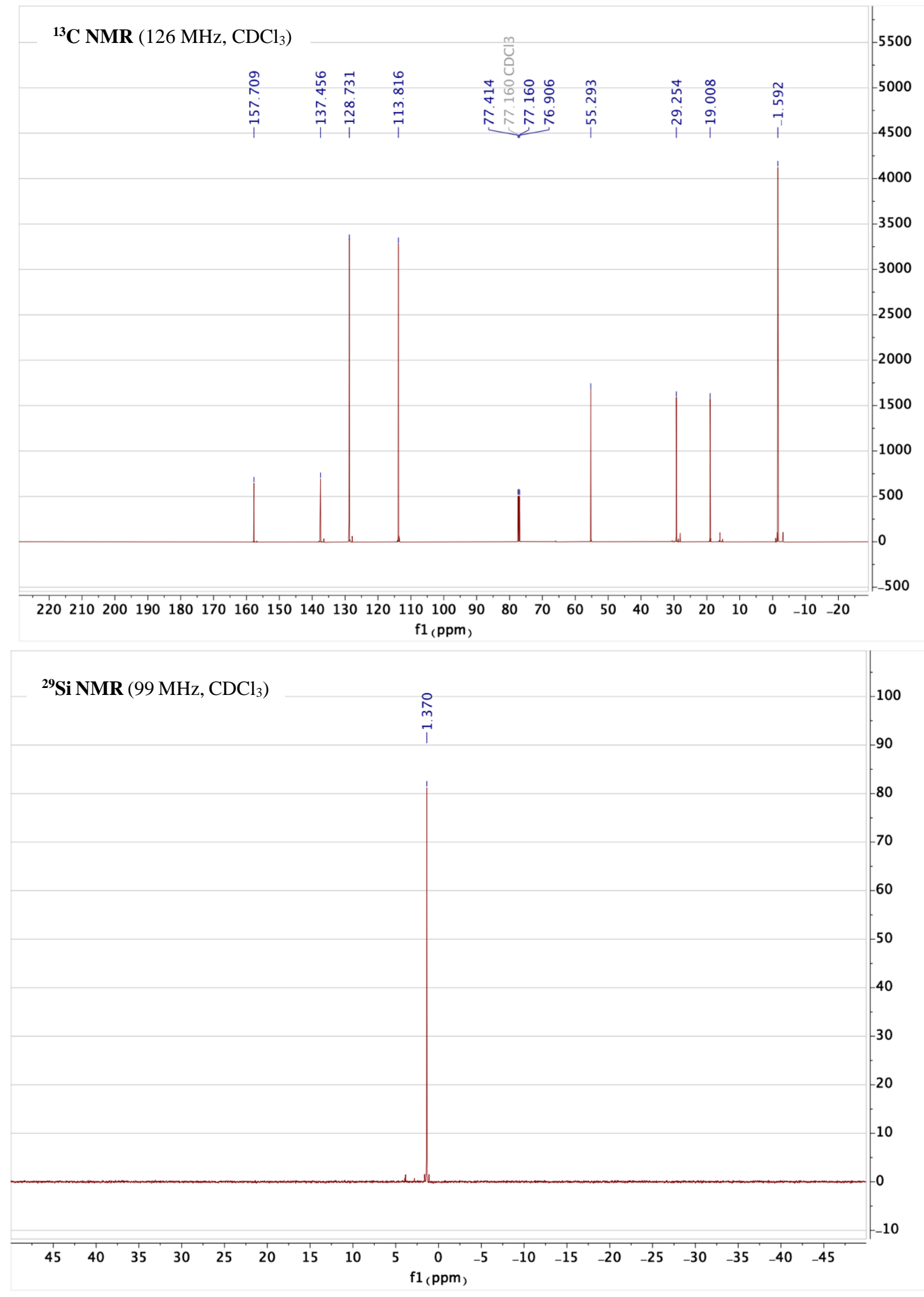


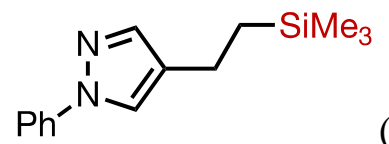

(44)
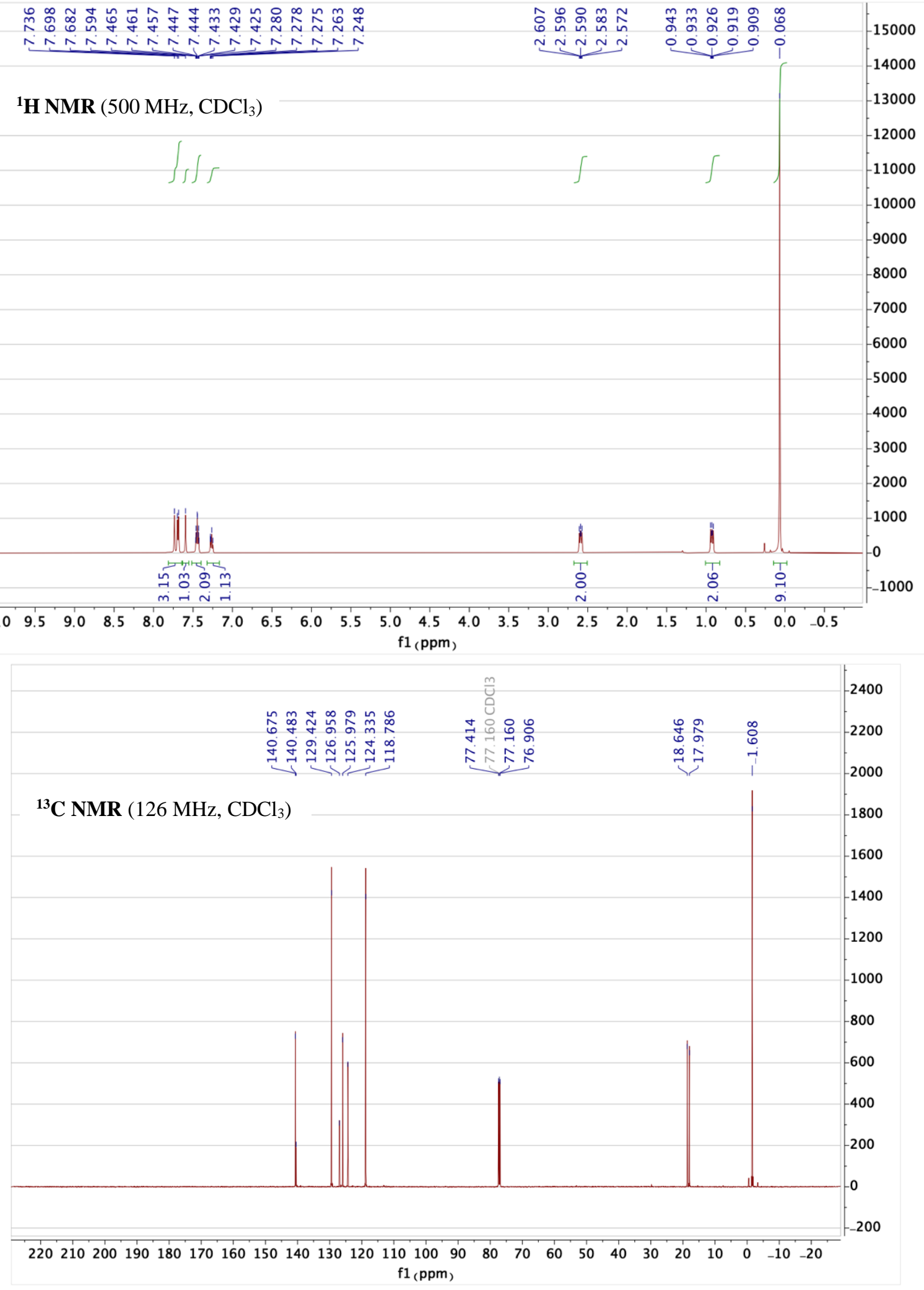

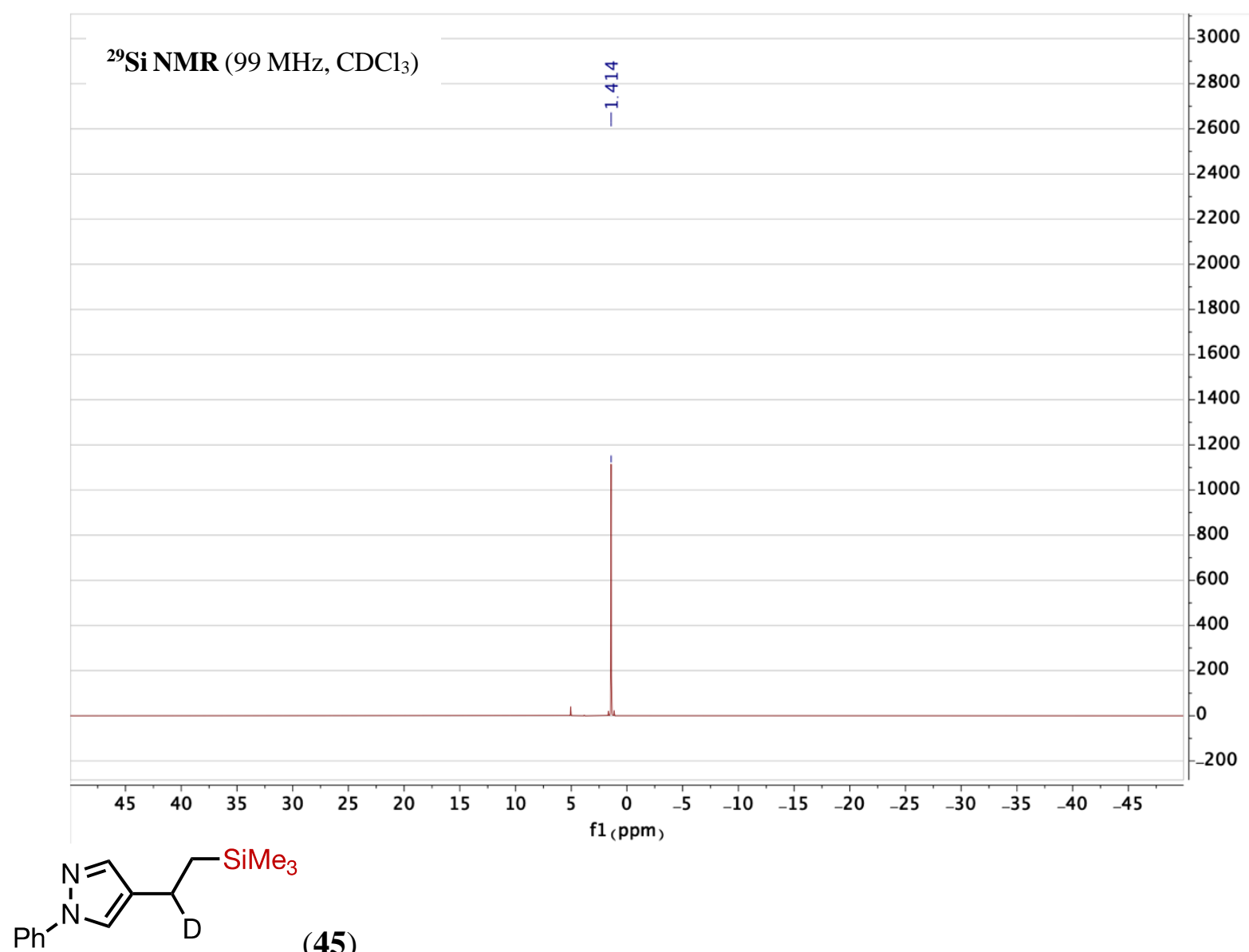

(45)

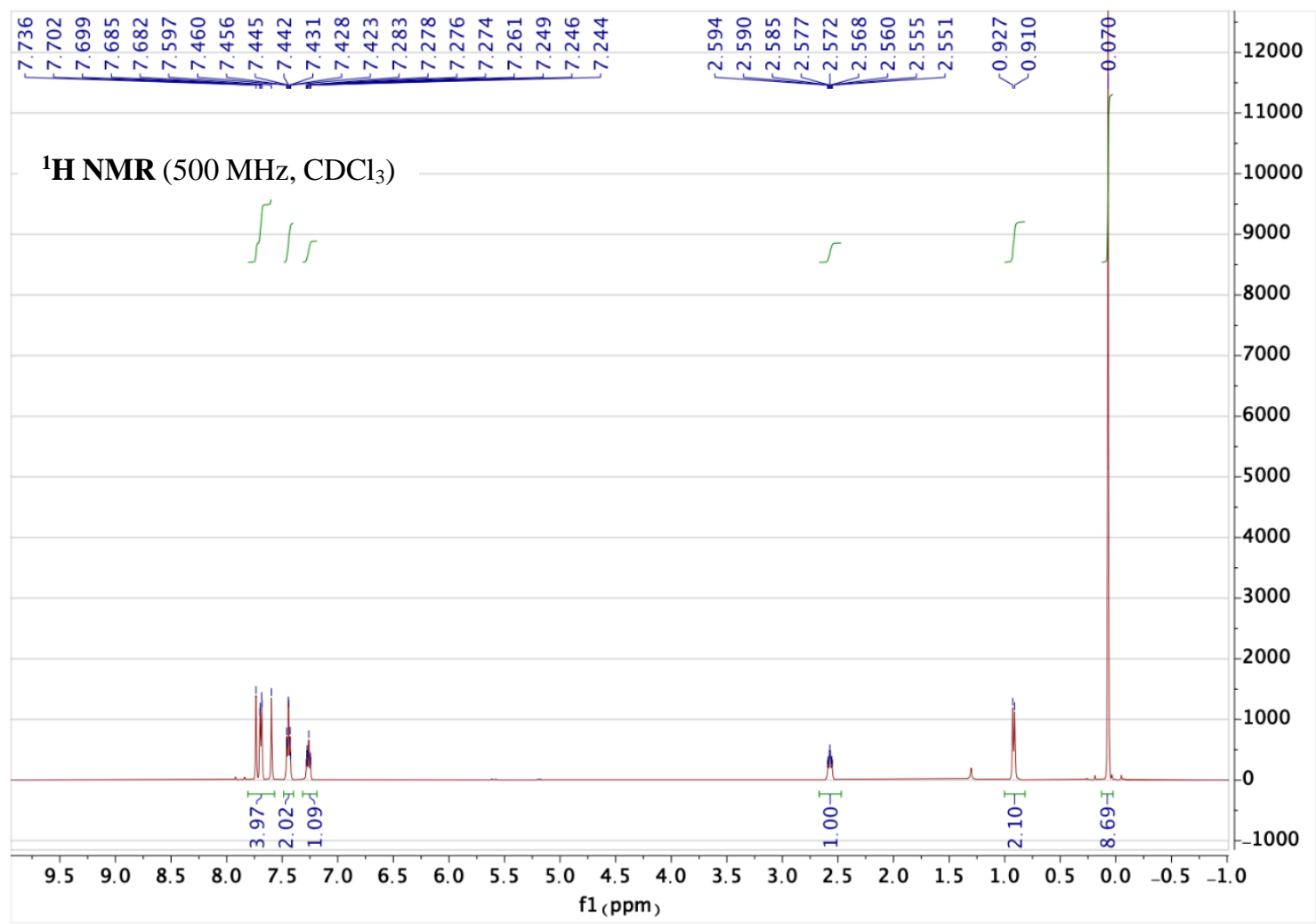




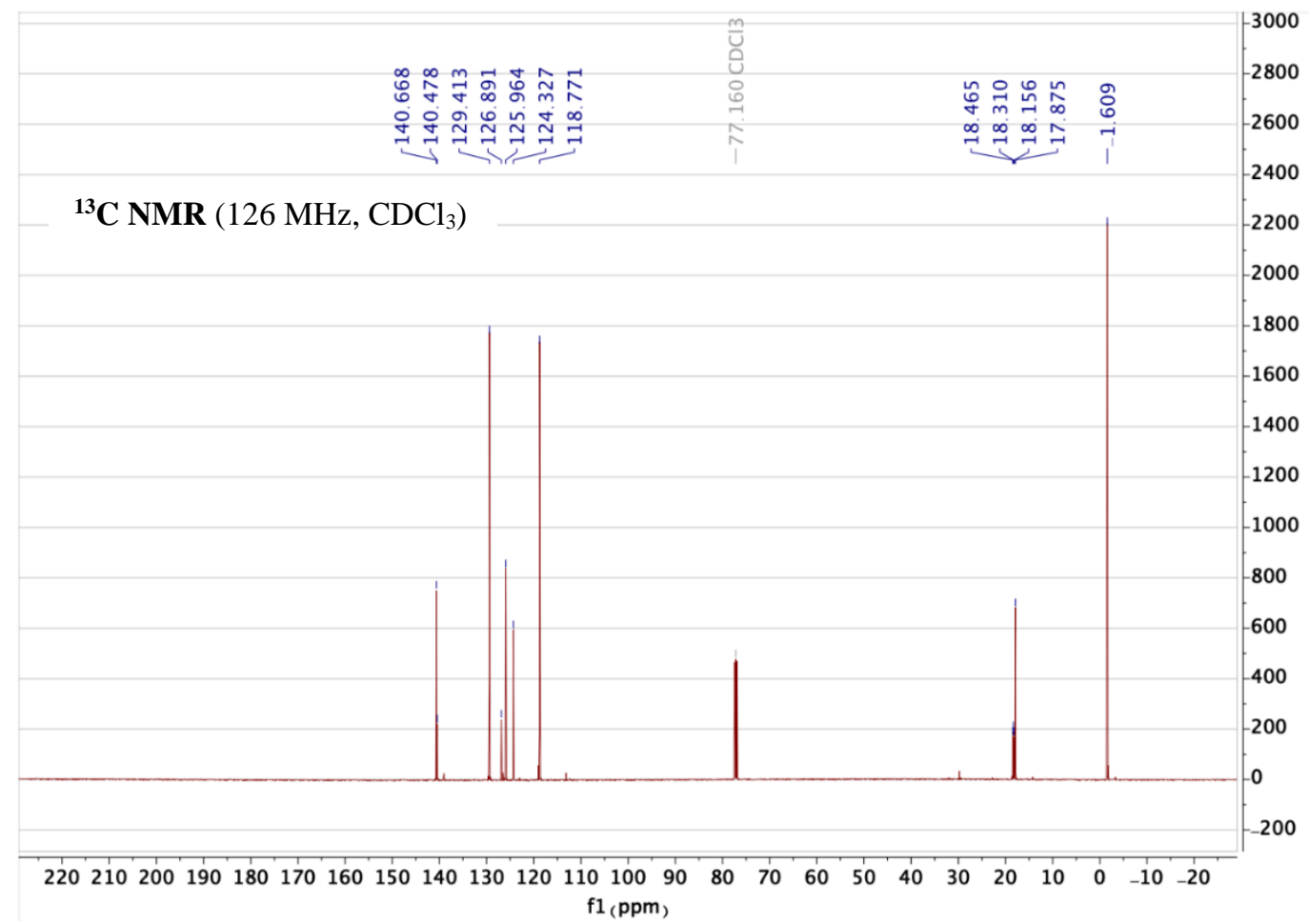<smiles>C[SiH3]C(=Cc1ccccc1)c1ccccc1</smiles>

(46)

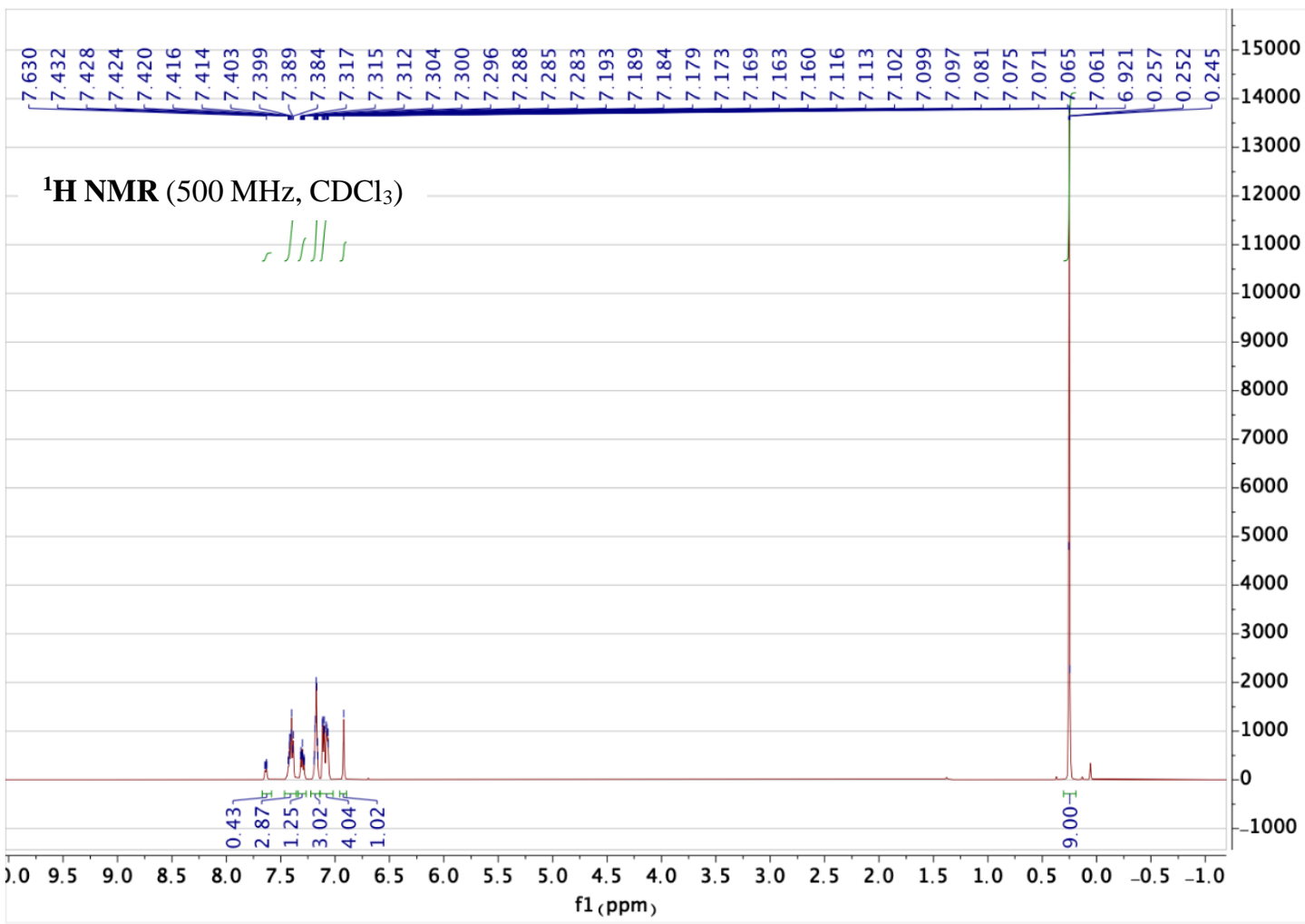



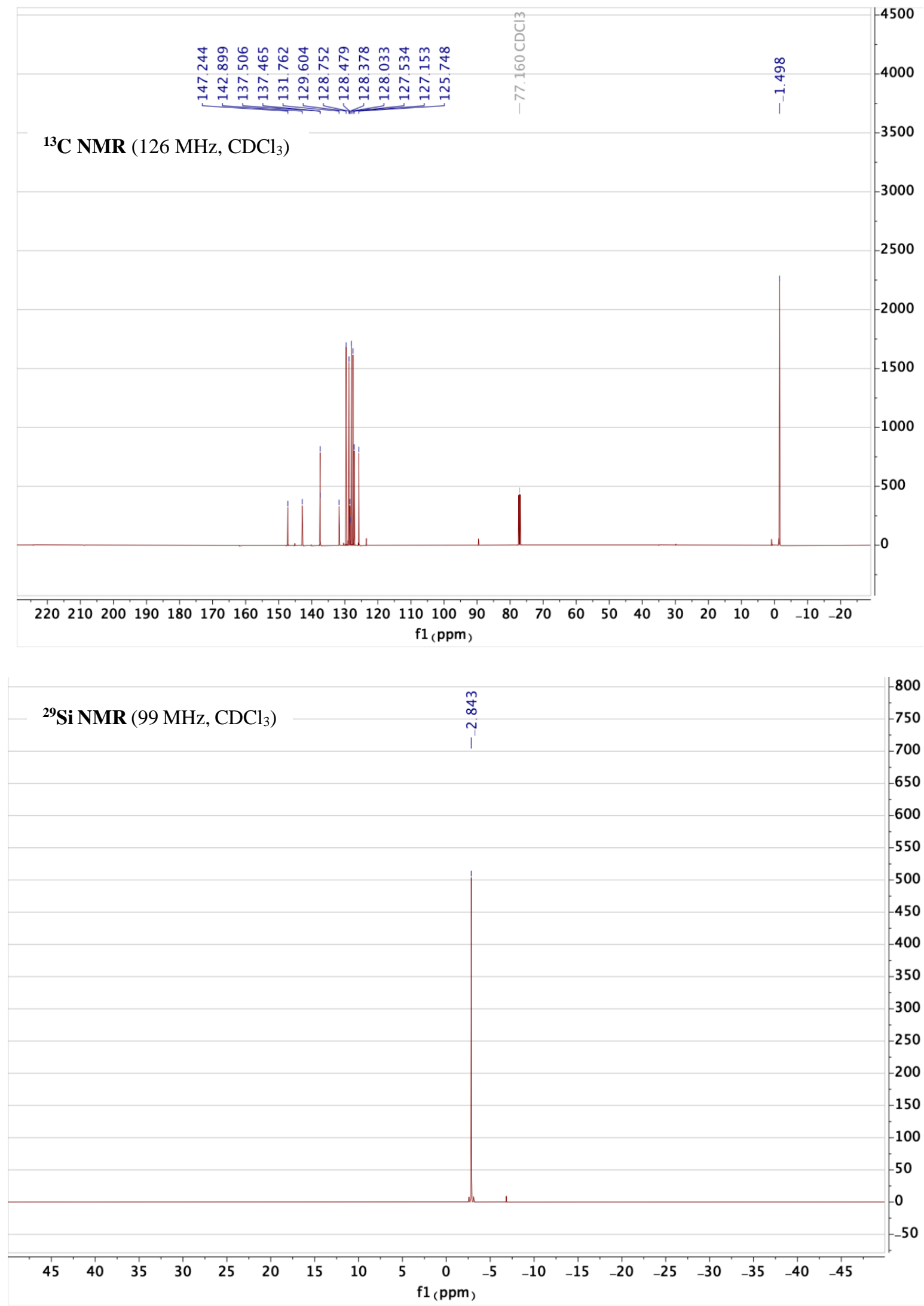

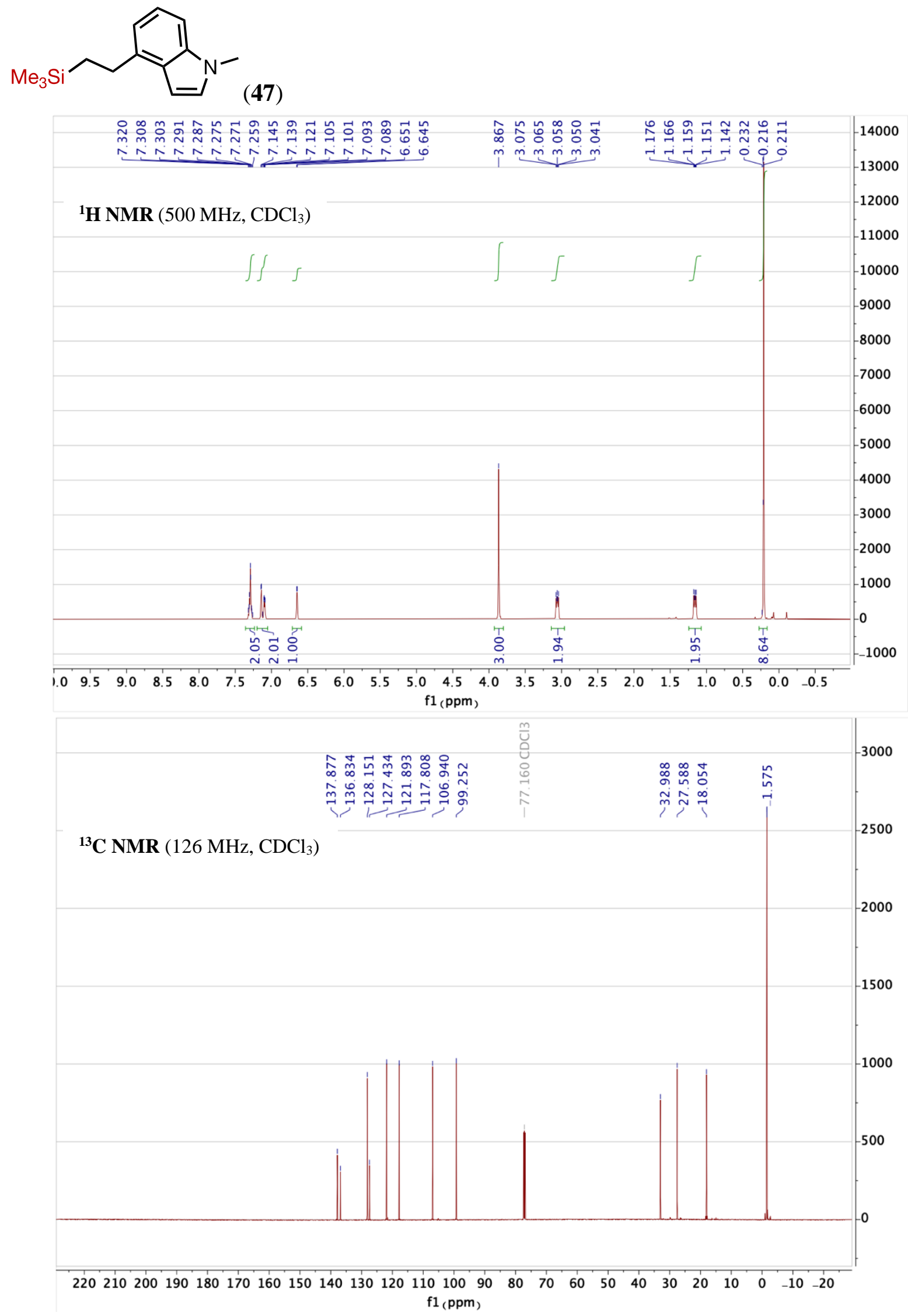

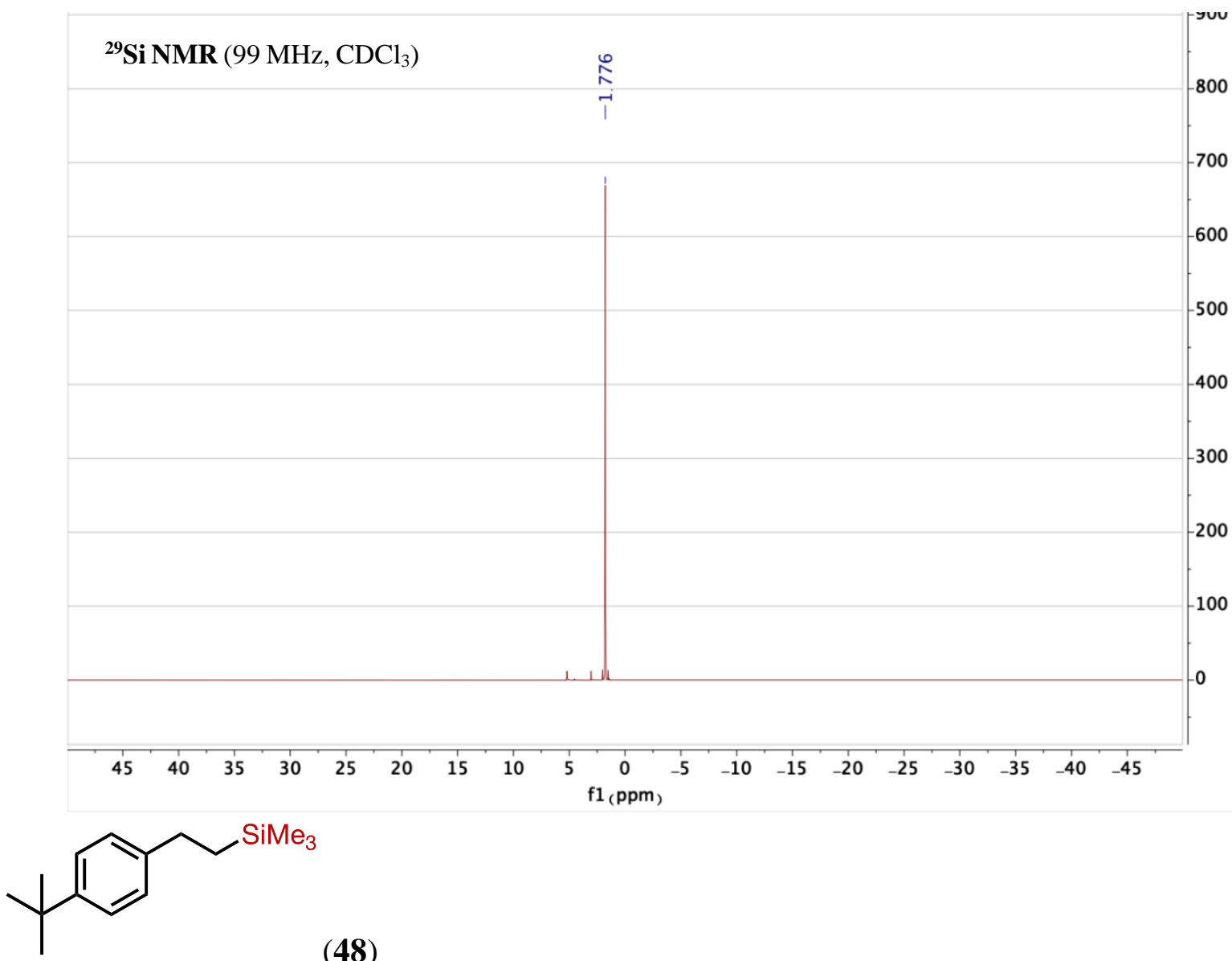

(48)

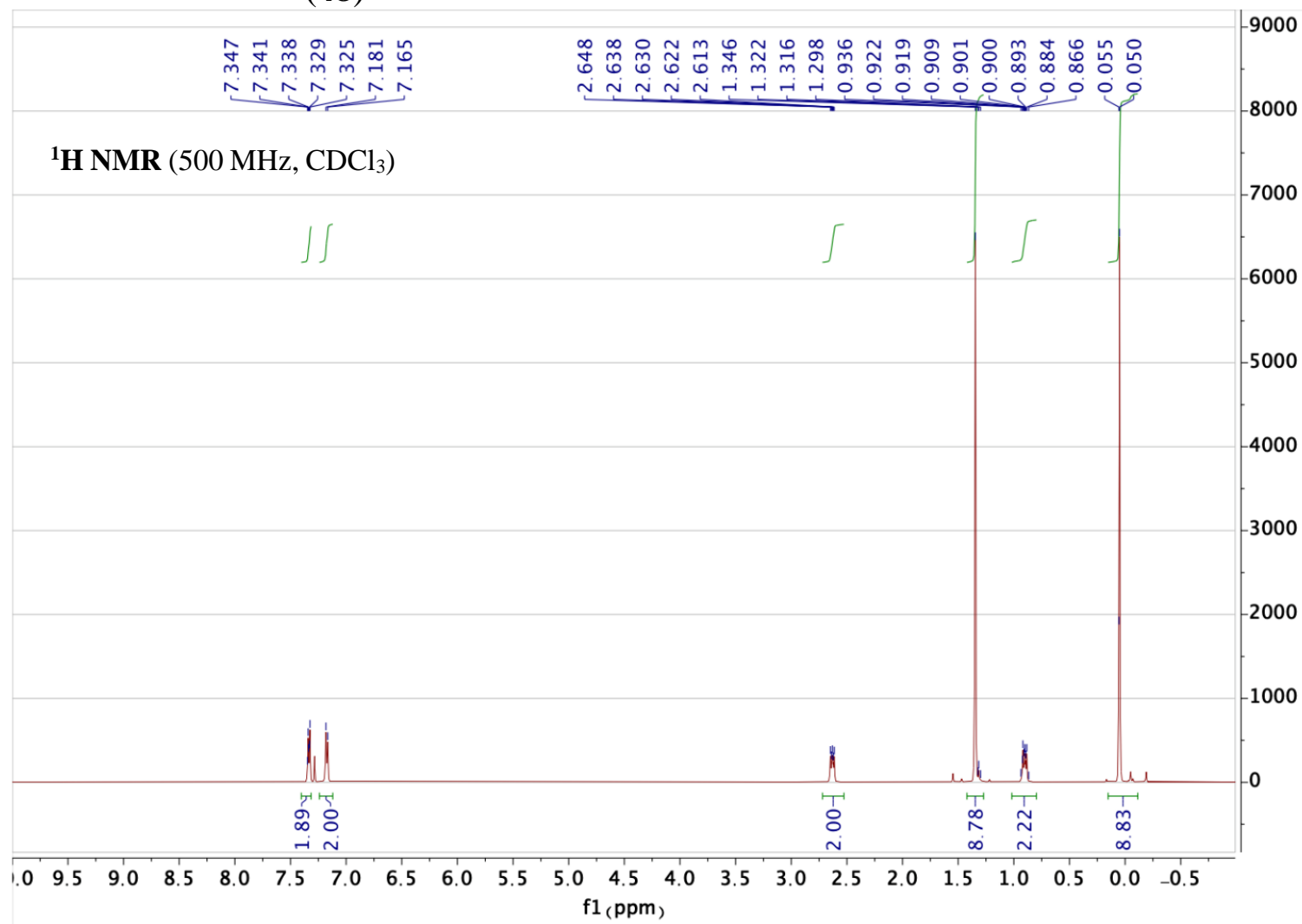




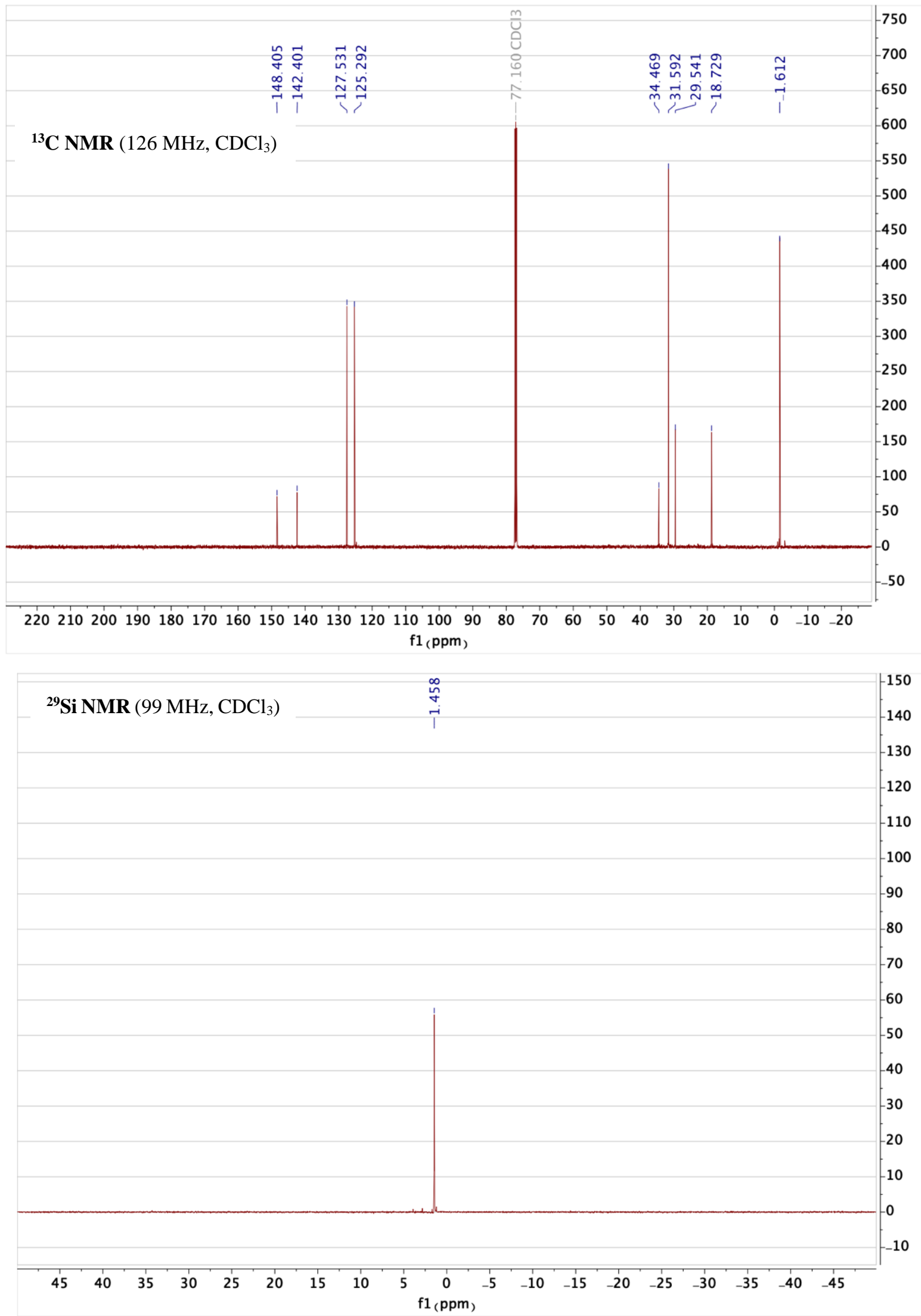



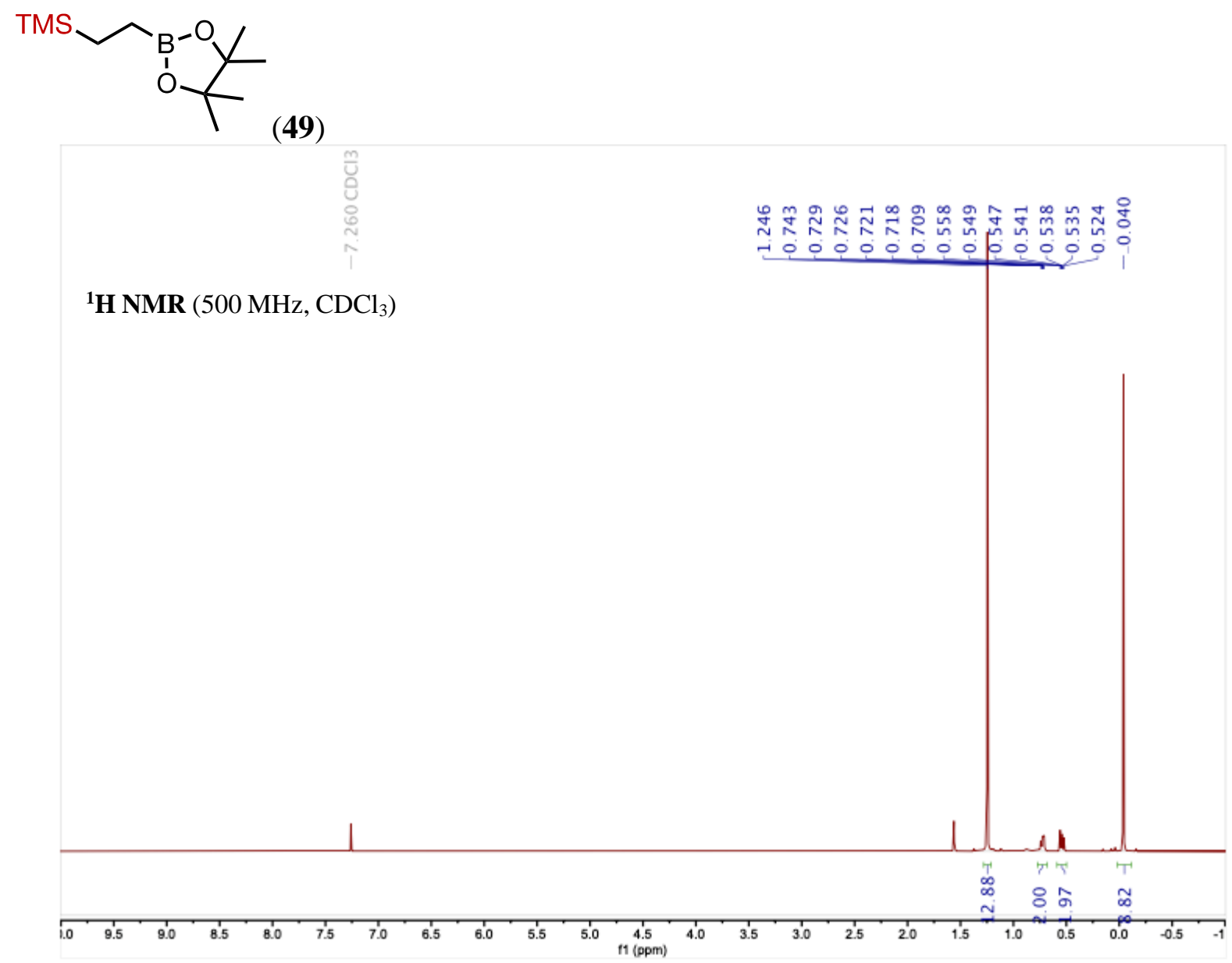

${ }^{13} \mathbf{C ~ N M R}\left(126 \mathrm{MHz}, \mathrm{CDCl}_{3}\right)$
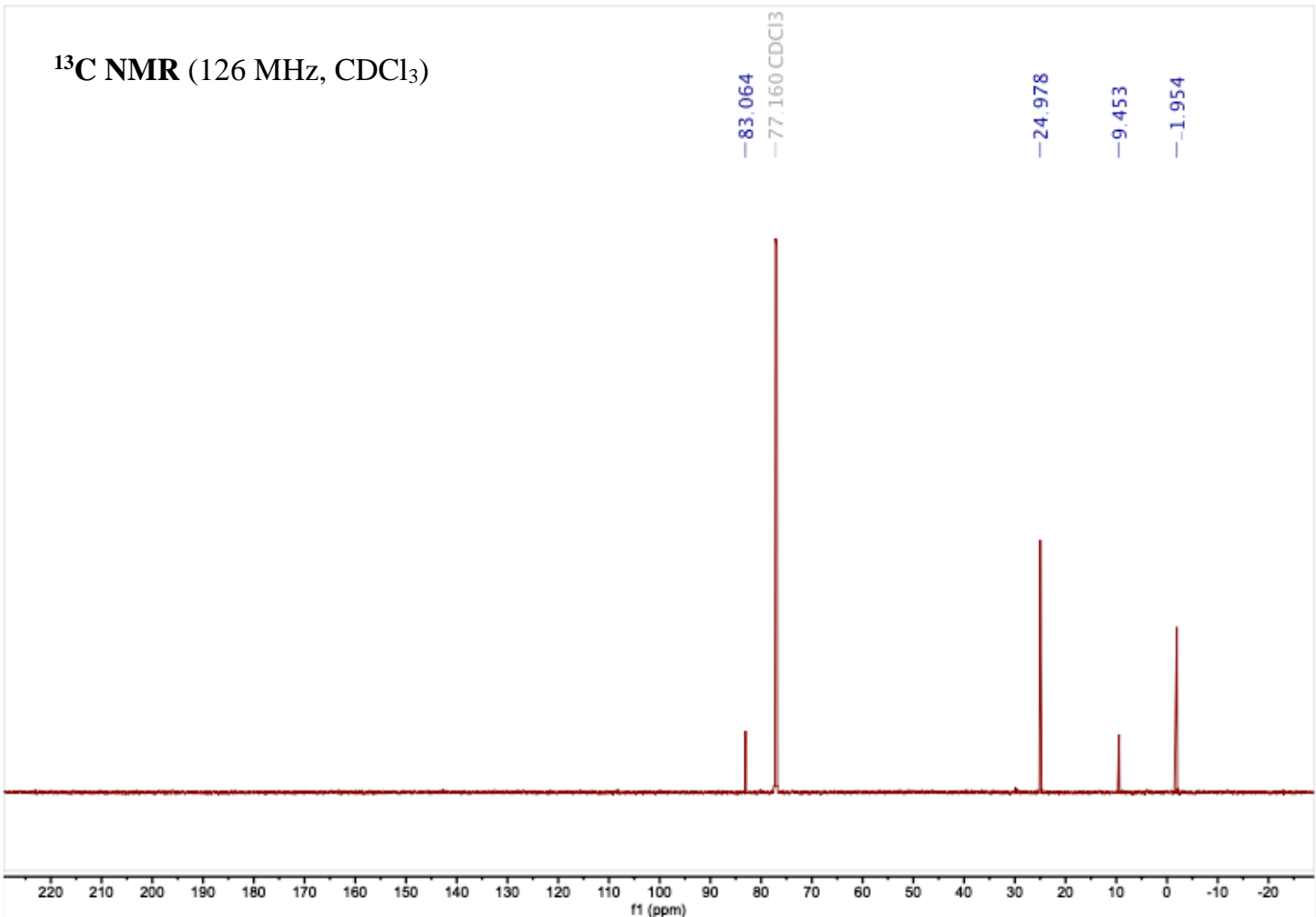
<smiles>C=C[Si](C)(C)CCc1csc2ccccc12</smiles>
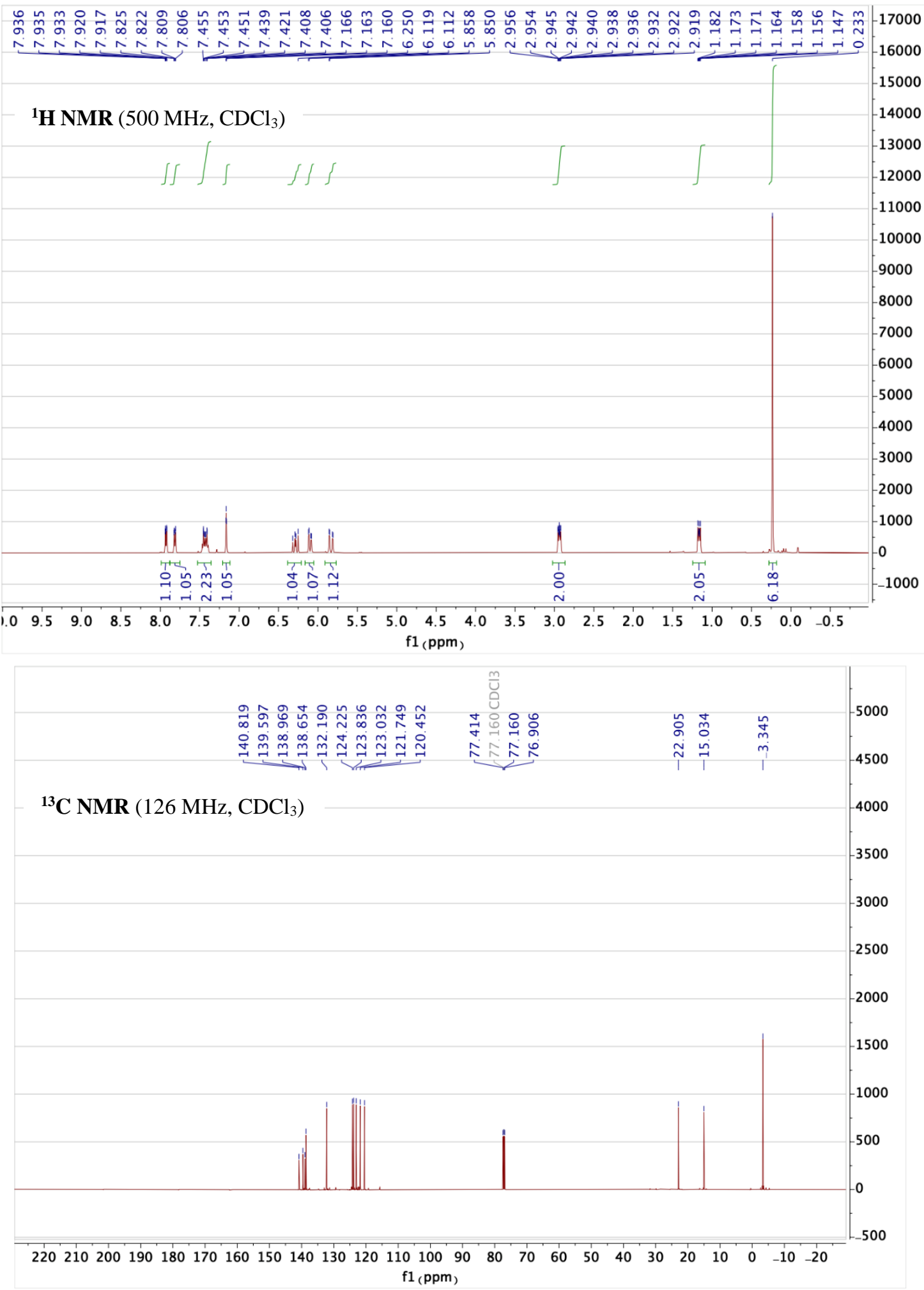


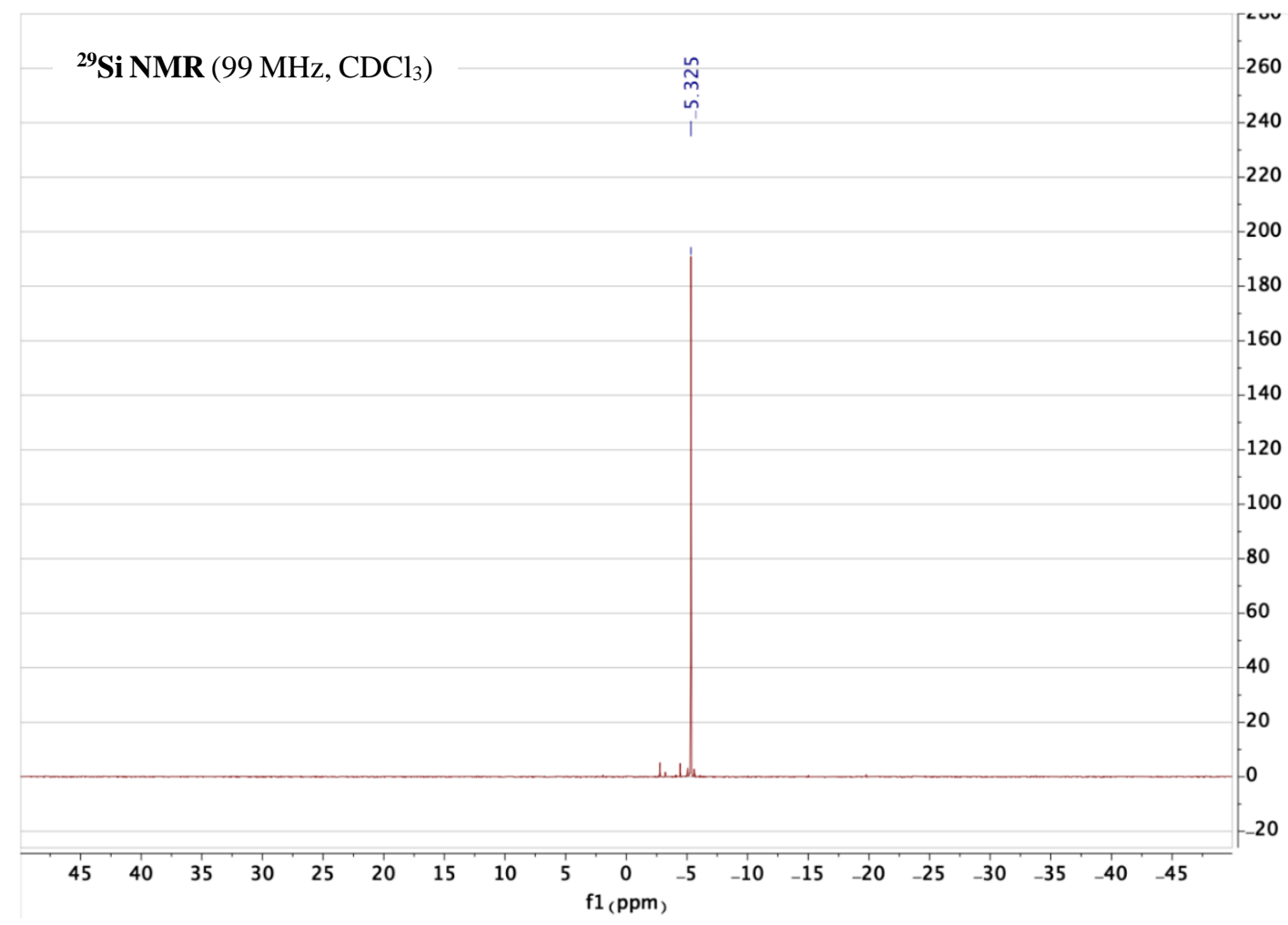<smiles>C=CC[Si](C)(C)CCc1ccc2sccc2c1</smiles>

(51)

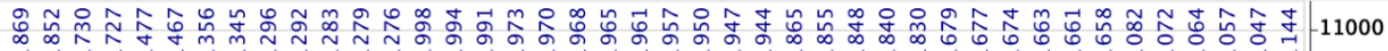

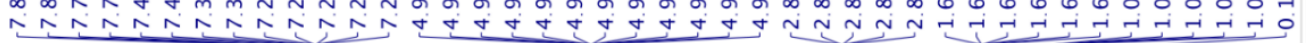

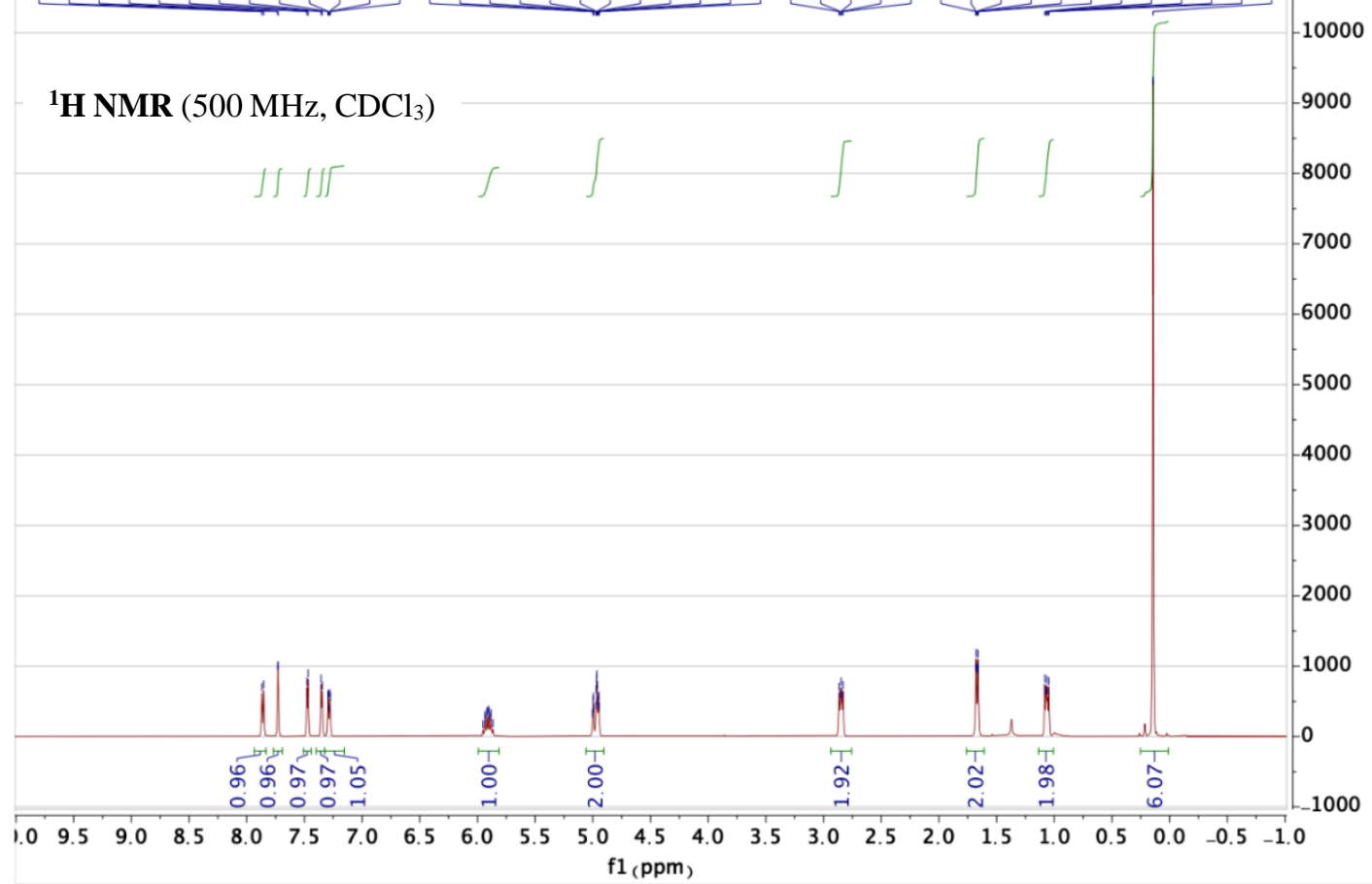



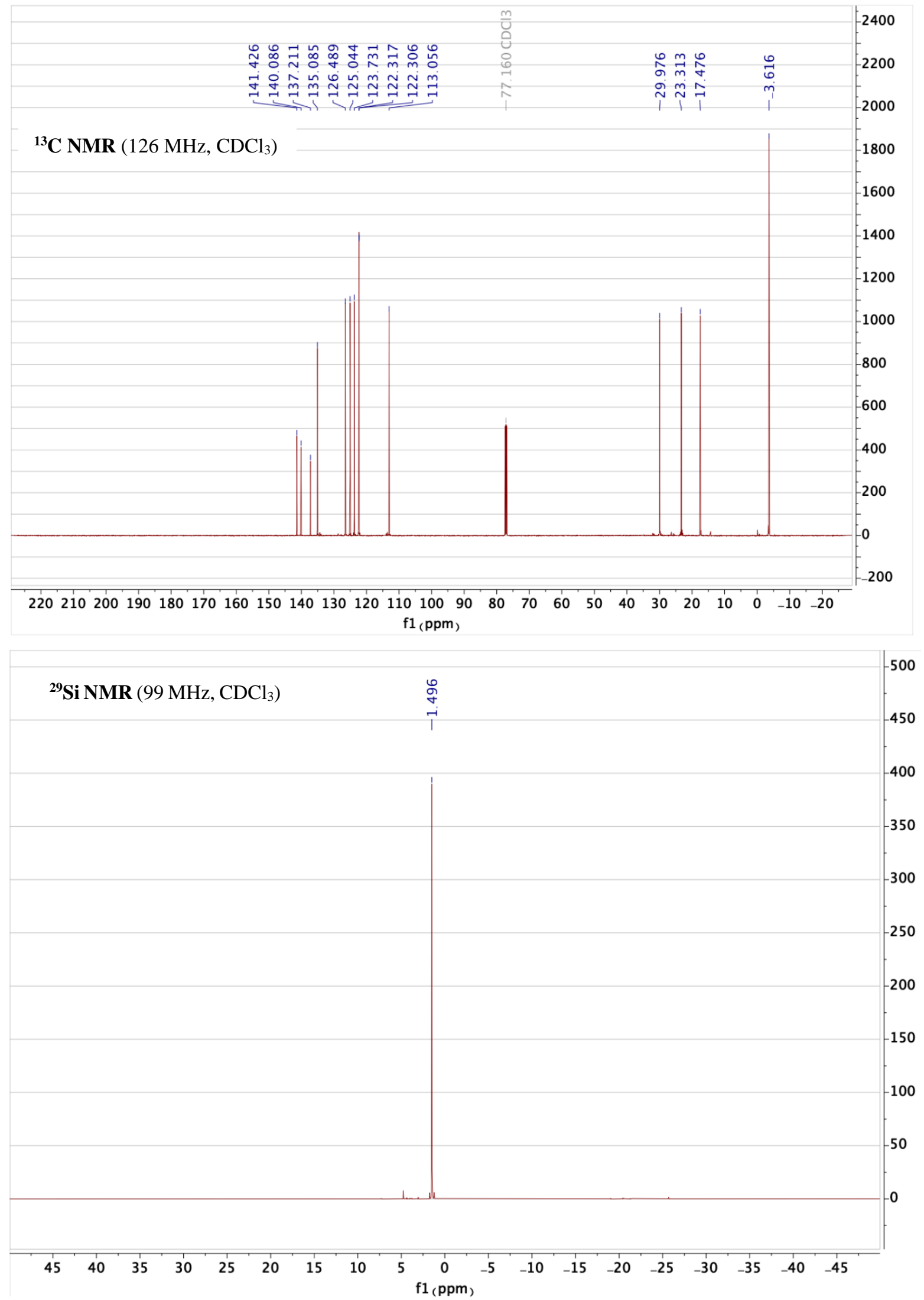


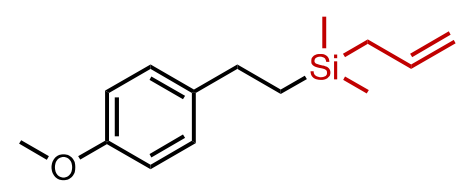

(52)
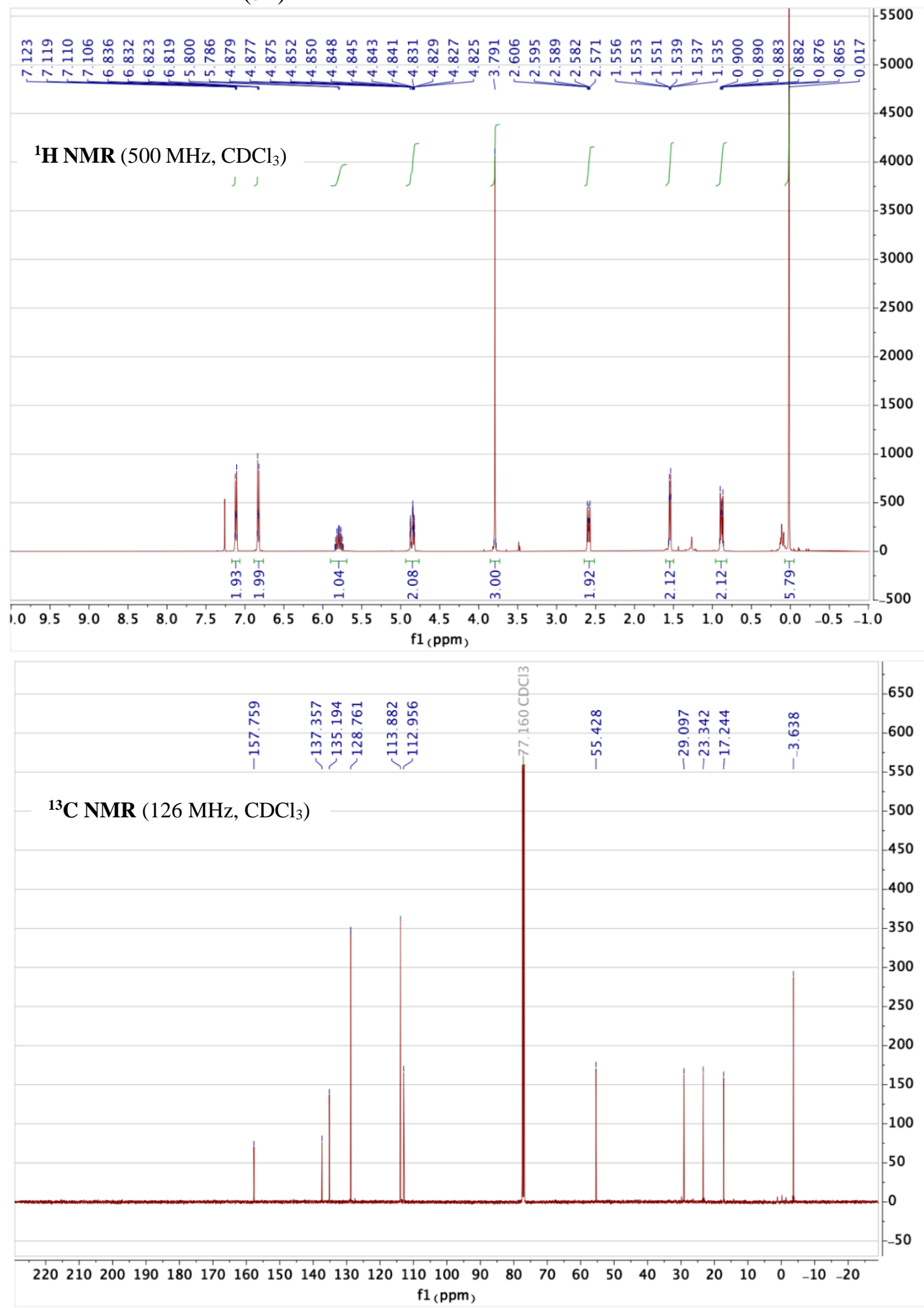


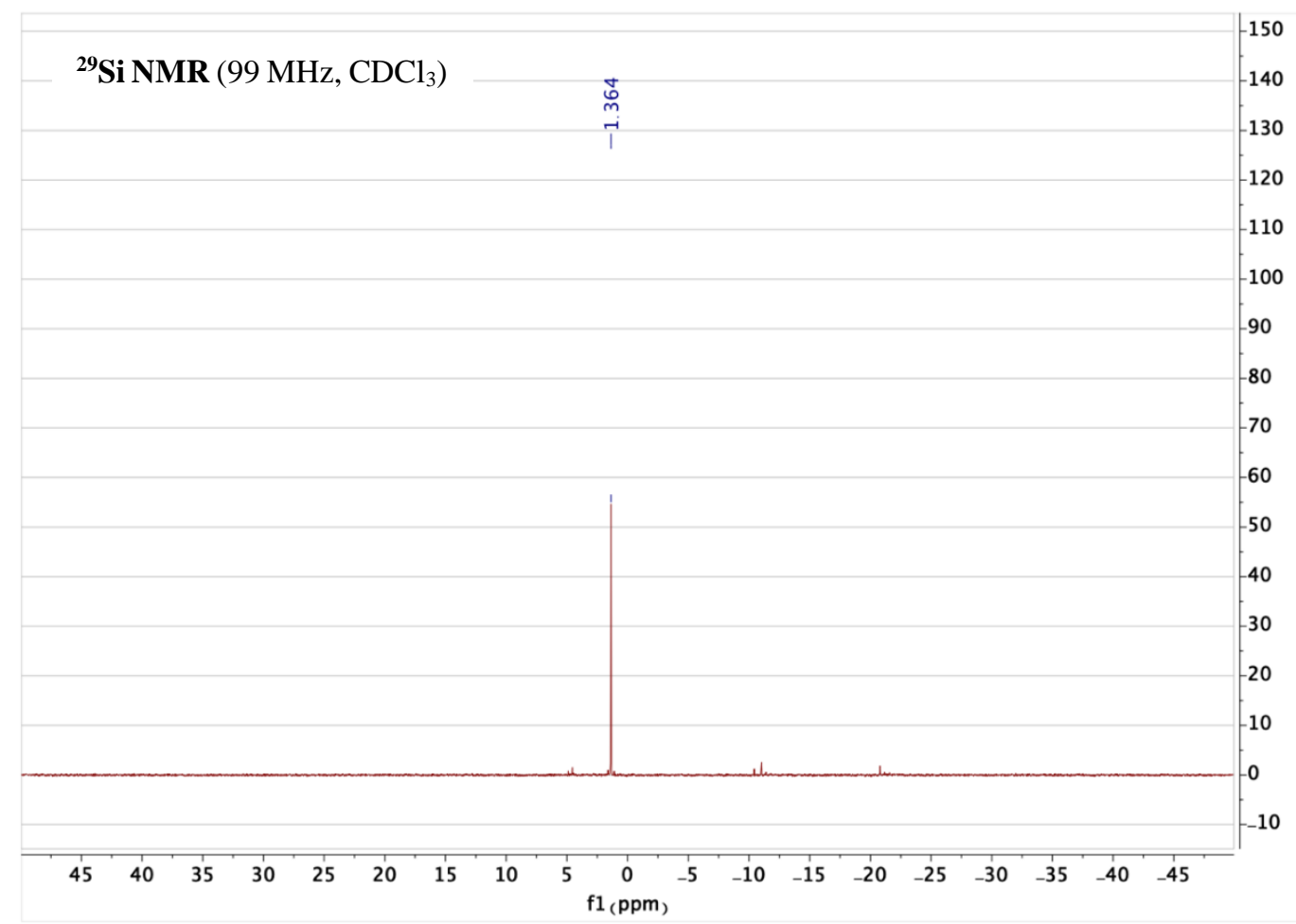

$\underbrace{\text { SiH }}_{\text {SiH }}$

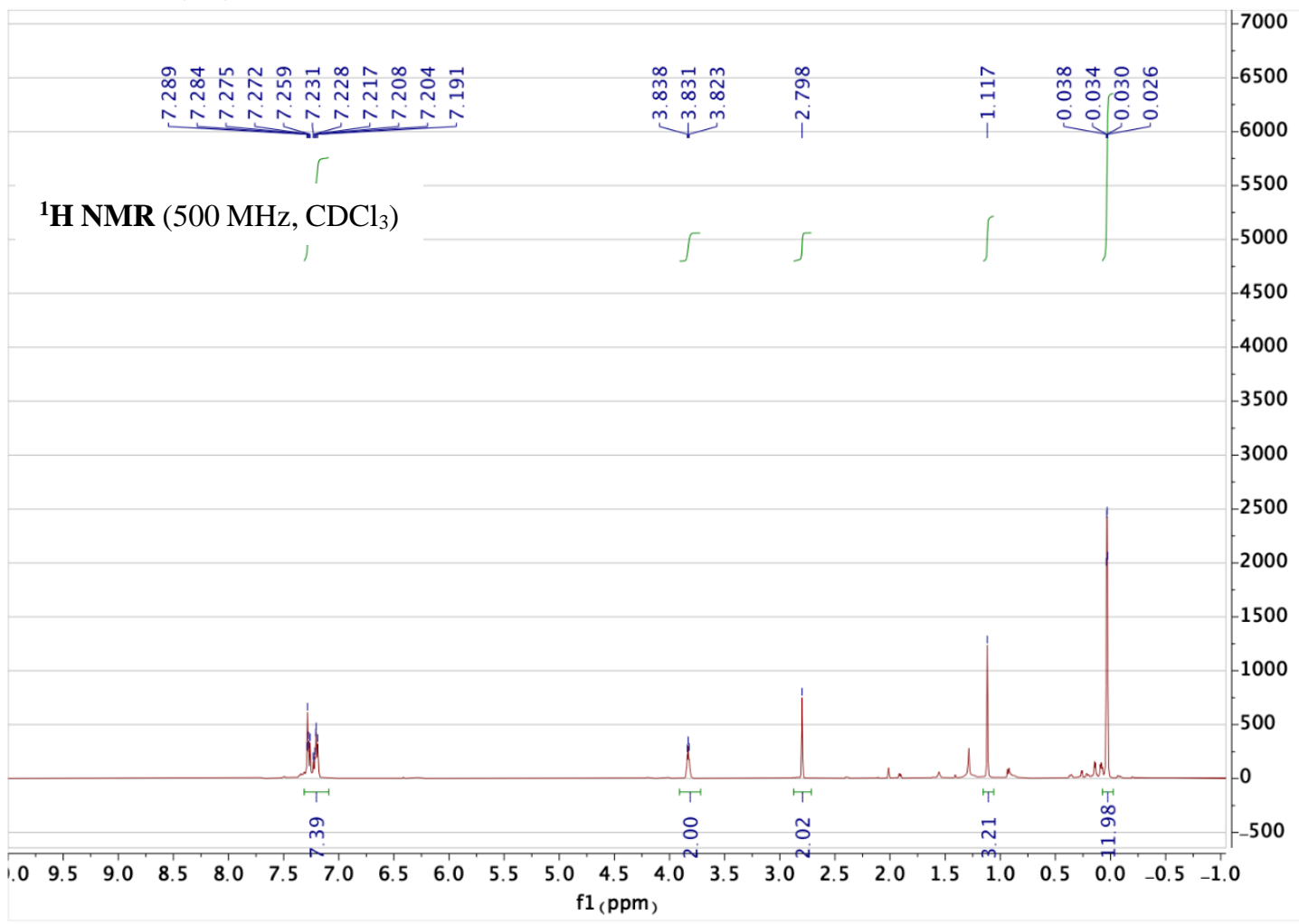




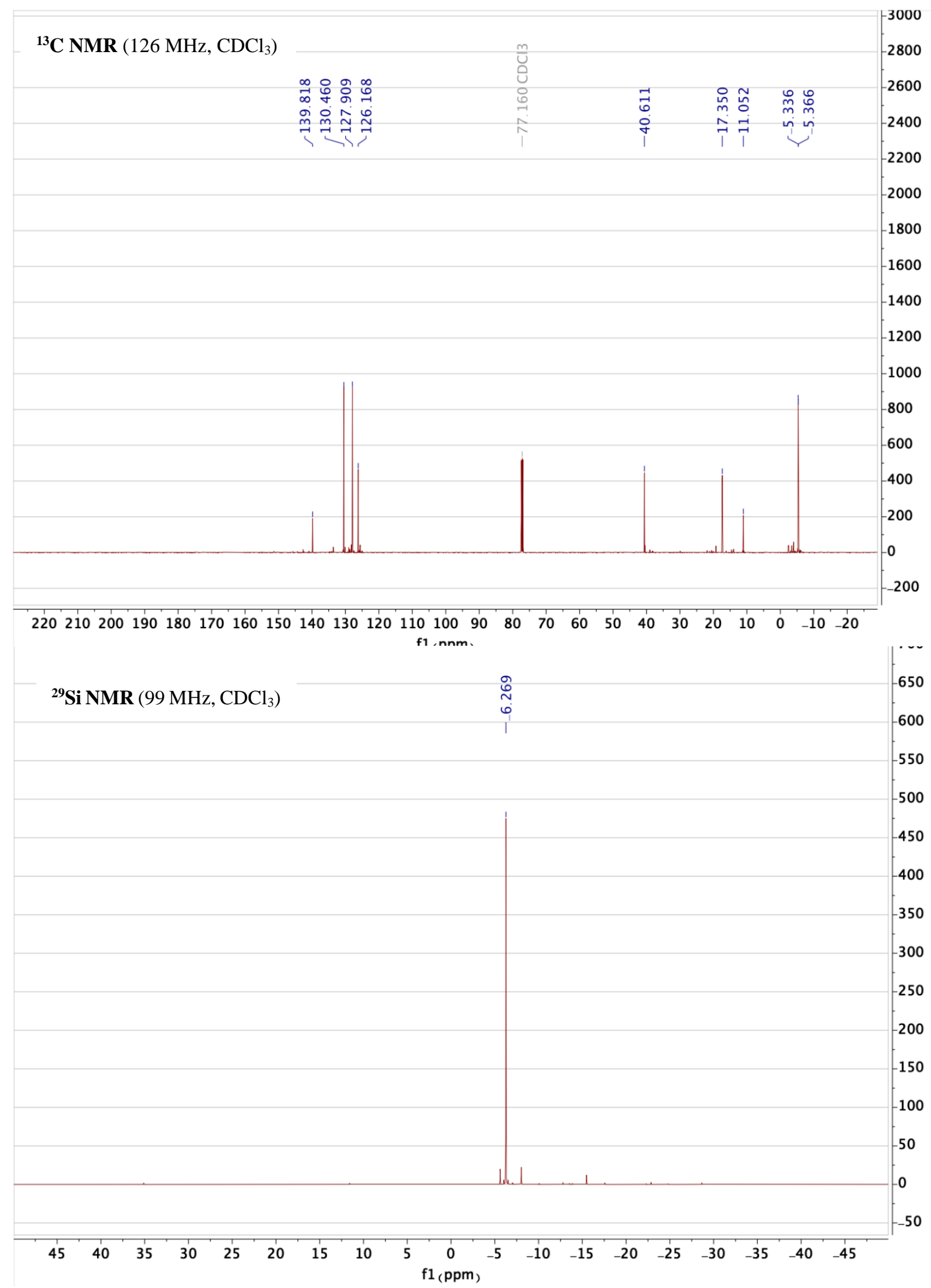


TMS $\mathcal{N O P h}_{(57)}$
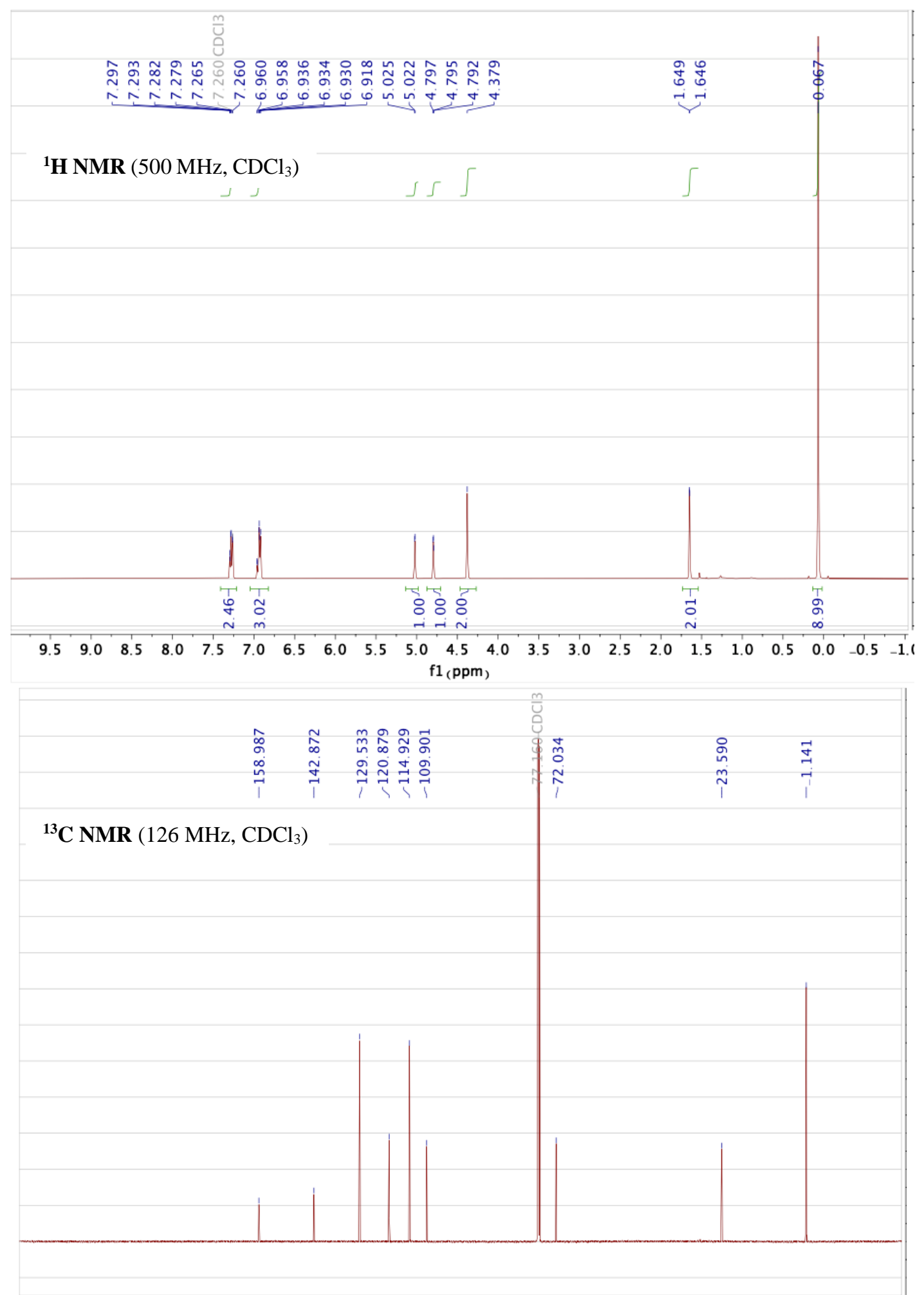

$\begin{array}{lllllllllllllllllllllllllllll}220 & 210 & 200 & 190 & 180 & 170 & 160 & 150 & 140 & 130 & 120 & 110 & 100 & 90 & 80 & 70 & 60 & 50 & 40 & 30 & 20 & 10 & 0 & -10 & -20\end{array}$ $\mathrm{f1}$ ( $\mathrm{ppm}$ ) 

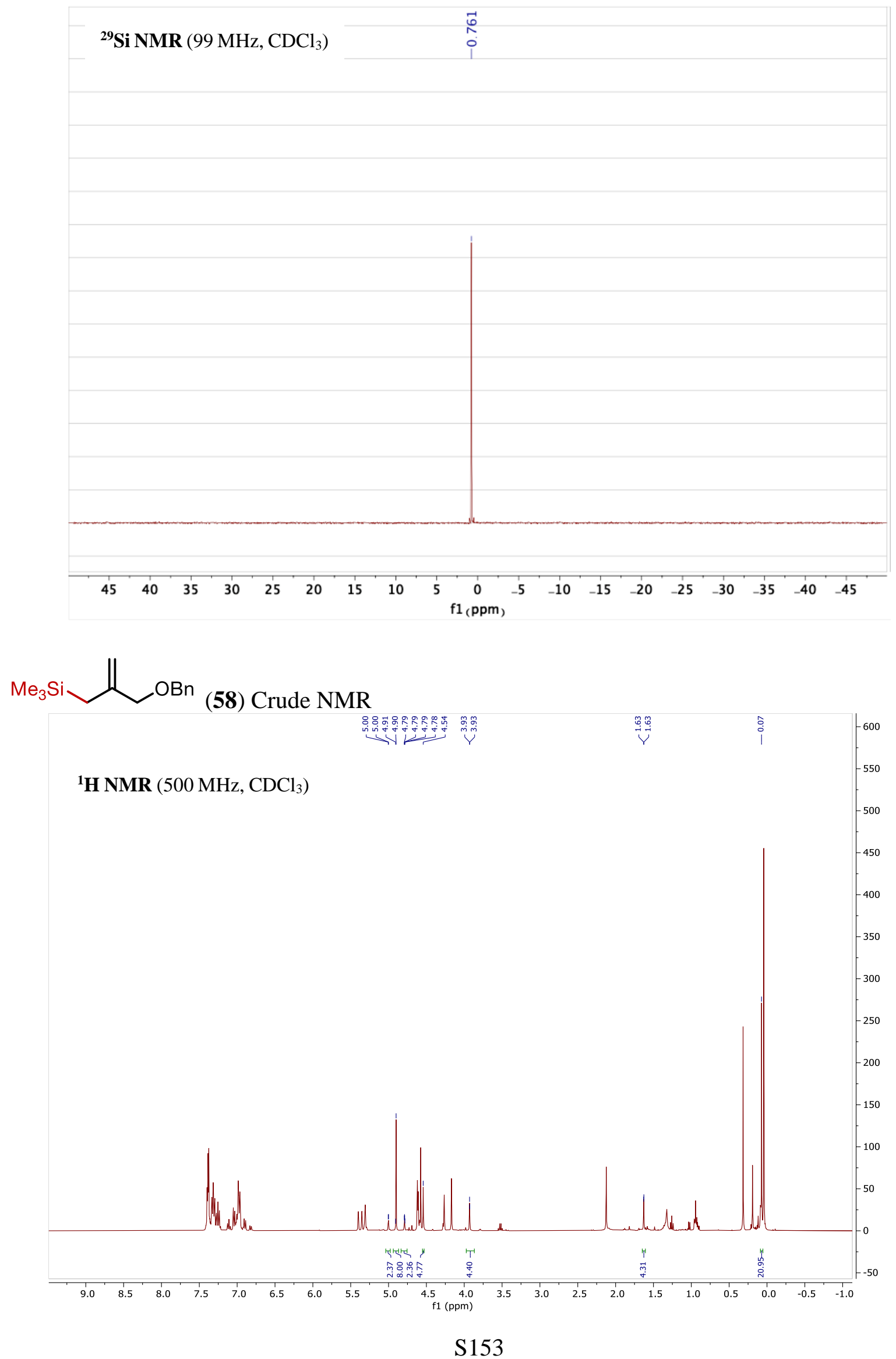


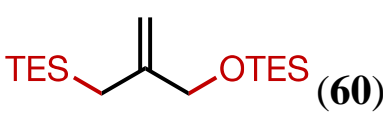

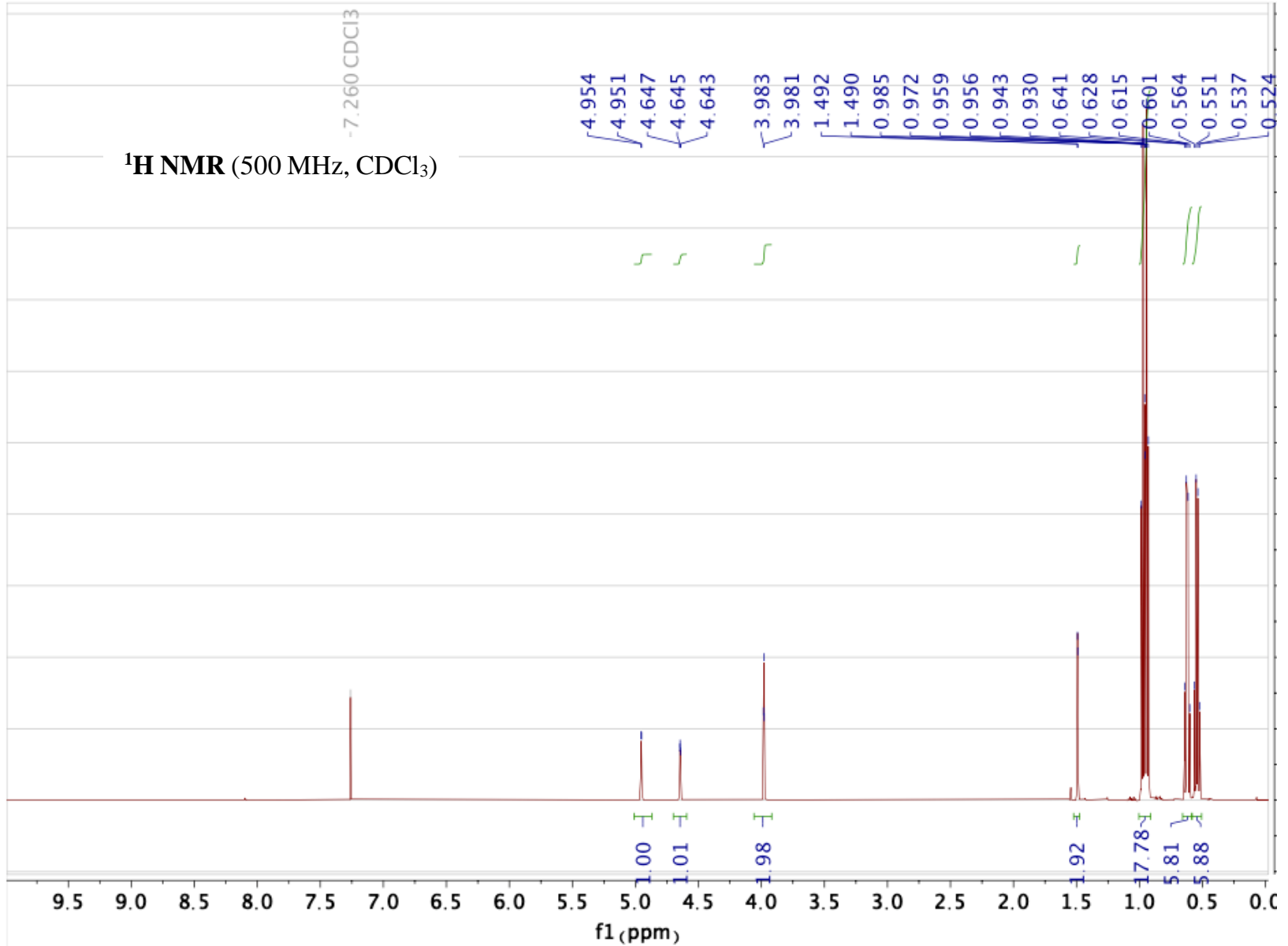




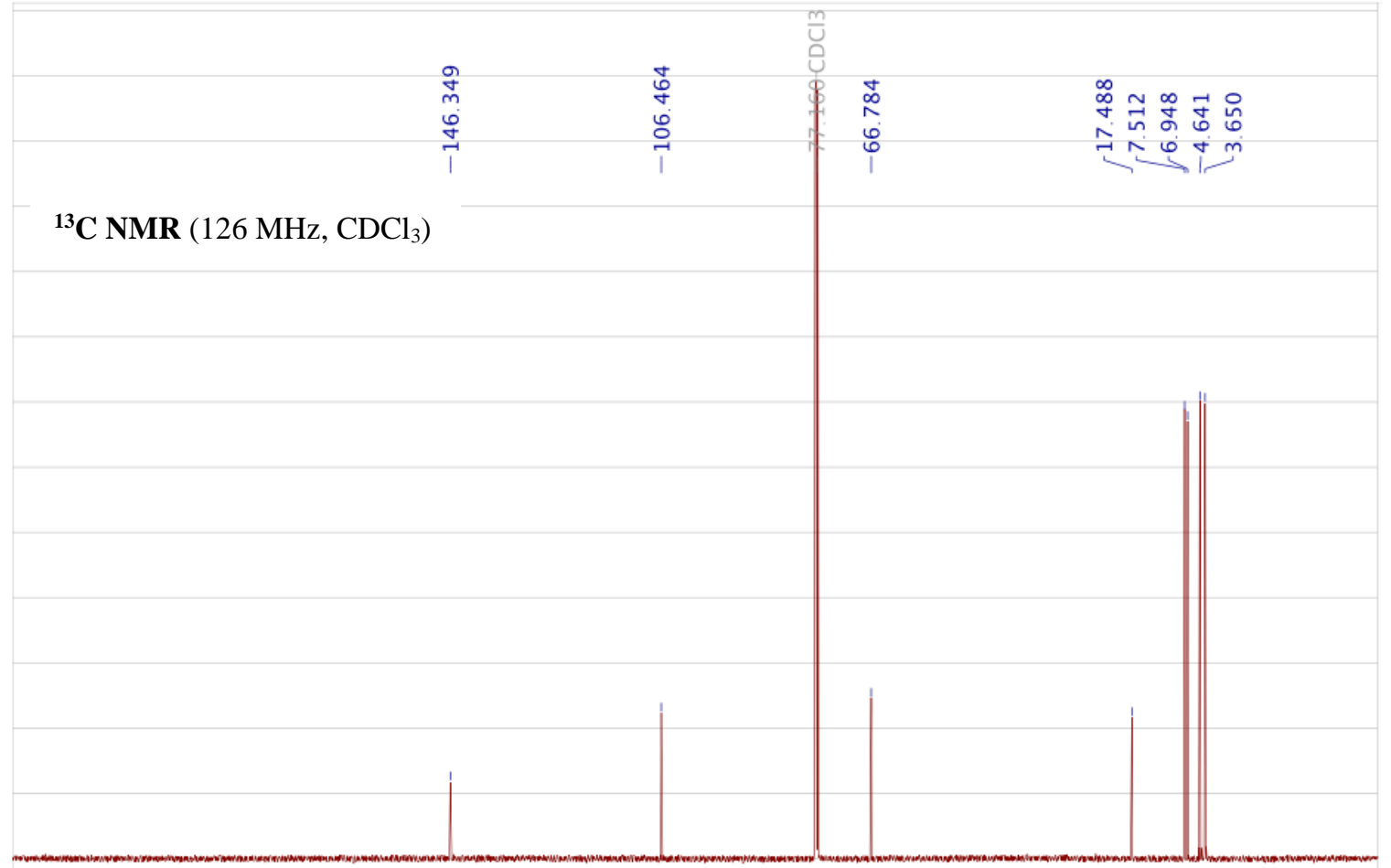

$\begin{array}{lllllllllllllllllllllllll}220 & 210 & 200 & 190 & 180 & 170 & 160 & 150 & 140 & 130 & 120 & 110 & 100 & 90 & 80 & 70 & 60 & 50 & 40 & 30 & 20 & 10 & 0 & -10 & -20\end{array}$ $\mathrm{f} 1(\mathrm{ppm})$

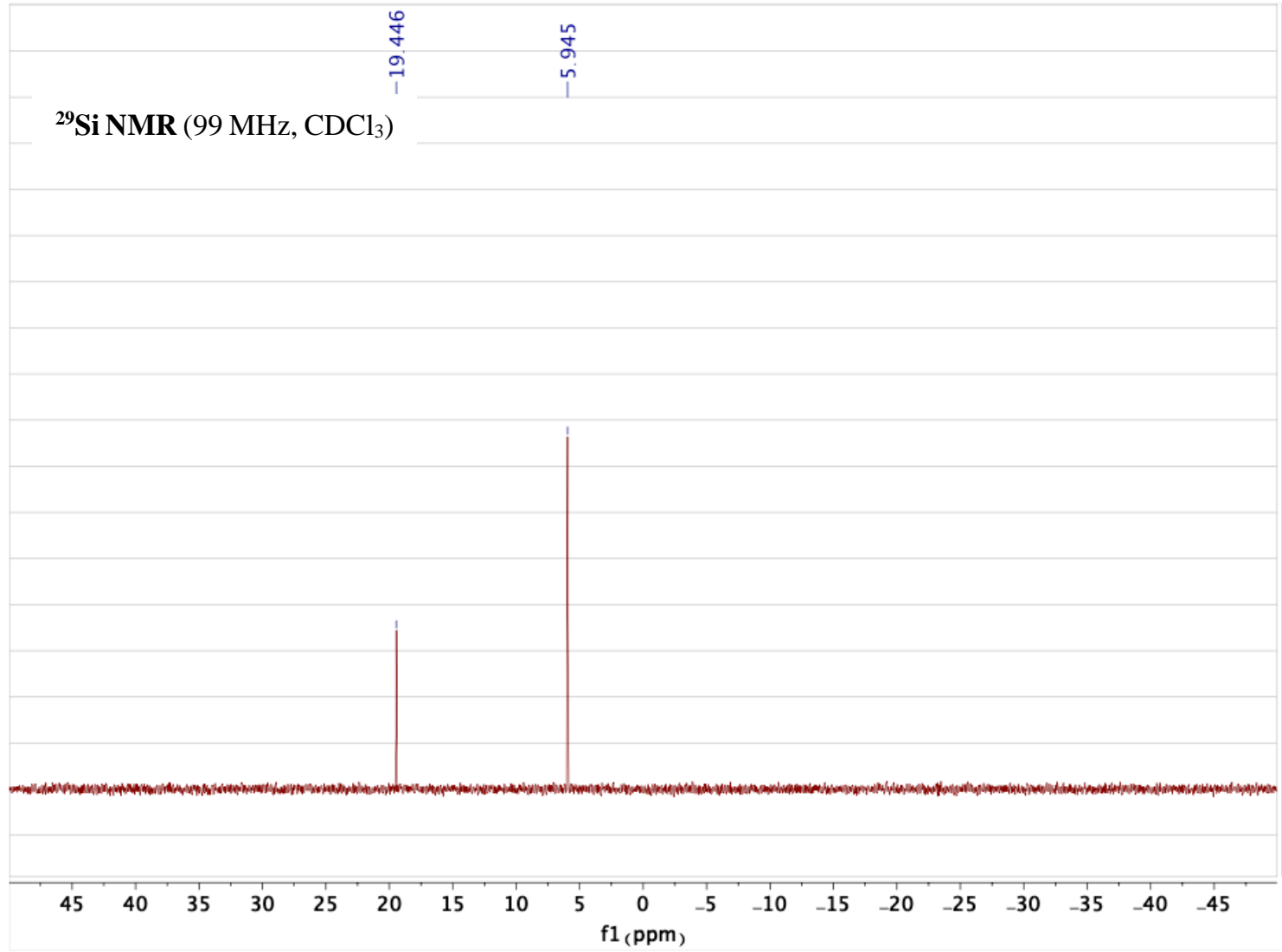




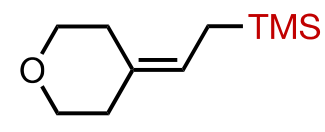

(64)

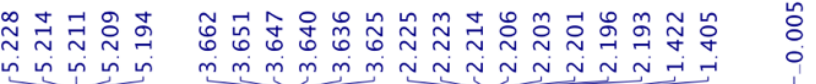

${ }^{1} \mathbf{H}$ NMR (500 MHz, $\left.\mathrm{CDCl}_{3}\right)$

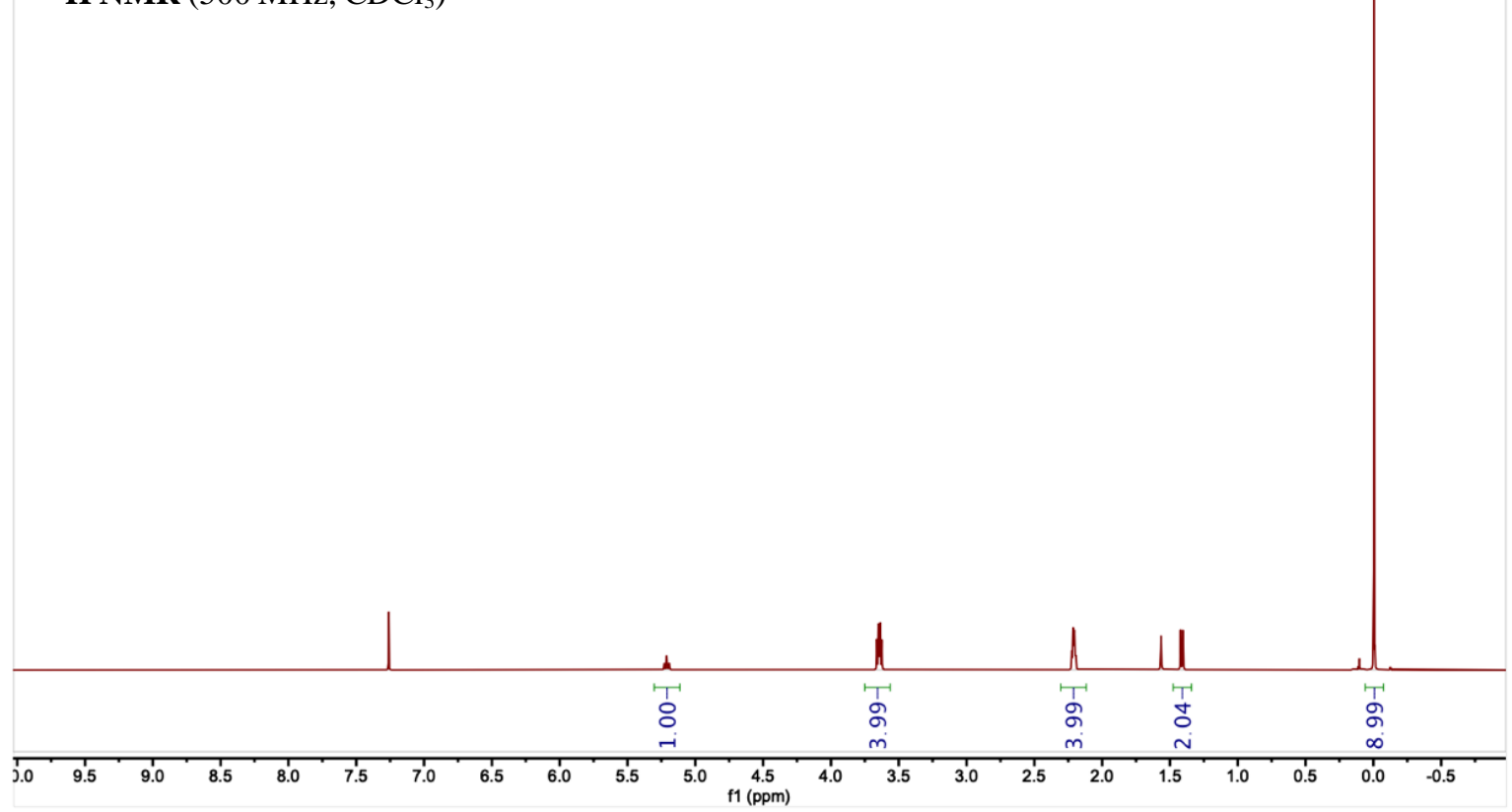

\begin{tabular}{|c|c|c|c|c|c|}
\hline${ }^{13} \mathbf{C ~ N M R}\left(126 \mathrm{MHz}, \mathrm{CDCl}_{3}\right)$ & 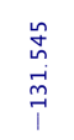 & 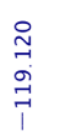 & 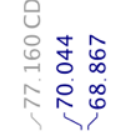 & 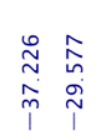 & $\begin{array}{l}\infty \\
\infty \\
\infty \\
\\
\mid 1\end{array}$ \\
\hline
\end{tabular}

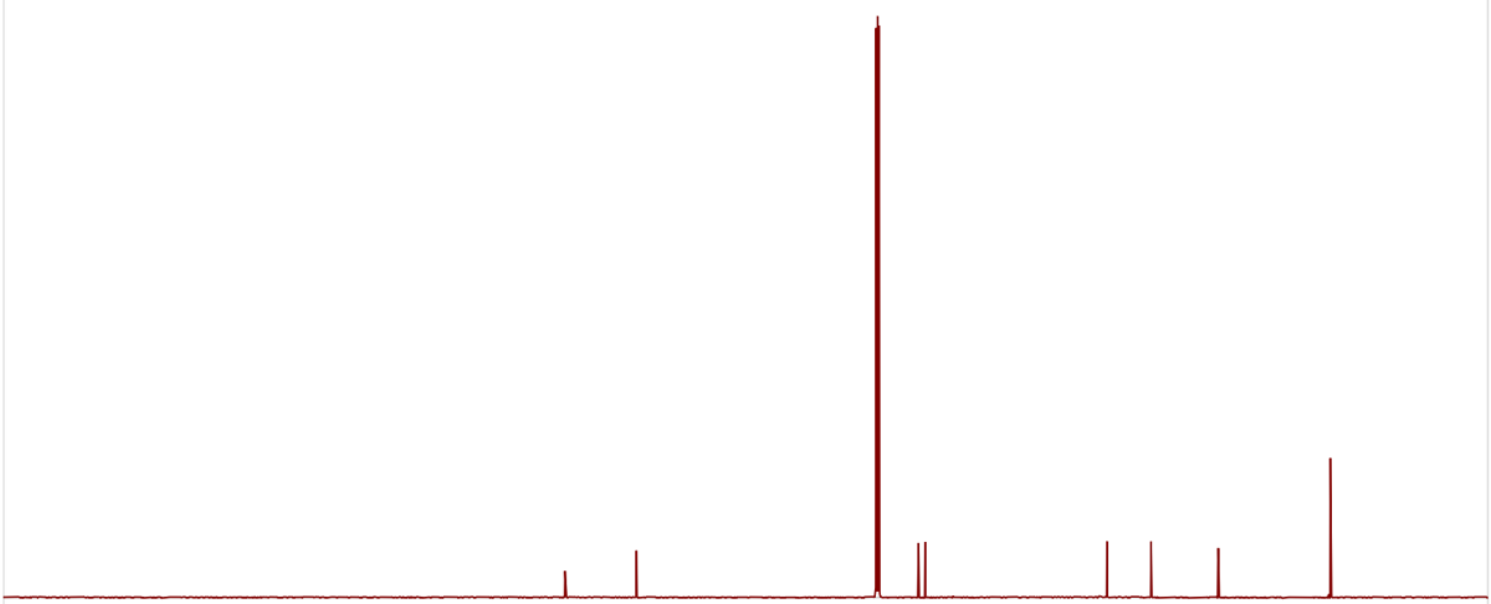

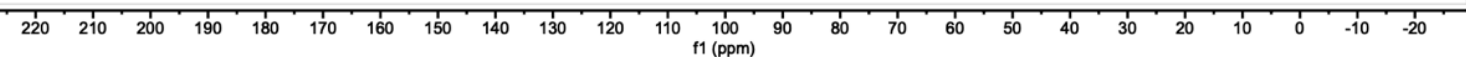




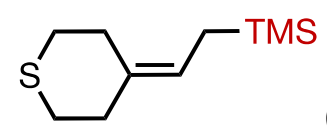

(65)

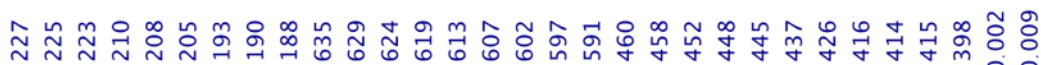
ni nu nu

${ }^{1} \mathbf{H}$ NMR $\left(500 \mathrm{MHz}, \mathrm{CDCl}_{3}\right)$
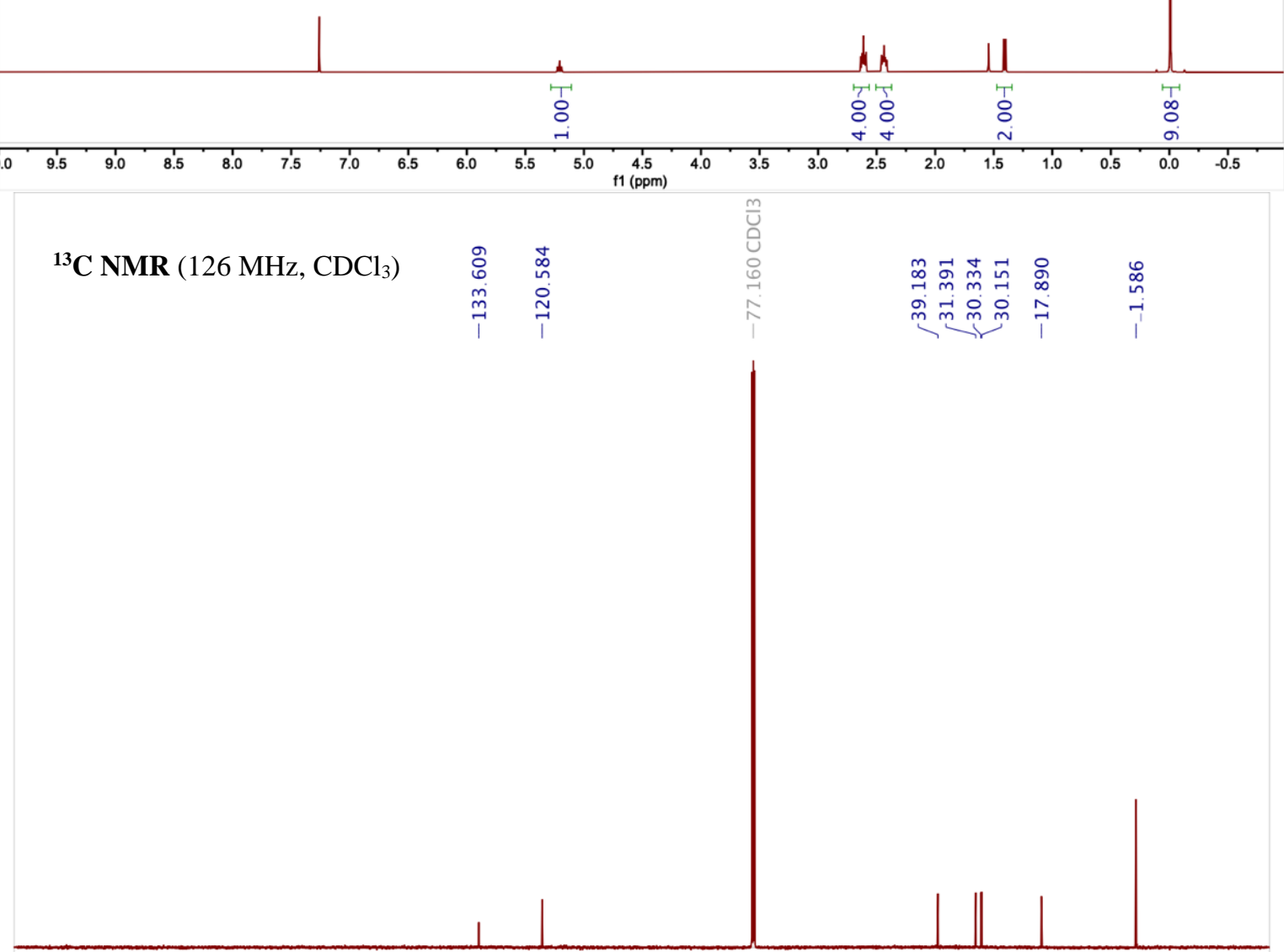

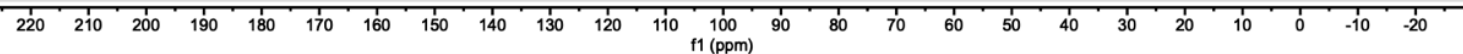



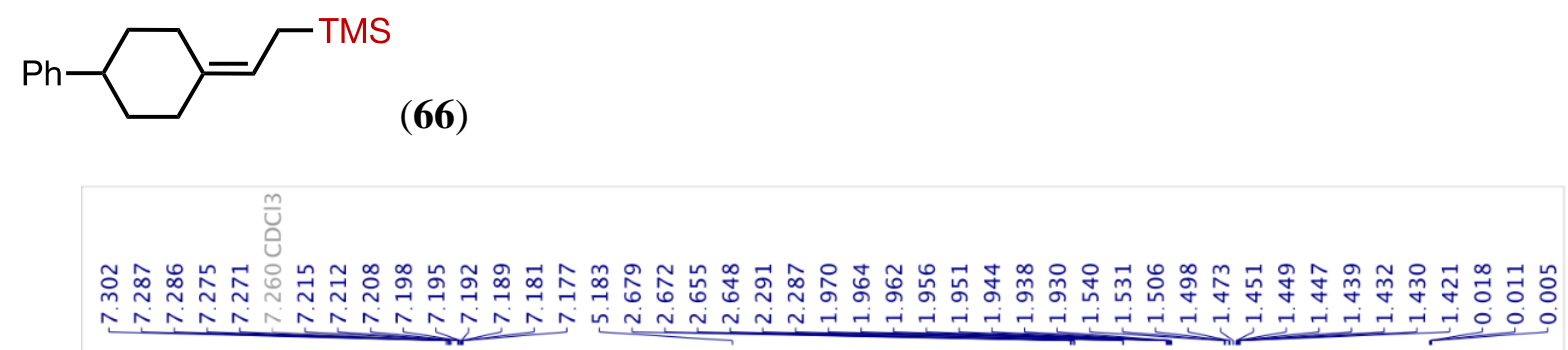

${ }^{\mathbf{1}} \mathbf{H} \mathbf{N M R}\left(500 \mathrm{MHz}, \mathrm{CDCl}_{3}\right)$
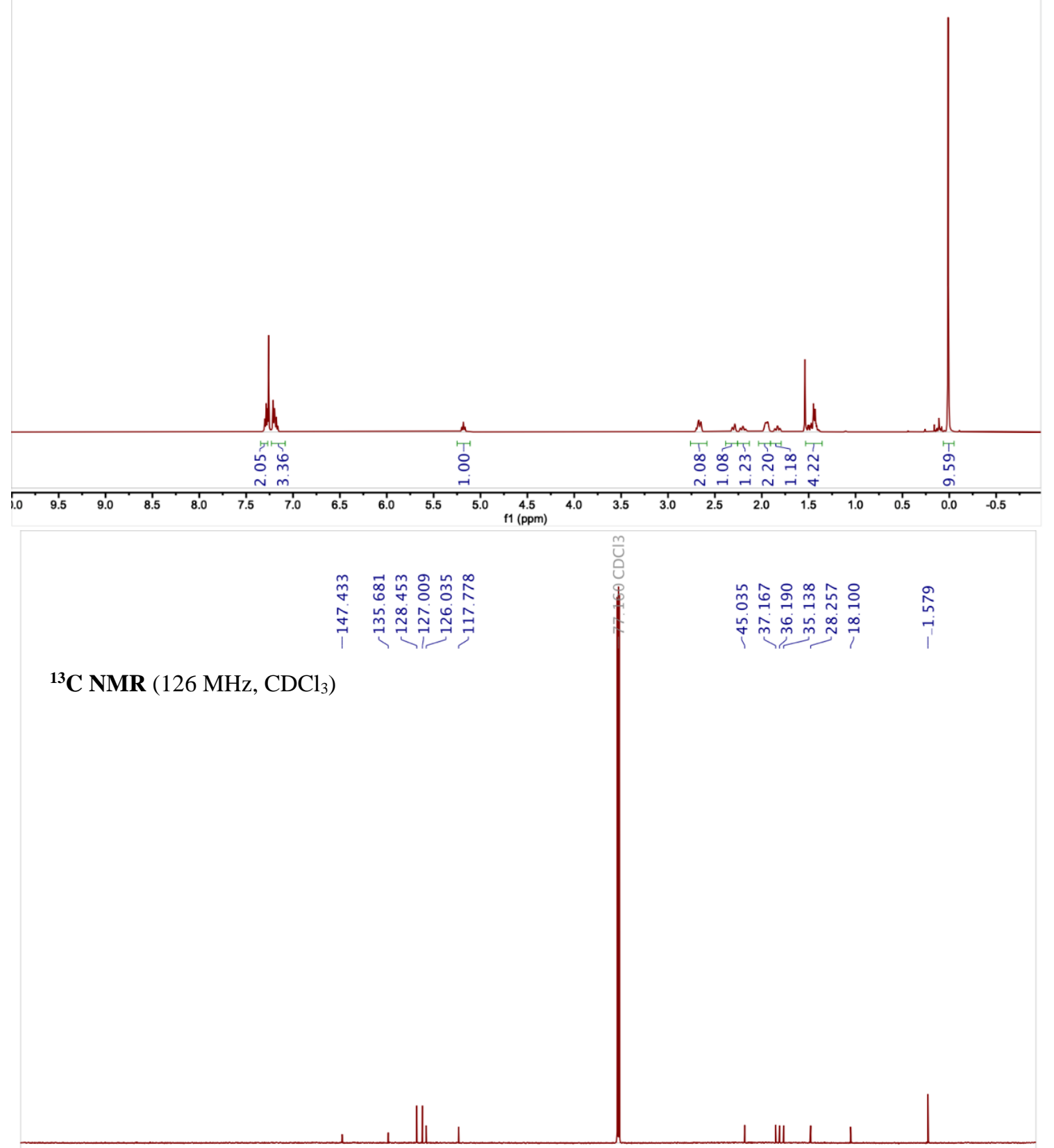

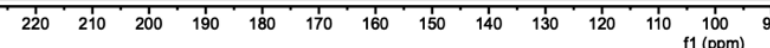


<smiles>CCC1(c2ccccc2)CCCC1</smiles>

${ }^{1} \mathbf{H} \mathbf{N M R}\left(500 \mathrm{MHz}, \mathrm{CDCl}_{3}\right)$
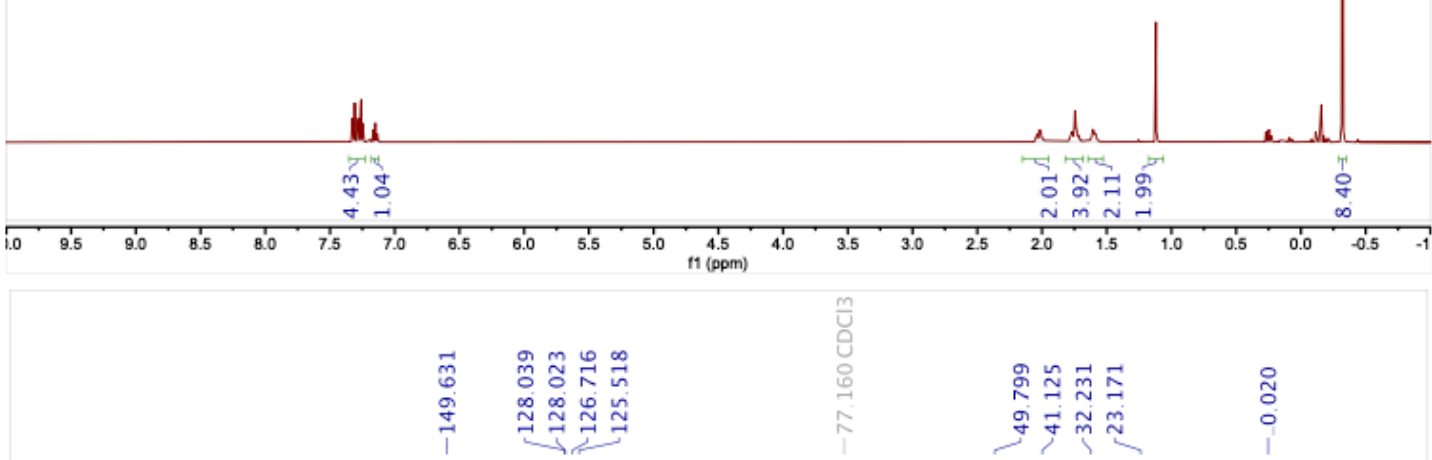

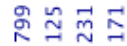

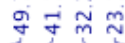

${ }^{13} \mathbf{C}$ NMR $\left(126 \mathrm{MHz}, \mathrm{CDCl}_{3}\right)$

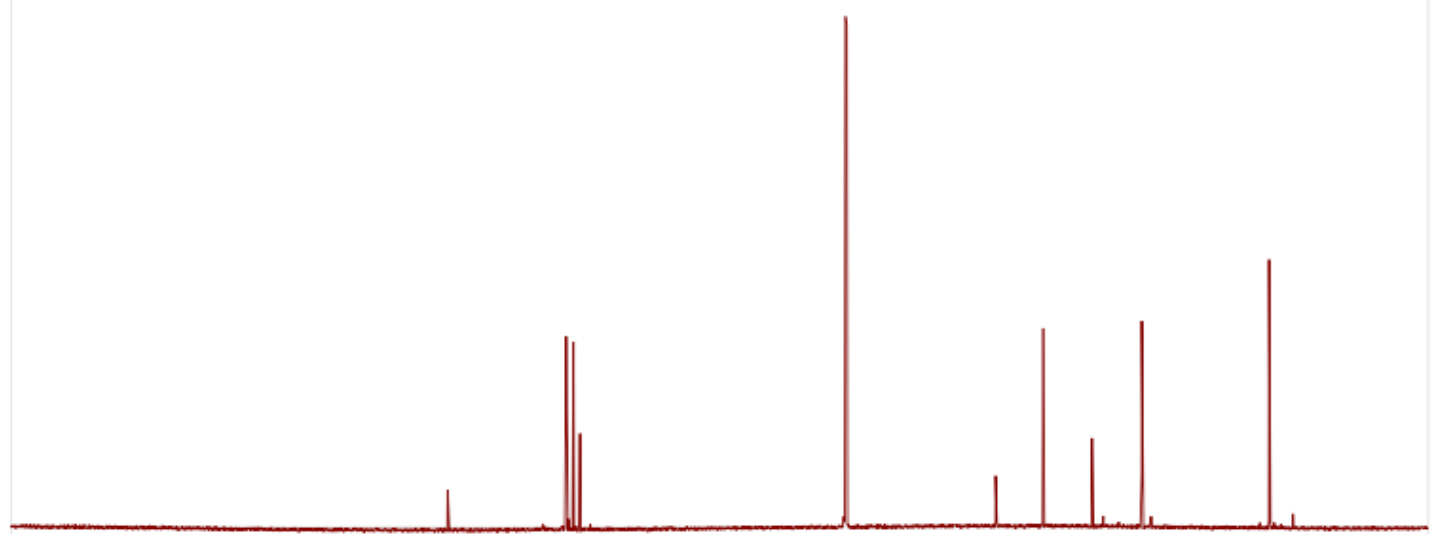

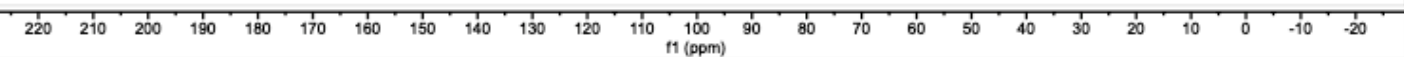




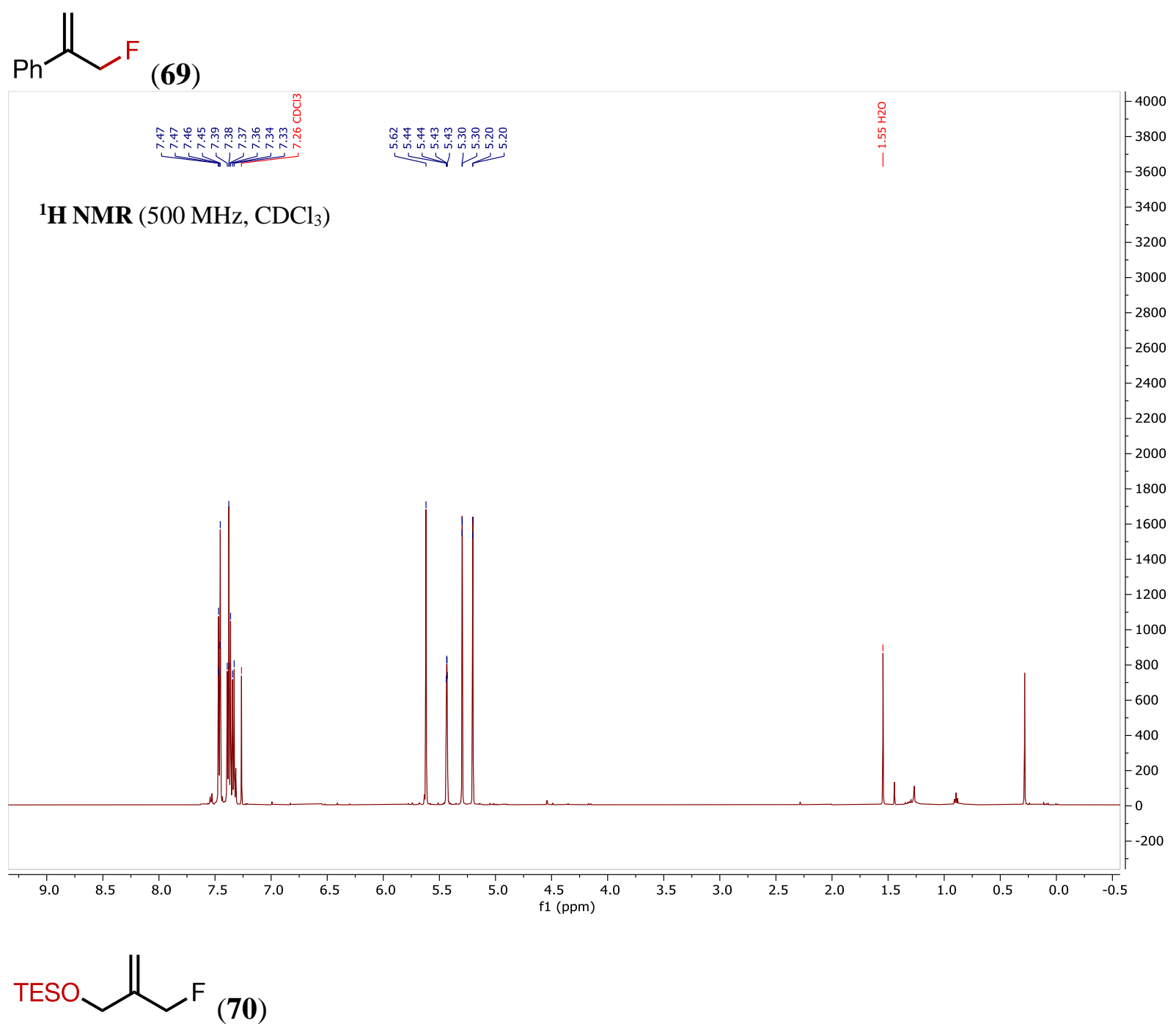




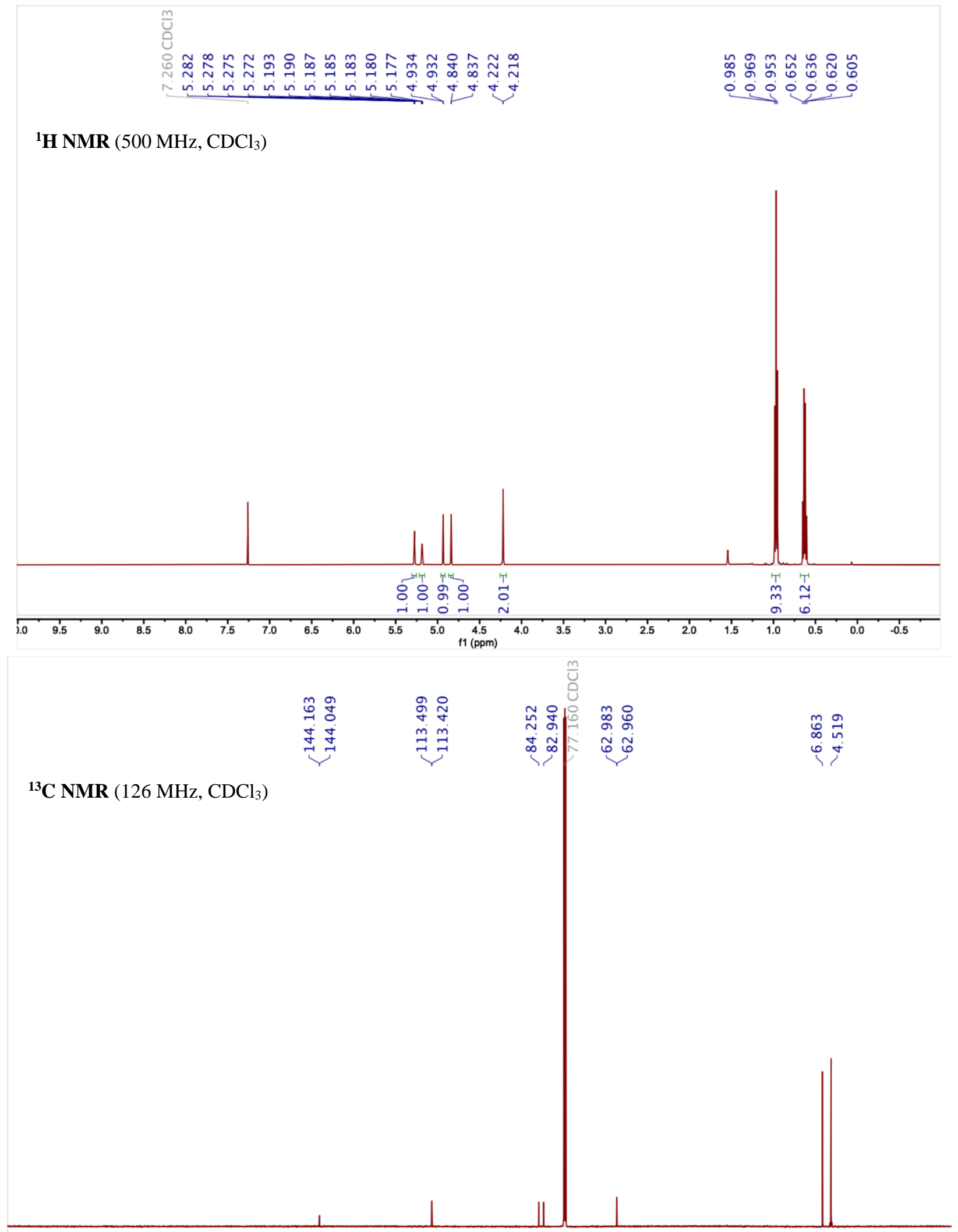

\begin{tabular}{lllllllllllllllllllllllll}
\hline & 1 \\
220 & 210 & 200 & 190 & 180 & 170 & 160 & 150 & 140 & 130 & 120 & 110 & 100 & 90 & 80 & 70 & 60 & 50 & 10 & 30 & 10 & 10 & 0 & -10 & -20
\end{tabular} S161 
${ }^{19} \mathbf{F} \mathbf{N M R}\left(470 \mathrm{MHz}, \mathrm{CDCl}_{3}\right)$

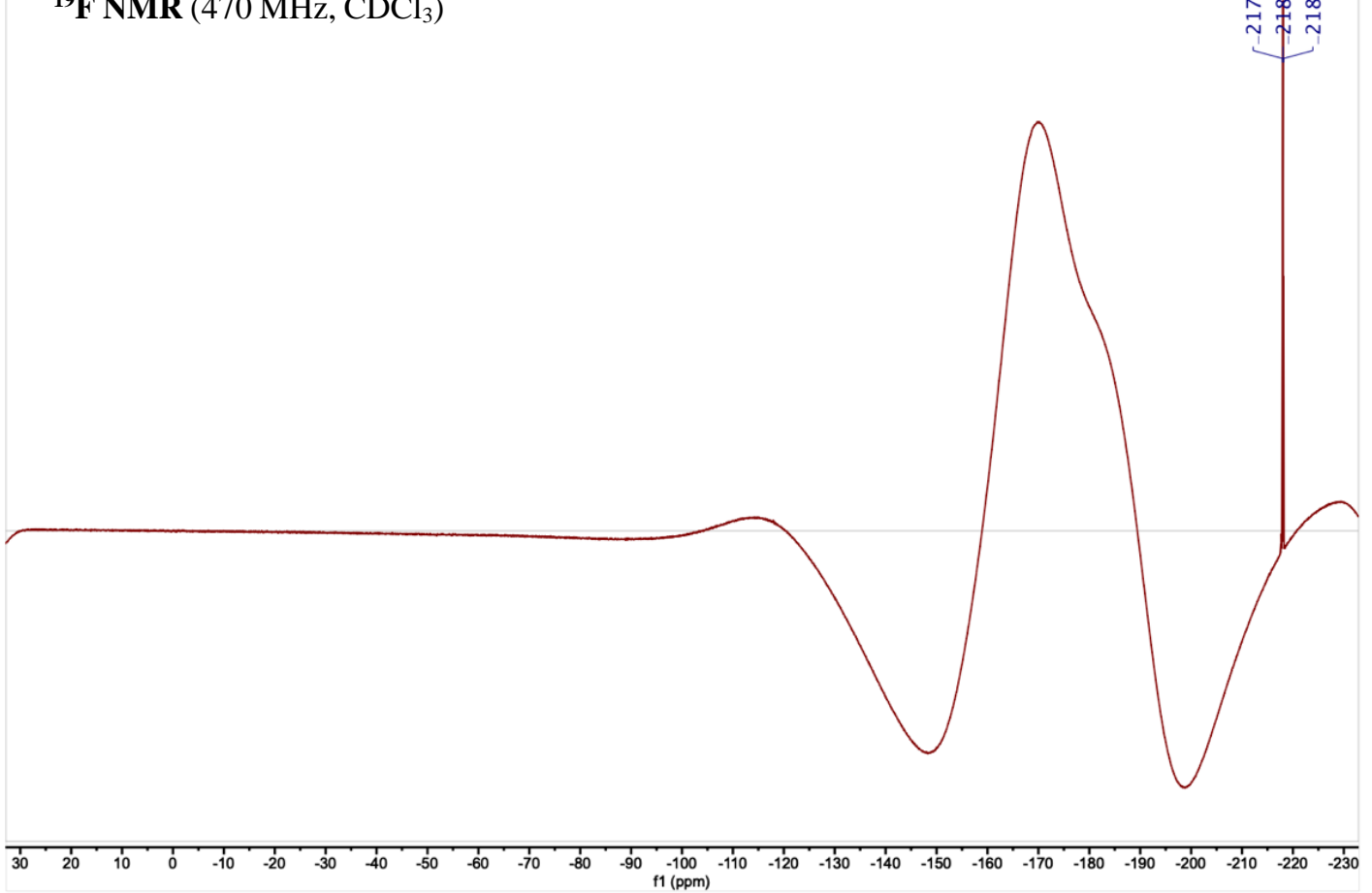<smiles>OC([Te])CS[Te]</smiles>

(71)

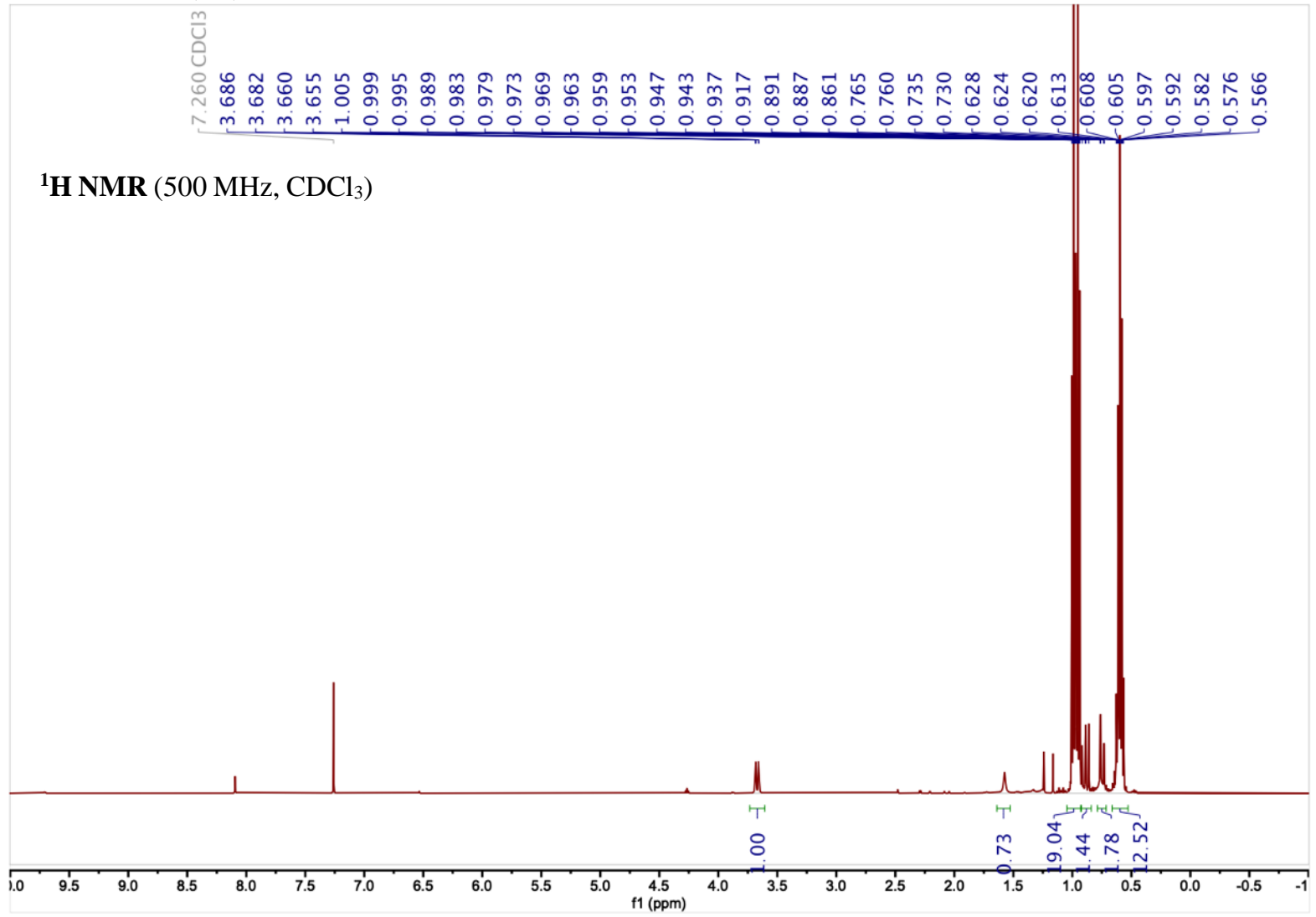


There is trace amount of impurity in ${ }^{1} \mathrm{H}$ NMR and cannot be removed via column chromatography (e.g. singlet peak at 8.1) and the satellite peaks of TES results in some integration deviation of some protons connected to the two carbons.
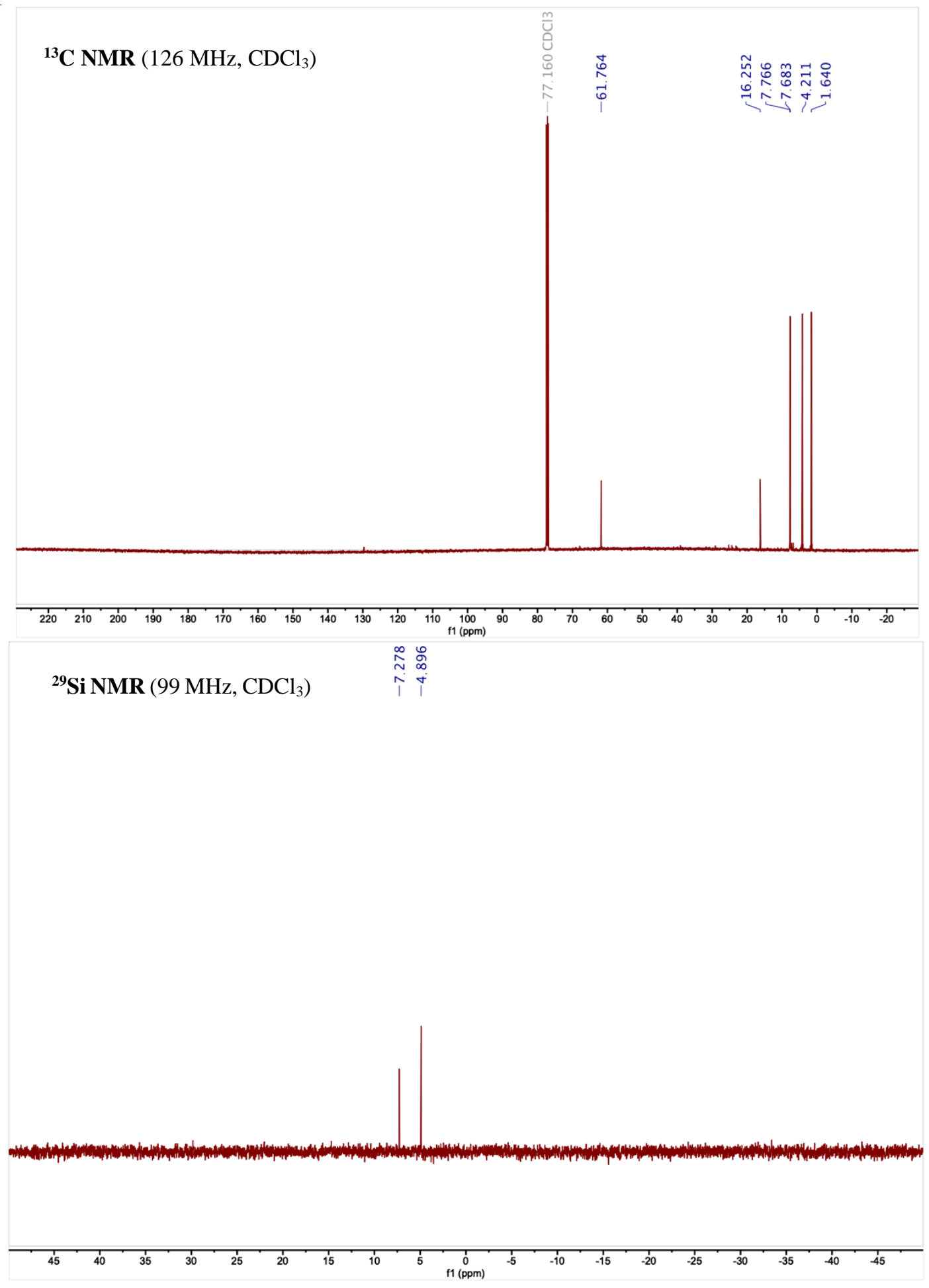


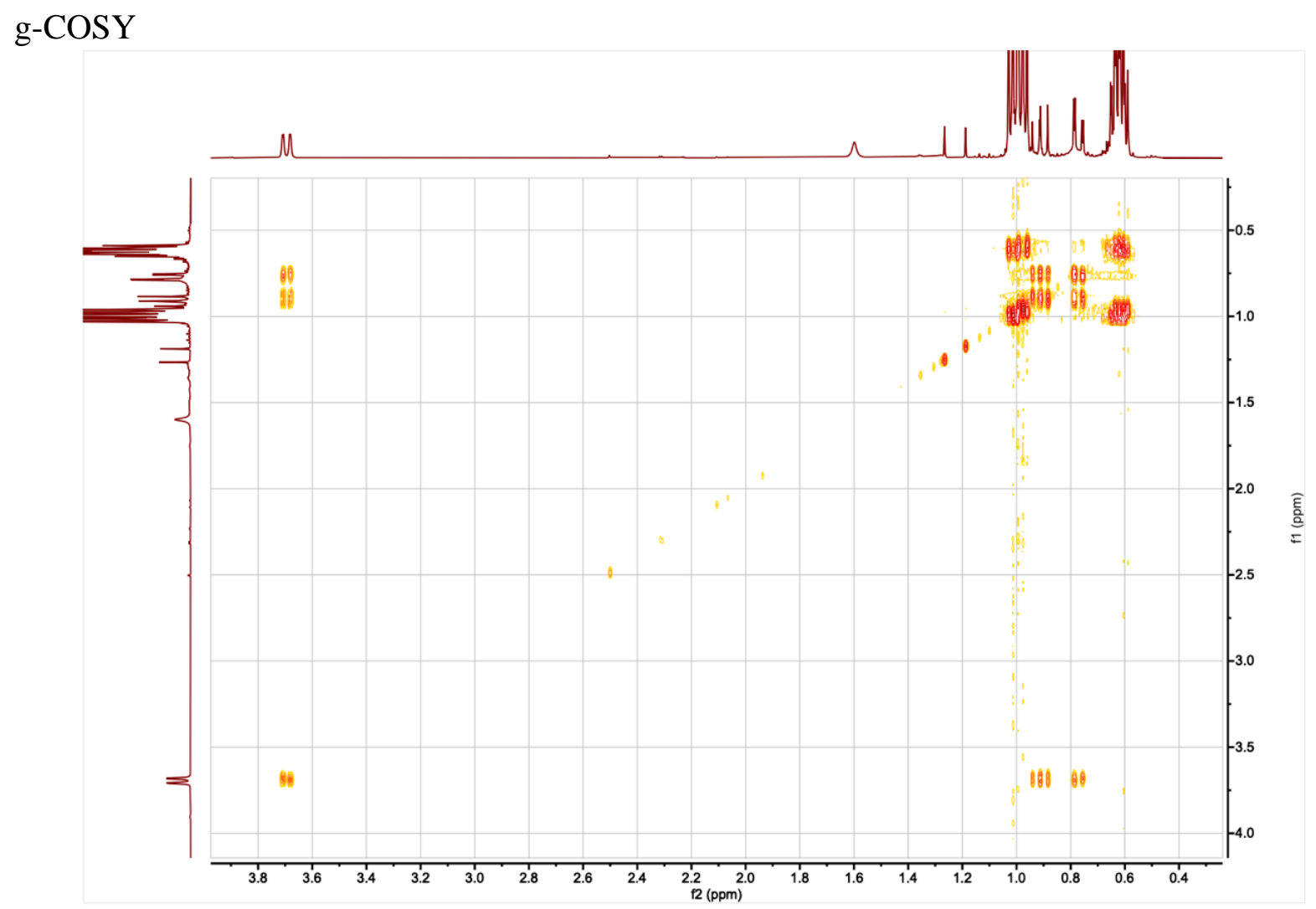

HSQC

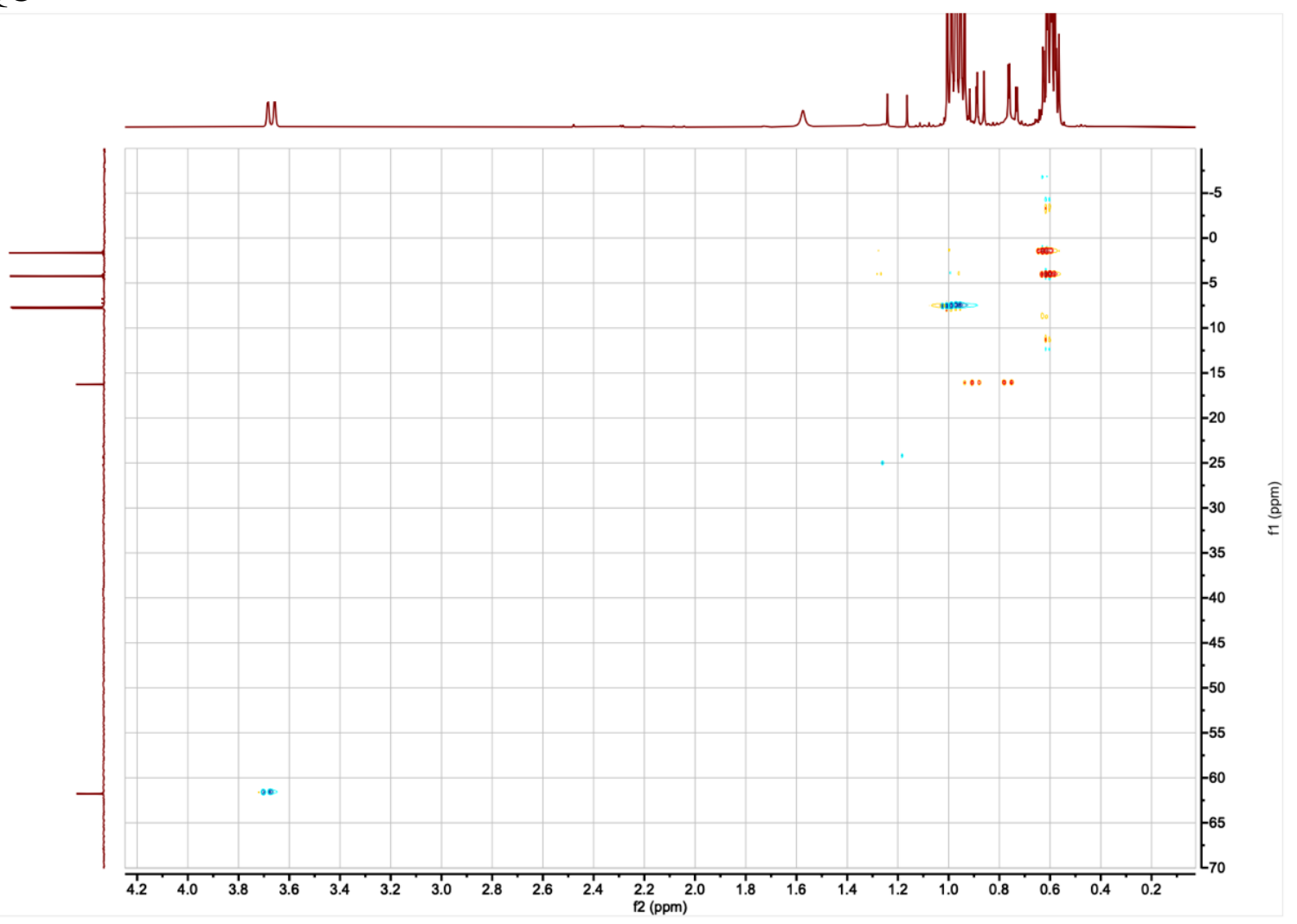



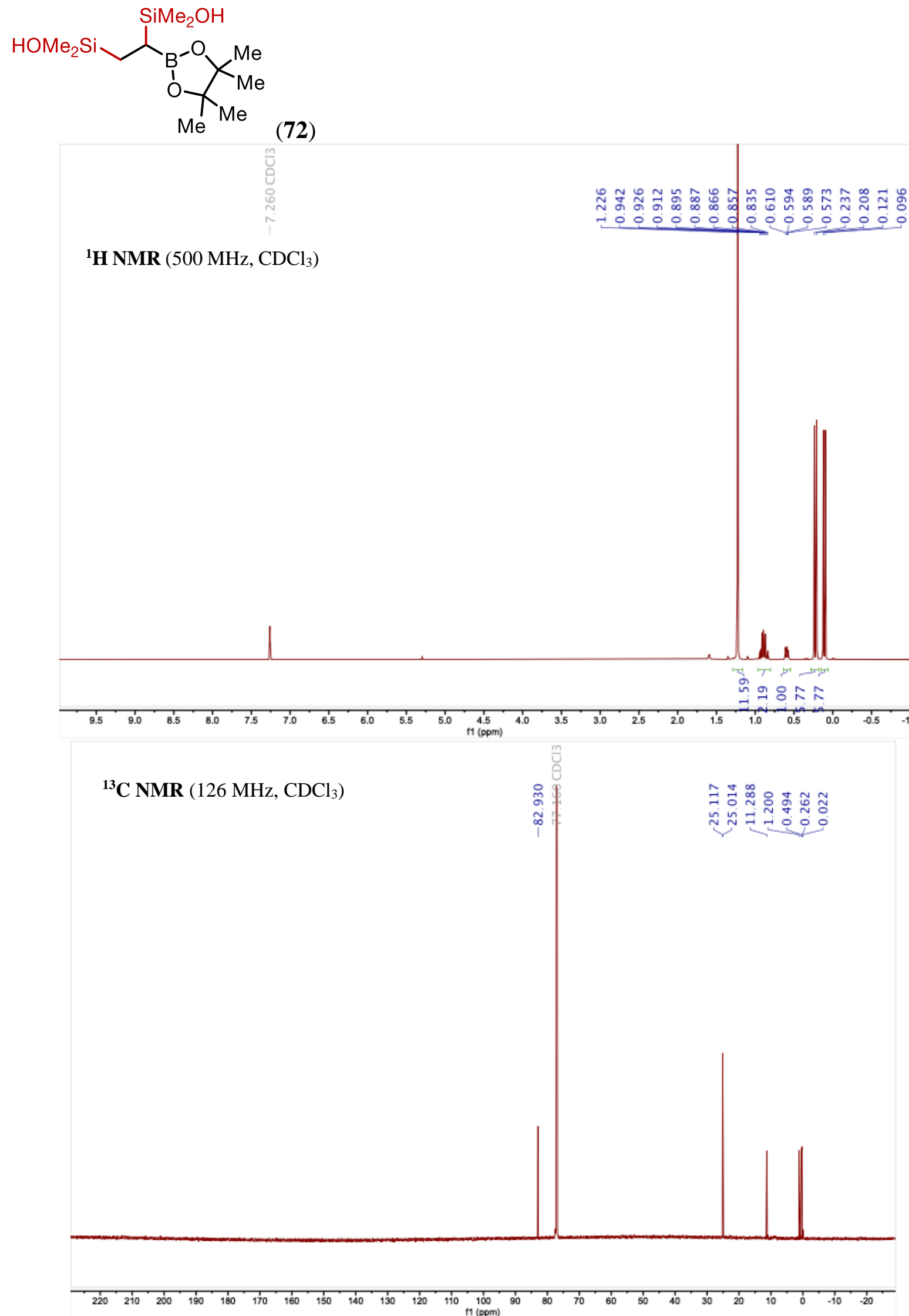


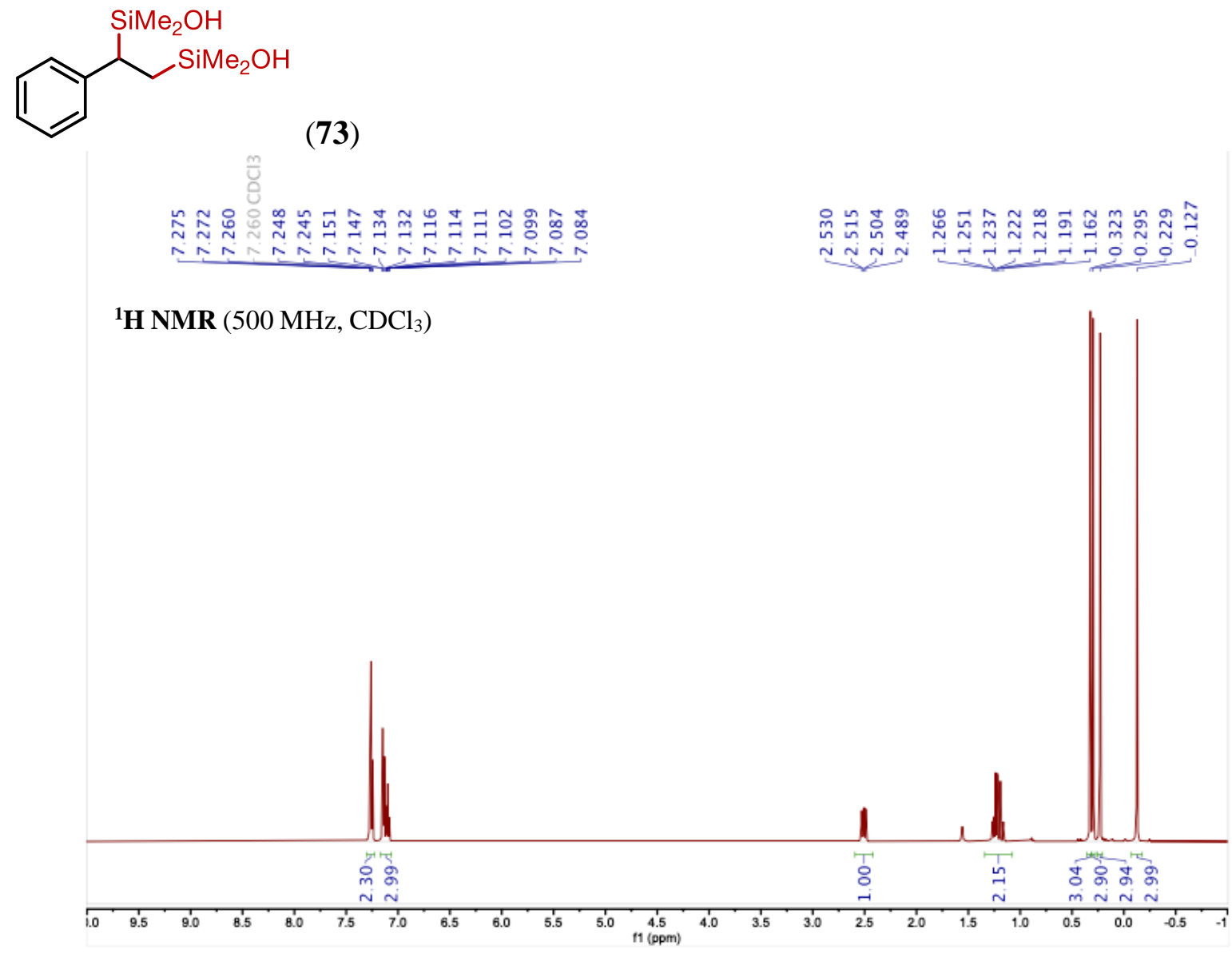

$$
\begin{aligned}
& \text { กิ } \\
& \text { 声 } \stackrel{\infty}{=} \underset{1}{\stackrel{0}{ \pm}}
\end{aligned}
$$

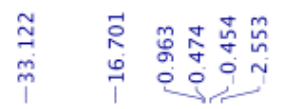

${ }^{13} \mathbf{C}$ NMR $\left(126 \mathrm{MHz}, \mathrm{CDCl}_{3}\right)$

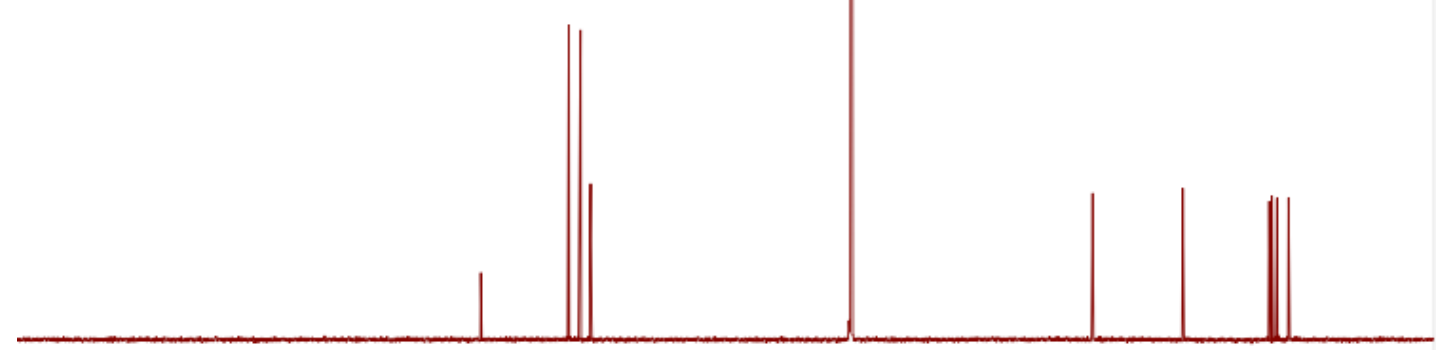

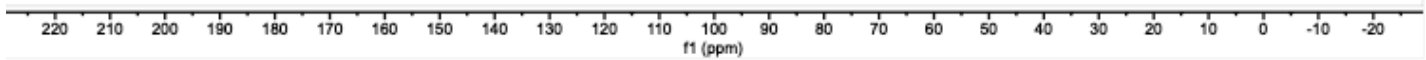



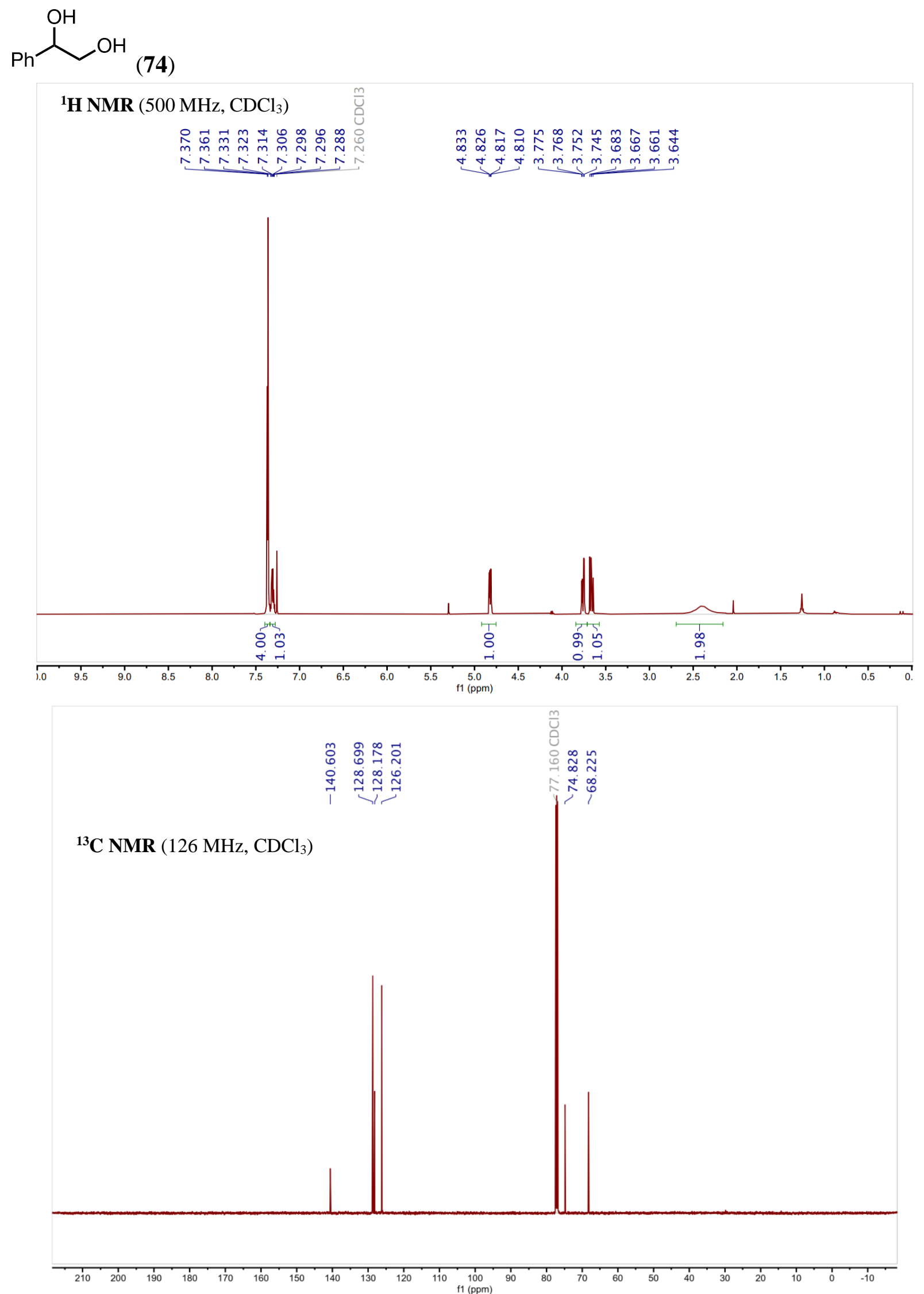

S167 


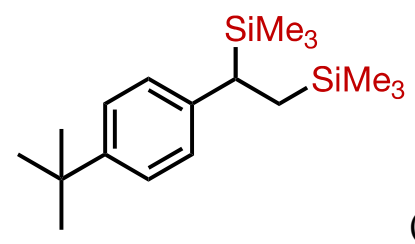

(76)
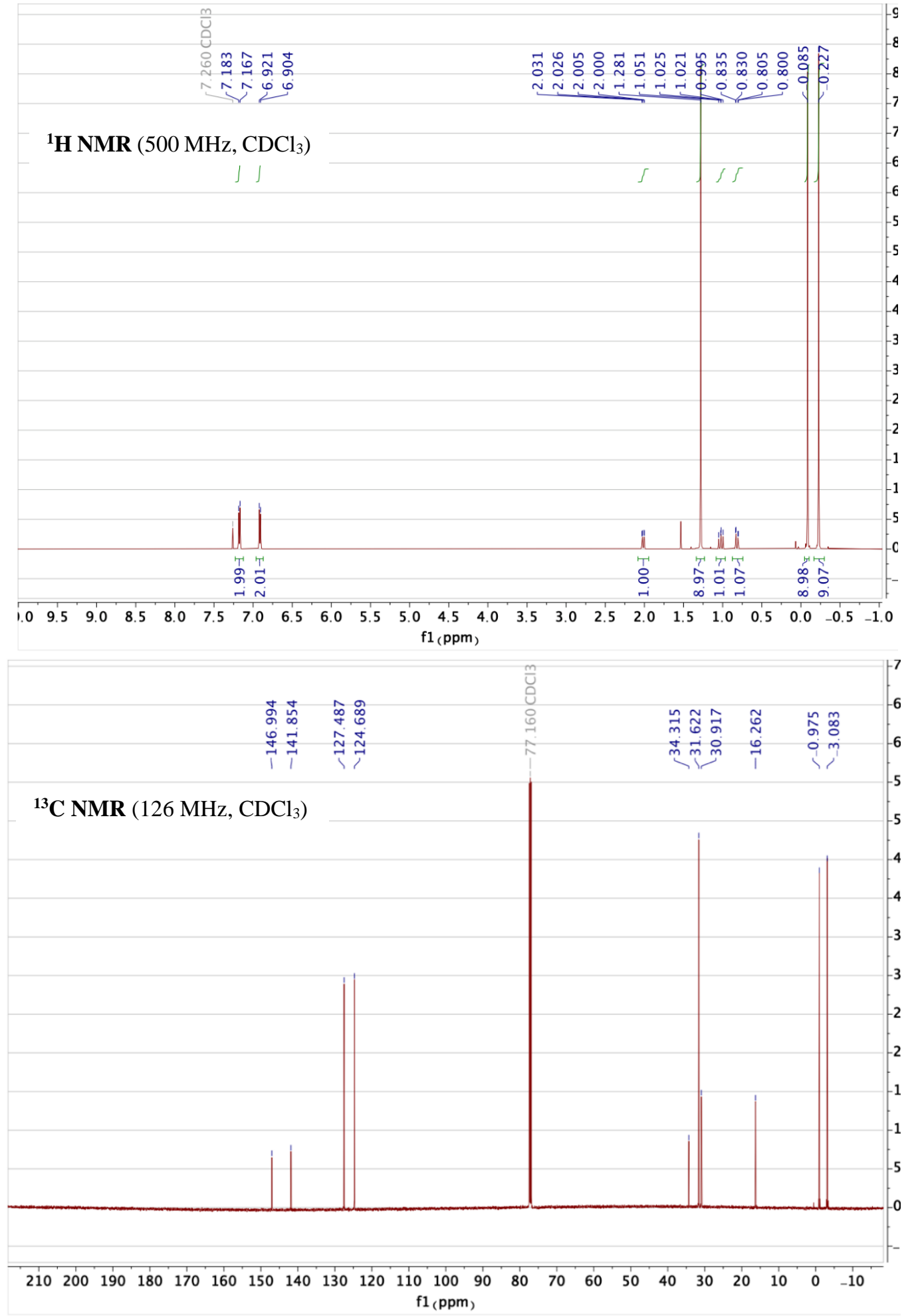

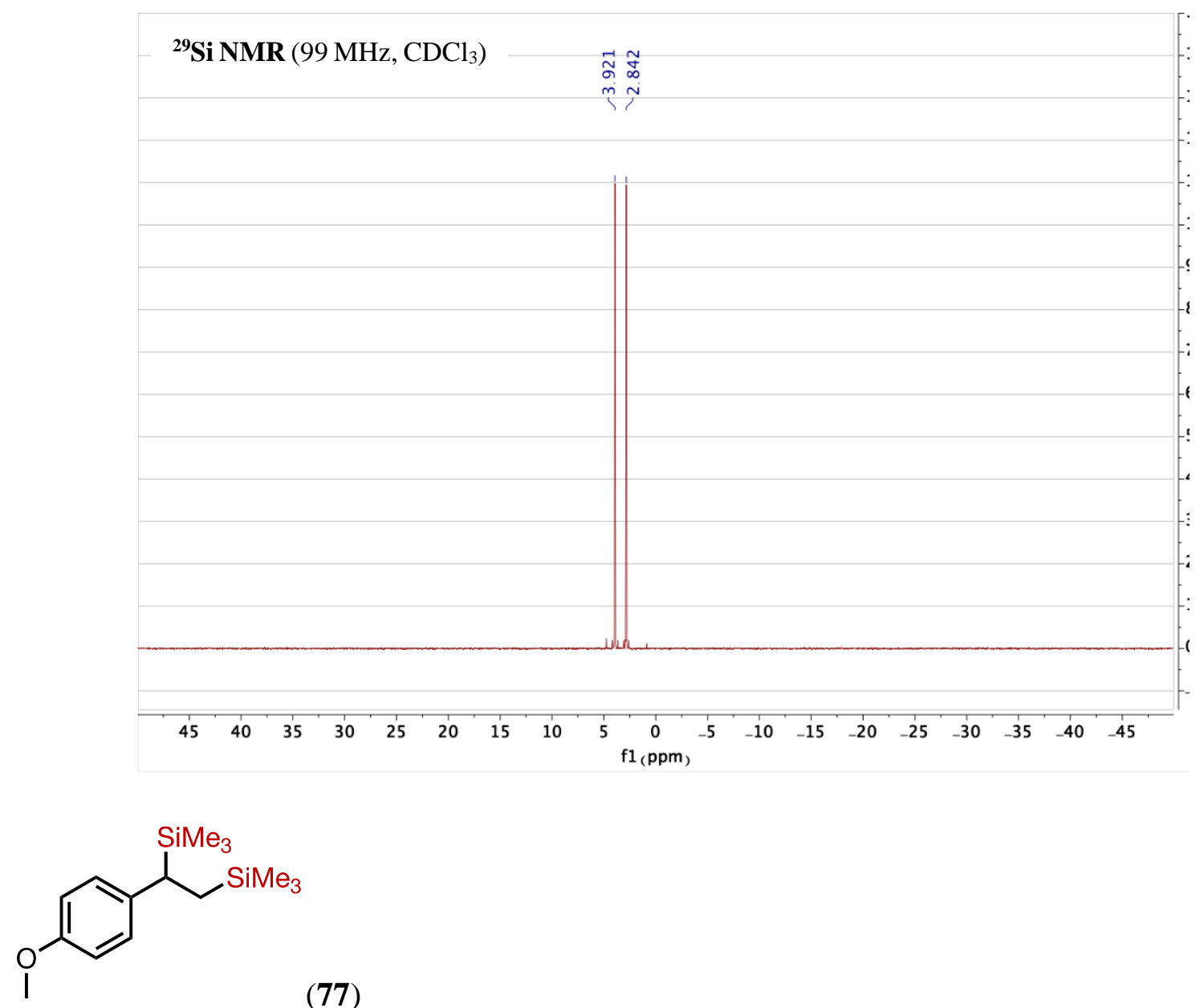

(77)

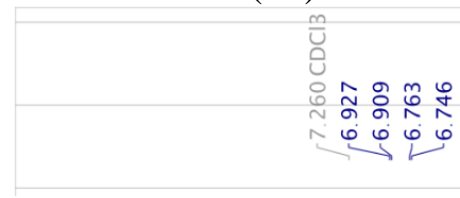

${ }^{\mathbf{1}} \mathbf{H} \mathbf{N M R}$ (500 MHz, $\mathrm{CDCl}_{3}$ )

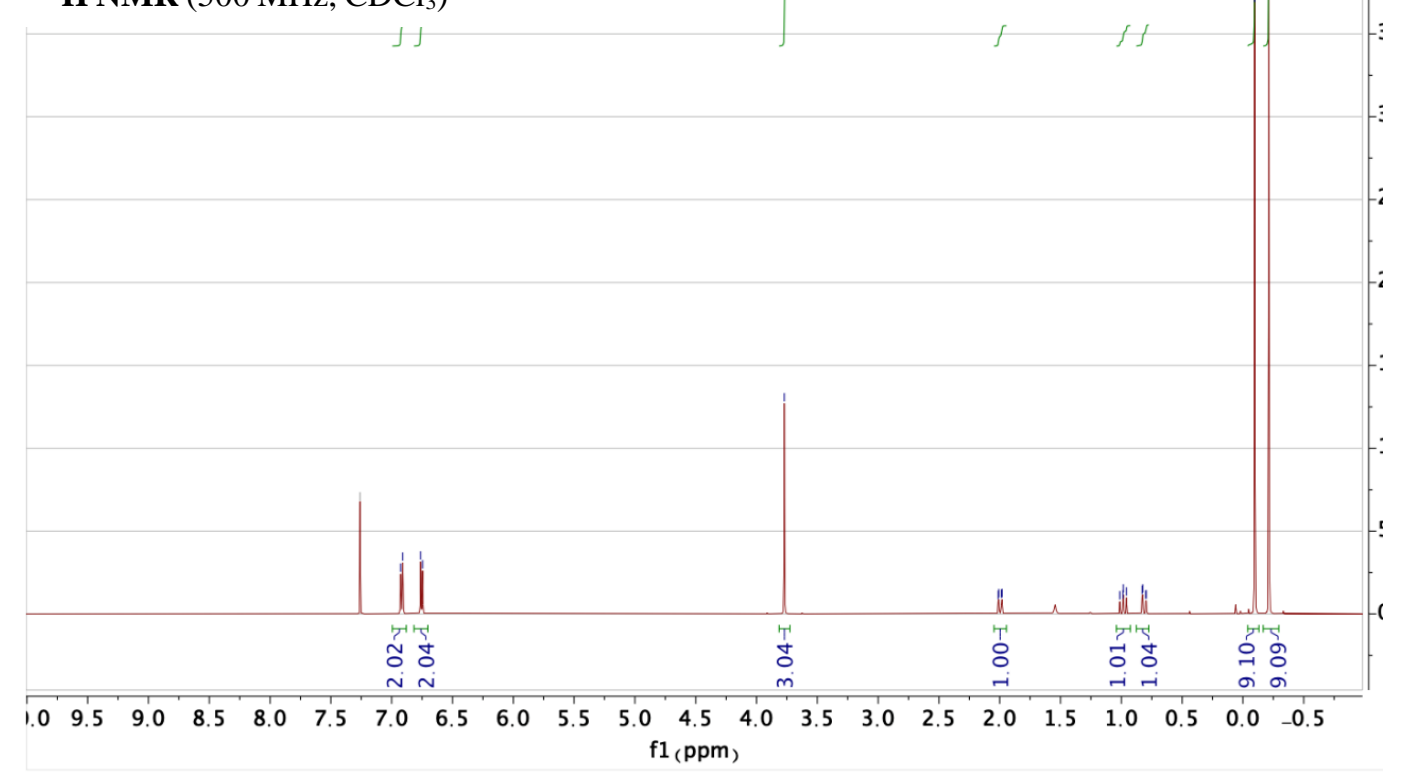




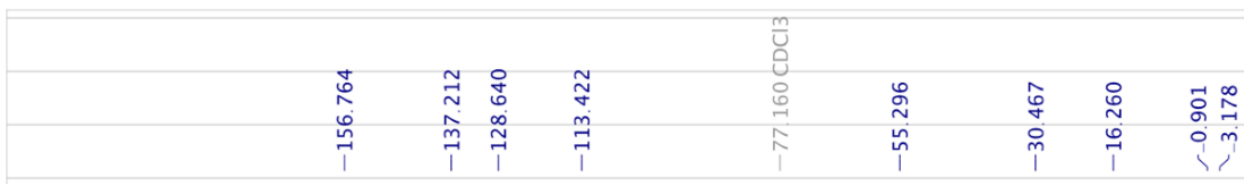

${ }^{13} \mathbf{C}$ NMR $\left(126 \mathrm{MHz}, \mathrm{CDCl}_{3}\right)$

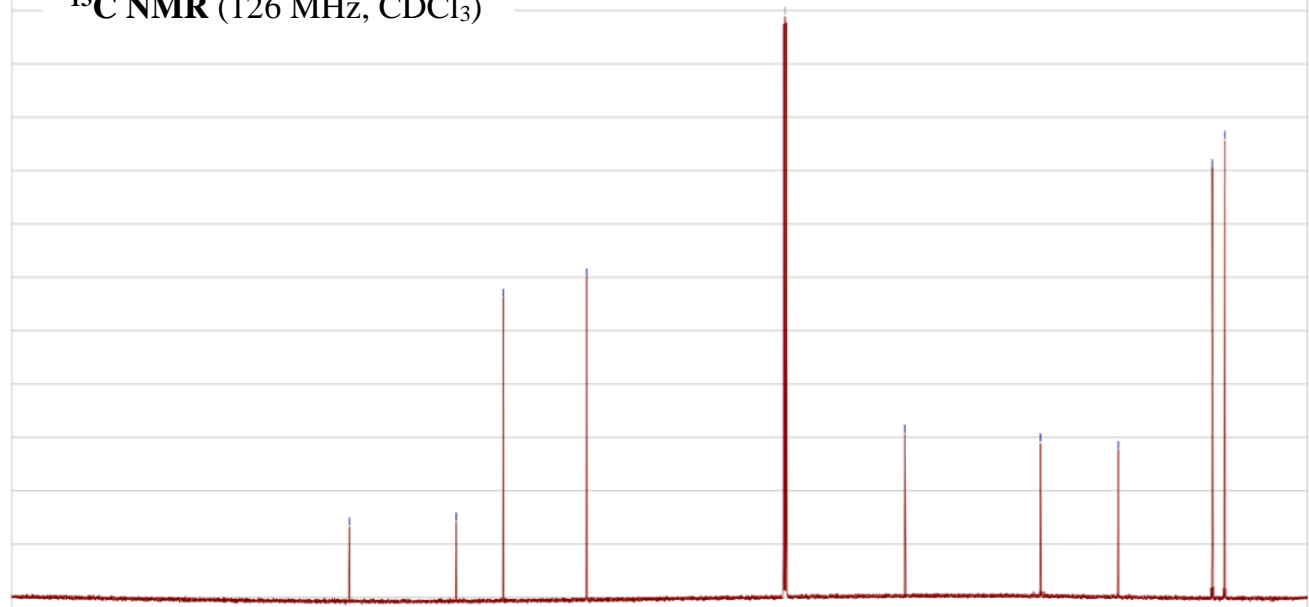

$\begin{array}{lllllllllllllllllllllllllllllllllll}210 & 200 & 190 & 180 & 170 & 160 & 150 & 140 & 130 & 120 & 110 & 100 & 90 & 80 & 70 & 60 & 50 & 40 & 30 & 20 & 10 & 0 & -10\end{array}$

f1 (ppm)<smiles>CCC(C)c1ccc(-c2ccccc2)cc1</smiles>

(78) 

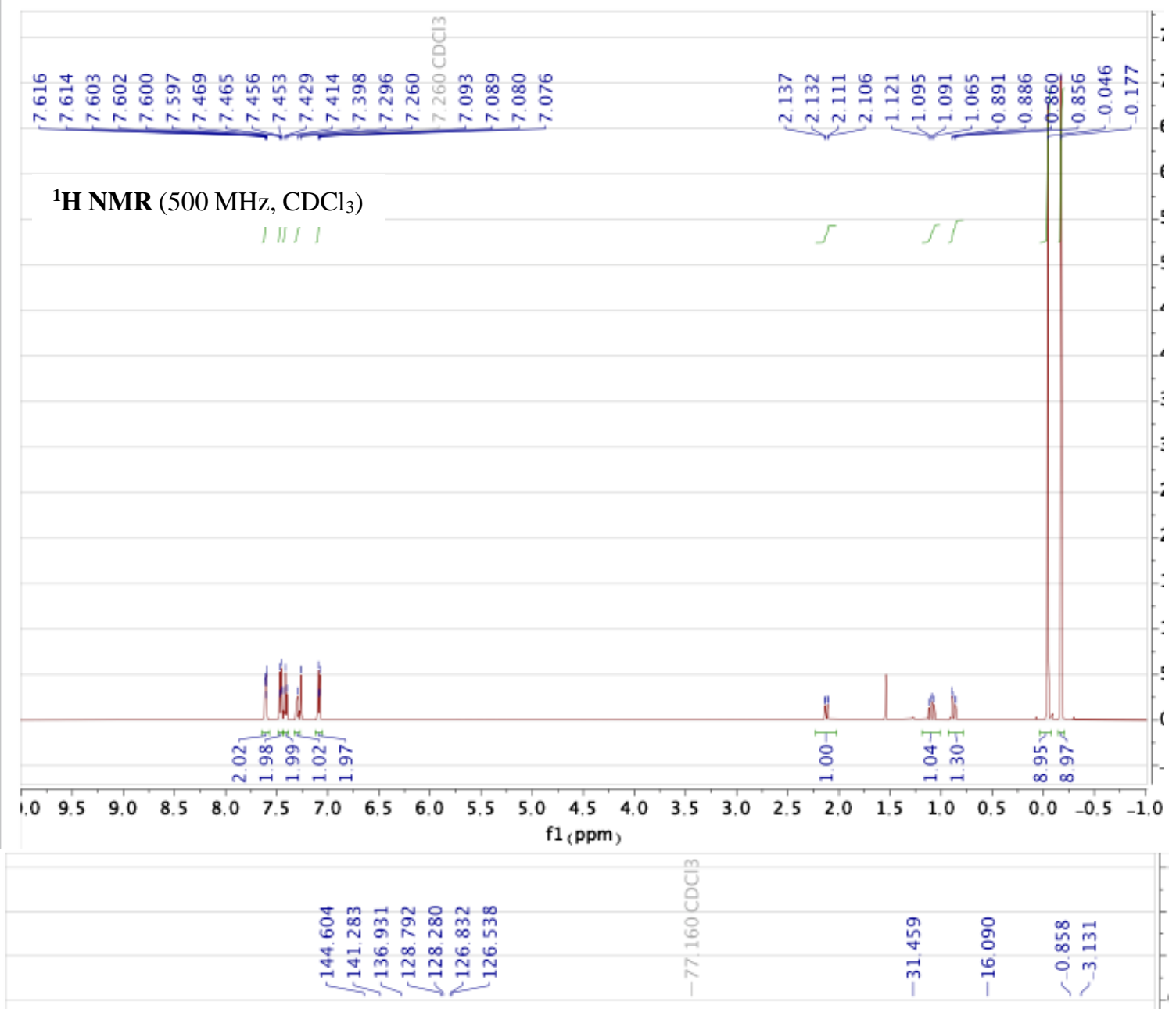

${ }^{13} \mathbf{C}$ NMR $\left(126 \mathrm{MHz}, \mathrm{CDCl}_{3}\right)$

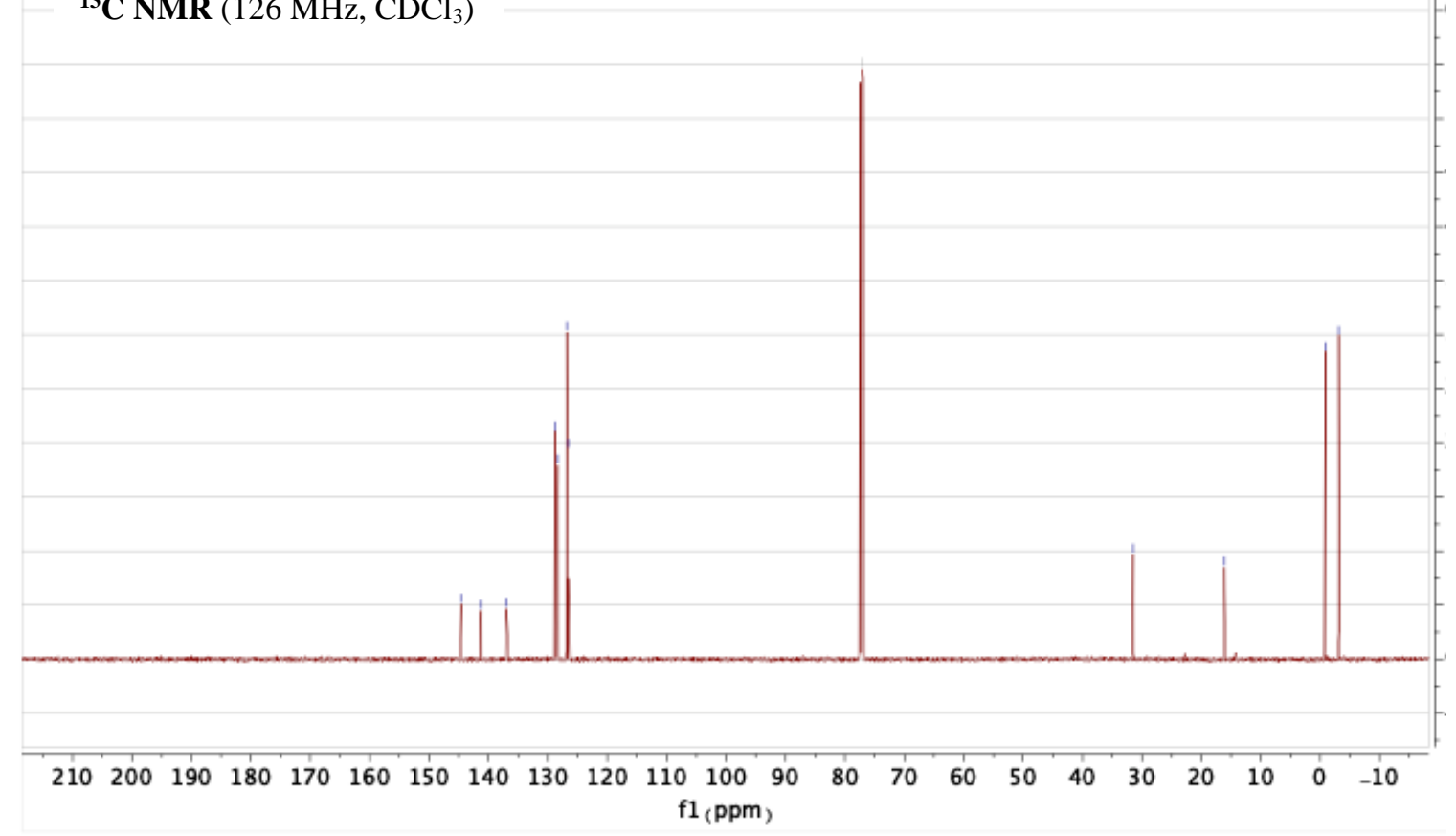


<smiles>CCCCC(c1ccccc1C)C(C)C</smiles>

(79)
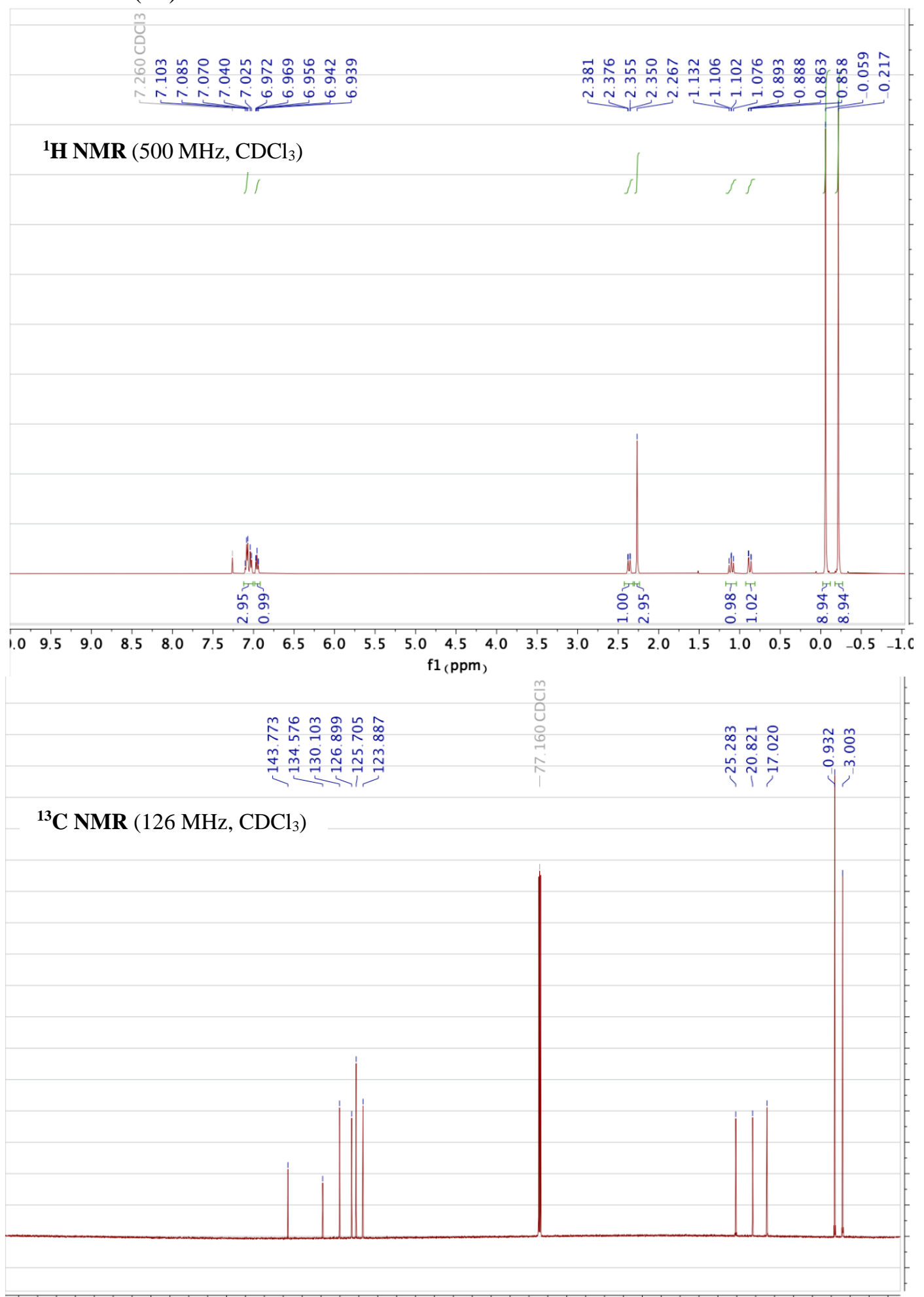

$\begin{array}{lllllllllllllllllllllllllll}210 & 200 & 190 & 180 & 170 & 160 & 150 & 140 & 130 & 120 & 110 & 100 & 90 & 80 & 70 & 60 & 50 & 40 & 30 & 20 & 10 & 0 & -10\end{array}$

$\mathrm{f1}(\mathrm{ppm})$ 


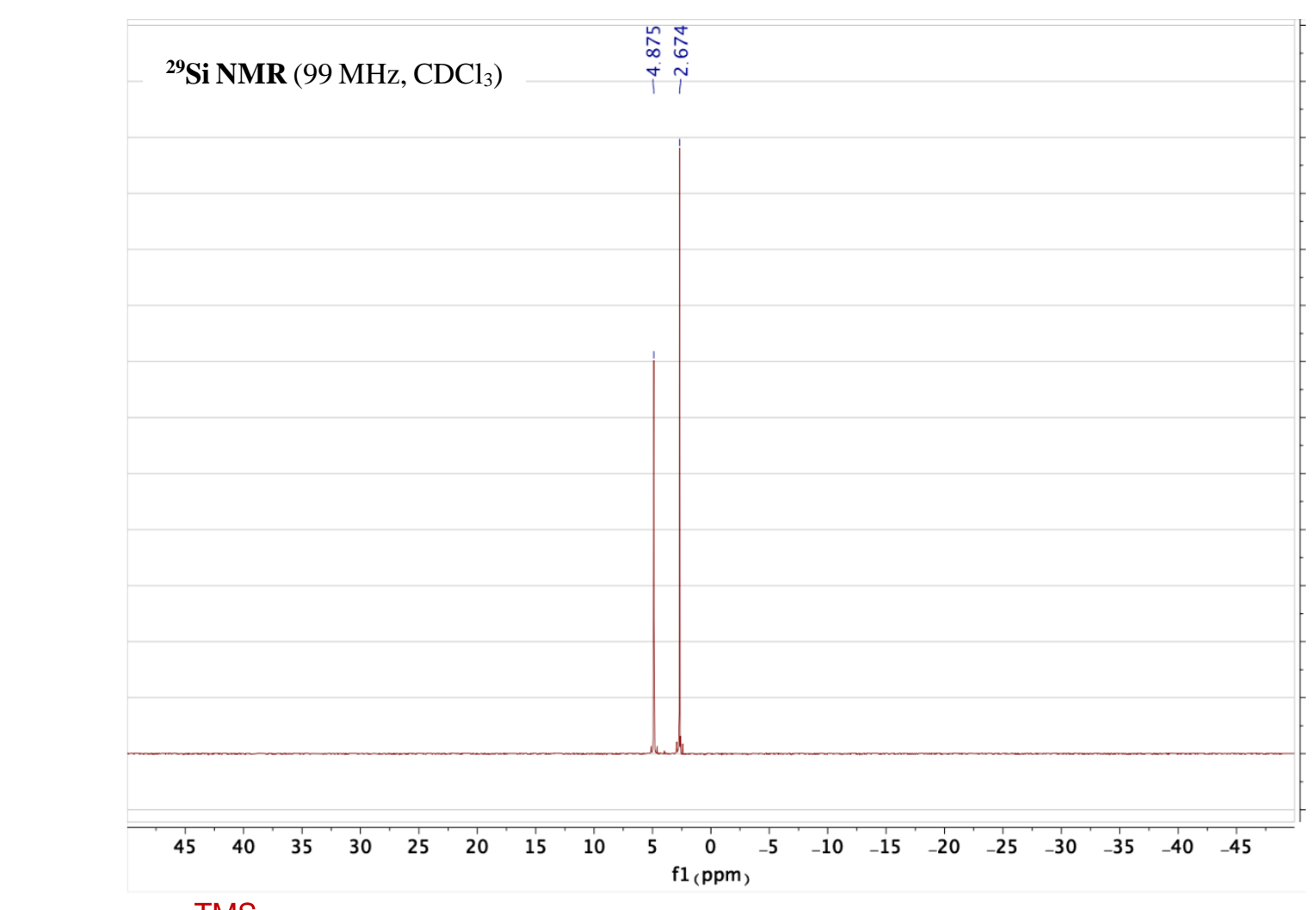<smiles>CCC(CC(C)(C)C)c1ccc2c(c1)OCO2</smiles>

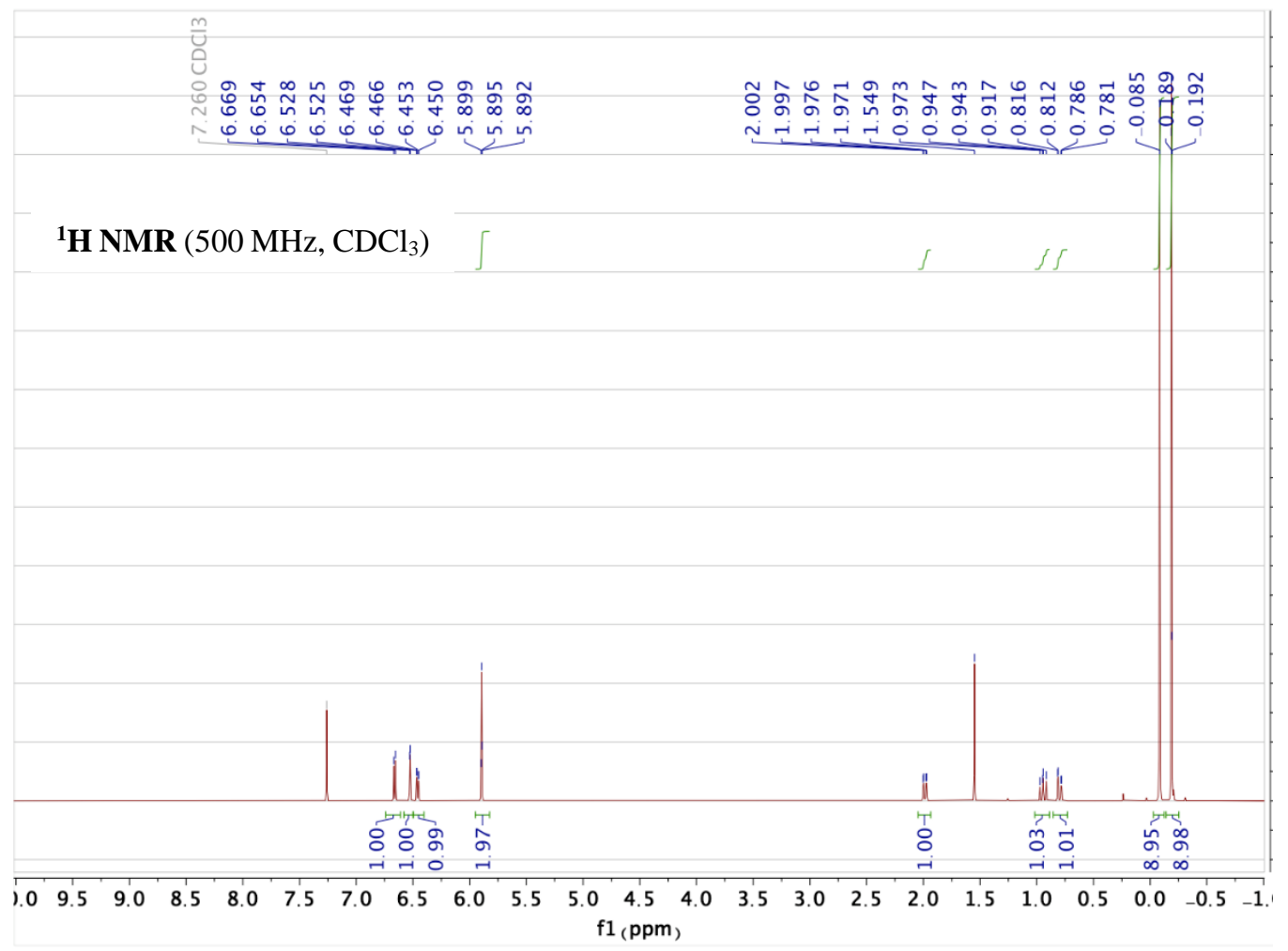




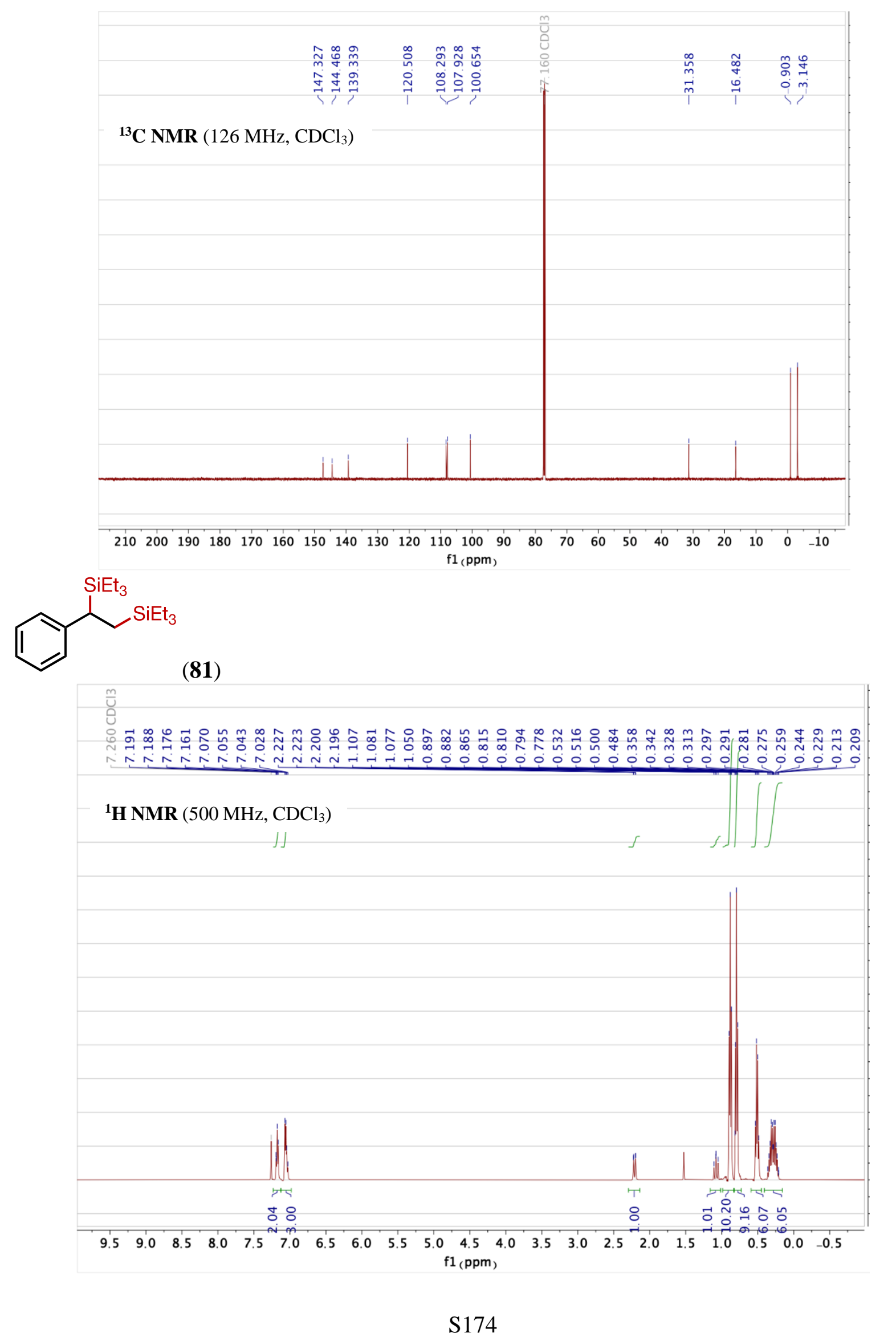




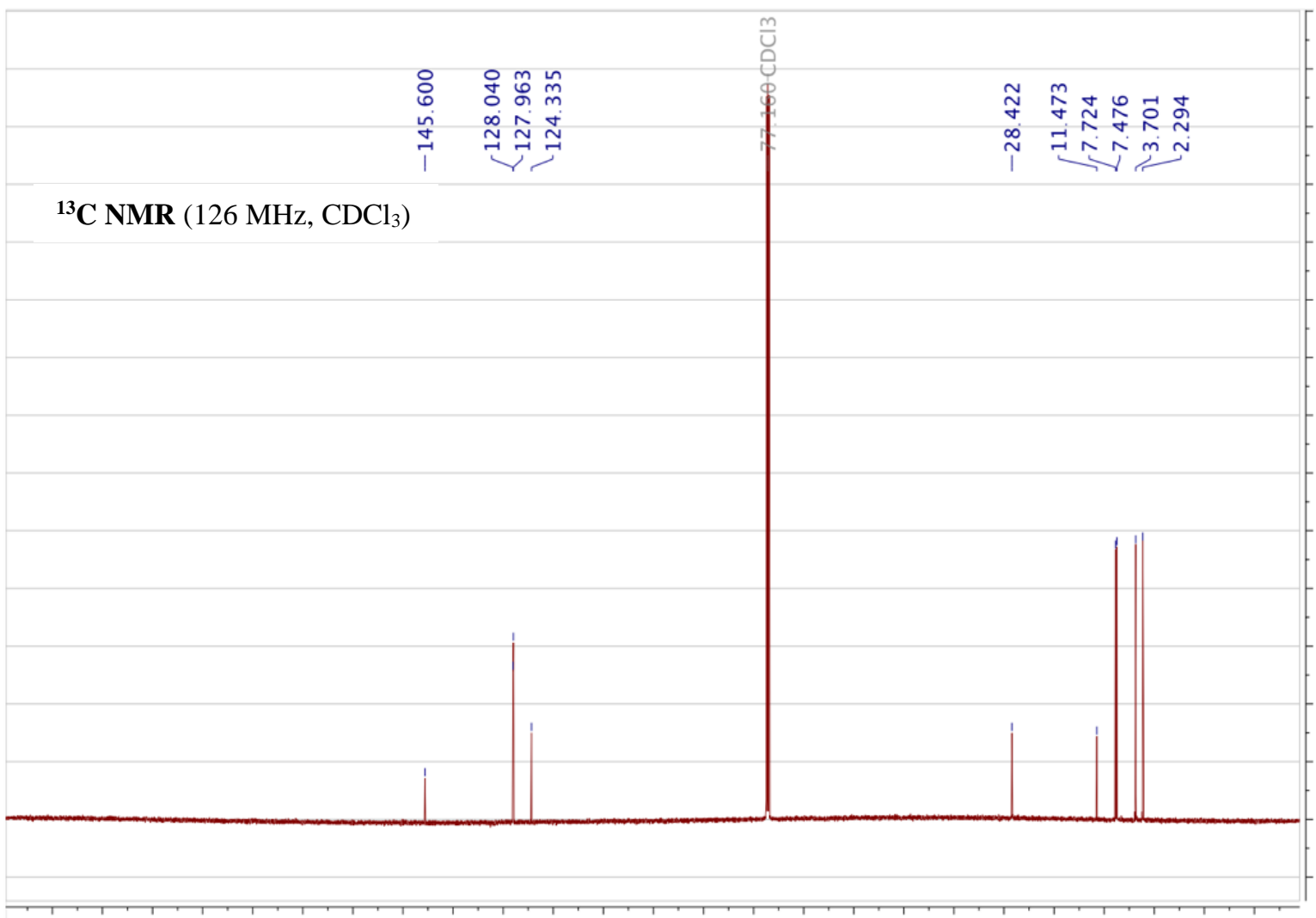

$\begin{array}{llllllllllllllllllllllllllllllllll}220 & 210 & 200 & 190 & 180 & 170 & 160 & 150 & 140 & 130 & 120 & 110 & 100 & 90 & 80 & 70 & 60 & 50 & 40 & 30 & 20 & 10 & 0 & -10 & -20\end{array}$ $\mathrm{f1}(\mathrm{ppm})$

${ }^{29} \mathrm{Si} \mathrm{NMR}\left(99 \mathrm{MHz}, \mathrm{CDCl}_{3}\right)$

유소

$\infty i$

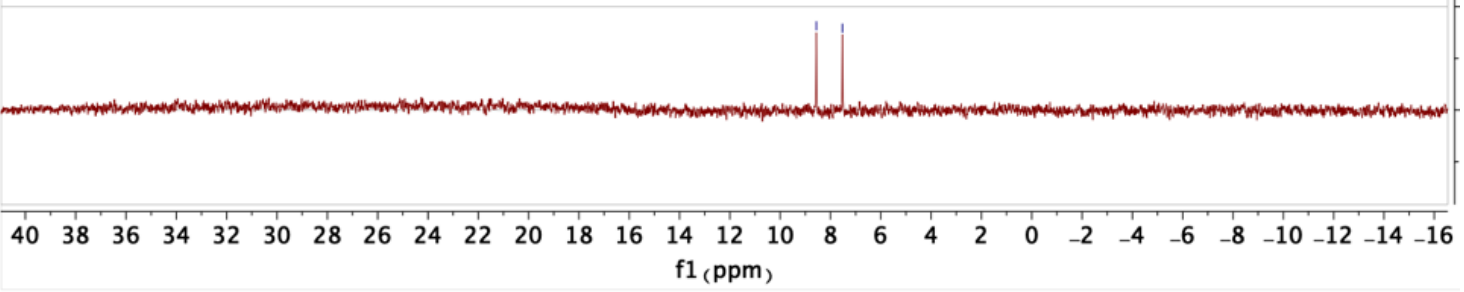

S175 


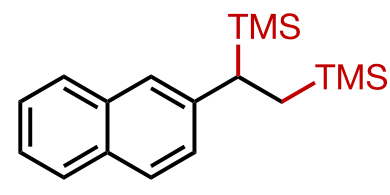

(82)

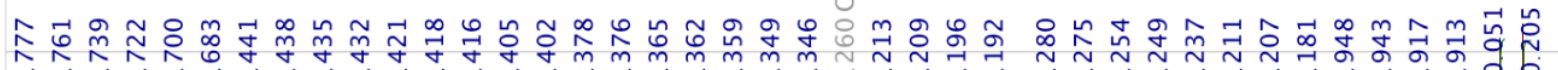

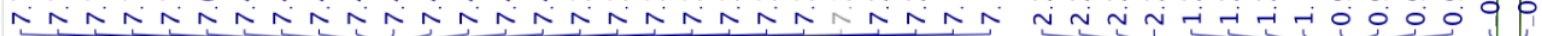

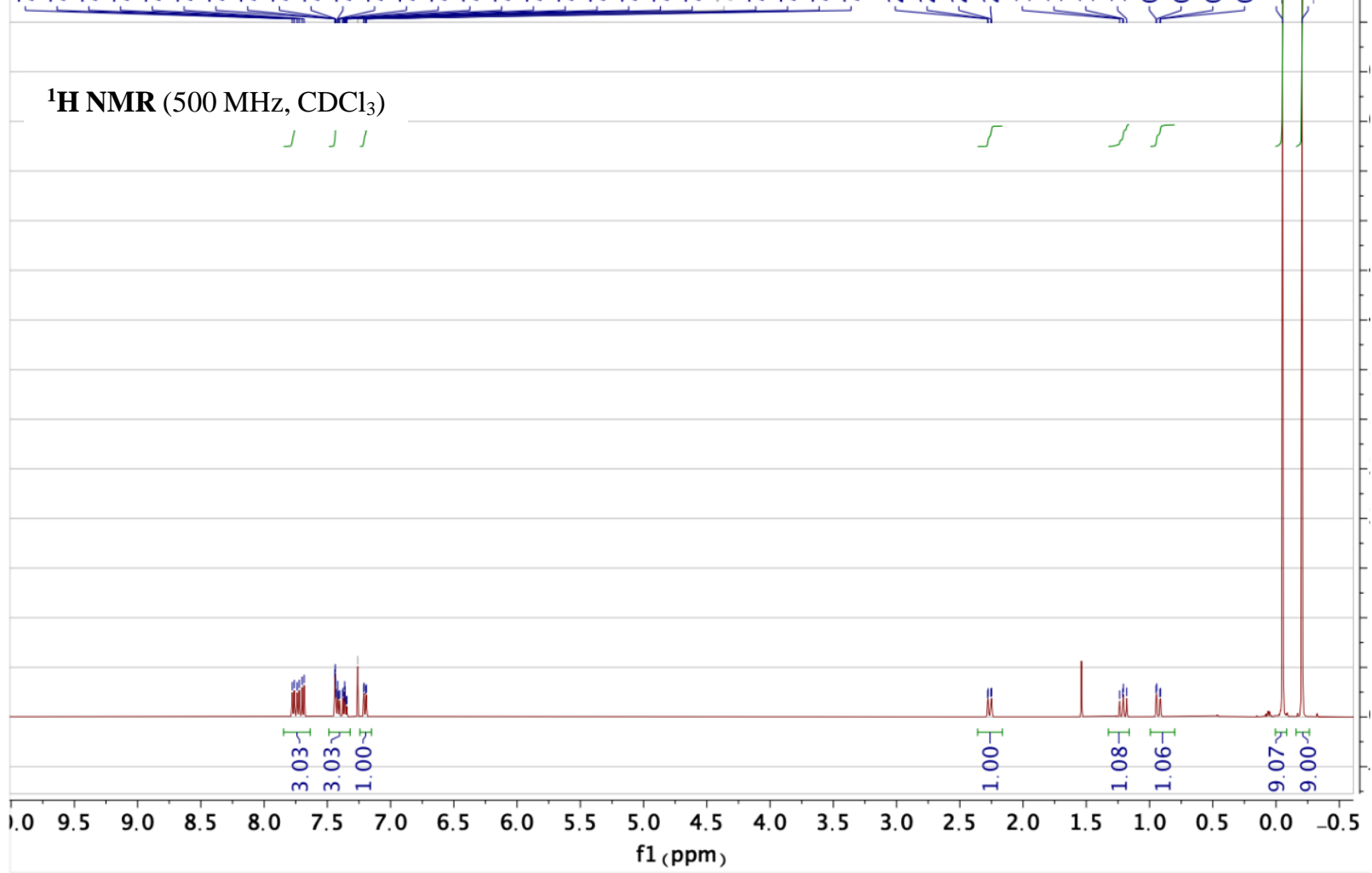



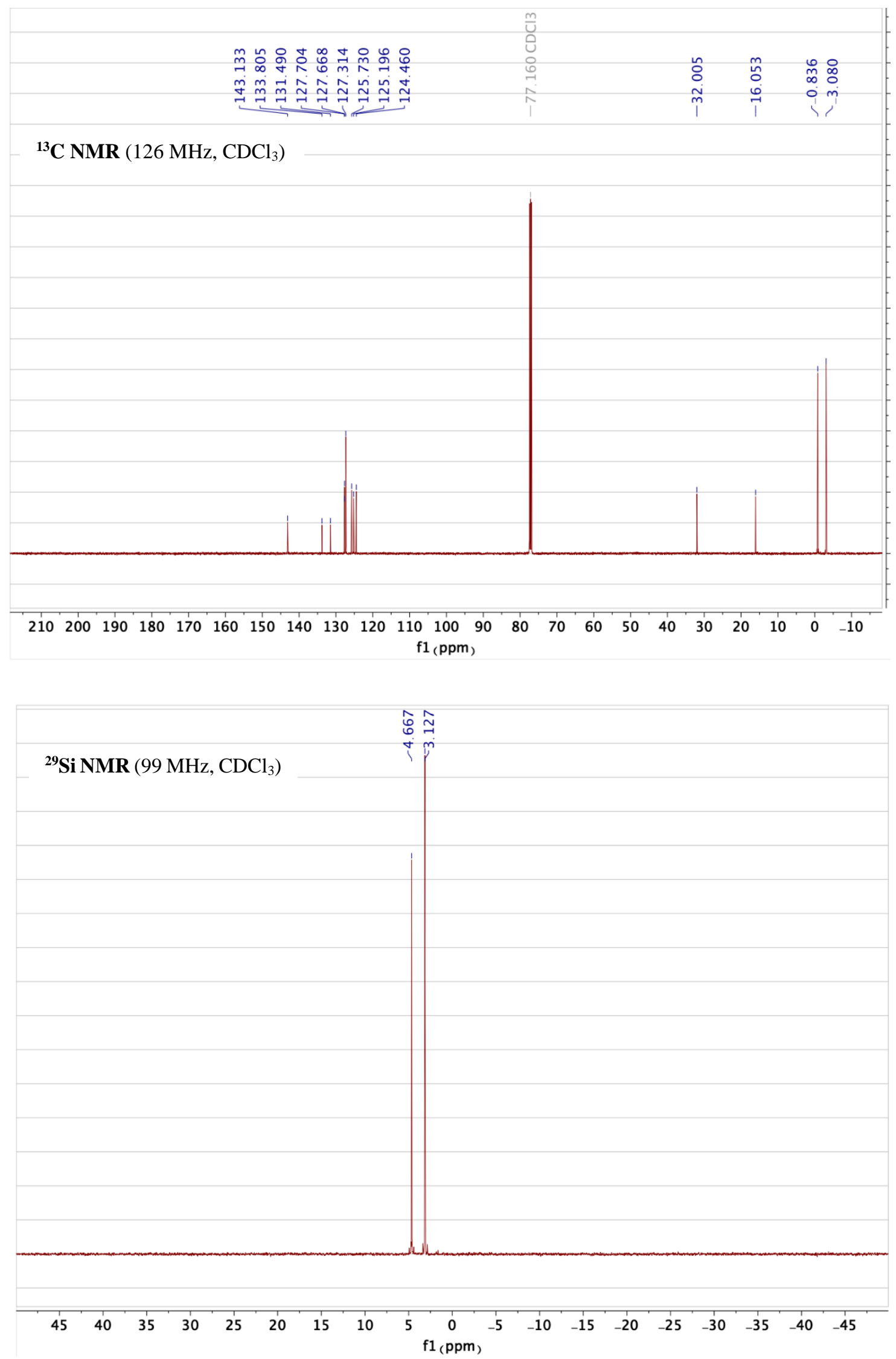
<smiles>CC(C)(C)C(=C(c1ccccc1)c1ccccc1)c1ccccc1</smiles>
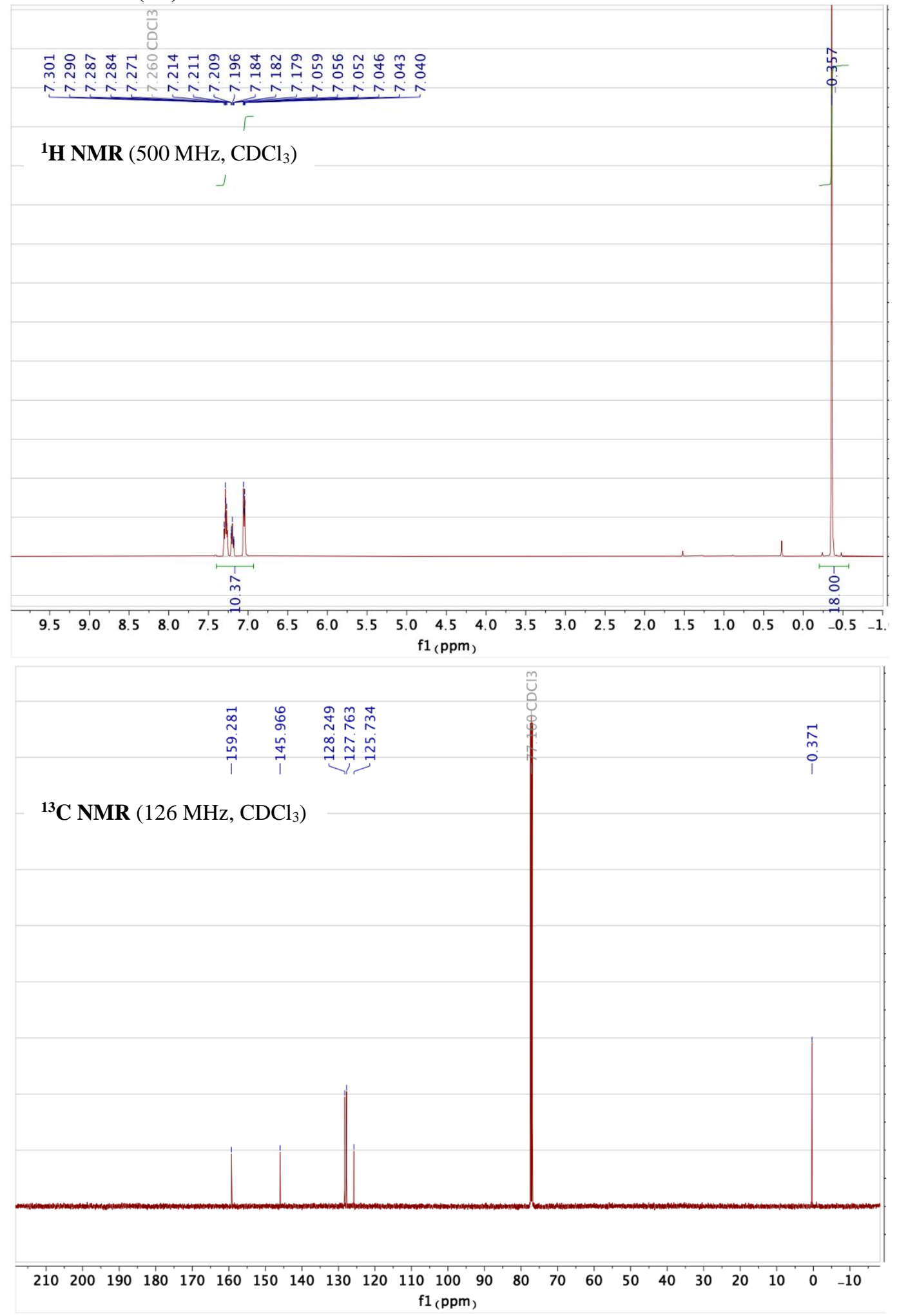
<smiles>CCC(C)c1cc2ccccc2o1</smiles>

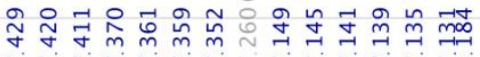

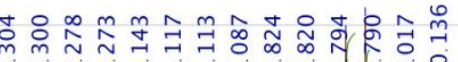

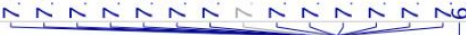

${ }^{1} \mathbf{H} \mathbf{N M R}\left(500 \mathrm{MHz}, \mathrm{CDCl}_{3}\right)$
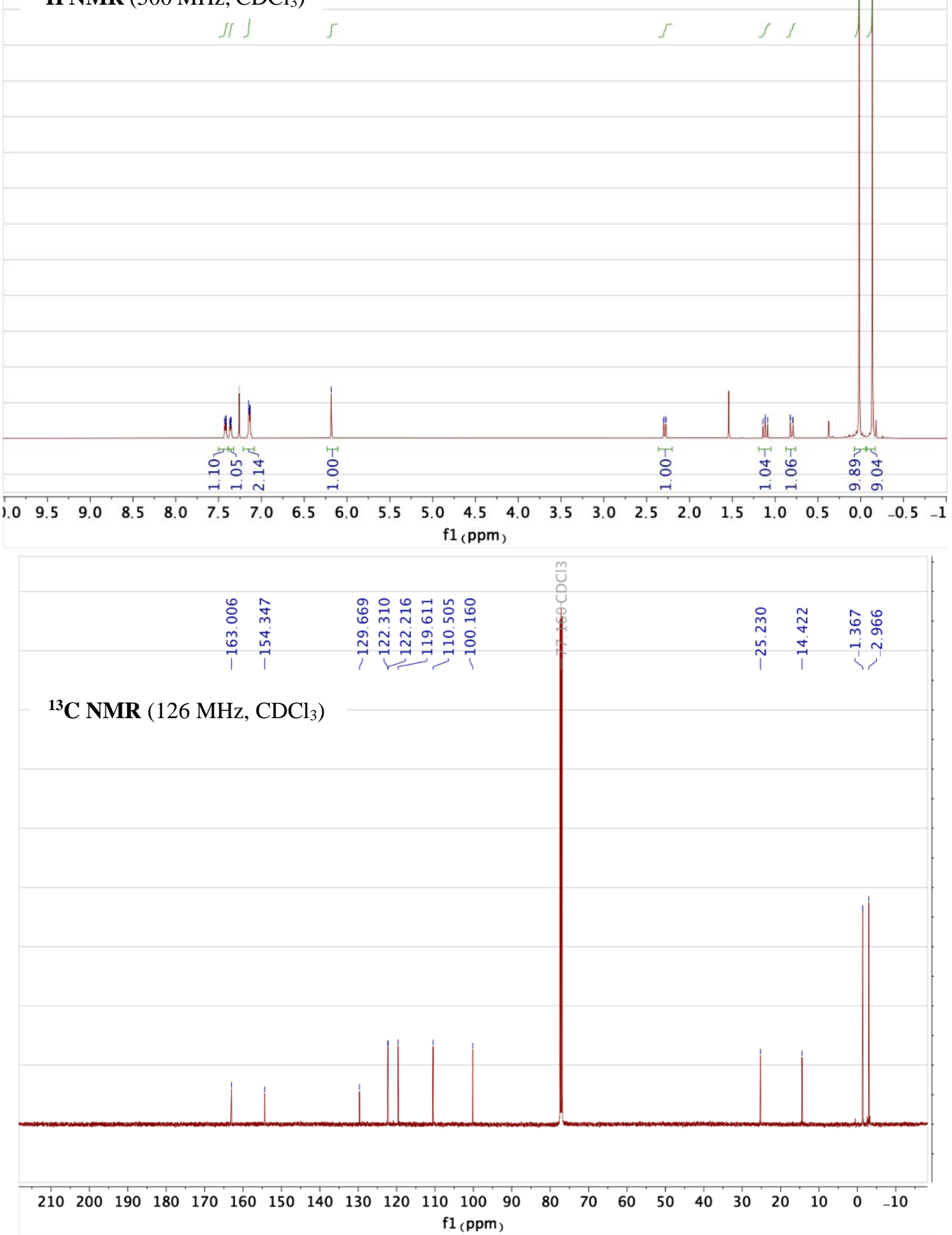


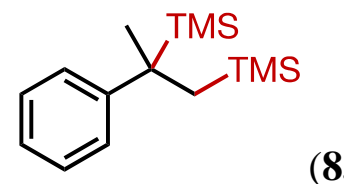

(85)

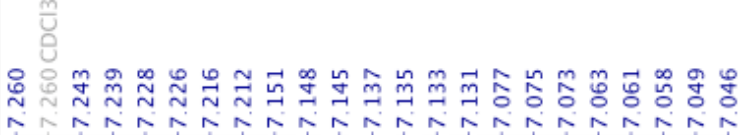

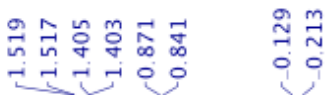

${ }^{1} \mathbf{H}$ NMR (500 MHz, $\left.\mathrm{CDCl}_{3}\right)$
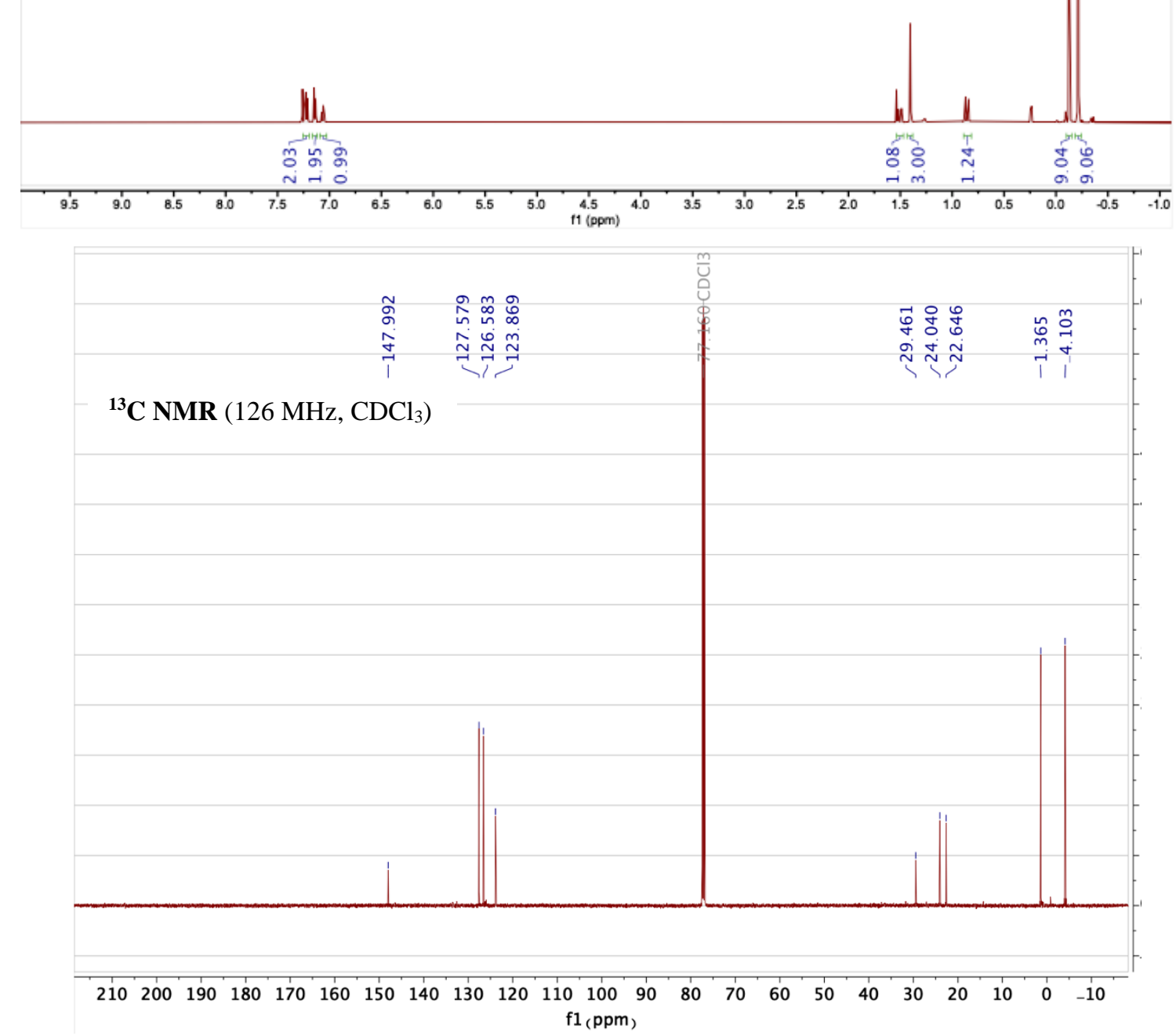

S180 
<smiles>CC[SiH2]CC([SiH2]CC)c1ccc(OC)cc1</smiles>

(86)

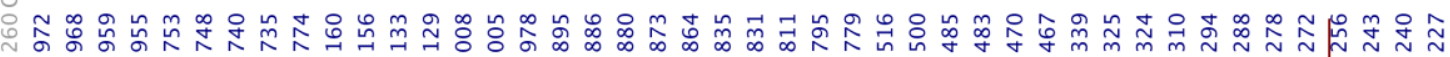
o

${ }^{1} \mathbf{H} \mathbf{N M R}\left(500 \mathrm{MHz}, \mathrm{CDCl}_{3}\right)$
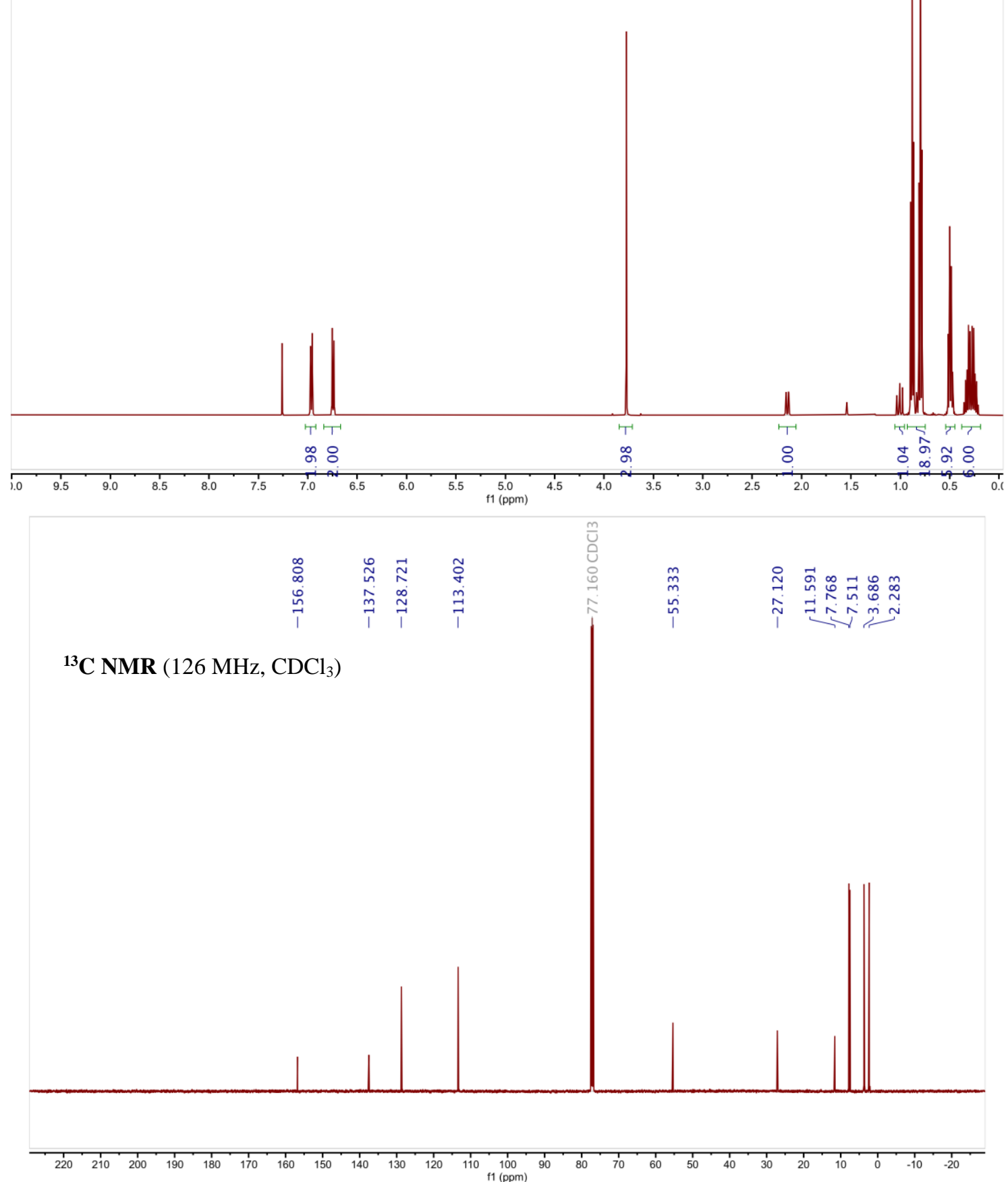
$\mathrm{Et}_{3} \mathrm{Si}-\mathrm{SiEt}_{3}(\mathbf{8 7})$

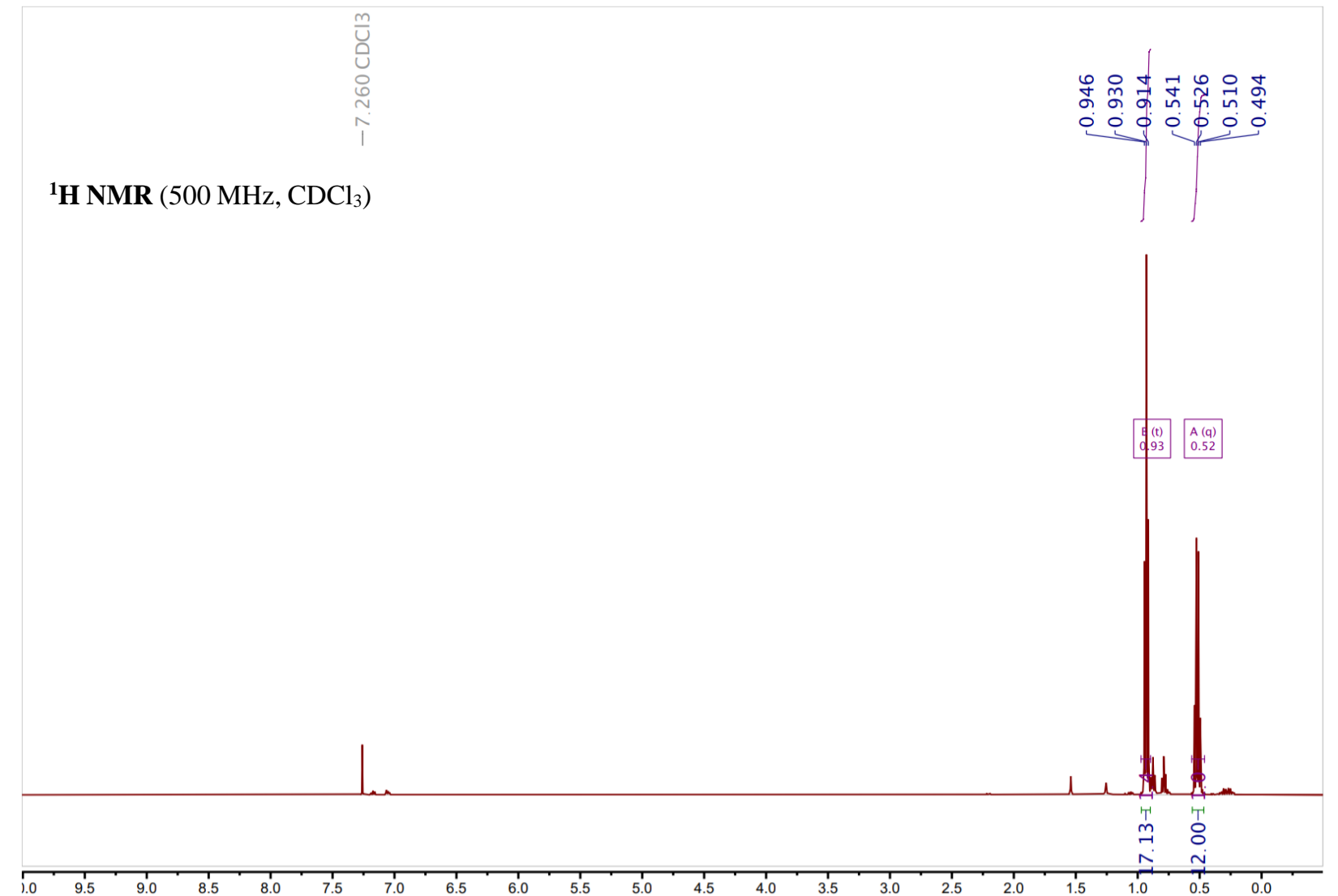

There is $<3 \%$ impurity that is inseparable with triethylsilyl dimer. 
${ }^{13}$ C NMR (126 MHz, $\left.\mathrm{CDCl}_{3}\right)$
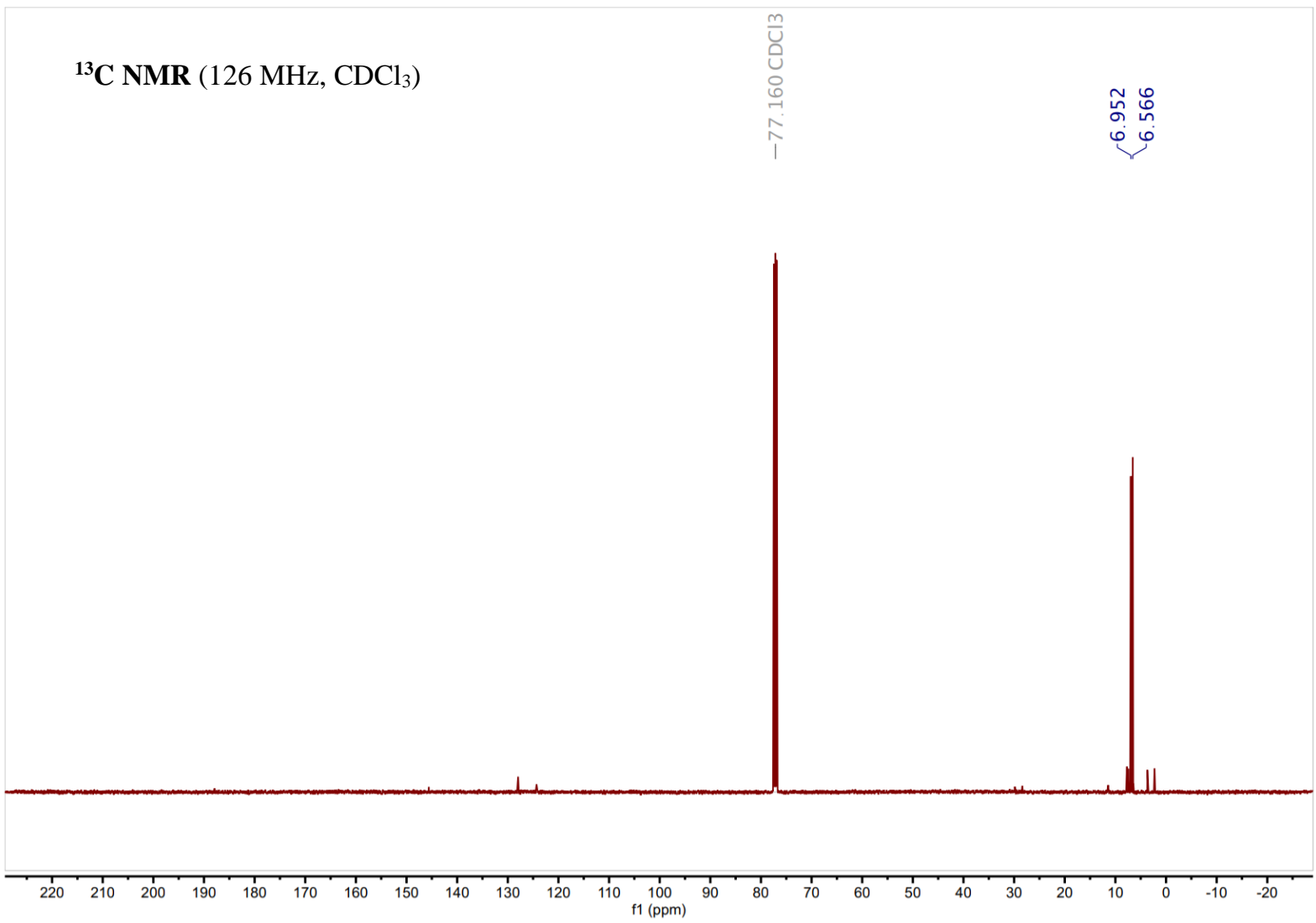

${ }^{29} \mathrm{Si} \mathrm{NMR}\left(99 \mathrm{MHz}, \mathrm{CDCl}_{3}\right)$

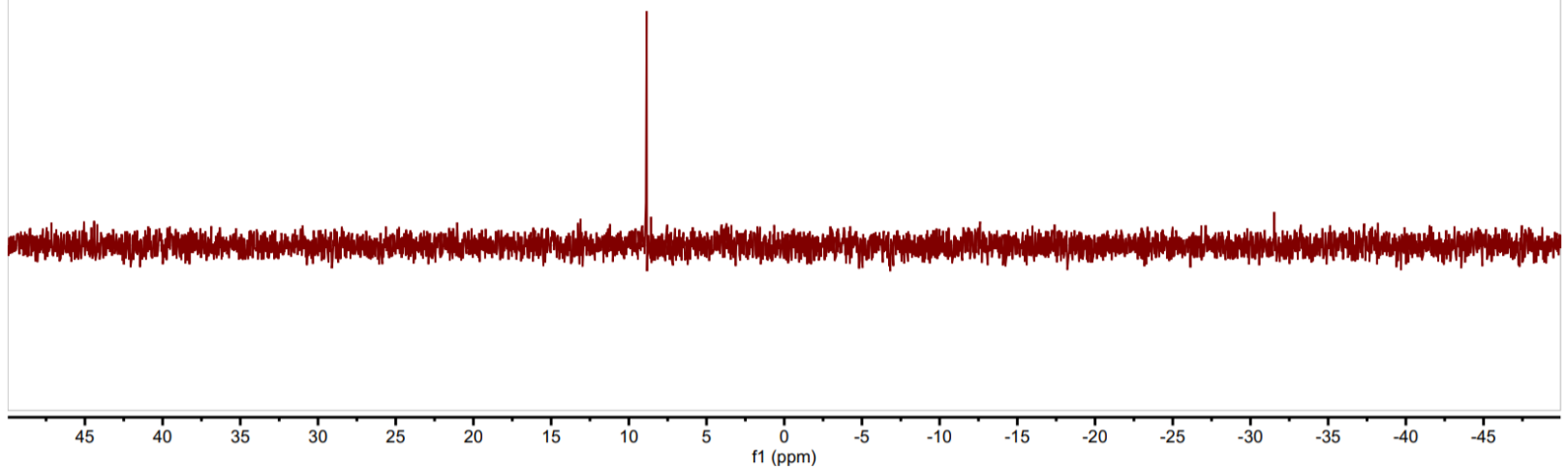

S183 


\section{Computational Coordinates}

Silyl radical addition to 4-tert-butylstyrene transition state (1)

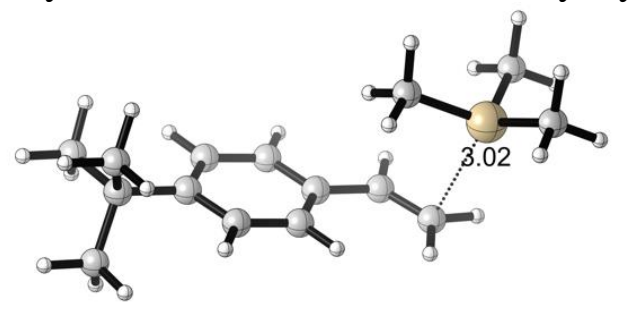

02

$\begin{array}{llll}\text { C } & 2.51798700 & -0.13242700 & -0.01029700\end{array}$

C $\quad 2.11009400 \quad-1.18437100 \quad 0.80381300$

C $\quad 0.85703500 \quad-1.75906400 \quad 0.66416800$

C $\quad-0.05050300 \quad-1.31616700 \quad-0.29950300$

C $\quad 0.35925100 \quad-0.25403900 \quad-1.11730400$

C $\quad 1.60524600 \quad 0.31585700 \quad-0.97199600$

$\mathrm{H} \quad 2.77409900 \quad-1.57099700 \quad 1.56582300$

$\mathrm{H} \quad 0.56966200 \quad-2.57712000 \quad 1.31570700$

$\mathrm{H} \quad-0.31721500 \quad 0.13631200 \quad-1.86854000$

$\mathrm{H} \quad \begin{array}{llll}1.88089100 & 1.13784100 & -1.62350900\end{array}$

C $\quad 3.88468100 \quad 0.53227600 \quad 0.10264400$

C $\quad 4.62840500 \quad 0.38506500 \quad-1.22929900$

$\mathrm{H} \quad 4.07939800 \quad 0.84539300 \quad-2.05027200$

$\mathrm{H} \quad 5.60744700 \quad 0.86420600 \quad-1.17628200$

$\mathrm{H} \quad 4.78104300 \quad-0.66487900 \quad-1.48070300$

$\begin{array}{llll}\mathrm{C} & 3.70199900 & 2.02214600 & 0.41103800\end{array}$

$\mathrm{H} \quad 3.18005200 \quad 2.16729900 \quad 1.35721900$

$\begin{array}{llll}\mathrm{H} & 4.67106700 & 2.51887200 & 0.48109200\end{array}$

$\mathrm{H} \quad 3.12778300 \quad 2.52743200 \quad-0.36491900$

$\begin{array}{llll}\mathrm{C} & 4.73781800 & -0.08793900 & 1.20349200\end{array}$

$\begin{array}{llll}\mathrm{H} & 4.27185600 & 0.01188800 & 2.18426300\end{array}$

$\mathrm{H} \quad 4.92382800 \quad-1.14756600 \quad 1.02530100$

$\begin{array}{llll}\mathrm{H} & 5.70591400 & 0.41131700 & 1.24862000\end{array}$

C $\quad-1.35509100 \quad-1.94201300 \quad-0.40527900$

$\mathrm{H} \quad-1.56687700 \quad-2.69011000 \quad 0.35432800$

C $\quad-2.30822000 \quad-1.67272900-1.31070200$

$\mathrm{H} \quad-2.15301200-0.99046600-2.13629700$

$\mathrm{H} \quad-3.24094800 \quad-2.21912200 \quad-1.32102500$

$\begin{array}{llll}\mathrm{Si} & -3.83602700 & 0.55836800 & 0.03496400\end{array}$

C $\quad-5.14811600 \quad 1.79466500 \quad-0.49611000$

$\mathrm{H} \quad-4.76655100 \quad 2.50060800 \quad-1.23235600$

$\mathrm{H} \quad-6.01696100 \quad 1.30220600 \quad-0.93102800$

$\mathrm{H} \quad \begin{array}{llll}\mathrm{H} & -5.49827500 & 2.37522600 & 0.36185200\end{array}$

C $\quad-2.35200300 \quad 1.44201000 \quad 0.75771400$

$\mathrm{H} \quad-2.64119100 \quad 2.02451200 \quad 1.63612700$

$\mathrm{H} \quad-1.58428000 \quad 0.73444000 \quad 1.07075900$ 


$\begin{array}{lrrr}\mathrm{H} & -1.89390800 & 2.12912200 & 0.04729000 \\ \mathrm{C} & -4.54236800 & -0.63871500 & 1.29138600 \\ \mathrm{H} & -5.39030200 & -1.19623100 & 0.89441100 \\ \mathrm{H} & -3.78904300 & -1.36123900 & 1.60476500 \\ \mathrm{H} & -4.88739200 & -0.11343000 & 2.18527900\end{array}$

Silyl radical addition to styrene transition state (2a) Ub3lyp/lanl2dz/SMD/THF

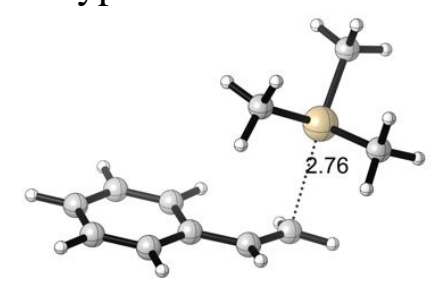

$\begin{array}{llll}02 & & & \\ \mathrm{C} & 3.834729 & 0.412238 & -0.858178 \\ \mathrm{C} & 2.709999 & 1.167474 & -0.482265 \\ \mathrm{C} & 1.710831 & 0.625987 & 0.370848 \\ \mathrm{C} & 1.87879 & -0.709553 & 0.830389 \\ \mathrm{C} & 3.002457 & -1.463658 & 0.453869 \\ \mathrm{H} & 4.58668 & 0.849929 & -1.511062 \\ \mathrm{H} & 2.598102 & 2.187336 & -0.846931 \\ \mathrm{H} & 1.130415 & -1.158653 & 1.478716 \\ \mathrm{H} & 3.112093 & -2.484022 & 0.815022 \\ \mathrm{C} & 0.557517 & 1.46551 & 0.735733 \\ \mathrm{H} & 0.534942 & 2.45019 & 0.266136 \\ \mathrm{C} & -0.476736 & 1.126627 & 1.566672 \\ \mathrm{H} & -0.491037 & 0.201496 & 2.137764 \\ \mathrm{H} & -1.243054 & 1.855709 & 1.817098 \\ \mathrm{Si} & -2.632707 & -0.161776 & -0.101099 \\ \mathrm{C} & -4.317223 & -0.626902 & 0.663844 \\ \mathrm{H} & -4.789268 & 0.236241 & 1.153109 \\ \mathrm{H} & -5.010861 & -0.99079 & -0.112135 \\ \mathrm{H} & -4.212966 & -1.421806 & 1.415185 \\ \mathrm{C} & -1.806937 & -1.694557 & -0.874937 \\ \mathrm{H} & -2.458499 & -2.138916 & -1.645079 \\ \mathrm{H} & -0.85343 & -1.435454 & -1.354095 \\ \mathrm{H} & -1.605795 & -2.46954 & -0.12272 \\ \mathrm{C} & -2.849879 & 1.207522 & -1.406621 \\ \mathrm{H} & -3.377203 & 2.077382 & -0.991087 \\ \mathrm{H} & -1.877704 & 1.552345 & -1.784317 \\ \mathrm{H} & -3.432584 & 0.842136 & -2.267442 \\ \mathrm{C} & 3.988363 & -0.909307 & -0.391459 \\ \mathrm{H} & 4.856601 & -1.497004 & -0.680151\end{array}$

Silyl radical addition to styrene transition state (2b) 


\begin{tabular}{|c|c|c|c|}
\hline \multicolumn{4}{|c|}{ Um061/def2tzvp/SMD/THF } \\
\hline \multicolumn{4}{|c|}{02} \\
\hline $\mathrm{C}$ & 3.618891 & 0.272616 & -0.982961 \\
\hline $\mathrm{C}$ & 2.539966 & 1.066049 & -0.63112 \\
\hline $\mathrm{C}$ & 1.620282 & 0.654418 & 0.341146 \\
\hline $\mathrm{C}$ & 1.822193 & -0.596125 & 0.943236 \\
\hline $\mathrm{C}$ & 2.899646 & -1.387816 & 0.591581 \\
\hline $\mathrm{H}$ & 4.316267 & 0.61582 & -1.737402 \\
\hline $\mathrm{H}$ & 2.396534 & 2.027422 & -1.111958 \\
\hline $\mathrm{H}$ & 1.120838 & -0.951719 & 1.688943 \\
\hline $\mathrm{H}$ & 3.03519 & -2.350191 & 1.070268 \\
\hline $\mathrm{C}$ & 0.500369 & 1.52122 & 0.669369 \\
\hline $\mathrm{H}$ & 0.440216 & 2.431127 & 0.078014 \\
\hline $\mathrm{C}$ & -0.454172 & 1.297261 & 1.584821 \\
\hline $\mathrm{H}$ & -0.439907 & 0.444282 & 2.252048 \\
\hline $\mathrm{H}$ & -1.230012 & 2.027988 & 1.770064 \\
\hline $\mathrm{Si}$ & -2.573204 & -0.209188 & -0.035972 \\
\hline $\mathrm{C}$ & -4.174353 & -1.101577 & 0.361696 \\
\hline $\mathrm{H}$ & -4.89662 & -0.445991 & 0.848534 \\
\hline $\mathrm{H}$ & -4.64141 & -1.474559 & -0.555058 \\
\hline $\mathrm{H}$ & -4.014102 & -1.95895 & 1.015542 \\
\hline $\mathrm{C}$ & -1.360921 & -1.358317 & -0.877622 \\
\hline $\mathrm{H}$ & -1.794976 & -1.769802 & -1.793801 \\
\hline $\mathrm{H}$ & -0.446838 & -0.834891 & -1.160145 \\
\hline $\mathrm{H}$ & -1.078795 & -2.203001 & -0.248712 \\
\hline $\mathrm{C}$ & -2.899016 & 1.284288 & -1.114251 \\
\hline $\mathrm{H}$ & -3.583577 & 1.990697 & -0.643901 \\
\hline $\mathrm{H}$ & -1.97473 & 1.818697 & -1.337464 \\
\hline $\mathrm{H}$ & -3.343167 & 0.988118 & -2.068774 \\
\hline $\mathrm{C}$ & 3.805784 & -0.959257 & -0.372303 \\
\hline $\mathrm{H}$ & 4.647946 & -1.582967 & -0.64414 \\
\hline
\end{tabular}

Silyl radical addition to styrene transition state (2c) uwb97xd/lanl2dz/SMD/THF

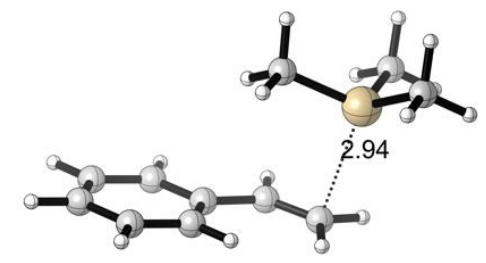

02 


$\begin{array}{llll}\mathrm{C} & 3.624846 & 0.276044 & -0.947274 \\ \mathrm{C} & 2.522291 & 1.081547 & -0.630387 \\ \mathrm{C} & 1.573666 & 0.673589 & 0.334552 \\ \mathrm{C} & 1.761299 & -0.575928 & 0.970444 \\ \mathrm{C} & 2.862643 & -1.381456 & 0.653698 \\ \mathrm{H} & 4.341441 & 0.609579 & -1.690992 \\ \mathrm{H} & 2.390885 & 2.035769 & -1.133839 \\ \mathrm{H} & 1.044775 & -0.925991 & 1.706825 \\ \mathrm{H} & 2.989369 & -2.338213 & 1.150586 \\ \mathrm{C} & 0.42337 & 1.551972 & 0.616209 \\ \mathrm{H} & 0.353442 & 2.441116 & -0.008935 \\ \mathrm{C} & -0.557951 & 1.329151 & 1.533228 \\ \mathrm{H} & -0.521365 & 0.51447 & 2.250767 \\ \mathrm{H} & -1.345584 & 2.062226 & 1.681805 \\ \mathrm{Si} & -2.468739 & -0.238337 & -0.05166 \\ \mathrm{C} & -3.907821 & -1.37631 & 0.455085 \\ \mathrm{H} & -4.604284 & -0.865206 & 1.130718 \\ \mathrm{H} & -4.47326 & -1.691594 & -0.434679 \\ \mathrm{H} & -3.546914 & -2.279847 & 0.960923 \\ \mathrm{C} & -1.265239 & -1.160957 & -1.191616 \\ \mathrm{H} & -1.780724 & -1.485838 & -2.10758 \\ \mathrm{H} & -0.430359 & -0.51498 & -1.487961 \\ \mathrm{H} & -0.847435 & -2.051719 & -0.708115 \\ \mathrm{C} & -3.141356 & 1.302095 & -0.933957 \\ \mathrm{H} & -3.801703 & 1.885007 & -0.280372 \\ \mathrm{H} & -2.321735 & 1.956605 & -1.25471 \\ \mathrm{H} & -3.716503 & 1.021075 & -1.828237 \\ \mathrm{C} & 3.800949 & -0.960967 & -0.305579 \\ \mathrm{H} & 4.652114 & -1.588398 & -0.549381\end{array}$

Silyl radical addition to styrene transition state (2d) ub3lyp/6-311++g(d,p)SMD/THF

02

$\begin{array}{llll}\mathrm{C} & 3.823677 & 0.36451 & -0.857084 \\ \mathrm{C} & 2.697939 & 1.118063 & -0.535099 \\ \mathrm{C} & 1.705496 & 0.617941 & 0.329447 \\ \mathrm{C} & 1.882946 & -0.678878 & 0.851409 \\ \mathrm{C} & 3.006873 & -1.431338 & 0.527821 \\ \mathrm{H} & 4.574318 & 0.776214 & -1.52341 \\ \mathrm{H} & 2.579612 & 2.113055 & -0.953129 \\ \mathrm{H} & 1.13587 & -1.103783 & 1.511994 \\ \mathrm{H} & 3.120918 & -2.428186 & 0.940826 \\ \mathrm{C} & 0.545838 & 1.455532 & 0.641108 \\ \mathrm{H} & 0.496769 & 2.391866 & 0.089707 \\ \mathrm{C} & -0.455491 & 1.173125 & 1.505022 \\ \mathrm{H} & -0.440318 & 0.30711 & 2.155878\end{array}$




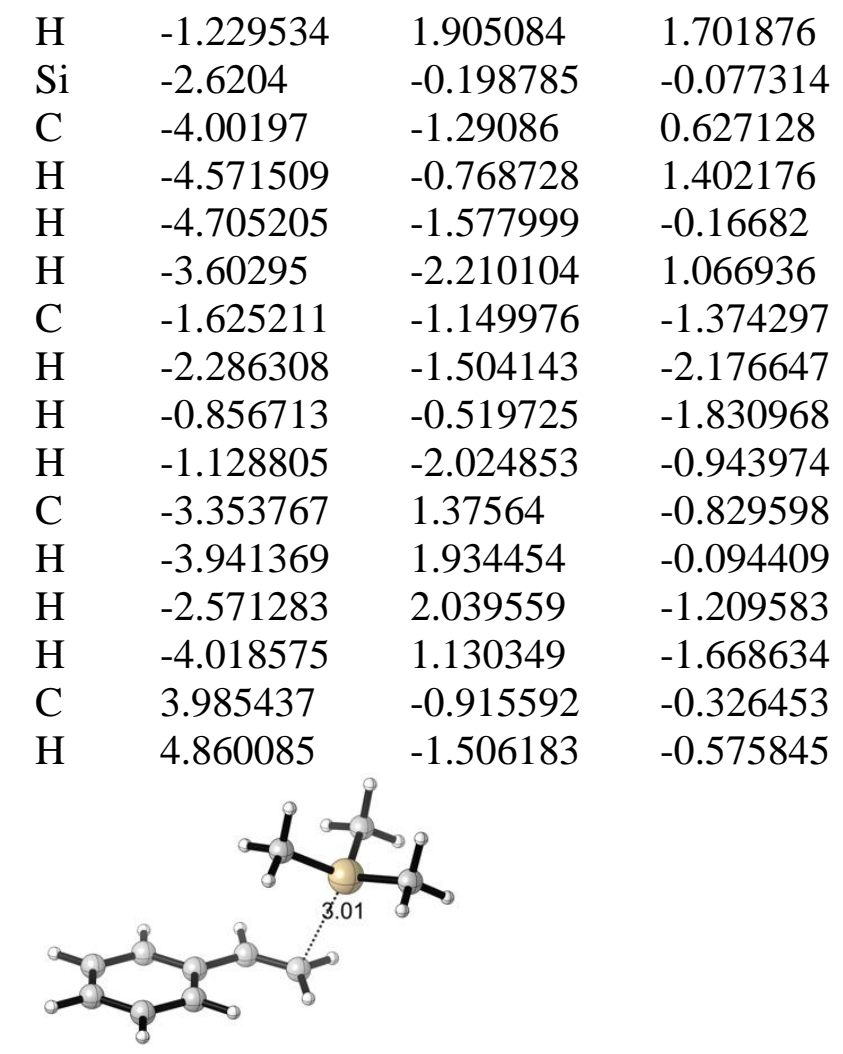

Silyl radical addition to styrene transition state (2e) ub3lyp/6-311++g(d,p)SMD/THF

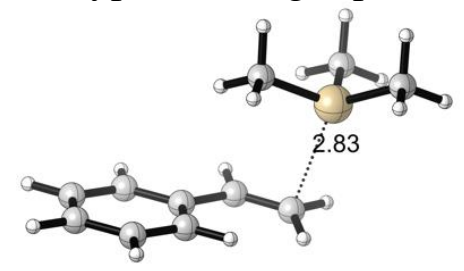

02

$\begin{array}{llll}\mathrm{C} & 3.595624 & 0.305417 & -0.889424 \\ \mathrm{C} & 2.486751 & 1.084599 & -0.595989 \\ \mathrm{C} & 1.521386 & 0.654199 & 0.32049 \\ \mathrm{C} & 1.696904 & -0.597358 & 0.925509 \\ \mathrm{C} & 2.804071 & -1.376689 & 0.630178 \\ \mathrm{H} & 4.331005 & 0.66246 & -1.600057 \\ \mathrm{H} & 2.359206 & 2.046449 & -1.080297 \\ \mathrm{H} & 0.958091 & -0.97008 & 1.625142 \\ \mathrm{H} & 2.921353 & -2.342605 & 1.106617 \\ \mathrm{C} & 0.363834 & 1.508071 & 0.586679 \\ \mathrm{H} & 0.27347 & 2.378872 & -0.0563 \\ \mathrm{C} & -0.591896 & 1.28046 & 1.504901 \\ \mathrm{H} & -0.51266 & 0.492068 & 2.242677 \\ \mathrm{H} & -1.388405 & 1.999126 & 1.654 \\ \mathrm{Si} & -2.404228 & -0.240915 & -0.038569\end{array}$




$\begin{array}{llll}\mathrm{C} & -3.847 & -1.367739 & 0.394247 \\ \mathrm{H} & -4.548168 & -0.875298 & 1.071331 \\ \mathrm{H} & -4.395926 & -1.64404 & -0.512735 \\ \mathrm{H} & -3.50643 & -2.288253 & 0.872827 \\ \mathrm{C} & -1.226863 & -1.126255 & -1.198051 \\ \mathrm{H} & -1.757214 & -1.400842 & -2.116537 \\ \mathrm{H} & -0.383925 & -0.490231 & -1.476846 \\ \mathrm{H} & -0.830248 & -2.043209 & -0.757611 \\ \mathrm{C} & -3.050892 & 1.313247 & -0.867435 \\ \mathrm{H} & -3.717872 & 1.874373 & -0.20921 \\ \mathrm{H} & -2.230165 & 1.97204 & -1.160015 \\ \mathrm{H} & -3.613201 & 1.056293 & -1.771194 \\ \mathrm{C} & 3.760805 & -0.929936 & -0.275689 \\ \mathrm{H} & 4.623949 & -1.54242 & -0.50432\end{array}$

Silyl anion addition to styrene transition state (3a) m061/def2tzvp/SMD/THF

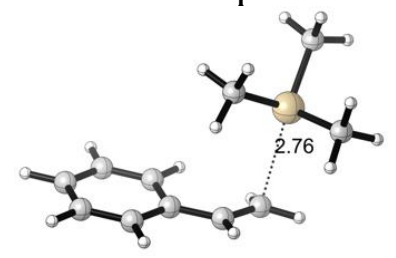

$-11$

$\begin{array}{llll}\text { C } & 3.757075 & -1.036063 & 0.141321\end{array}$

$\begin{array}{llll}\text { C } & 2.803273 & -1.27238 & -0.843138\end{array}$

$\begin{array}{llll}\text { C } & 1.730199 & -0.417511 & -1.015477\end{array}$

$\begin{array}{llll}\text { C } & 1.560939 & 0.726116 & -0.201442\end{array}$

$\begin{array}{llll}\text { C } & 2.532795 & 0.934646 & 0.801372\end{array}$

$\begin{array}{llll}\text { C } & 3.604859 & 0.07914 & 0.964163\end{array}$

$\begin{array}{llll}\mathrm{H} & 4.596742 & -1.707178 & 0.271493\end{array}$

$\mathrm{H} \quad 2.895692 \quad-2.144002 \quad-1.481978$

$\mathrm{H} \quad 0.993871 \quad-0.639083 \quad-1.778861$

$\begin{array}{llll}\mathrm{H} & 2.424638 & 1.792706 & 1.457664\end{array}$

$\mathrm{H} \quad 4.331646 \quad 0.2774 \quad 1.744156$

C $\quad 0.438454 \quad 1.60543 \quad-0.348602$

$\begin{array}{llll}\mathrm{H} & 0.293828 & 2.333114 & 0.445722\end{array}$

C $\quad-0.534199 \quad 1.473434 \quad-1.304012$

$\mathrm{H} \quad-1.329455 \quad 2.202363 \quad-1.379708$

$\mathrm{H} \quad-0.364714 \quad 0.900515 \quad-2.203144$

$\begin{array}{llll}\mathrm{Si} & -2.35977 & -0.271453 & -0.201018\end{array}$

$\begin{array}{llll}\text { C } & -3.349768 & 1.189768 & 0.554909\end{array}$

$\begin{array}{llll}\mathrm{H} & -3.900049 & 0.898439 & 1.457389\end{array}$

$\begin{array}{llll}\mathrm{H} & -2.694492 & 2.019039 & 0.836499\end{array}$

$\mathrm{H} \quad-4.084186 \quad 1.598771 \quad-0.144921$

$\begin{array}{llll}\mathrm{C} & -1.308423 & -0.815871 & 1.303455\end{array}$

$\mathrm{H} \quad-1.923433 \quad-0.897841 \quad 2.207273$ 


$\begin{array}{llll}\mathrm{H} & -0.836108 & -1.789698 & 1.151165 \\ \mathrm{H} & -0.507652 & -0.107976 & 1.530535 \\ \mathrm{C} & -3.71235 & -1.650633 & -0.181693 \\ \mathrm{H} & -4.171024 & -1.752785 & 0.810072 \\ \mathrm{H} & -4.522213 & -1.45075 & -0.888028 \\ \mathrm{H} & -3.303425 & -2.629144 & -0.446215\end{array}$

Silyl anion addition to styrene transition state (3b) m062x/def2tzvp/SMD/THF

$\begin{array}{llll} & & & \\ & & & \\ & & & \\ & & & \\ & & & \\ & & & \\ \mathrm{C} & 3.692833 & -1.058209 & 0.1211 \\ \mathrm{C} & 2.726226 & -1.278509 & -0.852907 \\ \mathrm{C} & 1.663801 & -0.400583 & -1.012217 \\ \mathrm{C} & 1.533439 & 0.739811 & -0.196845 \\ \mathrm{C} & 2.513989 & 0.935108 & 0.794981 \\ \mathrm{C} & 3.573527 & 0.058256 & 0.947467 \\ \mathrm{H} & 4.519537 & -1.746593 & 0.242465 \\ \mathrm{H} & 2.794603 & -2.150944 & -1.492752 \\ \mathrm{H} & 0.914456 & -0.608475 & -1.765483 \\ \mathrm{H} & 2.428107 & 1.79445 & 1.451904 \\ \mathrm{H} & 4.312597 & 0.240053 & 1.719353 \\ \mathrm{C} & 0.411405 & 1.645121 & -0.323033 \\ \mathrm{H} & 0.274941 & 2.359272 & 0.483428 \\ \mathrm{C} & -0.546277 & 1.53373 & -1.285367 \\ \mathrm{H} & -1.352821 & 2.254021 & -1.336655 \\ \mathrm{H} & -0.376724 & 0.967695 & -2.188588 \\ \mathrm{Si} & -2.302734 & -0.272611 & -0.214873 \\ \mathrm{C} & -3.292922 & 1.168273 & 0.603619 \\ \mathrm{H} & -3.807468 & 0.845532 & 1.517578 \\ \mathrm{H} & -2.635371 & 2.000845 & 0.875629 \\ \mathrm{H} & -4.052729 & 1.569442 & -0.07598 \\ \mathrm{C} & -1.241568 & -0.856866 & 1.281575 \\ \mathrm{H} & -1.849307 & -0.917789 & 2.193738 \\ \mathrm{H} & -0.812827 & -1.84835 & 1.105287 \\ \mathrm{H} & -0.411673 & -0.1752 & 1.488624 \\ \mathrm{C} & -3.67364 & -1.647308 & -0.187678 \\ \mathrm{H} & -4.109873 & -1.768165 & 0.813094 \\ \mathrm{H} & -4.491541 & -1.413335 & -0.876717 \\ \mathrm{H} & -3.270503 & -2.618845 & -0.490875\end{array}$

Silyl anion addition to styrene transition state (3c) 


$\begin{array}{llll}\text { c97xd/lanl2dz/SMD/THF } & \\ & & & \\ & & & \\ & & & \\ & & & \\ & & & \\ -1 & & & \\ \mathrm{C} & 3.797834 & -1.040217 & 0.182605 \\ \mathrm{C} & 2.80968 & -1.349023 & -0.768217 \\ \mathrm{C} & 1.71608 & -0.495179 & -0.974919 \\ \mathrm{C} & 1.57494 & 0.711976 & -0.233616 \\ \mathrm{C} & 2.579857 & 0.999353 & 0.732855 \\ \mathrm{C} & 3.668651 & 0.144823 & 0.934874 \\ \mathrm{H} & 4.641656 & -1.704998 & 0.33932 \\ \mathrm{H} & 2.88685 & -2.265883 & -1.34668 \\ \mathrm{H} & 0.959007 & -0.771715 & -1.702111 \\ \mathrm{H} & 2.491541 & 1.907072 & 1.325827 \\ \mathrm{H} & 4.418156 & 0.396961 & 1.680241 \\ \mathrm{C} & 0.442903 & 1.612649 & -0.407939 \\ \mathrm{H} & 0.326332 & 2.392324 & 0.343975 \\ \mathrm{C} & -0.547707 & 1.445745 & -1.358804 \\ \mathrm{H} & -1.338645 & 2.184321 & -1.453091 \\ \mathrm{H} & -0.406233 & 0.812555 & -2.226792 \\ \mathrm{Si} & -2.390581 & -0.253202 & -0.197214 \\ \mathrm{C} & -3.315578 & 1.224178 & 0.669645 \\ \mathrm{H} & -3.82458 & 0.895726 & 1.591128 \\ \mathrm{H} & -2.603947 & 2.016944 & 0.946412 \\ \mathrm{H} & -4.07284 & 1.673453 & 0.00874 \\ \mathrm{C} & -1.307994 & -0.89292 & 1.28004 \\ \mathrm{H} & -1.897116 & -0.95459 & 2.210431 \\ \mathrm{H} & -0.893593 & -1.890999 & 1.074754 \\ \mathrm{H} & -0.463989 & -0.211717 & 1.461768 \\ \mathrm{C} & -3.821317 & -1.587402 & -0.20266 \\ \mathrm{H} & -4.254558 & -1.715418 & 0.804305 \\ \mathrm{H} & -4.63479 & -1.294856 & -0.884245 \\ \mathrm{H} & -3.449206 & -2.568255 & -0.536172\end{array}$

Silyl radical addition to styrene transition state (3d) uB31yp/6-311++g(d,p)/SMD/THF

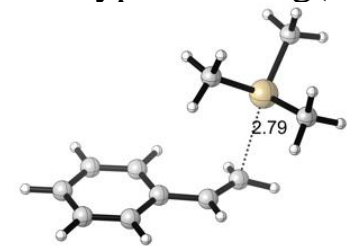

$-11$

$\begin{array}{llll}\mathrm{C} & 3.989212 & -0.925576 & 0.275492 \\ \mathrm{C} & 3.029602 & -1.370582 & -0.639414\end{array}$ 


$\begin{array}{llll}\mathrm{C} & 1.890694 & -0.618669 & -0.909738 \\ \mathrm{C} & 1.660628 & 0.630115 & -0.273315 \\ \mathrm{C} & 2.640415 & 1.050745 & 0.663939 \\ \mathrm{C} & 3.775551 & 0.295589 & 0.928101 \\ \mathrm{H} & 4.875671 & -1.515841 & 0.480071 \\ \mathrm{H} & 3.169801 & -2.320576 & -1.147282 \\ \mathrm{H} & 1.163937 & -1.000281 & -1.61814 \\ \mathrm{H} & 2.493221 & 1.993425 & 1.183931 \\ \mathrm{H} & 4.501914 & 0.657906 & 1.649751 \\ \mathrm{C} & 0.504033 & 1.45389 & -0.537407 \\ \mathrm{H} & 0.385235 & 2.317754 & 0.113458 \\ \mathrm{C} & -0.494326 & 1.184294 & -1.448654 \\ \mathrm{H} & -1.246579 & 1.9341 & -1.654895 \\ \mathrm{H} & -0.361104 & 0.452209 & -2.233175 \\ \mathrm{Si} & -2.529645 & -0.2141 & -0.152395 \\ \mathrm{C} & -3.288115 & 1.354477 & 0.700247 \\ \mathrm{H} & -3.843549 & 1.100942 & 1.615885 \\ \mathrm{H} & -2.511877 & 2.077058 & 0.980441 \\ \mathrm{H} & -3.985182 & 1.875045 & 0.031202 \\ \mathrm{C} & -1.576041 & -1.04329 & 1.311401 \\ \mathrm{H} & -2.199078 & -1.115561 & 2.21605 \\ \mathrm{H} & -1.255645 & -2.059635 & 1.05237 \\ \mathrm{H} & -0.674083 & -0.482056 & 1.581253 \\ \mathrm{C} & -4.1013 & -1.362387 & -0.272538 \\ \mathrm{H} & -4.613211 & -1.473055 & 0.697305 \\ \mathrm{H} & -4.835039 & -0.967329 & -0.986162 \\ \mathrm{H} & -3.831528 & -2.368342 & -0.617221\end{array}$

Silyl anion addition to styrene transition state (3e) B3lyp/lanl2dz/SMD/THF

$\begin{array}{llll} & & \\ & & & \\ \mathrm{C} & 4.07152 & -0.931867 & 0.269476 \\ \mathrm{C} & 3.092053 & -1.394647 & -0.63799 \\ \mathrm{C} & 1.934442 & -0.643142 & -0.902192 \\ \mathrm{C} & 1.705947 & 0.619862 & -0.263693 \\ \mathrm{C} & 2.706412 & 1.062383 & 0.661137 \\ \mathrm{C} & 3.860132 & 0.307558 & 0.919466 \\ \mathrm{H} & 4.966083 & -1.518053 & 0.468108 \\ \mathrm{H} & 3.232872 & -2.351303 & -1.140091 \\ \mathrm{H} & 1.194526 & -1.034058 & -1.596667 \\ \mathrm{H} & 2.560372 & 2.013133 & 1.173769 \\ \mathrm{H} & 4.598724 & 0.680442 & 1.628066\end{array}$




$\begin{array}{llll}\mathrm{C} & 0.535462 & 1.444353 & -0.519779 \\ \mathrm{H} & 0.425492 & 2.324472 & 0.116759 \\ \mathrm{C} & -0.471844 & 1.170634 & -1.440894 \\ \mathrm{H} & -1.238326 & 1.913275 & -1.64355 \\ \mathrm{H} & -0.350634 & 0.415199 & -2.211374 \\ \mathrm{Si} & -2.589551 & -0.229474 & -0.158582 \\ \mathrm{C} & -3.364668 & 1.386983 & 0.612647 \\ \mathrm{H} & -3.978796 & 1.16538 & 1.503487 \\ \mathrm{H} & -2.57579 & 2.09196 & 0.921181 \\ \mathrm{H} & -4.008508 & 1.912369 & -0.11189 \\ \mathrm{C} & -1.671681 & -0.988137 & 1.380303 \\ \mathrm{H} & -2.269721 & -0.894995 & 2.304142 \\ \mathrm{H} & -1.460311 & -2.058666 & 1.225537 \\ \mathrm{H} & -0.707542 & -0.484652 & 1.55361 \\ \mathrm{C} & -4.172236 & -1.383764 & -0.263085 \\ \mathrm{H} & -4.681287 & -1.47213 & 0.714419 \\ \mathrm{H} & -4.905512 & -0.99074 & -0.986259 \\ \mathrm{H} & -3.901159 & -2.400286 & -0.591484\end{array}$

\section{References}

\footnotetext{
${ }^{1}$ Kang, S. H.; Han, J. S.; Lim, W. C.; Jung, I. N.; Lee, M. E.; Yoo, B. R. Double Silylation of Olefin with HSiCl3 in the Presence of Bu4PCl. New Synthetic Method for $\alpha$, $\beta$-Bis (trichlorosilyl) alkanes. Organometallics, 2006, 25, 318319.

${ }^{2}$ Zhang, T., Zhang, Z., Nishiyama, Y., \& Maekawa, H. Facile and highly selective silylation of vinylpyridines at the $\beta$-olefinic carbon by magnesium-promoted reduction. Tetrahedron 2016, 72, 2293-2299.

${ }^{3}$ Jouikov, V.; Grigorieva, L. Electrochemically induced silylation of unsaturated compounds. Electrochim. Acta 1996, $41,469-470$.

${ }^{4}$ Nii, S., Terao, J., Kambe, N. Titanocene-catalyzed formation of allylsilanes from allyl ethers and chlorosilanes. Tetrahedron lett. 2004, 45, 1699-1702.

${ }^{5}$ Terao, J., Watabe, H., Watanabe, H., Kambe, N. Novel Nickel-Catalyzed Coupling Reaction of Allyl Ethers with Chlorosilanes, Alkyl Tosylates, or Alkyl Halides Promoted by Vinyl-Grignard Reagent Leading to Allylsilanes or Alkenes. Adv. Synth. Catal. 2004, 346, 1674-1678.

${ }^{6}$ Selander, N.; Paasch, J. R.; Szabo, K. J.' Palladium-Catalyzed Allylic C-OH Functionalization for Efficient Synthesis of Functionalized Allylsilanes. J. Am. Chem. Soc. 2011, 133, 409-411.

${ }^{7}$ Nonhebel, D. C. The chemistry of cyclopropylmethyl and related radicals. Chem. Soc. Rev. 1993, 22, 347-359.

${ }^{8}$ Still. W. C. Conjugate Addition of Trimethylsilyllithium. A Preparation of 3-Silyl Ketones. J. Org. Chem. 1976, 41, 3063-3064.

${ }^{9}$ Santelli-Rouvier, C., Lefrère, S.; Santelli, M. Addition of Lithium Metal to But-1-En-3-Ynes. Synthesis and Epoxidation of 1,4-Bis(Trimethylsilyl)Buta-1,2-Dienes. Tetrahedron Lett. 1999, 40, 5491-5494.

${ }^{10}$ Hansch, C., Leo, A. \& Taft, R. W. A survey of Hammett substituent constants and resonance and field parameters. Chem. Rev. 1991, 91, 165-195.

${ }^{11}$ Sandford, C.; Fries, L. R.; Ball, T. E.; Minteer, S. D.; Sigman,M. S. Mechanistic Studies into the Oxidative Addition of Co(I) Complexes: Combining Electroanalytical Techniques with Parameterization. J. Am. Chem. Soc. 141, 2019, $18877-18889$.

12 Thibaudeau, S.; Gouverneur, V. Sequential cross-metathe-sis/electrophilic fluorodesilylation: A novel entry to functionalized allylic fluorides. Org. Lett. 2003, 5, 4891-4893

${ }^{13} \mathrm{Yu}$, M., Jing, H. \& Fu, X. Highly Efficient Generation of Hydrogen from the Hydrolysis of Silanes Catalyzed by $\left[\mathrm{RhCl}(\mathrm{CO})_{2}\right]_{2}$. Inorg. Chem. 2013, 52, 10741-10743.
} 
${ }^{14}$ Szymaniak, A. A.; Zhang, C.; Coombs, J. R.; Morken, J. P. Enantioselective Synthesis of Nonracemic Geminal Silylboronates by Pt-Catalyzed Hydrosilylation. ACS Catal. 2018, 8, 2897-2901.

${ }^{15}$ Shimada, T.; Mukaide, K.; Shinohara, A.; Han, J.; Hayashi, T. Asymmetric Synthesis of 1-Aryl-1,2-ethanediols from Arylacetylenes by Palladium-Catalyzed Asymmetric Hydrosilylation as a Key Step. J. Am. Chem. Soc. 2002, $124,1584-1585$.

${ }^{16}$ Yoshida, J.; Murata, T.; Isoe, S. Electrochemical oxidation of organosilicon compounds I. Oxidative cleavage of carbon-silicon bond in allylsilanes and benzylsilanes. Tetrahedron Lett. 1986, 27, 3373-3376.

${ }^{17}$ US patent : US5142056A

${ }^{18}$ Kippo, T.; Fukuyama T.; Ryu, I. Regioselective radical bromoallylation of allenes leading to 2-bromo-substituted 1, 5-dienes. Org. Lett. 2011, 13, 3864-3867.

${ }^{19}$ Pagano, M.; Castagnolo, D.; Bernardini, M.; Fallacara, A. L.; Laurenzana, I.; Deodato, D.; Kessler, U.; Pilger, B.; Stergiou, L.; Strunze, S.; Tintori, C.; Botta, M. The fight against the influenza A virus H1N1: synthesis, molecular modeling, and biological evaluation of benzofurazan derivatives as viral RNA polymerase inhibitors. ChemMedChem 2014, 9, 129- 150.

${ }^{20}$ Narayanarao, M.; Koodlur, L.; Revanasiddappa, V. G.; Gopal, S.; Kamila, S. Multicomponent synthesis of spiropyrrolidine analogues derived from vinylindole/indazole by a 1,3-dipolar cycloaddition reaction.

Beilstein J. Org. Chem. 2016, 12, 2893-2897.

${ }^{21}$ Yang, B.; Xu, X. H.; Qing, F. L. Copper-Mediated Radical 1,2-Bis(trifluoromethylation) of Alkenes with Sodium Trifluoromethanesulfinate. Org. Lett. 2015, 17, 1906-1909.

${ }^{22}$ Mato, M.; Herlé, B.; Echavarren, A. M. Cyclopropanation by Gold- or Zinc-Catalyzed Retro-Buchner Reaction at Room Temperature. Org. Lett. 2018, 20, 4341-4345.

${ }^{23}$ Cooper, P.; Crisenza, G. E. M.; Feron, L. J.; Bower. J. F. Iridium-Catalyzed $\alpha$-Selective Arylation of Styrenes by Dual C-H Functionalization. Angew. Chem. 2018, 130, 14394-14398.

${ }^{24}$ Green, S. A.; Vásquez-Céspedes S.; Shenvi, T. A. Iron-Nickel Dual-Catalysis: A New Engine for Olefin Functionalization and the Formation of Quaternary Centers. J. Am. Chem. Soc. 2018, 140, 11317-11324.

${ }^{25}$ Budai, B.; Leclair, A.; Wang, Q.; Zhu. J. Copper-Catalyzed 1,2-Methoxy Methoxycarbonylation of Alkenes with Methyl Formate. Angew. Chem. Int. Ed. 2019, 58, 10305-10309.

${ }^{26}$ Tweedie, V. L.; Cuscurida, M. Hydrogenolysis by Metal Hydrides I. Hydrogenolysis of Aryl Allyl Ethers by Lithium Aluminum Hydride J. Am. Chem. Soc. 1957, 79, 5463-5466.

${ }^{27}$ Huang, J.; Hu, G.; An, S.; Chen, D.; Li, M.; Li, P. Synthesis of N-Alkylpyridin-4-ones and Thiazolo[3,2-a]pyridin5-ones through Pummerer-Type Reactions. J. Org. Chem. 2019, 84, 9758-9769.

${ }^{28}$ Gockel, S. N.; Buchanan, T. L.; Hull, K. L. Cu-Catalyzed Three-Component Carboamination of Alkenes. J. Am. Chem. Soc. 2018, 140, 58-61.

${ }^{29}$ Klausen, R. S.; Widawsky, J. R.; Steigerwald, M. L.; Venkataraman, L.; Nuckolls, C. Conductive molecular silicon. J. Am. Chem. Soc. 2012, 134, 4541- 4544.

${ }^{30}$ Russell, A. G.; Guveli, T.; Kariuki, B. M.; Snaith, J. S. Synthesis and characterisation of two new binaphthyl trisilanes. 2009, J. Organomet. Chem. 694, $137-141$.

${ }^{31}$ Orcel, U.; Waser, J. One-Pot Three-Component Synthesis of Vicinal Diamines via In Situ Aminal Formation and Carboamination Angew. Chem., Int. Ed. 2016, 55, 12881-12885.

${ }^{32}$ Hoang, G. T.; Reddy, V. J.; Nguyen, H. H. K.; Douglas, C. J. Insertion of an Alkene into an Ester: Intramolecular Oxyacylation Reaction of Alkenes through Acyl C-O Bond Activation. Angew. Chem., Int. Ed. 2011, 50, 1882-1884.

${ }^{33}$ Gensini, M.; Altamura, M.; Dimoulas, T.; Fedi, V.; Giannotti, D.; Giuliani, S.; Guidi, A.; Harmat, N. J. S.; Meini, S.; Nannicini, R.; Pasqui, F.; Tramontana, M.; Triolo, A.; Maggi, C. A. Modulation on C- and N-Terminal Moieties of a Series of Potent and Selective Linear Tachykinin NK2 Receptor Antagonists ChemMedChem 2010, 5, 65-78.

${ }^{34}$ Touney, E. E.; Foy, N. J.; Pronin, S. V. Catalytic radical-polar crossover reactions of allylic alcohols. J. Am. Chem. Soc. 2018, 140, 16982-16987.

${ }^{35}$ Riaz, M. T.; Pohorilets, I.; Hernandez, J. J.; Rios, J.; Totah, N. I. Preparation of 2-(trimethylsilyl) methyl-2-propen1-ol derivatives by cobalt catalyzed sp2-sp3 coupling. Tetrahedron Lett. 2018, 59, 2809-2812.

${ }^{36}$ Singleton, D. A.; Thomas, A. A. High-precision simultaneous determination of multiple small kinetic isotope effects at natural abundance. J. Am. Chem. Soc.1995, 117, 9357.

${ }^{37}$ Frisch, M. J.; Trucks, G. W.; Schlegel, H. B.; Scuseria, G. E.; Robb, M. A.; Cheeseman, J. R.; Scalmani, G.; Barone, V.; Mennucci, B.; Petersson, G. A.; Nakatsuji, H.; Caricato, M.; Li, X.; Hratchian, H. P.; Izmaylov, A. F.; Bloino, J.; Zheng, G.; Sonnenberg, J. L.; Hada, M.; Ehara, M.; Toyota, K.; Fukuda, R.; Hasegawa, J.; Ishida, M.; 
Nakajima, T.; Honda, Y.; Kitao, O.; Nakai, H.; Vreven, T.; Montgomery, J. A.; Peralta, J. E.; Ogliaro, F.; Bearpark, M.; Heyd, J. J.; Brothers, E.; Kudin, K. N.; Staroverov, V. N.; Kobayashi, R.; Normand, J.; Raghavachari, K.;

Rendell, A.; Burant, J. C.; Iyengar, S. S.; Tomasi, J.; Cossi, M.; Rega, N.; Millam, J. M.; Klene, M.; Knox, J. E.; Cross, J. B.; Bakken, V.; Adamo, C.; Jaramillo, J.; Gomperts, R.; Stratmann, R. E.; Yazyev, O.; Austin, A. J.;

Cammi, R.; Pomelli, C.; Ochterski, J. W.; Martin, R. L.; Morokuma, K.; Zakrzewski, V. G.; Voth, G. A.; Salvador, P.; Dannenberg, J. J.; Dapprich, S.; Daniels, A. D.; Farkas; Foresman, J. B.; Ortiz, J. V.; Cioslowski, J.; Fox, D. J. Gaussian 09; Gaussian Inc.: Wallingford, CT, 2009.

${ }^{38}$ Zhao, Y.; Truhlar, D. G. The M06 suite of density functionals for main group thermochemistry, thermochemical kinetics, noncovalent interactions, excited states, and transition elements: two new functionals and systematic testing of four M06-class functionals and 12 other functionals. Theor. Chem. Acc. 2008, 120, 215.

${ }^{39}$ Anderson, T.L.; Kwan, E.E. PyQuiver 2016, www.github.com/ekwan/PyQuiver.

${ }^{40}$ CrysAlisPro; Rigaku OD, The Woodlands, TX, 2015.

${ }^{41}$ Sheldrick, G. M. Acta Crystallogr. 2015, A71, 3.

${ }^{42}$ Müller, P. Practical suggestions for better crystal structures. Crystallogr. Rev. 2009, 15, 57. 